\title{
IntechOpen
}

\section{Advances in \\ Endoscopic Surgery}

Edited by Cornel Iancu

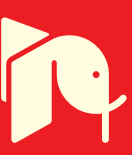





\section{ADVANCES IN \\ ENDOSCOPIC SURGERY}

Edited by Cornel lancu 


\section{Advances in Endoscopic Surgery}

http://dx.doi.org/10.5772/1774

Edited by Cornel lancu

\section{Contributors}

Ivana Pajic-Penavic, Marco Berlucchi, Michele Sessa, Piero Nicolai, Barbara Pedruzzi, Rohan R Walvekar, Waleed F Ezzat, Katsuhiro Yorioka, Marwan Najjar, Ali Turkmani, David W.J. Côté, Erin Wright, Kin Fah Chin, Peter Grunert, Joachim Oertel, Durval Moraes De Carvalho, Durval M. Carvalho Jr., Francisco Lima, Michele Molinari, Karim Mohamed Eltawil, Miroslav Andric, Giuseppe Paolo Ferulano, Saverio Dilillo, Michele D’Ambra, Ruggero Lionetti, Piero Di Silverio, Stefano Capasso, Domenico Pelaggi, Michele Rutigliano, Georgios Giourgos, Alessandro Pusateri, Elina Matti, Fabio Pagella, Claudio Coco, Gianluca Rizzo, Alessandro Verbo, Claudio Mattana, Donato Paolo Pafundi, Alberto Manno, Jan Betka, Martin Chovanec, Eduard Zverina, Lukes Petr, Jan Kluh, Jiri Skrivan, Zdenek Fik, Oliver Profant, Filippo Tosato, Salvatore Marano, Stefano Mattacchione, Leoluca Vasapollo, Giulia Paltrinieri, Barbara Luongo, Valentina Mingarelli, Benedikt Josef Folz, Claus-Günther Konnerth, John Calleary, Ratislav Hejj, Marie McNulty, Emmanuel Jouanneau, Mahmoud Messerer, Moncef Berhouma, Sumeet M Anand, Saul Frenkiel, Rickul Varshney, Kris R. Jatana

\section{(c) The Editor(s) and the Author(s) 2011}

The moral rights of the and the author(s) have been asserted. All rights to the book as a whole are reserved by INTECH. The book as a whole (compilation) cannot be reproduced, distributed or used for commercial or non-commercial purposes without INTECH's written permission. Enquiries concerning the use of the book should be directed to INTECH rights and permissions department (permissions@intechopen.com).

Violations are liable to prosecution under the governing Copyright Law.

\section{(cc) BY}

Individual chapters of this publication are distributed under the terms of the Creative Commons Attribution 3.0 Unported License which permits commercial use, distribution and reproduction of the individual chapters, provided the original author(s) and source publication are appropriately acknowledged. If so indicated, certain images may not be included under the Creative Commons license. In such cases users will need to obtain permission from the license holder to reproduce the material. More details and guidelines concerning content reuse and adaptation can be foundat http://www.intechopen.com/copyright-policy.html.

\section{Notice}

Statements and opinions expressed in the chapters are these of the individual contributors and not necessarily those of the editors or publisher. No responsibility is accepted for the accuracy of information contained in the published chapters. The publisher assumes no responsibility for any damage or injury to persons or property arising out of the use of any materials, instructions, methods or ideas contained in the book.

First published in Croatia, 2011 by INTECH d.o.o.

eBook (PDF) Published by IN TECH d.o.o.

Place and year of publication of eBook (PDF): Rijeka, 2019.

IntechOpen is the global imprint of IN TECH d.o.o.

Printed in Croatia

Legal deposit, Croatia: National and University Library in Zagreb

Additional hard and PDF copies can be obtained from orders@intechopen.com

Advances in Endoscopic Surgery

Edited by Cornel lancu

p. $\mathrm{cm}$.

ISBN 978-953-307-717-8

eBook (PDF) ISBN 978-953-51-6586-6 


\section{We are IntechOpen, \\ the world's leading publisher of Open Access books}

Built by scientists, for scientists

\section{$4,100+$}

Open access books available

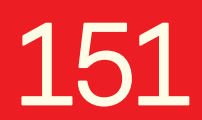

Countries delivered to
$116,000+$

International authors and editors
$120 \mathrm{M}+$

Downloads

Our authors are among the

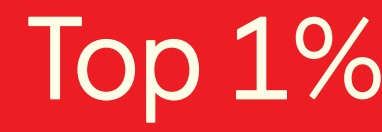

most cited scientists

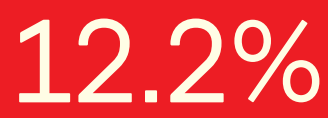

Contributors from top 500 universities

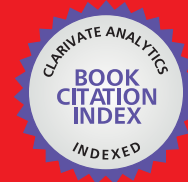

WEB OF SCIENCE ${ }^{\mathrm{TM}}$

Selection of our books indexed in the Book Citation Index in Web of Science ${ }^{\mathrm{TM}}$ Core Collection (BKCI)

Interested in publishing with us?

Contact book.department@intechopen.com

Numbers displayed above are based on latest data collected.

For more information visit www.intechopen.com

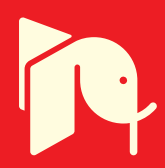





\section{Meet the editor}

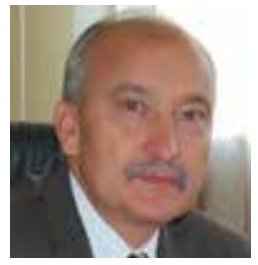

Cornel Iancu, professor and chairman of the of 3rd Department of Surgery at "Iuliu Hatieganu" University of Medicine Cluj-Napoca is a pioneer in laparoscopic surgery. A highly respected surgeon, teacher and researcher, he is one of the leading authorities on advanced laparoscopic surgery in Romania. Beside laparoscopic cholecystectomy and anti-reflux surgery Dr. Iancu has extensive experience in performing advanced laparoscopic procedures for gastric tumors, colo-rectal malignancies, pancreatic and liver tumors and complicated hydatic disease. Among research in the field of digestive surgical oncology and endoscopic surgery, Dr. Iancu's research was also focused in the area of nanotechnology, bio-nano-medical research and treatment by bioconjugated nanomaterials with priority on the selective laser ablation and optical damage of the pancreatic and liver cancer cells mediated by antibodies functionalized nanoparticles. 



\section{Contents}

\section{Preface XIII}

Part 1 Endoscopy of the Paranasal Sinuses and the Skull Base 1

Chapter 1 Endoscopic Endonasal Skull Base Surgery:

Current State of the Art and Future Trends 3

Jouanneau Emmanuel, Messerer Mahmoud

and Berhouma Moncef

Chapter 2 Endoscopic Surgery of Maxillary Sinuses

in Oral Surgery and Implantology 39

Miroslav Andrić

Chapter 3 Objective Outcomes

in Endoscopic Sinus Surgery $\mathbf{5 7}$

David W.J. Côté and Erin D. Wright

Chapter 4 Endoscopy in Nasopharyngeal Adenoid Surgery $\mathbf{7 1}$

W. F. Ezzat

Chapter 5 Endoscopically Guided Balloon Dilatation of

Recurrent Choanal Stenosis 89

B.J. Folz and C.-G. Konnerth

Chapter 6 Sialendoscopy: Endoscopic Approach to Benign Salivary Gland Diseases 101

Meghan Wilson, Kyle McMullen

and Rohan R. Walvekar

Chapter 7 Virtual Endoscopy of the Nasal Cavity and the Paranasal Sinuses 117

Sumeet Anand, Rickul Varshney and Saul Frenkiel

Chapter 8 Evolution of the Adenoidectomy

in the Endoscopic Era 131

Fabio Pagella, Alessandro Pusateri,

Georgios Giourgos and Elina Matti 
Part 2 Endoscopy of the Central Nervous System 155

Chapter 9 Endoscopy in Intracranial Pathology 157

Marwan Najjar and Ali Turkmani

Chapter 10 Technical and Clinical Evolution of

Modern Neuroendoscopy 175

P. Grunert and J. Oertel

Chapter 11 Minimally Invasive Endoscopic and Endoscopy-Assisted Microsurgery of Vestibular Schwannoma 191

Betka Jan, Chovanec Martin, Zverina Eduard, Profant Oliver, Lukes Petr, Skrivan Jiri, Kluh Jan and Fik Zdenek

Part 3 Endoscopy in Ophtalmology 217

Chapter 12 Ocular Endoscopy 219

Durval Moraes de Carvalho, Francisco Eduardo Lima

and Durval Moraes de Carvalho Jr

\section{Part 4 Digestive Endoscopy 235}

Chapter 13 Laparoscopy in Diagnosis and Treatment of Small Bowel Diseases 237

Coco Claudio, Rizzo Gianluca, Verbo Alessandro, Mattana Claudio, Pafundi Donato Paolo and Manno Alberto

Chapter 14 Surgical Treatment of Gastroesophageal Reflux Disease 259

Filippo Tosato, Salvatore Marano, Stefano Mattacchione, Barbara Luongo, Giulia Paltrinieri, Valentina Mingarelli and Leoluca Vasapollo

Chapter 15 Laparoscopic One-Stage vs Endoscopic Plus Laparoscopic Management of Common Bile Duct Stones - A Prospective Randomized Study 291 Giuseppe P. Ferulano, Saverio Dilillo, Michele D'Ambra, Ruggero Lionetti, Piero Di Silverio, Stefano Capasso, Domenico Pelaggi and Michele Rutigliano

Chapter 16 Endoscopic Ultrasound for Solid and Cystic Neoplasms of the Pancreas 307

Karim M. Eltawil and Michele Molinari

Part 5 Urogynecological Endoscopy 327

Chapter 17 Urology: The Home of Endoscopy 329

Rastislav Hejj, Marie McNulty and John G. Calleary 
Part 6 Pediatric Endoscopy 343

Chapter 18 Diagnostic and Therapeutic Sinonasal Endoscopy in Pediatric Patients $\mathbf{3 4 5}$

Marco Berlucchi, Barbara Pedruzzi, Michele Sessa and Piero Nicolai

Chapter 19 Endoscopy in the Evaluation

and Management of the Pediatric Airway $\mathbf{3 7 5}$

Kris R. Jatana and Jeffrey C. Rastatter

Part 7 Concepts in Endoscopic Surgery 399

Chapter 20 Laparoscopic Surgery: An Almost Scarless Approach 401

Peng Soon Koh and Kin Fah Chin

Chapter 21 Endoscopic Monitoring of Postoperative Sinonasal Mucosa Wounds Healing 419 Ivana Pajić-Penavić

Chapter 22 Microbial Contamination of Suction Tubes Attached to Suction Instrument and Its Preventive Methods 437 Katsuhiro Yorioka and Shigeharu Oie 



\section{Preface}

The efforts to treat diseases through the human body's orificies and cavities date back to antiquity when Hippocrates (460-374 BC) used a speculum to inspect and treat an inflammatory lesion of the rectum. One major limitation of these attempts was represented by poor illumination. Only in the 9th century did Arabian physician Abukasim developed a method of cervix inspection using a mirror to reflect light. Later, Leonardo da Vinci used a lense to direct sun rays into the nasal cavity, but the major advance came in 1805 when Bozzini, a physician from Frankfurt introduced the first light conductor to visually examine the interior of the urethra.

Jacobaeus, a Swedish physician (1910) used a cytoscope to examine the abdominal cavity in humans after first insuflating the abdominal cavity a techinque that was termed "laparoscopy". Subsequently, this technique has evolved, the instruments became smaller, more flexible, cold light illumination was added favoring the transmission of abundant light with minimal heat and consequently the obtainance of sharp and clear images. Another significant progress in the field of operative endoscopy was the introduction of bipolar electrocoagulation (1968) and the invention of computer-chip video camera in 1986.

Since then, surgeons from various domains have become fascinated by endoscopy with its very low complication rates, high diagnostic yields and the possibility to perform a large variety of therapeutic procedures. Therefore, the number and diversity of surgical endoscopic procedures have proliferated enormously over last 30 years. Currently there are several "standard" procedures that are used at work on daily basis. There are also few recognized "advanced procedures" practiced by only a small amount of surgeons who require an increased learning curve and intensive training.

This book is a comprehensive didactic material covering all aspects of both standard and advanced endoscopic techniques applied in surgical diseases starting from diagnosis and ending with therapy. 
$\times$ Preface

We intend to provide our readers with easy-to-use information on latest topics in surgical endoscopy provided by over 30 distinguished experts in each specific area from all over the world.

Cornel Iancu, MD, PhD

Professor of General Surgery, 3rd Department of Surgery at

"Iuliu Hatieganu" University of Medicine Cluj-Napoca,

Romania 




\section{Part 1}

Endoscopy of the Paranasal Sinuses and the Skull Base 



\title{
Endoscopic Endonasal Skull Base Surgery: Current State of the Art and Future Trends
}

\author{
Jouanneau Emmanuel, Messerer Mahmoud \\ and Berhouma Moncef \\ Department of Neurosurgery \\ Skull Base Surgery Unit Pierre Wertheimer \\ Neurological and Neurosurgical Hospital \\ Lyon \\ France
}

\section{Introduction}

Endoscopic endonasal skull base surgery (EESBS) is undergoing a remarkable evolution as in the last two decades it shifted from pituitary surgery to a myriad of approaches extending from the cribriform plate to $\mathrm{C} 2$ and laterally to the petrous apex and to the infratemporal fossa. The collaboration with ENT surgeons, technological advances in the field of instrumentation and endoscopic systems, a better comprehension of the skull base anatomy as seen from below and recent innovations in reconstruction techniques have led to obvious improvements in the management of cranial base lesions. EESBS is now becoming the goldstandard approach to the sellar, retrosellar and clival regions; however, its role in the management of anterior skull base tumors is still debated.

Through their experience of more than 400 endoscopic endonasal skull base procedures, the authors expose their modus operandi and discuss the current controversies as well as future trends.

\section{A brief history of endoscopic procedures in neurosurgery}

EESBS represents the recent meeting of endoscopic techniques, developed mainly by urologists, and pituitary transsphenoidal surgery.

\subsection{First endoscopes}

Endoscopic explorations of hollow organs appeared during the 19th century. A German physician, Philipp Bozzini (1773-1809), is considered as the inventor of the first endoscope which he named "Lichtleiter" (Figure 1, left). The latter was very difficult to handle and painful for the patients. Jean Desormeaux (1815-1894), a renowned French urologist, improved Bozzini's Lichtleiter using all the advances made during the second half of the $19^{\text {th }}$ century in the fields of lighting (Castelnuovo et al., 2010a; Leger, 2004). The development of 
optic lenses led to the ergonomy optimization and the manoeuvrability improvement of endoscopes used mainly by urologists such as Max Nitze (1848-1906) (Herr, 2006; Litynski, 1999; Rathert, 1967). Paradoxically, the first ventricular neuroendoscopy with cauterization of choroid plexuses has been performed by an American urologist, Victor Darwin Lespinasse (1878-1946) (Grant JA, 1996), followed during the first half of the 20th century by Walter Dandy, one of the leading pioneers of neurosurgery (Dandy, 1926, 1932). Numerous improvements of optical systems made during the first half of the $20^{\text {th }}$ century led to the modernization of endoscopic procedures. ENT surgeons like Messerklinger, Draf and Stammberger developed these endoscopic techniques in the management of paranasal sinuses diseases (Draf, 1973; Reuter, 2000; Wigand, 1981). After being used initially for cerebral third ventriculostomy, endoscopy changed dramatically the approach to the pituitary region at the end of the last century and initiated neurosurgeons to endoscopic endonasal skull base surgery.
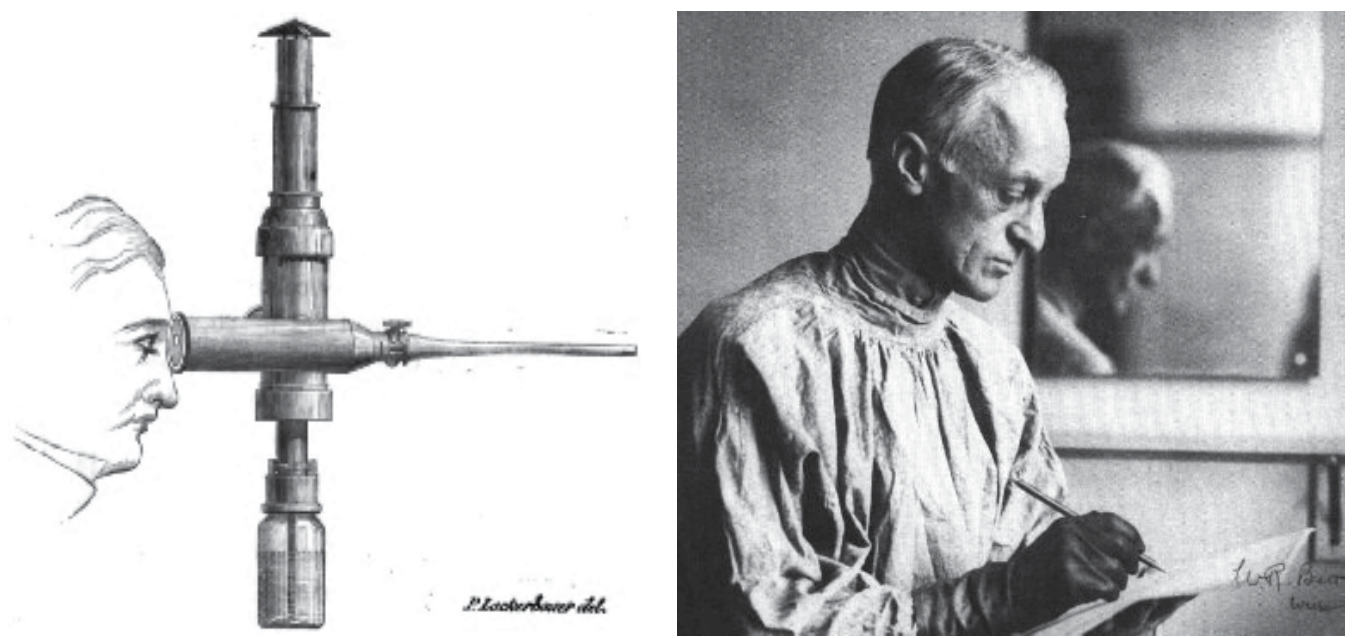

Fig. 1. Left: The "Lichtleiter", first endoscope designed by Philipp Bozzini (1773-1809)

Right: Harvey Cushing (1869-1939), the pioneer founder of the transsphenoidal pituitary surgery

\subsection{Pituitary surgery}

Modern pituitary surgery began with Harvey Cushing (Figure 1, right) during the early years of the $20^{\text {th }}$ century, with the development of the sublabial transsphenoidal approach (Figure 2) and the use of a frontal light (Cushing, 1909). The initial poor results of this technique and the reduced visualization of the sella led to abandon the transsphenoidal route in favor of the transcranial approaches (Caton, 1893; Landolt, 2001; Lindholm, 2007; Pollock et al., 2003).

Gerard Guiot induced a rebirth of the trans-sphenoidal surgery in the middle of the last century with the introduction of the peroperative fluoroscopy and he first used an endoscope within the sella (Guiot et al., 1963a, 1963b). Later, Jules Hardy normalized the current pituitary surgery practice with the use of the operative microscope (Hardy, 2010; Liu et al., 2001). The application of endoscopic techniques in pituitary surgery and then in skull 
base surgery started progressively in the late 1980's before experiencing an exponential success during the last decade (Carrau et al., 2001).
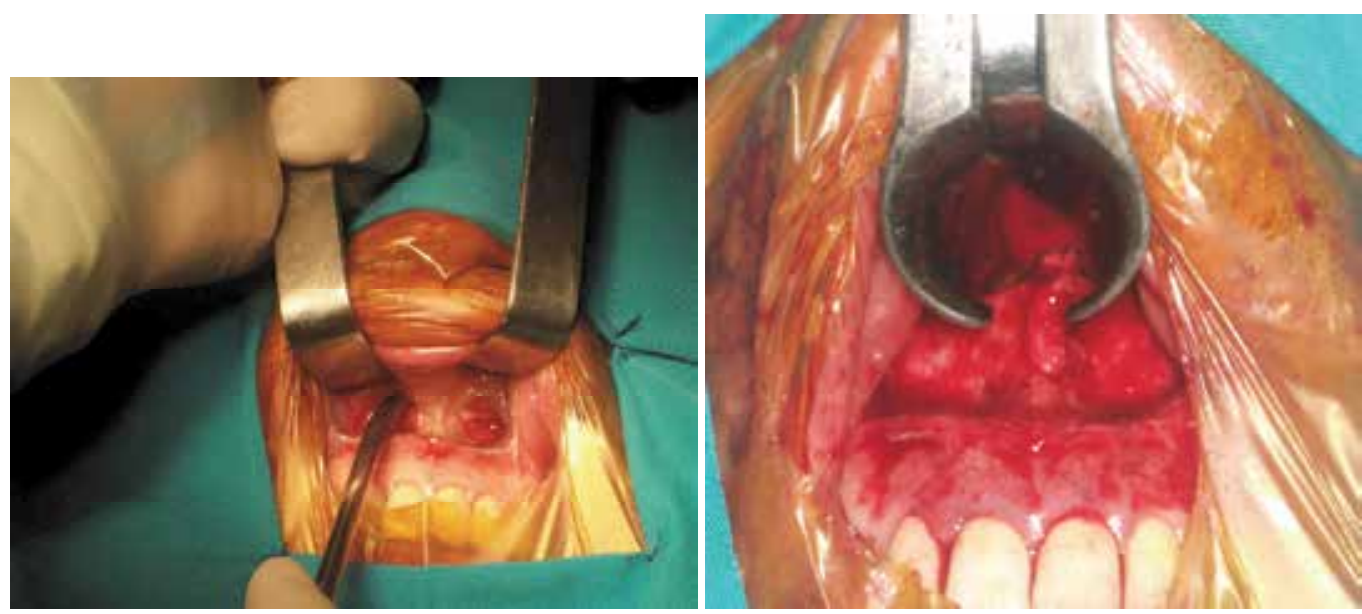

Fig. 2. Classical sublabial trans-sphenoidal microsurgical approach for pituitary adenomas.

\section{Endoscopic endonasal anatomy of the skull base}

The point of this section is to give the reader the main anatomical landmarks the surgeon has to deal with when using endonasal approach to the skull base (for more detailed and specific anatomical considerations, readers are invited to consult the references list at the end of this chapter).

\subsection{Nasal fossa}

The nasal cavity is marked medially by a rigid structure, the septum (S) (association of the perpendicular plate of the ethmoidal bone on the superior aspect, the vomer on the inferior aspect and anteriorly the quadrangular cartilage), and laterally by three longitudinal folds named the turbinates or conchae. Posteriorly both choanae (Ch) give access to the cavum. Placed immediately at the entry and below, the first seen turbinate is the inferior turbinate (IT) attached to the ethmoidal bone. Up, above and more posterior is the middle turbinate (MT), a part of the ethmoid (Figure 3, left).

The head of the middle turbinate is free and may be pneumatised (concha bullosa) and then narrows the surgical corridor (in such cases its complete or partial resection may be required). Septal deviation or spines can also complicate the surgical approach and have to be studied scrupulously on the preoperative imaging. During the nasal step, the middle turbinate has to be pushed gently aside laterally placing a spatula up to its posterior part (Figure 3, right). Caution will be taken when mobilizing those turbinates to avoid any skull base fracture and CFS leaks. Thereafter, the superior turbinate and medially the sphenoid ostium will appear (Figure 3). This key landmark is the entry point to the sphenoid sinus, placed about one and a half centimeter above the choanae (Ch). A branch of the sphenopalatine artery, the posterior nasal artery (dotted red arrow) passes just below to the sphenoid ostium to join the septum and may be cauterized to avoid postoperative nasal hemorrhages. 


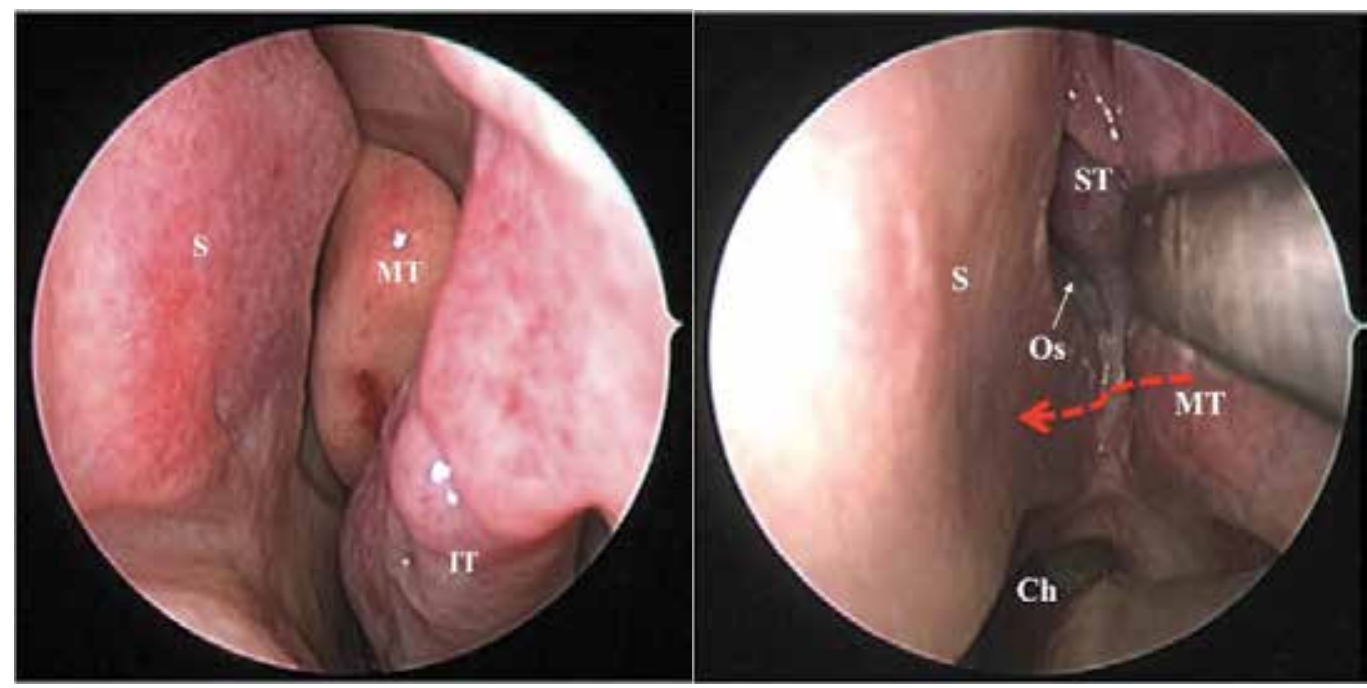

Fig. 3. Nasal step of a left endoscopic endonasal approach: Middle (MT) and inferior (IT) turbinates with the nasal septum (S) on the midline. Note the sphenoid ostium (Os) boarded by the posterior nasal branch of the sphenopalatine artery and superiorly the superior turbinate (ST)

\subsection{Sphenoid sinus}

The degrees of pneumatization of the sphenoid sinus and its septa have numerous variations which have to be studied on the preoperative MRI and/or CT-Scan. More often, the sphenoid sinus is largely pneumatized (sellar type). After removing the septa, the following landmarks are identified (Figure 4):

- $\quad$ Up and anteriorly the tuberculum of the sella (TS) and the planum (Planum)

- Centrally, the sella and the pituitary fossa.

- Laterally and up the optic nerve, paraclinoid carotid processes (C5) and the medial (OCR med) and lateral (OCR lat) opticocarotid recesses.

- Laterally, the anterior wall of the cavernous sinus and below the paraclival carotid processes (C3).

- Below the clivus corresponding posteriorly to the brainstem.

\subsection{Perisellar anatomy}

\subsubsection{Parasellar compartment}

Opening up laterally both sphenoid recesses gives access to the superior orbital fissure and straight below to the infratemporal fossa (Figure 5). After removing the bone, passing laterally and below to the cavernous sinus, the surgeon can get access to Meckel's cave with the V2 and V3 branches of the trigeminal nerve (Alfieri et al., 2001a, 2001b; RiveraSerrano et al., 2010).

An important key for safer surgery of this area is to perfectly control the internal carotid artery (ICA). Within a sphenoid sinus of sellar type, the paraclival segment C3 is usually directly visible into the sinus just below and laterally to the sella as shown in figure 5 . It is much more difficult to individualize the $\mathrm{C} 2$ segment of the ICA which can be retrieved following the vidian nerve in its canal (Figure 5, right) (Prevedello et al., 2010). 


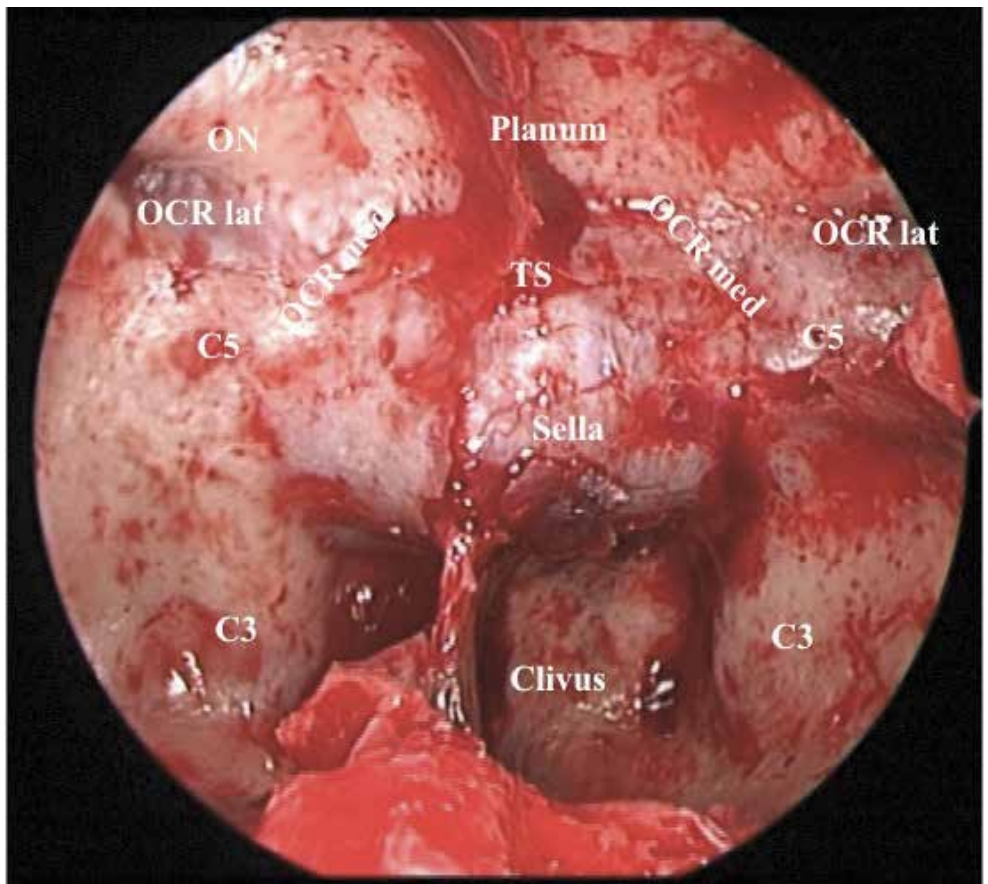

Fig. 4. Endoscopic operative view of the sphenoid sinus. Internal carotid arteries (paraclival segment C3, paraclinoid segment C5); right optic nerve: ON; Opticocarotid recesses medial (OCR med) and lateral (OCR lat).
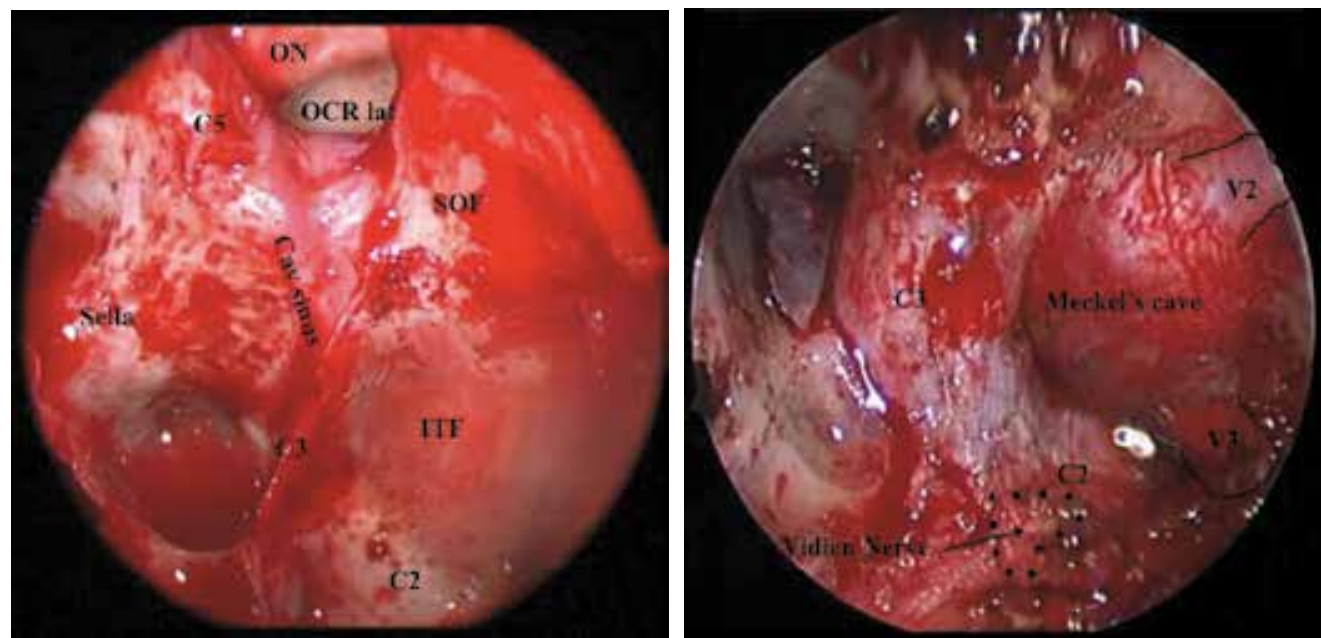

Fig. 5. Operative views of the parasellar compartment after opening the lateral sphenoid recesses.

Left (bone wall preserved): Optic nerve $(\mathrm{ON})$; internal carotid artery (ICA) paraclinoid segment C5, paraclival segment C3, petrous segment C2; lateral opticocarotid recess (OCR lat); superior orbital fissure (SOF); infratemporal fossa (ITF); cavernous sinus (Cav sinus). Right: the bone has been removed especially on the ITF exposing the Meckel's cave and the branches V2 and V3 of the trigeminal nerve. 


\subsubsection{Transplanum approach anatomy}

Opening the sella with its tuberculum and the planum provides an access to the suprasellar cistern and the optic tract. The C6 segment of the ICA and the ON will be seen laterally as well as the pituitary stalk posteriorly. Above the optic chiasm are the anterior cerebral arteries and on the midline the anterior communicating artery (Figure 6).
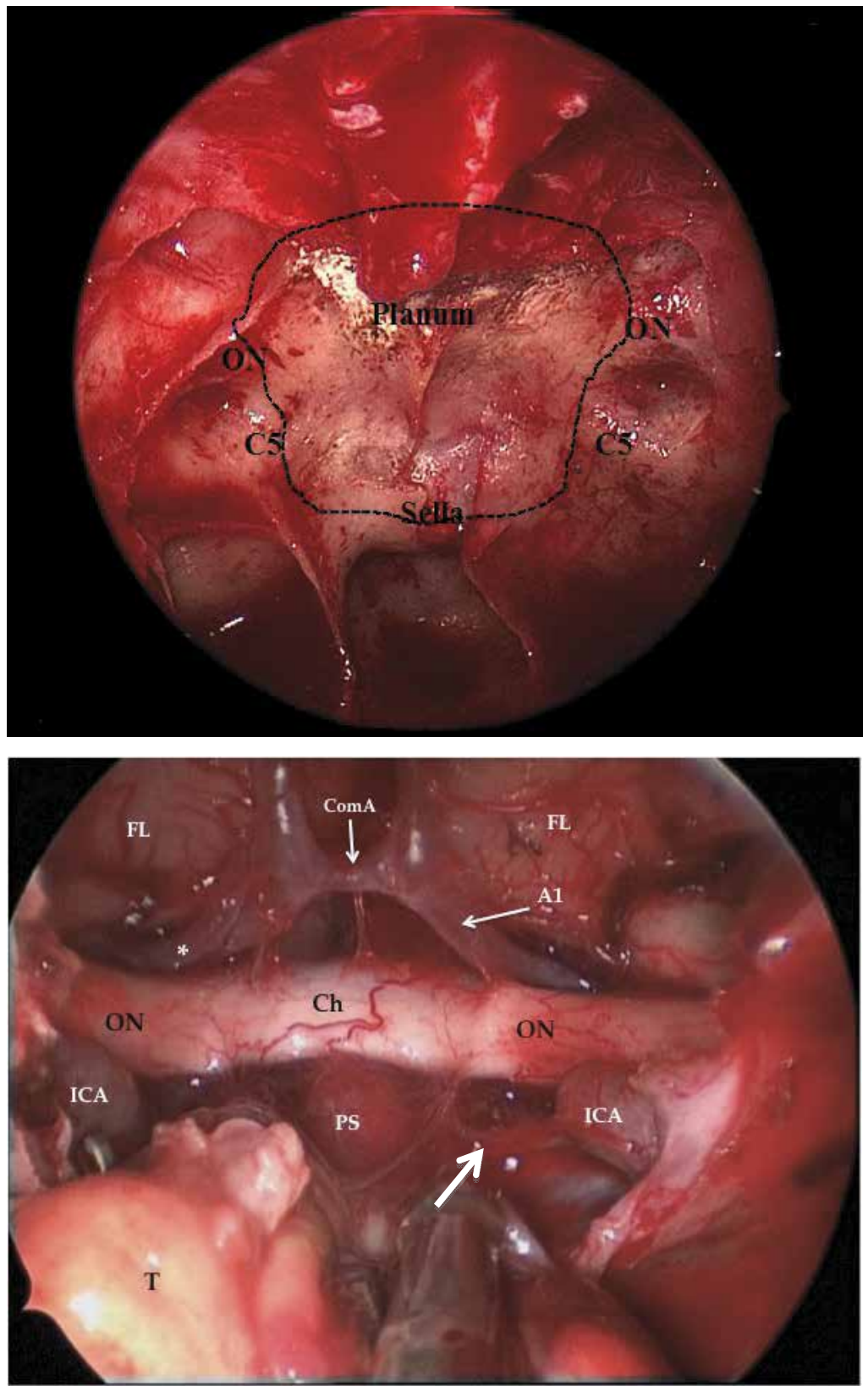

Fig. 6. Endoscopic endonasal resection of a tuberculum sellae meningioma through a transplanum transtubercular approach. 
Up: Sphenoid sinus and bone aperture (dotted line). Internal carotid artery (ICA) C5; optic nerve $(\mathrm{ON})$.

Down: Intradural dissection: meningioma $(\mathrm{T})$; optic nerves $(\mathrm{ON})$, the chiasm $(\mathrm{Ch})$, the pituitary stalk (PS), the internal carotid arteries (ICA), the A1 artery (A1) and Heubner artery $\left({ }^{*}\right)$, the anterior communicating artery (ComA) and frontal lobes (FL). A useful mark to find the PS when removing a tumor is to follow the ICA and until after the superior hypophyseal arteries (SHA, white arrow) which always lead to the PS.

\subsubsection{Transclival - transodontoid approach anatomy}

Going through the choanae and below to the sphenoid sinus (white dotted arrow, figure 7 left) gives access to the cavum. An important landmark is the Eustachian tube as the ICA passes laterally very closely (Alfieri, 2002). The mucosa can be opened as a flap that can be placed in the cavum during the surgery. After removing the lower third of the clivus bone as well as the anterior arch of $\mathrm{C} 1$ and the odontoid process, an intradural and anterior access of the brainstem is easily obtained, making the removal of lesions located in this critical area possible (Cavallo et al., 2007).

Drawing a line from the nasal bone to the palatine one (Kassam's line, orange line, figure 7 left) gives the surgeon a good evaluation of how far he can go inferiorly by an endonasal approach (usually odontoid process and upper part of C2).
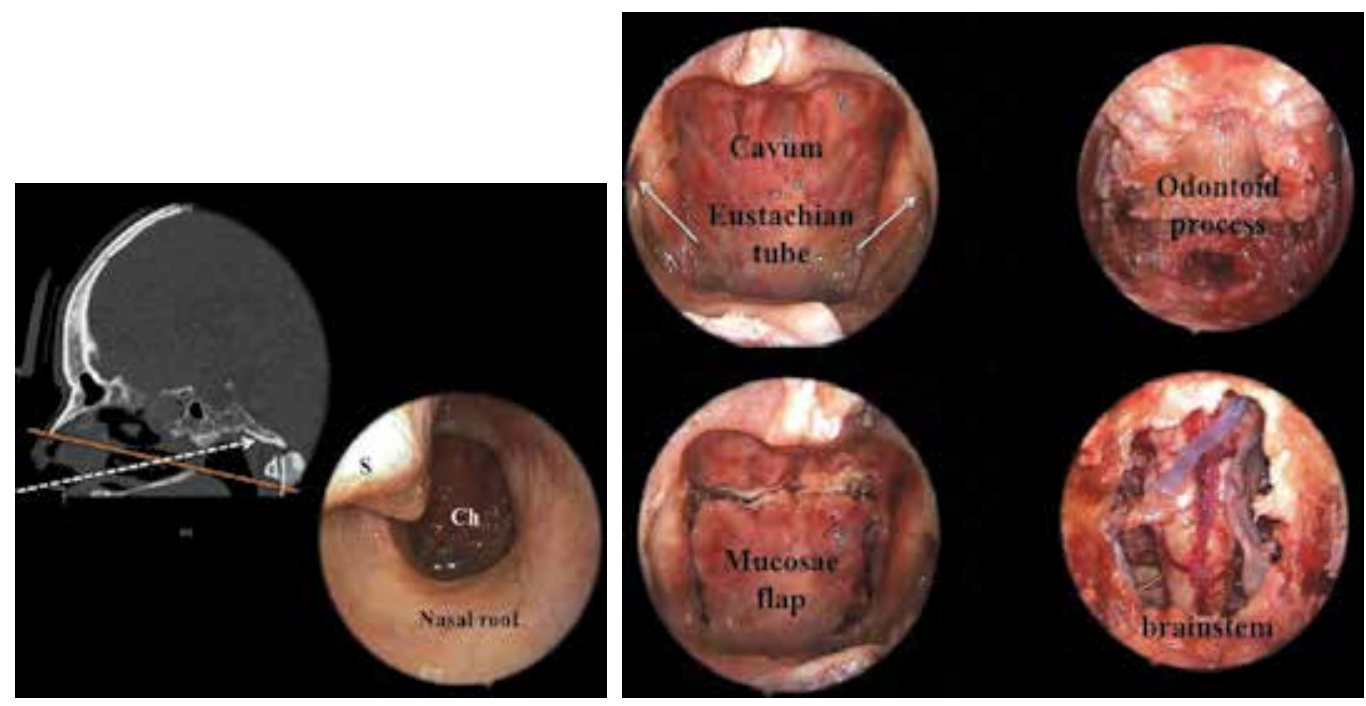

Fig. 7. Anatomic views of the endonasal approach to the clivus and cranio-cervical junction.

\section{Operative characteristics, anesthetic considerations and instrumentation}

\subsection{Preoperative protocol}

Preoperative craniofacial CT-Scan and MRI (Gardner et al., 2008) are systematically done as well as endocrinological assessment for sellar or perisellar tumors and ophthalmological examination. For cavernous sinus or Meckel's cave tumors, a cerebral angiography with occlusion tests is mandatory to avoid any unacceptable peroperative arterial occlusion in case of vascular injury during the surgery (vascular occlusion, clipping alone or associated with extra-intracranial anastomosis). 
The preoperative protocol includes polyvidone shower and nasal disinfection with polyvidone cream, the day before surgery, and also on the morning of surgery. For extended approaches, vaccines against pneumococcus, meningococcus and haemophilus are usually used to prevent postoperative meningitis risk, whenever possible.

\subsection{Anesthetic considerations}

Specific considerations for anesthesia have to be tailored to endonasal endoscopic surgery, as well as to the extent of the approach (Ramachandran et al., 2011).

Under general anesthesia, an orotracheal intubation is used, with the tube placed on the left side. Invasive blood pressure monitoring is discussed with the surgeon, depending on the invasiveness of the approach and the vascularization of the tumor.

A bloodless surgical field is of paramount importance to the neurosurgeon. Many means help to achieve this goal: preoperative planning with rigorous study of the vasculature of the tumor, which may be embolized, total intravenous anesthesia (TIVA) with propofol and short-acting opioids, the use of sympathetic blockers as hypotensive agents, the use of diluted adrenalin-soaked pledgets before endoscope introduction.

In selected cases of extended endoscopic approaches, direct submucosal infiltration with diluted adrenalin (or other vasoconstrictor) may be useful to minimize peroperative bleeding especially if a nasoseptal flap is planned.

The use of peroperative hypopharyngeal packing should be systematic to avoid the pooling of blood and to minimize the risk of postoperative vomiting related to intragastric blood accumulation.

\subsection{Dedicated instrumentation and operative room organization}

Our endonasal endoscopic technique is derived from the one described by Jho (Jho \& Carrau, 1997) and Cappabianca (Cappabianca et al. 1998).

Zero, $30^{\circ}$ and more rarely $45^{\circ}, 4 \mathrm{~mm}$ diameter rigid endoscopes are currently used during the surgery, the short one $(18 \mathrm{~cm})$ for the nasal step and the longer one $(30 \mathrm{~cm})$ during the tumor removal stage. For pediatric patients or for narrowed nostrils, small endoscopes (2.7 $\mathrm{mm}$ of diameter) have been designed.

The endoscope column is composed of a HD screen and camera, a Xenon light with an automated irrigation system to clean the endoscope during surgery without removing the endoscope.

Kassam, Cappabianca and Frank have designed dedicated sets of instrumentation (Storz®). High-speed bone drills and a mucosal automated shaver are useful. A microprobe Doppler is also used for extended approaches and for some specific pituitary surgery to localize accurately the nasoseptal flap pedicle or to individualize internal carotid arteries.

The neuronavigation system with CT-scan/MRI fusion is used for almost all our surgeries with a major role for recurrent tumors or when extended transbasal approaches have to be performed to remove intradural tumors.

The operative room organization is depicted in figure 8. The patient is in a supine position, the trunk slightly elevated $\left(30^{\circ}\right)$ with the head flexed up and turned to the right side to face the surgeon (figure 9). We prefer to raise both the trunk and the head so that the blood accumulates into the sphenoid sinus, falling down into the cavum during surgery to improve the visualization. This position also enables a better venous drainage from the head and less peroperative venous bleeding. A real supine position is preferred for anterior skull base tumors and a more flexed position when dealing with clival tumors. 
Nevertheless, other teams work with a strict supine position whatever the type of surgery and strictly at the head of the patient. The lateral side of the right thigh is systematically draped in case of fascia lata and/or fat are needed for closure in specific cases of peroperative CSF leaks.

During the patient's positioning and after disinfection with polyvidone iodine, cotton pledgets soaked with diluted adrenalin and lidocain are placed on both nostrils. Those cottons, kept in place several minutes, allow retraction of the middle turbinate and minimize the bleeding during the nasal step.
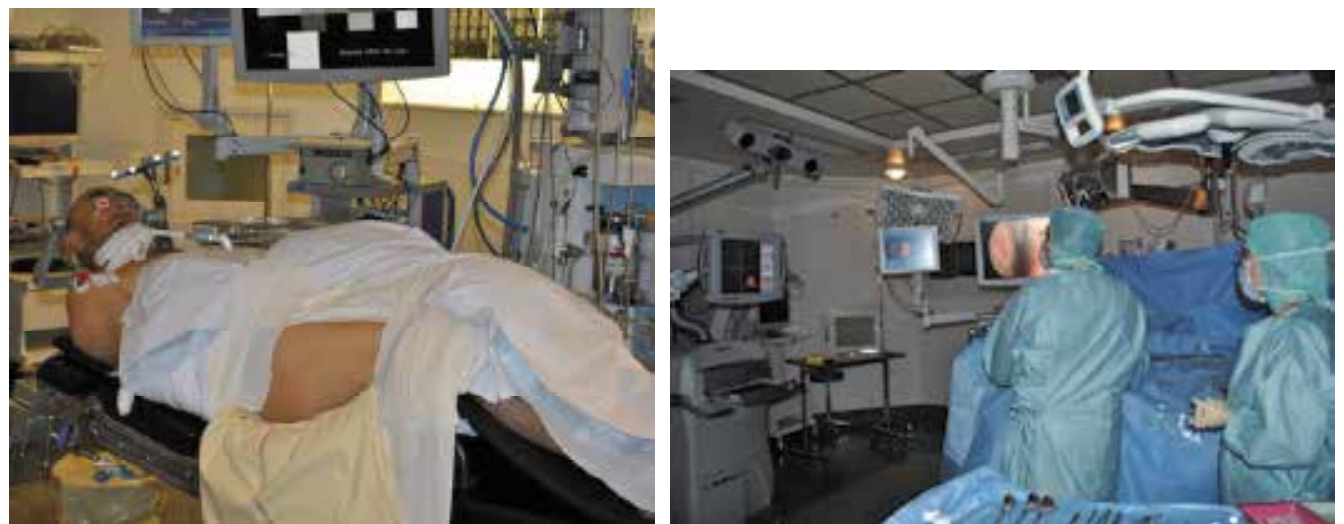

Fig. 8. Left: Patient's positioning. Right: Room organization with the neuronavigation system on the left surgeon's side and on the right the endoscopic screen. The scrub nurse is placed beside the surgeon and the anesthesiologist at the patient's left side.
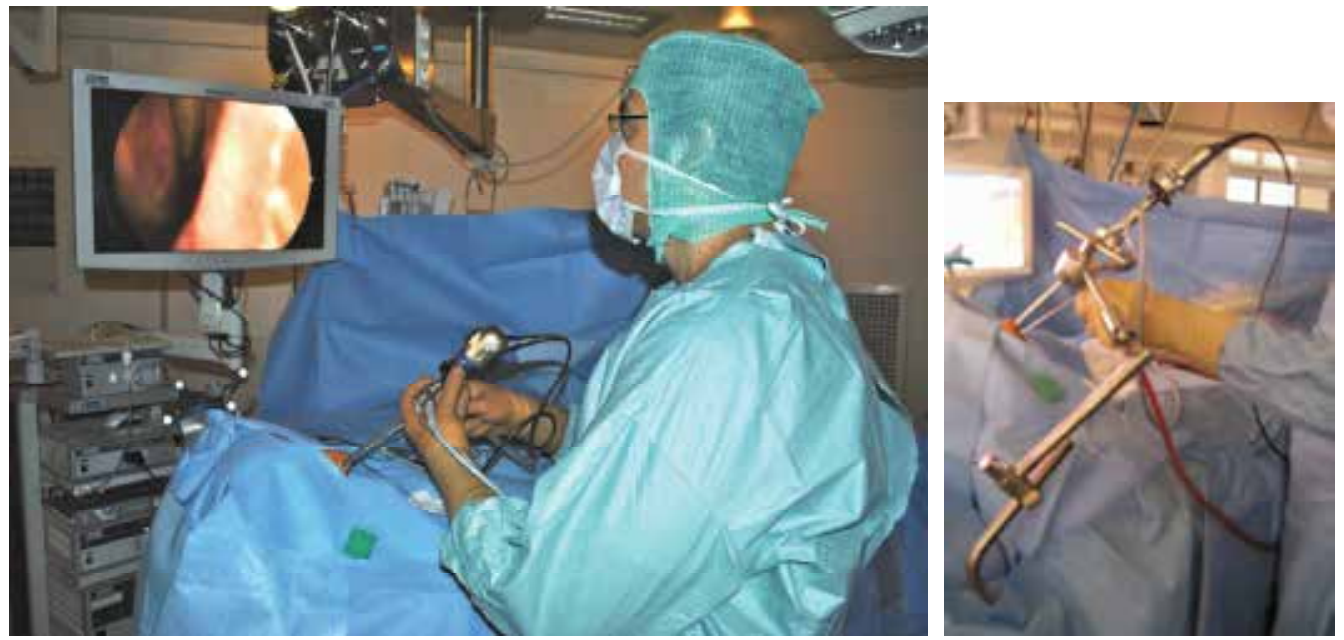

Fig. 9. Endoscope holding. Left: initial naso-sphenoidal step with a handed short endoscope. Right: Skull base opening and further steps with a rigid arm fixed to the operating table to hold the long endoscope. This allows a two hands working as with microscope. The tools are most of time introduced below the endoscope. 


\subsection{Surgical steps}

\subsubsection{Regular procedure for pituitary surgery}

The choice of the side of the nasal fossa to be used is determined by the nasal anatomy (septal deviation, megaturbinate...), lateral extension (contralateral approach to a lateral extension) and size of the tumor (binostril approach for large tumors).

In most cases of pituitary adenomas, a unilateral approach is used except for large tumors. The entire endonasal procedure until the opening of the sellar floor is performed with a hand-held short $0^{\circ}$ angle endoscope $\left(4 \mathrm{~mm}, 18 \mathrm{~cm}\right.$, Karl Storz ${ }^{\circledR}$, Tuttlingen, Germany). The superior and middle turbinates are identified and gently pushed laterally aside. It is very rare to have to remove the middle turbinate except in the case of hypertrophy as in some cases of acromegalic patient. The mucosa from the sphenoidal ostium to the choanae at the base of the vomer is coagulated and thereafter opened up pushing away the vomer until the contralateral ostium appears (figure 10). A large sphenoidotomy is performed by removing the posterior part of the vomer and the sella turcica aperture is done by performing a small bone flap from one cavernous sinus to the other, and from the tuberculum of the sella to the clivus with bone scissors (figure 11). This bone flap, kept in place at the bottom of the sphenoid sinus during the surgery, is used at the end of the surgical procedure to close the sella.

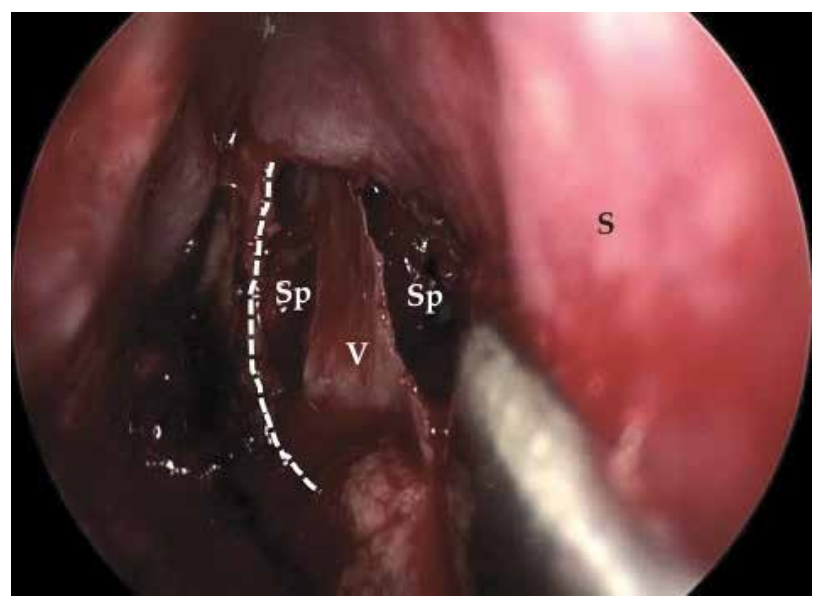

Fig. 10. Anterior sphenoidotomy via a right endonasal route. A $1 \mathrm{~cm}$ mucosa aperture (dotted white line) is done vertically after cauterization. After a sub-mucosa dissection pushing aside the bone septum (S), the vomer (V) appears and has to be removed to achieve the opening of the sphenoid sinus (Sp).

After opening of the dura mater, a long $0^{\circ}$ endoscope $(4 \mathrm{~mm}, 30 \mathrm{~cm})$ fixed on a tablemounted endoscope holder is placed up into the nostril thus allowing the use of both hands for tumor dissection and removal (figure 9, right). The surgical tools are passed through the nose below the endoscope. Other teams work with four hands (neurosurgeon with ENT surgeon), an assistant playing the role of a smart holder. The latter can move freely to help more efficiently the operator without losing time but this needs 2 surgeons of the same level of experience, a condition not available in all institutions.

Adenomas are removed using a piecemeal technique similar to that used with microscopic surgery. In rare cases of firm adenomas, an en bloc removal with an extracapsular dissection may be used. At the end of the procedure, the sellar and suprasellar regions are explored using $0^{\circ}, 30^{\circ}$ and rarely $45^{\circ}$ endoscopes pushed up within the sella turcica (figure 11). 


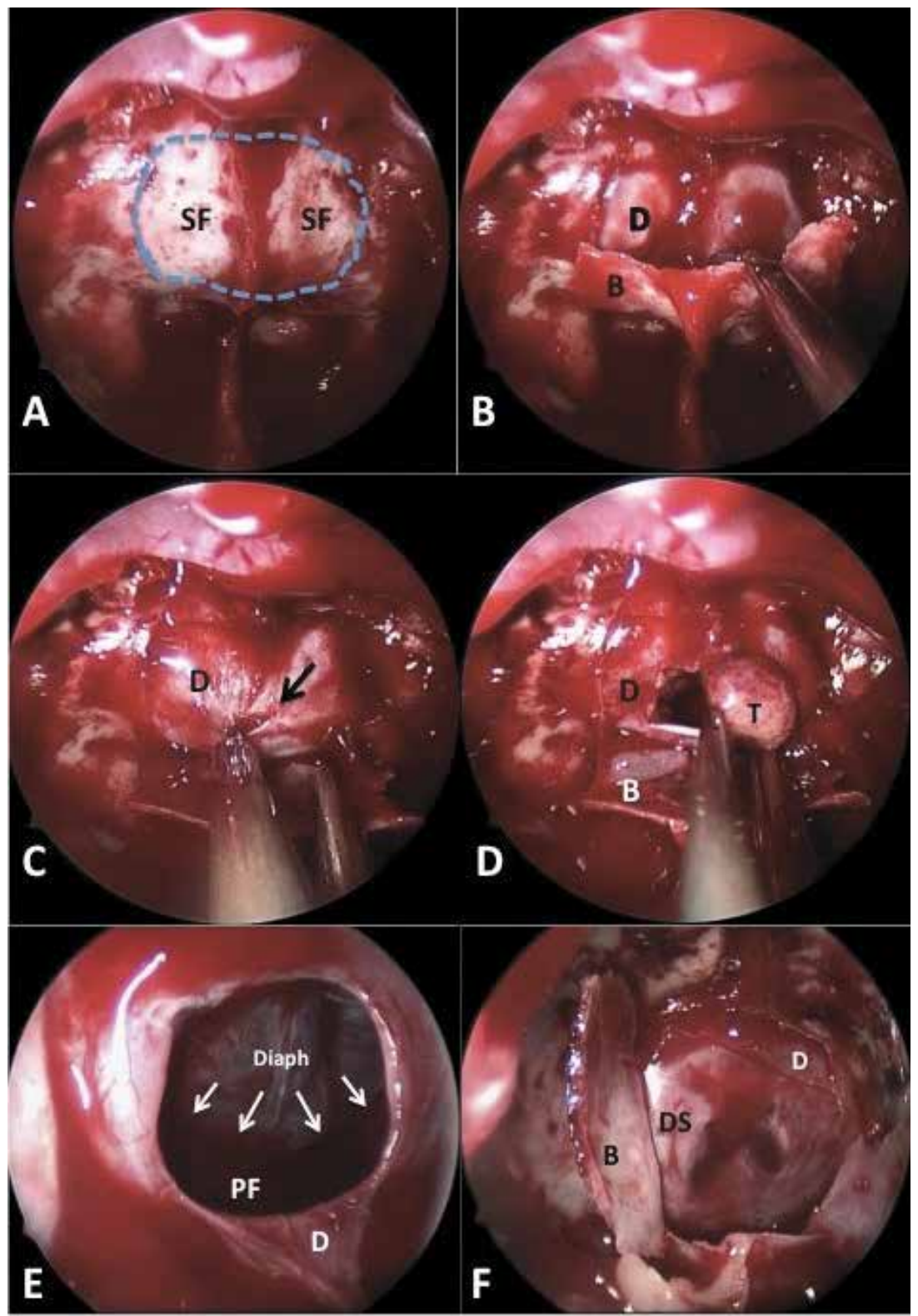

Fig. 11. Steps of the endoscopic endonasal approach to pituitary non-functioning adenoma. A: Exposition of the sphenoid sinus and the sellar floor (SF)

B: Sellar floor craniotomy leaving a bone flap (B) and exposing pituitary dura (D)

C: Dural (D) opening with a blade knife

D: Piece-meal removal of the adenoma $(T)$ with ring curettes

E: End of removal allowing a drop of the sellar diaphragm (Diaph) and verification of the vacuity of the pituitary fossa $(\mathrm{PF})$

F: End of the procedure after verification of the hemostasis, interposition of dural substitute (DS) before closure of the bony sellar floor (B) 
A jugular compression is applied by the anesthesiologist at the patient's neck to detect a CSF leak before closure. Except in the case of a huge fistula, our preference is to avoid any plugging of the sella to facilitate the analysis of postoperative MRI. The closure technique did not differ from the one used during microscopic surgery with a combination of a dura mater substitute placed extradurally and covered by the bone flap embedded in fibrin glue. Should a peroperative CSF leak occurred, additional autologous material such as fat or fascia lata will be placed respectively into the sella and the sphenoid sinus with postoperative CSF lumbar drainage or puncture performed. The repositioning of the middle turbinate without any nasal packing ends the procedure.

\subsubsection{Extended approaches}

For anterior skull base tumors, a mucosa nasoseptal flap pediculated on the sphenopalatine artery or its posterior nasal branches is usually prepared and pushed down into the cavum during the surgery (Hadad et al., 2006). A Doppler probe can be used to find and keep intact the posterior nasal branches of the sphenopalatine artery which vascularize the mucosa flap. For suprasellar or retrochiasmatic tumors, an opening of the sella and the tuberculum of the sella is sufficient to expose the tumors as well as both the ICA and the optic tract. The surgeon may have to keep in mind that the wider the opening is, higher is the risk of postoperative CSF leak. We always try to perform a bone flap as we do for adenomas, to restore a rigid bone plane for the closure time. The dura mater is therefore incised on both parts of the anterior intercavernous sinus. The latter is coagulated, while small clips are placed on both extremities to prevent bleeding. The intradural time does not differ much from that of regular microsurgery.

Closure time is essential. A multilayer technique (refer to the closure paragraph), restoring all the anatomic planes, has to be done meticulously. Fibrin glue is injected onto the intradural space to seal the arachnoid. Others authors prefer to fill up the cavity with fat. Two layers of a bio-absorbable dura are thereafter placed extradurally and the bone flap flips back on the aperture sealed with fibrin glue. The mucosa flap is placed in such a manner to cover entirely the bone defect and glue is injected over to reinforce the watertight of the closure. A last layer with fat and/or fascia lata is finally used to fill up the sphenoid sinus. To prevent any layer migration during the first hours and days after surgery, an inflatable balloon can be placed inside the sphenoid sinus but only for patients who can be woken up rapidly to have their vision checked.

The same steps are respected for a transcribriform or transplanum surgery, the extension of the bone removal particularly the ethmoidectomy is dictated by the tumor extension and facilitated by the use of neuronavigation.

For parasellar approaches, after preparing the nasoseptal flap (if an intradural time or a CSF leak are expected), a lateral opening of the sphenoid sinus recess on the side of the lesion is done. The optic canal, the superior orbital fissure and orbital apex, the infratemporal fossa (behind, are Meckel's cave and the petrous apex) and medially, the cavernous sinus can be exposed, after opened up the bone, by drilling or with punches (Cebula et al., 2010). Working close to the cavernous sinus and to the Meckel's cave will require a proximal and distal control of the ICA from the $\mathrm{C} 2$ to the $\mathrm{C} 5$ segments. The $\mathrm{C} 2$ segment and the genu of the ICA at the foramen lacerum can be exposed following the vidian canal. 
For transclival approaches, anatomical landmarks have been described previously. A midline access from the upper clivus to the superior part of $\mathrm{C} 2$ is possible and provides a useful corridor to the tumors located anteriorly to the brainstem.

\subsection{Postoperative management}

Extended approaches with grafts and/or recovering flaps are the only approaches requiring postoperative nasal packing. The latter may be uncomfortable and painful.

If a balloon is used at the end of the surgery to maintain the grafts, it will usually be remove after 3 to 5 days, depending on the degree of skull base bone removal.

Patients are asked to avoid nose blowing as well as any activities that may raise intracranial pressure for 2 or 3 weeks after surgery and not to wash their nostrils during the first week.

To avoid germ selection, we do not give any antibiotics postoperatively whatever the type of surgery (whether extended approach or not, with or without CSF leak).

After an extended approach with CSF cisterns' opening (or after a moderate to major CSF leak during adenoma surgery), a 3 to 5 days lumbar puncture or drainage is systematically done to prevent a permanent nasal CSF fistula. The first few days after the skull base reconstruction are critical but CSF leak can be observed as much as 8 to 10 days after surgery. In our own experience, there were very few problems thereafter. Thus, the patient is usually discharged on day 4 for pituitary surgery but not before day 8 for extended approaches.

For sellar or perisellar tumors surgery, blood and urinary parameters are closely monitored for 4 days following surgery to detect diabetes insipidus. Corticosteroids hormone substitution is kept until the results of the postoperative hormonal assessment.

It may be useful to control nasal healing under local anesthesia, washing and removing crusts for 2 or 3 weeks after surgery especially for extended approaches.

\section{Classification of endoscopic endonasal approaches to skull base}

Endoscopic endonasal skull base surgery (EESBS) was initially dedicated to treat anterior and middle skull base CSF leaks, mainly by ENT surgeons but also neurosurgeons. During the 1990's, EESBS indications extended to pituitary surgery and to anterior skull base tumors, parasellar tumors and clival lesions (figure 12), shifting to what is now called extended EESBS (Kassam et al., 2006, 2007a, 2007b, 2008; Schwartz et al., 2008). Even if it is very attractive because it is considered as less invasive and adds new routes for deeply located tumors, these techniques are limited by a long learning curve (Snyderman et al., $2007,2008)$ and many closure challenges.

\subsection{CSF leaks and skull base defects}

Since the publication by Dandy in 1926, in which he reported the repair of a cranio-nasal fistula through a frontal craniotomy, few advances have been described in this field, until Malte Erik Wigand, a German ENT surgeon, opened the way to endoscopic endonasal skull base fistulas' management in the early 1980's (Hirsch, 1952). Nowadays, endoscopic endonasal approach is the gold standard in this field, regarding anterior, middle or posterior skull base defects (Castelnuovo et al., 2007, 2008; Martin \& Loehrl, 2007; Nyquist et al., 2010). Closure techniques have recently benefited from biomaterial innovations (synthetic dura, fibrin glue) and pediculated flaps, and help to deal with closure issues in tumoral pathologies managed by EESBS. 


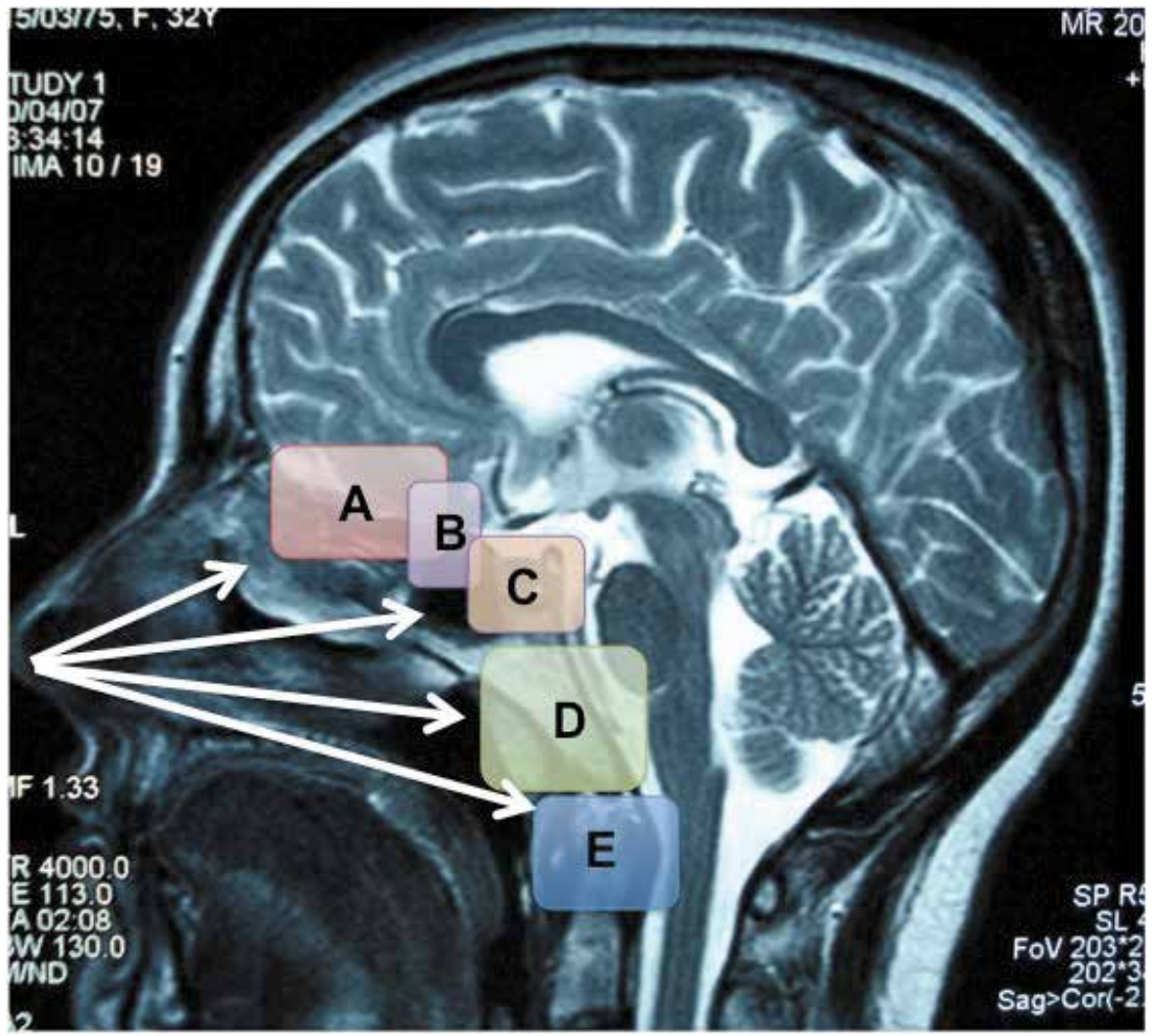

Fig. 12. Sagittal classification of endoscopic endonasal corridors.

A: Transcribriform transethmoidal

B: Transtubercular transplanum

C: Transsphenoidal sellar

D: Transclival

E: Transodontoid

\subsection{Endoscopic endonasal pituitary surgery}

The immediate position of the pituitary fossa posterior to the sphenoid sinus naturally led to the widespread use of the transsphenoidal route either by sublabial or by nasal corridors. One must keep in mind the pioneering influence of the Pittsburgh school (Jho and colleagues) and Neapolitan school (Paolo Cappabianca and colleagues) among others in the development of the endonasal endoscopic pituitary surgery during the last 2 decades (Cappabianca et al., 1998; Jankowski et al., 1992; Jho et al., 1996, 1997). Nowadays, this approach is becoming the first-line technique and most of pituitary surgeons are shifting to the endoscopic technique because of the excellent visualization of supra and para-sellar compartments it provides and a painless shorter postoperative course. Despite the absence of randomized studies proving definitely the superiority of endoscopic surgery upon microsurgery, the recent literature is accumulating evidence in favor of endoscopy especially for non-functioning pituitary adenomas (Dehdashti et al., 2008; Kassis et al. 2009 ; Frank et al., 2006, Higgins et al., 2008; Messerer et al., 2011; Schaberg et al., 2010). The rate of 
gross total removal is definitely higher in endoscopic series than in microscopic ones, for such adenomas. Data regarding functional adenomas are still debated and it seems that their results are at least comparable if not better with endoscopy (D'haens et al., 2009; Har-El, 2005).
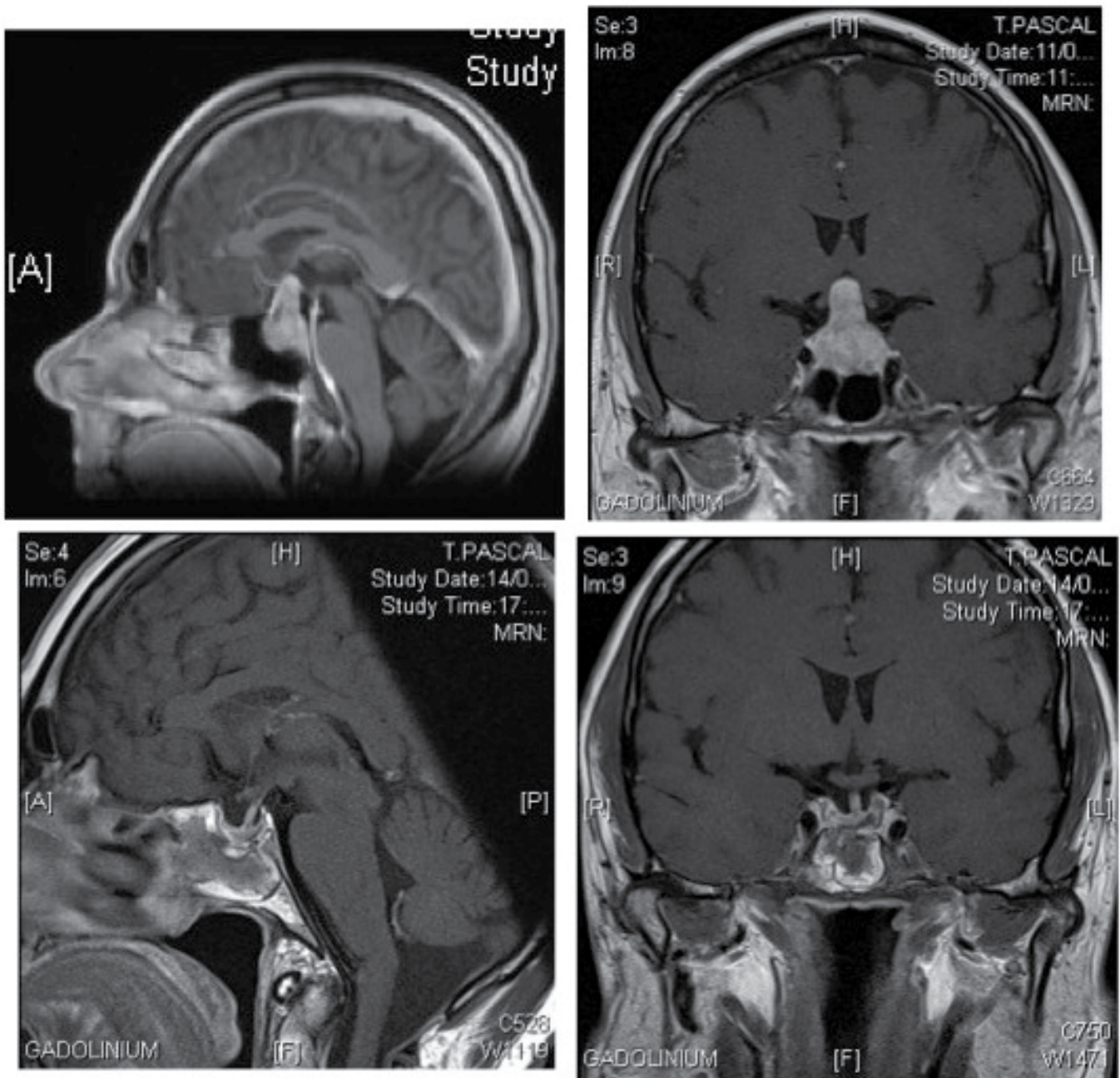

Fig. 13. Complete resection of non-functioning pituitary adenoma by endonasal endoscopic approach. Preoperative (up) and postoperative (down) MRI, T1 sequence with gadolinium.

It is important to consider that anatomic training in the lab followed by a regular practice of pituitary surgery is the first step towards endoscopic technique. The first level in the learning curve is obviously dealing with extradural cases, which have less closure and vascular issues. Surgeons will also have to learn to work with a $2 \mathrm{D}$ system and to watch a screen. After having gathered enough experience, (roughly more than 100 cases of endoscopic pituitary surgery according to almost all the renowned endoscopic experts), the training of the surgeon will enable him to perform further operations such as intradural works and extended approaches (Snyderman et al., 2007). 


\subsection{Extended endoscopic endonasal surgery}

\begin{tabular}{|c|c|}
\hline Anterior skull base & $\begin{array}{l}\text { Olfactory groove meningioma } \\
\text { Planum sphenoidale meningioma } \\
\text { Tuberculum sellae meningioma } \\
\text { Ethmoidal carcinoma } \\
\text { Nasopharyngeal fibroma } \\
\text { Esthesioneuroblastoma }\end{array}$ \\
\hline Sellar and suprasellar regions & $\begin{array}{l}\text { Diaphragm sellae meningioma } \\
\text { Pituitary adenomas } \\
\text { Craniopharyngiomas } \\
\text { Rathke cleft cyst } \\
\text { Inclusion tumors (epidermoids, dermoids, } \\
\text { teratomas) } \\
\text { Germinal tumors } \\
\text { Suprasellar tumor, "(biopsy)" }\end{array}$ \\
\hline Meckel's cave & $\begin{array}{l}\text { Chondrosarcoma and Chondroma } \\
\text { Trigeminal schwannoma } \\
\text { Metastasis (biopsy in case of doubt) }\end{array}$ \\
\hline Cavernous sinus & $\begin{array}{l}\text { Extension of pituitary adenoma or of others soft } \\
\text { tumors such as chondrosarcomas } \\
\text { Meningioma or metastasis (biopsy in case of doubt) }\end{array}$ \\
\hline Orbital apex and optic canal & $\begin{array}{l}\text { Orbital tumor located medially to the optic nerve } \\
\text { (biopsy or resection) } \\
\text { Exophtalmos } \\
\text { Canal optic decompression }\end{array}$ \\
\hline Petrous apex and clivus & $\begin{array}{l}\text { Chordoma } \\
\text { Chondroma and chondrosarcoma } \\
\text { Epidermoid cyst } \\
\text { Meningiomas }\end{array}$ \\
\hline Anterior craniocervical junction & $\begin{array}{l}\text { Rheumatoid pannus } \\
\text { Spondylodiscitis } \\
\text { Malformative anomaly } \\
\text { Bone tumors } \\
\text { Chordoma }\end{array}$ \\
\hline
\end{tabular}

Table 1. Topographical classification of skull base lesions accessible to EESBS

\subsubsection{Anterior skull base neoplasms}

Meningiomas remain the more frequent neoplasms in this region, arising between the crista galli process and the tuberculum of the sella (Fernadez-Miranda et al., 2009). Herein, the true advantages of EESBS are to provide a direct devascularization of the tumor 
(cauterization of anterior and posterior ethmoidal arteries) and avoid any retraction of the frontal lobes. The limits are detailed further in the controversies' discussion and include a high risk of fistula, a higher risk of olfactory nerves injury and a lower control of the lateral margins of the tumor. For such a location, intracranial mini-invasive keyhole approaches represent serious alternatives.

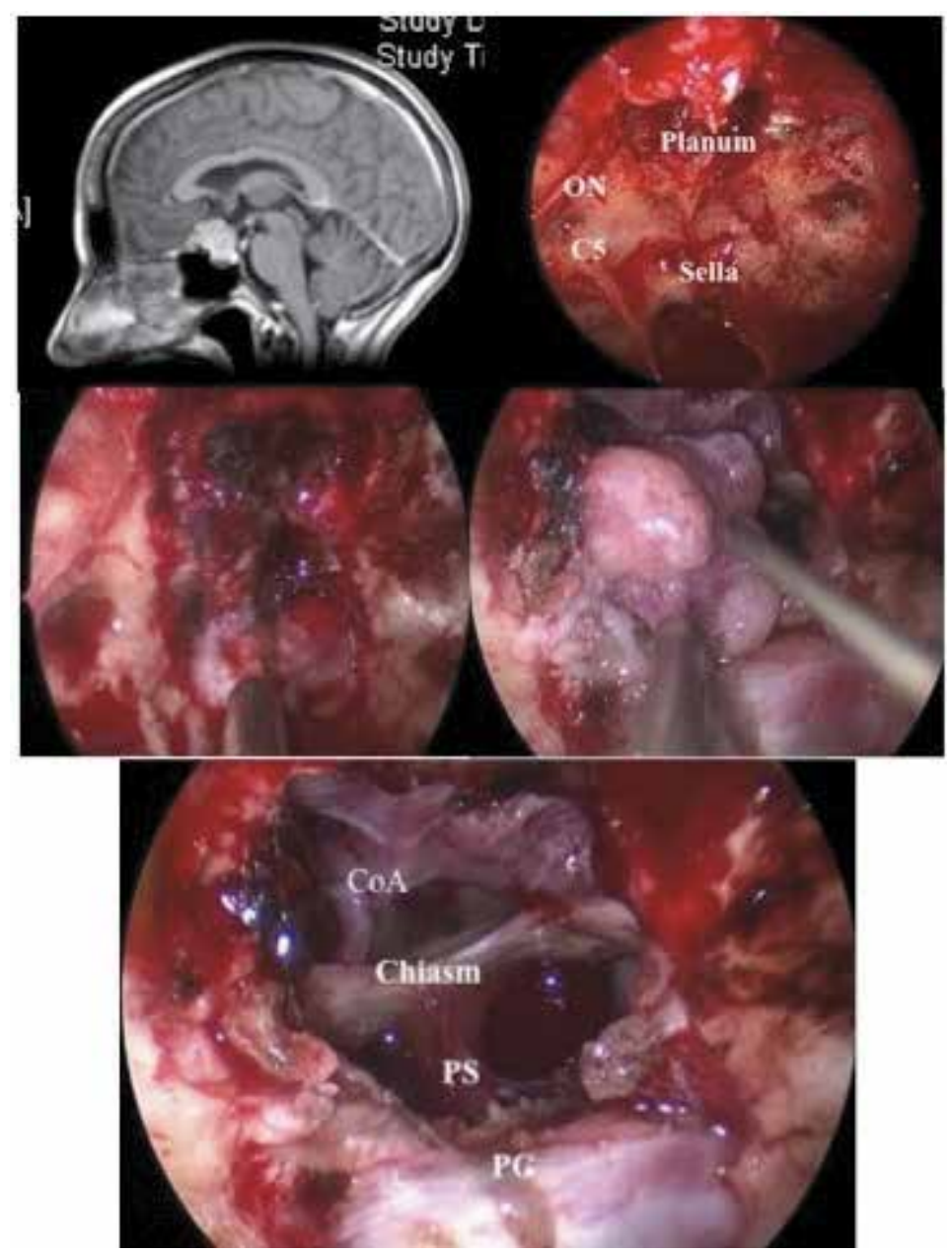

Fig. 14. Endonasal endoscopic resection of planum sphenoidale meningioma by a transtubercular approach. A one and half centimeter bone window is done with bone punch or by drilling to remove the roof of the sella, the tuberculum of the sella and the adjacent planum. After coagulation of the insertion basal dura, this latter is resected and the meningioma is progressively debulked and dissected from critical neurovascular structures (ON: optic nerve; Chiasm; CoA: anterior communicating artery; PS: pituitary stalk; PG: pituitary gland).

Many other neoplasms involving the anterior skull base have been approached through the endonasal route such as esthesioneuroblastomas (Suriano et al., 2007), nasopharyngeal fibromas and carcinomas (Batra et al., 2005). 


\subsubsection{Sellar and suprasellar non-adenomatous tumors}

Endoscopic endonasal route has been naturally and progressively applied to the suprasellar lesions (craniopharyngiomas (Cavallo et al., 2009; Gardner et al., 2008a, 2008b), Rathke cleft cyst (Alfieri, 2002), epidermoid cysts, teratomas, etc,) with an obvious absence of frontal lobe retraction and a better corridor to the retro-chiasmatic region (de Divitiis et al, 2002). An approach from below provides a direct view of the tumor and its extension into the third ventricle (figures $15 \& 16$ ). Soft or non-adherent tumors can be more completely removed under direct visualization, what cannot be done through the intracranial route. However, identifying the tumor extension does not involve its systematic removal. Tumors adherent to the third ventricle like some craniopharyngiomas have to be kept in place considering the important risk of poor postoperative outcome.

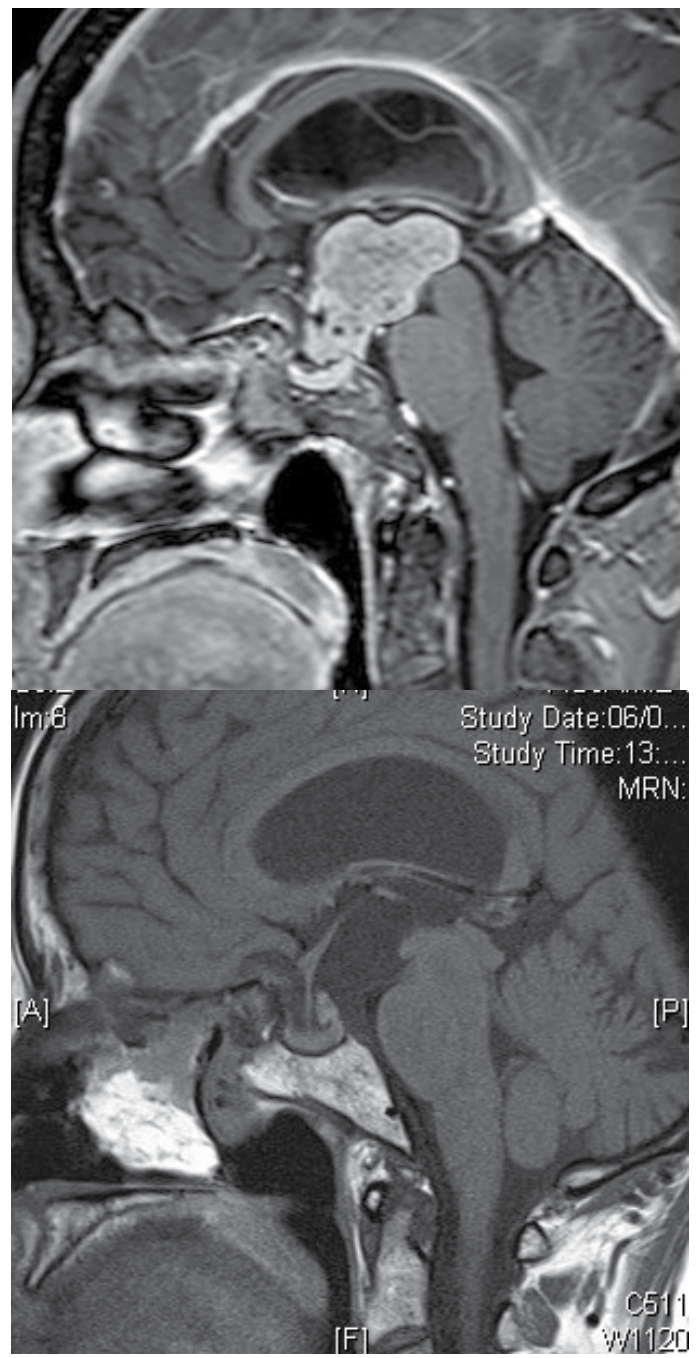

Fig. 15. Resection via an endoscopic endonasal approach of a retrochiasmatic and intraventricular suprasellar epidermoid cyst. Preoperative sagittal T1 gado MRI (left), postoperative control (right) with uneventful postoperative course. 


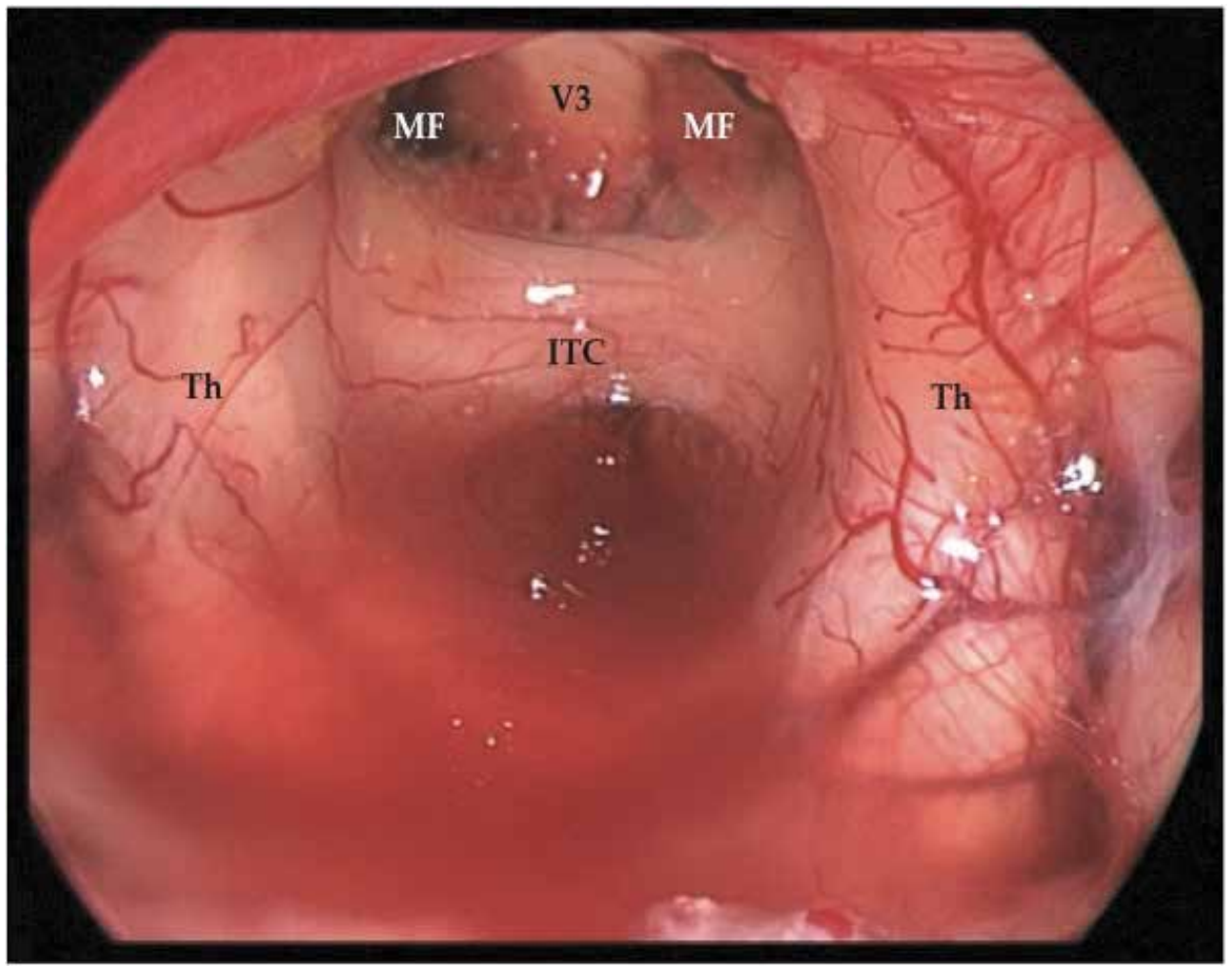

Fig. 16. Same patient as in figure 15. End of the resection showing the cavity of the $3^{\text {rd }}$ ventricle, its roof (V3), both Monro foraminas (MF), and the 2 thalami bridged by the interthalamic commissure (ITC).

\subsubsection{Meckel's cave tumors}

Mainly represented by trigeminal shwannomas (figure 17), their approach requires classically an extensive orbitozygomatic frontopterional craniotomy. Meckel's cave can be approached by EESBS through its anterior aspect laterally to the para-clival portion of the internal carotid artery (Kassam et al., 2009). By this route, we can either remove the tumor or take a sample for a biopsy, the main advantage being not crossing cranial nerves, usually pushed laterally when the tumor grows.

\subsubsection{Cavernous sinus lesions}

There are very few indications of direct surgery for cavernous sinus tumors. Primarily represented by meningiomas, the current strategy is to treat them with radiotherapy or radiosurgery, when imaging is typical. However, when some soft tumors project into the cavernous sinus such as adenomas or chondrosarcomas, the panoramic view offered by the endoscopic endonasal route allows us to complete the removal safely. In our own experience (112 cases of pituitary adenomas extending into the cavernous sinus), the endoscope allowed us to cure more than one third of functioning or non-functioning adenomas without additional morbidity (figure 18). This route can also be used for biopsy when there is any doubt about the nature of a cavernous disease. 

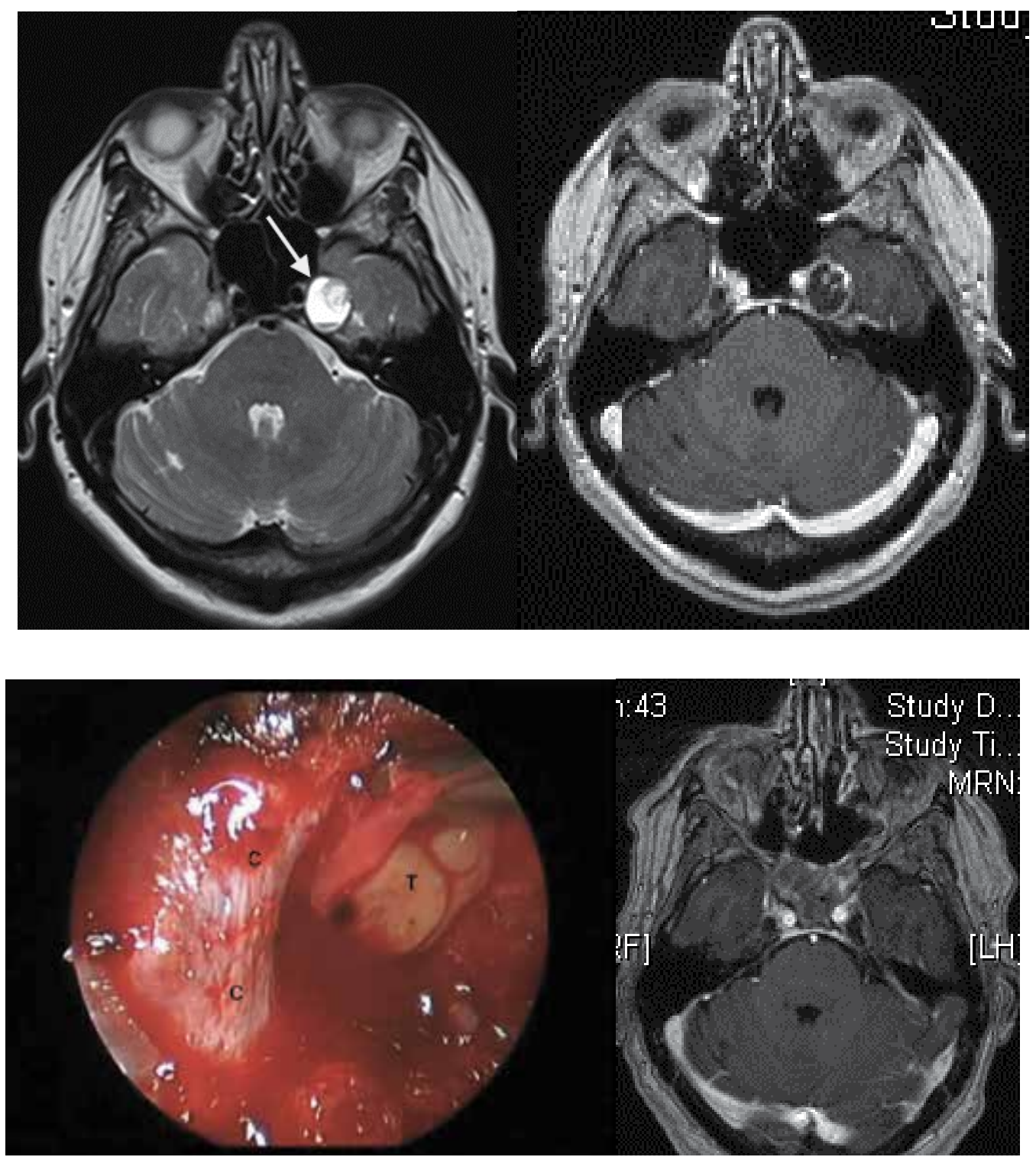

Fig. 17. Removal of a trigeminal schwannoma by an endoscopic endonasal approach. Top pictures, axial T2 and gadolinium T1 MRI showing a partially cystic tumor. Anterior approach of Meckel's cave with on the medial and the inferior side respectively the $\mathrm{C} 3$ and C2 portion of the ICA (C) and a typical aspect of a schwannoma (T) (down left picture). Down right: the postoperative MRI that shows a complete removal. 


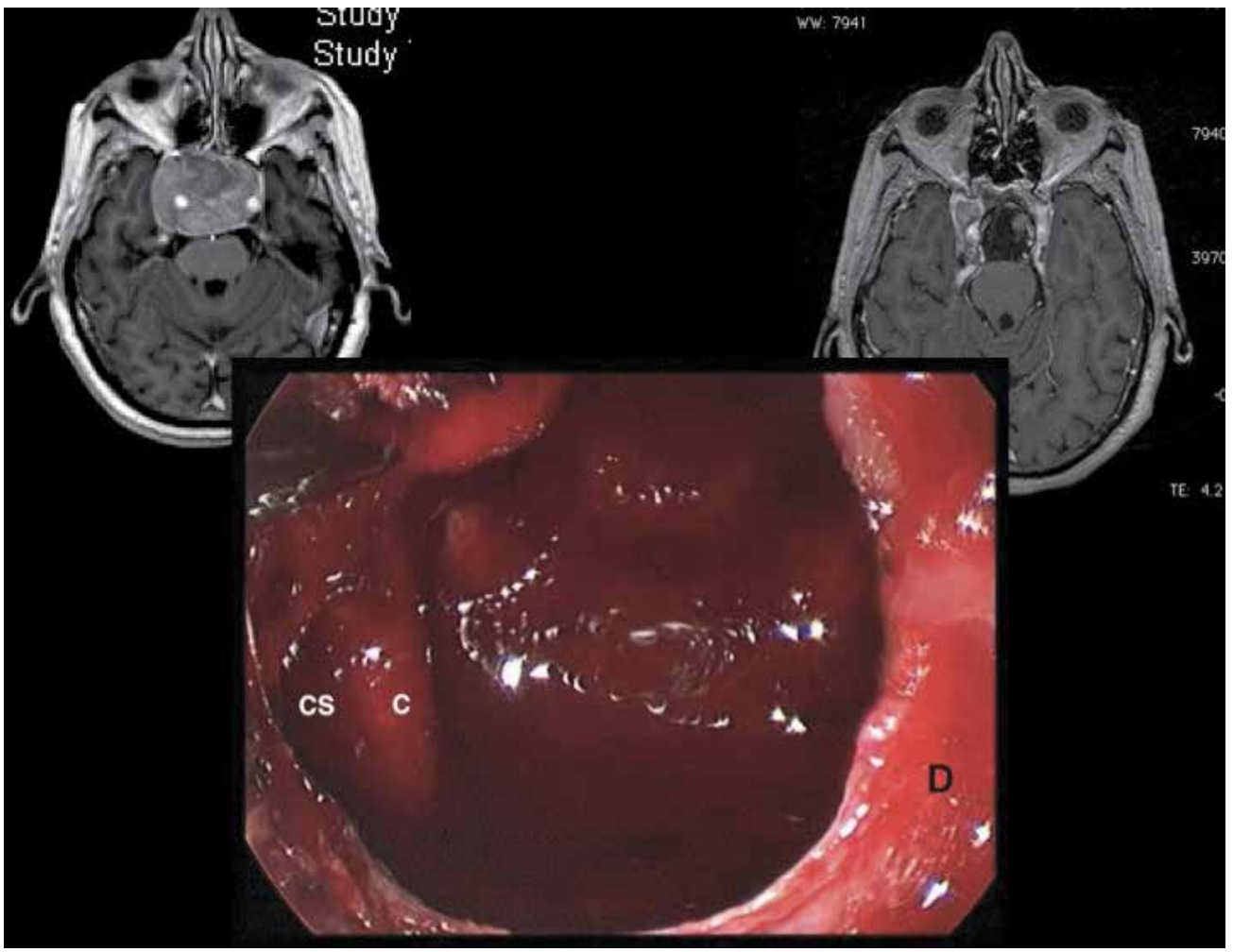

Fig. 18. Giant non-functioning adenoma invading the right cavernous sinus operated with a pure endoscopic endonasal approach (top left). During the surgery with a $30^{\circ}$ angle endoscope pushed at the entry of the pituitary fossa (D), the cavernous portion of the carotid (C) was clearly seen with a perforation on the cavernous sinus wall (CS). Under visualization and with a two suction technique, a large part of the cavernous portion of the adenoma was removed without additional morbidity. The remnant tumor is therefore minimized and accessible to radiotherapy (top right).

\subsubsection{Petrous apex and clival tumors}

Petrous apexes as well as clival tumors are very challenging lesions because of their deepness and their neurovascular environment. EESBS provides a direct access to such tumors replacing progressively the traditional subtemporal, transpetrosal or retrosigmoid approaches (Griffith \& Terrel, 1996; Kassam et al., 2005). Combination of both endonasal and intracranial procedures may be performed depending of the tumor conformation (figure 19).

\subsubsection{Anterior craniocervical junction}

Midline anterior lesions are good candidates for a direct approach by an endonasal endoscopic route, as standard far lateral approaches require crossing nerves and vessels before reaching the tumor. Rheumatoid pannus are fortunately rare with modern treatment but endoscopic endonasal resection has been described in the literature (Nayak et al., 2007). Skull base malformative anomalies and tumors can also be more easily approached through the endonasal route (Leng et al., 2009; Magrini et al., 2008). 


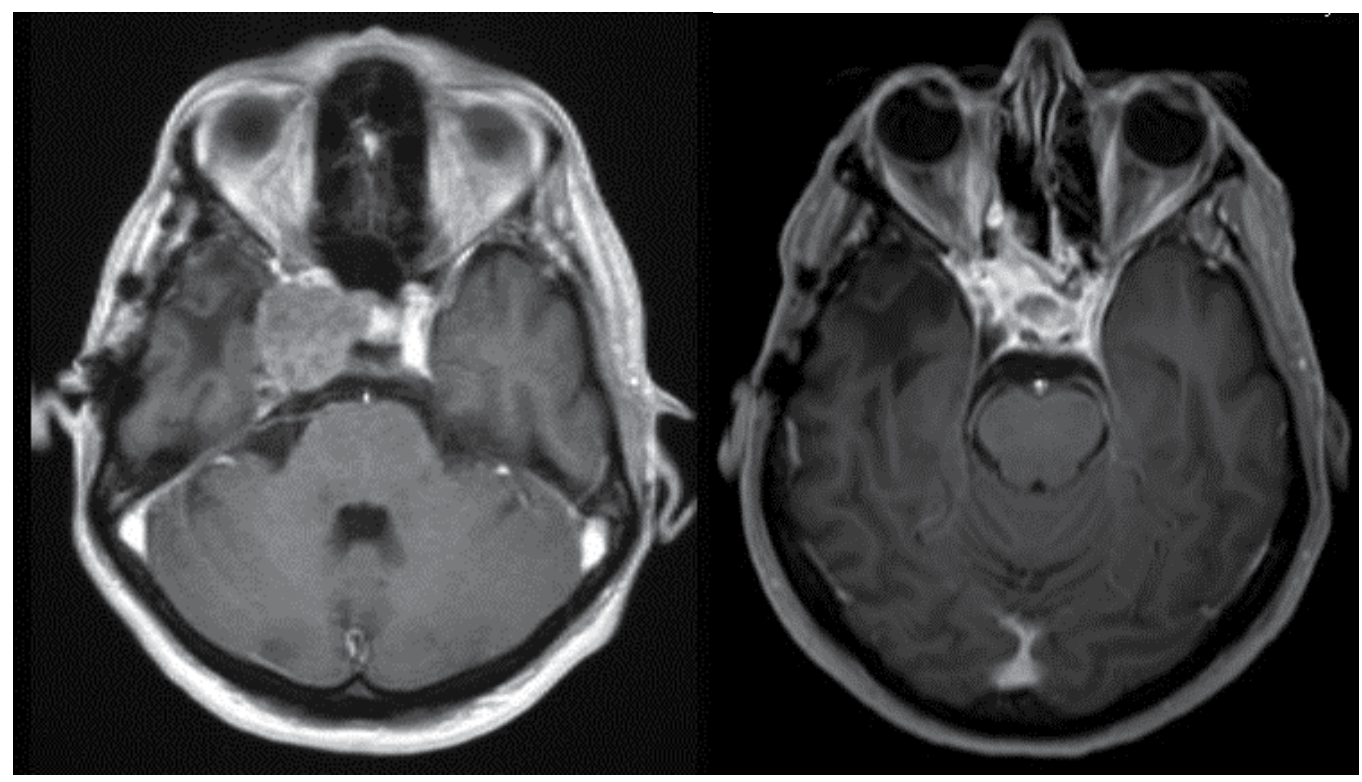

Fig. 19. Left: Petrocavernous chondrosarcoma operated on first via a subtemporal approach with a cavernous sinus tumor remnant. Right: A second step via a transsellar endonasal approach completed the removal without any postoperative cranial nerve or pituitary deficit.

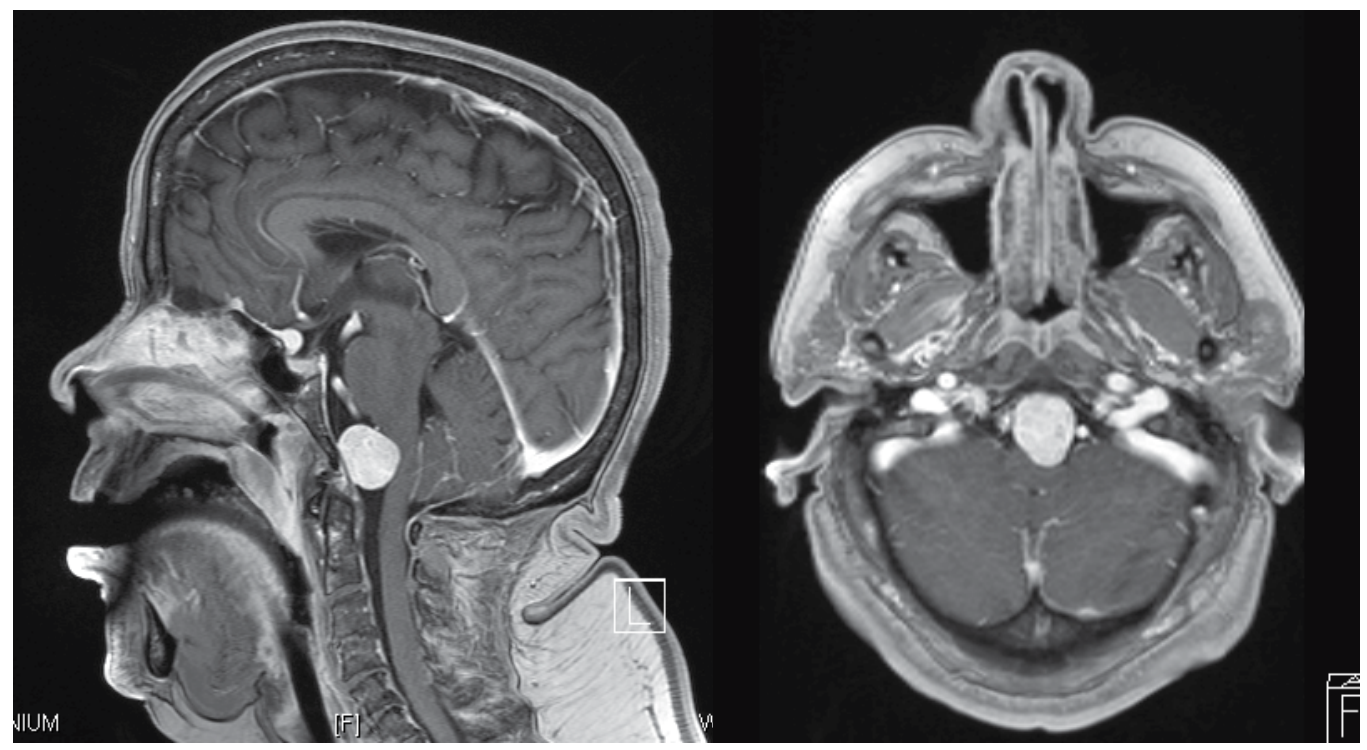

Fig. 20. Example of an anterior midline craniocervical meningioma that would be a good candidate for an endonasal endoscopic surgery because situated above the Kassam's line. 
Drawing a line from the nasal bone to the palatine bone will help the surgeon to visualize the inferior limits of the surgical field (de Almeida et al., 2009). The endonasal route usually gives access to the odontoid and the upper third of C2 (figures $20 \& 21$ ). A lesion located inferiorly is an indication of a transoral surgery where the endoscope can also be useful as the surgical field is deep and narrow (Crockard, 1985).

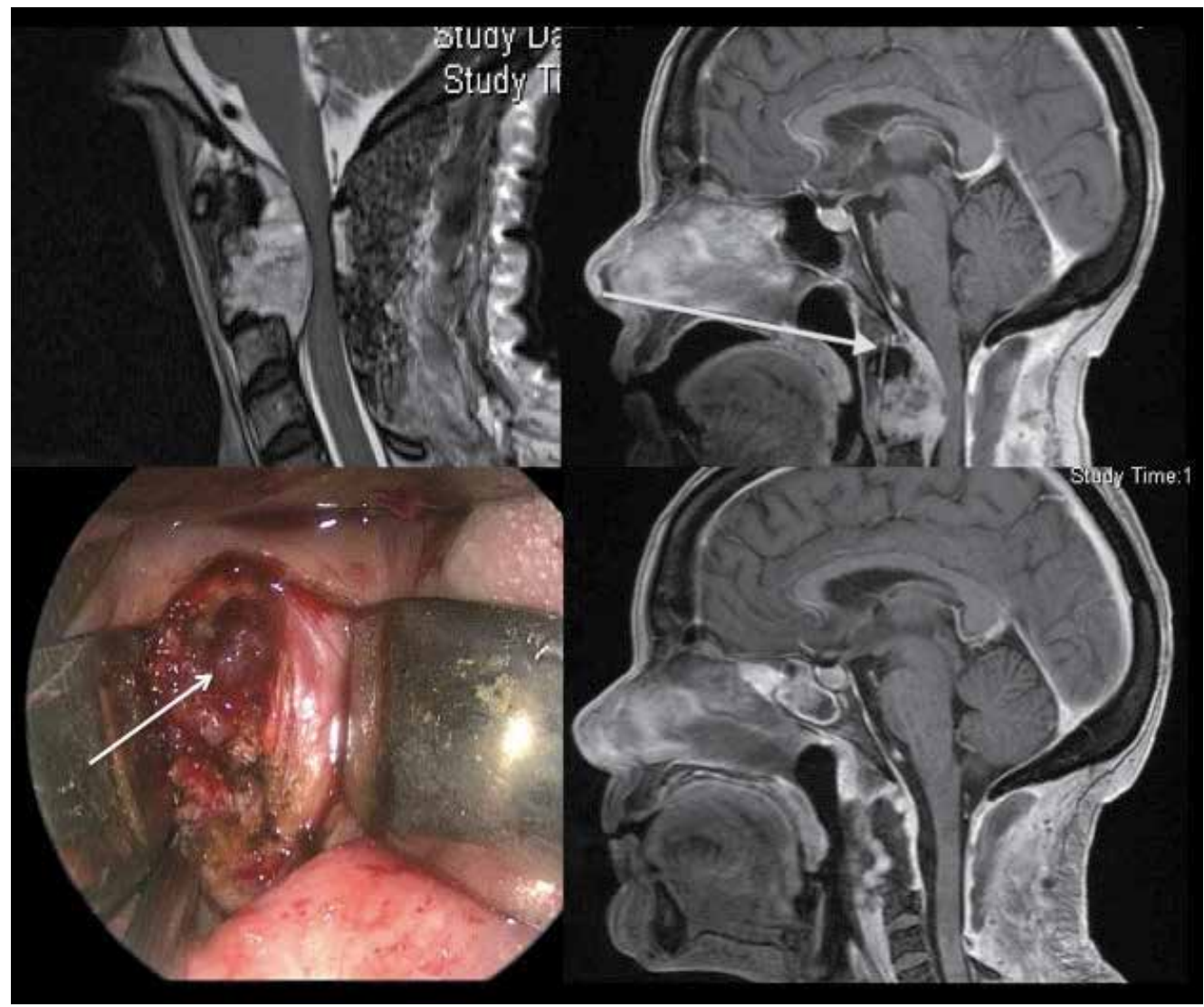

Fig. 21. Example of a C2 chordoma (top left). The tumor is located below Kassam's line indicating a transoral approach instead of the endonasal route (top right). The endoscope is a vast improvement on the microscope and provides a better visualization of this deep and narrow surgical field. Bottom left: an operative view with a typical aspect of chordoma, soft purplish tissue (white arrow). Bottom right, the postoperative MRI showing a macroscopic complete removal.

\subsubsection{Infratemporal and pterygopalatine fossas}

Herein, the endonasal approach competes with the usual transfacial approaches like Lefort I to remove tumors in this area (paragangliomas, shwannomas, meningiomas, juvenile nasopharyngeal angiofibromas) (figure 22). This region represents the lateral and inferior limits of endoscopic endonasal approaches with issues related to the multiples branches of the external carotid artery and the trigeminal nerve (Rivero-Serrano et al., 2010; Robinson et al., 2005). 


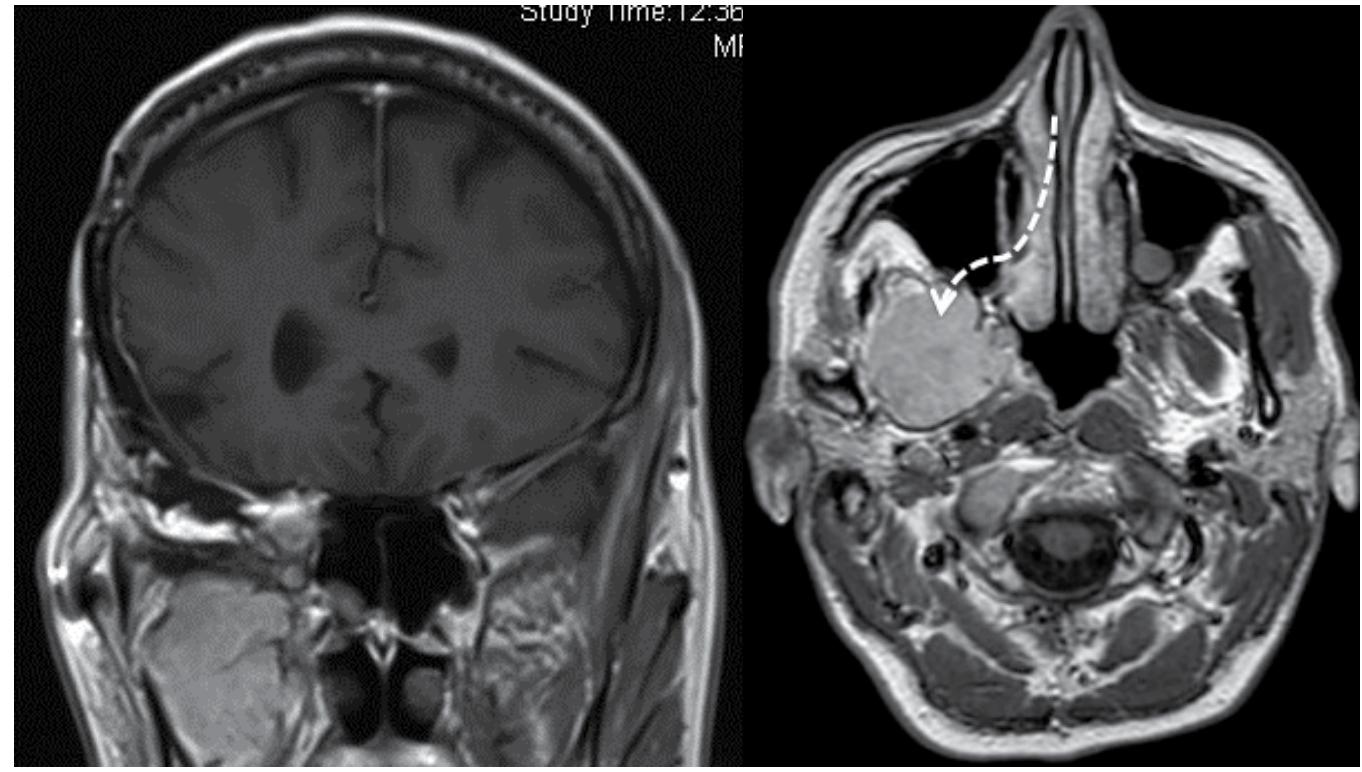

Fig. 22. Example of an extension of a sphenocavernous meningioma into the pterygopalatine fossa. Opening the maxillary sinus by an endonasal route will give a direct access to the lesion (white dotted arrow). It is a variant of the classic Lefort I approach.

\section{Complications}

\subsection{Nasal morbidity}

EESBS is constantly associated with postoperative nasal morbidity including mainly nasal crusting and blocked nose sensation lasting for the first few weeks. The intensity of these symptoms varies from one patient to another, and is usually moderate for pituitary surgery and perfectly managed by local treatments given to patients on discharge from hospital. Nasal consequences of extended approaches are far more complicated, with frequent chronic crusting rhinosinusitis. Indeed in such approaches, turbinectomies and wide sinus aperture impair the nasal airflow which leads to crusting and obstruction. With the spread of these kinds of approaches, many teams are studying the postoperative quality of life using questionnaires such as the SNOT-22 (Sino-Nasal Outcome Test-22) and the anterior skull base questionnaire. Nasal crusting remains the most frequent postoperative complaint while no risk factors have been identified (98\% of the patients had nasal crusting at one month in Almeida's series). According to Almeida et al., the use of nasoseptal flap for skull base closure is not a significant risk factor of nasal morbidity (de Almeida et al., 2011).

Patients have to be informed preoperatively of this nasal morbidity and particularly the high rate of nasal crusting and possible loss of smell. They should also know that these morbidities are transitional in almost all cases and will improve within 3 to 6 months postoperatively. A postoperative nasal care including sinus humidification and saline irrigation is highly recommended.

Postoperative infectious sphenoid sinusitis may occur in about $2 \%$ of patients, while it can reach $9 \%$ in microsurgical series of pituitary adenomas. A large opening of the sphenoid sinus and a lesser use of artificial packing material within the sphenoid sinus may prevent these infections (Balaker et al., 2010). 


\subsection{CSF leak and meningitis}

CSF leak is the most common complication during endoscopic endonasal approaches to the skull base, with initial high rates decreasing progressively with the learning curve and the closure methods with pediculated flaps. Higher experience in pituitary surgery means rarer CSF leaks, less than 5\% (Messerer et al., 2011). Obviously, extensive approaches involving the anterior skull base are more likely to lead to per and postoperative CSF leaks. We also experienced more frequent CSF leaks for planum meningiomas than for other types of tumors. A postoperative leakage was observed in $15.9 \%$ of the 800 patients treated by Kassam et al., but for anterior skull base meningiomas it can reach up to $50 \%$ of cases (Kassam et al., 2010). An accurate multilayer closure of the skull base is of a paramount importance to avoid postoperative CSF leaks, especially when a peroperative leak is observed. The use of pedicle flaps dramatically decreased the rate of these postoperative CSF leaks (Hadad et al., 2006; Kassam et al., 2005).

Postoperative infection risk is comparable to intracranial open skull base surgery with a rate reaching $1.8 \%$ of patients. Risk factors of meningitis are mainly peroperative CSF leaks and postoperative external ventricular or lumbar drains (Gondim et al., 2010, Kono et al., 2010).

\subsection{Vascular complications}

Early or delayed nasal hemorrhages may occur postoperatively in up to $7 \%$ of patients. These hemorrhages related to small mucosal arteries, are usually of small volume and respond easily to transitory nasal tamponade. An injury or insufficient coagulation of branches of the sphenopalatine artery in the sphenoethmoidal recess may rarely lead to important potentially life-threatening epistaxis, requiring reoperation and/or endovascular management (Gondim et al., 2010).

An internal carotid artery injury is a striking peroperative complication. This artery may be encased within an invasive skull base tumor as well as in redo surgeries, conditions in which neuronavigation and microdoppler probe are very useful tools. In such difficult cases of Meckel's cave or cavernous sinus surgery, a preoperative angiogram should be done and an extra-intracranial anastomosis discussed in the case of a non-functional Willis polygon.

One must be particularly aware of "kissing" carotid arteries sometimes observed in acromegalic patients.

Pseudoaneurysms and carotid-cavernous fistulas may result months or years after aggressive tumoral dissection and curettage within the cavernous sinuses (Cappabianca et al., 2001).

A careful analysis of preoperative MRI and eventually angiographic studies is mandatory to avoid such dramatic complications (Solares et al., 2010).

\subsection{Endocrinological issues}

Hydro-electrolytic balance monitoring is compulsory during the early postoperative course in order to detect diabetes insipidus, either transient (2-20\% in pituitary adenomas) or permanent $(1-5 \%)$, usually secondary to surgical manipulation or injury to posterior pituitary gland and/or pituitary stalk.

Postoperative anterior pituitary dysfunction may occur in $13 \%$ to one third of patients with pituitary adenomas managed endoscopically, depending of the preoperative condition (Messerer et al., 2011). Endocrine results are clearly poorer for craniopharyngiomas while unclear or sparse for the other sellar tumors. 


\subsection{Ocular complications}

Orbital hemorrhages may be caused by an injury to the ethmoidal artery during an endoscopic endonasal approach, and subsequent retraction of this artery within the orbit may occur. In such a case, an emergency orbital decompression can be needed (Charalampaki et al., 2009). Elsewhere, a direct injury to the globe or to the medial rectus muscle or even to the nasolacrymal duct may occur, specially during the initial learning curve (May et al., 1994).

\subsection{Pituitary apoplexy}

Postoperative apoplexy usually occurs when an obvious suprasellar adenomatous remnant is left in place. The major risk of this complication is the acute compression of the visual apparatus, acute pituitary insufficiency and obstructive hydrocephalus. Emergent reoperation may be necessary, either by the same endonasal route or intra-cranial approach according to apoplexy configuration.

\section{Closure techniques: The multilayer technique as key point}

The CSF leak is the main issue of EESBS, thus making closure time as important as the tumor removal itself. Many studies have been pusblished on this topic, each progress leading to a decrease in the percentage of postoperative CSF leak. One must understand that each layer has to be rebuilt (figure 23).

The arachnoid plane can be reinforced with intradural injection of fibrin glue and/or using a soft inlay graft covering the dural aperture. Extraduraly, the removed dura mater has to be replaced by a substitute. We prefer a bio-absorbable one as we experienced 3 cases of sellar space-occupying infections following pituitary surgery, where the dural plane had been rebuilt with a non-resorbable dural substitute. The bone plane is recreated using synthetic material (titanium plate) or using autologous bone (ie what we called the bone flap previously). This latter has our preference, as the long term tolerance of synthetic material is not known. The turbinates can be also used specially in locations where a bone flap cannot be done (clivus for instance).

The bone flap has to be covered after its repositionning. Autologous but inert material such as fat graft and fascia lata alone are disapointing with 30 to $50 \%$ of postoperative CSF leaks (Kassam et al., 2008). Vascular pediculated flaps (figure 24) is the biggest advance in endoscopic surgery leading to a dramatic decrease in the rate of CSF leak from $30 \%$ to around $4 \%$ (Kassam et al., 2008). The most easy to prepare is the nasoseptal flap (Hadad et al., 2006) but many other flaps are possible: pericranial, buccinator and temporoparietal fascia flap (Caicedo-Granados et al., 2010 ; Patel et al., 2010 ; Rivera-Serrano et al., 2010). To reinforce the watertight of the reconstruction, a fibrin glue is applied all over the bone and mucosa. The sinus cavities are therefore filled up with fat embedded in glue.

When repairing the anterior skull base, the risk of graft migration is higher during the first few days. A balloon can be used in prevention, as a support kept in place for 3 or 5 days. This must not be used in patients where visual function cannot be monitored closely.

Finally, CSF drainage to decrease intracranial pressure is still being discussed. External drain should be avoided because of the risk of contamination but we perform 3 to 5 postoperative lumbar punctures for high-risk surgeries such as anterior skull base, meningiomas and intraventricular surgeries. 


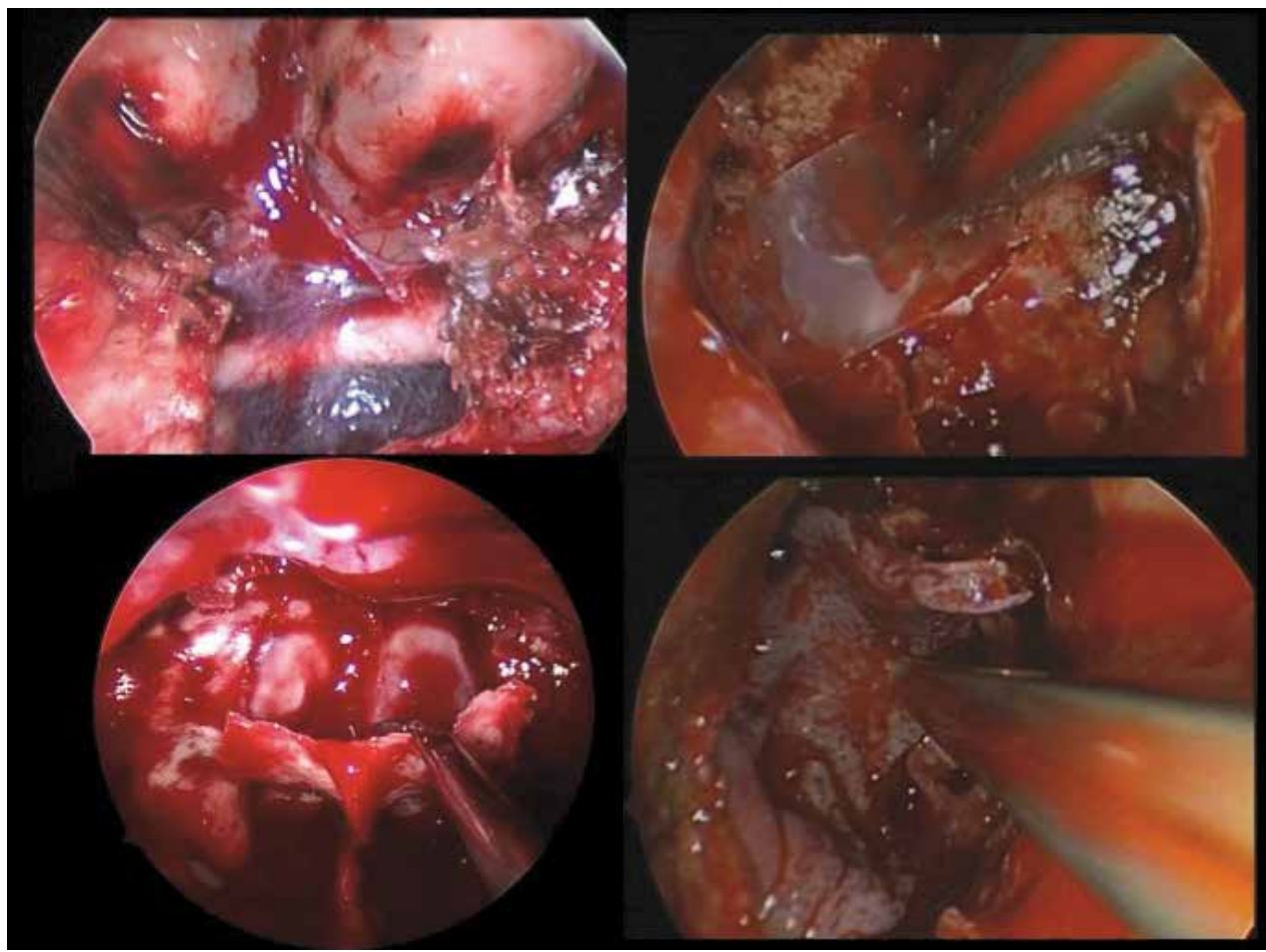

Fig. 23. Multilayered closure. Arachnoid with fibrin glue (top left), dura mater substitute (top right), bone flap positioning (bottom left) and mucosa flap( bottom right).

\section{Controversies and limits: Extended endonasal approaches versus trans- eyebrow keyhole endoscopic approaches for anterior skull base tumors}

If no one disputes the benefit of the endonasal endoscopic approach for retrochiasmatic parasellar or clival tumors, its use for anterior skull base tumors is still being discussed (i.e. from the cribriform plate to the tuberculum of the sella) (de Devitiis et al., 2008).

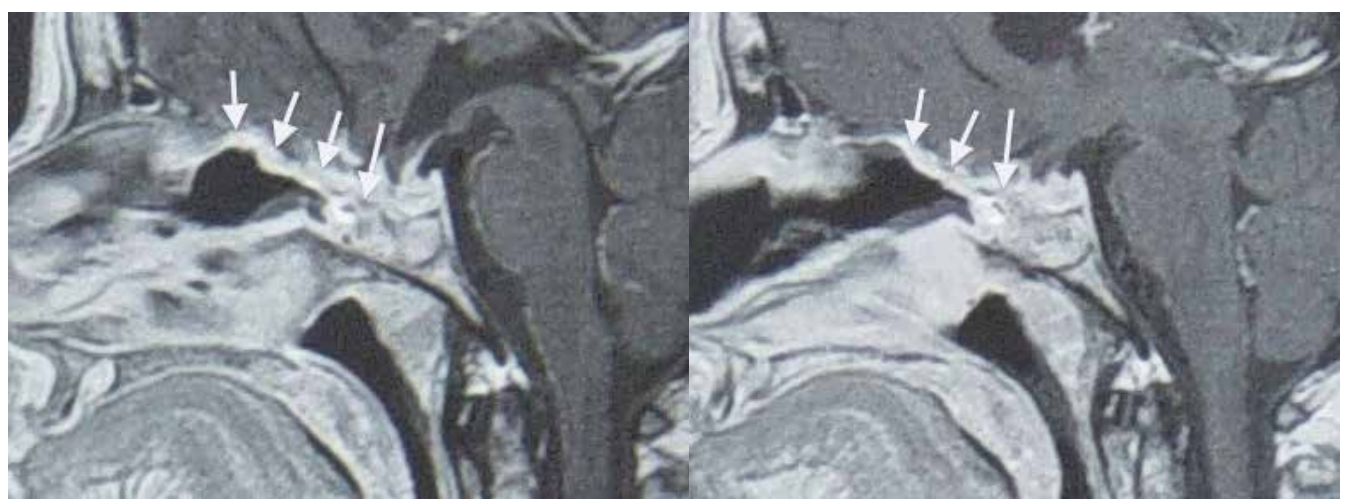

Fig. 24. Complete removal of planum meningioma. Postoperative MRI (sagittal T1 weighted sequence with gadolinium). The nasoseptal flap used to reconstruct the skull base is clearly visible enhanced after contrast injection (arrows). 
Indeed, the endonasal route presents many advantages: no brain retraction, a useful suppression of the blood supplies of the tumour always interesting specially for meningiomas and a better control of the inferomedial part of the optic canal. Other arguments argue against the endonasal approach in case of anterior skull base tumors.

The first one is a risk of CSF fistula higher than in others locations as the dura mater and bone graft might migrate down more easily when repairing the anterior skull base compared to middle cranial base. As reported by others (de devitiis et al. 2008), in our own experience of extended approaches (more than 100 cases to date comprising all locations), around half of the procedures done for anterior skull base (20 cases) was complicated by postoperative CSF leaks. Even though, we obtain clearly better results when using the bone and nasal flaps, this point was the first to exhort us to find an alternative mini-invasive keyhole approach.

The second one was the absence of control of the tumor margins when tumors grow over the optic nerves and carotid arteries, a common feature of meningiomas that usually extend largely on the anterior skull base. This may lead to tumor remnant (figure 25). On that particular point, it will be interesting to consider the frequency of tumor recurrence in series of anterior skull base meningiomas operated from below with long-term follow up before giving a definitive conclusion.

Finally, anterior skull tumors without olfactory impairment are another reason to choose the intracranial instead of the endonasal route. There are, of course, better chances to preserve olfactory nerves by an intracranial route.

The supraorbital trans-eyebrow approach was initially introduced for vascular clipping or skull base tumors removal primarily with the use of microscope (Reisch et al., 2003).

Since 2010, we use this keyhole approach purely with the endoscope instead of microscope. This allows a real keyhole approach (bone aperture of $2 \mathrm{~cm}$ ), the straight and angle endoscopes give a panoramic view of the entire anterior skull base but also of the opticocarotid recess and of the medial part of the optic canal (figure 26). With such a technique, we are able to remove meningiomas with as uneventful postoperative course as after endonasal endoscopic approaches (even with shorter hospital stay), without the risk of CSF fistula.

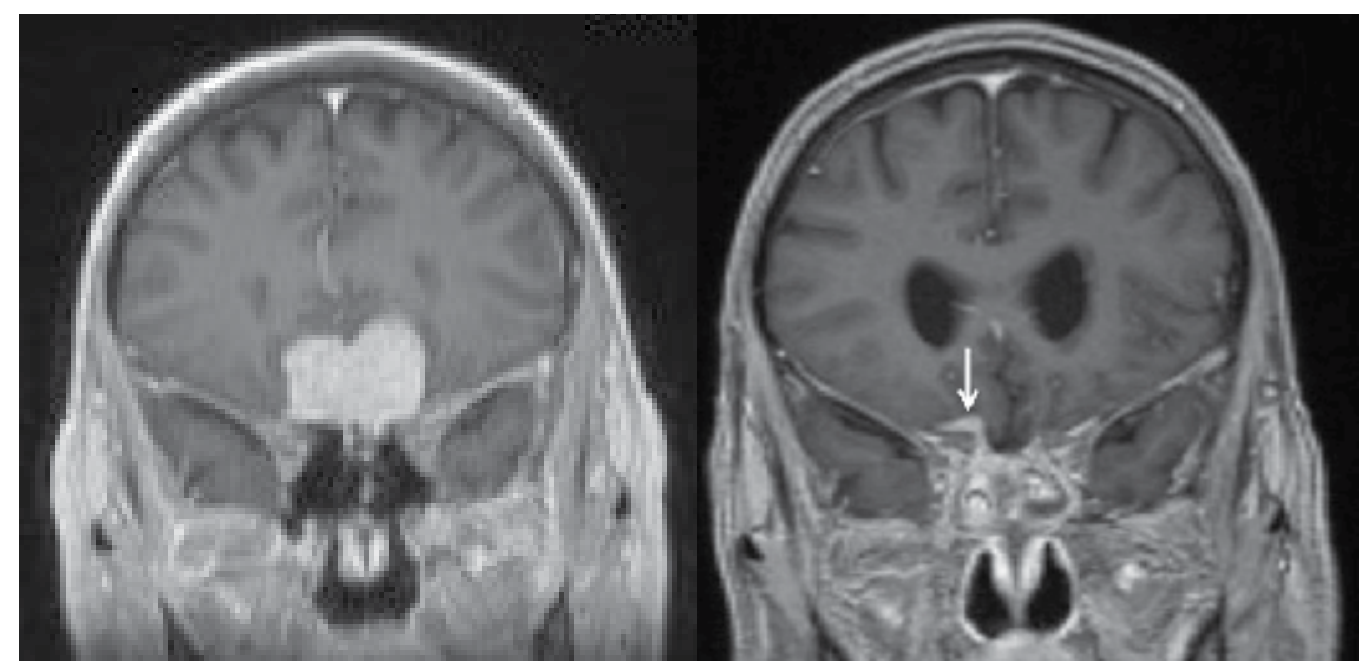

Fig. 25. Example of an anterior skull base meningioma removed from below. The postoperative MRI clearly indicated a tumor remnant on the top of the right clinoidal process (white arrow) that has not been seen during the endonasal route. 


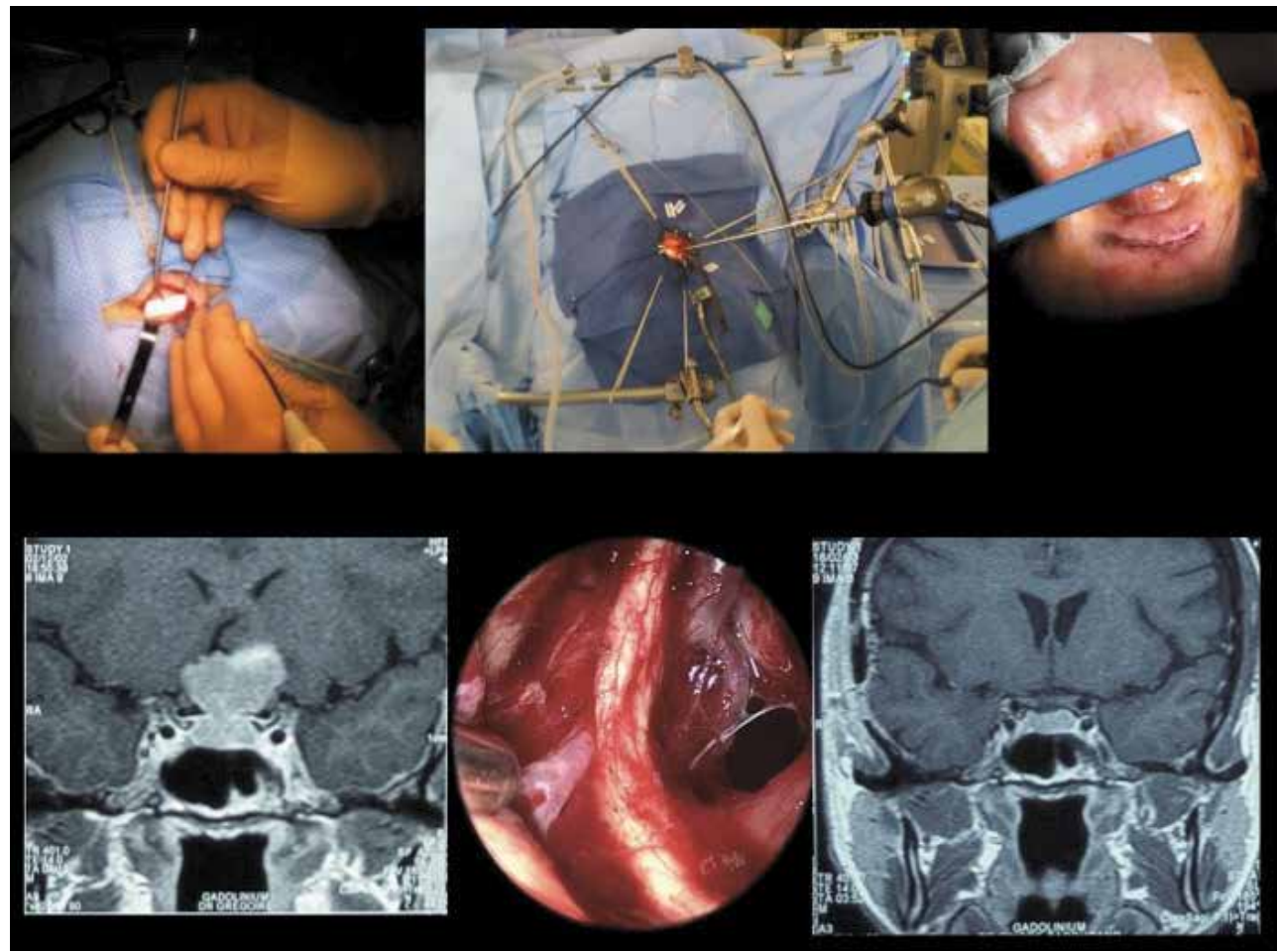

Fig. 26. Eyebrow supraorbital surgery for a suprasellar meningioma. This keyhole approach (frontal craniotomy of $2 \mathrm{~cm}$, top left) can be done with the endoscope instead of the microscope (middle top). Angled endoscopes give the possibility of exploring all the corners of the surgical field like the medial opticocarotid recess (middle bottom) but also the entire anterior skull base, what cannot be done with the endonasal route.

For the authors, this mini invasive endoscopic eyebrow approach should be considered for large tumors grown beyond the suprasellar carotid arteries but also for small anteriorly based tumors, thus avoiding any CSF leak.

\section{Conclusion}

The part of the endoscopic endonasal approach is getting more and more important in skull base surgery since the beginning of the nineties. Under the impulsion of the Pittsburgh and Neapolitan neurosurgical teams, the last two decades saw an increasing number of publications on that topic either on anatomy or surgical techniques (more than 800 entries on Pubmed using 2 key words, "endoscopy" and "skull base"). Thus, new surgical corridors to approach especially midline skull base tumors have been described.

However, the exact role and indications of the endonasal endoscopic surgery are still being discussed in the absence of large or randomized series. Compared to intracranial microsurgical approaches, the endoscopic endonasal routes offer genuine benefits for pituitary surgery as well as for retrochiasmatic or midline clival tumors. For parasellar lesions, the endonasal endoscopic technique is also interesting to remove or to biopsy Meckel's cave lesions or tumors extending into the cavernous sinus. This will not change 
much the current management of cavernous sinus lesions where most of the time radiotherapy is the treatment of choice and for which no biopsy is required when imaging is typical.

Its use for anterior skull base tumors is more controversial. Indeed, the high percentage of CSF leaks, specially for meningiomas, and the absence of control of the lateral tumour margins raise interrogation about the real benefit of this endonasal approach. In their experience, the authors think that other mini-invasive approaches such as the supraorbital eyebrow one are likely to be more seductive.

To date, the endonasal endoscopic surgery provides interesting and complementary routes for skull base tumors but will not replace the standard intracranial approaches. Large multicentric studies with long-term follow up are being done to define precisely the real indications and benefits of such endonasal surgery. Technical refinements are also expected in a close future using 3D endoscope and robotics (Hanna et al., 2007; Levy et al., 2006; O'Malley \& Weinstein, 2007).

\section{References}

Alfieri A, Jho HD. (2001). Endoscopic endonasal cavernous sinus surgery: an anatomic study. Neurosurgery 48: 827-836; discussion 836-837.

Alfieri A, Jho HD. (2001). Endoscopic endonasal approaches to the cavernous sinus: surgical approaches. Neurosurgery 49: 354-360; discussion 360-362.

Alfieri A, Jho H, Tschabitscher M. (2002). Endoscopic endonasal approach to the ventral cranio-cervical junction: anatomical study. Acta Neurochir (Wien) 144: 219-225; discussion 225.

Alfieri A, Schettino R, Tarfani A, Bonzi O, Rossi GA, Monolo L. (2002). Endoscopic endonasal removal of an intra-suprasellar Rathke's cleft cyst: case report and surgical considerations. Minim Invasive Neurosurg 45: 47-51.

de Almeida JR, Zanation AM, Snyderman CH, Carrau RL, Prevedello DM, Gardner PA, et al. (2009). Defining the nasopalatine line: the limit for endonasal surgery of the spine. Laryngoscope 119: 239-244.

de Almeida JR, Snyderman CH, Gardner PA, Carrau RL, Vescan AD. (2011). Nasal morbidity following endoscopic skull base surgery: A prospective cohort study. Head Neck 33(4):547-51.

Balaker AE, Bergsneider M, Martin NA, Wang MB. (2010). Evolution of sinonasal symptoms following endoscopic anterior skull base surgery. Skull Base 20(4):245-51.

Batra PS, Citardi MJ, Worley S, Lee J, Lanza DC. (2005). Resection of anterior skull base tumors: comparison of combined traditional and endoscopic techniques. Am J Rhinol 19: 521-528.

Caicedo-Granados E, Carrau R, Snyderman CH, Prevedello D, Fernandez-Miranda J, Gardner P, et al. (2010). Reverse rotation flap for reconstruction of donor site after vascular pedicled nasoseptal flap in skull base surgery. Laryngoscope 120: 1550-1552.

Cappabianca P, Alfieri A, de Divitiis E. (1998). Endoscopic endonasal transsphenoidal approach to the sella: towards functional endoscopic pituitary surgery (FEPS). Minim Invasive Neurosurg 41: 66-73. 
Cappabianca P, Briganti F, Cavallo LM, de Divitiis E. (2001). Pseudoaneurysm of the intracavernous carotid artery following endoscopic endonasal transsphenoidal surgery, treated by endovascular approach. Acta Neurochir (Wien) 143: 9596.

Carrau RL, Kassam AB, Snyderman CH. (2001). Pituitary surgery. Otolaryngol. Clin. North Am 34: 1143-1155, ix.

Castelnuovo P, Dallan I, Pistochini A, Battaglia P, Locatelli D, Bignami M. (2007). Endonasal endoscopic repair of Sternberg's canal cerebrospinal fluid leaks. Laryngoscope 117: 345-349.

Castelnuovo P, Dallan I, Bignami M, Pistochini A, Battaglia P, Tschabitscher M. (2008). Endoscopic endonasal management of petroclival cerebrospinal fluid leaks: anatomical study and preliminary clinical experience. Minim Invasive Neurosurg 51: 336-339.

Castelnuovo P, Dallan I, Battaglia P, Bignami M. (2010). Endoscopic endonasal skull base surgery: past, present and future. Eur Arch Otorhinolaryngol 267: 649-663.

Caton R. (1893). Notes of a case of acromegaly treated by operation. Br Med J 2: 14211423.

Cavallo LM, Cappabianca P, Messina A, Esposito F, Stella L, de Divitiis E, et al. (2007). The extended endoscopic endonasal approach to the clivus and cranio-vertebral junction: anatomical study. Childs Nerv Syst 23: 665-671.

Cavallo LM, Prevedello DM, Solari D, Gardner PA, Esposito F, Snyderman CH, et al. (2009). Extended endoscopic endonasal transsphenoidal approach for residual or recurrent craniopharyngiomas. J Neurosurg 111: 578-589.

Charalampaki P, Ayyad A, Kockro RA, Perneczky A. (2009). Surgical complications after endoscopic transsphenoidal pituitary surgery. J Clin Neurosci. 16(6):786-9.

Cebula H, Lahlou A, De Battista JC, Debry C, Froelich S. (2010). [Endoscopic approaches to the orbit]. Neurochirurgie 56: 230-235.

Crockard HA. (1985). The transoral approach to the base of the brain and upper cervical cord. Ann R Coll Surg Engl 67: 321-325.

Cushing H.(1909). III. Partial Hypophysectomy for Acromegaly: With Remarks on the Function of the Hypophysis. Ann. Surg 50: 1002-1017.

Dandy W. (1926). Pneumocephalus (intracranial pneumatocoele or aerocoele). Arch Surg 12: 949-982.

Dandy W. (1932). Practice of surgery. Dans: The brain. Hagerstown; p. 247-252.

de Divitiis E, Cappabianca P, Cavallo LM. (2002). Endoscopic transsphenoidal approach: adaptability of the procedure to different sellar lesions. Neurosurgery 51: 699-705; discussion 705-707.

de Divitiis E, Esposito F, Cappabianca P, Cavallo LM, de Divitiis O, Esposito I. (2008). Endoscopic transnasal resection of anterior cranial fossa meningiomas. Neurosurg Focus 25(6):E8.

Dehdashti AR, Ganna A, Karabatsou K, Gentili F. (2008). Pure endoscopic endonasal approach for pituitary adenomas: early surgical results in 200 patients and comparison with previous microsurgical series. Neurosurgery. 62(5):1006-15; discussion 1015-7. 
D'Haens J, Van Rompaey K, Stadnik T, Haentjens P, Poppe K, Velkeniers B. (2009). Fully endoscopic transsphenoidal surgery for functioning pituitary adenomas: a retrospective comparison with traditional transsphenoidal microsurgery in the same institution. Surg Neurol. 72(4):336-40. Epub 2009 Jul 14.

Draf W. (1973). [Clinical value of sinus endoscopy (author's transl)]. Z Laryngol Rhinol Otol 52: 890-896.

Fernandez-Miranda JC, Gardner PA, Prevedello DM, Kassam AB. (2009). Expanded endonasal approach for olfactory groove meningioma. Acta Neurochir (Wien) 151: 287-288; author reply 289-290.

Frank G, Pasquini E. (2003). Approach to the cavernous sinus. Dans: Endoscopic endonasal transsphenoidal surgery. Vienna: de Devitiis E, Cappabianca P; p. 159-175.

Frank G, Pasquini E, Farneti G, Mazzatenta D, Sciarretta V, Grasso V, et al. (2006). The endoscopic versus the traditional approach in pituitary surgery. Neuroendocrinology 83: 240-248

Gardner PA, Kassam AB, Rothfus WE, Snyderman CH, Carrau RL. (2008). Preoperative and intraoperative imaging for endoscopic endonasal approaches to the skull base. Otolaryngol. Clin. North Am 41: 215-230, vii.

Gardner PA, Kassam AB, Snyderman CH, Carrau RL, Mintz AH, Grahovac S, Stefko S. (2008). Outcomes following endoscopic, expanded endonasal resection of suprasellar craniopharyngiomas: a case series. J. Neurosurg 109: 6-16.

Gardner PA, Prevedello DM, Kassam AB, Snyderman CH, Carrau RL, Mintz AH. (2008). The evolution of the endonasal approach for craniopharyngiomas. J. Neurosurg 108: 1043-1047.

Gondim JA, Almeida JP, Albuquerque LA, Schops M, Gomes E, Ferraz T, Sobreira W, Kretzmann MT. (2010). Endoscopic endonasal approach for pituitary adenoma: surgical complications in 301 patients. Pituitary. 2010 Dec 23. [Epub ahead of print]

Grant JA. (1996). Victor Darwin Lespinasse: a biographical sketch. Neurosurgery 39: 12321233.

Griffith AJ, Terrell JE. (1996). Transsphenoid endoscopic management of petrous apex cholesterol granuloma. Otolaryngol Head Neck Surg 114: 91-94.

Guiot G, Bouche J, Hertzog E, Vourc'h G, Hardy J. (1963). [Hypophysectomy by transsphenoidal route.]. Ann Radiol (Paris) 6: 187-192.

Guiot J, Rougerie J, Fourestier M, Fournier A, Comoy C, Vulmiere J, et al. (1963). [Intracranial endoscopic explorations.]. Presse Med 71: 1225-1228.

Hadad G, Bassagasteguy L, Carrau RL, Mataza JC, Kassam A, Snyderman CH, et al. (2006). A novel reconstructive technique after endoscopic expanded endonasal approaches: vascular pedicle nasoseptal flap. Laryngoscope 116: 18821886.

Hanna EY, Holsinger C, DeMonte F, Kupferman M. (2007). Robotic endoscopic surgery of the skull base: a novel surgical approach. Arch. Otolaryngol. Head Neck Surg 133: 1209-1214.

Har-El G. (2005). Endoscopic transnasal transsphenoidal pituitary surgery--comparison with the traditional sublabial transseptal approach. Otolaryngol. Clin. North Am 38: 723735 . 
Hardy J. (2010). [History of pituitary surgery]. Neurochirurgie 56: 358-362.

Herr HW. (2006). Max Nitze, the cystoscope and urology. J. Urol 176: 1313-1316.

Higgins TS, Courtemanche C, Karakla D, Strasnick B, Singh RV, Koen JL, et al. (2008). Analysis of transnasal endoscopic versus transseptal microscopic approach for excision of pituitary tumors. Am J Rhinol 22: 649-652.

Hirsch O. (1952). Successful closure of cerebrospinal fluid rhinorrhea by endonasal surgery. AMA Arch Otolaryngol 56: 1-12.

Jankowski R, Auque J, Simon C, Marchal JC, Hepner H, Wayoff M. (1992). Endoscopic pituitary tumor surgery. Laryngoscope 102: 198-202.

Jho HD, Carrau RL, McLaughlin ML, Somaza SC. (1996). Endoscopic transsphenoidal resection of a large chordoma in the posterior fossa. Case report. Neurosurg Focus 1: e3; discussion $1 p$ following e3.

Jho HD, Carrau RL. (1997). Endoscopic endonasal transsphenoidal surgery: experience with 50 patients. J. Neurosurg 87: 44-51.

Kassam A, Carrau RL, Snyderman CH, Gardner P, Mintz A. (2005). Evolution of reconstructive techniques following endoscopic expanded endonasal approaches. Neurosurg Focus 19: E8.

Kassam AB, Gardner P, Snyderman C, Mintz A, Carrau R. (2005). Expanded endonasal approach: fully endoscopic, completely transnasal approach to the middle third of the clivus, petrous bone, middle cranial fossa, and infratemporal fossa. Neurosurg Focus 19: E6.

Kassam AB, Mintz AH, Gardner PA, Horowitz MB, Carrau RL, Snyderman CH. (2006). The expanded endonasal approach for an endoscopic transnasal clipping and aneurysmorrhaphy of a large vertebral artery aneurysm: technical case report. Neurosurgery 59: ONSE162-165; discussion ONSE162-165.

Kassam AB, Thomas AJ, Zimmer LA, Snyderman CH, Carrau RL, Mintz A, et al. (2007). Expanded endonasal approach: a fully endoscopic completely transnasal resection of a skull base arteriovenous malformation. Childs Nerv Syst 23: 491-498.

Kassam AB, Gardner PA, Mintz A, Snyderman CH, Carrau RL, Horowitz M. (2007). Endoscopic endonasal clipping of an unsecured superior hypophyseal artery aneurysm. Technical note. J. Neurosurg 107: 1047-1052.

Kassam AB, Gardner PA, Snyderman CH, Carrau RL, Mintz AH, Prevedello DM. (2008). Expanded endonasal approach, a fully endoscopic transnasal approach for the resection of midline suprasellar craniopharyngiomas: a new classification based on the infundibulum. J. Neurosurg 108: 715-728.

Kassam AB, Prevedello DM, Carrau RL, Snyderman CH, Gardner P, Osawa S, et al. (2009). The front door to meckel's cave: an anteromedial corridor via expanded endoscopic endonasal approach- technical considerations and clinical series. Neurosurgery 64: 71-82; discussion 82-83.

Kassam AB, Prevedello DM, Carrau RL, Snyderman CH, Thomas A, Gardner P, Zanation A, Duz B, Stefko ST, Byers K, Horowitz MB. (2010). Endoscopic endonasal skull base surgery: analysis of complications in the authors' initial 800 patients. J Neurosurg. 2010 Dec 17. [Epub ahead of print]

Kassis S, De Battista JC, Raverot G, Jacob M, Simon E, Rabilloud M, Froehlich P, Trouillas J, Borson-Chazot F, Perrin G, Jouanneau E. (2009). [Endoscopy versus microsurgery: 
results in a consecutive series of nonfunctioning pituitary adenomas]. Neurochirurgie 55: 607-615.

Kono Y, Prevedello DM, Snyderman CH, Gardner PA, Kassam AB, Carrau RL, Byers KE. (2011). One thousand endoscopic skull base surgical procedures demystifying the infection potential: incidence and description of postoperative meningitis and brain abscesses. Infect Control Hosp Epidemiol. 32(1):77-83. Epub 2010 Dec 1.

Landolt AM. (2001). History of pituitary surgery from the technical aspect. Neurosurg. Clin. N. Am 12: 37-44, vii-viii.

Leng LZ, Anand VK, Hartl R, Schwartz TH. (2009). Endonasal endoscopic resection of an os odontoideum to decompress the cervicomedullary junction: a minimal access surgical technique. Spine 34: E139-143.

Levy ML, Nguyen A, Aryan H, Jandial R, Meltzer HS, Apuzzo MLJ. (2006). Robotic virtual endoscopy: development of a multidirectional rigid endoscope. Neurosurgery 59: ONS134-141; discussion ONS134-141.

Léger P. (2004). [Antonin Jean Desormeaux]. Prog. Urol 14: 1231-1238.

Lindholm J. (2007). A century of pituitary surgery: Schloffer's legacy. Neurosurgery 61: 865867; discussion 867-868.

Litynski GS. (1999). Endoscopic surgery: the history, the pioneers. World J Surg 23: 745753.

Liu JK, Das K, Weiss MH, Laws ER, Couldwell WT. (2001). The history and evolution of transsphenoidal surgery. J. Neurosurg 95: 1083-1096.

Magrini S, Pasquini E, Mazzatenta D, Mascari C, Galassi E, Frank G. (2008). Endoscopic endonasal odontoidectomy in a patient affected by Down syndrome: technical case report. Neurosurgery 63: E373-374; discussion E374.

Martin TJ, Loehrl TA. (2007). Endoscopic CSF leak repair. Curr Opin Otolaryngol Head Neck Surg 15: 35-39.

May M, Levine HL, Mester SJ, Schaitkin B. (1994). Complications of endoscopic sinus surgery: analysis of 2108 patients--incidence and prevention. Laryngoscope 104: 1080-1083.

Messerer M, De Battista JC, Raverot G, Kassis S, Dubourg J, Lapras V, Trouillas J, Perrin G, Jouanneau E. (2011). Evidence of improved surgical outcome following endoscopy for nonfunctioning pituitary adenoma removal. Neurosurg Focus. 30(4):E11.

Nayak JV, Gardner PA, Vescan AD, Carrau RL, Kassam AB, Snyderman CH. (2007). Experience with the expanded endonasal approach for resection of the odontoid process in rheumatoid disease. Am J Rhinol 21: 601-606.

Nyquist GG, Anand VK, Mehra S, Kacker A, Schwartz TH. (2010). Endoscopic endonasal repair of anterior skull base non-traumatic cerebrospinal fluid leaks, meningoceles, and encephaloceles. J. Neurosurg 113: 961-966.

O'Malley BW, Weinstein GS. (2007). Robotic skull base surgery: preclinical investigations to human clinical application. Arch. Otolaryngol. Head Neck Surg 133: 1215-1219. 
Patel MR, Shah RN, Snyderman CH, Carrau RL, Germanwala AV, Kassam AB, et al. (2010). Pericranial flap for endoscopic anterior skull-base reconstruction: clinical outcomes and radioanatomic analysis of preoperative planning. Neurosurgery 66: 506-512; discussion 512.

Pollock JR, Akinwunmi J, Scaravilli F, Powell MP. (2003). Transcranial surgery for pituitary tumors performed by Sir Victor Horsley. Neurosurgery 52: 914-925; discussion 925926.

Prevedello DM, Pinheiro-Neto CD, Fernandez-Miranda JC, Carrau RL, Snyderman CH, Gardner PA, Kassam AB. (2010). Vidian nerve transposition for endoscopic endonasal middle fossa approaches. Neurosurgery. 67(2 Suppl Operative):47884.

Ramachandran R, Singh PM, Batra M, Pahwa D. (2011). Anaesthesia for endoscopic endonasal surgery. Trends in Anaesthesia and Critical Care 1:79-83

Rathert P. (1967). Max Nitze (1848-1906). Invest Urol 5: 327-330.

Reisch R, Perneczky A, Filippi R. (2003). Surgical technique of the supraorbital key-hole craniotomy. Surg Neurol 59: 223-227.

Reuter M. (2000). The historical development of endophotography. World J Urol 18: 299302.

Rivera-Serrano CM, Oliver CL, Sok J, Prevedello DM, Gardner P, Snyderman CH, et al. (2010). Pedicled facial buccinator (FAB) flap: a new flap for reconstruction of skull base defects. Laryngoscope 120: 1922-1930.

Rivera-Serrano CM, Terre-Falcon R, Fernandez-Miranda J, Prevedello D, Snyderman CH, Gardner P, Kassam A, Carrau RL. (2010). Endoscopic endonasal dissection of the pterygopalatine fossa, infratemporal fossa, and post-styloid compartment. Anatomical relationships and importance of eustachian tube in the endoscopic skull base surgery. Laryngoscope. 120 Suppl 4:S244.

Robinson S, Patel N, Wormald PJ. (2005). Endoscopic management of benign tumors extending into the infratemporal fossa: a two-surgeon transnasal approach. Laryngoscope 115: 1818-1822.

Schaberg MR, Anand VK, Schwartz TH, Cobb W. (2010). Microscopic versus endoscopic transnasal pituitary surgery. Curr Opin Otolaryngol Head Neck Surg 18: 814.

Schwartz TH, Fraser JF, Brown S, Tabaee A, Kacker A, Anand VK. (2008). Endoscopic cranial base surgery: classification of operative approaches. Neurosurgery 62: 9911002; discussion 1002-1005.

Snyderman C, Kassam A, Carrau R, Mintz A, Gardner P, Prevedello DM. (2007). Acquisition of surgical skills for endonasal skull base surgery: a training program. Laryngoscope 117: 699-705.

Snyderman CH, Carrau RL, Kassam AB, Zanation A, Prevedello D, Gardner P, et al. (2008). Endoscopic skull base surgery: principles of endonasal oncological surgery. J Surg Oncol 97: 658-664.

Solares CA, Ong YK, Carrau RL, Fernandez-Miranda J, Prevedello DM, Snyderman CH, et al. (2010). Prevention and management of vascular injuries in endoscopic 
surgery of the sinonasal tract and skull base. Otolaryngol. Clin. North Am 43: 817-825.

Suriano M, De Vincentiis M, Colli A, Benfari G, Mascelli A, Gallo A. (2007). Endoscopic treatment of esthesioneuroblastoma: a minimally invasive approach combined with radiation therapy. Otolaryngol Head Neck Surg 136: 104-107.

Wigand ME. (1981). Transnasal ethmoidectomy under endoscopical control. Rhinology 19: 715. 


\title{
Endoscopic Surgery of Maxillary Sinuses in Oral Surgery and Implantology
}

\author{
Miroslav Andrić \\ University of Belgrade, Faculty of Dentistry \\ Serbia
}

\section{Introduction}

Due to the close anatomical relationship of the maxillary sinuses to the dentoalveolar complex, surgical procedures involving these anatomical structures represent a part of a everyday practice in oral surgery and implantology. Those interventions are directed mainly towards the treatment of odontogenic infections of the sinuses as well as surgical treatment of oroantral fistulae and odontogenic cysts and tumors of the upper jaw invading the antrum. Traditional approach under such circumstances is a Caldwell-Luc type of surgery, comprising osteotomy of the anterior sinus wall and creation of artificial opening of the sinus into the inferior nasal meatus. Although high success rates are reported, this kind of surgery may result in significant long-term complications, such as sclerosis of the antral walls, collapse of the sinus and creation of the postoperative cysts of maxilla (Nemec at al., 2009).

Also, widespread use of dental implants resulted in development of numerous techniques of alveolar bone augmentation in order to provide adequate amount of bone for implant placement. Many of those techniques involve the maxillary sinuses. However, complications of dental implant placement and augmentation procedures may include injuries to the maxillary sinuses, infections of the sinuses and displacement of augmentation material or implants themselves into the sinus. Again, the primary treatment of those complications includes surgery of the sinus, most commonly a Caldwell-Luc procedure.

Recent technological advances in field of endoscopy resulted in substantial improvement in techniques of endoscope-controlled surgery of nose and paranasal sinuses. Those techniques proved to be very useful in this area of complex anatomy and limited access to the surgical field. In particular, endoscopic surgery of the maxillary sinuses is widespread and has a long track of good results in ENT surgery. This is especially true for functional endoscopic sinus surgery (FESS). This technique, proved to be safe and effective for treatment of both chronic rhino-sinusitis and nasal polyposis (Chiu \& Kennedy, 2004), nowadays is widely accepted as a standard of care for patients who require surgical treatment of chronic rhino-sinusitis.

In contrast to this, surgical treatment of odontogenic diseases of the maxillary sinus still predominantly comprises traditional surgical techniques. Recently, several studies reported results of FESS for treatment of odontogenic sinusitis. Also, new techniques of endoscopeassisted surgery of odontogenic cysts and tumors were described. Finally, endoscope- 
controlled sinus augmentation and endoscopic treatment of implant-related complications involving the maxillary sinuses were reported in the literature.

Therefore, the aim of this article was to describe clinical problems encountered in this field, to briefly discuss indications for the surgery and to analyze available treatment options and criteria for selection of optimal treatment modality. Also, a literature review will present available data on endoscopic surgery for treatment of odontogenic diseases of the maxillary sinuses, as well as for sinus augmentation procedures and treatment of implant-related complications affecting the maxillary sinuses. Finally, the level of current scientific evidence on these issues will be evaluated.

\section{Odontogenic maxillary sinusitis}

Apart from more common rhinogenic sinusitis, some cases of maxillary sinus infections are related to odontogenic sources. This entity, designated as odontogenic maxillary sinusitis (OMS) accounts for approximately $10-12 \%$ of all cases of maxillary sinusitis (Mehra \& Murad, 2004).
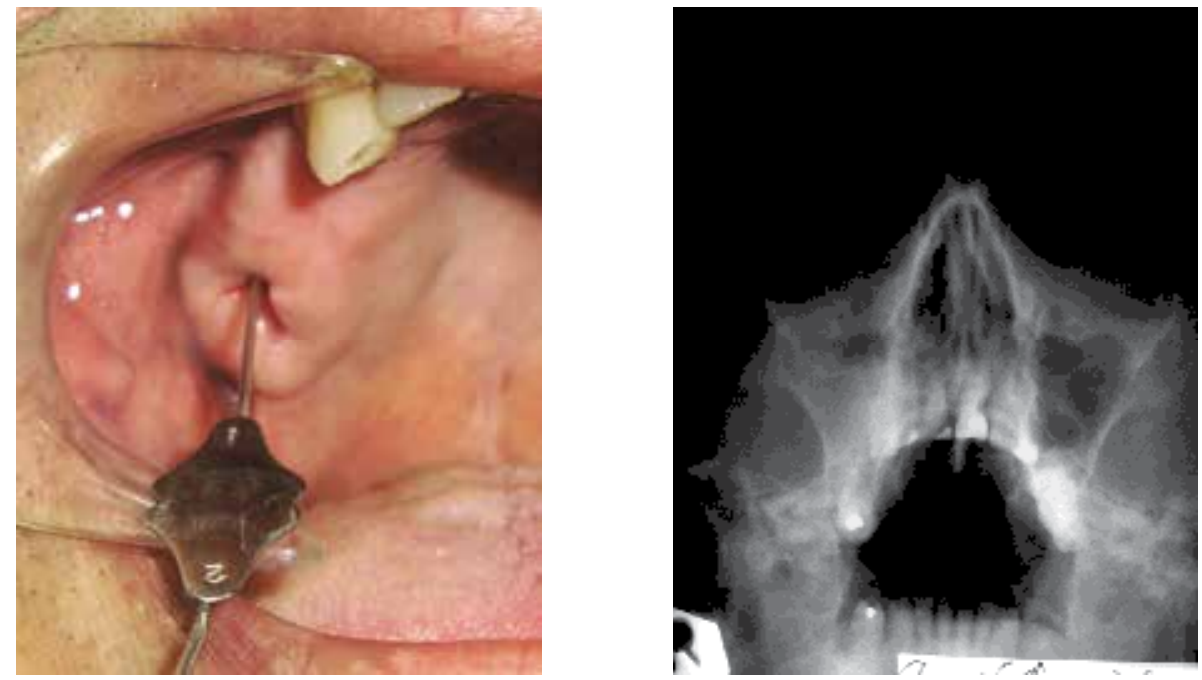

Fig. 1. a. Clinical photograph of a right oroantral fistula. A probe is inserted through the fistula into the sinus. $b$. Waters' view of the same patient indicating acute maxillary sinusitis.

The most common cause of OMS are oroantral fistulae (OAF) (Figures 1a and 1b) which are defined as pathological communications between the oral cavity and the maxillary sinus (Brook, 2006). Usually, they result from unsuccessful healing of Schneidarian membrane perforations that occur during tooth extractions and other surgical procedures involving alveolar processes of the upper jaw. In contrast to oroantral communications, which occasionally may heal spontaneously, most cases of OAF and subsequent maxillary sinusitis will require some kind of surgical treatment. This treatment varies from simple fistula closure by local flap to endoscopically assisted surgery of the maxillary sinus.

Besides oroantral fistulae, other common causes of OMS are chronic periapical or periodontal odontogenic infections, odontogenic cysts of the maxilla and iatrogenic factors, including placement of dental implants, sinus augmentations and intra-antral foreign bodies 
(Brook, 2006; Zimbler et al., 1998) (Figure 2). What is common for all these conditions is that disruption of the sinus membrane results in creation of a pathway through which oral microorganisms invade the antrum, resulting in sinus inflammation.
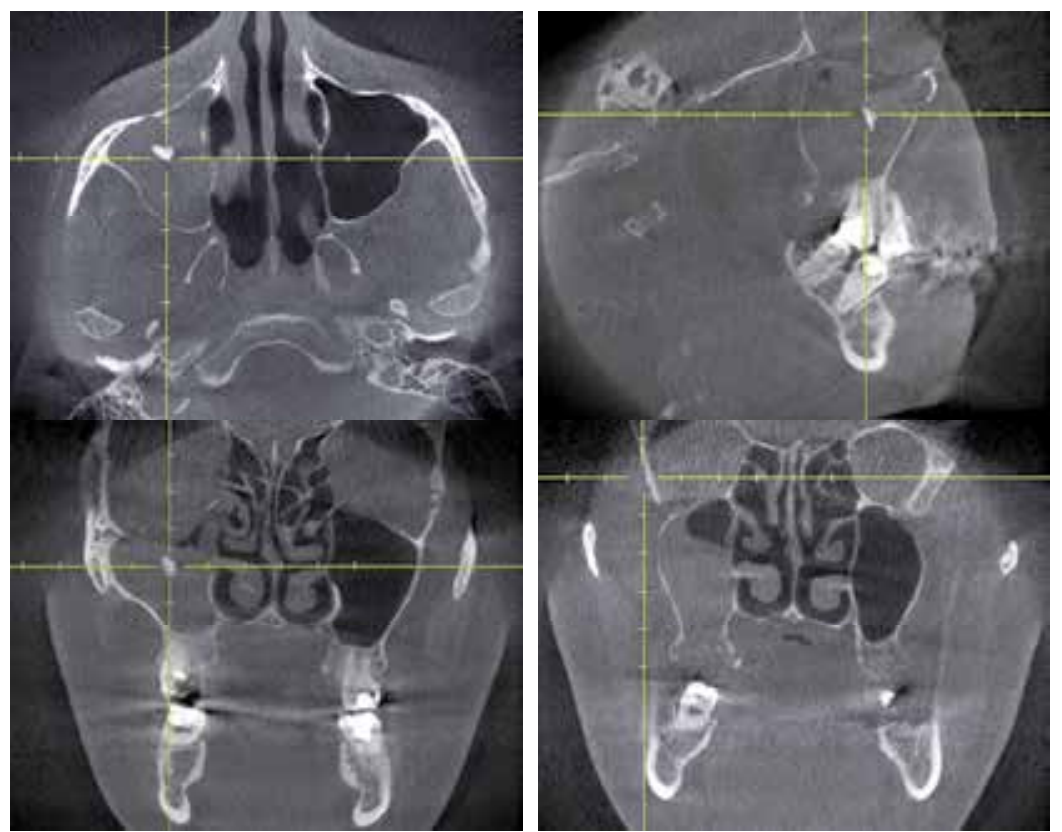

Fig. 2. Cone-beam CT of a patient with OAF and a root tip pushed into the sinus. Such situation is usually followed by pronounced inflammatory reaction.

Traditionally, OMS is treated by a Caldwell-Luc type of surgery (Guven, 1998) or by conservative procedures, including antibiotics and sinus irrigation, followed by surgical treatment of odontogenic source of infection (Dolanmaz et al., 2004). Caldwell-Luc operation is criticized as aggressive surgery with relatively high incidence of complications (De Freiatas \& Lucente, 1988; Ikeda et al., 1996). Most important of all, efficacy of inferior meatal antrostomy, typically performed in this kind of surgery, is doubtful, as the mucociliary clearance remains directed toward the natural sinus ostium in the middle meatus (Kennedy \& Shaalan, 1989). Car \& Juretić (1998) and Al-Belasy (2004), showed that inferior meatal antrostomy might be avoided, at least in some patients. Nevertheless, opening of the sinus on its anterior wall has to be performed, which results in permanent defect of anterior maxilla, sclerosis of the antral walls and collapse of the sinus cavity (Nemec at al., 2009). This kind of defect could complicate future prosthetic rehabilitation of these patients, regarding possible use of dental implants.

\section{Functional endoscopic sinus surgery for treatment of odontogenic sinusitis}

Principles of functional endoscopic sinus surgery (FESS) are based on actual understanding of sinus physiology, particularly regarding mucociliary clearance of the sinus. If effective drainage of the maxillary sinus is to be established, the optimal location to do so is at the 
level of the natural sinus ostium, in the middle nasal meatus. Therefore, FESS is directed towards establishment and preservation of osteomeatal complex patency. It aims to restore sinus ventilation and reestablish mucociliary clearance, which in turn results in resolution of sinus disease and maintenance of healthy sinus mucosa (Stammberger, 1986; Kennedy, 1985). It was demonstrated that such therapeutical approach is a viable option for treatment of both maxillary sinusitis and other inflammatory conditions affecting this anatomical structure (Busaba \& Kieff, 2002). In contrast to rhino-sinusitis, most cases of OMS are still treated by a Caldwell-Luc type of surgery. However, several studies were published, reporting results of FESS for treatment of chronic odontogenic sinusitis (Lopatin et al., 2002; Costa et al., 2007; Andric et al., 2010; Albu \& Baciut, 2010; Hajiioannou et al., 2010), which will be discussed in details later in this chapter.

\subsection{Surgical technique}

Endoscopic surgery of the maxillary sinuses is most commonly based on usage of rigid endoscopes, usually of $4.0 \mathrm{~mm}$ diameter. These endoscopes, providing different angles of vision, from 0 to 120 degrees, allow for good visualization of all parts of the osteomeatal complex, sinus ostium and sinus itself. Also, a set of specially designed surgical instruments includes different biting and grasping forceps, cutting knives and microdebriders.

This kind of surgery is usually done with patient under the general anesthesia. If possible, carefully controlled hypotension is useful to minimize bleeding during the procedure (Eberhart et al., 2007) since it was shown that pronounced intraoperative bleeding correlates with higher failure rates in endoscopic sinus surgery (Albu \& Baciut, 2010). Also, infiltration of surgical field with local anesthetic solution containing epinephrine is very helpful for this purpose.

Procedures starts with careful medial dislocation of the middle turbinate which allows good visualization of the middle nasal meatus, uncinate process and ethmoidal bulla. Uncinate process is cut by a sickle-knife in a direction parallel to its upper edge and removed by grasping forceps (Figures 3a, 3b and 3c).

After that, natural sinus ostium is identified and subsequently enlarged in a postero-inferior direction. Ostium is enlarged to the size that should allow access to the sinus with appropriate instruments and also effective drainage of the sinus after the surgery (Figure 4). At this point care must be taken, as extremely anterior enlargement of the ostium might result in an injury to the nasolacrimal duct. Through this enlarged ostium it is possible to inspect entire maxillary sinus, particularly when angled-view endoscopes are used (Figure 5). Also, using long curved antrum forceps and suction tips, most of intra-antral pathology and foreign bodies can be removed by this approach too.

What is specific for odontogenic sinusitis is that most of mucosal inflammation, as well as majority of foreign bodies of dental origin, are located in the alveolar recess of the sinus. Depending on local anatomy and position of sinus ostium, access to this part of the sinus might be difficult, even when large middle meatal antrostomy is performed. Still, in cases with OAF it is possible to reach alveolar recess through the fistula itself. Beside this, additional small puncture of the sinus in canine fossa, with an endoscope introduced through it, can provide better visualization of this part of the sinus. However, in most of the cases, angled view endoscopes will ensure good visual control in the entire sinus, even through middle meatal antrostomy. 

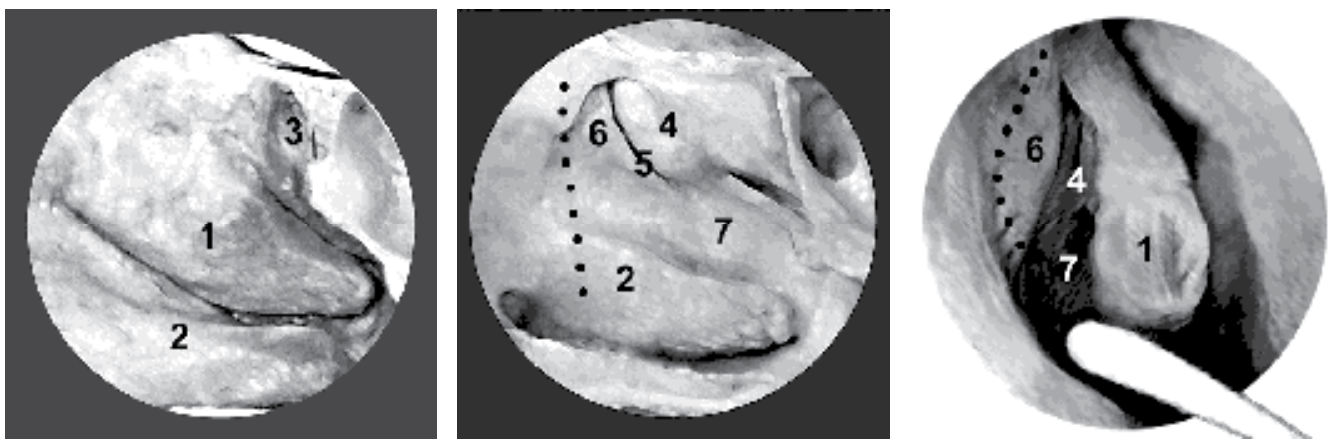

Fig. 3. Schematic presentation of the right lateral nasal wall. a. Middle turbinate (1), inferior turbinate (2) and spheno-ethmoidal recess (3) are seen. b. Upon removal of the middle turbinate, middle nasal meatus (7) is visible. Semilunar hiatus (5) is bounded by ethmoidal bulla (4) and uncinate process (6). Aperture of the maxillary sinus can not be seen, as it is located behind these structures. Dotted line illustrates path of the nasolacrimal duct. c. The same anatomic structures as seen through a $0^{\circ}$ endoscope. Middle turbinate (1) is dislocated medially by a Freer elevator. Dotted line represents line of resection of the uncinate process (6). Previously published in ref: Andric et al, 2010.

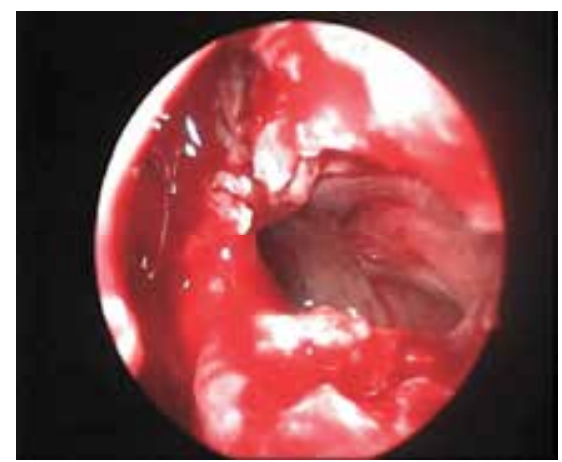

Fig. 4. A view of the enlarged natural ostium of the left maxillary sinus ( $0^{\circ}$ endoscope).

Previously published in ref: Andric et al, 2010.

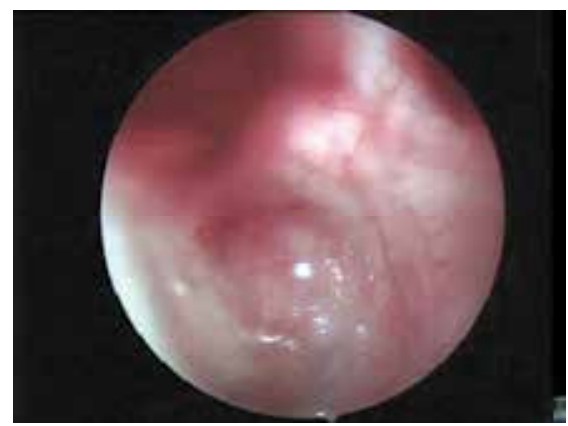

Fig. 5. A view of the alveolar recess of the maxillary sinus through the enlarged ostium $\left(70^{\circ}\right.$ endoscope). Previously published in ref: Andric et al, 2010. 
The most important advantage of FESS, compared to other treatment options for odontogenic sinusitis, is a possibility to surgically treat inflammation of other paranasal sinuses as well, in particular that of anterior ethmoidal cells. Residual inflammation in this area is one of common causes of failure in treatment of odontogenic sinusitis. Therefore, if necessary, removal of ethmoidal bulla and opening of the anterior ethmoidal cells may be performed too (Figures $6 a$ and $6 b$ ).
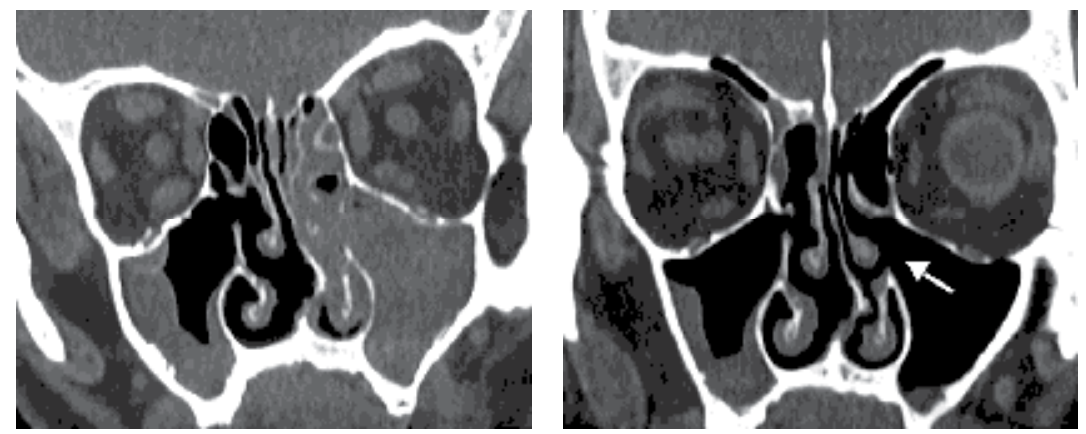

Fig. 6. a. Coronal CT scan of odontogenic sinusitis demonstrating pronounced inflammation of left maxillary sinus and anterior ethmodial cells. $b$. Six months postoperatively reduction in extent of sinus inflammation, as well as resection of the uncinate process (arrow) are clearly visible. Previously published in ref: Andric et al, 2010.

Finally, successful treatment of odontogenic sinusitis is based on efficient elimination of odontogenic source of infection. Therefore, closure of oroantral fistula (if present) and extraction of causative teeth should be performed in the same surgical act.

\subsection{Literature data}

Recently, several authors reported results of FESS for treatment of chronic odontogenic sinusitis (Lopatin et al., 2002; Costa et al., 2007; Andric et al., 2010; Albu \& Baciut, 2010; Hajiioannou et al., 2010). Although there were some technical differences, all studies have reported essentially the same surgical procedure, comprising middle meatal antrostomy, access to the maxillary sinus through enlarged natural sinus ostium and endonasal approach for removal of intra-antral foreign bodies, such are root tips and dental implants. The most important results of these studies are summarized in table 1.

Lopatin (Lopatin et al., 2002) was the first who reported 70 cases of odontogenic sinusitis treated by endoscopic sinus surgery. Apart from 39 cases with OAF, he presented 10 cases with odontogenic cysts and 6 with fungal balls in the sinuses. Also, he presented 21 cases with foreign bodies inside the sinus. Surgical technique included usage of microdebrider, resection of the uncinate process and removal of ethmoidal bulla. Removal of foreign bodies was accomplished by curved suction tip through enlarged sinus ostium. Authors commented on difficulty in reaching alveolar recess of the sinus through middle meatal antrostomy. In initial cases they used additional puncture in canine fossa, and later on, access to alveolar recess was established through OAF itself. Out of 70 cases, after the follow-up period of up to three years, there were 4 failures. In 3 patients recurrence of OAF was noticed and in one patient stenosis of antrostomy resulted in recurrent sinusitis. Besides facial edema and nasal discharge, which were described as less pronounced compared to Caldwell-Luc procedure, no other complications were reported. 


\begin{tabular}{|c|c|c|c|c|c|c|}
\hline & $\begin{array}{l}\text { Total } \\
\text { cases } \\
(\mathrm{n})\end{array}$ & $\begin{array}{c}\text { Cases } \\
\text { with OAF } \\
\text { (n) }\end{array}$ & $\begin{array}{c}\text { OAF } \\
\text { reccurence } \\
\mathrm{n}(\%)\end{array}$ & $\begin{array}{l}\text { Revision } \\
\text { surgery } \\
\mathrm{n}(\%)\end{array}$ & $\begin{array}{l}\text { Follow up } \\
\text { period }\end{array}$ & $\begin{array}{l}\text { Overall sucess } \\
\text { rate } \\
\mathrm{n} / \text { all cases }(\%)\end{array}$ \\
\hline Lopatin et al., 2002 & 70 & 39 & $3(8 \%)$ & $3(4 \%)$ & $1-3 y r$ & $66 / 70(95 \%)$ \\
\hline Costa et al., 2007 & 17 & 5 & No & No & $6 \mathrm{mo}-2 \mathrm{yr}$ & $17 / 17(100 \%)$ \\
\hline Andric et al., 2010 & 14 & 14 & No & No & $6 \mathrm{mo}-2 \mathrm{yr}$ & $14 / 14(100 \%)$ \\
\hline $\begin{array}{l}\text { Albu \& Baicut, } \\
2010\end{array}$ & 104 & 30 & $4(13 \%)$ & $9(9 \%)$ & Min. 6 mo & $95 / 104(91 \%)$ \\
\hline $\begin{array}{l}\text { Hajiioannou et al., } \\
2010\end{array}$ & 4 & 4 & No & No & $6 \mathrm{mo}-3 \mathrm{yr}$ & $4 / 4(100 \%)$ \\
\hline
\end{tabular}

Table 1. Results of clinical studies on FESS for treatment of odontogenic sinusitis. Mo months, Yr - years. Previously published in ref: Andric, 2010.

Costa (Costa et al., 2007) presented 17 cases of OMS, including 5 OAF, 7 cases with odontogenic cysts, 2 cases of sinusitis related to placement of dental implants and 3 patients with root-canal sealing materials pushed into the sinus. Again, microdebrider was used and access to alveolar recess was obtained through OAF. In cases with odontogenic cysts, a bone window in the anterior sinus wall was created for removal of the cyst, while middle meatal antrostomy was performed to enhance resolution of corresponding sinus inflammation. After a 2-years follow up period, good long - term results (defined as absence of sinusitis symptoms and healing of OAF) were obtained in all cases. In one case nasal synechiae were observed postoperatively, which was corrected under the local anesthesia.

Another retrospective study (Andric et al., 2010) reported results of FESS in 14 patients with OAF and corresponding OMS. Similarly to previous studies, middle meatal antrostomy was performed by resection of the uncinate process, and most of instrumentation inside the sinus was accomplished through the enlarged sinus ostium, including removal of intra-antral foreign bodies. Closure of OAF was achieved by Rehrmann's buccal advancement flap or buccal fat pad. During the follow up period of up to two years, clinical examination and control CT scans showed good results in all cases. No significant complications were reported.

The only prospective study on this issue included 104 patients with OMS and 307 with rhinogenic sinusitis (Albu \& Baciut, 2010). It is of interest that no statistically significant differences in failure rates was noted comparing these two groups of patients $(7 \%$ in odontogenic vs. $9 \%$ in rhinogenic group). Still, somewhat higher failures rates $(13 \%)$ were noticed in cases with OAF. Surgical technique was similar to other studies, including puncture of the sinus in the canine fossa for better visualization of the alveolar recess. Again, there were not significant intraoperative and postoperative complications.

Finally, in a case series from Hajiioannou, four cases of odontogenic sinusitis with OAF were successfully treated by endoscopic sinus surgery and fistula closure by synthetic 
surgical glue and buccal advancement flap (Hajiioannou et al., 2010). Also, retrospective analysis of 27 cases of odontogenic sinusitis revealed that most common causes of this condition were complications of dental implants placement and tooth extraction. Treatment included transnasal endoscopic sinus surgery in 19 (70.4\%) patients, Caldwell-Luc operation in two $(7.4 \%)$ patients and only antibiotics in $4(14.8 \%)$ cases. During the follow-up period of two to six months, no recurrences were recorded (Lee, KC. \& Lee, SJ., 2010).

In summary, all these studies reported essentially the same surgical procedure, applying principles of FESS for treatment of rhinogenic sinusitis in patients with OMS. In all cases middle meatal antrostomy was performed which presents substantial difference to Caldwell-Luc procedure. It is interesting that most of the surgeons, at least in some cases, used additional puncture of the sinus in canine fossa, creating better access to the alveolar recess of the sinus. This is very specific for treatment of odontogenic sinusitis in which most of the intra-antral pathology is located in the lower third of the sinus. Still, it was also demonstrated that access to this part of the maxillary sinus can be achieved through oroantral fistula, if present.

It is important that practically no significant intraoperative or postoperative complications occurred in any case. Although endoscopic sinus surgery might result in broad range of major and minor complications (McMains, 2008), rare occurrence of complications in the treatment of odontogenic maxillary sinusitis might be related to the relatively limited extent of endo-nasal surgery, compared to the treatment of rhinosinusitis and nasal polyps.

Although all studies presented very good short to mid-term results (follow up period lasted up to three years), there is a lack of objective measures of improvement in patients condition after the surgery. All studies reported lack of symptoms and subjective patients' perception of improvement as outcome measures. In one study (Andric et al., 2010) control CT scans were performed, but without attempt to use some scoring system of CT findings in sinusitis. The only result which can be objectively validated is recurrence rate of OAF. It ranged from 0 to $13 \%$ which is similar or somewhat higher comparing to Caldwell-Luc procedure (Guven, 1998; Yilmaz et al., 2003). Still, it has to be addressed that closure of OAF is a delicate procedure, requiring vast experience specific to this kind of surgery. Although it is a common procedure for oral and maxillofacial surgeons, some of otorhinolaryngologists rarely perform this intervention. In fact, in a study with highest recurrence rate reported (Albu \& Baciut, 2010), authors were able to demonstrate "learning curve" since three recurrences occurred in the first 15 cases and only one in remaining patients.

Finally, it can be concluded that use of FESS for treatment of OMS is clinically well documented procedure with good results and low incidence of complications. Still, from the scientific point of view, the main shortcoming of all these studies is that results of FESS are not compared with results of some traditional treatment options for odontogenic sinusitis, such is Caldwell-Luc procedure. Therefore, prospective and randomized studies are needed to establish efficacy of this kind of treatment, as well to provide criteria for individual selection of most suitable treatment of odontogenic sinusitis.

\section{Endoscopic surgery for treatment of odontogenic cysts and tumors}

The most common odontogenic cysts and tumors involving the upper jaw include periapical and dentigerous cysts, keratocystic odontogenic tumors (previously known as odontogenic keratocysts) and ameloblastomas. Their expansive growth within the upper jaw might result in destruction of the bony walls of the sinus and subsequent sinus infection (Figure 7). 
Clinical course of these lesions tends to be relatively asymptomatic and it is not uncommon that symptoms of maxillary sinusitis are among the first signs indicating the presence of an odontogenic cyst or tumor.

Selection of the most appropriate surgical treatment is based on hystological type of the lesion, but also having in mind its size and relationship to the neighboring anatomical structures, including maxillary sinuses. Inflammatory periapical and residual cysts, as well as dentigerous cysts are effectively treated by simple enucleation and extraction or endodontic treatment of the causative tooth. On the other hand, keratocystic odontogenic tumors (KCOT) and ameloblastomas require more aggressive type of surgery due to infiltrative growth and high recurrence rates. Still, while most of ameloblastomas of the upper jaw are treated by partial maxillectomy, surgical options for KCOT include decompression followed by complete enucleation, enucleation in combination with Carnoy solution or cryosurgery and, finally, resection of the involved jaw (Ghali \& Connor, 2003).
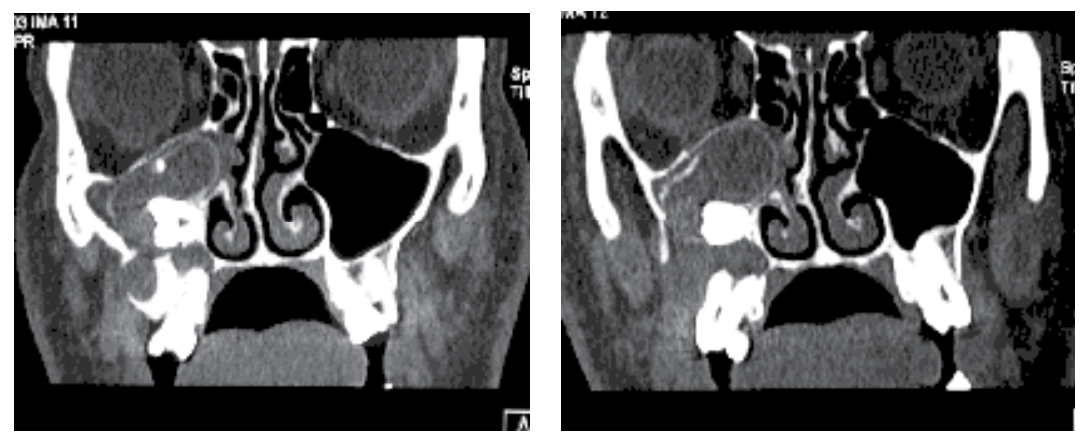

Fig. 7. Coronal CT scan of keratocystic odontogenic tumor of the right maxilla invading the maxillary sinus and resulting in secondary inflammation of antral mucosa.

In cases of odontogenic cysts or tumors occupying the sinus, common surgical approach includes removal of the lesion by a Caldwell-Luc type of surgery. Still, while such procedure results in permanent defect of anterior maxilla, visualization of the posteroinferior part of the upper jaw (which is a common site of occurrence of odontogenic lesions) might be difficult even when large antrostomy in the canine fossa is performed. Endoscopeassisted surgery for those lesions has a potential to provide better overview of the surgical field and still to allow less aggressive surgical approach.

Few articles were published describing techniques of endoscope-assisted removal of odontogenic cysts from the maxillary sinus. Cedin (Cedin et al., 2005) presented 4 cases of periapical cysts with oroantral fistulae, occupying alveolar recess of the sinus. Surgical technique comprised combined approach through canine fossa and inferior meatal antrostomy, cystectomy under the endoscopic control and closure of oroantral fistula by local flaps. Successful closure of OAF was obtained in all cases and there were no recurrences during the 2-years follow- up period.

In a case-series including 10 periapical and 3 dentigerous cysts (Seno et al., 2009), after partial resection of anterior portion of the inferior turbinate, inferior meatal antrostomy was performed to gain access to the alveolar recess and to allow removal of the cyst. Interestingly, although authors stated that in five cases of periapical cysts only partial resection of the cystic wall was performed, in a follow-up period ranging from 11 to 72 
months no recurrences were noted. Also, several case reports were published, describing similar surgical techniques (Micozkadioglu \& Erkan, 2007; Lamb et al., 2009; Di Pasquale \& Shermetaro, 2006; Hasbini et al., 2001; Christmas et al., 2008).

It is interesting that in this studies access to the sinus was obtained through inferior nasal meatus, which reflects difficulties in reaching inferior portion of the maxillary sinus through middle meatal antrostomy. Such difficulties are best described in an article from Lamb and coworkers (Lamb et al., 2009), who reported that attempt to remove odontogenic cyst and the tooth from the sinus through middle meatal antrostomy was unsuccessful, so conversion to Caldwell-Luc approach had to be performed. On the other hand, several authors reported successful removal of dentigerous cysts through middle meatal antrostomy (Di Pasquale \& Shermetaro, 2006; Hasbini et al., 2001; Christmas et al., 2008). As an alternative technique, Costa (Costa et al., 2007) used intraoral approach for removal of the cyst and middle meatal antrostomy was performed to treat corresponding sinusitis.

At this point, it seems reasonable to use inferior meatal antrostomy for removal of cysts from the sinus, if patency of natural sinus ostium is preserved. If not, widening of the natural sinus ostium, according to FESS principles, should enhance resolution of sinus inflammation. However, there is some concern that simultaneous persistence of more than one opening of the sinus might actually decrease its drainage, due to "circular flow" phenomenon (Coleman \& Duncavage, 1996). Still, in a prospective study including 72 patients with severe maxillary sinusitis (as determined by Lund-Mackay CT score), combined inferior and middle meatal antrostomy shown superior results in reduction of sinus inflammation, compared to middle meatal antrostomy alone (Albu et al., 2011).

Regarding odontogenic tumors, several cases of endoscopic surgery for treatment of ameloblastomas of the upper jaw were reported (London \& Schlosser, 2002; Bray et al., 2007; Leong et al., 2010). Although in most of them entire tumor was removed by an endoscopic approach, a technique of combined maxillectomy and endoscopic ethmoidectomy was described, too (Bray et al., 2007).

Also, few cases of fibro-osseous lesions involving ethmoidal region were presented (Akao et al 2003, Cansiz et al 2004, Lopatin and Kapitanov 2005). In all of them transnasal endoscopic approach was used with variable success, since in one case only reduction of tumor was achieved which required additional surgery for complete removal of the lesion (Akao et al, 2003).

At this point it has to be noted that follow up periods for cases of odontogenic tumors were variable in these reports, which rises the question of possible recurrences. While an average time for diagnosis of ameloblastoma recurrence is approximately 5 years, late recurrences are well documented in the literature (Carlson \& Marx, 2006). Therefore, long term followup, as well as prospective studies comparing endoscopic and traditional surgical techniques for removal of odontogenic tumors are needed.

\section{Endoscopic sinus surgery and dental implant treatment}

An increasing number of dental implants is placed in posterior parts of the upper jaw. Although advances in surgical techniques and implant design resulted in high success rates, complications are still encountered, many of them involving maxillary sinuses. Such complications include creation of oroantral fistulae, sinus infections and displacement of implants or grafting material into the sinus. Also, in unfavorable anatomical situations, sinus augmentation procedures are needed prior or simultaneously to implant placement. 
Recently, endoscopic techniques were described, both for treatment of sinus related complications of dental implants and for sinus augmentation.

\subsection{Endoscopic surgery for treatment of implant-related complications}

Occasionally, displacement of implants into the sinus may occur, either during implant insertion or during functional loading period. Although some of cases may remain asymptomatic, such situation usually results in inflammation of the corresponding sinus, obviously requiring some kind of treatment. Even in asymptomatic cases, removal of displaced implant should not be postponed, since it may result in migration of implant into distant spaces, such are nasal cavity (Kitamura, 2007), sphenoid sinus (Felisati et al., 2007), orbit (Griffa et al., 2010) or even anterior cranial fossa (Cascone et al., 2010).

Once the diagnosis has been made, surgical procedure for implant removal may include opening of the anterior sinus wall via Caldwell-Luc approach, or endoscopic sinus surgery. In available literature, two endoscopic techniques for removal of implants were described, creating access to the sinus through canine fossa or through middle meatal antrostomy.

Nakamura (Nakamura et al., 2004) reported endoscopic removal of displaced implant through a $10 \times 10 \mathrm{~mm}$ window in the anterior sinus wall, using urological retrieval basket. Similar technique was described by Varol and colleagues in a series of 3 cases, except that curved hemostat was used for implant removal (Varol et al., 2006). Also, it was shown that endoscopic removal of implant from the sinus can be successfully combined with simultaneous sinus lift procedure (Ucer, 2009), facilitating future placement of a new implant. What is common for all these reports is that essentially conventional approach through canine fossa was used, but due to the use of endoscopes much smaller opening of the sinus wall had to be performed, compared to traditional Caldwell-Luc procedure.

Another possibility is to remove the implant by transnasal approach, usually through middle meatal antrostomy (Ramotar et al., 2010; Lubbe 2008). Such approach is particularly indicated if concomitant sinusitis is present, since it provides both an opportunity to remove displaced implant and to improve ventilation and drainage of affected sinus (Kim et al., 2007). Still, actual position of an implant has to be determined when decision regarding access through canine fossa or middle meatal antrostomy is to be made. In a case report from El Charkawi et al. (2005), it was shown that when an implant was in a more anterior and medial position in the maxillary sinus, transnasal access was unsuccessful so a Caldwell-Luc approach had to be used. Besides this, a clinician should have in mind that position of an implant within the sinus might change in a short period of time, so once appropriate radiographs are taken, surgery should not be delayed.

In a retrospective multi-center report of 27 patients with implants displaced into the sinus, Chiapasco proposed a treatment protocol based on presence of sinusitis and oroantral communication (OAC). In 17 patients who didn't have signs of sinusitis and in whom patency of the sinus ostium was preserved, intraoral approach through canine fossa was used for retrieval of implant and closure of OAC. In 6 patients who presented with maxillary sinusitis and obstruction of the sinus ostium, but without OAC, treatment consisted of partial uncinectomy, middle meatal antrostomy and removal of implant through enlarged ostium. Finally in 4 cases with sinusitis, ostium obstruction and oroantral communication, operative procedure comprised combined FESS and intraoral approach for closure of OAC by buccal advancement flap (Chiapasco et al., 2009). 
It seems that in cases of sinus related complications in implantology, several factors have to be considered before the decision regarding the most suitable treatment option can be made. Chronic sinusitis with obstruction of the sinus ostium probably should be treated by FESS, and middle meatal antrostomy can be used for removal of foreign bodies, including implants and grafting material, as well. In situations with no or minimal signs of sinusitis and with preserved patency of the ostium, access via canine fossa is simple and effective for retrieval of displaced implants. When such an intervention is performed under the endoscopic control, it is possible to create significantly smaller bony window in the sinus wall, which should provide uneventful healing of the defect, without long-term effect on the corresponding sinus health. Finally, in a study from Aimeti and colleagues, it was shown that inferior meatal antrostomy and insertion of nasosinus tube might be useful in treatment of sinus lift complications (Aimeti et al, 2001).

\subsection{Endoscopic surgery for sinus augmentation procedures}

Reduction in hight of residual alveolar ridge of posterior maxilla might be the result of either increased pneumatization of the upper jaw or pronounced resorption of alveolar process following tooth extraction. Whatever is the reason, such situation must be resolved before placement of implants is considered. Regarding this, several sinus augmentation techniques are used, comprising crestal or lateral approach to the sinus. Although these techniques are well established, use of endoscopes has a potential to further improve results of this procedures.

A prospective study on osteotome sinus floor elevation under the endoscopic control was published in 2002 (Nkenke at al., 2002). In 14 patients a total of 22 implants were placed and the endoscopes with view angles of 70, 90 and 120 degrees were inserted through the canine fossa. After sinus floor elevation with appropriate osteotomes, $\beta$-tricalcium phosphate or autogenous bone from the retromolar region were used for sinus augmentation. At a second stage surgery, 6 months after the implant placement, follow-up sinusoscopy was performed. Out of 22 implant sites, perforation on sinus membrane was noted in one case, which was immediately repaired by periosteal patch. In all patients postoperative course was uneventful, without signs of sinusitis. However, at a second stage surgery mobility of 2 implants was noted (in a same patient who had sinus perforation) and implants were removed, while control sinusoscopy revealed migration of grafting material into the sinus. Also, in one patient polyposis of antral mucosa was noted in control endoscopy, but the implants were stable and the patient was free of symptoms, so no further treatment was performed. Authors commented on complexity of procedure and extended length of surgery (average time of surgery was 67 minutes), so they concluded that such approach is more of scientific than clinical interest.

Berengo and colleagues reported results of endoscopic controlled osteotome sinus floor elevation in 8 patients in whom 16 Osseotite implants were placed (Berengo et al., 2004). Endoscopes were introduced through a $4 \mathrm{~mm}$ diameter opening in the canine fossa and BioOss ${ }^{\circledR}$ particles were used for sinus augmentation. Lacerations of sinus membrane with minimal displacement of grafting material were noted in 2 cases. Authors commented on pattern of sinus membrane distension and concluded that it is possible that mucosal lacerations are more likely to occur when detachment of sinus membrane is confined to the small area around the implant site in contrast to situations when mucosa is elevated on the broader base. It is important that after a 14 months follow-up period all implants were 
considered to be successful, including two cases in whom membrane lacerations had occurred. Also, no signs of sinusitis were noted in these cases.

Apart from endoscope-controlled osteotome sinus lift procedure, a technique of minimally invasive lateral-window sinus augmentation was described by Engelke and colleagues (Engelke \& Deckwer, 1997; Engelke et al., 2003). Basically, surgical technique comprised only a small $5 \mathrm{~mm}$-diameter osteotomy at the lateral sinus wall, through which elevation of sinus membrane and placement of augmentation material was performed under the control of a 2,7 mm-diameter endoscope. A total of 118 sinus augmentations were performed in 83 patients and 211 implants were placed, most of them simultaneously with augmentation procedure. Perforation of sinus membrane was noted in 28 cases, which were repaired immediately using polyglactine mesh except of 1 case with large perforation, who required second surgical intervention. Postoperatively, one case of wound dehiscence was noted, resulting in creation of oroantral fistula and concomitant sinusitis. Out of 211 implants, 11 implants were lost, most of them before the prosthetic loading (Engelke et al., 2003). Same author (Engelke \& Capobianco, 2005) described technique of flapless sinus augmentation and simultaneous implant placement using three-dimensional surgical template. Twenty one implants were placed in six patients using this approach. One case of sinus perforation was noted and out of 21 implants one failure occurred during the healing period.

Based on pertinent literature, it seems that endoscope-controlled sinus augmentation yields similarly good results compared to conventional approach. However, need for expensive equipment and prolonged time of surgery are the factors that have to be considered when discussing this issue. Also, regarding minimally invasive lateral window augmentation, prospective study comparing this technique to the traditional surgical procedure will be necessary before its clinical use can be recommended.

\section{Conclusion}

Functional endoscopic sinus surgery for treatment of odontogenic sinusitis seems as a safe and predictable treatment option. Although scientific evidence is low (since there are not any studies comparing FESS to other treatment possibilities), this is a clinically well documented procedure with low incidence of complications. Also, it has to be noted that criteria for selection of optimal treatment modality for specific clinical situations are not established. Regarding this, FESS might be particularly indicated in cases of odontogenic sinusitis with severe ethmoidal inflammation and/or obstruction of sinus ostium. On the other hand, if patency of osteomeatal complex is preserved, which is not a rarity with odontogenic sinusitis, it is questionable if middle meatal antrostomy is of any benefit for the patient. Still, available scientific data are not sufficient to answer these questions.

In contrast to odontogenic sinusitis, endoscopic surgery for odontogenic cysts and tumors is documented only in limited case series reports, so at this moment it is difficult to discuss possible advantages and shortcomings of such procedures. However, it is clear that endoscope-assisted removal of these lesions should provide better visualization of the surgical field which is very important for prevention of recurrences. Until more scientific data are available, traditional treatment options with endoscopic control of poorly visualized regions (such is postero-inferior part of the sinus) can be recommended.

Also, regarding treatment of implant-related complications involving the maxillary sinus, endoscopic surgery has a potential to provide effective treatment of those cases, similarly to treatment of odontogenic sinusitis of non-implant etiology. On the other hand, endoscopic 
sinus augmentation needs scientific evidence of superiority to conventional techniques before it can be introduced into clinical practice.

\section{Acknowledgment}

This work was supported by grant no. 175021 from The Ministry of science of Republic of Serbia.

\section{References}

Aimetti, M., Romagnoli, R., Ricci, G. \& Massei, G. (2001). Maxillary sinus elevation: the effect of macrolacerations and microlacerations of the sinus membrane as determined by endoscopy. The International journal of periodontics $\mathcal{E}$ restorative dentistry, Vol.21, No.6, (December 2001), pp. 581-589, ISSN 0198-7569

Akao, I., Ohashi, T., Imokawa, H., Otsuka, T., Taguchi, Y. \& Takagi, M. (2003). Cementifying fibroma in the ethmoidal sinus extending to the anterior cranial base in an 11-yearold girl: a case report. Auris Nasus Larynx, Vol.30, Suppl.1, (15 February 2003), pp. 123-126, ISSN 0385-8146

Al-Belasy, FA. (2004). Inferior meatal antrostomy: is it necessary after radical sinus surgery through the Caldwell-Luc approach? Journal of oral and maxillofacial surgery : official journal of the American Association of Oral and Maxillofacial Surgeons Vol.62, No.5, (May 2004), pp. 559-562, ISSN 0278-2391

Albu, S. \& Baciut, M. (2010). Failures in endoscopic surgery of the maxillary sinus. Otolaryngology--head and neck surgery: official journal of American Academy of Otolaryngology-Head and Neck Surgery, Vol.142, No.2, (February 2010), pp. 196-201, ISSN 0194-5998

Albu, S., Gocea, A. \& Necula, S. (2011). Simultaneous inferior and middle meatus antrostomies in the treatment of the severely diseased maxillary sinus. American journal of rhinology $\mathcal{E}$ allergy. Vol.25, No.2, (March/April 2011), pp. e80-e85(6), ISSN 1945-8924

Andric, M. (2010). Endoscopic surgery of the maxillary sinuses in oral and maxillofacial surgery practice: a literature review. Hellenic Archives of Oral \& Maxillofacial Surgery, Vol.2, No.2, (August 2010), pp. 57-68, ISSN 1108-829 X

Andric, M., Saranovic, V., Drazic, R., Brkovic, B. \& Todorovic L. (2010). Functional endoscopic sinus surgery as an adjunctive treatment for closure of oroantral fistulae: a retrospective analysis. Oral surgery, oral medicine, oral pathology, oral radiology, and endodontics, Vol.109, No.4, (April 2010), pp. 510-516. ISSN 1079-2104

Berengo, M., Sivolella, S., Majzoub, Z. \& Cordioli, G. (2004). Endoscopic evaluation of the bone-added osteotome sinus floor elevation procedure. International journal of oral and maxillofacial surgery, Vol.33, No.2, (March 2004), pp. 189-194, ISSN 0901-5027

Bray, D., Michael, A., Falconer, DT \& Kaddour, HS. (2007). Ameloblastoma: a rare nasal polyp. The Journal of laryngology and otology, Vol.121, No.1, (January 2007), pp. 72-75, ISSN 0022-2151

Brook I. (2006). Sinusitis of odontogenic origin. Otolaryngology--head and neck surgery: official journal of American Academy of Otolaryngology-Head and Neck Surgery, Vol.135, No.3, (September 2006), pp. 349-355, ISSN 0194-5998 
Busaba, NY. \& Kieff, D. (2002). Endoscopic sinus surgery for inflammatory maxillary sinus disease. Laryngoscope, Vol.112, No.8, (August 2002), pp. 1378-1383. ISSN 0023-852X

Cansiz, H., Tuskan, K., Karaman, E. \& DervisoÄŸlu, S. (2004). Endoscope assisted removal of cementoossifying fibroma in the paranasal sinuses in a five-year-old girl. International journal of pediatric otorhinolaryngology, Vol.68, No.4, (April 2004), pp. 489-493, ISSN 0165-5876

Car, M. \& Juretić, M. (1998) Treatment of oroantral communications after tooth extraction. Is drainage into the nose necessary or not? Acta oto-laryngologica, Vol.118, No.6, (November 1998), pp. 844-846. ISSN 0001-6489

Carlson, ER. \& Marx, RE. (2006). The ameloblastoma: primary, curative surgical management. Journal of oral and maxillofacial surgery: official journal of the American Association of Oral and Maxillofacial Surgeons, Vol.64, No.3, (March 2006), pp. 484494, ISSN 0278-2391

Cascone P., Ungari, C., Filiaci, F., Gabriele, G. \& Ramieri, V. (2010). A dental implant in the anterior cranial fossae. International journal of oral and maxillofacial surgery, Vol.39, No.1, (January 2010), pp. 92-93, ISSN 0901-5027

Chiapasco, M., Felisati, G., Maccari, A., Borloni, R., Gatti, F. \& Di Leo, F. (2009). The management of complications following displacement of oral implants in the paranasal sinuses: a multicenter clinical report and proposed treatment protocols. International journal of oral and maxillofacial surgery, Vol.38, No.12, (December 2009), pp. 1273-1278, ISSN 0901-5027

Chiu, AG. \& Kennedy, DW. (2004) Surgical management of chronic rhinosinusitis and nasal polyposis: a review of the evidence. Current allergy and asthma reports, Vol.4, No.6, (November 2004), pp. 486-489, ISSN 1529-7322

Christmas DA, Mirante JP, Yanagisawa E. (2008). Endoscopic view of a maxillary dentigerous cyst. Ear, nose, \& throat journal, Vol.87, No.6, (June 2008), pp. 316, ISSN 0145-5613

Coleman, JR Jr. \& Duncavage, JA. (1996). Extended middle meatal antrostomy: the treatment of circular flow. Laryngoscope, 1996; Vol.106, No.10, (October 1996), pp. 1214-1217, ISSN 0023-852X

Costa, F., Emanuelli, E., Robiony, M., Zerman, N., Polini, F. \& Politi, M. (2007). Endoscopic surgical treatment of chronic maxillary sinusitis of dental origin. Journal of oral and maxillofacial surgery: official journal of the American Association of Oral and Maxillofacial Surgeons, Vol.65, No.2, (February 2007), pp. 223-228. ISSN 0278-2391

DeFreitas, J. \& Lucente, FE. (1988). The Caldwell-Luc procedure: institutional review of 670 cases: 1975-1985. Laryngoscope, Vol.98, No.12, (December 1988), pp. 1297-1300, ISSN 0023-852X

Di Pasquale, P. \& Shermetaro, C. (2006). Endoscopic removal of a dentigerous cyst producing unilateral maxillary sinus opacification on computed tomography. Ear, nose, E throat journal, Vol.85, No.11, (November 2006), pp. 747-748, ISSN 0145-5613

Dolanmaz, D., Tuz, H., Bayraktar, S., Metin, M., Erdem, E. \& Baykul, T. (2004). Use of pedicled buccal fat pad in the closure of oroantral communication: analysis of 75 cases. Quintessence international, Vol. 35, No.3, (March 2004), pp. 241-246, ISSN 0033-6572

Eberhart, LH., Kussin, A., Arndt, C., Lange, H., Folz, BJ., Werner, JA., Wulf H. \& Kill, C. (2007). Effect of a balanced anaesthetic technique using desflurane and remifentanil 
on surgical conditions during microscopic and endoscopic sinus surgery. Rhinology, Vol.45, No.1, (March 2007), pp. 72-78, ISSN 0300-0729

El Charkawi, HG., El Askary, AS \& Ragab, A (2005). Endoscopic removal of an implant from the maxillary sinus: a case report. Implant dentistry, Vol.14, No.1, (March 2005), pp. 30-35, ISSN 1056-6163

Engelke, W. \& Capobianco, M. (2005). Flapless sinus floor augmentation using endoscopy combined with CT scan-designed surgical templates: method and report of 6 consecutive cases. The International journal of oral \& maxillofacial implants, Vol.20, No.6, (November - December 2005), pp. 891-897, ISSN 0882-2786

Engelke, W. \& Deckwer, I. (1997). Endoscopically controlled sinus floor augmentation. A preliminary report. Clinical oral implants research, Vol.8, No.6, (December 1997), pp. 527-531, ISSN 0905-7161

Engelke, W., Schwarzwäller, W., Behnsen, A. \& Jacobs, HG. (2003). Subantroscopic laterobasal sinus floor augmentation (SALSA): an up-to-5-year clinical study. The International journal of oral $\mathcal{E}$ maxillofacial implants, Vol.18, No.1, (January - February 2003), pp. 135-143, ISSN 0882-2786

Felisati, G., Lozza, P., Chiapasco, M. \& Borloni, R. (2007). Endoscopic removal of an unusual foreign body in the sphenoid sinus: an oral implant. Clinical oral implants research, 2007; Vol.18, No.6, (December 2007), pp. 776-780, ISSN 0905-7161

Ghali, GE. \& Connor, MS. (2003). Surgical management of the odontogenic keratocyst. Oral and maxillofacial surgery clinics of North America, Vol.15, No.3, (August 2003), pp. 383-392. ISSN 1042-3699

Griffa A., Viterbo, S. \& Boffano, P. (2010). Endoscopic-assisted removal of an intraorbital dislocated dental implant. Clinical oral implants research, Vol.21, No.7, (July 2010), pp. 778-780, ISSN 0905-7161

Güven O. (1998). A clinical study on oroantral fistulae. Journal of cranio-maxillofacial surgery, Vol.26, No.4, (August 1998), pp. 267-271, ISSN 1010-5182

Hajiioannou, J., Koudounarakis, E., Alexopoulos, K., Kotsani, A. \& Kyrmizakis, DE. (2010). Maxillary sinusitis of dental origin due to oroantral fistula, treated by endoscopic sinus surgery and primary fistula closure. The Journal of laryngology and otology, Vol.124, No.9, (September 2010), pp. 986-989, ISSN 0022-2151

Hasbini, AS., Hadi, U. \& Ghafari, J. (2001). Endoscopic removal of an ectopic third molar obstructing the osteomeatal complex. Ear, nose, \& throat journal, Vol.80, No.9, (September 2001), pp. 667-670. ISSN 0145-5613

Ikeda, K., Hirano, K., Oshima, T., Shimomura, A., Suzuki, H., Sunose, H., Kondo, Y. \& Takasaka, T. (1996). Comparison of complications between endoscopic sinus surgery and Caldwell-Luc operation. The Tohoku journal of experimental medicine, Vol.180, No.1, (September 1996), pp. 27-31, ISSN 0040-8727

Kennedy, DW. \& Shaalan, H. (1989). Reevaluation of maxillary sinus surgery: experimental study in rabbits. The Annals of otology, rhinology, and laryngology, Vol.98, No.11, (November 1989), pp. 901-906, ISSN 0003-489

Kennedy, DW. (1985). Functional endoscopic sinus surgery: technique. Archives of otolaryngology, Vol.111, No.10, (October 1985), pp. 643-649. ISSN 0003-9977

Kim, JW., Lee, CH., Kwon, TK. \& Kim, DK. (2007). Endoscopic removal of a dental implant through a middle meatal antrostomy. The British journal of oral $\mathcal{E}$ maxillofacial surgery, Vol.45, No.5, (July 2007), pp. 408-409, ISSN 0266-4356 
Kitamura A. (2007). Removal of a migrated dental implant from a maxillary sinus by transnasal endoscopy. The British journal of oral $\mathcal{E}$ maxillofacial surgery, Vol.45, No.5, (July 2007), pp. 410-411, ISSN 0266-4356

Lamb, JF., Husein, OF. \& Spiess, AC. (2009). Ectopic molar in the maxillary sinus precipitating a mucocele: a case report and literature review. Ear, nose, $\mathcal{E}$ throat journal, Vol.88, No.8, (August 2009), pp. E6-E11, ISSN 0145-5613

Lee, KC. \& Lee, SJ. (2010). Clinical Features and Treatments of Odontogenic Sinusitis, Yonsei Medical Journal, Vol.51, No.6, (November 2010), pp. 932-937. ISSN 0513-5796

Leong, SC., Karkos, PD., Krajacevic, J., Islam, R. \& Kent, SE. (2010). Ameloblastoma of the sinonasal tract: A case report. Ear, nose, \& throat journal, Vol.89, No.2, (February 2010), pp. 70-71, ISSN 0145-5613

London, SD., Schlosser, RJ. \& Gross, CW. (2002). Endoscopic management of benign sinonasal tumors: a decade of experience. American journal of rhinology, Vol.16, No.4, (July - August 2002), pp. 221-227, ISSN 1050-6586

Lopatin, AS. \& Kapitanov, DN. (2005). Endonasal removal of a large ethmoidal cementoblastoma. Rhinology, Vol.43, No.2, (June 2005), pp. 156-158, ISSN 0300-0729

Lopatin, AS., Sysolyatin, SP., Sysolyatin, PG. \& Melnikov, MN. (2002). Chronic maxillary sinusitis of dental origin: is external surgical approach mandatory? Laryngoscope, Vol.112, No.6, (June 2002), pp. 1056-1059, ISSN 0023-852X

Lubbe, DE., Aniruth, S., Peck, T. \& Liebenberg, S. (2008). Endoscopic transnasal removal of migrated dental implants. British dental journal, Vol.204, No.8, (April 26th, 2008), pp. 435-436, ISSN 0007-0610

McMains, KC. (2008). Safety in endoscopic sinus surgery. Current opinion in otolaryngology $\mathcal{E}$ head and neck surgery, Vol.16, No.3, (June 2008), pp. 247-251. ISSN 1068-9508

Mehra, P. \& Murad, H. (2004). Maxillary sinus disease of odontogenic origin. Otolaryngologic clinics of North America, Vol.37, No.2, (April 2004), pp. 347-364, ISSN 030-6665

Micozkadioglu, SD \& Erkan, AN. (2007). Endoscopic removal of a maxillary dentigerous cyst. B-ENT, Vol.3, No.4, pp.213-216. ISSN 1781-782X

Nakamura, N., Mitsuyasu, T. \& Ohishi, M. (2004). Endoscopic removal of a dental implant displaced into the maxillary sinus: technical note. International journal of oral and maxillofacial surgery, Vol.33, No.2, (March 2004), pp. 195-197, ISSN 0901-5027

Nemec, SF., Peloschek, P., Koelblinger, C., Mehrain, S., Krestan, CR. \& Czerny, C. (2009). Sinonasal imaging after Caldwell-Luc surgery: MDCT findings of an abandoned procedure in times of functional endoscopic sinus surgery. European Journal of Radiology, Vol.70, No.1, (April 2009), pp. 31-34, ISSN 0720-048X

Nkenke, E., Schlegel, A., Schultze-Mosgau, S., Neukam, FW. \& Wiltfang, J. (2002). The endoscopically controlled osteotome sinus floor elevation: a preliminary prospective study. The International journal of oral $\mathcal{E}$ maxillofacial implants, Vol.17, No.4, (July - August 2002), pp. 557-566, ISSN 0882-2786

Ramotar, H., Jaberoo, MC., Koo Ng, NK., Pulido, MA. \& Saleh, HA. (2010). Image-guided, endoscopic removal of migrated titanium dental implants from maxillary sinus: two cases. The Journal of laryngology and otology, Vol.124, No.4, (April 2010), pp. 433436, ISSN 0022-2151

Stammberger, H. (1986). Endoscopic endonasal surgery--concepts in treatment of recurring rhinosinusitis. Part II. Surgical technique. Otolaryngology--head and neck surgery: 
official journal of American Academy of Otolaryngology-Head and Neck Surgery, Vol.94, No.2, (February 1986), pp. 147-156. ISSN 0194-5998

Ucer, TC. (2009). A modified transantral endoscopic technique for the removal of a displaced dental implant from the maxillary sinus followed by simultaneous sinus grafting. The International journal of oral $\mathcal{E}$ maxillofacial implants, Vol.24, No.5, (September - October 2009), pp. 947-951, ISSN 0882-2786

Varol, A., Türker, N., Göker, K. \& Basa, S. (2006). Endoscopic retrieval of dental implants from the maxillary sinus. The International journal of oral $\mathcal{E}$ maxillofacial implants, Vol.21, No.5, (September - October 2006), pp. 801-804. ISSN 0882-2786

Yilmaz, T., Suslu, AE. \& Gursel, B. (2003). Treatment of oroantral fistula: experience with 27 cases. American journal of otolaryngology, Vol.24, No.4, (July - August 2003), pp. 221223. ISSN 0196-0709

Zimbler, MS., Lebowitz, RA., Glickman, R., Brecht, LE. \& Jacobs, JB. (1998). Antral augmentation, osseointegration, and sinusitis: the otolaryngologist's perspective. American journal of rhinology, Vol.12, No.5, (September - October 1998) , pp. 311-316, ISSN 1050-6586 


\title{
Objective Outcomes in Endoscopic Sinus Surgery
}

\author{
David W.J. Côté and Erin D. Wright \\ University of Alberta \\ Canada
}

\section{Introduction}

Use of endoscopes in the sinonasal cavity dates as far back as the turn of the $20^{\text {th }}$ century with Hirschmann and Reichert performing the first sino-endoscopies and sinus surgeries, respectively. Widespread use was limited until H.H. Hopkins helped address illumination difficulties with the rod optic system in the 1960s and Walter Messerklinger began systematic use of the endoscope to evaluate the lateral nasal wall and mucociliary clearance in the late 1970s (Lee \& Kennedy 2006). With the advent of modern endoscopic sinus surgery instruments and techniques in the 1980s, the endoscope has radically altered the surgical approach and management of inflammatory and neoplastic sinonasal disease rendering many of the open approaches nearly obsolete.

Successful outcomes in endoscopic sinus surgery have often been largely based on subjective qualifiers by the patient. Significant improvements in patient perceived nasal congestion, obstruction, facial pressure, rhinorrhea, headache, postnasal drainage have been the impetus for the widespread growth of functional endoscopic sinus surgery, while modest improvements in olfaction, taste, allergic symptoms and tooth pain have also been reported. (Lee \& Kennedy 2006). Some objective measures of outcomes previously proposed include acoustic rhinometry, mucociliary measures using saccharine transit times and ciliary beat clearance, and olfactory thresholds using butanol testing and the UPSIT-University of Pennsylvania Smell Identification Test (Min et al 1995; Lund \& Scadding 1994). Radiologic evidence of polyp disease on CT scanning has also been studied with validated scoring systems, but with poor correlation with clinical symptoms and as such a poor indicator of outcomes (Newman et al 1994; Friedman 1990; Giklich 1994; Jorgensen 1991; Browne et al 2006; Newton \& Ah-See 2008). Increasingly, the rhinologic community looks to standardized objective endoscopic measures in scientific communications to evaluate success in managing sinonasal disease. These various grading schemes have been targeted at eliciting objective reproducible measures of: (1) polyp grade, (2) sinus cavity status, and (3) surgical field visibility. We present the first complete review of all objective published endoscopic scoring schemes for sinonasal disease.

\section{Objective endoscopic measures of polyp disease}

\subsection{Objective endoscopic measures of polyp disease}

Objective, standardized endoscoping scoring systems to communicate disease burden of nasal polyposis dates back at least to the late 1980s with staging systems being proposed by various international clinical groups over the years (Table 1). 


\begin{tabular}{|c|c|c|c|c|}
\hline YEAR & AUTHOR(S) & COUNTRY & SCALE & TYPE \\
\hline 1990 & Levine & United States of America & 6 point & Polyp staging \\
\hline 1992 & Kennedy & United States of America & 5 point & $\begin{array}{l}\text { Sinus cavity } \\
\text { staging }\end{array}$ \\
\hline 1992 & Gaskins & United States of America & 5 point & $\begin{array}{l}\text { Sinus cavity } \\
\text { staging }\end{array}$ \\
\hline \multirow{4}{*}{1993} & \multirow{4}{*}{ Johansen et al. } & \multirow{4}{*}{ Denmark } & $\begin{array}{c}5 \text { point } \\
\text { inflammation }\end{array}$ & \multirow{4}{*}{$\begin{array}{l}\text { Sinus cavity } \\
\text { staging }\end{array}$} \\
\hline & & & $\begin{array}{c}5 \text { point } \\
\text { previous } \\
\text { surgery } \\
\end{array}$ & \\
\hline & & & $\begin{array}{l}5 \text { point } \\
\text { infection }\end{array}$ & \\
\hline & & & $\begin{array}{l}4 \text { point polyp } \\
\text { staging }\end{array}$ & \\
\hline 1993 & Lund \& Mackay & United Kingdom & $\begin{array}{c}3 \text { point } \\
\text { including } \\
\text { polyp, } \\
\text { discharge, } \\
\text { edema, scars, } \\
\text { crusting }\end{array}$ & $\begin{array}{l}\text { Sinus cavity } \\
\text { staging }\end{array}$ \\
\hline 1993 & May \& Levine & United States of America & 5 point & Polyp staging \\
\hline 1995 & Lildholdt et al. & Sweden & 4 point & Polyp staging \\
\hline 1995 & $\begin{array}{l}\text { Lund \& } \\
\text { Kennedy }\end{array}$ & $\begin{array}{l}\text { United Kingdom, United } \\
\text { States of America }\end{array}$ & $\begin{array}{c}3 \text { point polyp } \\
\text { edema } \\
\text { discharge, } \\
\text { scarring } \\
\text { crusting } \\
\end{array}$ & $\begin{array}{l}\text { Sinus cavity } \\
\text { staging }\end{array}$ \\
\hline 1996 & $\begin{array}{l}\text { Mackay \& } \\
\text { Nacleiro }\end{array}$ & United Kingdom & 4 point & Polyp staging \\
\hline 2000 & Johansson et al. & Sweden & $0-100 \mathrm{VAS}$ & Polyp staging \\
\hline 2000 & Rasp & Germany & 4 point & Polyp staging \\
\hline 2003 & Passali et al & Italy & 4 point & Polyp staging \\
\hline 2006 & Meltzer et al & United States of America & 5 point & Polyp staging \\
\hline 2007 & $\begin{array}{l}\text { Wright \& } \\
\text { Agrawal }\end{array}$ & Canada & 20 point & $\begin{array}{l}\text { Sinus cavity } \\
\text { staging }\end{array}$ \\
\hline \multirow[t]{3}{*}{2009} & \multirow{3}{*}{ de Sousa et al } & \multirow{3}{*}{ Brazil } & $\begin{array}{c}4 \text { point } \\
\text { horizontal }\end{array}$ & \multirow{3}{*}{ Polyp staging } \\
\hline & & & $\begin{array}{l}5 \text { point } \\
\text { vertical }\end{array}$ & \\
\hline & & & 5 point $\mathrm{AP}$ & \\
\hline
\end{tabular}

Table 1. Staging Systems for Endoscopic Polyp Disease and Sinonasal Cavaties.

Howard Levine from Cleveland presented his 6 point staging system at the VIIth International Symposium on Infection and Allergy of the Nose in Baltimore, 1989 (Table 2). This system was employed to evaluate outcome in a series of 250 patients undergoing endoscopic 
sinus surgery and followed long term up to 42 months post-operatively to advocate for the utility of nasal endoscopy to diagnose and monitor sinonasal disease (Levine, 1990).

\begin{tabular}{|l|l|}
\hline 0 & no polyps \\
\hline 1 & polyps totally confined to the middle meatus \\
\hline 2 & $\begin{array}{l}\text { anterior to the turbinate, extending inferiorly to the inferior turbinate but not } \\
\text { covering it }\end{array}$ \\
\hline 3 & medial and posterior to the middle turbinate in addition to being anterior to it \\
\hline 4 & extending to the floor of the nose, but with parts of the turbinates visible \\
\hline 5 & filling the nasal cavity with no portion of the turbinate visible \\
\hline $\begin{array}{l}\text { Adapted from Levine HL. Functional endoscopic sinus surgery: evaluation, surgery, and } \\
\text { follow-up of } 250 \text { patients. Laryngoscope 1990; 100:79-84. }\end{array}$ \\
\hline
\end{tabular}

Table 2. Endoscopic grading of polyp systems proposed by Levine, 1990

In 1993, a group from Aarhus, Denmark, under Lars Johansen proposed a 4-point staging system they employed in their study to evaluate the efficacy of intranasal budesonide in treating small and medium sized nasal polyps (See table 3) (Johansen et al, 1993). Simpler than the system proposed by Levine in 1989, the Johansen system outlined parameters to divide eosinophilic sinonasal polyp disease between mild, moderate and severe.

\begin{tabular}{|l|l|}
\hline 0 & no polyps \\
\hline 1 & $\begin{array}{l}\text { mild polyposis- small polyps not reaching the upper edge of the inferior } \\
\text { turbinate, causing only slight obstruction }\end{array}$ \\
\hline 2 & $\begin{array}{l}\text { moderate polyposis- medium-sized polyps reaching between the upper and the } \\
\text { lower edge of the inferior turbinate and causing troublesome obstruction }\end{array}$ \\
\hline 3 & $\begin{array}{l}\text { severe polyposis- large polyps reaching below the lower edge of the inferior } \\
\text { turbinate and causing total or almost total obstruction }\end{array}$ \\
\hline *total score = sum of scores for each nasal cavity \\
\hline $\begin{array}{l}\text { Adapted from Johansen VL, Illum P, Kristensen S, Winther L, Petersen S, } \\
\text { Synnerstad B. The effect of Budesonide (Rhinocort }{ }^{\circledR)} \text { in the treatment of small and } \\
\text { medium sized nadal polyps. Clin Otolaryngol 1993; 18:524-7. }\end{array}$ \\
\hline
\end{tabular}

Table 3. Endoscopic grading of polyp systems proposed by Johansen et al, 1993.

That same year, Howard Levine along with Mark May published staging systems aimed at facilitating quantifying objectively outcomes in sinus surgery (May et al, 1993). Among the various staging systems proposed including staging of the endoscopic sinus surgical intervention, anatomical abnormalities on CT scans, patient subjective measures, etc., a five point scheme was proposed (table 4).

Also in 1993, an overall staging system for sinonasal disease was published by Lund and Mackay from University College of London. In addition to scoring systems for the nasal cavity and of the radiographic appearance on sinus CT, a simple 3 point staging system for endoscopic appearance of nasal polyps was proposed with 0 correlating to no polyps, 1 for polyps confined to the middle meatus and 2 for polyps beyond the middle meatus (Lund \& Mackay, 1993). Moreover, the Danish/Swedish Study Group carried out a double-blind placebo-controlled study of topical budesonide for nasal polyps and presented a 4 point scoring scheme (table 5) which expanded on the simple classification presented by Lund and Mackay (Lildholdt et al, 1995). 


\begin{tabular}{|l|l|}
\hline $1+$ & anterior attachment of middle turbinate visible \\
\hline $2+$ & anterior attachment of middle turbinate obscured \\
\hline $3+$ & nasal cavity filled to vestibule \\
\hline $4+$ & nasal cavity filled to nares \\
\hline $5+$ & nasal cavity filed to lip \\
\hline Adapted from May M, Levine HL, Schaitkin B, Mester SJ. Results of surgery. In: \\
Levine H, May M, editors. Endoscopic sinus surgery. New York: Thieme Medical \\
Publishers, Inc., 1993:176-92.
\end{tabular}

Table 4. Endoscopic grading of polyp systems proposed by May and Levine, 1993

\begin{tabular}{|l|l|}
\hline 0 & no polyposis \\
\hline 1 & $\begin{array}{l}\text { mild polyposis - (small polyps not reaching the upper edge of the inferior } \\
\text { turbinate) }\end{array}$ \\
\hline 2 & $\begin{array}{l}\text { moderate polyposis - (medium sized polyps reaching between the upper and } \\
\text { lower edge of the inferior turbinate) }\end{array}$ \\
\hline 3 & $\begin{array}{l}\text { severe polyposis - (large polyps reaching below the lower edge of the inferior } \\
\text { turbinate) }\end{array}$ \\
\hline $\begin{array}{l}\text { Adapted from Lildholdt T, Rundkrantz H, Lindqvist N. Efficacy of topical } \\
\text { corticosteroid powder for nasal polyps: a double-blind, placebo-controlled study of } \\
\text { budesonide. Clin Otolaryngol 1995; 20(1): 26-30. }\end{array}$ \\
\hline
\end{tabular}

Table 5. Endoscopic grading of polyp systems proposed by Lildholdt et al., 1995

In March 1996, an international workshop on nasal polyposis in Davos, Switzerland, the International Conference on Sinus Disease, proposed a polyp staging scheme somewhat adapted from the polyp staging system based on Lund and MacKay (Lund \& MacKay 1993; Lund \& Kennedy 1995). This staging system, sometimes referred to as Mackay \& Nacleiro, includes an endoscopic polyp grading system with grading from 0 to 3 depending upon the polyp burden (table 6) where a score of 0 indicates to visible polyp disease on endoscopy, 1 polyps confined to the middle meatus, 2 polyps not completely obstructing the nasal cavity and 3 polyps completely obstructing the nasal cavity (Malm, 1997). This system has since been employed several times in the rhinology literature as a validated scale for outcomes measures (Andrews et al, 2005; Browne et al, 2006). Multicentre validation of this system demonstrated a strong correlation between its scores and symptom reduction using the 22question Sinonasal Outcome Test-SNOT 22, as well as a correlation with complication rates and revision rates (Hopkins et al, 2007).

Johansson et al from the Central Hospital in Skövde, Sweden conducted an evaluation of 5 various endoscopic measures of polyp burden and proposed their own Visual Analog Scale from 0-100 where 0 refers to a total absence of polyps and 100 a nasal cavity completely filled with polyps. They conducted a study to evaluate the reproducibility of this system along with evauation of the Lildholdt scoring system and the Lund-Mackay scoring systems as well as lateral imaging (where polyps are expressed on a schematic picture of the lateral nasal wall and expressed as a percentage of total area) and their 0-100 visual analog scale for nasal patency. They found that their visual analog scale, along with the Lund-Mackay, and nasal patency score yielded poor inter-rater reproducibility; rather, the Lildholdt score and 


\begin{tabular}{|l|l|}
\hline 0 & absence of polyps \\
\hline 1 & $\begin{array}{l}\text { polyps that do not prolapse beyond the middle turbinate and may require an } \\
\text { endoscope for visualization }\end{array}$ \\
\hline 2 & $\begin{array}{l}\text { polyps that are extended below the middle turbinate and are visible with a } \\
\text { nasal speculum }\end{array}$ \\
\hline 3 & polyps are massive and occlude the entire nasal cavity \\
\hline $\begin{array}{l}\text { Adapted from Malm L. Assessment and staging of nasal polyposis. Acta Otolaryngol } \\
\text { (Stockh) 1997; 117:465-467. }\end{array}$ \\
\hline
\end{tabular}

Table 6. Endoscopic grading of polyp systems proposed by Mackay \& Nacleiro, 1996

lateral imaging were found to be superior for reliability and reproducibility (Johansson et al, 2000). After finding poor inter-rater agreement using the Lund-Mackay polyp scoring but a high correlation using lateral imaging and the four step scoring system proposed by Lildholdt et al, that same group then conducted a study in 2002 to identify the sensitivity of grading systems for detect early changes in polyp disease with topical budesonide treatments in a prospective, randomized placebo controlled trial. Lateral imaging showed statistically significant changes in polyp size was detectable after 14 days of topical corticosteroid use and found to be more sensitive than the Lildholdt staging (Johansson et al, 2002).

Rasp et al from the Ludwig-Maximilians-Universität in Munich proposed a four grade polyp score to include early polypoid changes and was again validated and employed to evaluate effect of topical and systemic steroid therapy (see table 7) (Rasp et al, 2000, Kramer\&Rasp, 1999).

\begin{tabular}{|l|l|}
\hline I & polyposal swelling of the mucosa of the middle meatus \\
\hline II & nasal polyps within the middle or lower meatus \\
\hline III & polyps extending over the middle turbinate \\
\hline IV & nasal polyposis with protrusion into the anterior nose \\
\hline $\begin{array}{l}\text { Adapted from Kramer MF, Rasp G. Nasal polyposis: eosinophils and interleukin-5. } \\
\text { Allergy 1999; 54:669-680. }\end{array}$
\end{tabular}

Table 7. Endoscopic grading of polyp systems proposed by Rasp 1999.

Passali et al from the University of Siena conducted a prospective randomized controlled study of 170 patients evaluating the efficacy of intranasal furosemide compared to intranasal mometasone for chronic sinusitis with polyposis. They evaluated subjective patient outcomes and for quantifying objective outcomes proposed a four point staging system very much like the Mackay - Nacleiro system, but taking into account endoscopic appearance as well as nasal volumes on acoustic rhinomanomatry (Table 8) (Passali et al 2003).

The multinational Rhinosinusitis Initiative with representation from national societies of the USA, Belgium, Netherlands, United Kingdom and Japan, in 2006 developed guidelines for facilitating clinical trials for rhinosinusitis. Among the recommendations put forth by the guidelines was a 5-point polyp grading (table 9) scheme which the group advocated to be used in all subsequent rhinologic literature (Meltzer et al, 2006). 


\begin{tabular}{|l|l|}
\hline 0 & no polyps seen \\
\hline 1 & polyps confined to the middle meatus with AR values in normal range \\
\hline 2 & $\begin{array}{l}\text { polyps prolapsing beyond the middle turbinate, with less than } 10 \% \text { reduction in } \\
\text { volume by AR }\end{array}$ \\
\hline 3 & $\begin{array}{l}\text { subobstructive forms requiring another operation }(>50 \% \text { reduction of nasal } \\
\text { volumes })\end{array}$ \\
\hline $\begin{array}{l}\text { Adapted from Passali D, Bernstein JM, Passali FM, Damiani V, Passali GC, Bellusi L. } \\
\text { Treatment of recurrent chronic hyperplastic sinusitis with nasal polyposis. Arch Otol Head } \\
\text { Neck 2003; 129: 656-659. }\end{array}$ \\
\hline
\end{tabular}

Table 8. Grading of polyp system proposed by Passali et al, 2003.

\begin{tabular}{|l|l|}
\hline 0 & no visible polyps seen \\
\hline 1 & small amount of polypoid disease confined within the middle meatus \\
\hline 2 & multiple polyps occupying the middle meatus \\
\hline 3 & $\begin{array}{l}\text { polyps extending beyond the middle meatus, within the sphenoethmoid recess but } \\
\text { not totally obstructing, or both }\end{array}$ \\
\hline 4 & polyps completely obstructing the nasal cavity \\
\hline \multicolumn{2}{|l}{ Adapted from Meltzer et al. Rhinosinusitis: developing guidance for clinical trials. J All } \\
Clin Immun 2006; 118(suppl): 17-61.
\end{tabular}

Table 9. Grading of polyps system proposed by Meltzer et al, 2006.

A group from Brazil proposed a novel endoscopic staging system using three-dimensional nasal polyp assessment and nasal endoscopy with polyp scales in vertical, horizontal and antero-posterior planes (see Table 10) but in the end was found to show less inter-rater agreement than the polyp systems of Johanssen et al and the Lund-Mackay polyp scores (de Sousa et al, 2009).

Overall, a common theme seems to emerge amongst all polyp scores regarding the degree polyp disease obstructs the middle meatus and the overall nasal cavity. Agreeing upon a single polyp system that is reliable, reproducible with high intra and inter-rater reliability and touches on clinically important factors pertaining to extent of polyp disease continues to challenge the rhinologic community. 


\begin{tabular}{|l|l|}
\hline \multicolumn{2}{|l|}{ Horizontal Plane $(H)$} \\
\hline H0 & no polyps \\
\hline H1 & polyps restricted to the middle meatus \\
\hline H2 & polyps expand beyond the middle meatus \\
\hline HT & polyps expand beyond the middle meatus and touch the septum \\
\hline Vertical Place $(V)$ \\
\hline V0 & no polyps \\
\hline V1 & polyps in the middle meatus only \\
\hline VI & $\begin{array}{l}\text { polyps extending inferiorly to the middle meatus, going beyond the upper border } \\
\text { of the inferior turbinate }\end{array}$ \\
\hline VS & $\begin{array}{l}\text { polyps extending superiorly to the middle meatus, between the septum and the } \\
\text { middle turbinate }\end{array}$ \\
\hline VT & polyps occupying the entire vertical aspect of the nasal cavity \\
\hline Antero-posterior plane (P) \\
\hline P0 & no polyps \\
\hline P1 & polyps in the middle meatus only \\
\hline PA & $\begin{array}{l}\text { polyps extending anteriorly to the middle meatus, reaching the head of the inferior } \\
\text { turbinate }\end{array}$ \\
\hline PP & $\begin{array}{l}\text { polyps extending posterior to the middle meatus, reaching the tail of the inferior } \\
\text { and middle turbinate }\end{array}$ \\
\hline PT & polyps occupying the entire antero-posterior aspect of the nasal cavity \\
\hline $\begin{array}{l}\text { Adapted from de Sousa, MCA, Becker HMG, Becker CG, de Castro MM, de Sousa NJA, } \\
\text { dos Santos Guimaraes RE. Reproducibility of the three-dimensional endoscopic staging } \\
\text { system for nasal polyposis. Braz J Otorhinolaryngol 2009;75(6):814-20. }\end{array}$ \\
\hline
\end{tabular}

Table 10. Grading of polyps system proposed by de Sousa et al, 2009.

\subsection{Objective endoscopic measures of the sinonasal cavity}

Scoring systems for endoscopic findings in the sinonasal cavity beyond simple polyp grading schemes have been used increasingly in the literature to objectively measure outcomes in interventions involving sinonasal disease (Cote \& Wright, 2010). As early as the late 1980's, efforts to classify severity of sinus pathology based on endoscopic findings was attempted. A rudimentary staging system was proposed by Jacobs et al relying on CT and endoscopic findings to classify severity of chronic sinusitis (Jacobs et al, 1990). At the first International Symposium: Contemporary Sinus Surgery in Pittsburgh, 1990, Ralph Gaskins of Atlanta, GA, presented a staging system for chronic sinusitis that incorporated endoscopic, radiologic findings, and patient immunologic factors, polyp severity, prior surgeries, and infection history into a complex staging system (table 11) to facilitate prediction of surgical response and guide selection of surgical procedure. Gaskins et al recommended Messerklinger technique functional endoscopic middle meatal surgery for stages 1 and early stage 2, with a Wigand total sphenoethmoidectomy for late stage 2 and stage 3 disease and external techniques for stage 4 disease (Gaskins, 1990). 


\begin{tabular}{|c|c|}
\hline Stage & \\
\hline 0 & No surgical sinus disease \\
\hline I & Score: $<1.3$ \\
\hline Site & Inflammation limited to the ostiomeatal area \\
\hline Surgery & $\begin{array}{l}\text { No prior sinus/nasal surgery except septoplasty and/or inferior metal } \\
\text { antrostomies }\end{array}$ \\
\hline Polyps & No polyps or localized to $<10 \%$ of the sinus space \\
\hline Infection & Well-controlled infection with no active mucopurulen drainage \\
\hline $\begin{array}{r}\text { Immune } \\
\text { status }\end{array}$ & No underlying immunologic disease except well-controlled allergy \\
\hline II & Score: $1.3-2.3$ \\
\hline Site & Inflammation confined to the maxillary/ethmoid/ostiomeatal areas \\
\hline Surgery & Prior Caldwell-Luc or polypectomy \\
\hline Polyps & Polyp disease, with involvement of $10 \%-50 \%$ of the nasal/sinus cavities \\
\hline Infection & Persistent, localized infection with some active purulent drainage \\
\hline $\begin{array}{r}\text { Immune } \\
\text { status } \\
\end{array}$ & Low-grade immune disorder or fair allergy control \\
\hline III & Score: $>2.3$ \\
\hline Site & Pansinus involvement, unilateral or bilateral; isolated sphenoid disease \\
\hline Surgery & Prior anterior ethmoidectomy/middle turbinate surgery \\
\hline Polyps & Nasal/sinus polyposis filling more than $50 \%$ of the nasal and sinus cavities \\
\hline Infection & $\begin{array}{l}\text { Poorly controlled multisinus infection with active mucopurulent drainage; } \\
\text { active fungal disease }\end{array}$ \\
\hline $\begin{array}{r}\text { Immune } \\
\text { status } \\
\end{array}$ & $\begin{array}{l}\text { Poorly controlled allergic rhinitis or significant immune disorder; history of } \\
\text { long term steroid treatment }\end{array}$ \\
\hline IV & Any score: 4 \\
\hline Site & $\begin{array}{l}\text { Sinus disease with extranasal/sinus extension; orbital or intracranial; frontal } \\
\text { disease above the nasofrontal duct }\end{array}$ \\
\hline Surgery & Prior complete ethmoidectomy or sphenoidectomy \\
\hline Polyps & Inverting papilloma or other potentially malignant nasal/sinus neoplasm \\
\hline Infection & Osteomyelitis or infection eroding into the orbit or cranium; mucormycosis \\
\hline $\begin{array}{r}\text { Immune } \\
\text { status } \\
\end{array}$ & Endstage immunologic disease/profoundly immunocompromised patient. \\
\hline \multicolumn{2}{|c|}{$\begin{array}{l}\text { Adapted from Gaskins RE. A surgical staging system for chronic sinusitis. Am J Rhinol } \\
\text { 1992; 6:5-12. }\end{array}$} \\
\hline
\end{tabular}

Table 11. Stages of Surgical Sinus Disease by Gaskins, 1990

The University of Pennsylvania's David Kennedy, in his 1992 thesis to the American Laryngological, Rhinological and Otological Society, attempted to classify extent of sinonasal inflammatory disease into 8 groups based on disease found at time of endoscopic surgery (see Table 12). In his study, he reviewed over 240 data fields for each of the 120 patient subjects to establish correlation with outcomes. Extent of preoperative disease and 


\begin{tabular}{|l|l|}
\hline 1 & Unilateral or bilateral anatomic abnormality \\
\hline 2 & Unilateral ethmoid disease \\
\hline 3 & Unilateral ethmoid diseas and involvement of 1 dependent sinus \\
\hline 4 & Bilateral ethmoid disease \\
\hline 5 & Unilateral ethmoid disease and involvement of 2 or 3 dependent sinuses \\
\hline 6 & Bilateral ethmoid disease and involvement of 1 dependent sinus \\
\hline 7 & Bilateral ethmoid disease and involvement of 2 or more dependent sinuses \\
\hline 8 & Diffuse sinonasal polyposis \\
\hline $\begin{array}{l}|l| \\
\text { Adapted from Kennedy DW. Prognostic factors, outcomes and staging in ethmoid sinus }\end{array}$ \\
\hline \multicolumn{2}{|l|}{ surgery. Laryngoscope 1992;102(Suppl 57):1-18. } \\
\hline
\end{tabular}

Table 12. Classification of the extent of disease by Kennedy, 1992.

surgical outcomes was found to be strongly correlated and as such, a staging system for chronic sinusitis was presented to help facilitate prognosis and comparison in inflammatory sinus disease (Table 13).

\begin{tabular}{|l|l|}
\hline \multirow{2}{*}{ I } & Anatomic abnormailities \\
\cline { 2 - 3 } & All unilateral sinus disease \\
\cline { 2 - 3 } & Bilateral disease limited to ethmoid sinuses \\
\hline II & Bilateral ethmoid disease with involvement of one dependent sinus \\
\hline III & $\begin{array}{l}\text { Bilateral ethmoid disease with involvement of two or more dependent sinuses on } \\
\text { each side }\end{array}$ \\
\hline IV & Diffuse sinonasal polyposis \\
\hline \multicolumn{2}{|l|}{$\begin{array}{l}\text { Adapted from Kennedy DW. Prognostic factors, outcomes and staging in ethmoid sinus } \\
\text { surgery. Laryngoscope 1992;102(Suppl 57):1-18. }\end{array}$} \\
\hline
\end{tabular}

Table 13. Chronic sinusitus staging system proposed by Kennedy, 1992.

Other endoscopic fields including mucosal hypertrophy, inflammation, discharge, crusting adhesions and polyp recurrence were examined but not incorporated into the staging scheme (Kennedy, 1992). Valerie Lund and Ian Mackay of University College London, in 1993, proposed a preoperative and postoperative inventory of the endoscopic appearance of the nasal cavities with a score of $0-2$ for polyps (0: none; 1 : confined to middle meatus; 2 : polyps beyond the middle meatus), as well as $0-2$ for discharge (0: none; 1 : clear and thin; 2 : thick and purulent) as well as observations for edema, scarring and crusting (Lund-Mackay, 1993). In 1995, the Staging and Therapy Group, headed by Valerie Lund and David Kennedy, proposed an endoscopic staging system for non-neoplastic sinonasal to evaluate therapeutic outcomes that was complex enough to incorporate the most important measures of the sinonasal cavity but simple enough to facilitate regular clinical use. Characteristics are assessed endoscopically of each sinonasal cavity to provide a score - polyp disease, mucosal edema/crusting/scarring and nasal secretion each receiving a score from 0 to 2 (Table 14)(Lund \& Kennedy, 1997).

This scoring system has since been the instrument of choice to endoscopically evaluate outcomes of interventions in non-neoplastic sinonasal disease prospectively over time in research and clinical practice. 


\begin{tabular}{|l|l|}
\hline Polyp & $\begin{array}{l}0=\text { absence of polyp, } 1=\text { polyps in middle meatus only, 2=beyond middle } \\
\text { meatus }\end{array}$ \\
\hline Edema & $0=$ absent, $1=$ mild, $2=$ severe \\
\hline Discharge & $0=$ no discharge, $1=$ clear, thin discharge, $2=$ thick, purulent discharge \\
\hline Scarring & $0=$ absent, $1=$ mild, $2=$ severe \\
\hline Crusting & $0=$ absent, $1=$ mild, $2=$ severe \\
\hline Adapted from Lund VJ, Kennedy DW. Quantification for staging sinusitis. In: Kennedy \\
DW, editor. International Conference on Sinus Disease: Terminology, Staging, Therapy. \\
Ann Otol Rhinol Laryngol 1995; 104(Suppl 167):17-21.
\end{tabular}

Table 14. Sinus endoscopy scoring system proposed by Lund and Kennedy, 1995.

A newer sinonasal scoring system, the Perioperative Sinus Endoscopy (POSE) scoring system was employed by Wright \& Agrawal to evaluate the outcomes in a randomized trial of perioperative systemic steroids on surgical patients with chronic rhinosinusits with polyposis (Wright \& Agrawal, 2007). POSE scoring was introduced to enhance face validity and responsiveness to change by providing richer measures of the inflammation in the ethmoid cavity, scarring and obstruction in outflow, as well as evaluation of secondary sinuses and included instructions for baseline assessments (table 15).

\begin{tabular}{|c|c|c|c|}
\hline Middle Turbinate & & Right & Left \\
\hline Normal & 0 & & \\
\hline Synechia/Lateralized & $1-2$ & & \\
\hline Middle Meatus/MMA & & Right & Left \\
\hline Healthy & 0 & & \\
\hline Narrowing/Closure & $1-2$ & & \\
\hline Maxillary Sinus Contents & $1-2$ & & \\
\hline Ethmoid Cavity & & Right & Left \\
\hline Healthy & 0 & & \\
\hline Crusting & $1-2$ & & \\
\hline Mucosal Edema & $1-2$ & & \\
\hline Polypoid Change & $1-2$ & & \\
\hline Polyposis & $1-2$ & & \\
\hline Secretions & $1-2$ & & \\
\hline \multicolumn{4}{|l|}{ Total (16) } \\
\hline \multicolumn{4}{|l|}{ Secondary Sinuses } \\
\hline Frontal Recess/Sinus & $0-2$ & & \\
\hline Sphenoid Sinus & $0-2$ & & \\
\hline Overall Total & 16 18F 18S 20 & & \\
\hline \multicolumn{4}{|c|}{$\begin{array}{l}18 \mathrm{~F}=\text { middle meatal antrostomy }+ \text { ethmoidectomy }+ \text { frontal sinusotomy; } \\
18 \mathrm{~S}=\text { middle meatal antrostomy }+ \text { ethmoidectomy }+ \text { sphenoidotomy } \\
20 \text { = middle meatal antrostomy }+ \text { ethmoidectomy }+ \text { sphenoidotomy }+ \text { frontal sinusotomy }\end{array}$} \\
\hline \multicolumn{4}{|c|}{$\begin{array}{l}\text { Adapted from Wright ED, Agrawal S. Impact of perioperative systemic steroids on surgical } \\
\text { outcomes in patients with chronic rhinosinusitis with polyposis: evaluation with the novel } \\
\text { perioperative sinus endoscopy (POSE) scoring system. Laryngoscope 2007; 117(suppl):1-28. }\end{array}$} \\
\hline
\end{tabular}

Table 15. Peri-Operative Sinus Endoscopy (Pose) Score by Wright and Agrawal, 2007. 
In that study, both the Lund - Kennedy Endoscopic score and POSE score were shown to be sensitive to changes over time but the POSE seemed to be more sensitive to subtle changes over time (fig 1) and correlated better with symptom scores. (Wright \& Agrawal, 2007). We found employing both measures simultaneously has merit in exploiting the established reliability of the Lund-Kennedy score while benefiting from the added information gleaned from the POSE score (Cote \& Wright, 2010). With further use and validation of the POSE score, it may perhaps become the staging system of choice to prospectively stage sinonasal cavities over time.

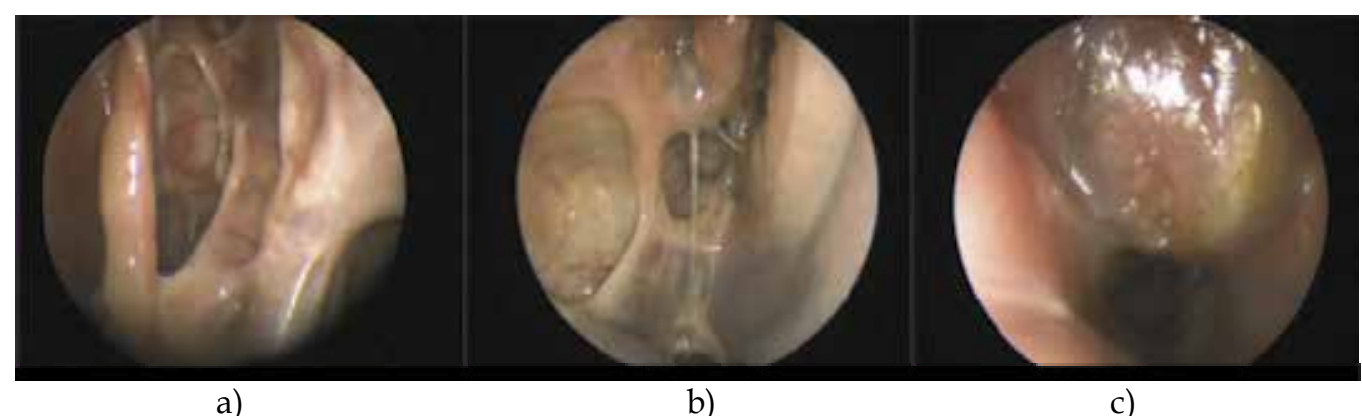

a)

b)

c)

Fig. 1. Three cavites: a) Left cavity, POSE $=0$ (normal middle turbinate, healthy middle meatal antrostomy, healthy ethmoid cavity); b) Right cavity, POSE $=3$ ( 2 points for edema, 1 point for mild secretions); c) Right cavity, POSE $=10$ ( 2 points for closure of middle meatus 2 for edema, 2 for polypoid changes, 2 for polyposis, 2 for secretions)

\subsection{Objective endoscopic measures of surgical field visibility}

With novel technologies and procedures being developed with the aim of facilitating visibility during endoscopic surgery, objective measures to evaluate such techniques are being proposed. The first proposed endoscopic surgical field grading scale (table 16) was published by Boerzaart et al in 1995 to objectively evaluate controlled hypotension with sodium nitroprusside in esmolol to facilitate sinus surgery and found that controlled esmolol-induce hypotension yielded superior surgical conditions.

\begin{tabular}{|l|l|}
\hline 0 & No bleeding. \\
\hline 1 & Slight bleeding - no suctioning of blood required. \\
\hline 2 & Slight bleeding- occasional suctioning required. Surgical field not threatened. \\
\hline 3 & $\begin{array}{l}\text { Slight bleeding- frequent suctioning required. Bleeding threatens surgical field a few } \\
\text { seconds after suction is removed. }\end{array}$ \\
\hline 4 & $\begin{array}{l}\text { Moderate bleeding- frequent suctioning required. Bleeding threatens surgical field } \\
\text { directly after suction is removed. }\end{array}$ \\
\hline 5 & $\begin{array}{l}\text { Severe bleeding- constant suctioning required. Bleeding appears faster than can be } \\
\text { removed by suction. Surgical field severely threatened and surgery not possible. }\end{array}$ \\
\hline $\begin{array}{l}\text { Adapted from Boerzaart AP, van der Merwe J. Comparison of sodium nitroprusside- and } \\
\text { esmolol-induced controlled hypotension for functional endoscopic sinus surgery. Can J } \\
\text { Anaesth 1995;42:373-376. }\end{array}$ \\
\hline
\end{tabular}

Table 16. Assessment of intra-operative surgical field by Boezaart et al, 1995 
This six point scale was aimed at quantifying the amount of bleeding in the surgical field that hindered progression of the surgical intervention -0 no bleeding, 1 slight bleeding no suctioning, 2 slight bleeding occasional suctioning, 3 slight bleeding frequent suctioning, 4 moderate bleeding frequent suctioning with bleeding threatening the surgical field, 5 severe bleeding constant suctioning (Boezaart 1995).

An eleven point grading scale was then proposed by PJ Wormald's group from Adelaide which sought to address some of the limitations with the Boezaart scale with grades 1-6 varying by number of points of ooze and 7-10 by severity of hemorrhage .

\begin{tabular}{|l|l|}
\hline 0 & No bleeding. \\
\hline 1 & $1-2$ points of ooze \\
\hline 2 & $3-4$ points of ooze \\
\hline 3 & $5-6$ points of ooze \\
\hline 4 & $7-8$ points of ooze \\
\hline 5 & $9-10$ points of ooze (sphenoid fills in 60 seconds) \\
\hline 6 & $>10$ points of ooze, obscuring surface (sphenoid fills in 50 seconds) \\
\hline 7 & $\begin{array}{l}\text { Mild bleeding/oozing from entire surgical surface with slow accumulation of blood } \\
\text { in the post nasal space (sphenoid fills by } 40 \text { seconds) }\end{array}$ \\
\hline 8 & $\begin{array}{l}\text { Moderate bleeding from entire surgical surface with moderate accumulation of } \\
\text { blood in the post nasal space (sphenoid fills by 30 seconds) }\end{array}$ \\
\hline 9 & $\begin{array}{l}\text { Moderately severe bleeding with rapid accumulation of blood in the post nasal } \\
\text { space (sphenoid fills by } 20 \text { seconds) }\end{array}$ \\
\hline 10 & Severe bleeding with nasal cavity filling rapidly (sphenoid fills in <10 seconds) \\
\hline $\begin{array}{l}\text { Adapted from Athanasiadis T, Beule A, Embate J, Steinmeier E, Field J, Wormald PJ. } \\
\text { Standardized video-endoscopy and surgical field grading scale for endoscopic sinus } \\
\text { surgery: a multi-centre study. Laryngoscope 2008; } 118: 314-319 .\end{array}$ \\
\hline
\end{tabular}

Table 17. Intra-operative surgical field grading by Wormald, 2008

By employing a standardized video-endoscopy technique both the Boerzaart and Wormald scores were found to have improved intra and inter-rater reliability; the Wormald scale, however, was found to be more sensitive to bleeding changes in endoscopic sinus surgery and demonstrated slightly better inter-rater reliability (Athanasiadis 2008). Further application and evaluation of these two systems must be undertaken before the rhinologic community decides a gold standard and establishes their strengths and limitations.

\section{Conclusion}

With increased refinement of endoscopic interventions for sino-nasal disease, there is simultaneous refinement in objective measures to audit the outcomes of these interventions. While each grading system has inherent limitations, they represent efforts to create a means to objectively communicate a richness in observations and outcomes that is both reliable and reproducible by practitioners treating sinonasal disease. In addition, many centres around the world are using these objective measures to monitor inflammatory sinus disease that, based simply on subjective measures, would be occult. This provides the opportunity to intervene with topical or less invasive therapies at a point where the disease may be more easily managed. 


\section{References}

Andrews AE, Bryson JM, Rowe-Jones JM. Site of origin of nasal polyps: Relevance toPathogenesis and management. Rhinology 2005; 43(3) 180-184.

Athanasiadis T, Beule A, Embate J, Steinmeier E, Field J, Wormald PJ. Standardized videoendoscopy and surgical field grading scale for endoscopic sinus surgery: a multicentre study. Laryngoscope 2008; 118:314-319.

Boerzaart AP, van der Merwe J. Comparison of sodium nitroprusside- and esmolol-induced controlled hypotension for functional endoscopic sinus surgery. Can J Anaesth 1995;42:373-376.

Browne JP, Hopkins C, Slack R et al. Health related quality of life after polypectomy with and without additional surgery. Laryngoscope 2006; 116:297-302.

Cote DWJ, Wright ED. Triamcinolone-impregnated nasal dressing following endoscopic sinus surgery: a randomized, double-blind, placebo-controlled study. Laryngoscope 2010; 120:1269-1273. de Sousa, MCA, Becker HMG, Becker CG, de Castro MM, de Sousa NJA, dos Santos

Guimaraes RE. Reproducibility of the three-dimensional endoscopic staging system for nasal polyposis. Braz J Otorhinolaryngol 2009;75(6):814-20.

Friedman WH, Katsantonis GP, Sivore M, Kay S. Computed tomography staging of the paranasal sinuses in chronic hyperplastic rhinosinusitis. Laryngoscope 1990; 100:1161-1665.

Gaskins RE. A surgical staging system for chronic sinusitis. Am J Rhinol 1992; 6:5-12.

Giklich RE, Metson R. A comparision of sinus computed tomography (CT) staging systems for outcomes research. Am J Rhinol 1994; 8:291-7.

Hopkins C, Browne JP, Slack R, Lund V, Brown P. The Lund-Mackay staging system for chronic rhinosinusitis: How is it used and what does it predict? Otolaryngol Head Neck Surg 2007; 137(4):555-61.

Jacobs JB, Gittelman P, Holliday R. Endoscopic sinus surgery for ostiomeatal disease. Am J Rhinol 1990; 4:41-43.

Johansen VL, Illum P, Kristensen S, Winther L, Petersen S, Synnerstad B. The effect of Budesonide (Rhinocort ${ }^{\circledR}$ ) in the treatment of small and medium sized nasal polyps. Clin Otolaryngol 1993; 18: 524-7.

Johansson L, Akerlund A, Holmberg K, MelenI, Stierna P, Bende M. Evaluation of methods for endoscopic staging of nasal polyposis. Acta Otolaryngol 2000; 120(1):72-6.

Johansson L, Holmberg K, Melen I, Stierna P, Bende M. Sensitivity of a new grading system for studying nasal polyps with the potential to detect early changes in polyp size after treatment with a topical corticosteroid (budesonide). Acta Otolaryngol 2002; 122:49-53.

Jorgensen RA. Endoscopic and computed tomographic findings in ostiomeatal sinus disease. Arch Otolaryng Head Neck Surg 1991; 117: 279-287.

Kennedy DW. Prognostic factors, outcomes and staging in ethmoid sinus surgery. Laryngoscope 1992;102(Suppl 57):1-18.

Kramer MF, Rasp G. Nasal polyposis: eosinophils and interleukin-5. Allergy 1999; 54:669680.

Lee JT, Kennedy DW. Endoscopic sinus surgery. In: Bailey B, Johnson J, Newlands SD, editors. Head $\mathcal{E}$ Neck Surgery - Otolaryngology, $4^{\text {th }}$ Edition. Lippincott Williams \& Wilkins, 2006: 459-475. 
Levine HL. Functional endoscopic sinus surgery: evaluation, surgery, and follow-up of 250 patients. Laryngoscope 1990; 100:79-84.

Lildholdt T, Rundkrantz H, Lindqvist N. Efficacy of topical corticosteroid powder for nasal polyps: a double-blind, placebo-controlled study of budesonide. Clin Otolaryngol 1995; 20(1): 26-30.

Lund V, Mackay IS. Staging in chronic rhinosinusitis. Rhinology 1993; 31: 183-4.

Lund VJ, Scadding GK. Objective assessment of endoscopic sinus surgery in themanagement of chronic rhinosinusitis: an update. J Laryngol Otol 1994; 108(9): 749-53.

Lund VJ, Kennedy DW. Quantification for staging sinusitis. In: Kennedy DW, editor. International Conference on Sinus Disease: Terminology, Staging, Therapy. Ann Otol Rhinol Laryngol 1995; 104(Suppl 167):17-21.

Lund VJ, Kennedy DW. Staging for rhinosinustis. Otolaryngol Head Neck Surg 1997; 117:S35S40.

Malm L. Assessment and staging of nasal polyposis. Acta Otolaryngol (Stockh) 1997; 117:465467.

May M, Levine HL, Schaitkin B, Mester SJ. Results of surgery. In: Levine H, May M, editors. Endoscopic sinus surgery. New York: Thieme Medical Publishers, Inc., 1993:176-92.

Meltzer EO, Hamilos DL, Hadley JA, Lanza DC, Marple BF, Nicklas RA, Adinoff AD,Bachert C, Borish L, Chinchilli VM, Danzig MR, Ferguson BJ, Fokkens WJ, Jenkins SG, Lund VJ, Mafee MF, Nacleiro RM, Pawankar R, Ponikau JU, Schubert MS, Slavin RG, Stewart MG, Togias A, Wald ER, Winther B. Rhinosinusitis: developing guidance for clinical trials. J All Clin Immun 2006; 118(suppl): 17-61.

Min Y, Yun Y, Song B et al. Recovery of nasal physiology after functional endoscopic sinus surgery: olfaction and mucociliary transport. Otorhinolaryngology 1995; 57:264-268.

Newton JR, Ah-See KW. A review of nasal polyposis. Therapeutics and Clinical Management2008; 4(2): 507-512.

Newman LJ Platts-Mills TAE, Phillips CD, Hazen KC, Gross CW. Chronic sinusitis: relationship of computed tomographic findings to allergy, asthma, and eosinophilia. JAMA 1994;271(5): 363-367.

Passali D, Bernstein JM, Passali FM, Damiani V, Passali GC, Bellusi L. Treatment of recurrent chronic hyperplastic sinusitis with nasal polyposis. Arch Otol Head Neck 2003; 129: 656-659.

Rasp. [A new system for he classification of ethmoid polyposis. Effect of combined local and systemic steroid theray]. Laryngorhinootologie. 2000; 79(5):266-72.

Wright ED, Agrawal S. Impact of perioperative systemic steroids on surgical outcomes in patients with chronic rhinosinusitis with polyposis: evaluation with the novel perioperative sinus endoscopy (POSE) scoring system. Laryngoscope 2007; 117(suppl):1-28. 


\title{
Endoscopy in Nasopharyngeal Adenoid Surgery
}

\author{
W. F. Ezzat \\ ORL- Head and Neck Surgery, \\ Consultant Pediatric Otolaryngologist, \\ Ain-Shams University, Cairo \\ Egypt
}

\section{Introduction}

Adenoid diseases include acute adenoiditis, recurrent acute adenoiditis, chronic adenoiditis, and obstructive adenoid hyperplasia. The latter, that constitutes a triad of symptoms including chronic nasal obstruction (with snoring and obligate oral breathing), nasal discharge, and nasal intonation of voice, is the most common cause necessitating surgical intervention. Differentiating adenoid infection from that of the sinuses may be challenging due to similarity of signs and symptoms, and the high incidence of coexistence of both diseases adds to the dilemma, as one may even lead to the other. An additional factor that has been recently recognized is the effect of extraesophageal reflux disease and its role in inducing both adenoid and sinus infection, when this is identified and treated, and treatment fails, surgery should intervene, usually adenoidectomy, putting in mind that the associated sinus affection may take a few weeks to months to clear out.

The diagnosis of adenoid hyperplasia and hypertrophy needing surgery is best achieved by both history and physical examination, the aforementioned triad of symptoms is quite nonspecific, as it may be present in other conditions, as allergic rhinitis, non allergic rhinitis, sinusitis, and reflux esophagitis. The physical examination should guide to the possible disease, and indicate if further investigations are needed. The classic "adenoid facies" appearance, luckily enough, is rarely seen now, as both the parents and physicians diagnose and treat such conditions early enough to avoid such drastic affection of prolonged nasal obstruction. One of the important investigations that are frequently needed is a sleep study, and a variety of tests are used according to need and facilities, starting from simple overnight oximetry, to a full sleep laboratory test, but these are used only in cases where more severe conditions are suspected, such as in cases of resistant nocturnal enuresis without definite history or physical findings of obstructive condition. When the condition is also not clear cut, a CT scan of the nasopharynx and sinuses may be done. It is the authors personal experience that for symptoms of nasal obstruction to occur in the very young child (below one year of age), to be attributed to simple adenoid hypertrophy, and to be severe enough to need surgery, a lateral radiograph of the nasopharynx would not suffice, but a CT scan should be done to exclude other more serious conditions that can cause nasal obstruction, as -and not restricted to- meningeocles, encephaloceles, dermoid cysts, and 
unilateral cases of choanal atresia. These conditions may present by mild nasal obstruction since birth but become aggravated during the next few months of life.

Till relatively recent, the well decided upon treatment to adenoid hypertrophy was surgical removal, but the understanding of the role of environmental inflammation from allergies and reflux, understanding the role of chronic infection, alterations in the relationship with commensal microorganisms, and the appearance of alternative therapeutic modalities has led us to revisit this concept. Alternatives to surgical interference in mild to moderate cases of adenoid hypertrophy implies judicious use of antibiotics and other possible contributing factors as allergy or reflux. The antibiotics used usually involve those acting on beta lactamase producing organisms, aiming to regain normal nasopharyngeal flora, and this was found very useful in some studies, especially when there is associated otitis media with effusion (Bernstein et al., 2002), of course this should not give the impression that adenoidectomy should be postponed as much as possible, but a judicious assessment should be done, to avoid the known "adenoid facies" of chronic nasal obstruction, which -as aforementioned- luckily enough is rarely encountered nowadays.

Adenoidectomy may be the most widely performed otolaryngologic procedure done worldwide, whether alone or in combination with other procedures, with rates reaching 65 per 10,000 children in England, and 50 per 10,000 children in the United States (Van Den Akker et al., 2004). Although it might seem as a very simple procedure, it is not without complications. Changes in technique have progressed over the years parallel to the advances in instrumentation and technology used in surgery.

Still, the classic technique of adenoidectomy is widely performed and adopted by many, if not most, otolaryngologic surgeons all over the world, regardless the economic status. This classic technique of adenoidectomy implies blind curettage of the adenoid tissue, which may or may not be followed by digital -still blind- palpation of the nasopharynx by the surgeon, to assure removal. A survey was performed among otolaryngologic surgeons in the United Kingdom, and showed -despite all the facilities and the total coverage of health services by insurance- that still approximately $80 \%$ of the surgeons performed this blind adenoidectomy, and approximately $70 \%$ performed digital palpation of the nasopharynx at the end of the procedure, even though about $40 \%$ of them recognize a possible need for revision surgery (Dhanasekar et al., 2010). Even mirror examination during or after the curettage procedure is not agreed upon nor done universally.

The most common complication of adenoidectomy is recurrence. The recurrence rates of adenoid regrowth needing revision surgery are so diverse and un-agreed upon, Tolczynski (1955) published a paper in 1955 reviewing earlier studies which showed recordings or recurrence ranging from as low as $4-8 \%$ as reported by Lundgren, to $23.7-50 \%$ according to Hill's investigation, and may even reach above $70 \%$ according to Crowe!!!

Residual adenoid tissue is now becoming to be recognized as a cause of persistence of symptoms, or early recurrence of symptoms with regrowth of adenoid tissue. Several studies have shown significant remaining adenoid tissue after the classic "blind" adenoid curettage technique, and Ark (2010) reported that only about $20 \%$ of patients had complete removal of their adenoid tissue when only digital palpation was done without any type of visualization. A study conducted by Saxby and Chappel (2009), where they performed nasopharyngoscopic examination postoperatively, showed that $68 \%$ of cases had some residual adenoid tissue evident of which $24 \%$ had significant obstruction (grade 2 or 3), Bross-Soriano D et al. (2004) even stated that less than $30 \%$ of adenoidectomies are complete in absence of use of endoscopy! 
The place of residual adenoid tissue with conventional curettage is also controversial, and Regmi et al (2011) reported that when curettage was used alone, it failed to completely remove adenoid tissue from the superiomedial choanae and anterior vault in all cases; incomplete removal was also seen in other parts of the choanae in $67.2 \%$ of patients, the Eustachian tube opening in $63 \%$, the nasopharyngeal roof in $61.78 \%$ and the fossa of Rosenmuller in $61 \%$. Ezzat (2010) reported that even with the aid of a mirror, $14.5 \%$ of patients had residual adenoid tissue, $35 \%$ of which had remnants at the vault of the nasopharynx, $47 \%$ at the lateral walls of the nasopharynx (peritubal), and $18 \%$ at the posterior choana.

Although the rates of residual adenoid tissue, even in modern literature when postoperative assessment is done, is relatively significant, the number or incidence of patients actually needing revision surgery is not as high as would be expected, thus other factors must be present that would favor or induce further regrowth of the residual adenoid tissue, but to date these factors are still to be determined, although theories as reflux or infection by Helicobacter pylori have been proposed by some as Bulut et al., (2006).

Although the history of endoscopy goes back to the early nineteenth century when it was introduced by Bozzini, it was not practically used for nasal and nasopharyngeal assessment until the early nineteen seventies, and the earliest published papers in this field addressed the use of endoscopy in merely assessing the size of the adenoid tissue as a survey (Weymuller E, 1974), later on with advancement of scopes and refinement of technique the uses were widened.

Adenoidectomy is not only indicated in cases of enlarged and obstructing adenoid tissue, other indications may be any type of chronic otitis media, resistant to treatment, whether secretory otitis media, or chronic suppurative otitis media, where any adenoid tissue should be removed, and especially from the area of the opening of the nasopharynx. Also in cases of sinusitis, especially in children, whether recurrent or chronic, adenoid enlargement must be suspected. Other conditions where adenoid tissue is suspected and should be excluded or removed even if not extensively enlarged are cases of obstructive sleep apnoea, or nocturnal enuresis.

Evaluation of the adenoid status includes symptoms, signs and some investigations (outlined in table 1), in order to differentiate adenoid hyperplasia from other causes presenting with similar symptoms such as rhinitis, sinusitis, deviated septum, reflux disease, or even lymphoproliferative disorders.

The general indications for adenoidectomy are usually classified into obstructive, infective, and neoplastic, the various indications are enumerated in table 2.

Preoperative preparation of a candidate for adenoidectomy does not differ than any other type of surgery, the general status of the patient should be assessed for any contraindications of surgery, but otherwise, especially in children, a blood picture and the bleeding profile usually suffices, although it is still a controversial matter (Hartnick and Ruben, 2000; Wei et al., 2000)

Since most patients are children, an addition to the preoperative assessment is to check the status of teeth, as the mouth gag may dislodge loose teeth, and risk of aspiration may occur. Also checking for overt or submucous cleft palate should be done preoperatively, even by simple palpation, to avoid the risk of developing velopharyngeal insufficiency if a complete adenoidectomy is done in presence of such condition. 


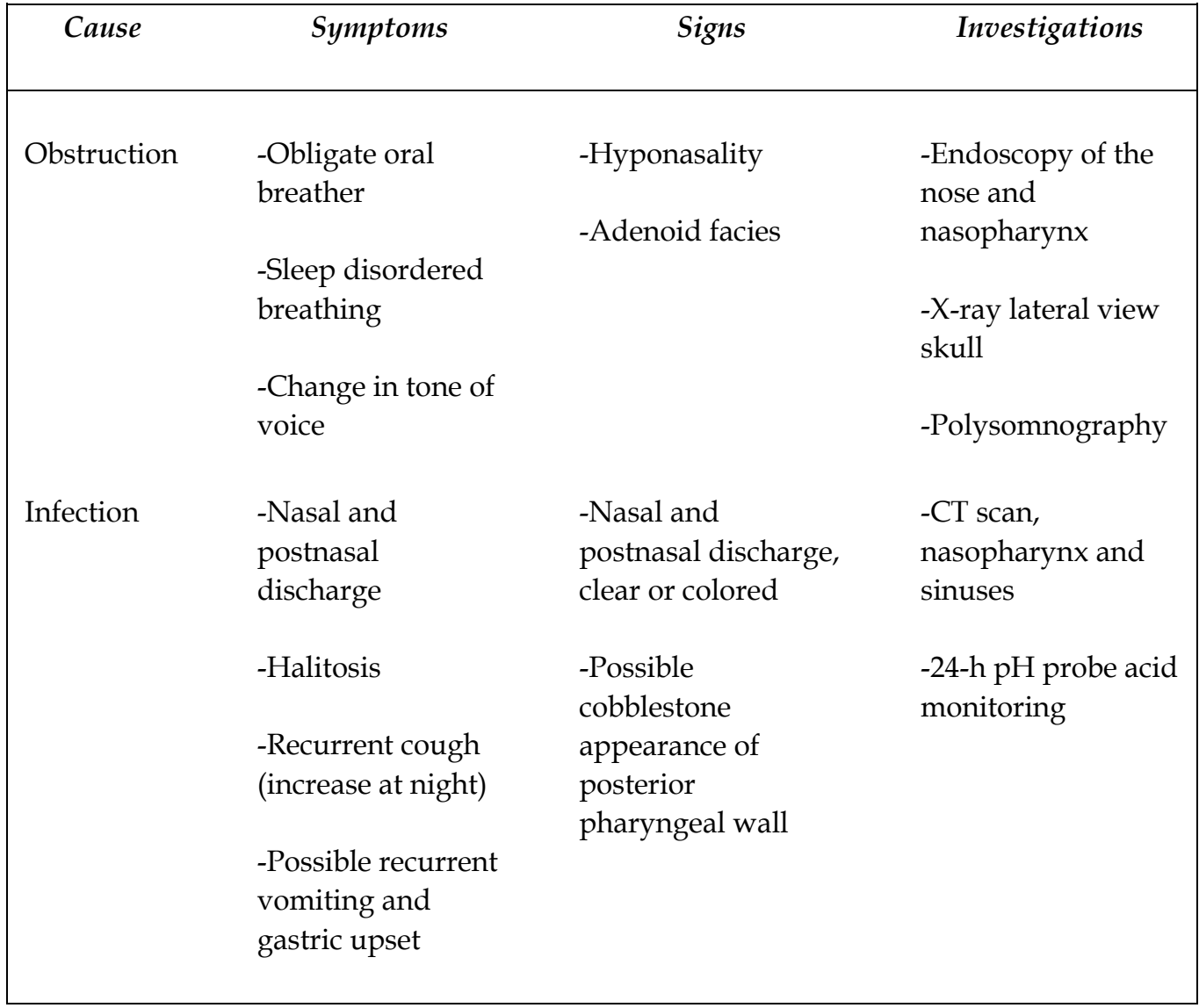

Table 1. Evaluation of adenoid status

A factor that seems overt but sometimes missed by junior staff is putting in mind the status of the atlantoaxial joint and the cervical spine, which can be affected by the hyper extension done during exposure, and the author has personally seen two cases with postoperative acute disc prolapse of the cervical spine in adults undergoing adenoidectomy. In young patients this is quite rare except in cases with congenital laxity of the ligaments, which is usually overt and diagnosed previously, or in cases of Down's syndrome.

Depending on the type of adenoidectomy to be performed, one or more techniques can be used for tissue removal:

- Primary adenoidectomy can be performed using a curette, suction coagulator, or a microdebrider. The suction coagulator is ideal for small adenoids, although it can be used routinely regardless of adenoid size.

- Secondary (revision) adenoidectomy can also be performed using curette, suction coagulator, or microdebrider; however, greater precision is achieved by the latter two methods. The suction coagulator generally results in the least bleeding.

- Partial superior adenoidectomy is performed in children at risk for Velo-PharyngealInsufficiency. The suction coagulator and microdebrider are best suited for this procedure (Kakani et al., 2000). 
- OBSTRUCTIVE

- Chronic nasal obstruction attributed to adenoid tissue hyperplasia, proven by endoscopy, X -ray, or CT scan

- Obligate nasal breathing attributed to adenoid tissue hyperplasia, proven by endoscopy, X -ray, or CT scan.

- Sleep disordered breathing attributed to adenoid tissue hyperplasia

- Obstructive sleep apnoea/hypopnoea syndrome

- Obstructive hypoventilation syndrome

- Upper airway resistance syndrome

- Speech abnormalities (closed nasality) attributed to adenoid tissue hypertrophy.

- Dental and orofacial abnormalities attributed to chronic nasal obstruction

- Failure to thrive attributed to chronic nasal obstruction

- Cor-pulmonale attributed to chronic nasal obstruction

- Lymphoproliferative disorders

- INFECTIVE

- Repeated, or chronic adenoiditis

- Recurrent otitis media

- Chronic otitis media with effusion, non responsive to medical treatment

- Chronic suppurative otitis media

- Chronic sinusitis

- NEOPLASTIC

○ Documented or suspected tumor, benign or malignant

Table 2. Indications for adenoidectomy

\section{Endoscopy in nasopharyngeal adenoid surgery}

Indications, uses and benefits of endoscopic use in nasopharyngeal adenoid surgery have varied over the years, but still to date, it has not become routine in this type of surgery, in spite of all the evidence and published studies that show the benefit of such use!!!

The uses of uses of endoscopy in nasopharyngeal adenoid surgery imply use in diagnosis, during surgery, and in follow up.

The types of rigid endoscopes used in adenoid surgery are illustrated in figure 1, and an example of the photo documentation systems that is optional but very beneficial is illustrated in figure 2.

\subsection{Use of endoscopy in diagnosis of nasopharyngeal adenoid size}

Although the most important factor in diagnosing adenoid size is the symptomatology the patient presents with, which is mainly nasal obstruction, mouth breathing, and/or snoring, other tools have been used to verify or exclude adenoid as the cause of nasal obstruction, the most commonly used is the X-ray of a lateral view of the skull, but this is not flawless. X- 
rays have over and under estimated sizes of adenoid tissue in many instances. Another issue is that adenoid tissue may be present in areas causing obstruction and not that well apparent in the X-ray, especially in recurrent or persistent cases after adenoidectomy, such as choanal adenoids. Therefore, a more realistic and accurate estimate is to perform an office endoscopy by a rigid sinoscope or flexible nasopharyngoscope, this is quite simple and applicable in adults, adolescents and cooperative older children, which are rarely the case where adenoid hypertrophy is a major problem. In younger children, which compromise most cases, to perform such office type endoscopy is quite difficult and challenging, but it can be used using short acting sedatives such as chloral hydrate which has been used safely in children and infants since 1869 (Buck, 1992), the recommended dose of chloral hydrate is 50 to $75 \mathrm{mg} / \mathrm{kg}$ given orally or rectally. In other studies, higher single doses of up to 100 $\mathrm{mg} / \mathrm{kg}$ have been used with increased success in children and infants over 1 month of age (Steinberg, 1993). It is the author's belief that such endoscopy is much more beneficial in assessing the whole upper airway for proper planning of the treatment modality, whether surgical, medical or both.

Endoscopy is also valuable in case symptoms are present other than obstruction, such as loss of appetite, slower development than peers, and decreased hearing which is related to Eustachian Tube dysfunction, in absence of apparent $X$-ray enlargement of the adenoid tissue, in such cases surgery may be indicated to remove tubal adenoid tissue even in absence of X-ray evidence of enlargement or significant nasal obstruction. Another important issue to be addressed is that there may be an element of septal deviation that is causing the obstruction, which was be missed by anterior rhinoscopy and X-ray, although this is quite rare below the age of 5 (Reitzen et al. 2011).

\subsection{Use of endoscopy in nasopharyngeal adenoid surgery}

Although many studies have reached a prevailed conclusion that endoscopic aided adenoidectomy has benefits covering any added cost or time, in terms of better exposure or complete removal, still regrettably it is not the standard teaching in many parts of the world, not only in developing countries, which might have some concern for cost, but also in developed countries. The application of endoscopy in adenoidectomy has several techniques, from full endoscopic power aided adenoidectomy to adenoidectomy by conventional curettage but with endoscopic visualization, to simple endoscopic examination at the end of conventional adenoidectomy to assess complete excision.

\subsubsection{Simple endoscopic examination after conventional adenoidectomy}

One of the simplest ways of using an endoscope for assurance of complete surgery and total removal of adenoid tissue is the mere transnasal endoscopic examination of the posterior choanal and the nasopharynx after conventional curettage adenoidectomy, with or without mirror assistance, and to proceed with completion adenoidectomy -if needed- under endoscopic guidance. In such case, after the surgeon has removed the main bulk of the adenoid tissue and established hemostasis, the endoscope -size of which is determined by age of patient- is introduced through BOTH SIDES of the nasal fossae to visualize the posterior choana and the nasopharynx (Ezzat 2010) (figure 3), and if any adenoid tissue is found it is removed either with a smaller adenoid curette, with a Blakesly-Wigand Nasal Forceps ${ }^{\circledR}$ (figure 4), or a Blakesly Rhinoforce ${ }^{\circledR}$ Ethmoid forceps (figure 5), straight or with upward curve. Further hemostasis should be accomplished if needed. An important sign of 
complete adenoid removal, without injury of the underlying pharyngeal bed, at least to the author's experience, is the lack of need for packing the nasopharyngeal bed after surgery, although there has been no published literature highlighting this fact

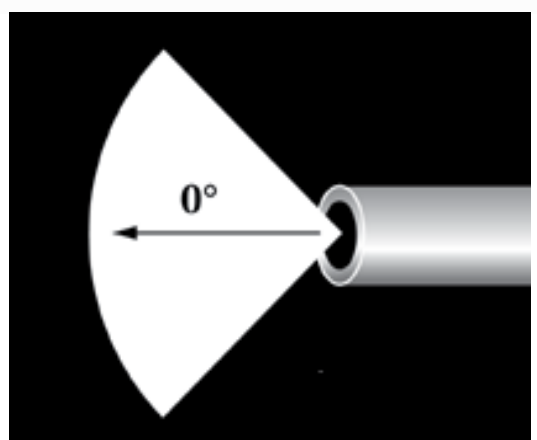

A)

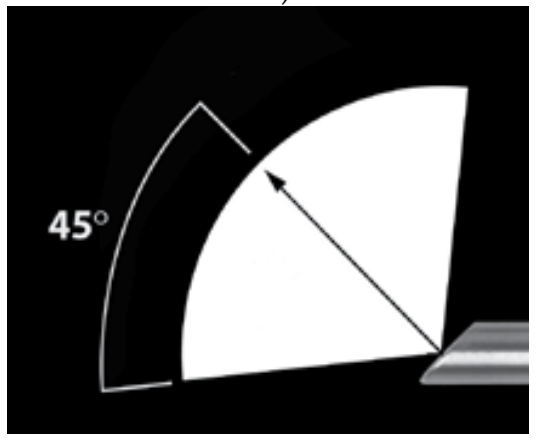

C)

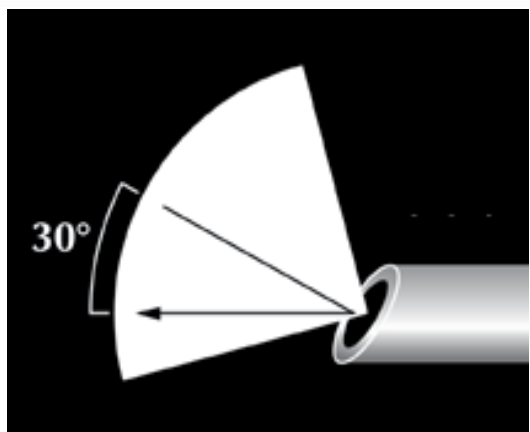

B)

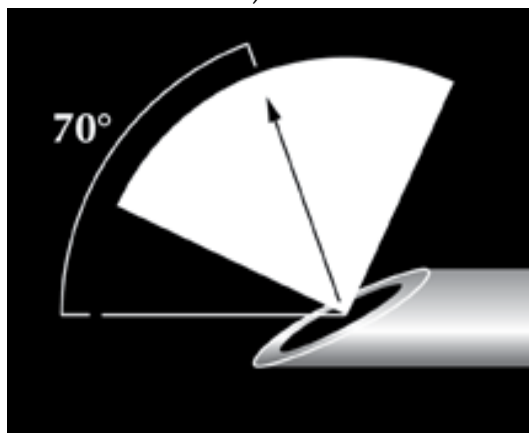

D)

The endoscope used can be the conventional $4.5 \mathrm{~mm}$ or the pediatric $2.3 \mathrm{~mm}$ sinoscope.

The angulations of the endoscope used is determined by the approach it is used in, when the endoscope is introduced and used through the nasal fossa, a $0^{\circ}$, or $30^{\circ}$ endoscope can be used, as they give a more direct view of the nasopharynx (A, and B), but when the endoscope is used transorally, a $45^{\circ}$ or $70^{\circ}$ endoscope is needed to obtain a better view by angulating the view $(\mathrm{C}$, and $\mathrm{D})$

Fig. 1. Endoscopes used in adenoid surgery

\subsubsection{Endoscopic visualization with routine curettage adenoidectomy}

To start the surgery of adenoidectomy under direct endoscopic visualization has been described by many (Wan et al, 2005, Songu et al, 2010), this technique implies the introduction of the endoscope endonasally at the beginning of surgery, attachment to a monitor, then using the regular mouth gag and conventional curette in excision of the adenoid tissue. 


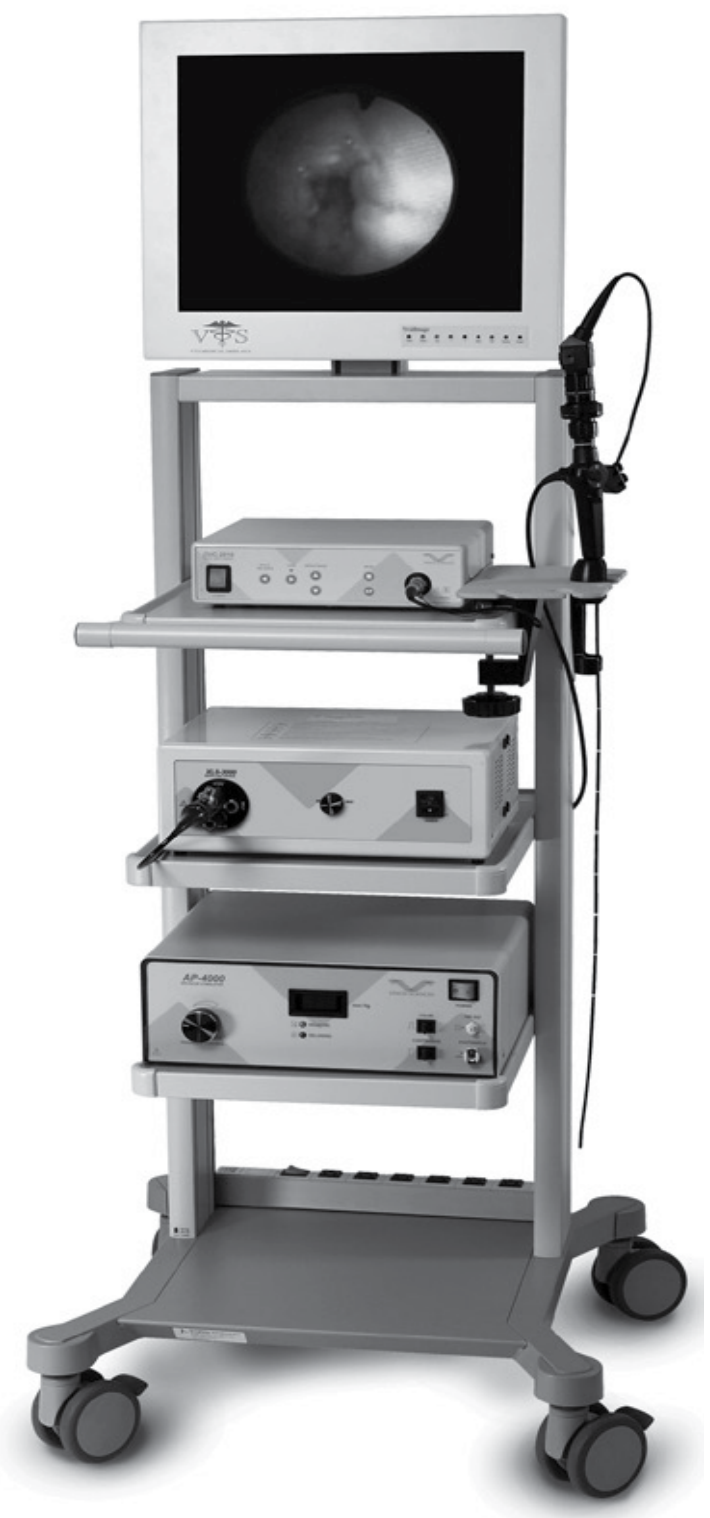

The system is optional, but helps in documentation, illustration to the patient and for teaching purposes.

Fig. 2. The photo documentation system of use with endoscopes.

According to the size of the adenoid tissue as assessed endoscopically, a curette is chosen and should fit snugly over the adenoid and is inserted up to the septal vomer, the curette is swept inferiorly with a side-to-side rocking motion to completely remove all adenoid tissue. Care is taken to avoid deep muscular or vertebral injury, injury to the torus region, and injury to the choana. Again, if endoscopy reveals any residual tissue, a smaller curette or a St Clair adenoid forceps or a Blakesly-Wigand Nasal Forceps ${ }^{\circledR}$ (figure 4), or a Blakesly 
Rhinoforce ${ }^{\circledR}$ Ethmoid forceps (figure 5), straight or with upward curve is used to remove any retained tissue noted.

Although this technique might seem appealing, in practice it is quite cumbersome, too many instruments and connections, and lack of direct eye-to-hand sense is difficult for beginners, although it is very useful in teaching and monitoring trainees. The cost of such a setting might also be a burden in less funded establishments, and no additional benefit over the routine adenoidectomy followed by mere examination by the endoscope at the end of surgery has been documented in any study published over the past years.

Some have described using the a $45^{\circ}$ or $70^{\circ}$ endoscope through the oral cavity to guide the excision of adenoid tissue all through the surgical procedure (Jong \& Gendeh, 2008), this might seems more appealing, especially in younger children, as their nasal cavities are smaller, but even with the transoral approach to insert the endoscope, the field may be somewhat narrow.

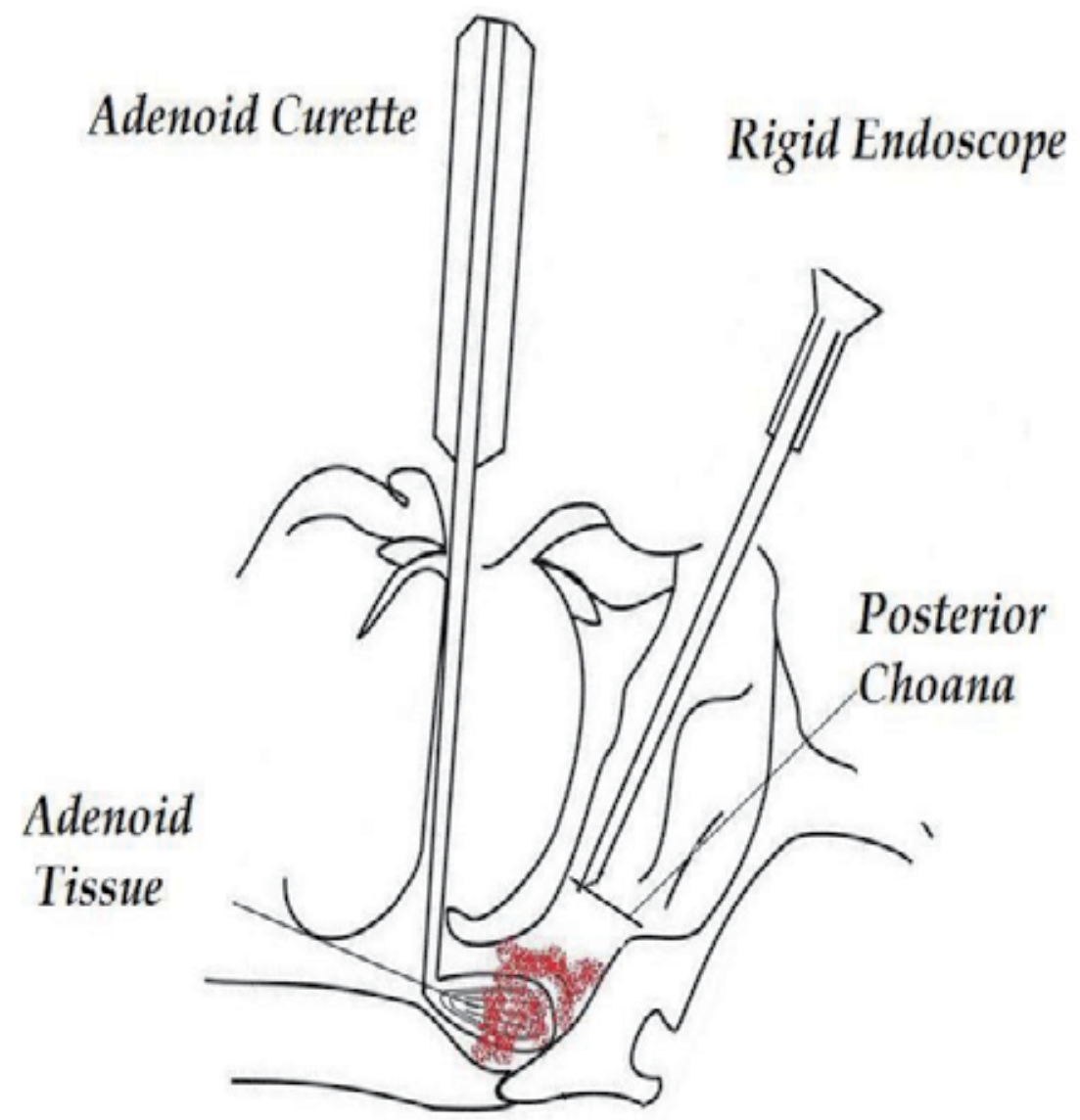

Fig. 3. Simultaneous use of the endoscope and adenoid curette

\subsubsection{Fully endoscopic diathermy aided ablation adenoidectomy}

The use of diathermy in adenoidectomy has been used since the nineteen sixties (Remington-Hobbs C, 1968), but with the regular monopolar blade, and was not widely 
used, as issues of pharyngeal stenosis and post operative pain were addressed, and many used such a technique for control of bleeding in cases where simple packing was not enough. The suction diathermy was introduced in the late nineteen nineties and gained popularity rapidly due to several reasons, the procedure is simple, with minimal blood loss, not associated way any risk of pharyngeal stenosis (Walker P., 2001) or recurrence, although it is repeatedly reported to have longer working time than conventional curettage adenoidectomy (Jonas NE., 2007). The price is not an issue, as the disposable hand piece is relatively cheap, and is even cheaper than other disposable more sophisticated instruments used (Walker P., 2001). (figure 6)

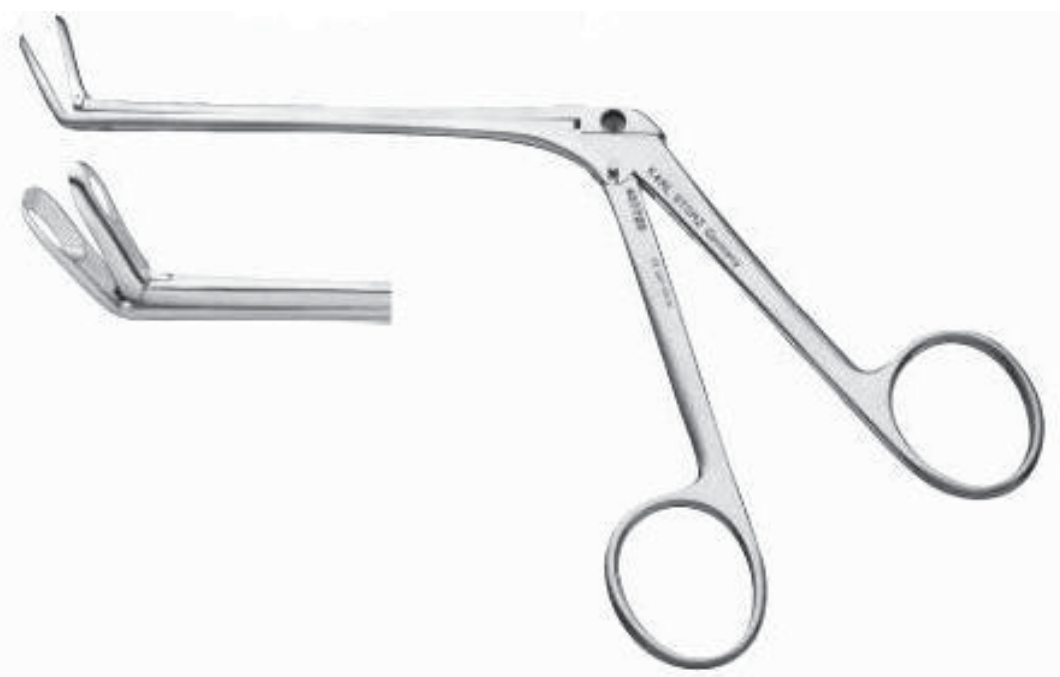

Fig. 4. Blakesly- Wigand Nasal Forceps

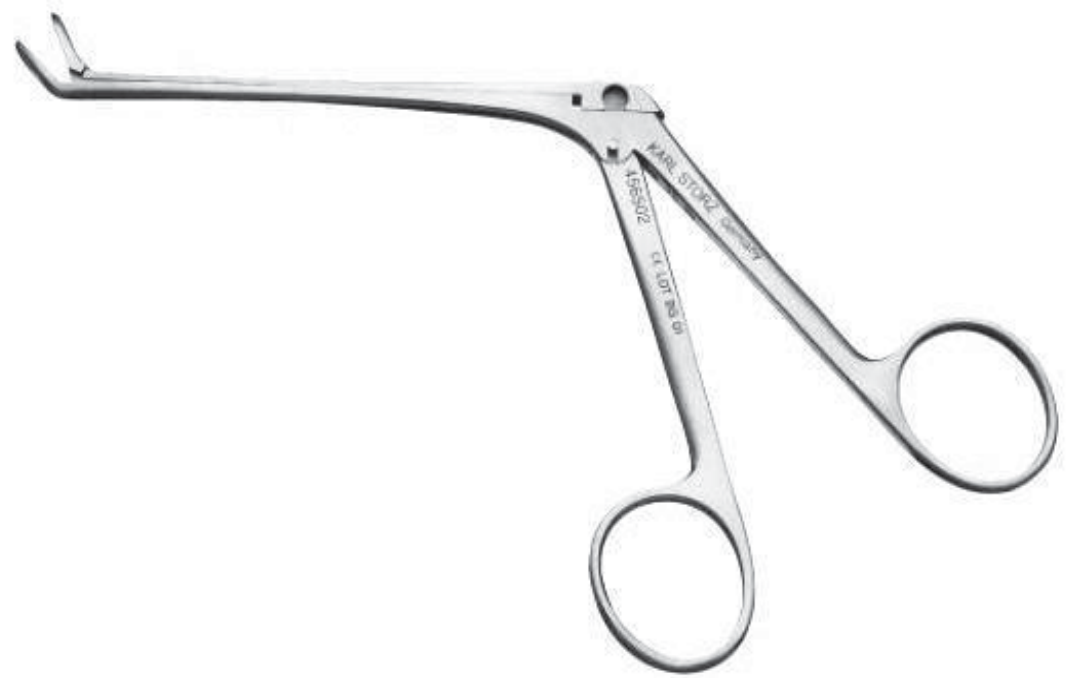

Fig. 5. Blakesly- Rhinoforce Forceps 


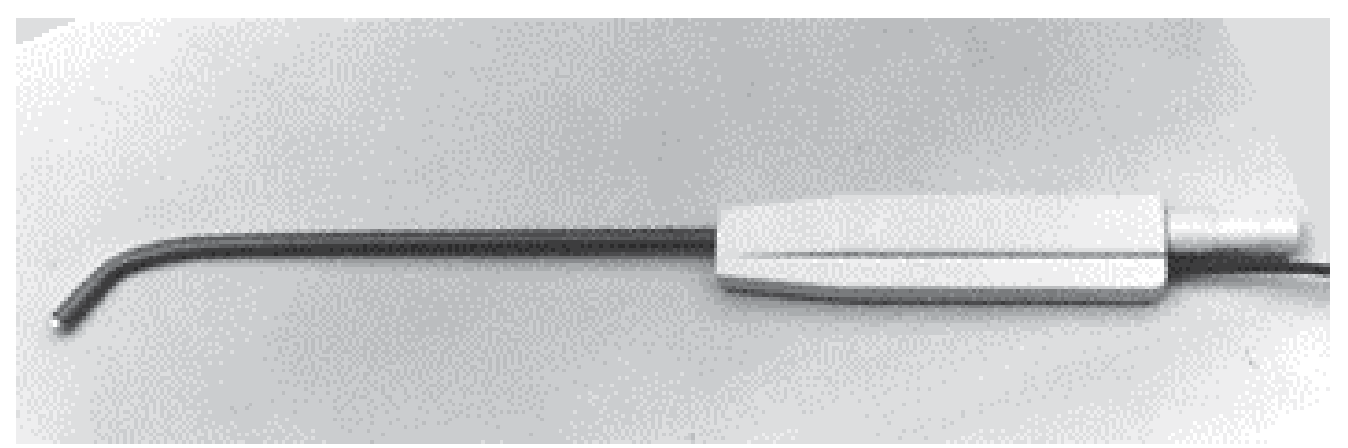

The tip of the handpiece is flexible and can be manipulated according to need

Fig. 6. The suction diathermy handpiece

The original description of such use dictated that the nasopharynx is exposed by means of two catheters introduced through the nose, and brought out of the mouth, retracting the soft palate. Often, a mirror was used to guide the suction diathermy to visualize the adenoid tissue, but the use of endoscopes with this suction diathermy technique is very beneficial. Endoscopes have been used by two approaches, the first of which a $45^{\circ}$ endoscope is introduced ORALLY for visualization, and then with the other hand the suction is introduced and surgery completed (Lo and Rowe-Jones, 2006), the second of which a fully transnasal endoscopic ablation adenoidectomy is done (Shin and Hartnick, 2003). Although this second type has the benefit of not needing hyperextension of the neck, it is not suitable for all age groups as it requires a certain wideness of the nasal fossa, but it is optimal in certain situations -if applicable- such as lack of neck stability as in children with Down's syndrome.

\subsubsection{Endoscopic coblation (hydrodebrider) adenoidectomy}

Coblation adenoidectomy (figure 7) is a relatively recent introduction in the field of otolaryngologic surgery. Coblation, that uses lower temperatures than electrocautery to remove tissue and achieve hemostasis, has been shown to reduce pain and decrease postoperative narcotic use, leading to shorter recovery times and a quicker return to normal in children (Benninger $\mathrm{M}$ and Walner D, 2007). The use of coblation can be used by guidance of mirror viewing, but of course endoscopic guidance gives much more accurate view, whether used transnasally to transorally, and does not add any cumbersome to the surgical field, as the hand that uses the mirror uses the endoscope. As with most guided techniques, this approach dictates the partial exposure of the surgical field by using 2 catheters introduced transnasal and delivered orally, to retract the soft palate.

\subsubsection{Endoscopic micro-debrider-aided adenoidectomy}

Microdebrider (power) aided adenoidectomy was introduced and started to be common in the early $21^{\text {st }}$ century, the use of the microdebrider (figure 8 ) has been used for adenoidectomy in several modes and approaches, the more common approach and practical one is introduction of the endoscope through the nasal fossa and the hand piece of the microdebrider through the mouth, and under endoscopic vision, shaving the adenoid tissue piece by piece. Both the hand piece and the endoscope can be introduced through the oral cavity if the nasal fossa is too narrow, in younger children. 


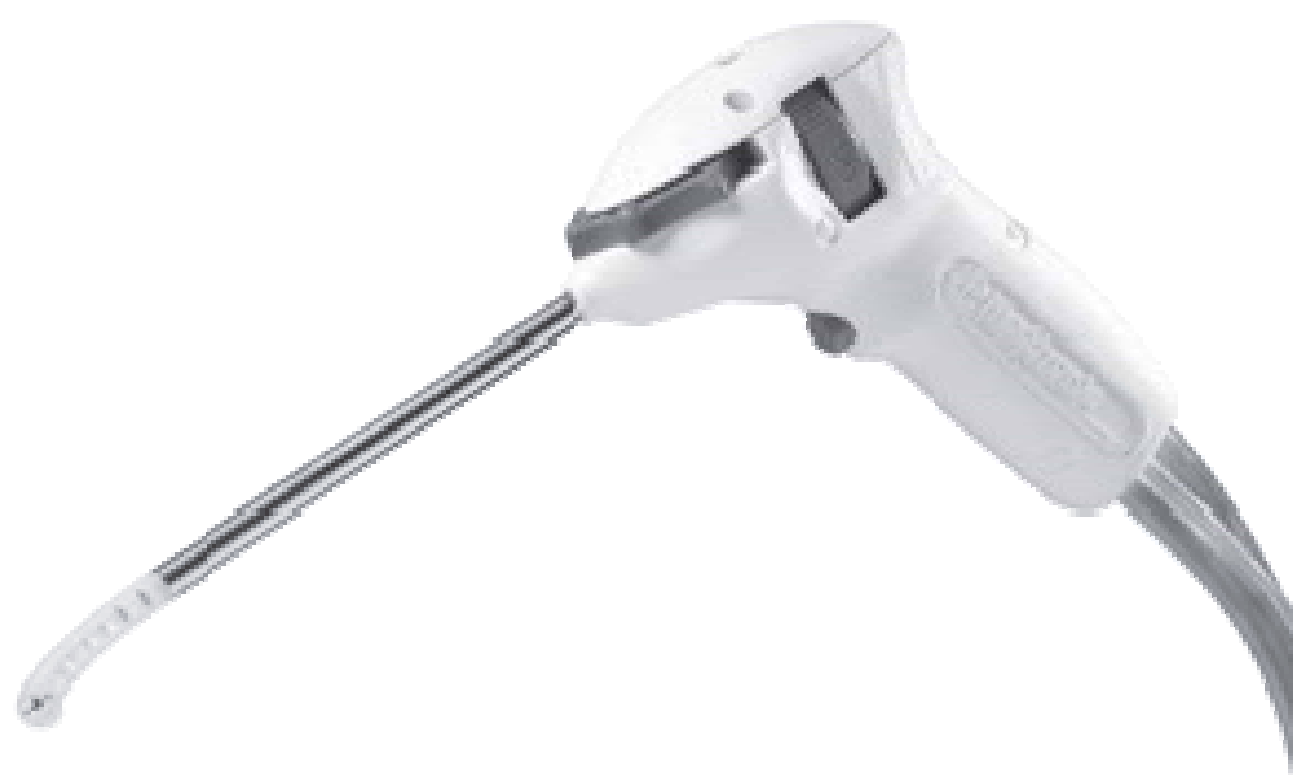

Fig. 7. Hydrodebrider Hand piece

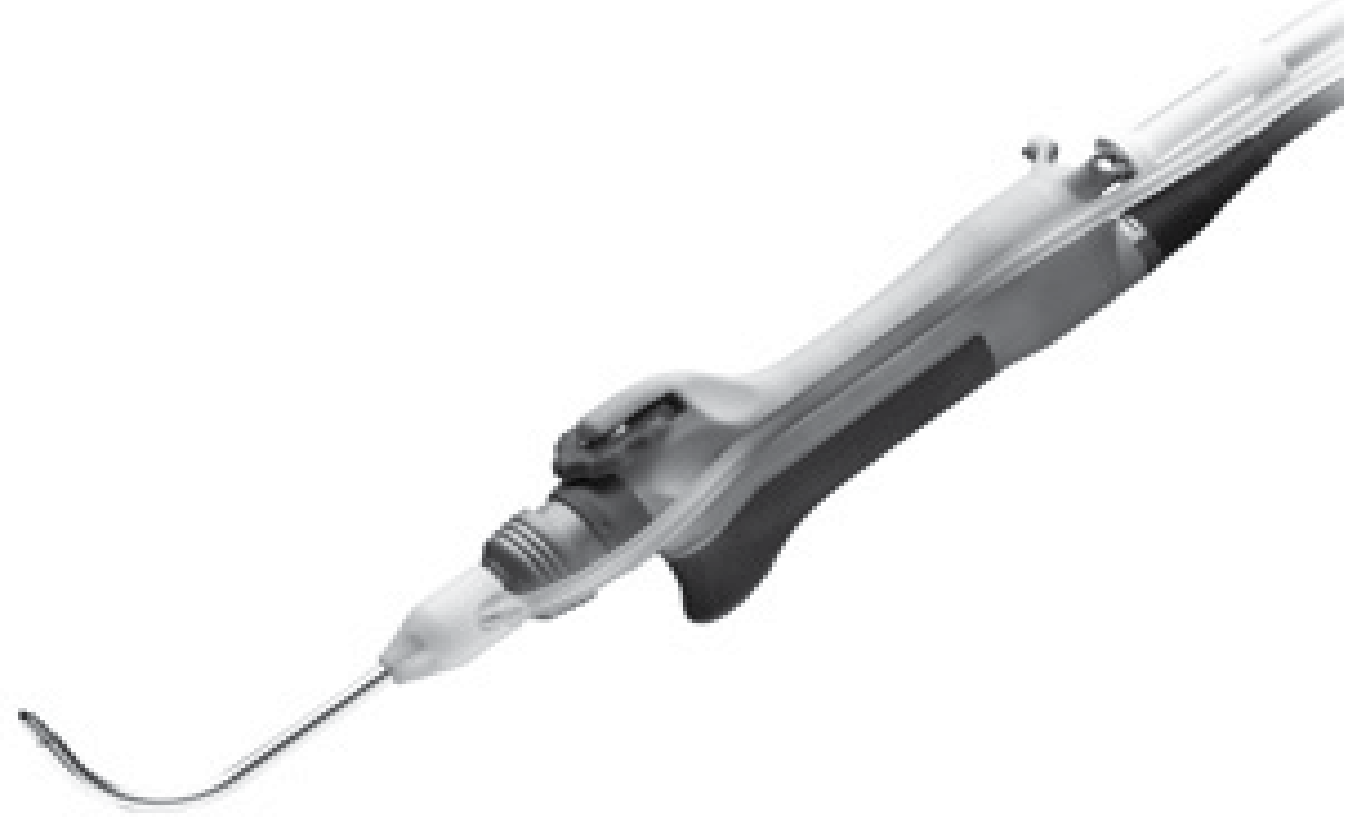

Fig. 8. Microdebrider hand piece

One of the major advantages of using the microdebrider is having the option to remove adenoid tissue only partially (Rodriguez et al., 2002; Koltai et al., 2002), as in cases of insufficient velopharyngeal valve, in cases of repaired cleft palate, and those with submucous cleft palate. 
Blood loss is comparable to other methods used, and although the time used may be more than with conventional curette adenoidectomy, the clearer filed and more complete surgery seems to justify the extra time, to save time, some have used such powered instrumentation after removal of the main bulk of the adenoid tissue by conventional curettage (Pagella F et al., 2009). The main limitation is the price of the disposable hand piece that exceeds by far all other modes of adenoidectomy. Figure 9 shows the typical setting for use of combined endoscopic and powered instrumentation adenoidectomy.
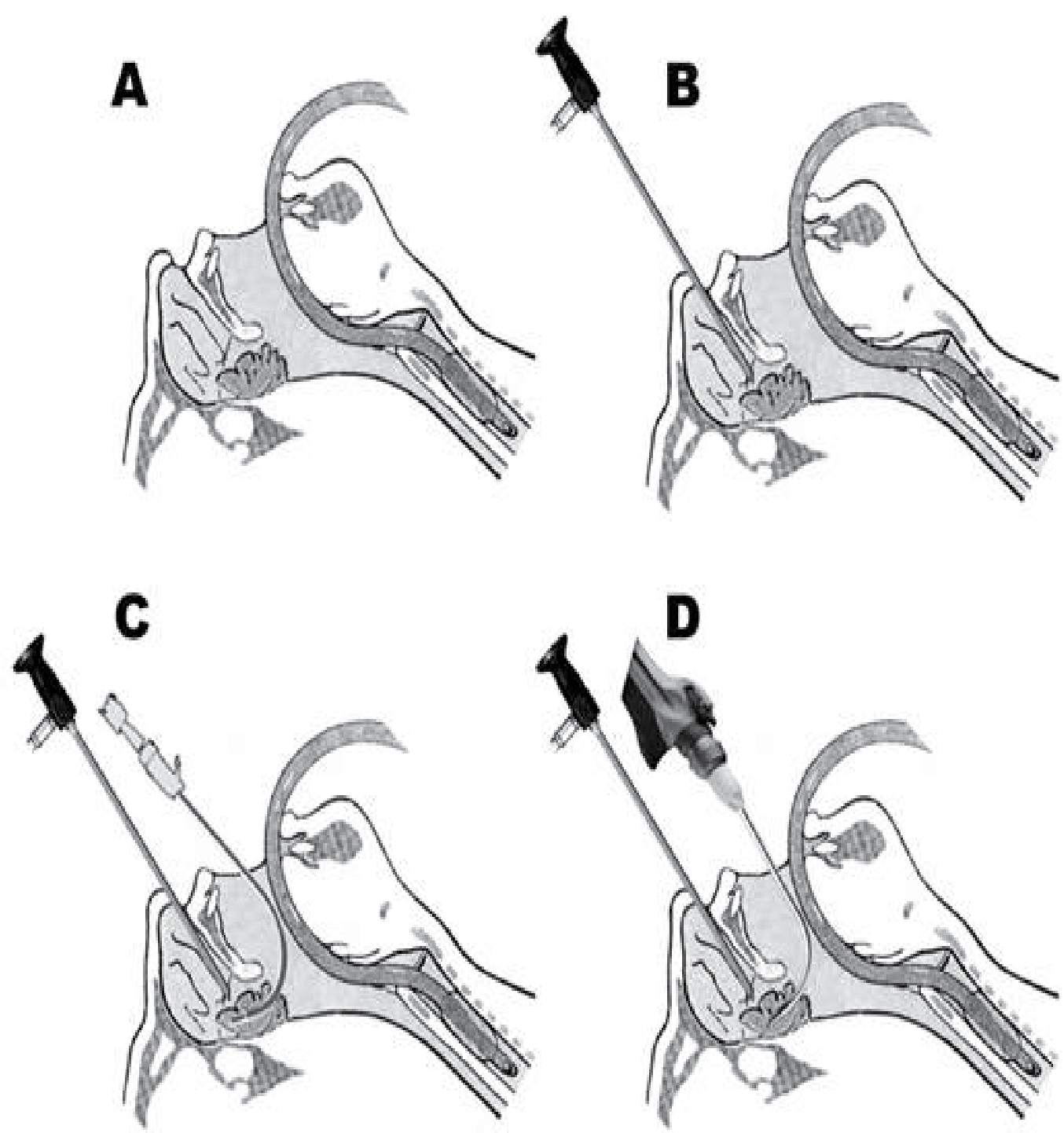

Fig. 9. Typical setting for use of combined endocopic guided powered adenoidectomy a; positioning of the patient with endotracheal tube inserted orally, $b$; introduction of the endoscope trans-nasally, c; introduction of the microdebrider hand piece, $\mathrm{d}$; attachment of the hand piece to the micromotor and commencing surgery (Courtsy of Prof Dr MZ Helal with permission) 


\subsection{Follow up after adenoidectomy}

Postoperative follow up after adenoidectomy does not entail any special proceedings; the child is usually discharge from the hospital on the same day of surgery. The usual regime is to advice soft diet for 24 hours, and then regular diet allowed after that, usually no antibiotics are used. A mild analgesic may be prescribed. Nasal decongestant drops may be used with caution for 2-3 days, as the child is more liable to toxicity from the raw surface of the adenoid bed. The child is usually scheduled for a post operative visit after 3-4 days to assure that everything is going in order. Some have advised postoperative endoscopy to assess any residual adenoid tissue, but this is of no value except if the patient develops symptoms of regrowth of the adenoid.

\subsection{Limitations of use of endoscopy in adenoid surgery}

In younger children with small nasal fossae, the introduction of the endoscope nasally can by quite difficult, if not impossible, but this can be overcomed if the endoscope is used trans-oral, otherwise there are no limitations. Excessive bleeding in the operative field may limit the view, but this should not be an indication to abort the surgery except in very rare cases, and if such case occurs it can be managed by regular packing for a few minutes, and then continuing surgery.

\subsection{Complications of endoscopy use in adenoid surgery}

No additional complications during adenoid surgery have been recorded with the additional use of endoscopy to guide the surgical procedure. Complications -if occurwould be those of the technique, and not from use of endoscopy. Complications related to surgery are outlined in table 3, with their presentation and possible management lines.

Noniatrogenic complications after adenoidectomy include (Randall and Hoffer, 1998)

- Regrowth of adenoid tissue, particularly in very young children, which may require revision (secondary) adenoidectomy.

- Hypernasality, because of temporary pain splinting. Persistent hypernasality is rare and probably caused by unrecognized pre-existing velopharyngeal weakness. Management includes speech therapy or a sphincter pharyngoplasty, if refractory.

- Atlantoaxial subluxation (Grisel's syndrome), which presents with persistent torticollis 1-2 weeks after surgery. Neurological or orthopedic consultation may be required.

Iatrogenic complications after adenoidectomy include

- Dental injury, from intubation or the mouth gag. Dentition should be checked prior to inserting and removing the mouth gag. Urgent laryngoscopy and bronchoscopy must be performed for any newly discovered missing teeth.

- Nasopharyngeal stenosis, caused by excessive tissue removal. Repair is difficult and may include dilation, steroid injection, or a tissue flap (rotational, advancement, or free flap) (Giannoni et al., 1998).

- Eustachian tube injury, if the torus tubarius is cauterized or denuded

- Meningitis, after injecting lidocaine and epinephrine into the posterior nasopharynx prior to adenoidectomy. Injections are unnecessary and should be avoided.

- Lingual nerve palsy, caused by pressure from the tongue blade of the mouth gag.

- Cautery burns, caused by operator error or equipment malfunction. (Zinder and Parker, 1996). 


\begin{tabular}{|c|c|c|}
\hline Complication & Presentation & Management \\
\hline Hemorrhage & Bleeding from mouth or nose & $\begin{array}{l}\text { Local control } \\
\text { (vasoconstricting } \\
\text { agents, cautery) }\end{array}$ \\
\hline Airway obstruction & $\begin{array}{l}\text { Stridor/stertor, palatal } \\
\text { swelling }\end{array}$ & $\begin{array}{l}\text { Nasopharyngeal } \\
\text { airway +/- steroids }\end{array}$ \\
\hline Dehydration & Dry mucous membrane & Hydration \\
\hline $\begin{array}{l}\text { Persistent velopharygneal } \\
\text { valve insufficiency }\end{array}$ & $\begin{array}{l}\text { Hypernasal speech (>2 } \\
\text { months postoperative) }\end{array}$ & $\begin{array}{l}\text { Speech therapy/ } \\
\text { palatal surgery }\end{array}$ \\
\hline Pulmonary edema & $\begin{array}{l}\text { Difficult oxygenation and pink } \\
\text { secretions from endotracheal } \\
\text { tube }\end{array}$ & $\begin{array}{l}\text { Posetive end } \\
\text { expiratory } \\
\text { ventilaton }\end{array}$ \\
\hline Nasopharyngeal stenosis & $\begin{array}{l}\text { Progressive nasal difficult } \\
\text { breathing and closed nasality }\end{array}$ & Palatal surgery \\
\hline Torticollis & $\begin{array}{l}\text { Neck pain and deviation to } \\
\text { one side }\end{array}$ & $\begin{array}{l}\text { Physiotherapy/ } \\
\text { surgery }\end{array}$ \\
\hline Cervical spine sublaxation & $\begin{array}{l}\text { Neck pain }+/ \text { - neurologic } \\
\text { deficit }\end{array}$ & $\begin{array}{l}\text { Neurological/ } \\
\text { orthopedic } \\
\text { consultation }\end{array}$ \\
\hline Dental injury & Defective teeth & ?? reinmplantation \\
\hline Eustachian Tube injury & Hearing loss and otitis media & ?? Ventilation tube \\
\hline Lingual nerve palsy & Poor tongue movement & Conservative \\
\hline Cautery burns & Burning sensation and ulcers & Surface anesthetics \\
\hline Nasal adhesions & $\begin{array}{l}\text { Nasal bleeding and } \\
\text { obstruction later on }\end{array}$ & ?? Lysis \\
\hline Recurrence & Progressive nasal obstruction & Revision surgery \\
\hline
\end{tabular}

Table 3. Complications related to adenoidectomy 
Complications related to use of endoscope

- Nasal mucosal injury, with subsequent scarring and adhesions, this is very rare, and occurs only after rough manipulation.

\section{Conclusion}

Adenoidectomy is a very widely and commonly performed surgery, although the standards for surgery have changed due to the better understanding of the etiopathology. There is no worldwide agreement on the "typical technique" for adenoidectomy, thus various procedures and aids for such surgery have been used, all aiming at better removal and reducing recurrence rates. The use of endoscopy to guide adenoidectomy aids in achieving better results -in terms of complete surgery- with all techniques of adenoidectomy, whether conventional curettage, or with more recent methods, and whether for obstructive or non obstructive causes, and whether for complete or partial adenoidectomies. It is simple to use, does not add to time or expenses of surgery.

\section{Acknowledgment}

- The companies of Storz, and Medtronic for supplying their catalogues for use of pictures.

- $\quad$ Professor MZ Helal for supplying the diagram for use of combined endocopic guided powered adenoidectomy.

\section{References}

Ark N., Kurtaran H., Ugur K.S., Yilmaz T., Ozboduroglu A.A., \& Mutlu C. (2010). Comparison of adenoidectomy methods: examining with digital palpation vs. visualizing the placement of the curette. International Journal of Pediatric Otorhinolaryngology. Jun;74(6):649-51.

Benninger M. \& Walner D. (2007). Coblation improving outcomes for children following adenotonsillectomy. Clinical Cornerstone. ; S Suppl 1:S13-23.

Bernstein JM, Faden HS, Scannapieco E, et al (2002). Interference of non-typeable Haemophilus Influenzae and MOraxella Catarrhalis by Streptococcus oralis in adenoid organ culture; possible strategy for the treatment of otitis prone child. Annals of Otorhinolaryngology; 111:696-700.

Bozzini. In, Nezhat's History of Endoscopy, Available from, http:/ / laparoscopy.blogs.com/endoscopyhistory/chapter_06/

Bross-Soriano D., Schimelmitz-Idi J., \& Arrieta-Gómez J.R. (2004). Endoscopic adenoidectomy; use or abuse of the technology. Cirugia Y Ciruganos. JanFeb;72(1):15-9; discussion 21-2.

Buck M.L. (1992). Chloral hydrate use during infancy. Neonatal Pharmacology Quarterly, 1:31-7

Bulut Y., Agacayak A., Karlidag T., Toraman Z.A., \& Yilmaz M. (2006). Association of cagA+ Helicobacter pylori with Adenotonsillar Hypertrophy, Tohoku Journal of Experimental Medicine. 209;229-233.

Carr MM, Poje CP, Ehrig D, Brodsky LS. (2001). Incidence of reflux in young children undergoing adenoidectomy. Laryngoscope. Dec;111(12):2170-2. 
Dhanasekar G., Liapi A., \& Turner N.(2010). Adenoidectomy techniques: UK survey. Journal of Laryngology and Otology. Feb;124(2):199-203.

Ezzat W.F., (2010) Role of endoscopic nasal examination in reduction of nasopharyngeal adenoid recurrence rates. International Journal of Pediatric Otorhinolaryngology, 74; 404-406

Giannoni C, Sulek M, Friedman EM, Duncan NO.(1998) Acquired nasopharyngeal stenosis. Archives of Otolaryngology Head \& Neck Surgery;124:163-7.

Hartnick CJ, Ruben RJ. (2000) Preoperative coagulation studies prior to tonsillectomy. Archives of Otolaryngology Head E Neck Surgery, 126:684-6.

Jonas N.E., Sayed R., \& Prescott C.A. (2007). Prospective, randomized, single-blind, controlled study to compare two methods of performing adenoidectomy. International Journal of Pediatric Otorhinolaryngology. Oct;71(10):1555-62.

Jong YH, Gendeh BS. (2008) Transoral endoscopic adenoidectomy: initial experience. Medical Journal of Malaysia 63:81.

Kakani RS, Callan ND, April MM. (2000) Superior adenoidectomy in children with palatal abnormalities. Ear Nose E Throat Journal; 79:300-5.

Koltai P.J., Chan J., \& Younes A. (2002). Power-assisted adenoidectomy: total and partial resection. Laryngoscope. Aug;112(8 Pt 2 Suppl 100):29-31.

Lo S., \& Rowe-Jones J. (2006). How we do it: Transoral suction diathermy adenoid ablation under direct vision using a 45 degree endoscope. Clinical Otolaryngology. Oct;31(5):440-2.

Pagella F., Matti E., Colombo A., Giourgos G., \& Mira E. (2009). How we do it: a combined method of traditional curette and power-assisted endoscopic adenoidectomy. Acta Otolaryngolgica. May;129(5):556-9.

Randall DA, Hoffer ME. (1998) Complications of tonsillectomy and adenoidectomy. Otolaryngology Head \& Neck Surgery;118:61-8.

Regmi D., Mathur N.N., \& Bhattarai M. (2011). Rigid endoscopic evaluation of conventional curettage adenoidectomy. Journal of Laryngology and Otology. Jan;125(1):53-8.

Reitzen S.D., Chung W., \& Shah A.R. (2011). Nasal septal deviation in the pediatric and adult populations. Ear Nose and Throat Journal. Mar;90(3):112-5.

Remington-Hobbs C. (1968) Diathermy in dissection tonsillectomy and retrograde dissection adenoidectomy. Journal of Laryngology and Otology. Nov;82(11):953-62.

Rodriguez K., Murray N., \& Guarisco J.L. (2002). Power-assisted partial adenoidectomy. Laryngoscope. Aug;112(8 Pt 2 Suppl 100):26-8.

Saxby A.J., \& Chappel C.A. (2009). Residual adenoid tissue post-curettage: role of nasopharyngoscopy in adenoidectomy ANZ J Surg. Nov;79(11):809-11.

Shin J.J., \& Hartnick C.J. (2003). Pediatric endoscopic transnasal adenoid ablation. Annals of Otology Rhinology and Laryngology. Jun;112(6):511-4.

Songu M., Altay C., Adibelli Z.H., \& Adibelli H. (2010). Endoscopic-assisted versus curettage adenoidectomy: a prospective, randomized, double-blind study with objective outcome measures. Laryngoscope. Sep;120(9):1895-9.

Steinberg A.D. (1993). Should chloral hydrate be banned? Pediatrics;92:442-6.

Tolczynski B., The recurrence of adenoids, Canadian Medical Association Journal. 72 (December) (1955) 672-673, Quoted from. 
Van Den Akker E.H., Hoes A.W., Burton M.J., \& Schilder A.G. (2004). Large international differences in adenotonsillectomy rates, Clinical Otolaryngology and Allied Sciences. 29 (2) 161-164.

Walker P. (2001). Pediatric adenoidectomy under vision using suction-diathermy ablation. Laryngoscope. Dec;111(12):2173-7.

Wan YM, Wong KC, Ma KH, (2005) Endoscopic guided adenoidectomy using a classic adenoid curette: a simple way to improve adenoidectomy Hong Kong Med $\mathrm{J} ; 11: 42-4$

Wei JL, Beatty CW, Gustafson RO. (2000). Evaluation of post tonsillectomy hemorrhage and risk factors. Otolaryngology Head \& Neck Surgery, 2000;123:229-35.

Weymuller E. (1974) Nasopharyngoscopic observations in the Alaskan native. Laryngoscope May;84(5):864-8.

Zinder DJ, Parker GS. (1996), Electrocautery burns and operator ignorance. Otolaryngology Head \& Neck Surgery;115:145-9. 


\title{
Endoscopically Guided Balloon Dilatation of Recurrent Choanal Stenosis
}

\author{
B.J. Folz and C.-G. Konnerth \\ Department of Otorhinolaryngology, \\ Karl Hansen Medical Center, Bad Lippspringe \\ Germany
}

\section{Introduction}

Balloon Sinuplasty is a treatment modality, which has gained much attention since its introduction in 2005. Nasal and paranasal sinus disease can be treated with this new method in a less traumatic way than by Functional Endoscopic Sinus Surgery (FESS) or conventional sinus surgery. One of the advantages of Balloon Sinuplasty is the preservation of anatomical structures within the nose. Also there is no loss of mucosal lining, i.e. ciliated nasal epithelium normally remains intact. The basic principle is to place a balloon catheter (Fig. 1) via a guidewire into a sinus ostium, to inflate the balloon and thus to dilate the sinus ostium. Improved ventilation and drainage of the sinus via the dilated ostium ensures healing of the diseased cavity. Dilatation catheters, which are now being used in Rhinology derive from vascular medicine, where these devices are used to open occluded or partially occluded arteries. In this field dilatation catheters have led to significant reduction of operative trauma compared to conventional vascular surgery.

FESS currently is the gold standard in the treatment of recurrent sinusitis, if conservative therapy fails to achieve a permanent relief. It provides good results with acceptable discomfort for the patient. In most cases postoperative packing is necessary after FESS to stop nasal bleeding. Packing is uncomfortable for the patient and thus there are ongoing endeavours to develop even less traumatic methods to treat nasal diseases than FESS. Balloon dilatation is supposed to have the following benefits compared to FESS: less bleeding, more tissue preservation, less trauma to surrounding tissue, less complications, faster patient recovery and less postoperative care.

Initially all Balloon Sinuplasty procedures were performed under fluoroscopy. This fact was bound to technical prerequisites, which not every ENT-Department was able to provide. In addition exposure of patients and surgeons to X-rays during the procedure was a matter of continuous debate, especially in paediatric patients. This problem was overcome, when the LUMA $^{\mathrm{TM}}$ technology was developed and catheters could be placed into the respective sinuses under endoscopic and diaphanoscopic control. We decided to use this technique for the restoration of the nasal airway passage in a child with recurrent choanal atresia. At the explicit wish of the parents no conventional surgery or FESS procedure was carried out. The parents reported, that the first operation had severely traumatized the child, therefore the parents asked for the least traumatic method, which was likely to give the child relief of his complaints. 


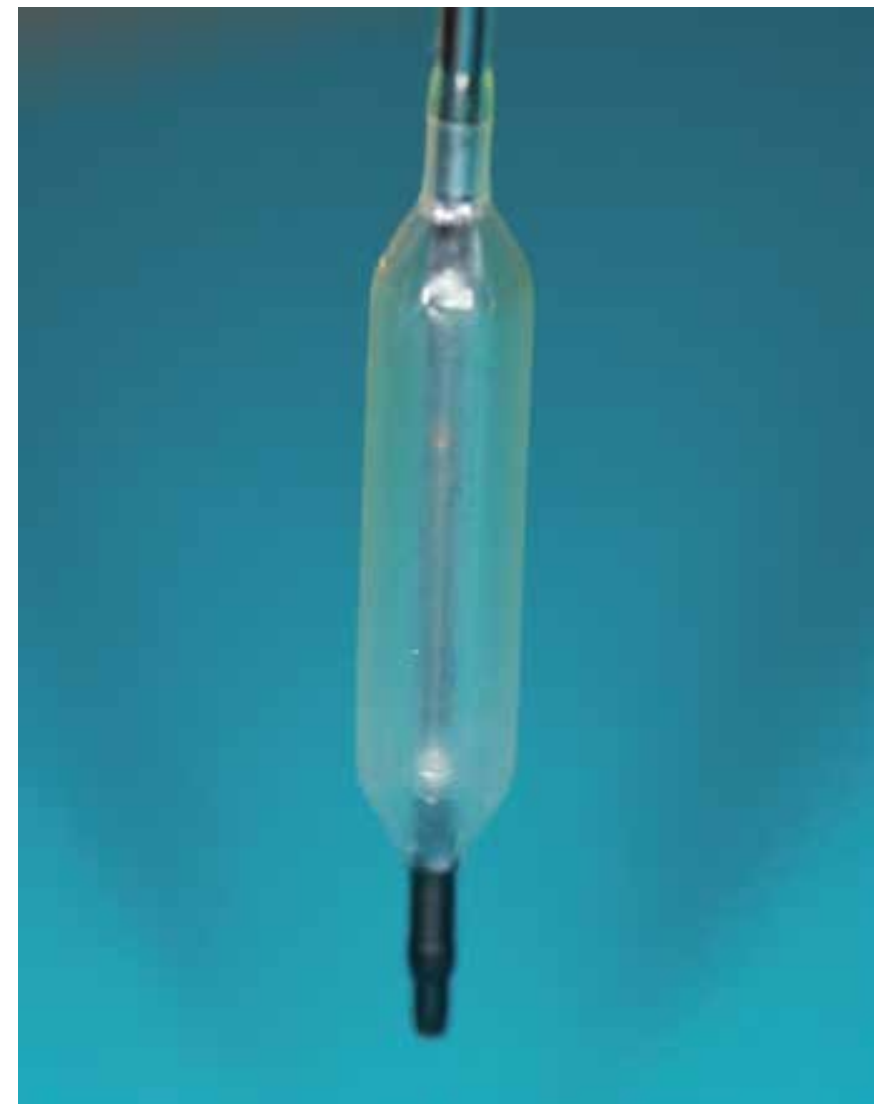

Fig. 1. Inflated Sinus Balloon. The size of the respective Balloon catheter was $7 \mathrm{~mm} \times 16 \mathrm{~mm}$ in diameter.

\section{Case report}

A 4 year-old boy had been operated on a right-sided choanal atresia via a transnasal approach at the age of two years. A silicone stent had been inserted for a period of three months postoperatively. Initially nasal breathing had been restored successfully, however within two years symptoms recurred. The child again suffered from persistent anterior rhinorrhoea, recurrent airway infections and snoring. Examination by flexible endoscopy and CT scanning revealed recurrent stenosis, which was partially bony and partially membranous (Fig.2). Repeated surgery was recommended, but the parents specifically asked for a less invasive treatment. The parents reported, that the child had suffered a lot after the initial operation and during the postoperative period, when the stent had to be kept in place for many months. The balloon method was explained to the parents and the interventional procedure was offered. Simultaneously it was pointed out that the balloon method was a treatment attempt and no standard therapy, as there were only few accounts in literature reporting that this method had already been used to open a choanal atresia. After weighing the potential advantages of the balloon dilatation method against their past experiences with endoscopic sinus surgery the parents explicitly asked for the balloon method. 


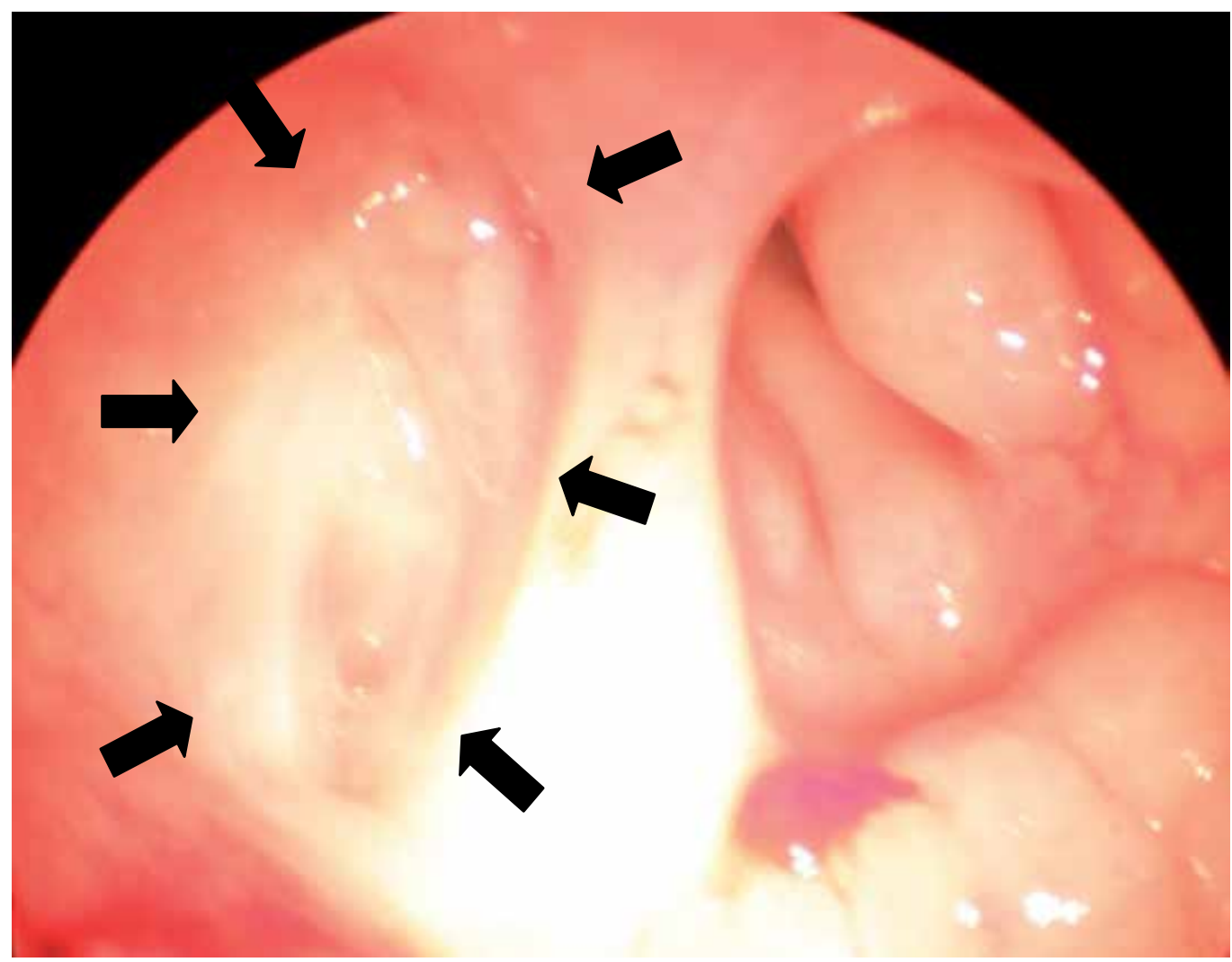

Fig. 2. Intraoperative view into the nasopharynx with a rigid $90^{\circ}$ angled endoscope (transoral view). The left-sided choana shows a normal appearance. The posterior ends of the inferior and middle turbinate can be identified. The right-sided choana is occluded by a membranous plate $(\rightarrow)$ and a bony stenosis .

\section{Method}

A balloon catheter was placed in the nasal cavity under control with a $0^{\circ}$-degree rigid endoscope (Karl Storz Company, Tuttlingen) while the child was under general anaesthesia (Fig. 3). After decongestion of the nasal mucosa with cottonoids soaked in decongestant nose drops, a pinpoint-sized lumen within the atretic plate could be identified. Under simultaneous transoral endoscopy of the nasopharynx with a rigid $90^{\circ}-$ angled endoscope (Karl Storz Company, Tuttlingen) (Fig 4.) a light source catheter was placed in the small choanal opening (Acclarent Company, Relieva LumaTM Sinus Illumination System, $7 \mathrm{~mm}$ diameter) (Fig. 5). The light source catheter was subsequently advanced into the nasopharynx. The sinuplasty balloon catheter was then gently rotated into the residual lumen, with the light source catheter as guide wire. The balloon was inflated with normal saline solution until a pressure of 8 atm. was reached (Fig. 6). The balloon remained inflated for 5 minutes and was then deflated. The residual lumen was thus enlarged to a diameter of $7 \mathrm{~mm}$ without any signs of hemorrhage or swelling (Fig.7). No packing was necessary and no postoperative bleeding occurred. After some 


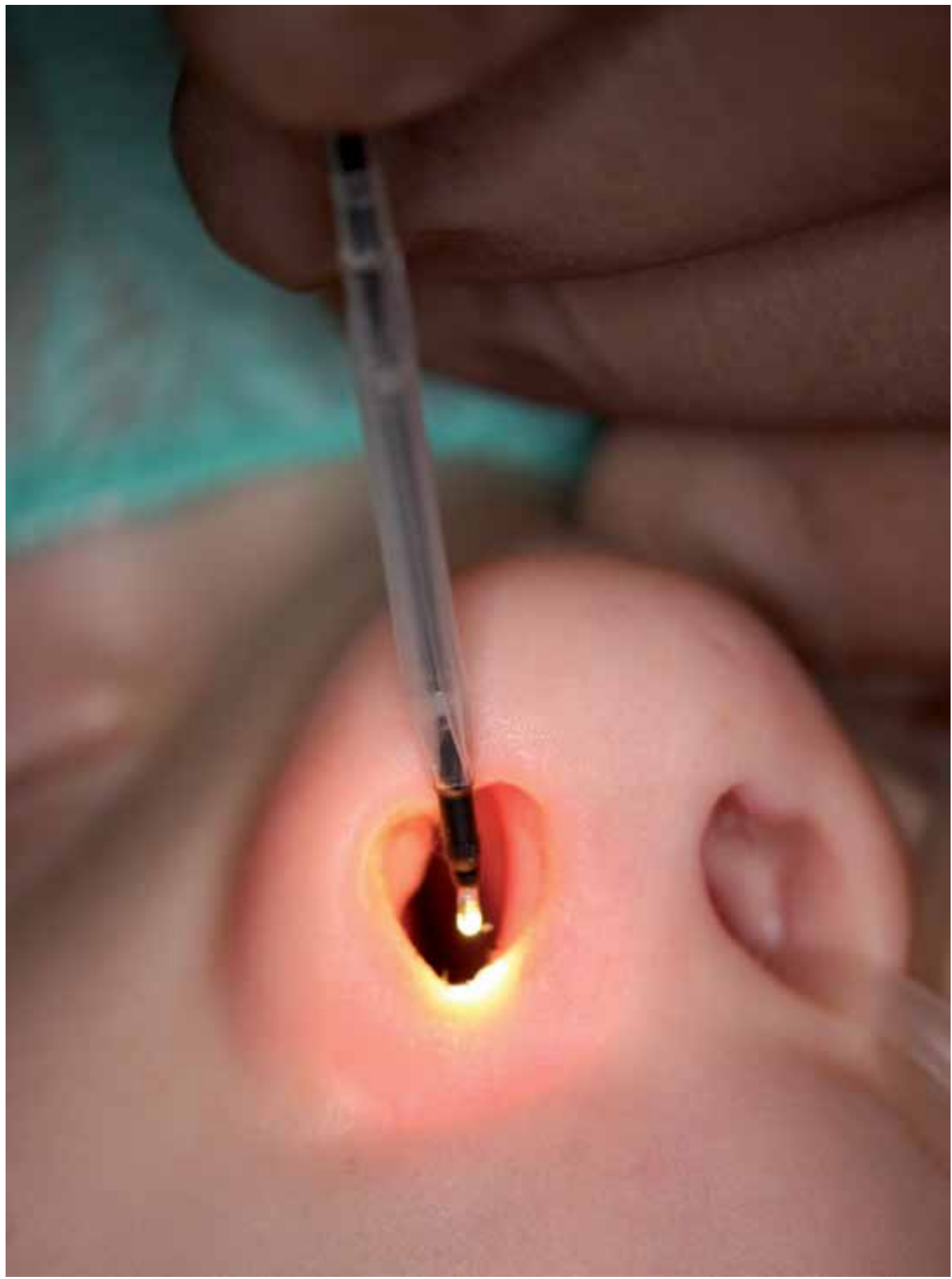

Fig. 3. The flexible light-fibre of the Relieva LUMA ${ }^{\mathrm{TM}}$ illumination system (straight tip) is the guide wire for the balloon catheter. The guide wire and the balloon catheter are inserted into the right nose and advanced to the stenosis. 


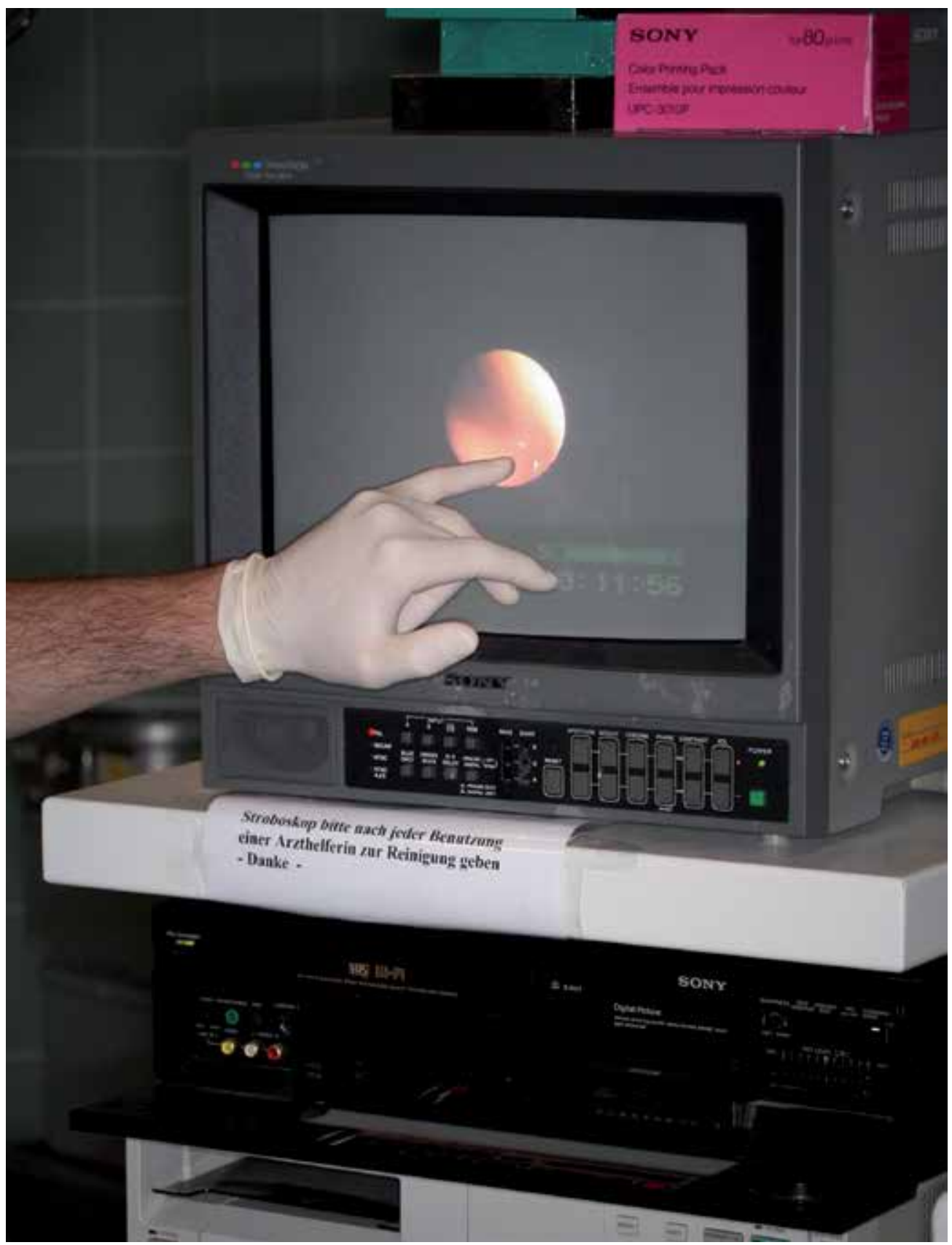

Fig. 4. Simultaneously the nasopharynx is controlled transorally with a $90^{\circ}$ endoscope coupled with a digital camera. The position of the catheter can be controlled on a monitor. The position of the catheter is correct, if the choana lightens up on diaphanoscopy. By transnasal endoscopy the residual lumen of the choana was identified and the catheter was placed into the lumen. 


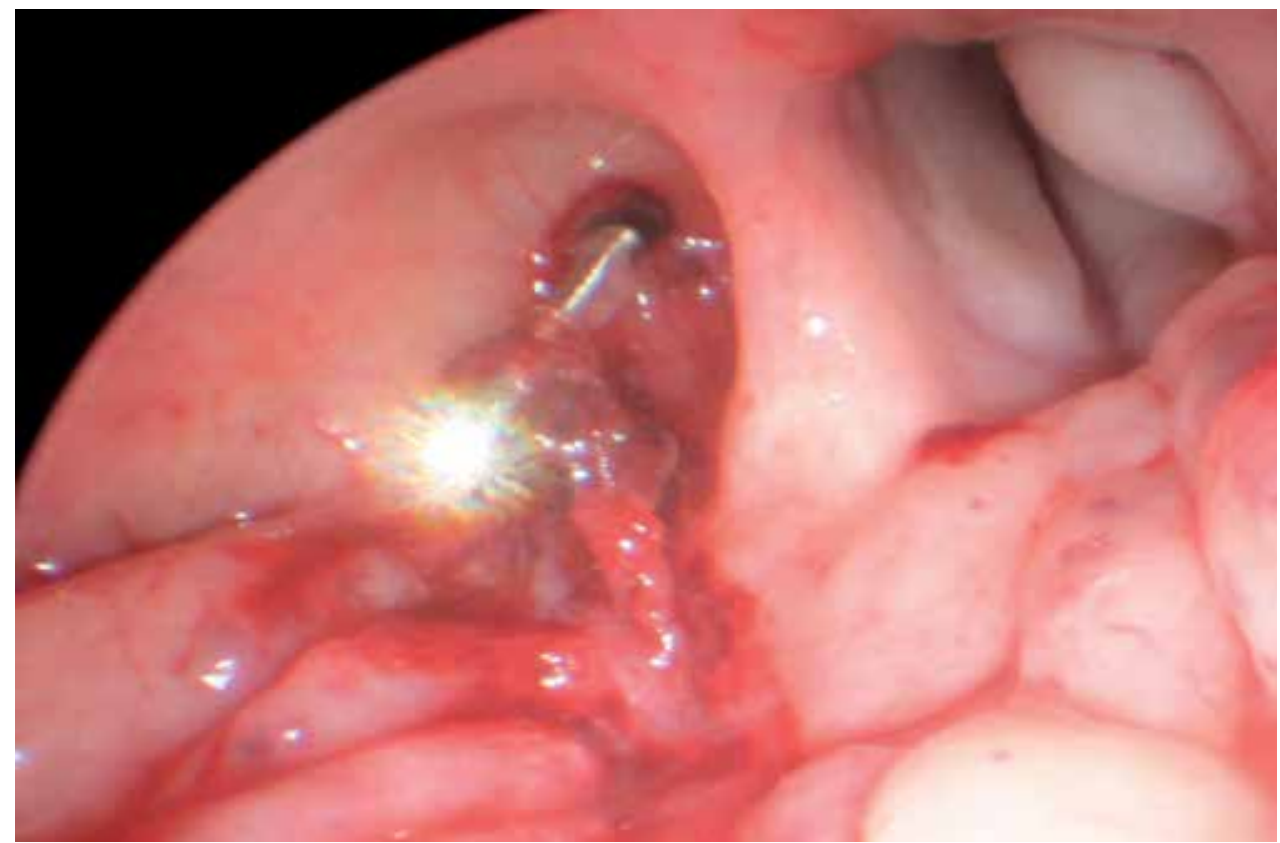

Fig. 5. The LUMA ${ }^{\mathrm{TM}}$ catheter is advanced into the nasopharynx (endoscopic image with a $90^{\circ}$-angled endoscope).

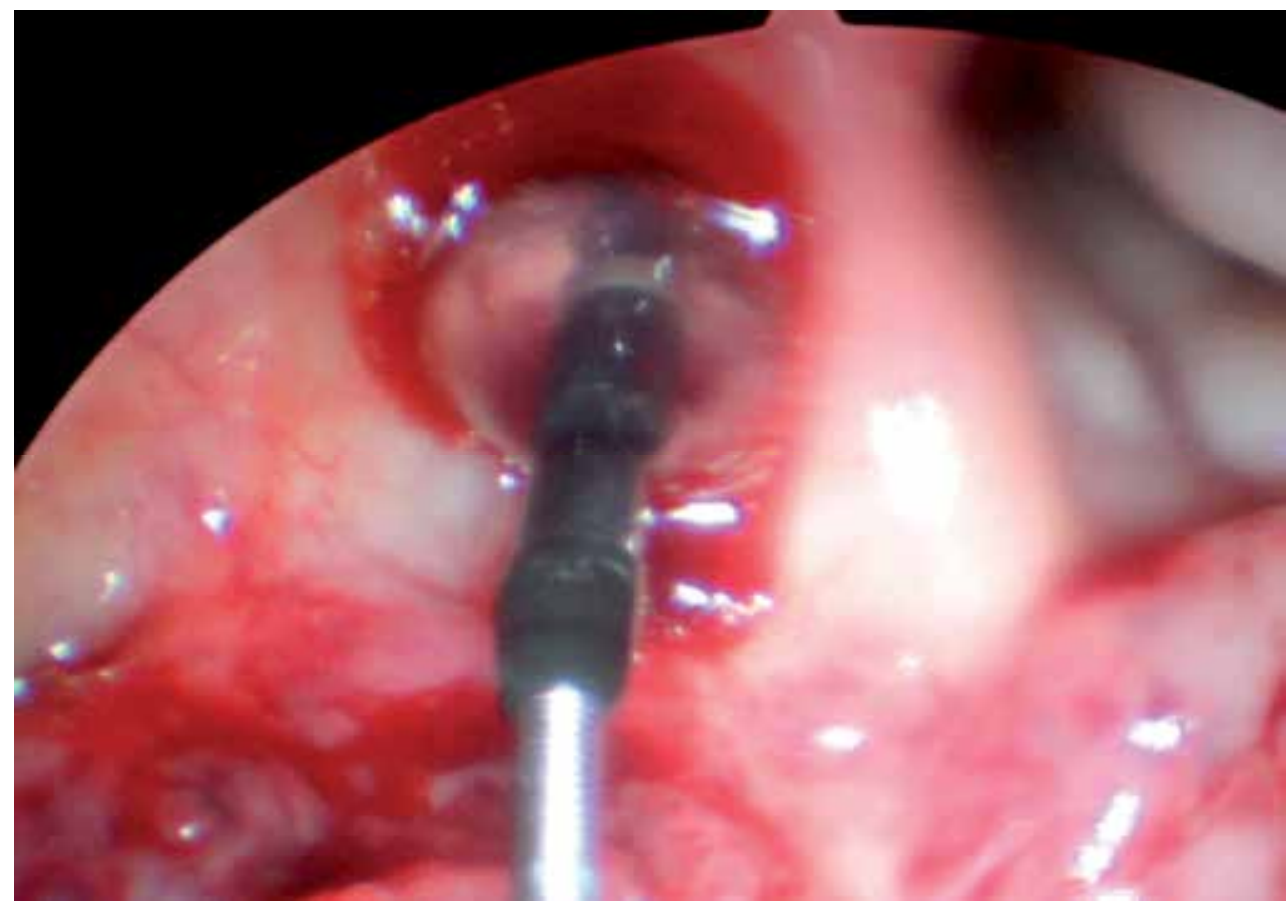

Fig. 6. Once the balloon catheter is in the correct position it is inflated with normal saline solution to a pressure of 8 atmospheres. The catheter stays in situ for 5 minutes. 


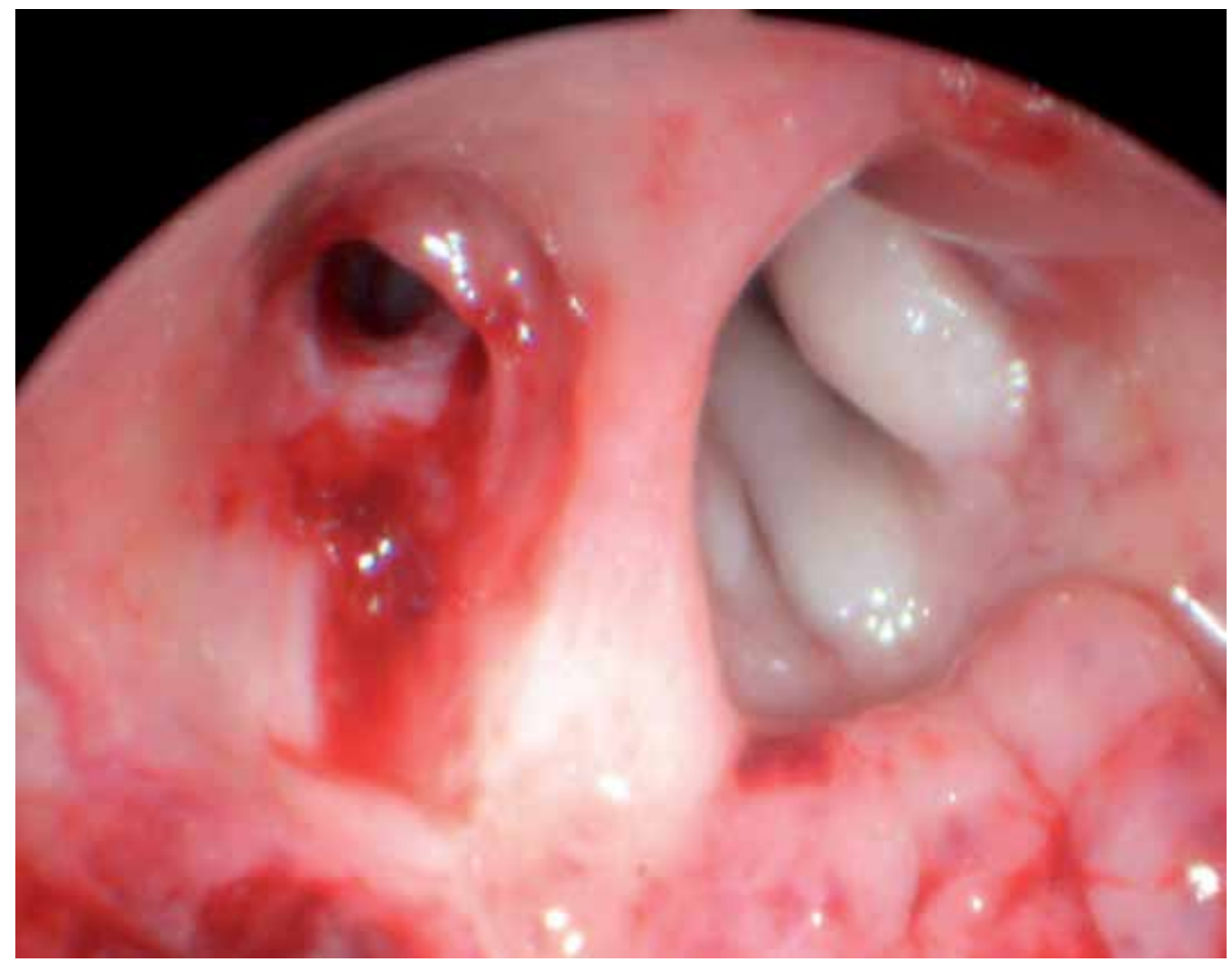

Fig. 7. After the removal of the catheter a circular dilatation of the atretic membrane is evident. The diameter of the choana is approximately $7 \mathrm{~mm}$. Stenting of the choana was not necessary.

uneventful hours in the recovery area the patient was discharged home. Normal saline solution for nasal rinsing and nasal antiseptic cream were prescribed two times daily for the treatment of possible crusting.

\section{Result}

Intraoperatively the procedure was performed as planned, no adverse events occured. The pressure in the balloon was successively increased at paces of 2 atms at each step until a target pressure of 8 atmospheres was reached. As shown in the respective image (Fig. 7), there was hardly any bleeding. The child had a normal recovery and did not complain about any pain or discomfort. There was no need for any specific postoperative therapy other than lubricating ointments, decongestive nose drops and rinsing the nose with normal saline. No antibiotics were administred. The child was under observation as an inpatient for 24 hours postoperatively. The boy was discharged on the following day. Postoperative packing or stenting was not necessary. On controls 6, 12, 18 and 24 months postoperatively the enlarged choanal lumen remained stable. Nasal breathing was restored successfully as could be shown by the mirror test (Fig. 8). There were no signs of rhinorrhoea, snoring or recurrent stenosis. The patient and his parents were extremely 
happy with the result and the fact that the operation and postoperative phase was considerably less traumatizing than the initial operation. No specific therapy other than the above mentioned topical treatments were prescribed. The child is currently observed at 6 month intervals in our outpatient department.

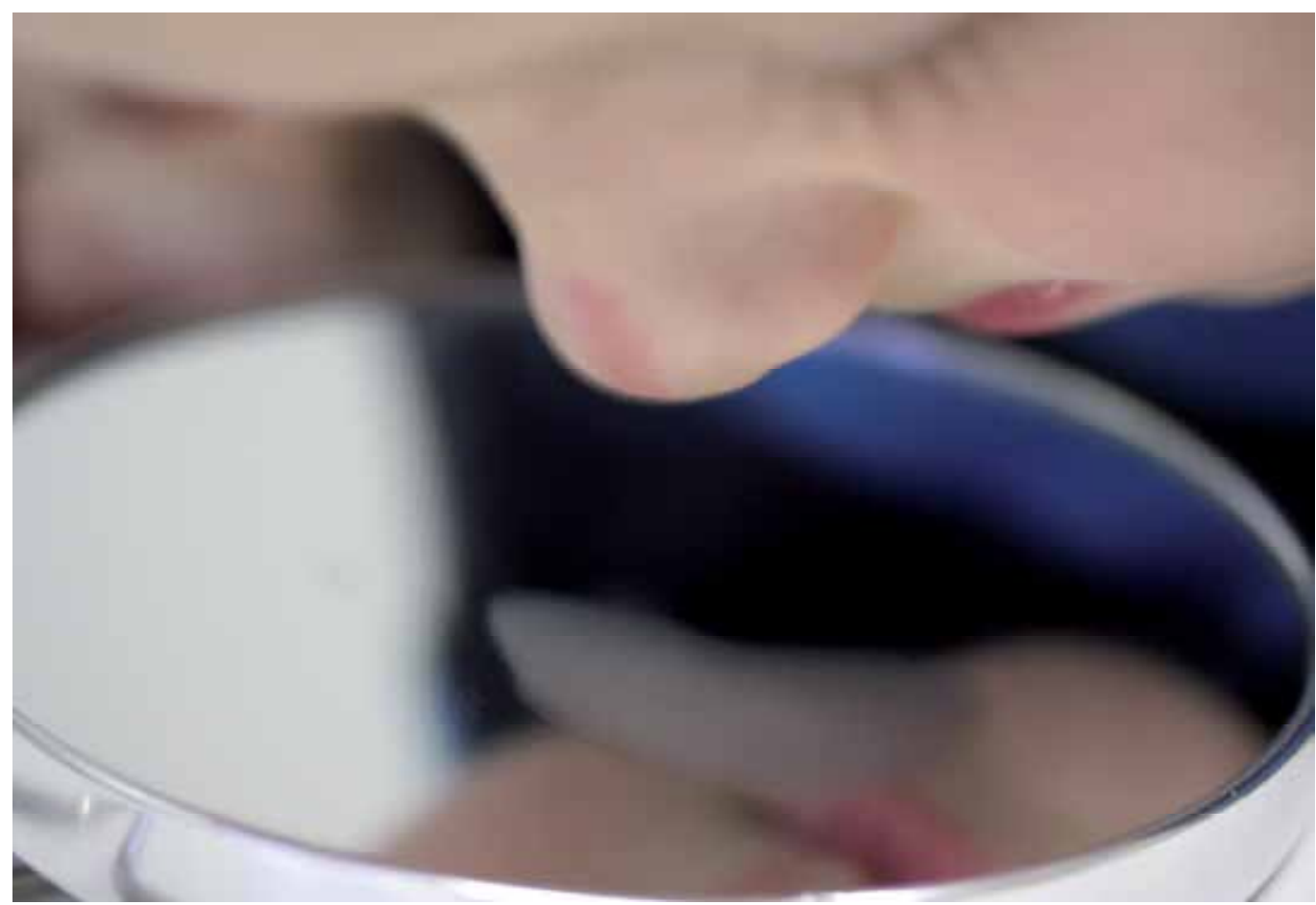

Fig. 8. The patient at 6 months postop. The mirror test proves patency of the nasal airway. During an observation period of $>24$ months the child has been free of complaints.

\section{Discussion}

Balloon catheter dilatation started in February 1974, when Andreas Grüntzig of Zürich/Switzerland used this method for the first time to dilate a vascular stenosis. Grüntzig had designed, constructed and produced the Balloon catheters all by himself. In September 1974 he used a catheter of an advanced design to dilate an occluded coronary artery for the first time in medical history. Today the method which has been developed by Grüntzig is the standard treatment for occluded vessels in interventional cardiology and vascular medicine. Since Grüntzig's invention, balloon catheters have become a well established technique in modern medicine (Schlupf, 2004). This applies not only to Cardiology and Angiology, but also to many other medical specialities including Otorhinolaryngology. Most frequently balloon dilatations are performed in Rhinology in socalled Balloon Sinuplasty procedures. The principle idea of this technique is to dilate intranasal or sinusoidal bottle-necks without any classical surgical intervention, like incisions, tissue removal, suturing or coagulation. Balloon Sinuplasty also allows to perform interventions in areas of the nose and sinuses, which are difficult to access by conventional 
endonasal techniques, like Functional Endoscopic Sinus Surgery (FESS). Based on these thoughts the idea of dilating a recurrent, predominantely membranous stenosis seemed to be promising. According to Meyer and Riemann stenoses and atresias of the choanae may be congenital, iatrogenic, post inflammatory or a result of radiation therapies for e.g. nasopharyngeal carcinoma (Meyer \& Riemann, 2010). The incidence of a congenital choanal atresia is estimated to occur in 1:8000 children, girls are twice as often affected than boys (Jacob, 2001). In up to $2 / 3$ of the cases choanal atresias may be combined with further congenital defects. The CHARGE syndrome (Coloboma of the eye, Heart disease, Atresia of choanae, Retarded growth and development and/or CNS anomalies, Genital hypoplasia, and Ear anomalies and/or deafness) may be an example for this observation (Pagon et al, 1981). Bilateral choanal atresia becomes evident in newborns immediately after birth. The healthy newborn usually breathes through the nose at rest. Nasal breathing is crucial for newborns especially while being fed. Newborns with bilateral choanal atresia show the clinical sign of "paradox cyanosis," while being fed, i.e. first they become cyanotic, then they stop swallowing and start to cry. At this point cyanosis resolves because the newborns can breathe orally again. In newborns with bilateral choanal atresia immediate action is therefore required. Unilateral choanal atresia on the other hand often is not recognized immediately. This defect usually becomes evident at a later point of time in life, when parents recognize permanent or recurrent unilateral rhinorrhoea. Therapy of unilateral choanal atresia mostly is surgical, either via the transnasal route or via the palatal route. Sometimes even a combined transnasal - transoral approach can be helpful. Although surgery nowadays is minimally invasive, large wounds and tissue defects may occur, necessitating intensive postoperative care and in some cases stenting. Despite all measures like excellent postoperative care, stenting or Mitomycin-C applications, the rate of secondary stenoses is high. Aside from the fact of a surgical intervention, insertion of a stent, which is supposed to keep the widend stenosis open, is often uncomfortable for the patient. Patients complain of foreign body sensation, rhinorrhaea and pain. Inflammation due to mucosal abrasions or foreign body reactions may occur. Many surgeons believe that only the reduction of trauma to surrounding tissue is likely to reduce the rate of recurrent stenoses. In 2006 Brown and Bolger described a new method for the dilation of sinus ostia for the treatment of recurrent nasal and paranasal sinusitis. One of the advantages of this new method was minimal trauma to surrounding tissues (Brown \& Bolger, 2006, Siow et al., 2009). When performing Balloon Sinuplasty the surgeon can generally choose between two different techniques. The correct position of the guide wire can be controlled by fluoroscopy in a similar fashion like in interventional cardiology or interventional radiology. However, in the head and neck region this leads to a considerable radiation exposure, first of all for the patient and secondly for the surgeon. Due to the fact that the hands of the surgeon manipulate in close proximity to the nose, they are regularly exposed to radiation during fluoroscopy, which is needed to control the position of the guide wire and the balloon. Patient's eyes and the lenses are exposed to the radiation beam and it is not uncommon that patients, who are frequently exposed to radiation may develop cataract. The estimated radiation dose during Balloon Sinuplasty accounts to approximately $730 \mathrm{mrem}$ (Bolger et al., 2007). In comparison the radiation dose of a CT scan of the head accounts to only 200 mrem, CT scan of the chest sums up to approximately 800 mrem. Angioplasty on the other hand exposes the patient to radiation between 750 and 5,700 mrem (Siow et al. 2008). 
Due to the problem of unwanted radiation exposure and due to the fact that not every hospital offers the technical requirements, which are needed for Balloon Sinuplasty under fluoroscopy a novel technique was developed. The presented patient was also treated with the new technique, which is known under the acronym LUMA technology. The principle of LUMA technology is that the position of the guide wire and the balloon is not controlled by fluoroscopy, but by endoscopy and diaphanoscopy. In this variation of Balloon Sinuplasty the guide wire contains a flexible light fiber, which can be coupled to a cold light source, which is usually present in every ENT operating room. The tip of the guide wire is extremely soft and emits light in an intensity, that it illuminates the frontal and maxillary sinus perfectly. Thus the position of the guide wire can be controlled from the outside by diaphanoscopy and from the inside by routine endoscopy with rigid endoscopes. Correct placement of the balloons in the frontal recess or in the maxillary ostium is thus possible without any radiation. The advantages of the LUMA technology rendered the treatment of our presented patient, who was four years old at the time of treatment. We feel that this technique allows minimally invasive dilatations in paediatric patients or even in pregnant women. The risk of perforating the orbit or the skull base is low, due to the flexibility of the guide wire/light fiber. Complications or undesired side effects are unlikely to occur. This method seemed ideal for the presented case and a thorough analysis of the medical literature showed that balloon dilatation had already been tried in a similar case. Goettman and coworkers were probably the first, who had treated recurrent choanal atresia by balloon dilatation. They used repeated balloon dilatations in a 16-year-old girl, which had presented herself with a restenosis 3 weeks after conventional and laser surgical therapy of a choanal atresia. In their publication from the year 2000 the authors reported about good results during a follow-up period of 2 years (Goettman et al., 2000). We could show similar results with only one dilatation. In addition, our method had the advantage that all manipulations were carried out under endoscopic control with the LUMA ${ }^{\mathrm{TM}}$ technique, i.e. no X-ray control was needed to position or control the balloon. Thus exposure of the patient or the surgeons to radiation was not necessary. The presented technique may be especially suited for membranous recurrent stenosis. A comparison between Balloon Sinuplasty for dilatation of a choanal stenosis with conventional transnasal, transoral or combined operations show that the Balloon technique requires more resources in disposable material (Fig. 9), the time for the actual intervention however is considerably shorter than operation time in conventional surgery. It seems to be recommendable to increase the pressure in the balloon stepwise. An increase of pressure of 2 atmospheres during each step is sufficient to build up adequate pressure in the balloon, as Brehmer recommended (Brehmer D., 2008). There are no uniform recommendations about the ideal pressure or the duration of the actual dilatation process in literature. We chose to build up a pressure of 8 atmospheres within 5 minutes to dilate the atresia. Meyer und Riemann reported on dilatation of a choanal atresia in an oncologic patient with 12 atmospheres within 12 seconds (Meyer and Riemann, 2010), Brown und Bolger used pressures between 10-16 atmospheres (mean maximum pressure $13 \mathrm{~atm}$ ) for about 5 seconds, with an inflation/deflation interval of 10 seconds each (Brown \& Bolger, 2006). Other authors like Brehmer estimate that the required pressure may be considerably lower, i.e. 8 atmospheres (Brehmer D., 2008).

Since it's initial description dilatation procedures in the nose have become more and more accepted in Otorhinolaryngology. The procedure are regarded to be safe, with hardly any side-effects. Bolger and coworkers could prove the safety and efficacy of Balloon Sinuplasty in a prospective multicentric trial with 115 patients. Patients were followed up for a period 


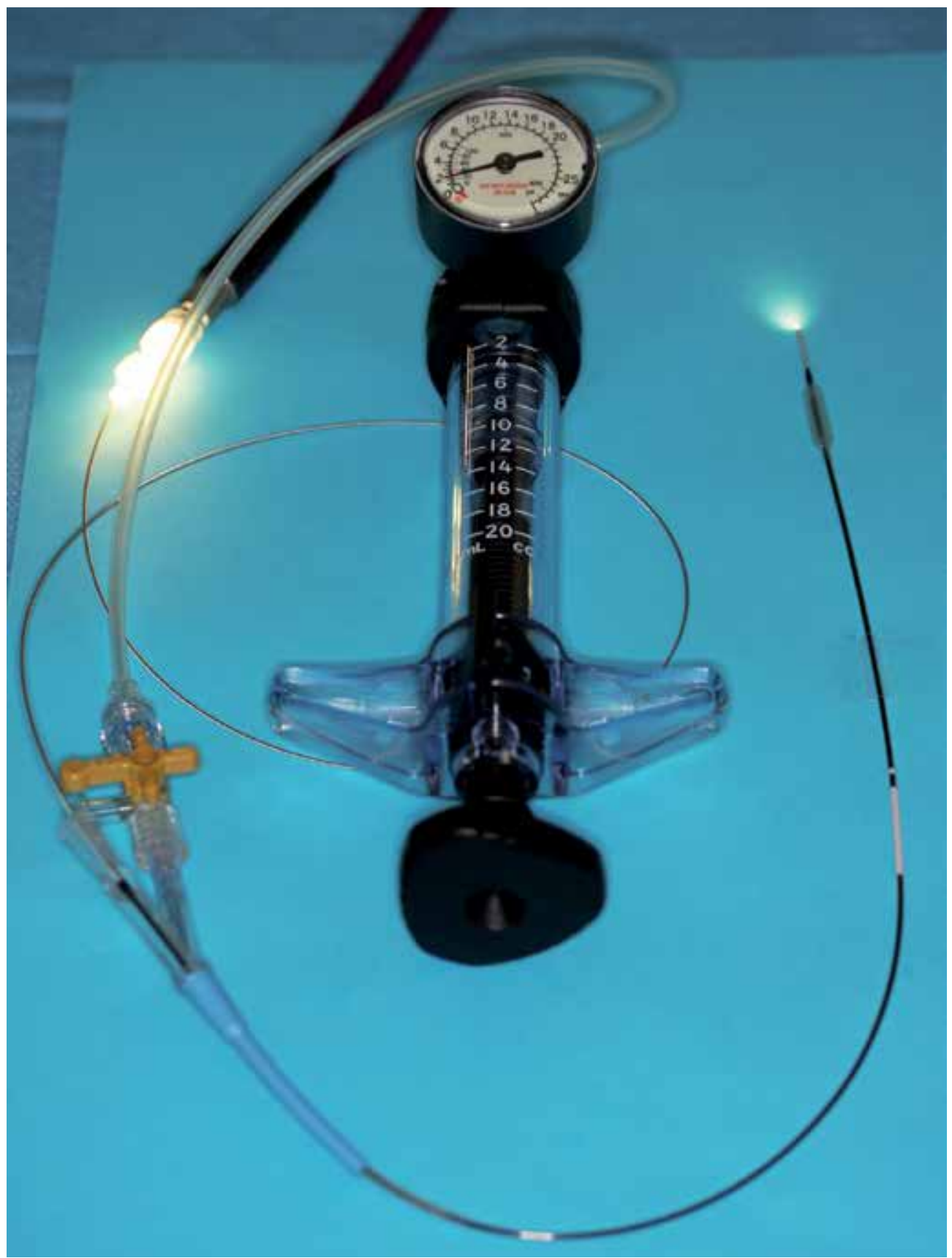

Fig. 9. System for Balloon dilatation as used for dilating the atresia of choanae. The guidewire is connected to a bright external light source with a detachable connector. Intraoperative the distal tip with the light fiber provides a direct visual confirmation via diaphanoscopic illumination. With the attached syringe-like Relieva ${ }^{\circledR}$ Sinus Balloon Inflation Device the balloon was inflated with normal saline solution until a pressure of 8 atm. was reached. 
of 24 weeks and postinterventional endoscopies were performed. The trial showed a successful and persistent dilatation of sinus ostia in $80,5 \%$ of the patients. In $1,6 \%$ of the cases a persitent ostium could not be seen on endoscopy, in 17,9\% of the cases the interior nose could not be assessed adequately by endoscopy (Bolger et al, 2007). Nine patients had developed bacterial sinusitis, which could be treated successfully by oral antibiotic therapy. Major complications had not occurred. In our case of a child with recurrent choanal atresia the results are similarily positive. Neither were there any undesired events during the intervention nor in the postinterventional period. No secondary hemorrhage or infection was observed. The good result encourages us to report this procedure as a possible alternative to conventional surgery in selected cases.

\section{Conclusion}

There is preliminary evidence that dilatation of membranous choanal atresia is feasible with balloon catheters. It is less invasive than FESS and requires only little postoperative care. Postoperatively no stents have to be inserted. This is of importance for patient's quality of life aspects. The patients does not have to endure any foreign bodies in his nasal airway. Additionally a second surgical procedure for removal of the stent is not necessary. Thus it seems to be of great value especially in the treatment of paediatric patients.

\section{References}

Brown CL, Bolger WE. Safety and feasibility of balloon catheter dilation of paranasal sinus ostia: a preliminary investigation. Ann Otol Rhinol Laryngol 2006; 115:293-299

Bolger WE, Brown CL, Church CA, Goldberg AN, Karanfilov B, Kuhn FA, Levine HL, Sillers MJ, Vaughan WC, Weiss RL. Safety and outcomes of balloon catheter sinustomy: a multicenter 24-week analysis in 115 patients. Otolaryngol Head Neck Surg 2007; 137:10-20

Brehmer D. Cathter-based balloon dilatation of the frontal, maxillary und sphenoid ostia: a new procedure in sinus surgery. HNO 2008; 56:65-70

Goettman D, Strohm M, Strecker EP. Treatment of a recurrent choanal atresia by balloon dilatation. Cardiovasc Intervent Radiol 2000; 23:480-481

Meyer HJ, Riemann R. Behandlung erworbener Choanalstenosen durch Ballon-Dilatation. Laryngorhinootologie 2010; 89:289-10

Siow JK, Al Kadah B, Werner JA. Balloon sinuplasty: a current hot topic in rhinology. Eur Arch Otorhinolaryngol. 2008; 265:509-11

Schlupf M. 30 Jahre Ballonkatheter: Andreas Grüntzig, ein Pionier in Zürich. Schweizerische Ärztezeitung, 2004; 85, 7: 346-351

Jacob R. 2001. Erkrankungen der Nase und des Pharynx: Pharynx. In: Praxis der HNOHielkunde, Kopf- u. Halschirurgie, J.Strutz \& W.Mann. p 396, Thieme-Verlag Stuttgart, New York; ISBN: 3-13-116971-0

Pagon RA, Graham JM Jr, Zonana J, Yong SL: Coloboma, congenital heart disease, and choanal atresia with multiple anomalies: CHARGE association. JPediatr.1981; 2:223-7. 


\title{
Sialendoscopy: Endoscopic Approach to Benign Salivary Gland Diseases
}

\author{
Meghan Wilson, Kyle McMullen \\ and Rohan R. Walvekar \\ Department of Otolaryngology Head \& Neck Surgery, \\ Louisiana State, University Health Science Center, \\ New Orleans, Louisiana \\ USA
}

\section{Introduction}

Sialadenitis, or recurrent salivary gland infection associated with pain and swelling of the major salivary glands, is a common presentation to emergency rooms and outpatient clinics. One of the most frequent causes of sialadenitis is obstruction in the salivary ductal system. Salivary calculi affect $1.2 \%$ of the population and account for $60-70 \%$ of salivary duct obstruction (Nahlieli 2004; Kim 2007; Bomeli 2009; Nahlieli 2006). Additional causes of obstruction to salivary flow include strictures in $25-25 \%$, inflammation (5-10\%) and other rare pathologies such as foreign bodies $(1 \%)$

Conservative treatment is the first line of therapy that includes treatment with antibiotics, salivary stimulants or sialogogues, and anti-inflammatory agents. However, conservative therapy fails in up to $40 \%$ of people with sialadenitis; in which case the recommended treatment is excision of the involved salivary gland. There as several important nerves that are in close proximity to the major salivary glands. The facial nerve, motor to facial muscles, runs through the parotid glandular system. Similarly, the submandibular gland is associated with the lingual nerve that is 1sensory to the anterior two thirds of the oral tongue; marginal mandibular nerve that allows movement of the angle of the mouth; and the hypoglossal nerve, motor to the tongue. Surgical excision of the gland carries numerous risks include but are not limited to paresis or palsy of the facial nerve, lingual nerve, and hypoglossal nerve. Other complications include Frey syndrome (gustatory sweating), sialoceles, salivary fistula, xerostomia, numbness in the distribution of the greater auricular nerve, infection, and hemorrhage. Consequently, although surgical resection in experienced hands is safe, it's often not desired due to the associated surgical risk and external scar in the neck associated with it.

In 1988, salivary duct endoscopes were introduced. Since their introduction, sialendoscopes have undergone technical refinements that have been instrumental in permitting clear and high definition visualization and manipulation of the salivary ductal system. Today, salivary duct endoscopy or "Sialendoscopy" allows the minimally invasive endoscopic visualization of major salivary gland ductal system and endoscopic interventions to treat chronic sialadenitis with or without sialolithiasis. 
As in many other surgical areas, the advent of sialendoscopy has added additional options to the diagnosis and management of non-neoplastic salivary pathology. Initially, sialendoscopy was created for diagnostic evaluation of the salivary glands and ductal system as well as treatment of obstruction through the removal of sialoliths or dilation of strictures. Nahlieli et al, in their study in $2004^{1}$ described successful endoscopic treatment of recurrent parotitis and juvenile recurrent parotitis. The scope of sialendoscopy was further expanded to treat radioactive iodine sialadenitis (Kim 2007; Bomeli 2009; Nhlieli 2006) and sialadenitis induced by autoimmune diseseases (Schacham 2011). Today, sialendoscopy is regarded as an acceptable and often preferred diagnostic and treatment tool for chronic sialadenitis and non-neoplastic obstruction of the salivary ductal system.

\section{Indications and contraindication}

Current evidence has validated sialendoscopy for the treatment of non-neoplastic disorders of the salivary glands, including sialolithiasis. Sialolithiasis is one of the most common nonneoplastic disorders of the major salivary glands and a major cause of sialadenitis and unilateral diffuse swelling of the major salivary glands (Marchal F, Dulguerov P. 2003; Nahlieli O. 2006). In general, stones less than $4 \mathrm{~mm}$ in the submandibular gland and less than $3 \mathrm{~mm}$ in the parotid gland are amenable to endoscopic removal. Intermediate size stones between 5-7 mm may need further fragmentation either using a Holmium laser or lithotripsy prior to endoscopic extraction. In general stones larger than $8 \mathrm{~mm}$ require a combined approach technique for stone removal (Karavidas K, Nahlieli O, Fritsch N, et al. 2010). The combined approach technique is a technique that uses the sialendoscope for stone localization and either an intra-oral or an external approach for removal of large submandibular or parotid gland stones, respectively (Bodner L. 2002; Lustmann J, Regev E, Melamed Y. 1990; Marchal F. 2007; Raif J, Vardi M, Nahlieli O, et al. 2006; Seldin HM, Seldin SD, Rakower W. 1953; Walvekar RR, Bomeli SR, Carrau RL, et al. 2009).

Sialendoscopy indications include diagnostic evaluation of recurrent or chronic sialadenitis, including unexplained swelling of the major salivary glands associated with meals, ductal stenosis, and intra-ductal masses (Nahlieli O. 2006; Walvekar RR, Razfar A, Carrau RL, et al. 2008). The authors of limited series have also suggested benefit in patients with radioiodineinduced sialadenitis (Kim JW, Han GS, Lee SH et al. 2007; Bomeli SR, Schaitkin B, Carrau RL, et al. 2009; Nahlieli O and Nazarian Y. Sialadenitis 2006). Recent studies and the authors' experience suggest benefit in children with recurrent sialadenitis (Nahlieli $\mathrm{O}$, Shacham R, Shlesinger M, et al. 2004; Jabbour N, Tibesar R, Lander T, et al. 2010; MartinsCarvalho C, Plouin-Gaudon I, Quenin S, et al. 2010; Faure F, Querin S, Dulguerov P, et al. 2007) and also in patients who have recurrent sialadenitis from autoimmune processes such as Sjogren's syndrome or Systemic Lupus Erythematosis (Shacham R, Puterman MB, Ohana N, et al. 2011).

The only absolute contraindication is acute sialadenitis. The use of the rigid dilator system and/or a semi-rigid endoscope during an acute episode increases the chance of ductal trauma and irrigation as well as ductal injury could increases the potential spread of infection in the head and neck soft tissues. Sialendoscopy can be challenging in patients with microstomia or trismus. These could be considered as relative contraindications for the procedure. 


\subsection{Sialendoscopy: Technique}

Sialendoscopy may be performed in the clinic setting with local anesthesia or in the operating room with sedation or general anesthesia. The decision of where to perform the procedure is physician and/or patient preferences. The basic work up for sialendoscopy is summarized in Table 1.

\begin{tabular}{|l|l|}
\hline History & Symptoms \\
\hline & Number of infections \\
\hline & Previous treatments \\
\hline & History of external beam radiation therapy \\
\hline & History of Radioactive iodine treatment \\
\hline Physical Examination & Full Head and Neck examination \\
\hline & Bimanual palpation of glands \\
\hline & Examine and note location of papilla \\
\hline & Note any anatomical limitations (trismus, small oral \\
& commissure, temporomandibular joint pathology, etc.) \\
\hline Imaging & Computed Tomography Scan (CT) \\
\hline & Ultrasound \\
\hline & Magnetic Resonance Imaging Scan (MRI) \\
\hline
\end{tabular}

Table 1. Preoperative Evaluation

\subsubsection{Basic workup}

Workup of all salivary disorders begins with history of present disease. Important questions to ask include symptoms, number of infections, previous treatments, and previous testing or other studies. Questions regarding history of external beam radiation or radioactive iodine therapy are important as well to understand possible etiologies of salivary disease.

Examination should first include a complete head and neck exam. Bimanual palpation of the salivary gland should be performed to examine for any masses or palpable stones, noting any asymmetry. Examine the papilla and its location. Assess the patency and saliva flow and quality of the saliva from the papilla. It is important to make notes regarding the papilla in the chart to aid in identification when ready to perform sialendoscopy; pictorial illustrations are particularly helpful. In addition, the surgeon must evaluate access to the oral cavity by paying close attention to the size of the oral commissure (e.g., microstomia), size of tongue, ability to open the mouth (e.g., preoperative trismus), and pathology of temporomandibular joints

Laboratory and imaging studies ordered will depend on the patient's symptoms and physical exam findings. Frequently used imaging studies include ultrasonography, computed tomography (CT), and magnetic resonance imaging (MRI), (Figures 1 and 2). The authors' preference for diagnosis and surgical planning is CT scan with and without contrast.

\subsubsection{Instrumentation}

The basic instrumentation includes a specialized dilator system that permits gradual serial dilation of the salivary duct papilla from the lowest No.0000 dilator to a maximum of No.8, 
(Figure 3). The sialendoscopes most frequently used are the "all-in-one" interventional sialendoscopes, which can range from 1.1. $\mathrm{mm}$ to $1.6 \mathrm{~mm}$ in diameter, (Figure 4). The sialendoscopes have an optic channel that transmits the image using fiberoptic channels, and an irrigation channel allows a continuous irrigation to be performed to maintain duct patency for endoscopic visualization of the salivary duct lumen and to allow working space for surgical intervention. A working channel is used to introduce instruments for intervention. The 1.1 and $1.3 \mathrm{~mm}$ sialendoscopes permit introduction of $0.4 \mathrm{~mm}$ stone wire baskets (Figure $5 \mathrm{~A}-\mathrm{C}$ ) and a hand-held micro burr to break stones. The $1.6 \mathrm{~mm}$ sialendoscope permits the use of $0.6 \mathrm{~mm}$ stone wire basket and a cup forceps in addition.

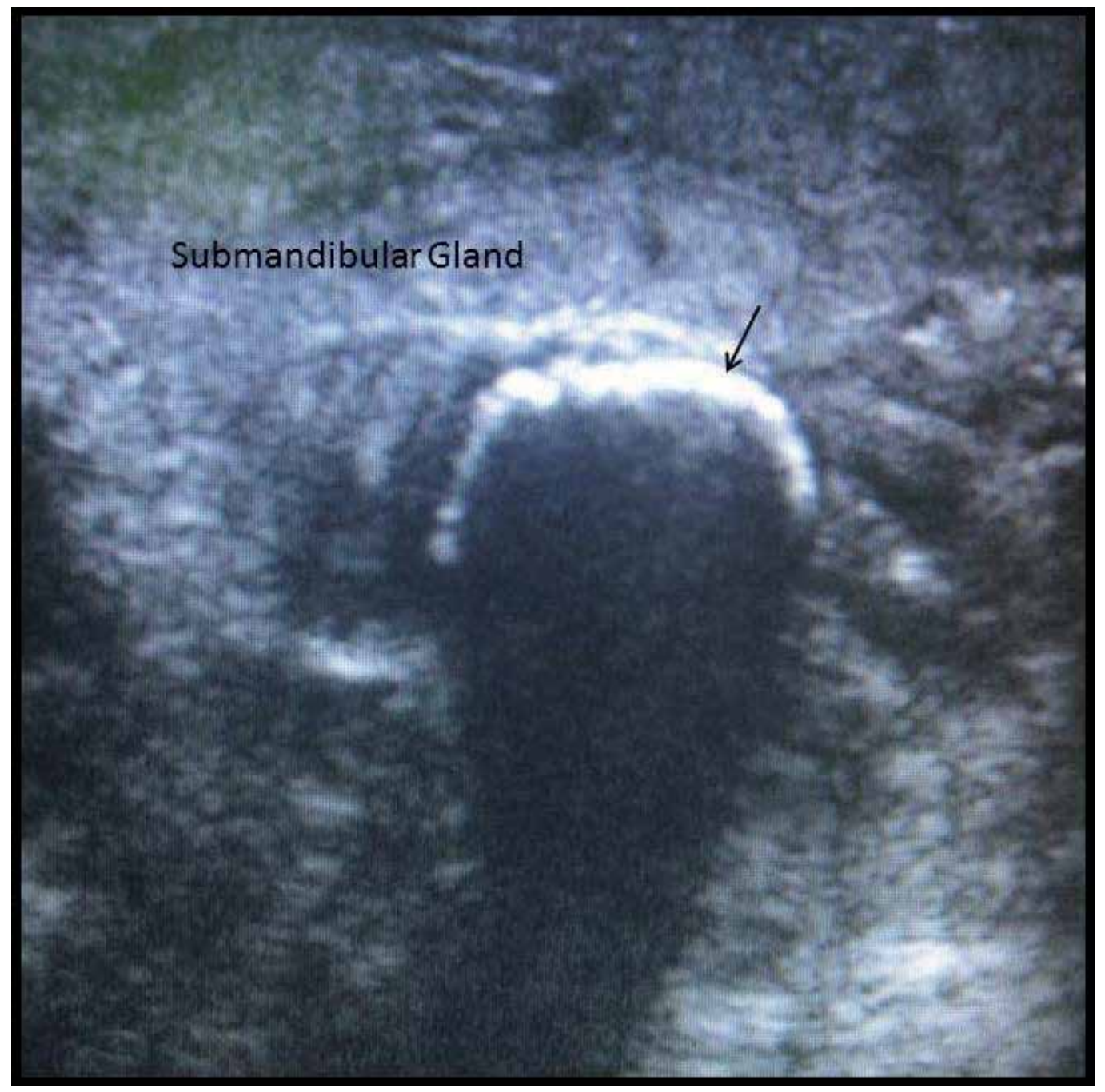

Fig. 1. Ultrasonography image of large submandibular stone. The stone appears as a hyperechoic rim while throwing a shadow that is a pathognomic finding of a salivary stone visualized via ultrasound, (black arrow points to the stone). 
Holmium laser fibers can be used as well through the interventional channels for intraductal laser fragmentation of stones, (Figure 6). Another important tool for sialendoscopy is the conical dilator that permits gradual dilation of the duct and allows one to transition from a smaller dilator to the next size, (Figure 7).

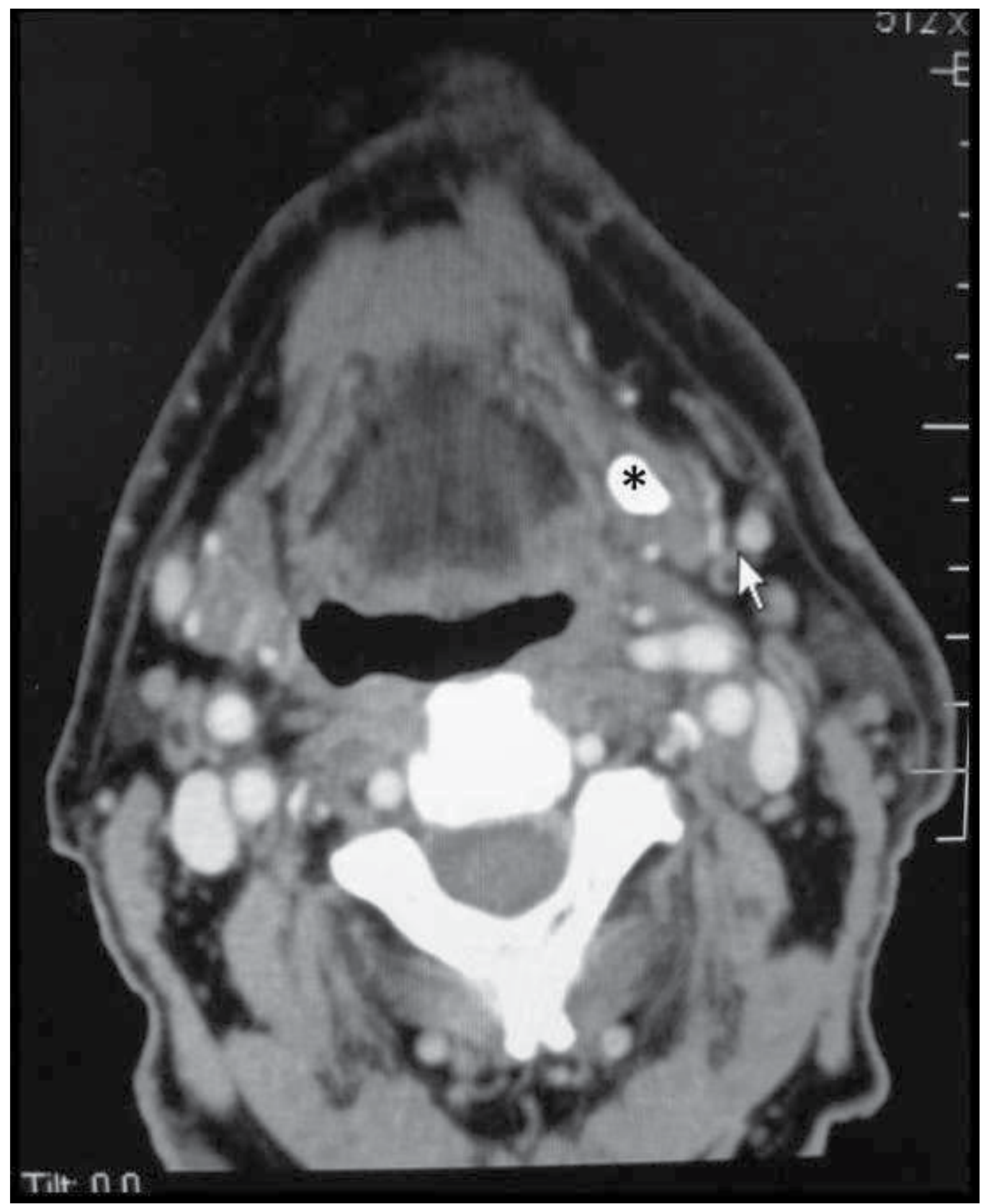

Fig. 2. An contrast enhanced axial CT scan showing a large irregular left submandibular stone in the hilum, (the asterix denotes the salivary stone). 


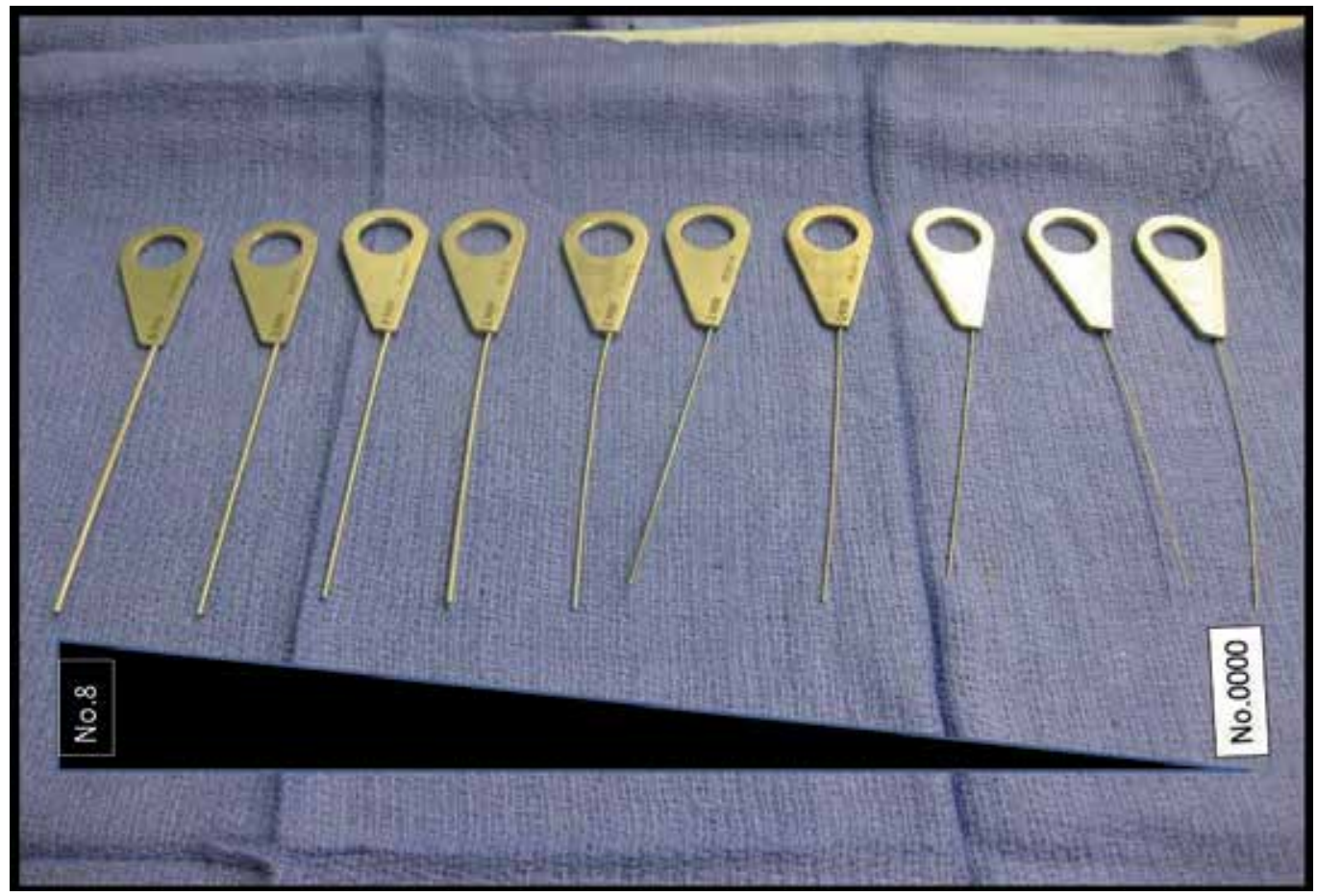

Fig. 3. Marchal Dilator System

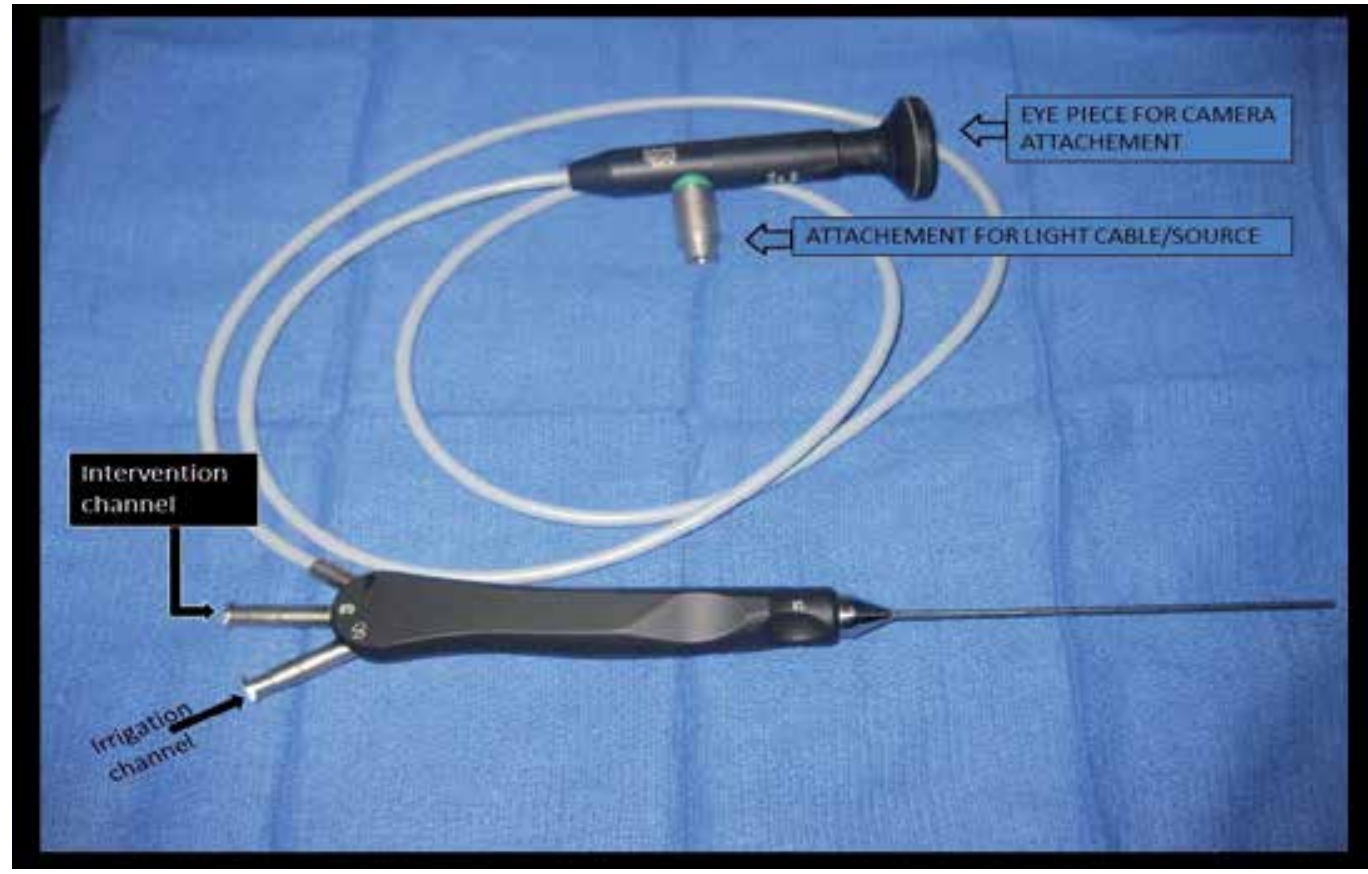

Fig. 4. 1.6 mm Erlangen "All-In-One" Sialendoscope 
Salivary duct stents or cannulae can usually left in place following the procedure to decrease the risk of scarring and papilla stenosis, (Figure 8)

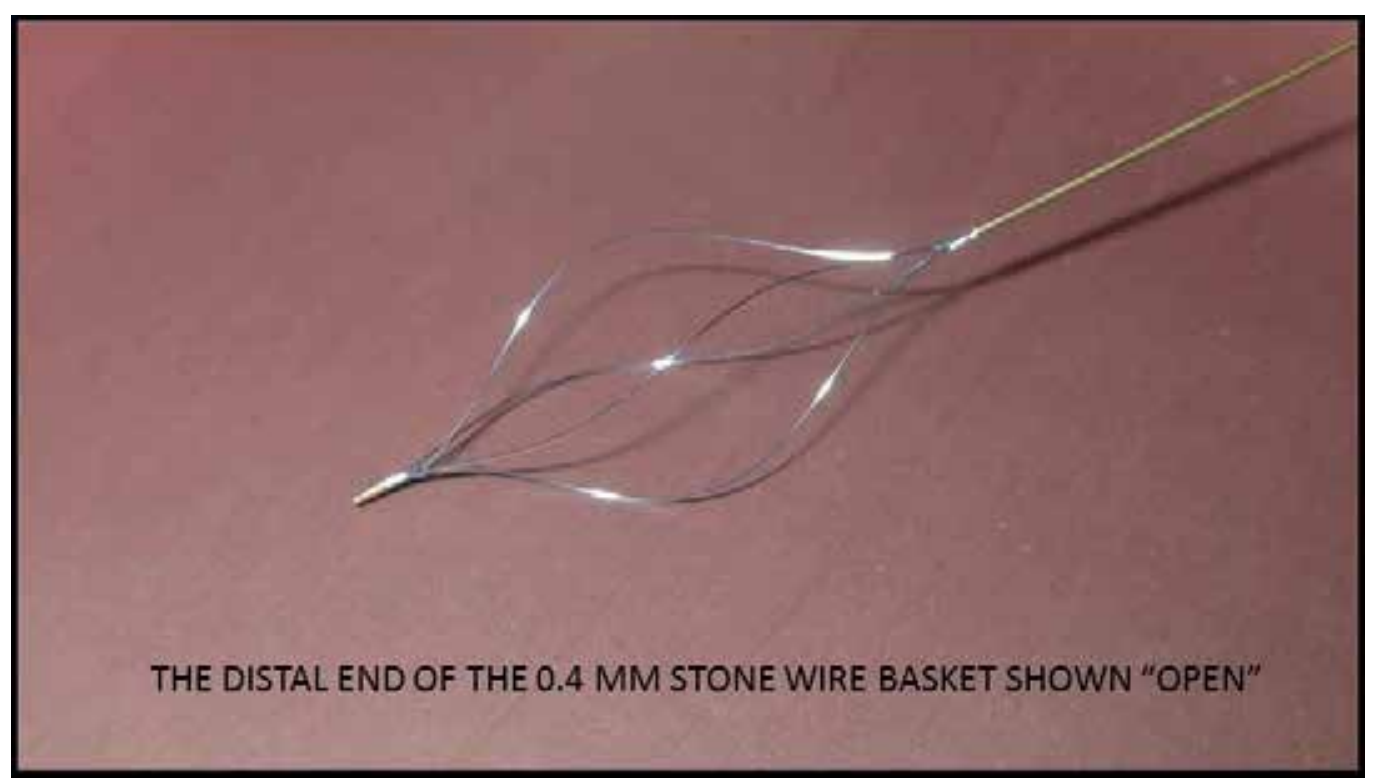

A)

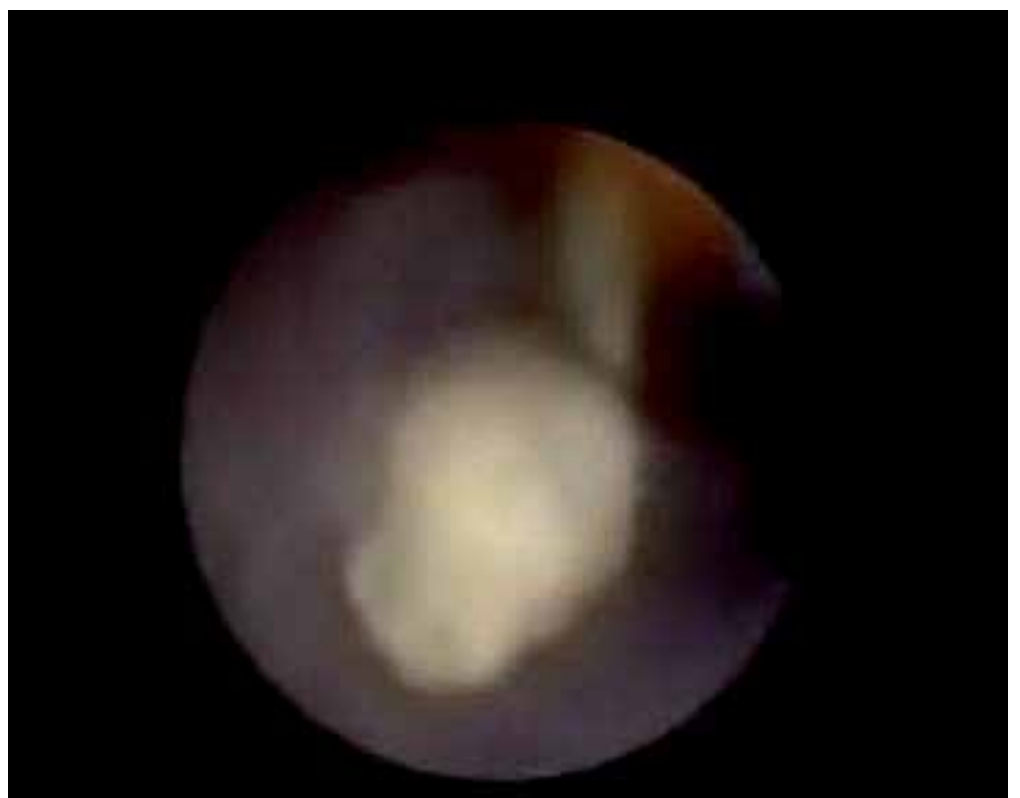

B) 


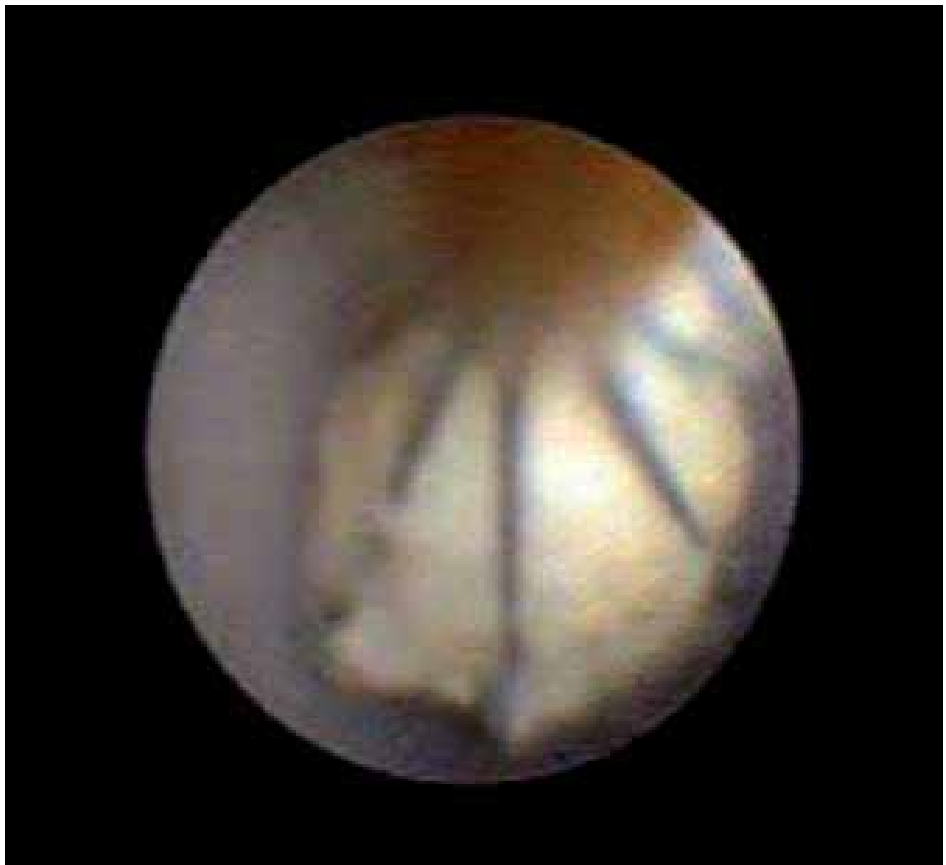

C)

Fig. 5. A) $0.4 \mathrm{~mm}$ stone wire basket. B) A free floating submandibular duct stone. C) The wire basket can be inserted through the interventional port of the sialendoscope and used for trapping the stone for endoscopic removal

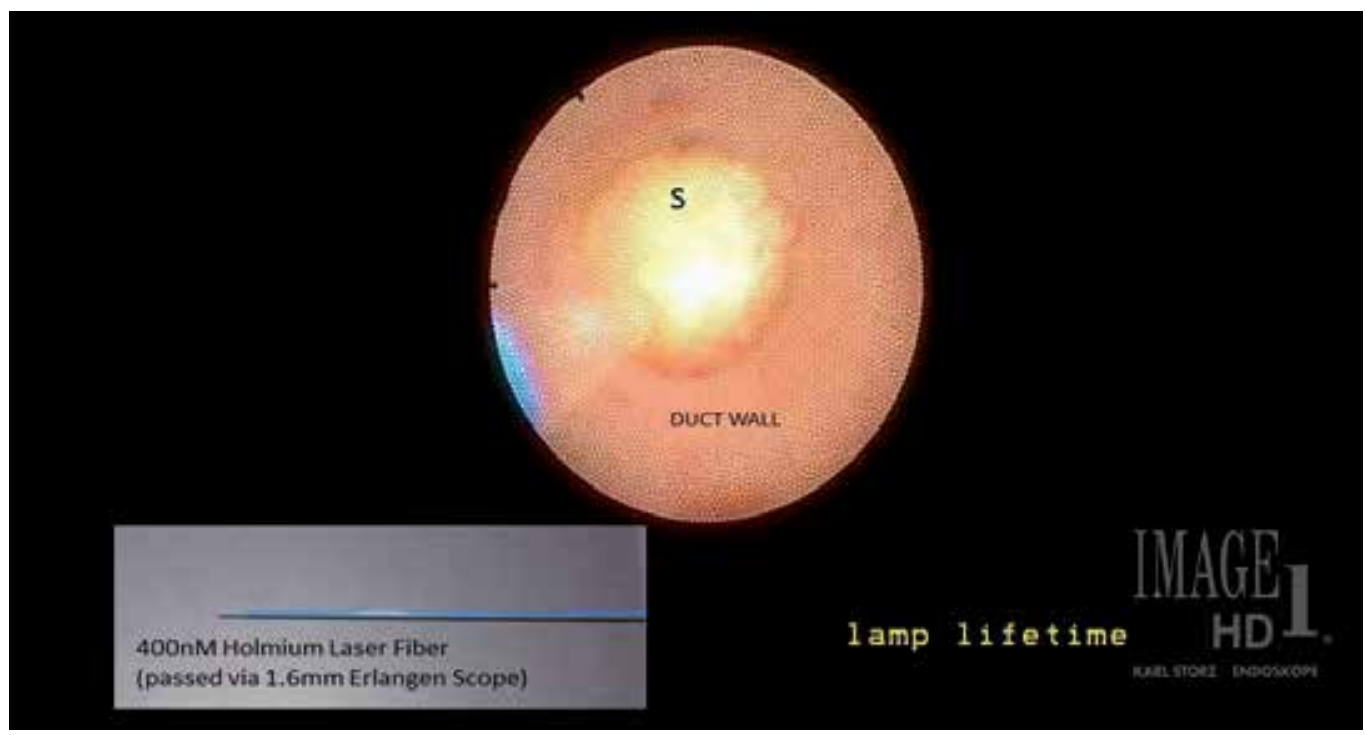

Fig. 6. A Holmium laser fiber being used for intra-ductal laser fulguration of an impacted $6 \mathrm{~mm}$ submandibular stone (S). Inset shows the tip of the $400 \mathrm{nM}$ Holmium laser fiber that can be passed via the interventional channel of the $1.6 \mathrm{~mm}$ Erlangen Sialendoscope. 


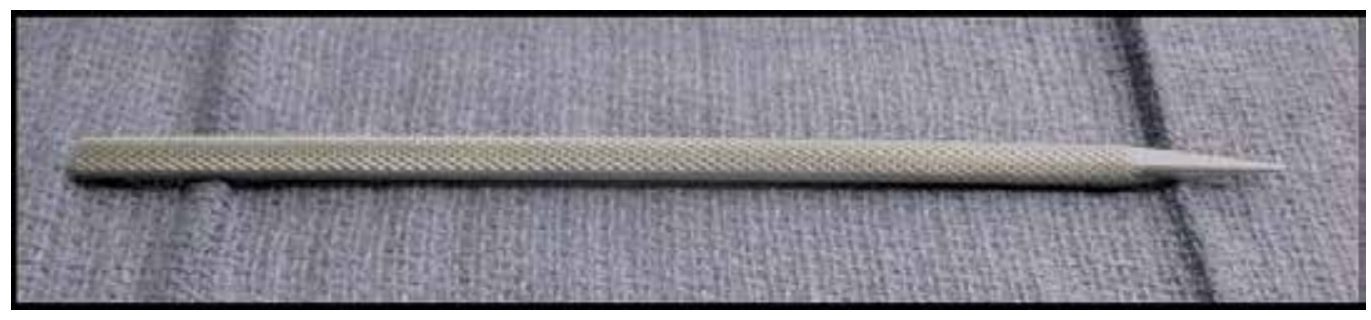

Fig. 7. Conical Dilator

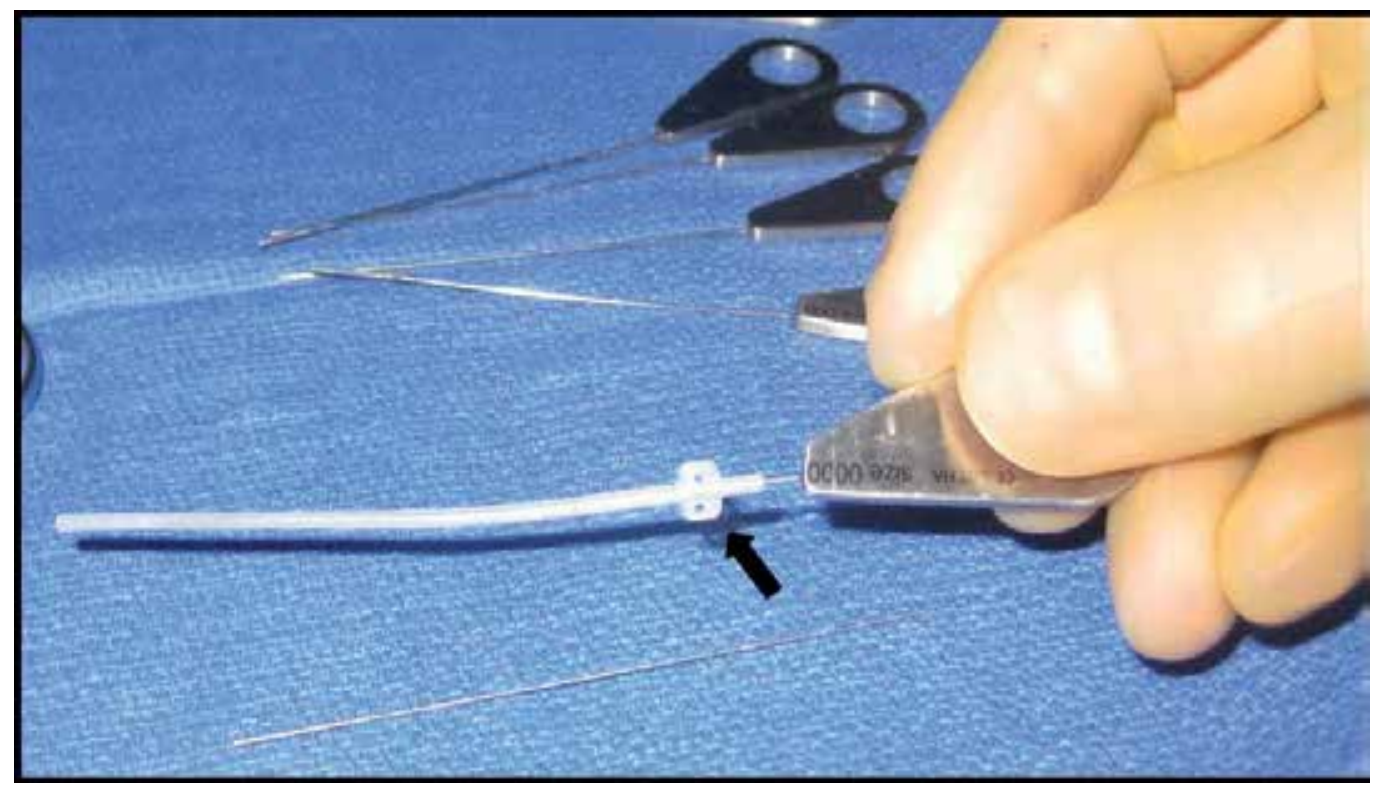

Fig. 8. The Schaitkin Salivary Canula (Hood Laboratories, Pembroke, MA) can be inserted over a No.0000 Marchal dilator to prevent subsequent stenosis of the duct or papilla. Black arrow points to distal end of stent that projects out of the papilla. Two holes on either side of the flange of the stent facilitate securing the stent to the floor of mouth or buccal mucosa via non-absorbable stitches.

\subsubsection{Operative technique}

Operative technique can be divided into two main components:

1. Access to the salivary duct i.e. dilation of the papilla

2. Endoscopy with or without intervention.

Management of papilla is the rate-limiting step of the procedure. Care must be taken to dilate the papilla. Poor handling of the papilla can lead to papillary stenosis in the future that can lead to obstructive sialadenitis. In addition, perforation of the duct (minor or major ductal tear) and creation of a false passage are potential complications of papilla dilation that add morbidity to the procedure and also are reasons for sialendoscopy failure.

Identification of the papilla can be aided by a using an operating microscope or surgical loupes. The author recommends the use of at least surgical loupes to identify and dilate the papilla. In office localization and documentation of the location of the papilla with imaging 
is very helpful in intra-operative identification of the papilla. Massage of the gland may also assist in localizing the papilla by denoting the point of saliva flow. After identification of the papilla, serial dilation of the papilla is then performed using the Marchal dilator system, (Figure 3). Injection of local anesthetic around the papilla often helps the process of serial dilation by making the ductal opening more prominent and by stiffening the surrounding tissues to facilitate serial cannulation of the duct. In this procedure, salivary probes of increasing diameter, beginning with the smallest (No. 0000), are sequentially introduced into the duct. Dilation up to No.3 or No.4 is adequate to allow introduction of a sialendoscope of $1.3 \mathrm{~mm}$ diameter. For the introduction of larger endoscopes $(1.6 \mathrm{~mm})$, dilation up to No.6 probe is necessary (Marchal F. 2003). Other techniques used to dilate the papilla or facilitate the identification of the papilla are the Seldinger's technique that uses a series of bougies of increasing diameter that are inserted over a guide wire that is placed in the salivary duct (Figure 9), the placement of a papillotomy or an incision at the entry point of the salivary duct to facilitate entry into the it, and use of Methylene blue to identify the duct orifice (Marchal F. 2003; Geisthoff UW. 2009; Papadaki ME, Kaban L, Kwolek C, et al. 2007; Iwai T, Matsui Y, Yamagishi M, et al. 2009; Luers JC, Vent J, Beutner D. 2008).

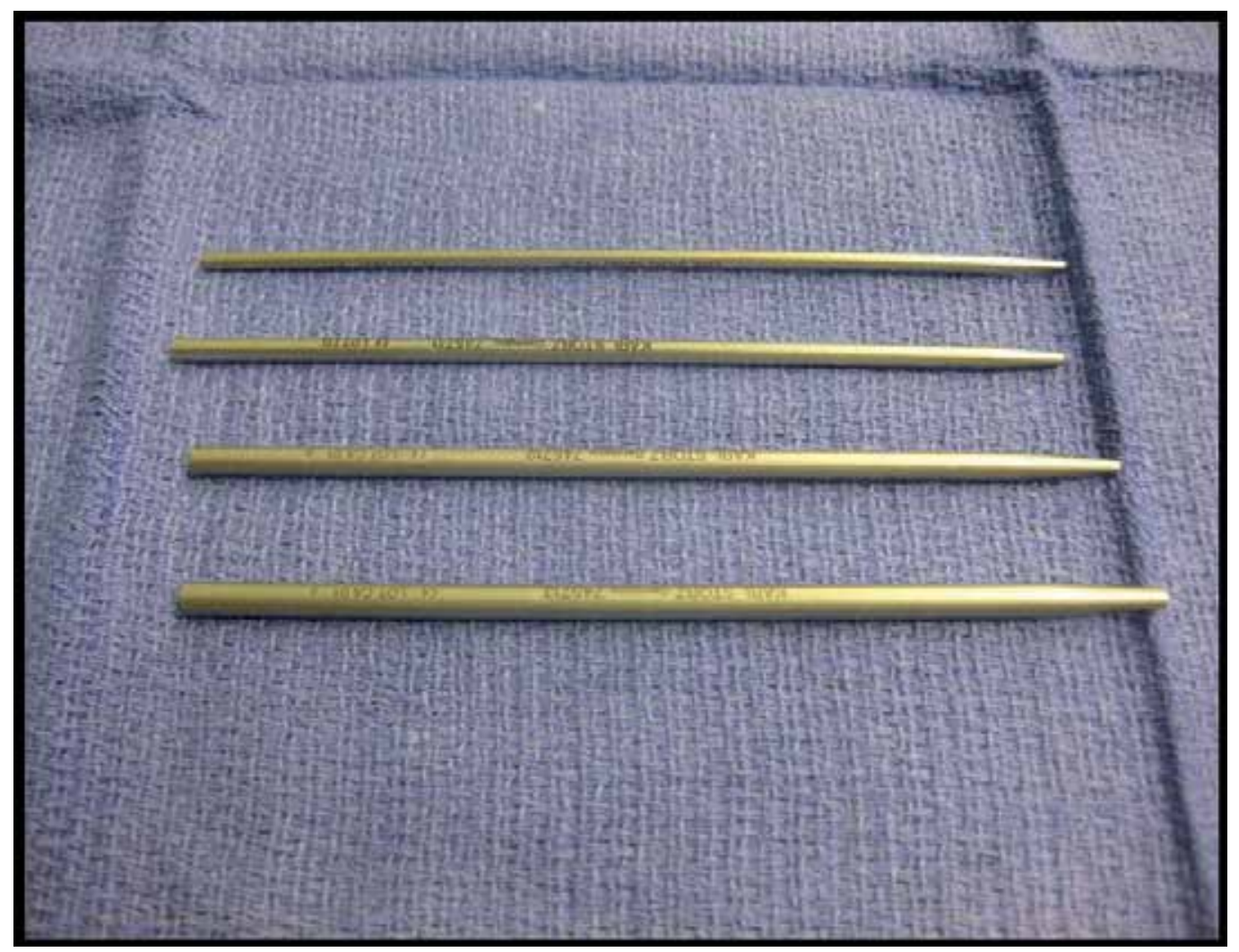

Fig. 9. Bougies of increasing diameter used in the Seldinger's technique of salivary duct dilation.

Nahlieli et al have suggested performing a papillotomy to allow introduction of the scope (Nahlieli O. 2006). However, in the authors' experience, a papillotomy prevents the creation 
of a mucosal seal around the endoscope, resulting in leakage of the irrigation, consequently preventing maximum dilation of the duct through hydraulic pressure. The authors reserve the use of a papillotomy for one of two scenarios: difficult cases in which standard dilation or the Seldinger's technique fails and if necessary for the delivery of larger stones at the conclusion of a procedure.

\subsubsection{Endoscopy with or without intervention}

Despite its apparent simplicity, sialendoscopy is a technically challenging procedure that requires organized and sequential learning(Marchal F. 2003). Once this is acquired, the success rates for diagnostic as well as interventional sialendoscopy can be more than $85 \%$ (Marchal F, Dulguerov P. 2003; Nahlieli O. 2006; Marchal F. 2003).

Sialendoscopy allows visualization of the entire ductal system with ability to navigate the scope into primary, secondary and at time tertiary branching systems, (Figure 10). With respect to diagnostic sialendoscopy, Marchal and Dulguerov reported a $98 \%$ success rate (Marchal F, Dulguerov P. 2003), whereas (Nahlieli O, Baruchin AM. 1999) reported a success rate of $96 \%$ in their case series. Though diagnostic sialendoscopy is possible in most patients, failure to pass the scope along the entire ductal system may result from ductal stenosis, inflammation, or because of the presence of an acute masseteric bend of the parotid duct, making navigation with the scope difficult.

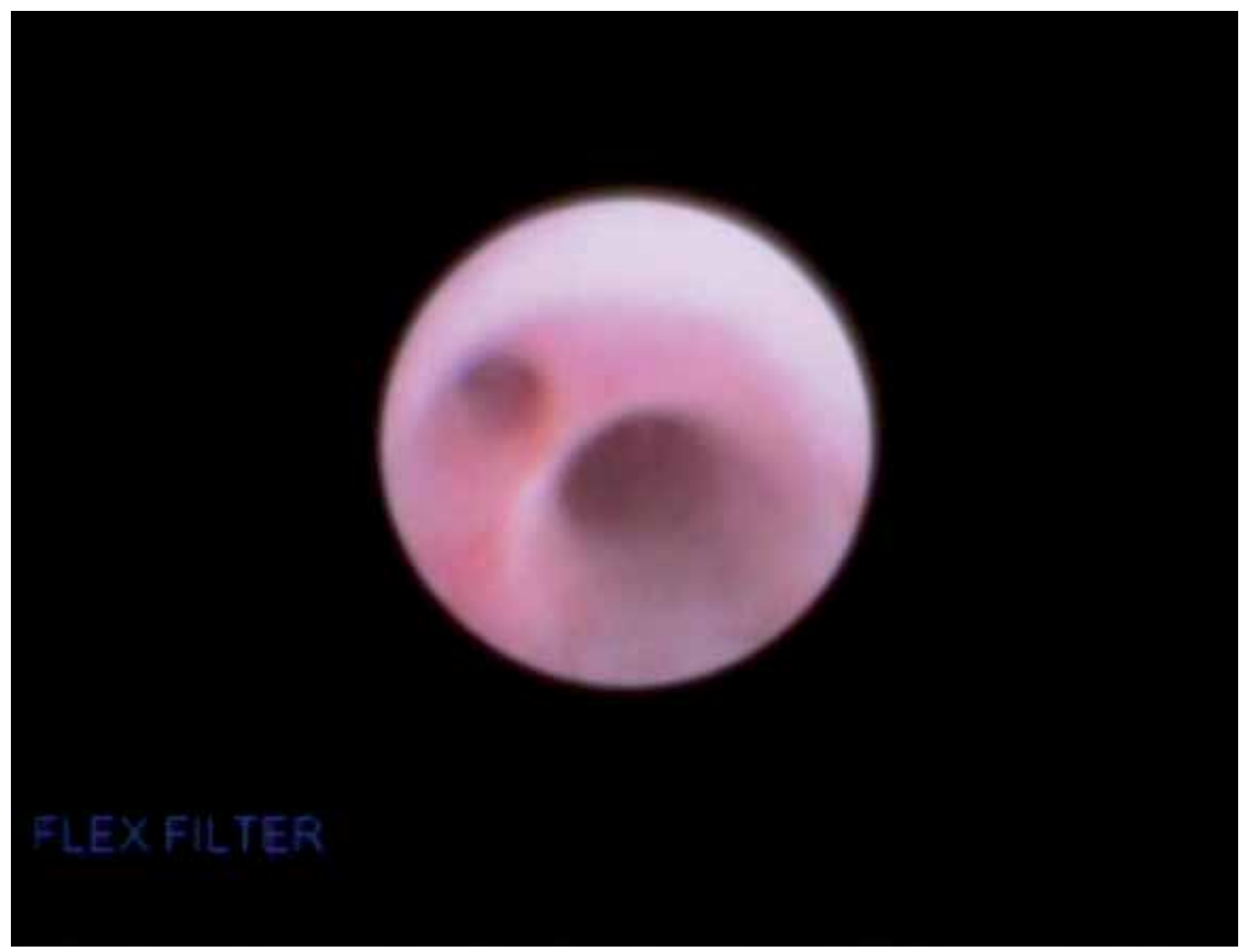

Fig. 10. Intraoperative view of the ductal lumen showing branching ductal system. 
In the interventional setting, stone removal is the most common indication for sialendoscopy. Success rates for endoscopic stone removal without adjuvant lithotripsy with forceps or laser fragmentation range from $74-89 \%$ in several case series(Marchal F, Dulguerov P. 2003; Nahlieli O. 2006; Marchal F. 2007; Walvekar RR, Razfar A, Carrau RL, et al. 2008; Bowen MA, Tauzin M, Kluka, EA, et al. 2010; Nahlieli O, Nakar LH, Nazarian Y, et al. 2008; Walvekar RR, Carrau RL, Schaitkin B. 2009). Nahlieli et al reported a success rate of $86 \%$ and $89 \%$, respectively, for endoscopic parotid and submandibular sialolithotomy in 736 cases of sialolithiasis (Nahlieli O. 2006). However, their success rate for endoscopic sialolithotomy was $80 \%$ in an earlier series of 3 years experience, during which they reported a total of 32 cases of sialolithiasis with 4 failures (Nahlieli O, Baruchin AM. 1999).

Stones larger than $3 \mathrm{~mm}$ in the parotid gland and $4 \mathrm{~mm}$ in the submandibular gland have a lower success rate for removal (Marchal F, Dulguerov P. 2003). Laser fragmentation and lithotripsy are useful for removal of intermediate-size stones (up to 6 or $7 \mathrm{~mm}$ ). External lithotripsy can be performed prior to sialendoscopy to fragment the stones into smaller fragments that can be more readily removed with wire baskets or forceps. External lithotripsy for the management of salivary stone is not currently FDA approved in the United States. Consequently, the authors have not incorporated this into their practice. Laser stone fragmentation and intra-ductal lithotripsy have been described. The Holmium has been used for stone fragmentation (Papadaki ME, McCain JP, Kim K, et al. 2008). For larger stones or stones that cannot be accessed endoscopically, a combined approach technique is required, (Figure 11). The use of the da Vinici robot has been described to assist in the

\section{Combined approach Technique for Parotid Stone}

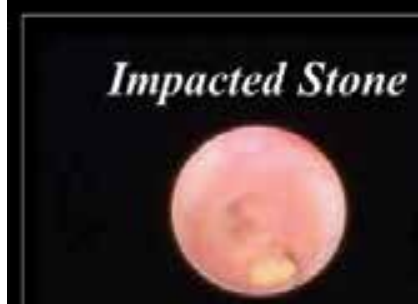

Stenson's duct
Endoscopic Localization with Trans-illumination and

External Parotid Approach via a Parotid Incision Permits Stone Localization and Removal With Gland Preservation

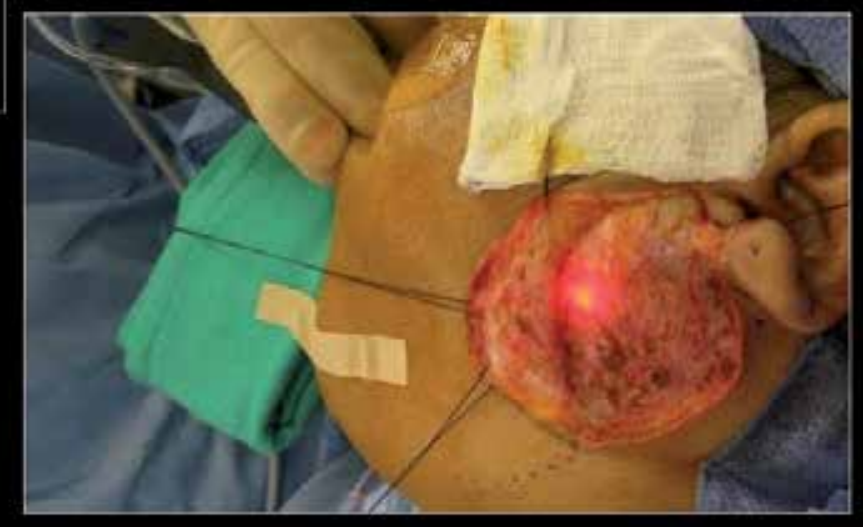

Fig. 11. Combined Approach Technique for Parotid Stones via an External Approach. 
management of large hilar and intra-glandular submandibular salivary stones are traditionally difficult to access via a transoral route, (Figure 12)(Walvekar RR, Tyler PD, Tammareddi N, et al. 2011).

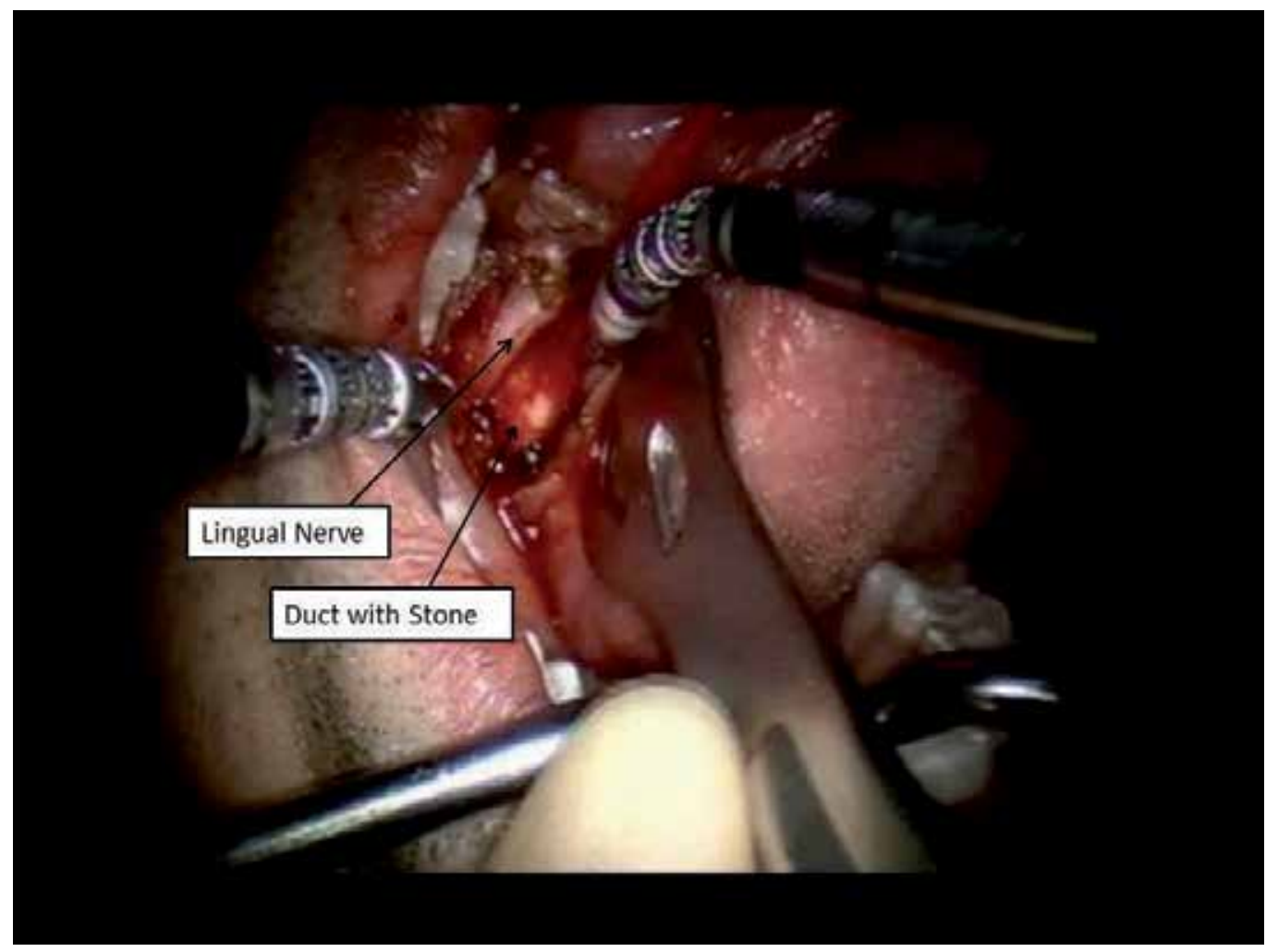

Fig. 12. Combined Approach Technique (Robot-Assisted) for Submandibular Stones via a Transoral Approach. Right submandibular stone is exposed with identification and dissection of the lingual nerve with excellent visualization via the da Vinci robotic system.

Radioactive iodine (RAI) treatment can induce sialadenitis with inflammation and consequently stenosis of the ductal system (Kim JW, Han GS, Lee SH et al. 2007; Bomeli SR, Schaitkin B, Carrau RL, et al. 2009; Nahlieli O and Nazarian Y. 2006). This occurs because the salivary glands in addition to the thyroid gland concentrate iodine. Up to $60 \%$ of patients treated with RAI can develop chronic sialadenitis (Kim JW, Han GS, Lee SH et al. 2007; Bomeli SR, Schaitkin B, Carrau RL, et al. 2009; Nahlieli O and Nazarian Y. 2006). Sialendoscopy has shown to be effective in the treatment of this disorder. Bomeli et al. (Bomeli SR, Schaitkin B, Carrau RL, et al. 2009) studied radioactive iodine induced sialadenitis in 12 patients. 37 glands were studied in these 12 patients. Mucous plugs were found in $44 \%$ of glands; strictures had developed in 30\%. Therapeutic maneuvers involve flushing the gland to expel mucous plugs, dilation of stenotic areas with an endoscopic balloon dilator, or , intra-ductal instillation of steroids during the procedure. Sialendoscopy was able to be performed in $84 \%$ of patients and $75 \%$ of patients had resolution of symptoms following the procedure. Overall success rates for resolution of symptoms range 
between 50 and $100 \%$. Failure is most commonly due to severe ductal stenosis(Kim JW, Han GS, Lee SH et al. 2007; Bomeli SR, Schaitkin B, Carrau RL, et al. 2009; Nahlieli O and Nazarian Y. 2006).

Juvenile recurrent sialadenitis most frequently affects the parotid gland. The etiology is unknown. Sialendoscopy can be used as both a diagnostic tool and treatment (Nahlieli O, Shacham R, Shlesinger M, et al. 2004; Jabbour N, Tibesar R, Lander T, et al. 2010; MartinsCarvalho C, Plouin-Gaudon I, Quenin S, et al. 2010; Faure F, Querin S, Dulguerov P, et al. 2007). Sialendoscopy with irrigation of the duct with or without injection steroids has been shown to be effective in treating this condition. Characteristic findings are seen on sialendoscopy originally include whitish appearance of the duct and lack of vascular markings seen on the ductal wall. Shacham et al. (Shacham R, Droma EB, London D, et al. 2009) studied 70 children with recurrent sialadenitis. In 56 of the children, symptoms were eliminated after one procedure; only 5 children required a repeat procedure.

\section{Complications}

Major complication rates are low with sialendoscopy. Gland swelling post-operatively is expected and usually resolves in approximately 24-48 hours. This is particularly important to consider in submandibular procedures, as swelling could cause airway compromise (Iwai T, Matsui Y, Yamagishi M, et al. 2009). Consequently, when performing bilateral submandibular gland procedures, it is important to examine the gland and oral cavity after completing one side and determine whether it is safe for the patient to proceed with the contralateral gland.

One of the more serious iatrogenic complications is avulsion of the duct. This complication can be prevented by avoiding excessive traction on the stone while it is engaged in the wire basket,. If duct avulsion or a major ductal tear occurs, subsequent gland excision could be necessary (Walvekar RR, Razfar A, Carrau RL, et al. 2008).

Lingual nerve paresthesias can occur in up to $15 \%$ of patients undergoing transoral combined procedures in the immediate post-operative period and resolves with time (Walvekar RR, Razfar A, Carrau RL, et al. 2008; Bowen MA, Tauzin M, Kluka, EA, et al. 2010; Nahlieli O. 2009). The development of a post-operative stricture has been reported (Bowen MA, Tauzin M, Kluka, EA, et al. 2010; Nahlieli O. 2009). Taking care not to cause trauma to the duct or papilla during the procedure minimizes the risk of this complication. In addition, in the event the duct is traumatized or a papillotomy is required, placement of a salivary stent for up to 2 weeks can help prevention of subsequent ductal or papillary stenosis. Salivary fistulas, sialoceles, minor ductal tears, development traumatic ranulas, minor bleeding, and infection have been reported (Nahlieli O. 2006; Walvekar RR, Razfar A, Carrau RL, et al. 2008; Bowen MA, Tauzin M, Kluka, EA, et al. 2010) $(4,21,31)$. Other complications may include inability to retrieve the stone and failure of the procedure due to ductal stenosis or an acute masseteric bend (Nahlieli O. 2006; Walvekar RR, Razfar A, Carrau RL, et al. 2008; Bowen MA, Tauzin M, Kluka, EA, et al. 2010; Nahlieli O. 2009).

\section{Future directions and conclusions}

Diagnostic and interventional sialendoscopy are safe and effective options for treating nonneoplastic disorders of the major salivary glands. Sialendoscopy is technically challenging and requires sequential learning in which success rates appear to be proportional to the surgeon's level of experience Marchal F, Dulguerov P. (2003). The authors recommend that 
future sialendoscopist's should familiarize themselves with the anatomy and physiology of the salivary glands and floor of the mouth. They should be competent in taking care of any potential complication and should be comfortable with major salivary gland resections if required. Sialendoscopy training via hands-on courses and case observations should be pursued prior to initiating a sialendoscopy practice

\section{Further resources for sialendoscopy-related information}

1. www.salivaryendoscopy.net

2. http://emedicine.medscape.com/article/1520153-overview

3. Marchal F. Sialendoscopy. In: E Myers, Ed. Salivary Gland Disorders. Berlin, Germany: Springer; 2007:127-48.

4. Marchal F, Dulguerov P. Sialolithiasis management: the state of the art. Arch Otolaryngol Head Neck Surg. Sep 2003;129(9):951-6.

5. Marchal F. Sialendoscopy: The Endoscopic Approach to Salivary Gland Ductal Pathologies. Tuttlingen, Germany: Endo Publishing; 2003.

6. Koch M, Zenk J, Iro H. Algorithms for treatment of salivary gland obstructions. Otolaryngol Clin North Am. 2009.

\section{References}

[1] Nahlieli O, Shacham R, Shlesinger M, et al.(2004). Juvenile recurrent parotitis: a new method of diagnosis and treatment. Pediatrics; Vol. 144,No. 1, (Jul 2004),pp. 9-12.

[2] Kim JW, Han GS, Lee SH et al.(2007). Sialendoscopic treatment for radioiodine induced sialadenitis. Laryngoscope Vol. 117, No. 1, (Jan 2007), pp. 133-136.

[3] Bomeli SR, Schaitkin B, Carrau RL, et al. (2009), Interventional sialendoscopy for treatment of radioiodine-induced sialadenitis. Laryngoscope, Vol 119, No.5, ( May 2009), pp. 864-867.

[4] Nahlieli O and Nazarian Y. Sialadenitis following radioiodine therapy - a new diagnostic and treatment modality. Oral Dis, 2006;12:476-479.

[5] Shacham R, Puterman MB, Ohana N, et al. (2011) Endoscopic treatment of salivary glands affected by autoimmune diseases. J Oral Maxillofac Surg, Vol. 69, No. 2 (Feb 2011), pp.476-481.

[6] Marchal F, Dulguerov P. (2003) Sialolithiasis management: the state of the art. Arch Otolaryngol Head Neck Surg, Vol. 129, No. 9 (Sept 2003), pp. 951-956.

[7] Nahlieli O. (2006) Sialendoscopy: a new approach to salivary gland obstructive pathology. J Am Dent Assoc, Vol. 137, No. 10, (Oct 2006), pp. :1394-1400.

[8] Karavidas K, Nahlieli O, Fritsch N, et al.(2010) Minimal surgery for parotid stones: a 7-year endoscopic experience. In J Oral Maxillofac Surg, Vol. 39, No. 1, (Jan 2010), pp. 1-4.

[9] Bodner L. (2002) Giant salivary gland calculi: diagnostic imaging and surgical management. Oral Surg Oral Med Oral Pathol Oral Radiol Endo,. Vol. 94, No. 2, (Sep 2002), pp. 320-233.

[10] Lustmann J, Regev E, Melamed Y. (1990) Sialolithiasis. A survey on 245 patients and a review of the literature. Int J Oral Maxillofac Surg, Vol. 19, No. 3, (Jun 1990), pp. 135-138.

[11] Marchal F. (2007) A combined endoscopic and external approach for extraction of large stones with preservation of parotid and submandibular glands. Laryngoscope, Vol. 117, No. 2 (Feb 2007), pp. 373-377.

[12] Raif J, Vardi M, Nahlieli O, et al. (2006) An Er:YAG laser endoscopic fiber delivery system for lithotripsy of salivary stones. Lasers Surg Med, Jul 2006;38(6):580-587. 
[13] Seldin HM, Seldin SD, Rakower W. (1953) Conservative surgery for the removal of salivary calculi. Oral Surg Oral Med Oral Pathol, Vol 6, No. 5, (May 1953), pp. 579-587.

[14] Walvekar RR, Bomeli SR, Carrau RL, et al. (2009) Combined approach technique for the management of large salivary stones. Laryngoscope. Vol. 119, No. 6, (Jun 2009), pp.1125-1129.

[15] Walvekar RR, Razfar A, Carrau RL, et al. (2008) Sialendoscopy and associated complications: a preliminary experience. Laryngoscope. Vol. 118, No. 5, (May 2008), pp. 776-779.

[16] Jabbour N, Tibesar R, Lander T, et al. (2010) Sialendoscopy in Children. Int J Pediatr Otorhinolaryngol, Vol 74, No. 4, (Apr. 2010), pp. 347-350.

[17] Martins-Carvalho C, Plouin-Gaudon I, Quenin S, et al. (2010) Pediatric Sialendoscopy: a 5-year experience at a single institution. Arch Otolaryngol Head Neck Surg, Vol 136, No. 1, (Jan 2010)pp. 33-36.

[18] Faure F, Querin S, Dulguerov P, et al. (2007) Pediatric salivary gland obstructive swelling: sialendoscopic approach. Laryngoscope, Vol 117, No. 8 (Aug 2007), pp. 1363-1367.

[19] Marchal F. Sialendoscopy: The Endoscopic Approach to Salivary Gland Ductal Pathologies. Tuttlingen, Germany: Endo-Publishing; 2003.

[20] Geisthoff UW. (2009) Technology of sialendoscopy. Otolaryngol Clin North Am Vol. 42, No. 6, (Dec 2009), pp. 1001-1028.

[21] Papadaki ME, Kaban L, Kwolek C, et al. (2007) Arterial stents for access and protection of the parotid and submandibular ducts during sialoendoscopy. J Oral Maxillofac Surg Vol 65, No. 9 (Sept 2007), pp. 1865-1868.

[22] Iwai T, Matsui Y, Yamagishi M, et al. (2009) Simple technique for dilatation of the papilla in sialoendoscopy. J Oral Maxillofac Surg, Vol. 67, No. 3, (Mar 2009) pp. 681-2.

[23] Luers JC, Vent J, Beutner D. (2008) Methylene blue for easy and safe detection of salivary duct papilla in sialendoscopy. Otolaryngol Head Neck Surg Vol. 139, No. 3 (Sept 2008), pp. 466-467.

[24] Nahlieli O, Baruchin AM. (1999) Endoscopic technique for the diagnosis and treatment of obstructive salivary gland diseases. J Oral Maxillofac Surg. Vol. 25, No. 12, (Dec 1999), pp. 1394-401; discussion 1401-1402.

[25] Bowen MA, Tauzin M, Kluka, EA, et al. (2010) Diagnostic and interventional sialendoscopy: a preliminary experience. Laryngoscope, Vol. 121, No. 2 (Feb 2010), pp. 299-303.

[26] Nahlieli O, Nakar LH, Nazarian Y, et al. (2008) Sialoendoscopy: a new approach to salivary gland obstructive pathology. J Am Dent Assoc, Vol. 137, No. 10, (Oct 2008), pp. 1394-1400.

[27] Walvekar RR, Carrau RL, Schaitkin B. (2009) Sialendoscopy: Minimally invasive approach to the salivary ductal system. Operative Techniques in Otolaryngology. Vol. 20, No. 2, (Jun 2009), pp. 131-135.

[28] Papadaki ME, McCain JP, Kim K, et al. Interventional sialoendoscopoy: Early Clinical Results. J Oral Maxillofac Surg, Vol. 66, No. 5, (May 2008), pp. 954-962.

[29] Walvekar RR, Tyler PD, Tammareddi N, et al. Robotic-assisted Transoral removal of a submandibular Megalith. Laryngoscope Vol. 131, No. 3, (Mar 2011), pp. 534-7.

[30] Shacham R, Droma EB, London D, et al. Long-term experience with endoscopic diagnosis and treatment of juvenile recurrent parotitis. J Oral Maxillofac Surg, Vol. 67, No. 1, (Jan 2009), pp. 162-167.

[31] Nahlieli O. Advanced Sialoendosocpy Techniques, Rare Findings and Complications. Otolaryngol Clinc North Am. Vol. 42, No. 6, (Dec 2009), pp. 1053-1072. 


\title{
Virtual Endoscopy of the Nasal Cavity and the Paranasal Sinuses
}

\author{
Sumeet Anand, Rickul Varshney and Saul Frenkiel \\ McGill University, Department of Otolaryngology - Head \& Neck Surgery
}

Canada

\section{Introduction}

The ideal approach to sinus surgery has been a mystery that many Otolaryngologists have attempted to discover throughout their career. The intricate and complex anatomy of the nasal cavity and paranasal sinuses has challenged many in terms of adequate exposure during surgery, as well as proper relief of patient symptoms. From the days of Hirschmann, who used a modified cystoscope to examine the sinuses in 1901 (Draf, 1983, as cited in Rice \& Schaefer, 2004), to the work of Messerklinger and Wigand in establishing operative techniques later in that century (Rice \& Schaefer, 2004), surgeons have appreciated the obscurity and complexity of endoscopic sinus surgery for decades.

Traditionally, pre-operative planning has consisted of imaging modalities, namely x-rays and computed tomography (CT) scans, as well as flexible or rigid endoscopy in the clinic. With the advent of helical CT in the late 1980s, pre-operative imaging has improved dramatically. It is faster than the conventional CT scanner and provides more details (Wood \& Razavi, 2002). In fact, helical CT has provided physicians with the ability to attain reconstruction of axial images into coronal and sagittal views with resolution quality equivalent to the original axial cuts. Imaging for the paranasal sinuses is ideally viewed in coronal slices as Otolaryngologists enter the nasal cavity in an anterior-posterior direction rather than the cephalocaudal direction represented by axial slices. Furthermore, the coronal view allows the examination of critical relationships such as the osteomeatal complex, not provided by axial imaging. The use of MRI for better soft tissue examination and less artifacts has also improved imaging of the head and neck (Kettenbach et al., 2008). The dependence of Otolaryngologists on proper pre-operative imaging highlights the strong relationship between radiologists and otolaryngologists in endoscopic sinus surgery in order to precisely locate numerous vital structures, such as the orbits and the internal carotid arteries, surrounding the nasal cavity and the paranasal sinuses and to identify benign and pathologic anomalies.

Recently, virtual endoscopy (VE) through reconstruction of CT images has interested Otolaryngologists that perform endoscopic sinus surgery. This contemporary technology facilitates the difficult task of mentally conceptualizing complex and intricate anatomy in a three-dimensional model from two-dimensional CT images, a task that requires years of experience to truly master. VE utilizes the technique of surface or volumetric rendering to process data provided by CT images to allow three-dimensional visualization of tubular 
anatomical structures (Rubin et al., 1996). This modality has been used in the past for imaging of the colon (Pineau et al., 2003) and more recently in imaging of the tracheobronchial tree (Vining et al., 1996), urinary tract, vessels, the middle ear and the paranasal sinuses (Han et al., 2000; Ossoff \& Reinisch, 1994; Rogalla et al., 1998). This chapter explores the use of $\mathrm{VE}$ in the imaging of the nasal cavity and the paranasal sinuses. The technical aspects of VE, the uses in Otolaryngology and Radiology, the benefits for preoperative and intra-operative planning, the roles in endoscopic sinus surgery training, as well as future directions will be discussed.

\section{Technical aspects of Virtual Endoscopy}

In essence, VE is a reformation of axial CT images into a 3D model using sophisticated software. In the present age where the medical system is influenced by a paucity of resources and increasing waiting times for diagnostic tests, VE provides a useful diagnostic adjunct for the surgeon, without the need of additional tests. There are no required particular CT specifications over the standard scanner and thus, any patient who can undergo a CT sinus can have a three-dimensional model created.

The process begins with a low-dose spiral CT scan, as routinely performed for sinus imaging. Axial slices of $2.5 \mathrm{~mm}$ thickness are acquired at an interval of $1.25 \mathrm{~mm}$, therefore providing an overlap of collected information. No intravenous contrast is used for the scan. The images are then reconstructed into thinner $0.625 \mathrm{~mm}$ slices at an increment of $0.625 \mathrm{~mm}$ using a standard algorithm. Thereafter, these images are processed through rendering into a three-dimensional model using software provided with a 64-multislice General Electrics (GE) LightSpeed scanner (Milwaukee, WI). VE software is accessed on a GE workstation in our radiology department, which was provided with the scanner without an additional fee. There are two main methods for post-processing, namely surface rendering and volume rendering. We use volume rendering at our institution for reasons that will be explained later in this chapter. Although the process of post-processing is complex, we present a brief overview of the two methods.

In order to understand the process of reformatting, it is useful to appreciate the significance of a voxel. The latter is defined as a volume element (Rogalla et al., 2000) or the smallest distinguishable volume part of a 3D space. These individual voxels merge to create the three-dimensional model, analogous to a two-dimensional image formed by pixels (Gilani et al., 1997).

\subsection{Surface rendering}

Surface rendering works by defining a certain threshold as to determine which pixels will be included in the model and which ones will not (Rogalla et al., 2000). Surface rendering then creates a 3D model by linking the contours of selected objects from one slice of a CT with the adjacent slices (Wood \& Razavi, 2002). Segmentation, as an intermediate step, is done to transform the volume into a mesh of polygons (Kettenbach et al, 2008; Rogalla et al., 2000). The polygons are then formed into a 3D model using specialized software often provided with the CT scan machine. Authors have suggested threshold ranges from -520 to -200 Hounsfield units (HU) in order to remove voxels denser than $-500 \mathrm{HU}$. They recommend that thresholds of -250 and $-400 \mathrm{HU}$ be used to optimally view the nasal anatomy in healthy patients or those with sinonasal disease (Kettenbach et al, 2008). Although it is faster than 
volume rendering, surface rendering has poorer definition (Wood \& Razavi., 2002), as there is data loss about the inner aspects of the polygons and artifacts. Furthermore, the axial images can be manipulated to show the surface structures modified by shading techniques, a process entitled surface shaded display. Surface shading can be done based on distance of the surface from the observer or the orientation of the surface in question. This differential shading pattern allows the surgeon to appreciate the position of one structure relative to another (Rogalla et al., 2000).

\subsection{Volume rendering}

Volume rendering consists of creating images by casting rays from an observation point from two possible locations, either outside the volume being visualized or from within it. The difference between surface and volume rendering is that it does not focus solely on the surface features, but also displays the structures as though they are partially transparent by modifying the percentage of light ray within a voxel (Kettenbach et al, 2008). In volume rendering, the volume data itself is analysed without the surface representation step of surface rendering.. (Rogalla et al., 2000) Also, all the pixels are kept in memory, as oppose to surface rendering which has a set threshold (Rogalla et al., 2000). This allows the acquisition of more information and increased detail with an expense, however, of increased need of data processing and time consumption. The quality of the processed three-dimensional model can be improved with increased quality of the original axial images, namely through thinner slices and greater pixel formatting (Kettenbach et al., 2008).

\subsection{Flight path}

The three-dimensional model provides the surgeons the ability to navigate through the created structure. VE software displays an optical electronic apparatus to dynamically navigate an organ lumen - the virtual endoscope. The navigation tool allows the operator to "fly through" or "sail through" the three-dimensional anatomy, traveling in any direction or any position in the nasal cavity and paranasal sinuses. The virtual endoscope is shown here (Figure 1) simultaneously traveling in the axial, sagittal and coronal planes. Orientation to the virtual endoscope is made easier to follow by these corresponding reference planes. Images are initially presented in a greyscale, though functions of the software allow subjective colour assignments to represent anticipated anatomic tissue hues.

The "flight path" is the route of flight as determined by the operator. This course can be set to emulate a course similar to conventional fiberoptic endoscopy or can be set to explore areas and obtain perspectives not available by routine fiberoptic exam. Pre-operatively, this has the potential to allow adequate planning and mapping of the operative approach in a safe manner, while localizing delicate and vital structures. Also, the surgeon can correlate the $3 \mathrm{D}$ images with the endoscopic view as to understand the limitations and imperfections of surface and volume rendering of the CT images, as there is a certain learning curve as with most novel technologies.

\section{Virtual Endoscopy in otolaryngology and radiology}

In the past two decades, multiplanar reformatting in coronal and sagittal planes of crosssectional axial imaging has offered a capability to visualize with detail complex bony and 


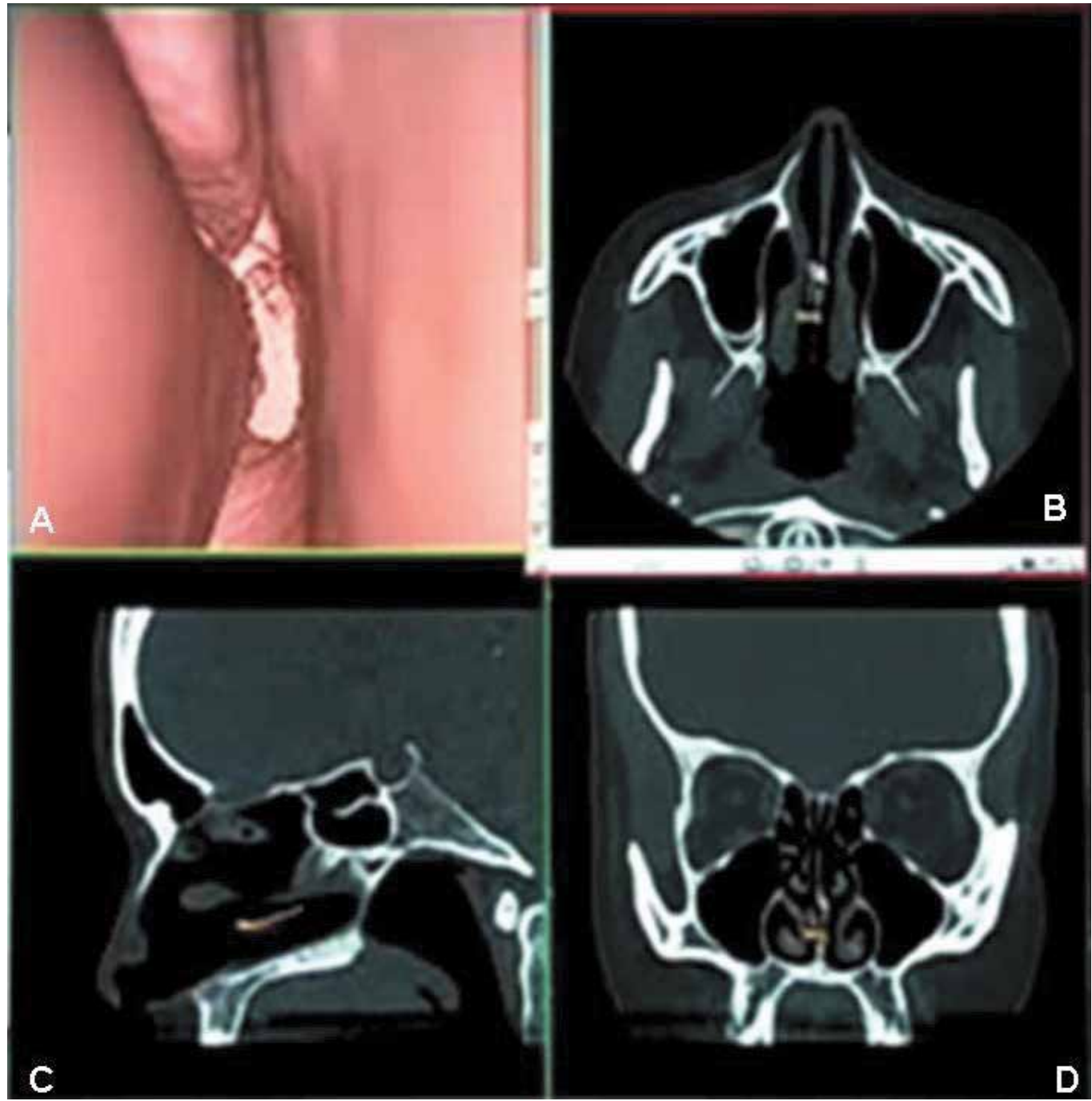

Fig. 1. Virtual Endoscopy

Virtual endoscope traveling in the right nasal cavity towards the nasopharynx (A), simultaneously being pursued in the axial (B), sagittal (C), and coronal planes (D); original figure.

soft tissue nasal structures. The otolaryngologist and radiologist, however, must routinely undertake a practice of mentally unifying two-dimensional images to accurately envision three-dimensional anatomy. VE offers a unique advantage of noninvasively carrying out this mental practice for the user to pictorially display the true anatomical site. Used in complement with high resolution thin-slice CT, VE significantly advances a users level of visualization of the paranasal sinuses, providing great utility to the clinical practice of both the otolaryngologist and radiologist. Many of the applications described here are relevant to the practice of both specialties, emphasizing the multidisciplinary utility of this technology. 


\subsection{Clinical applications}

In direct patient care, VE offers numerous advantages that can complement office-based rhinoscopy. Foremost, VE has the ability to surmount the anatomical boundaries of the sinuses, visualizing areas and obtaining perspectives that are not achievable with conventional fiberoptic rhinoscopy. The virtual endoscope can in effect be directed in an innumerable array of flight paths, limited only by the creativity of the operator. A user can instantly traverse across bony and soft tissue landmarks such as the medial maxillary wall or the curvature of the turbinates to visualize an adjacent structure. Systematically, the user can follow pathways of mucociliary clearance from the originating sinus, via the narrow ostia, to ultimately the area of natural drainage. For instance, drainage of the maxillary sinus can be followed from within the cavity, through the narrow maxillary sinus ostia to the ethmoid infundibulum and ultimately the maxillary sinus meatus at the semilunar hiatus (see figure 2A). Distinctively, VE allows the user to reach anatomically arduous to reach landmarks not amenable via conventional endoscopy such as the frontal and sphenoid sinus cavities or ostia (see figure $2 \mathrm{~B}$ ).

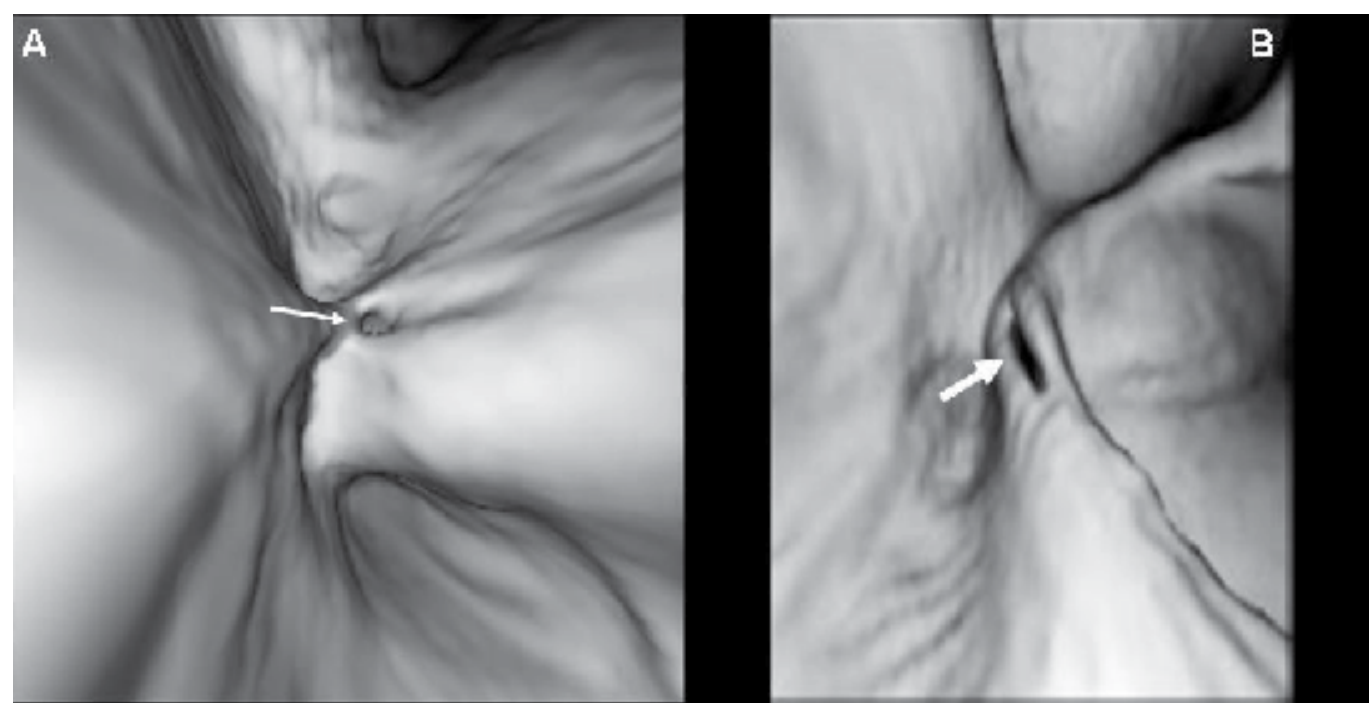

Fig. 2. Interior Views

En face three-dimensional view of a non-diseased right maxillary sinus ostium (A; thin arrow) and right sphenoethmoidal recess (B; thick arrow); original figure.

Unique perspectives, such as a retrograde evaluation of the nasal choanae via the nasopharynx or looking medially towards a maxillary sinus ostia from within the maxillary sinus, can be appreciated. VE also permits access to narrow and painful areas as small as 1-2 $\mathrm{mm}$ in diameter including the inferior meatus and stenotic pathology such as an obstructed osteomeatal complex or choanal atresia (Han et al., 2000). In each of these instances, VE affords superior visualization than fiberoptic rhinoscopy.

We have previously reported that VE has great utility in visualizing anatomical landmarks of the sinonasal tract (Anand et al., 2009). Similar to CT, in the presence of an air-tissue or airfluid interface, VE has equivocal utility in recognizing sinonasal pathology (Figures 3 and 4). Visualization is strongly dependent on this interface to delineate the surface anatomy of a structure as viewed en face by the virtual endoscope. In instances of soft tissue obliteration, like CT, VE finds difficulty in distinguishing mucinous secretions from soft-tissue anatomy. 


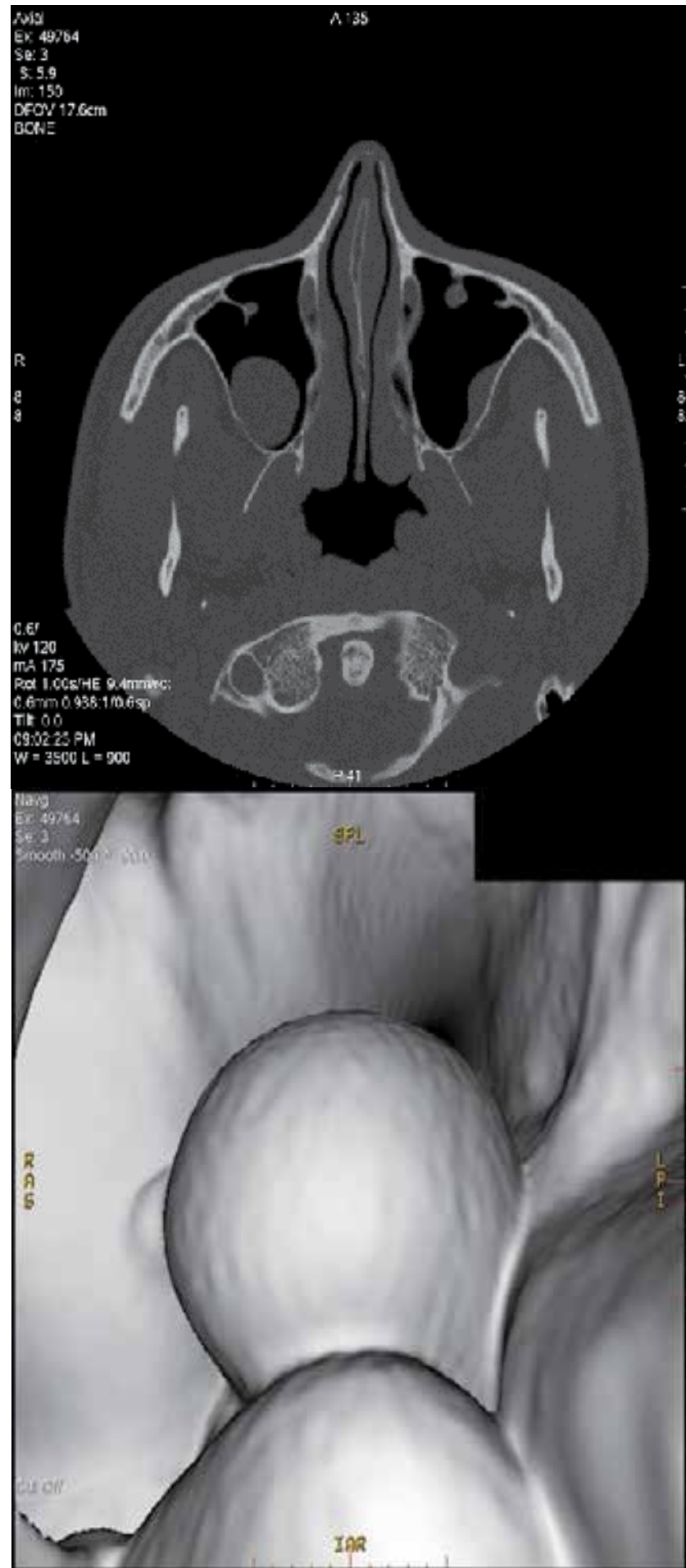

Fig. 3. Right maxillary sinus cyst; original figure. 


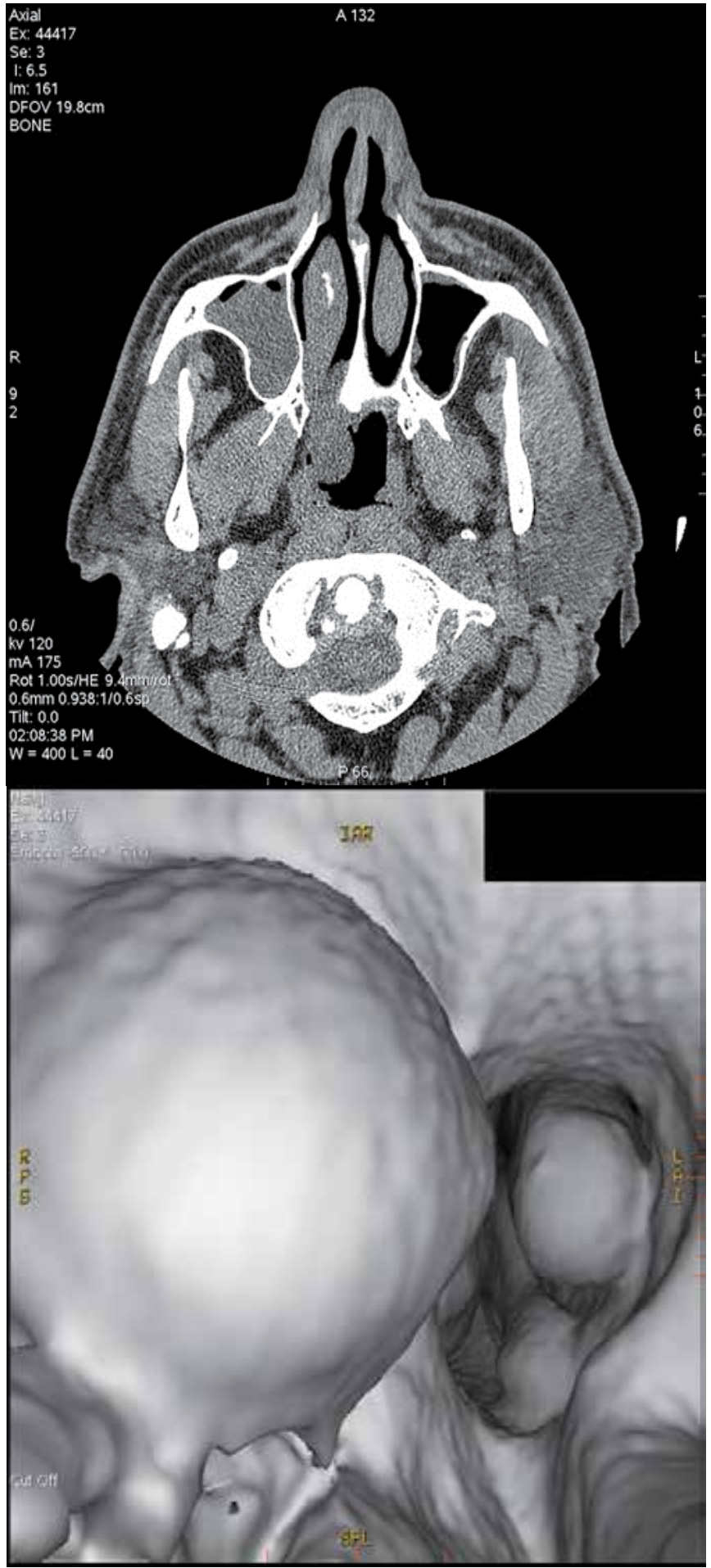

Fig. 4. Right choanal polyp; original figure. 
VE remains an imaging technology and unlike conventional rhinoscopy there is no opportunity for the otolaryngologist to simultaneously culture, lavage or biopsy pathology (Greess et al., 2000). Table 1 outlines the advantages and disadvantages of VE. Overall, the advanced visualization inherent in VE makes it an excellent complement to flexible or rigid clinic endoscopy.

\begin{tabular}{l|l} 
Advantages & Disadvantages \\
$\begin{array}{l}\text { Visualize areas inaccessible by nasal endoscopy (ostia, } \\
\text { paranasal cavities) }\end{array}$ & $\begin{array}{l}\text { Cannot appreciate quality } \\
\text { of the mucosa }\end{array}$ \\
$\begin{array}{l}\text { Antro- and retro-grade views, } 360^{\circ} \text { analysis } \\
\text { Traverse painful stenotic areas }\end{array}$ & $\begin{array}{l}\text { No density } \\
\text { measurements }\end{array}$ \\
$\begin{array}{l}\text { No additional morbidity (no special CT, no IV } \\
\text { contrast/aerolized medication/local trauma/discomfort or } \\
\text { complications) }\end{array}$ & $\begin{array}{l}\text { Cannot culture, lavage or } \\
\text { biopsy pathology }\end{array}$ \\
\hline
\end{tabular}

Table 1. Advantages and Disadvantages of virtual endoscopy

Unlike fiber-optic rhinoscopy, there is no additional morbidity with VE beyond that associated with a routine non-contrast CT. No particular CT specifications are required and any patient that can undergo a CT sinus can have VE completed using the same image dataset. For the Radiologist, VE can offer the unique advantages of superior visualization described above without additional imaging of the patient or a considerable amount of additional image processing. For communication amongst colleagues, VE can serve as an additional tool to $\mathrm{CT}$ for subjects on whom the Otolaryngologist has not performed rhinoscopy. If care is being provided in a remote area where there is limited or no access to fiberoptic rhinoscopy or for discussing a patient case across two or more centers, VE can increase communication amongst treating physicians.

There are limitations to the use of VE in both Otolaryngology and Radiology. As with other advances in medical technology there is a learning curve with the introduction of a new modality (Chin et al., 2007). Once the user is familiar with VE software, however, navigation of the virtual endoscope is instantaneous. Specific limitations of VE arise in evaluating mucosal surfaces and secretions. For the Otolaryngologist, it is difficult to appreciate the quality and colour of the mucosal lining, prohibiting a differentiation of erythema or leukoplakia. Radiologically, mucous in the nasal cavity can be mistaken for a false soft tissue density; however, this is a similar pitfall in conventional two-dimensional CT (Anand et al., 2009).

\subsection{Pre-operative and intra-operative planning}

As a preoperative tool in surgical planning VE holds widespread potential. Prior to undertaking either open or endoscopic sinus surgery, the operative approach can be mapped out with the virtual endoscope. The influence of removing or partially altering surgical landmarks can be evaluated from the direction of anticipated view during surgery, 
as well as from several perspectives adjacent to the anatomic landmark to evaluate the functional impact of the intended surgical step. This could assist in making both endoscopic and open nasal surgery even safer and minimally invasive by assisting in preservation of vital functional structures. A step-by-step anatomic prosection can be formulated with VE for each individual case, allowing the surgeon to plan out an entire procedure in the preoperative setting. This is a great assist for both active surgeons and in simulation-based teaching for trainees; the latter is an advantage that will be explored in detail later in this chapter. Several institutions have already begun using VE as a preoperative tool, and its use has been described in combination with multiplanar imaging, to preoperatively decide safe surgical entrance points for paranasal sinus mucoceles (Nakasato et al, 2000). The advantage of being able to traverse stenotic segments inaccessible by conventional rhinoscopy increases the utility of VE in advanced skull base surgery. Visualization and thorough preoperative localization of critical structures such as the carotid and optic canal within the sphenoid, frontal sinus recess, anterior and posterior ethmoid arteries, fovea ethmoidalis and cribriform plate at the skull base increases operative safety in endoscopic and open skull base approaches. Intra-operatively, VE can be used as a navigational aid with imageguided applications to identify complex anatomy. The surgeon can herein use VE in realtime for intraoperative decision making to help direct the surgery, increase efficiency, localize detailed landmarks and importantly reduce the complication rate of advanced sinonasal surgery.

\subsection{Anatomical correlation}

To date several reports have evaluated the capability of VE to identify nasal cavity and paranasal sinus landmarks and a high level of agreement has been seen between volume rendering virtual three-dimensional imaging methods and conventional fiber-optic rhinoscopy (Di Rienzo et al., 2003; Han et al., 2000). Reports also demonstrate considerable agreement among VE and intra-operative findings in the vast majority of patients (Bisdas et al., 2004). We completed a preliminary evaluation of our VE interpretation experience and compared this to operative identification of landmarks in yet unpublished data. In a retrospective series, VE of 25 patients undergoing CT imaging of the paranasal sinuses for chronic nasal symptoms was compared to operative fiberoptic endoscopy findings. None of the patients were given aerosolized nasal decongestant prior to their CT imaging. Nineteen anatomic landmarks relevant in the mucociliary clearance of paranasal sinus secretions were objectively evaluated to be either not, partially or very well identified and scored with a 0,1 , or 2 out of 2 respectively (Table 2 ).

VE outperformed operative findings in visualizing hard to access areas such as the superior turbinate, superior meatus, choanae, nasopharynx, sphenoid and sphenoethomoidal recess. VE also identified more clearly structures of the osteomeatal complex, namely the maxillary sinus ostium, uncinate process, and infundibulum, where most often pathology is localized in chronic sinonasal disease. Operative endoscopy better identified the middle and inferior meati and turbinates, as similarly described by Han et al. (2003). Surgeons are able to dissect away diseased mucosa or suction secretions to gain entry into a meatus, a feat not feasible with VE. Operative endoscopy also better visualized the ethmoid air cells, frontoethmoidal recess and frontal sinus, areas routinely addressed in dissection during endoscopic sinus surgery. 


\begin{tabular}{|l|c|c|c|c|c|c|}
\hline & & VE & & & FOE & \\
& 0 & 1 & 2 & 0 & 1 & 2 \\
\hline Septum & 0 & 0 & $25(100 \%)$ & 0 & 1 & $25(96.2 \%)$ \\
Nasal cavity & $1(4.0 \%)$ & $2(8.0 \%)$ & $22(88.0 \%)$ & $3(12.0 \%)$ & $3(12.0 \%)$ & $19(76.0 \%)$ \\
Infer Turbinate* & 0 & $2(8.3 \%)$ & $22(91.7 \%)$ & 0 & $1(4.2 \%)$ & $23(95.8 \%)$ \\
Mid Turbinate* & $6(25.0 \%)$ & $2(8.3 \%)$ & $16(66.7 \%)$ & $1(4.8 \%)$ & $2(9.5 \%)$ & $18(85.7 \%)$ \\
Super Turbinate & $13(52.0 \%)$ & 0 & $12(48.0 \%)$ & $25(100 \%)$ & 0 & 0 \\
Infer Meatus* & $1(4.2 \%)$ & $2(8.3 \%)$ & $21(87.5 \%)$ & 0 & $1(4.2 \%)$ & $23(95.8 \%)$ \\
Mid Meatus* & $4(16.7 \%)$ & $3(12.5 \%)$ & $17(70.8 \%)$ & $1(4.8 \%)$ & $1(4.8 \%)$ & $19(90.4 \%)$ \\
Super Meatus & $10(40.0 \%)$ & 0 & $15(60.0 \%)$ & $25(100 \%)$ & 0 & 0 \\
Choanac & $2(8.0 \%)$ & $1(4.0 \%)$ & $22(88.0 \%)$ & $2(8.0 \%)$ & $2(8.0 \%)$ & $21(84.0 \%)$ \\
Nasopharynx & $1(4.0 \%)$ & 0 & $24(96.0 \%)$ & $3(12.0 \%)$ & $3(12.0 \%)$ & $19(76.0 \%)$ \\
Uncinate process & $9(37.5 \%)$ & $7(29.2 \%)$ & $8(33.3 \%)$ & 0 & $20(83.3 \%)$ & $4(16.7 \%)$ \\
Infundibulum & $9(37.5 \%)$ & $10(41.7 \%)$ & $5(20.8 \%)$ & 0 & $20(83.3 \%)$ & $4(16.7 \%)$ \\
Max sinus ostium & $8(32.0 \%)$ & $4(16.0 \%)$ & $12(48.0 \%)$ & 0 & $21(84.0 \%)$ & $4(16.0 \%)$ \\
Maxillary Sinus & $3(12.0 \%)$ & $6(23.1 \%)$ & $17(68.0 \%)$ & 0 & $19(76.0 \%)$ & $6(24.0 \%)$ \\
Frontal Sinus* & $8(32.0 \%)$ & $6(24.0 \%)$ & $11(44.0 \%)$ & $2(8.0 \%)$ & $10(40.0 \%)$ & $13(52.0 \%)$ \\
Sphenoid Sinus & $7(28.0 \%)$ & $6(24.0 \%)$ & $12(48.0 \%)$ & $1(4.0 \%)$ & $16(64.0 \%)$ & $8(32.0 \%)$ \\
Ethmoid cells* & $6(24.0 \%)$ & $9(36.0 \%)$ & $10(40.0 \%)$ & 0 & $13(52.0 \%)$ & $12(48.0 \%)$ \\
FER* & $8(32.0 \%)$ & $6(24.0 \%)$ & $11(44.0 \%)$ & $2(8.0 \%)$ & $10(40.0 \%)$ & $13(52.0 \%)$ \\
SER & $8(32.0 \%)$ & $5(20.0 \%)$ & $12(48.0 \%)$ & $1(4.0 \%)$ & $16(64.0 \%)$ & $8(32.0 \%)$ \\
\hline
\end{tabular}

FER: frontoethmoidal recess; SER: sphenoidoethmoidal recess; "Only 24 were observed as opposed to 25 as these structures were surgically removed in the past.

Table 2. Total number and percentages of scores for each anatomical landmark observed by virtual endoscopy (VE) and by fiberoptic operative endoscopy (FOE).

\section{Virtual Endoscopy in resident and trainee education}

Apart from clinical uses for the Otolaryngologist, virtual endoscopy also has potential applications in resident training. In the past, resident education in most surgical disciplines has been heavily based on the apprenticeship model, where trainees learned various procedures through clinical encounters. This fact is particularly still prevalent in endoscopic sinus surgery, where technical expertise is gained mainly from practice on real patients. However, the intricate anatomy of the nasal cavity and paranasal sinuses, as well as the numerous surrounding vital structures, create the potential for significant morbidity of endoscopic sinus surgery in inexperienced hands. 
In the past decade, there has been an emergence of novel teaching modalities, such as simulation training and virtual reality. Numerous authors in the past have reported improved resident performance after simulation and virtual reality training (Grantcharov et al., 2005; Seymour et al., 2002). More recently, VE has become an innovative educational method for training residents. In terms of Gastroenterology, improvement in technical accuracy and time needed to reach technical competency has been shown for colonoscopies after virtual endoscopy training (Ferlitsch et al., 2010). Improved trainee performance with surgical simulation training in endoscopic sinus surgery has been demonstrated, although there is a lack of data with virtual endoscopy. Edmond published his work with an endoscopic sinus surgical simulator as a training device for Otolaryngology residents and suggested a positive impact in performance in certain procedures such as anterior ethmoidectomies and also increased surgical confidence (Edmond, 2002). Similar response to simulation in endoscopic sinus surgery was demonstrated by Glaser with his work with medical students, who praised the ability for simulation training to help with 3D visualization and understanding of nasal anatomy (Glaser et al., 2006). There is a lack of data on the use of the virtual endoscope in Otolaryngology training, which we hope will be addressed as VE becomes more popular amongst endoscopic sinus surgeons.

In our institution, similar to many programs, endoscopic sinus surgery is typically performed by senior residents, starting in the upper years of residency. The use of VE would allow exposure for younger junior residents to explore and properly understand the anatomy early in their training, potentially improving their ultimate performance and safety on real patients. It is common knowledge within the discipline of Otolaryngology, furthermore, that the anatomy of the paranasal sinuses is a challenge to truly comprehend. A realistic virtual portrayal of the anatomy can strengthen the comprehension of natural pathways of mucociliary clearance as well as rhinoscopy and operative assessment. As mentioned previously, users are not restricted to spaces defined by mucosal surfaces in the nasal cavity as they are during real endoscopy. They are able to permeate through mucosal and bony structures and therefore learn the complex anatomical relationships. The literature has shown on numerous occasions that an operator can better appreciate spatial relationships in a three-dimensional model than in a series of two-dimensional images (Greess et al., 2000; Remy-Jardin et al., 1998).

VE provides many advantages over surgical experience for early training. It is noninvasive, thus there is no compromise in patient care. The same approaches and technical exercises can be performed multiple times or variant approaches can be designed and practiced. A resident can thus attempt to discover numerous pathways to a certain anatomical structure, such as a specific meatus or the maxillary, sphenoid or frontal sinus ostia. VE also provides crucial information to the location of surrounding anatomical structures. This will provide teaching faculty with pre-operative knowledge of what procedures are safe to be performed by resident trainees at varying levels of experience, thus objectifying procedure delegation.

\section{Future directions}

Three-dimensional rendering of helical CT images has modified the way Otolaryngologists and Radiologists approach the investigation of tubular anatomical structures. It is evident 
that VE of the paranasal sinuses provides surgeons with a new tool for both pre-operative and intra-operative planning, as well as a teaching modality for residents. The limitations of $\mathrm{VE}$ are in its ability to assess mucosal surfaces and secretions. In particular, current VE processing does not allow adequate differentiation of color and density, thus mucus or other secretions can be mistaken for a false soft tissue density. The differentiation between erythema and leukoplakia also can not be addressed by VE as opposed to direct visualization of color by conventional rhinoscopy. Moreover, VE is currently not able to identify tissue densities with imaging. Ultimately understanding from density measurements the nature of individual structures will allow improved identification of critical landmarks and pathologic anomalies possibly without conventional invasive rhinoscopy. Further research and innovation of VE technology will likely address this issue in the future. Finally, prospective trials in its use in training can objectively explore the role of this innovation in resident education.

\section{Conclusion}

As we enter a new decade, we are fortunate to constantly being challenged by novel diagnostic and therapeutic modalities to enhance patient care. VE has the potential to improve pre-operative diagnosis of pathologies, intra-operative patient management, post-operative follow-up, as well as trainee education. In this chapter, we have explored this relatively new technology, with an overview of its numerous utilities in clinical practice and residency training. VE has already been implemented routinely in certain centers for imaging of the colon and bronchial tree. It is our belief that VE will continue to progress as a technology and will certainly be an essential armamentarium of Otolaryngologists in the near future.

\section{References}

Anand SM, Frenkiel S, Le BQH, Glikstein R. Virtual Endoscopy: Our Next Major Investigative Modality? J Otolaryngol Head Neck Surg. 2009 Dec;38(6): 642-5.

Bisdas S, Verink M, Burmeister HP, et al. Three-dimensional visualization of the nasal cavity and paranasal sinuses: clinical results of a standardized approach using multislice helical computed tomography. J Comput Assist Tomogr 2004; 28(5):661-669.

Chin JL, Luke PP, Pautler SE. Initial experience with robotic-assisted laparoscopic radical prostatectomy in the Canadian health care system. Can Urol Assoc J 2007; 1(2):97101.

Di Rienzo L, Coen Tirelli G, Turchio P, et al. Comparison of virtual and conventional endoscopy of nose and paranasal sinuses. Ann Otol Rhinol Laryngol 2003; 112:139142.

Edmond, C. V. Impact of the Endoscopic Sinus Surgical Simulator on Operating Room Performance. The Laryngoscope 2002;112:1148-1158.

Ferlitsch A, Schoefl R, Puespoek A, Miehsler W, Schoeniger-Hekele M, Hofer H, Gangl A, Homoncik M. Effect of virtual endoscopy simulator training on performance of 
upper gastrointestinal endoscopy in patients: a randomized controlled trial. Endoscopy 2010;42(12):1049-56.

Gilani S, Norbash AM, Ringl H, et al. Virtual endoscopy of the paranasal sinuses using perspective volume rendered helical sinus computed tomography. Laryngoscope 1997;107:25-9

Glaser AY, Hall CB, Uribe S JI, Fried MP. Medical students' attitudes toward the use of an endoscopic sinus surgery simulator as a training tool. Am J Rhinol 2006;2:177-9.

Grantcharov, TP, Kristiansen, VB, Bendix, J, Bardram, L, Rosenberg, J, Funch-Jensen, P. Randomized clinical trial of virtual reality simulation for laparoscopic skills training. Br J Surg 2005;91(2): 146-150.

Greess H, Nomayr A, Tomandl B, et al. 2D and 3D visualization of head and neck tumours from spiral-CT data. European Journal of Radiology 2000; 33:170-177.

Han P, Pirsig W, Ilgen F, et al. Virtual endoscopy of the nasal cavity in comparison with fiberoptic endoscopy. Eur Arch Otolaryngol 2000; 257:578-583.

Kettenbach, J., Birkfellner, W., Rogalla, P. (2008). Virtual Endoscopy of the Paranasal Sinuses, In: Image Processing in Radiology, Neri, E., Caramella, D., Bartolozzi, C., (151-171), Springer Berlin Heidelberg, 978-3-540-49830-8, Berlin Heidelberg.

Nakasato T, Katoh K, Ehara S, et al. Virtual CT Endoscopy in Determining Safe Surgical Entrance Points in Paranasal mucoceles. J Comput Assist Tomogr 2000; 24(3):486492.

Ossoff RH, Reinisch L. Computer-assisted surgical techniques: a vision for the future of otolaryngology, head and neck surgery. J Otolaryngol 1994; 23(5):354-359.

Pineau BC, Paskett ED, Chen GJ, et al. Virtual colonoscopy using oral contrast compared with colonoscopy for the detection of patients with colorectal polyps. Gastroenterology 2003; 125(2):304-10.

Remy-Jardin M, Artaud D, Fribourg M, et al. Volume rendering of the tracheobroncial tree: clinical evaluation of bronchographic images. Radiology 1998;208:761-70.

Rice, DH, Schaefer, SD. (2004). Introduction, In: Endoscopic paranasal sinus surgery, Rice, DH, Schaefer, SD. (XV), Lippincott Williams \& Wilkins, 0-7817-4077-0, Philadelphia.

Rogalla P, Nischwitz A, Gottschalk S,et al. Virtual endoscopy of the nose and paranasal sinuses. Eur Radiol 1998; 8(6): 946-950.

Rogalla, P. (2000). Virtual Endoscopy of the Nose and Paranasal Sinuses, In: Virtual endoscopy and related 3D techniques, Rogalla, P., Terwisscha van Scheltinga, J., Hamm, B., (17-38), Springer, 3-540-65157-8, Berlin Heidelberg New York.

Rubin GD, Beaulieu CF, Argiro V, et al. Perspective volume rendering of CT and MR images: applications for endoscopic imaging. Radiology 1996; 199(2):321330.

Seymour, NE, Gallagher, AG, Roman, SA, O’Brien, MK, Bansal, VK, Andersen, DK, Satava, RM. Virtual Reality Training Improves Operating Room Performance. Results of a Randomized, Double-Blinded Study. Ann Surg 2002; 236(4):458-464. 
Vining DJ, Liu K, Choplin RH, et al. Virtual bronchoscopy: Relationships of virtual reality endobronchial simulations to actual bronchoscopic findings. Chest 1996; 109(2):549-553.

Wood BJ, Razavi P. Virtual Endoscopy: A Promising New Technology. Am Fam Physician 2002;66:107-12. 


\title{
Evolution of the Adenoidectomy in the Endoscopic Era
}

\author{
Fabio Pagella, Alessandro Pusateri, \\ Georgios Giourgos and Elina Matti \\ Foundation IRCCS Policlinico San Matteo \\ and University of Pavia \\ Italy
}

\section{Introduction}

During the last 20 years, we have observed an increasing recognition of the high prevalence of sleep-disordered breathing (SDB) in children. Adenotonsillar enlargement, leading to a partial or a complete obstruction of the nasopharynx (or epipharynx, or rhinopharynx) and/or of the oropharynx, accounts for the vast majority of cases. Consequently, the number of adenoidectomies performed in children for SDB has increased significantly. An adenoidectomy can be performed as an isolated procedure or as part of an adenotonsillectomy operation. As a matter of fact, adenoidectomy with or without tonsillectomy is one of the most common surgical procedures performed by Otolaryngologists in the paediatric population. Historically, tonsil and adenoid surgery increasingly began to be carried out together in the early $20^{\text {th }}$ century, as the popular "focus on infection" theory attributed various systemic disorders to diseased tonsils and adenoids; thus, tonsillectomy plus adenoidectomy were recommended as a standard treatment for several different conditions as anorexia, mental retardation and enuresis, or simply as a general measure to promote good health (Hays, 1924; Kaiser, 1932). In certain communities the surgical procedure was performed widely in the entire population of scholars in public school buildings (Baker, 1953). Over the years, things have changed, and precise indications were proposed for tonsillectomy and adenoidectomy. Adenoid hypertrophy can lead to obstructive sleep apnea, otitis media with effusion, recurrent otitis media and nasal obstruction, and nowadays these remain the most common indications for adenoidectomy. Techniques and instruments have considerably evolved from the first techniques described by Cornelius Celsus in the first century A.D. (Thornval, 1969) and Paul of Aegina in 625 A.D. (Paul of Aegina, 1847) to the later contributions of surgeons such as Wilhelm Meyer of Copenhagen and Samuel J. Crowe in the last centuries (Curtin, 1987; Meyer, 1870; Wiatrak \& Wooley, 2005; Younis \& Lazar, 2002). Pioneers of tonsillectomy and adenoidectomy have developed novel techniques and instruments in order to increase the speed of the procedure, especially in the pre-anesthesia era, and to decrease the intra-operative complications and postoperative morbidity.

The classic surgical technique performed with an adenoid curette or an adenotome has recently evolved by the introduction of the endoscopic sinus surgery (ESS) 
instrumentation, with an improved patients' outcome and a better satisfaction of the surgeon (Havas \& Lowinger, 2002; Rodriguez et al., 2002; Stanislaw et al., 2000). The standard adenoidectomy technique is to remove the nasopharyngeal lymphatic tissue with an adenoid curette or an adenotome (Figure $1-3$ ), under general anaesthesia via oro-tracheal intubation, with the patient placed in the Rose position, and a mouth gag inserted (Kornblut, 1987; Paradise 1996).

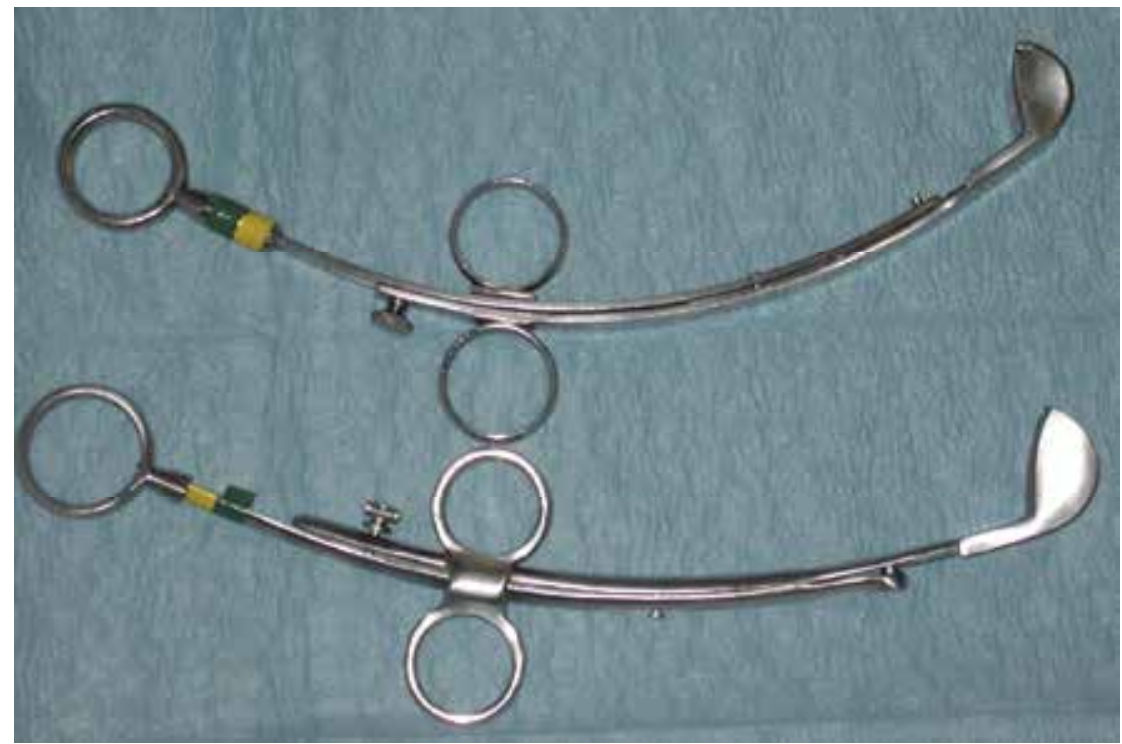

Fig. 1. Two, different sized, standard Shambaugh adenotomes.

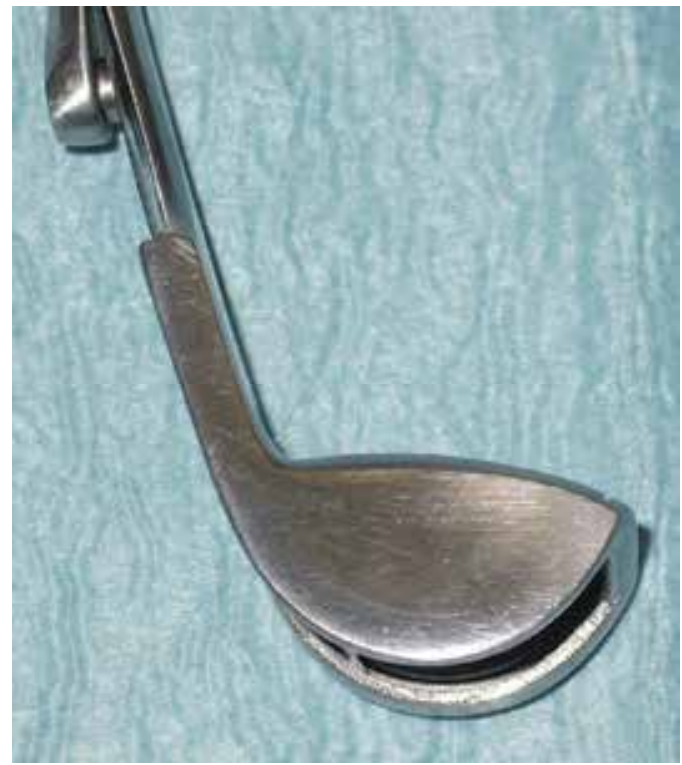

Fig. 2. Distal end of the Shambaugh adenotome. 


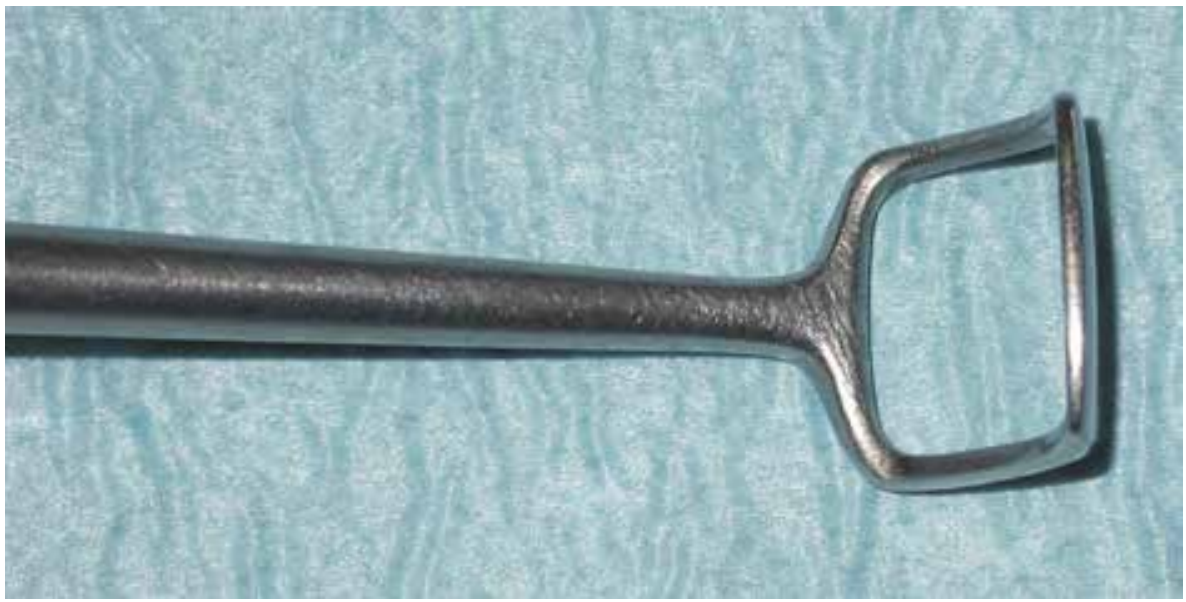

Fig. 3. Distal end of an adenoid curette.

The surgeon is placed at the head of the patient (Figure 4). The curette is transorally applied to the nasopharynx thus, by-passing the soft palate and the main bulk of the adenoids is removed, with a single or repeated passes. The majority of surgeons perform the procedure blindly, without a direct visualization of the nasopharynx (Figure 5). In some cases a partial visualization of the adenoid pad can be achieved by retracting the soft palate with rubber catheters or by using a laryngeal or a dental mirror. Bleeding is controlled by compression, through the placement of a gauze pack in the nasopharynx for several minutes.

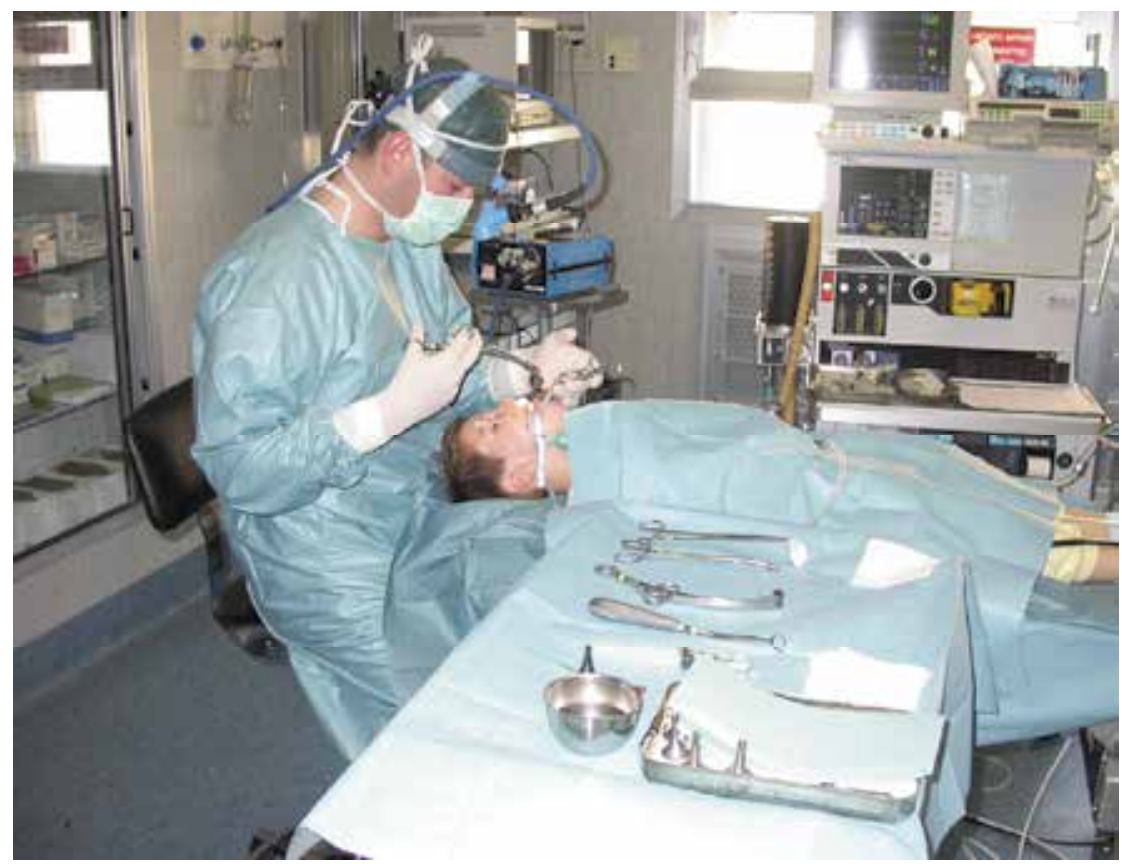

Fig. 4. Surgical position during a traditional adenoidectomy. 


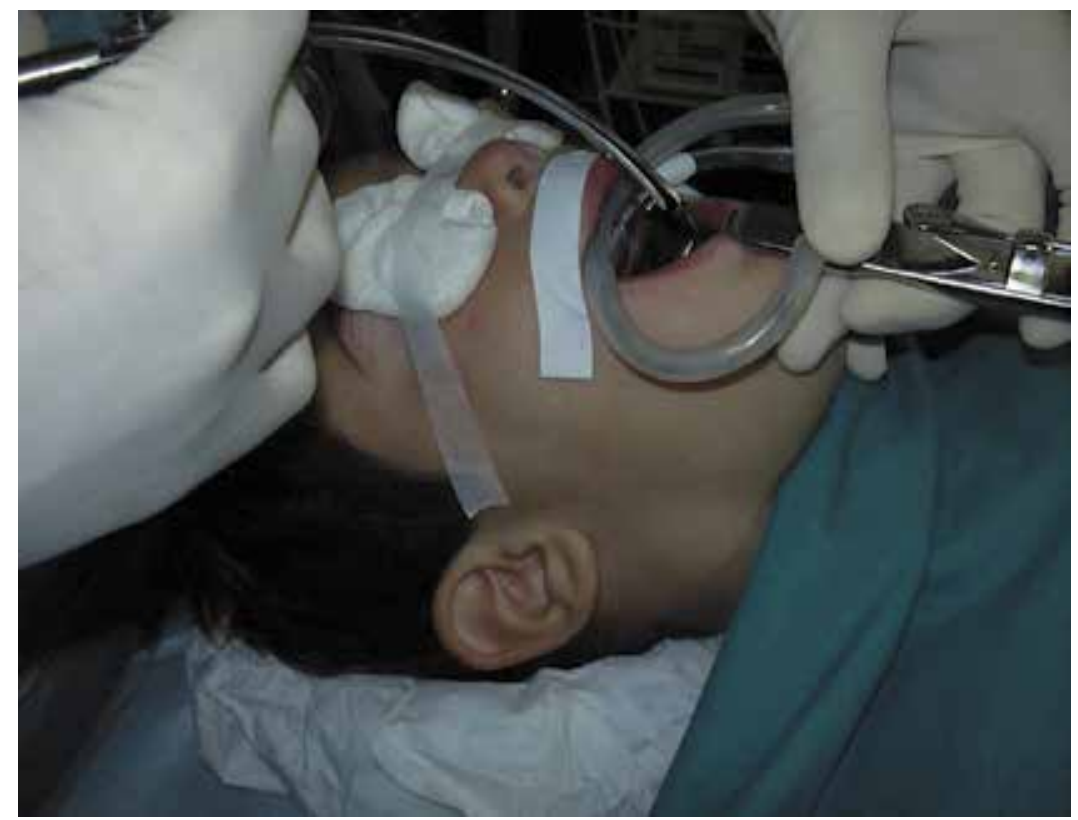

Fig. 5. External view of a traditional adenoidectomy performed with a Shambaugh adenotome; as underlined, the procedure is conducted without the visualization of the nasopharynx.

The visualization of the operative field is particularly useful in avoiding damages to important structures located nearby the adenoid tissue, such as the Eustachian tube and/or the pharyngeal muscles. Postoperative complications such as velopharyngeal insufficiency, tubaric stenosis and nasopharyngeal stenosis are rare but difficult to resolve when occurred. Several investigations prove that in up to one-third of children with clinically significant adenoid hypertrophy, conventional curettage adenoidectomy does not achieve an adequate removal of obstructive adenoid tissue, especially when there is an intranasal extension of the tissue, or a bulky mass of adenoids superiorly in the nasopharynx and in the peritubaric region (Buchinsky et al., 2000; Elluru et al, 2002; Havas \& Lowinger, 2002; Stanislaw et al., 2000).

To possibly reduce the morbidity linked to the adenoid tissue persistence and to prevent recurrences, total excision of the adenoids is the most important goal of this operation. Initially, the surgical clearance was confirmed by a digital palpation of the rhinopharynx and this procedure is still performed by some Otolaryngologists (Buchinsky et al., 2000). Subsequently, the use of an angled mirror or an endoscope during the adenoidectomy provided adequate visualization of the field, and these techniques are currently preferred by most surgeons (Brodsky, 1996; Cannon et al., 1999; Discolo et al., 2001). A recent study published by Ark et al. showed that in order to achieve a complete adenoid tissue removal, a direct/indirect visual assistance is necessary. The authors considered a group of patients that underwent a conventional adenoidectomy (surgical efficacy confirmed by digital palpation at the end of the procedure); subsequently, the surgical field was inspected through an indirect laryngeal mirror visualization. Their finding was that only one-fifth of the patients had no residual adenoid tissue. Instead, in the $81 \%$ of the patients a residue of lymphatic tissue was still present on the pharyngeal roof nearby the choanal 
opening; also, $11.4 \%$ of the patients had a residue along the torus tubarius on either side of the nasopharynx and in $6.3 \%$ the residual tissue was located at both cited sites (Ark et al., 2010).

The recurrence of adenoid hypertrophy after the surgical procedure is under debate in the literature, but several reports do not offer comparable results. According to Lundgren, the recurrence rate is $4-8 \%$, in Hill's series instead it is $23.7-50 \%$ and Crowe states a recurrence rate of over $75 \%$ (Tolczynski, 1955). More recent studies cite a significantly lower recurrency rate till a 0.5\% (Joshua et al., 2006; Monroy et al., 2008), and this success could be attributed to the evolution of the techniques. Historically, Tolczynski (Tolczynski, 1955) considered various situations to be responsible for the recurrence of adenoid hypertrophy, summarized in:

- Anatomical difficulties.

- Adenoidectomy is often performed in a hurry, and sometimes the inadequate anaesthesia could be responsible for an insufficient relaxation of the palato-pharyngeal muscles, whose contracture interferes with the manipulations of the adenoid pad.

- In many cases it is a "Surgical operation in the dark", without a visualization of the operatory field.

A recent paper in a cohort of over 300 patients reports that, when an endoscopic examination was performed at the end of the adenoidectomy, in the $14.5 \%$ of the cases some residual adenoid tissue that required additional removal was found; during the follow-up of these patients only $0.85 \%$ needed a revision adenoidectomy in the next 2 years. In contrast, when that endoscopic examination was not performed, there was a $6.7 \%$ persistence of the initial symptoms and a 5.6\% of the patients required a revision adenoidectomy. By comparing such results, it becomes evident that the endoscopic examination at the end of the procedure, significantly reduces the incidence of recurrence and thus the need for a revision surgery (Ezzat, 2010).

In the last years, several surgical techniques have been proposed to ensure a finer removal of the adenoid mass, as well as to achieve a better control of intraoperative bleeding.

Suction diathermy was initially introduced for haemorrhage control following conventional curettage of the adenoid pad (Kwok \& Hawke, 1987). Subsequently, the whole procedure was performed with this technique, always along a transoral approach and an indirect visualization by a laryngeal or dental mirror (Owens et al., 2005; Sherman, 1982; Skilbeck et al., 2007). As an evolution, in the following years suction diathermy was performed via a transnasal approach and under a transnasal endoscopic control, in the older paediatric population (Shin, 2003).

The introduction of powered instrumentation for sinus surgery prompted the use of a shaver system with an overbent cannula for a, power-assisted, total or partial adenoidectomy; however, the approach still was a transoral one, along with an indirect visualization through a laryngeal mirror (Heras et al., 1998; Koltai et al., 1997, 2002; Stanislaw et al. 2000).

In a recent article Walner et al presented a survey between 300 members of the American Society of Pediatric Otolaryngology, which examined the equipment used in adenotonsillectomy along the last 15 years in order to assess the surgical trends in instrument usage for paediatric adenotonsillectomy. About the adenoidectomy, there has been less consistency in the choice of an instrument over the years than for tonsillectomy. 
The most promising instrument 15 years ago was a monopolar electrocautery curette, the use of which instead declined over the years against the simple curette adenoidectomy. One other instrument with a decreasing usage over the years is the adenotome. On the other hand, three adenoidectomy instruments faced an increasing success over the last 15 years. Firstly, a monopolar electrocautery's usage passed from a 7.1\%, 15 years ago to a current $25.9 \%$. This upgrade is, most likely, due to the increased speed of the procedure, the better control of the blood loss and the lower cost of the instrument. Secondly, although the use of a simple debrider showed a modest rise in popularity, instead its model enriched with a monopolar electrocautery went from a $0 \%$ to a current $19.8 \%$ in the surgeon's preferences during the last 15 years. According to the present study, when combining the data from debrider alone and debrider with monopolar electrocautery, the instrument is currently used more commonly than curette with monopolar electrocautery touch-up and is second only to monopolar electrocautery in total usage for adenoidectomy. A major advantage of the microdebrider is the possibility to use the angled blades to within the nasopharynx, the anatomy of which is problematic for some instruments, such as the curette or the adenotome. In addition, debrider's usage showed a low incidence of complications. One study evidenced no long-term complications, including significant blood loss, in over 1000 procedures carried out with the powerassisted instrument (Rodriguez et al., 2002). Finally, coblation adenoidectomy gained significant popularity over the years. When combining the current data from pure coblation adenoidectomy and adenoidectomy with monopolar electrocautery coblation procedures, the instrument is used by the $8.6 \%$ of the surgeons in over $50 \%$ of their cases (Walner et al, 2007). We must recognize that the cited survey was conducted in a restricted national cohort (U.S.A.), and this may not reflect the international trend on the surgical equipment for the adenoidectomy.

In the '90s, the advent of ESS popularized the use of intranasal scopes and the endoscopic adenoidectomy became the natural evolution of the conventional adenoidectomy, permitting a direct visualization throughout the procedure (Becker et al., 1992; Cannon et al., 1999). By using this technique the adenoid remnants along the superior portion of the nasopharynx, the choanae and the peritubal region, can be clearly visualized and, thus, removed; moreover, the likelihood of damage of the Eustachian tube and/or of the pharyngeal muscles is reduced, and the haemorrhage may be effectively controlled by a direct identification of the bleeding point. In addition to the endoscopic-assisted adenoid curettage, residual adenoid tissue can be removed piece by piece using, transnasally, either a straight or a $45^{\circ}$ Blakesley forceps, always under an endoscopic view (Huang et al., 1998). In the following years, a power-assisted adenoidectomy conducted completely through a transnasal approach and under an endoscopic guidance was suggested by Parson in 1996 (Parson, 1996) and firstly reported by Yanagisawa in 1997 (Yanagisawa, 1997).

As reported in the literature, the usual approaches to the adenoid tissue with power-assisted instruments are: pure transnasal (Al-Mazrou et al., 2009; Havas \& Lowinger, 2002; Pagella et al., 2009), transorally inserted curved debrider under a transoral control (with laryngeal mirrors or with $45^{\circ}$ or $70^{\circ}$ scopes) (Koltai et al., 2002; Rodriguez et al., 2002; Stanislaw et al., 2000) or transorally inserted curved microdebrider combined with a transnasal endoscopic control (Pagella et al., 2010). 
In this chapter we expose the evolution of the adenoidectomy during the last decades, from the traditional procedures to the modern endoscopic-assisted methods, with a description of each surgical technique.

\section{Preoperative evaluation}

All children underwent a preoperative flexible fiberoptic nasal endoscopy; we used a paediatric flexible endoscope, diameter $3.6 \mathrm{~mm}$. Before the exam, nasal secretions were removed either by nasal-blowing or by a gentle aspiration through a small flexible rubber tube. No topical intranasal decongestant was used to avoid a misdiagnosis of an inferior turbinate hypertrophy or generalized nasal mucosa congestion; moreover, the National guidelines proposed by the Italian Drugs Agency, permit the use of intranasal vasoconstrictor drugs in children over 12 years of age. No local or general anesthesia was used. We preferred the presence of the parents in the examination room, for a better compliance of the patient. Most children fully collaborated during the nasal endoscopy; if necessary, the head of the patient was gently immobilized by the assistant/nurse during the performance. The entire procedure could be followed on the video screen and taped, which facilitated review and discussion of the disease along with the parents.

The procedure enables the visualization of the entire nasal fossae and, in particular, keyareas as the inferior and middle turbinates, the septum, the Osteo-Meatal Complex (OMC), the fontanella area, the Spheno-Ethmoidal Recess (SER) and the nasopharynx. The child is laid supine on an examination bed with his head bent by about $30^{\circ}-45^{\circ}$. The endoscopic evaluation can be divided into three main phases. At first, the endoscope is introduced along the nasal floor (between the inferior turbinate and the septum) so as to evaluate the inferior turbinate volume, the inferior meatus and the septum's morphology; then, the scope proceeds towards the nasopharynx to evaluate the presence of lymphatic tissue (adenoids), oedema, pathologic drainages and the condition of the Eustachian orifices. As a second step, the scope is gently retracted thus, permitting the investigation of the SER and the lateral nasal wall along with the fontanella area and the OMC. In this area, evidence of pathological drainage is crucial. Purulent secretions at the level of the OMC and of the posterior fontanella zone are an endoscopic sign of an anterior compartment rhinosinusitis (maxillary, anterior ethmoid and frontal sinuses), whereas secretions within the SER mean rhinosinusitis of the posterior compartment (posterior ethmoid and sphenoid sinuses). As a final step, if requested, an endoscopic visualization of the oropharynx and of the hypopharynx/larynx can be obtained by passing through the nasopharynx. During this step particular importance should be given to the posterior extension of the palatine tonsils within the oropharynx, as long as both the laryngeal morphology and motility. The entire procedure lasts about $30 \mathrm{sec}-1 \mathrm{~min}$.

The degree of obstruction by the adenoid tissue over the posterior choanae was estimated using the grading system proposed by Parikh et al. (Figure 6): grade 1 for adenoid tissue not in contact with adjacent structures; grade 2 for adenoid tissue in contact with torus tubarius, grade 3 for adenoid tissue in contact with vomer, and grade 4 for adenoid tissue in contact with soft palate (at rest) (Parikh et al., 2006). Other diseases usually noted during the examination, include inferior turbinate hypertrophy, septal deviation, choanal stenosis or atresia, mucosal infections and polypoid formations.

A clinical examination of the ear and the oropharynx is, usually, obtained in all children. Further exams as pure-tone audiometry (in children over 4 years-old) and timpanometry, are suggested in cases of referred hearing impairment or middle otitis. 


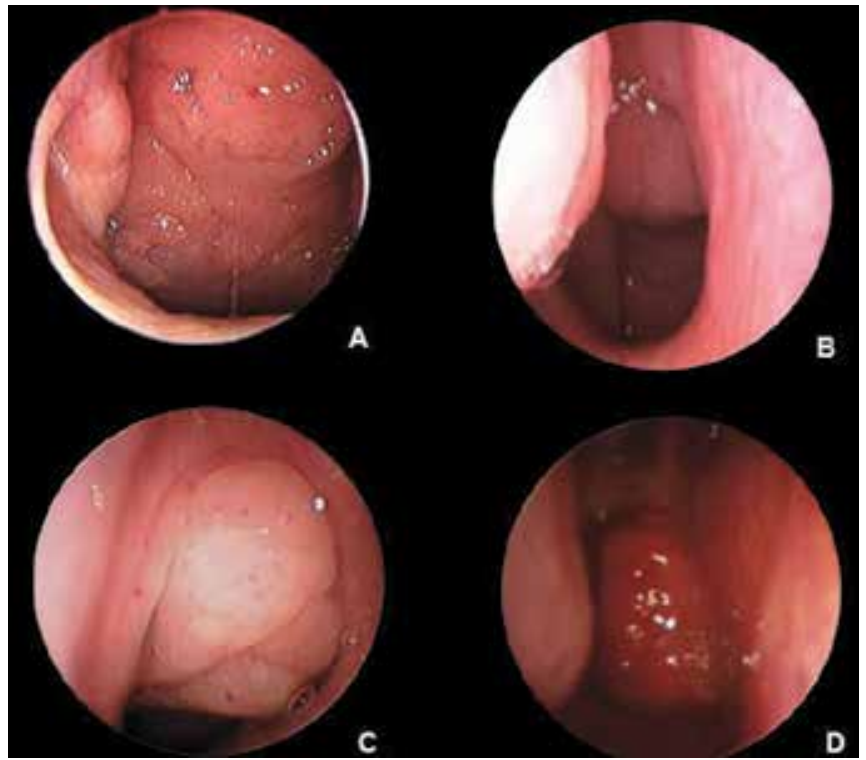

Fig. 6. Adenoid hypertrophy grading system proposed by Parikh et al. (Parikh et al., 2006): grade $1(\mathrm{~A})$, grade $2(\mathrm{~B})$, grade $3(\mathrm{C})$ and grade $4(\mathrm{D})$.

\section{Evolution of surgical techniques}

\subsection{Adenoidectomy in the pre - endoscopic era 3.1.1 Traditional adenoidectomy}

This is the "standard" surgical procedure of an adenoidectomy. As an initial step, the hard palate is inspected and palpated for the detection of an eventual submucous cleft (bifid uvula, zona pellucida, notching of the posterior hard palate). The palate's length should also be inspected. In the present procedure, the whole procedure is conducted without visualization of the nasopharynx. A complete set of three adenotomes (Shambaugh or LaForce) - small, medium and large size - is prepared, and an adenoid curette is chosen based on the dimension of the child's oropharynx and should fit between the tori (Figure 7).

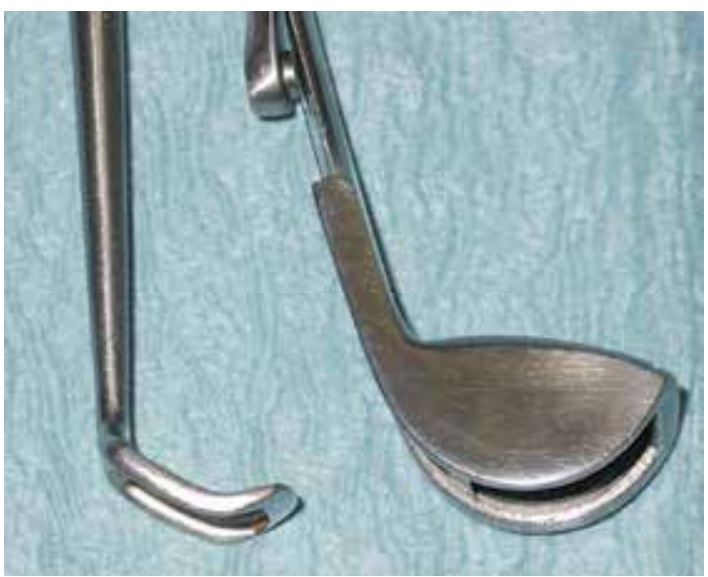

Fig. 7. An Adenoid curette (left) and a Shambaugh adenotome (right). 
The large-sized adenotome is inserted in the oral cavity by-passing the oropharynx in order to reach the nasopharynx and the adenoid pad; when the target is reached the adenotome is applied in order to remove the lymphatic nasopharyngeal tissue (Figure 8). The procedure is, then, repeated with both the medium and small sized adenotomes. After this passage, the curette is transorally inserted in the superior nasopharynx and in contact with the vomer; then is swept inferiorly with a side-to-side rocking motion to completely remove all the remaining adenoids. Care is taken to avoid injury to the deep muscular and vertebral plane, to the torus region or to the choanal area. A smaller curette can consequently be used to remove any retained tissue. After several saline solution irrigations, hemostasis is obtained by placing a tonsillar pack in the nasopharynx for some minutes. Complete removal of the adenoid tissue is confirmed by digital palpation.

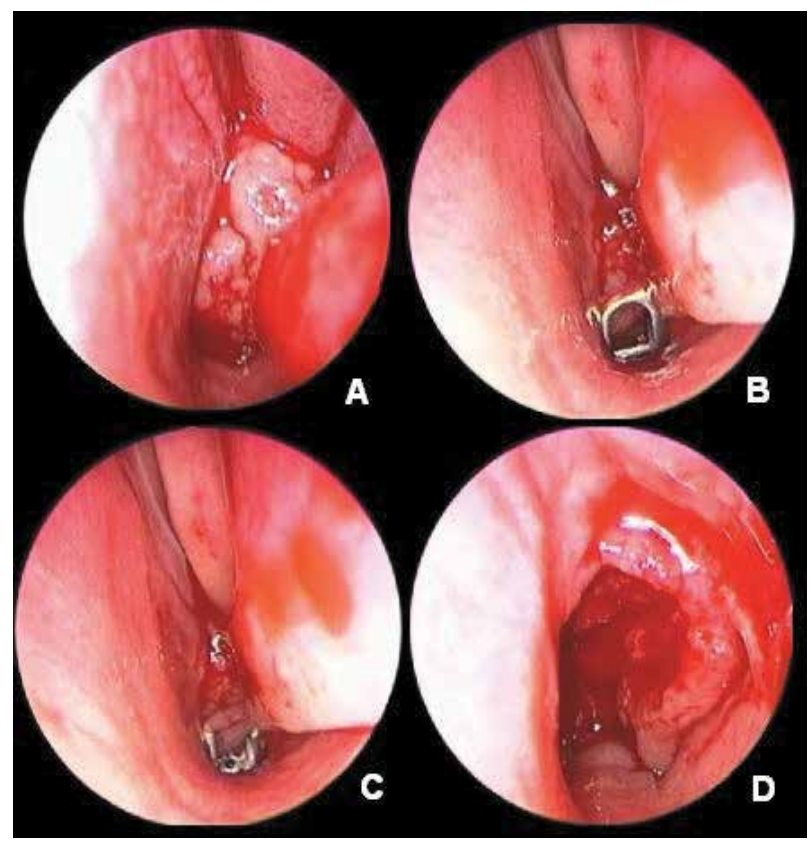

Fig. 8. Intraoperative steps of a traditional adenoidectomy with an adenotome. The procedure is showed under endoscopic view (transnasal $0^{\circ}$ endoscope) only for didactic purposes because, as mentioned, the procedure is usually performed blindly. In A the adenoid pad is visualized from the left nasal fossa in the nasopharynx. In B and C the adenotome is transorally inserted to reach the adenoid tissue, opened (B), and then closed (C) in order to remove the lymphatic tissue. In $\mathrm{D}$ the surgical field at the end of the adenoidectomy.

The main limitations of the traditional technique include: less precise removal and potentially less effective treatment, possible increased bleeding, risk of neck pain and velopharyngeal insufficiency, and lack of surgical visualization. As known, this procedure does not always remove completely the adenoid tissue (Cannon et al., 1999).

This method showed an efficacy of tissue removal only in 30\% of cases (Bross-Soriano et al., 2004). The superior and the peritubaric portions of the adenoid that obstruct the nasopharynx and the orifice of the Eustachian tube should be considered as zones of potential adenoid remnants. Moreover, excessive removal of adenoid tissue by curettage 
may provoke damage to the pharyngeal muscles, to the posterior choana, to the Eustachian tubes, or to other structures. As a result, several complications might result from an excessive traditional adenoidectomy, including velopharyngeal insufficiency and persistence of obstructive symptoms (Gelder, 1974).

\subsubsection{Laryngeal-mirror assisted adenoidectomy}

In all these surgical techniques the surgical field is controlled through a transorally placed laryngeal (or dental) mirror, in order to achieve an adequate view of the nasopharynx.

\subsubsection{Traditional adenoidectomy with transoral laryngeal-mirror control}

The already described traditional adenoidectomy may also been conducted under a transoral laryngeal mirror control; in this case the curette, under indirect visualization, is passed through the oral cavity in order to reach the adenoid tissue, and adenoidectomy is performed. Hemostasis can also be obtained with an electrocautery under the mirror control.

\subsubsection{Power assisted adenoidectomy with transoral laryngeal-mirror control (Koltai et} al., 2002; Rodriguez et al., 2002; Stanislaw et al., 2000)

This technique consists in performing the adenoidectomy with a debrider under an indirect visualization, through a laryngeal mirror. The shaver cannula is usually curved (mainly $45^{\circ}$ or $60^{\circ}$ ). The cannula is, transorally, introduced into the nasopharynx under indirect transoral mirror visualization, and the oscillating blade is then switched on. The adenoidectomy starts high in the nasopharynx, near the choanal sill. The resection is performed in a side-to-side manner, progressing on an even level until the inferior edge of the adenoid pad is reached. The depth of adenoid resection, as well as the resection around the choana and torus, is precisely controlled. The tip of the oscillating cannula is always under visual control via the laryngeal mirror. Electrocautery can be used in order to obtain an adequate hemostasis.

A complete adenoidectomy with a microdebrider was shown to be faster than, and as safe as, a traditional curettage adenoidectomy. A recent review evidenced that operative time was significantly less when applying the debrider and that blood loss, recovery time, and complications were comparable between those two techniques. Moreover, the main advantage of debrider adenoidectomy resides in its precision.

\subsubsection{Suction diathermy (Elluru et al., 2002;Walker, 2001)}

This technique is usually performed with an indirect visualization of the nasopharynx through a laryngeal mirror (but it can also be conducted under endoscopic control), as already seen. Diathermy ablation of the adenoid pad is accomplished by using an insulated, curved Frazier-type suction system or, more commonly, a disposable, malleable 10 French size, hand-switching suction coagulator (E2610-6, ValleyLab, Boulder, CO). When the Frazier-type system is used, a monopolar diathermy is applied. Typically, a setting of 30 to $45 \mathrm{~W}$ (depending on the patient's weight) is used to ablate the adenoid tissue.

The suction electrocautery device is applied to the adenoid pad, beginning at the most superior part of it (within the central bulk of the adenoid tissue, and not superficially). As the pad is cauterized, it shrinks and the suction device helps in evacuating the smoke. Thus, the obstructive adenoid tissue is ablated with care taken not to traumatize the soft palate, the opening of the Eustachian tube, or other adjacent pharyngeal structures. Surgery is completed when the choanae are clearly visible and the nasopharynx presents a smooth 
level contour. There should be no heat damages on the vomer, nasal turbinates, soft palate, or lateral nasopharyngeal walls. In the literature, this method is defined as precise, easy to perform, fast, bloodless and relatively inexpensive.

In a recent prospective study published by Jonas et al., a comparation between suctiondiathermy ablation and curettage adenoidectomy was performed. The majority of patients experienced symptom improvement after 6 months, regardless of the method used. Both techniques were very effective in controlling the symptoms of the adenoidal hypertrophy. As referred, suction diathermy technique was superior in reducing adenoidal regrowth at 6 months. Although this result was statistically significant, it is uncertain whether it is of a true clinical significance, as the symptom improvement for both groups were in excess of 95\% (Jonas et al., 2007).

\subsection{Adenoidectomy in the endoscopic era 3.2.1 The pure endoscopic approaches}

\subsubsection{Nasal endoscopic-guided curettage transoral adenoidectomy (EGA) (Wan et al.,} 2005)

The nasal cavities and nasopharynx are examined through a $0^{\circ}$ nasal scope. A throat pack is then inserted to prevent blood from entering the trachea. A Boyle- Davis mouth gag is used to open the mouth widely as during the classic adenoidectomy. A suitably sized Beckmann adenoid curette is transorally placed into the nasopharynx. Under nasal endoscopic guidance, the blade of the adenoid curette is placed just above the superior border of the adenoid. The lateral ends of the blade should just be away from the Eustachian tube area on both sides. The nasal endoscope is then taken out from the nose and the curette is used as in conventional curettage.

Transoral packing gauze is used for some minutes to control bleeding, which usually stops spontaneously without need for cauterization. Nasal endoscopy, simply, allows an assessment of the adenoid size and extension and improves the accuracy of the adenoidectomy with the curette. This method is particularly useful in younger patients with a small oral cavity; in fact, adenoid palpation, mirror examination and consequent laryngeal mirror-adenoidectomy is challenging in patients with narrow passages. These problems can be addressed by the rigid nasal endoscope, which allows accurate and safe placement of the curette at the superior border of the tissue. Thus, we obtain a complete removal of the main bulk of the adenoid without the need for nasal grasping forceps or a debrider. Moreover, teaching is much easier when combined with the real-time video presentation. Last but not least, as sophisticated instruments are not required, the cost-effectiveness of such method remains highly acceptable.

In all cases, the EGA curettage method is sufficient to remove the main bulk of the adenoids in one attempt. In contrast, the adenoid tissue is removed piece by piece during the classic curette adenoidectomy. In conclusion, the EGA allows a more complete and precise removal of the adenoid compared with the conventional method.

3.2.1.2 Transoral endoscopic adenoidectomy with adenoid curette and St. Claire Thomson forceps (El-Badrawy \& Abdel-Aziz, 2009)

Under general oro-tracheal anesthesia, a Boyle-Davis mouth gag is used. The soft palate is retracted with rubber catheters passed from the nose to the mouth. The $4 \mathrm{~mm}-70^{\circ}$ rigid 
nasal scope is introduced through the mouth, and the nasopharyngeal adenoid mass is identified. Curettage of the main adenoid mass is carried out using an adequate size adenoid curette; removal of any residual tissue is performed by a St. Claire Thomson forceps while suction is used to clear the field. At the end, a pack of gauze is inserted into the nasopharynx for some minutes.

According to the authors, the advantage of this method is the direct visualization of the operative field that would decrease the incidence of post-op adenoidal remnants, the reduced possibility of injury to the Eustachian tube with a consequent long-term fibrosis and the finer control of possible haemorrhage sites. Moreover, the procedure does not need any expensive equipment and it needs just a few minutes more than the conventional mirror-adenoidectomy method. However, the transoral endoscopic approach demands some experience in endoscopic surgery through curved scopes.

\subsubsection{Transoral adenoid ablation by suction diathermy under a $45^{\circ}$ scope vision (Lo \& Rowe- Jones, 2006)}

The procedure is undertaken under general anaesthesia. The patient is positioned and prepared as described in the latter technique. The surgeon stands on the right side of the patient's head. A $45^{\circ}$ rigid scope is introduced through the oral cavity to the oropharynx with the lens pointing towards the nasopharynx. The suction coagulator device is introduced alongside the endoscope. Using a setting of $30 \mathrm{~W}$ of monopolar coagulation combined with suction, the adenoidectomy is performed under direct endoscopic vision.

The known limitations of the traditional indirect mirror adenoidectomy are mainly associated to the poor visualization and manoeuvrability. Thus, the endoscopic transoral approach overwhelms those limitations. More specifically, by the $30^{\circ}$ endoscope and in particular in patients with small mouth opening, visualization of the nasopharynx, although better, could be limited. With a $90^{\circ}$ endoscope, although the whole nasopharynx could be effectively visualised, however a closer look into the nasopharynx would be very difficult, and the endoscope and suction coagulator would be oriented at different axis, thus limiting manoeuvrability.

By using a $45^{\circ}$ endoscope the entire nasopharynx can be easily visualised. In addition, the axis of introduction in both, scope and suction-coagulator, is the same so, bimanual coordination is easily achieved. As referred, with this technique and in patients with submucous cleft palate, adenoid tissue may be excised under direct vision preserving, however, a small pad inferiorly to avoid velopharyngeal insufficiency. In conclusion, this technique is both effective and presents a short learning curve.

\subsubsection{Transnasal power assisted adenoidectomy with transnasal endoscopic control (Al-} Mazrou et al., 2009; Havas \& Lowinger, 2002)

The shaver blade used is the XPS Xomed Power System with the lightweight magnumscaled handpiece and a 2.9-mm Tricut blade with straight-through suction irrigation (Medtronic Xomed Surgical Products, Jacksonville, Fl). The theater setup and positioning is as for a standard functional endoscopic sinus surgery. Using the $0^{\circ}, 2.7-\mathrm{mm}$ rigid telescope (4 mm scope is used in older children with larger nasal cavities), the posterior choanae and nasopharynx are assessed. Under endoscopic vision the shaver cannula is passed into the nose with the suction switched off to allow passage to the adenoids without traumatizing the turbinates or the septum. The suction is then turned on and the obstructive tissue is removed under constant endoscopic vision with care not to lacerate the torus tubarius. The 
cutting and aspirating action of the shaver removes both adenoid tissue and blood, providing a clear view. Tissue is removed at the site of the oscillating blade only, and the blade is kept under vision all the time using the telescope. Working from proximal to distal, intranasal adenoid and hypertrophic nasopharyngeal adenoid are removed until the surgeon is satisfied with the clearance. A small inferior rim of adenoid tissue can be left intact intentionally to preserve the velopharyngeal sphincter. With this technique a finer peritubal and perichoanal tissue clearance is possible, and a better control on the depth of the tissue resection is achieved. Careful tissue removal is carried out with the concomitant visual-protection of important nearby structures like Eustachian tubes, torus tubarius and the posterior pharyngeal wall. Moreover, this technique is reported to be faster and bloodless than the traditional curette adenoidectomy. A possible disadvantage, is the cost of the disposable blade of the shaver.

\subsubsection{Transoral power assisted adenoidectomy with transoral $70^{\circ}$ endoscopic control (Costantini et al., 2008)}

A general orotracheal anaesthesia is performed for the surgery. The patient is placed in a supine position with the neck slightly extended and the surgeon placed to the right of the patient. A mouth gag, the same as for tonsillectomy, is positioned; two rubber catheters are introduced through the nasal fossae to apply light upward traction to the soft palate, thereby increasing the forward-back diameter of the passage. The slight stretching of the palate achieved with this manoeuvre can also help to detect a possible soft palate cleft.

A $70^{\circ}$ endoscope with a video attachment is introduced through the mouth to visualize the nasopharynx, and consequently a $40^{\circ}$ curved blade microdebrider is introduced through the mouth (Figure 9).

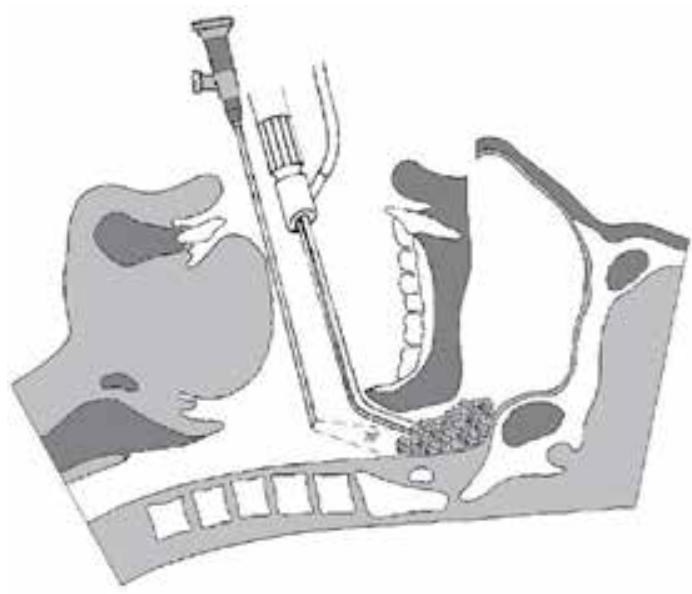

Fig. 9. Schematic representation of the transoral power assisted adenoidectomy with transoral $70^{\circ}$ endoscopic control

The instrument is connected to an aspirator, and set a rotational speed of $1200 \mathrm{rpm}$. Removal of the adenoid tissue starts from the choanal extension, and proceeds backwards along the vault towards the posterior wall of the nasopharynx. The smooth tip of the microdebrider can be introduced into the recess between the side vegetations and the tubaric ostium so that the tissue can be completely removed without damaging the mucosa covering the torus tubarius. 
The resection is interrupted at the passage from the regular adenoid vegetations to the chaotic aspect of the lymphatic tissue in this zone (Passavant ridge). From a functional point of view, the removal can be considered complete at this point. In doing so, such procedure prevents transitory velo-pharyngeal insufficiency and avoids excessive intraoperative bleeding.

At the end of the resection, a gauze packing could be placed and is maintained for some minutes. The packing is then removed and the cavity is checked for possible remnants and for the absence of bleeding. In the uneventful case of persistent bleeding, hemostasis can be established using a curved suction-coagulator, always under an endoscopic transoral control.

Several authors have described the high percentage of residual tissue remnant after traditional (adenotome or curette) adenoidectomy, especially in the choanal and the peritubaric region. A blindly performed surgical procedure is no longer satisfactory. A clear vision of the operating field is essential and this can be obtained, with excellent illumination and focus, with a transorally inserted $70^{\circ}$ endoscope. Compared to the image obtained by a laryngeal or dental mirror, the quality of the image is unquestionably better.

In some cases, introduction of the catheters for suspending the soft palate can be rather difficult (especially in the presence of an important choanal obstruction), but, according to these authors, they do offer considerable advantages. The safety and precision of the transoral curved microdebrider for adenoidectomy is well documented by the Rodriguez et al. (Rodriguez et al., 2002), Koltai et al. (Koltai et al., 2002), and Murray et al. (Murray et al., 2002). This method can be effective to remove the lateral (peritubaric) adenoidal tissue with a precision that is difficult to achieve with any other instrument, thereby minimizing the risk of damaging the surrounding structures. If a partial adenoidectomy is appropriate, it is also possible to perform very selective removal of the adenoid tissue. Moreover, the continuous suction generated by the microdebrider maintains a bloodless field.

Although the technique described may appear, at a first view, more difficult than those traditionally used, it is easier to learn, particularly for the surgeon who is, somewhat, familiar with the endoscopic nasal surgery. Moreover, it is easy to teach using the video images. The duration of the procedure is slightly longer than a standard adenoidectomy. The organization and preparation times are also longer, while ablation and haemostasis times are substantially the same. However, the slightly longer duration is more than compensated by the greater precision and confidence gained by the surgeon.

\subsubsection{The combined methods}

A recent report (Saxby et al., 2009) comments on the incidence of residual adenoid tissue after 425 consecutive traditional curette adenoidectomies. This residual tissue can lead to a combination of potential problems, including peritubaric obstruction, bacterial reservoirs and hyperplasia of the remnants with the persistence of obstructive symptoms; all these aspects highlight the importance of addressing a complete removal of the adenoid tissue. In this report, the majority $(73 \%)$ of patients who underwent to a traditional curettage adenoidectomy had some endoscopic evidence of residual adenoids, $26 \%$ of which presented also symptoms of nasal obstruction (grade 2 or 3 adenoid hypertrophy).

The incidence of residual adenoid tissue found in this study agrees with previously published results. (Havas \& Lowinger, 2002; Cannon et al., 1999). Residual tissue revealed by the endoscopic control of the nasopharynx, occurred in a significant proportion of cases and would have been missed by palpation alone; this fact highlights the advantage of 
endoscopically inspecting the nasopharynx after the curettage. However, it is still not clear whether lesser grades of residual tissue warrant further resection. In this study, and also according to our experience, all the residual lymphatic tissue should be removed. Some authors argue that grade 1 (up to one-third of choanal occlusion) is not clinically significant, but it appears interesting that this occurs in most cases. Herein, we report some of the different combined methods described in the literature.

\subsubsection{Conventional curettage adenoidectomy with transnasal endoscopic forceps' residual asportation (Huang et al., 1998)}

In this procedure, the patient is placed supine in Rose position with the head extended. The mouth is opened with a Dingmann mouth gag. Disinfection of the face and oral cavity is performed to avoid contamination. The soft palate is retracted with a Hurd tonsil retractor. After this passage, an adenoid curette is transorally applied to the nasopharynx and the main bulk of adenoid is removed. Care is required during this process in order to avoid injury to the choana and to the pharyngeal muscles, which could result in a massive bleeding.

Subsequently, a $4-\mathrm{mm}$ or $2.7-\mathrm{mm}, 0^{\circ}$ or $30^{\circ}$ endoscope is inserted transnasally till the nasopharynx. Residual adenoid tissue at the superior portion of the rhinopharynx and at the orifice of the Eustachian tube, is usually detected at this time. Under endoscopic guidance, the adenoidectomy is completed by removing the residual tissue piece by piece using either a straight or a $45^{\circ}$ Blakesley forceps. The nasopharynx and the orifice of the Eustachian tube can be inspected by a direct endoscopic visualization without damage to other structures. Adjunctively, control of the hemostasis can be performed through a direct endoscopic view.

The use of endoscopic equipment allows a piece by piece adenoid removal. However, in patients with important tissue volume, such approach requires more time than conventional surgery, which prolongs the anesthesia times and increase the peri-operative risks. In such cases, conventional surgical methods (curette and adenotome) may remove the main bulk of the adenoid mass. On the other hand, direct endoscopic visualization reduces the likelihood of damage to other structures by excessive excision and hemorrhage due to residual adenoid tissue. Direct visualization allows direct identification and treatment of the source of bleeding, thus sparing unaffected structures and is therefore highly suitable for children. If necessary, suction cautery or other hemostatic methods can be locally used under endoscopic control.

A similar method was proposed also by Cannon et al. in 1999 (Cannon et al., 1999) and called "Endoscopic-assisted adenoidectomy (EAA)"; according to this technique, at the end of a conventional adenoidectomy, both the nasal cavities and the rhinopharynx were inspected with a $4-\mathrm{mm} 0^{\circ}$ rigid telescope. Adenoid remnants in the rhinopharynx were removed under direct visualization by paediatric straight forceps or pituitary forceps.

\subsubsection{Endoscopic-assisted combined curettage adenoidectomy (Regmi et al., 2011)}

This is a simple but effective technique, as confirmed in our personal experience too. Surgery is conducted under general oro-tracheal anaesthesia, with the child placed in the Rose's position, and a mouth gag inserted to expose the oropharynx. A rubber catheter may be inserted through the mouth to obtain an adequate palatal retraction. As a first step a traditional curettage and / or adenotome adenoidectomy without a direct visualization of the nasopharynx is performed. Subsequently, the nasal cavities and nasopharynx are endoscopically examined with a rigid endoscope. If adenoid remnants are observed, those are removed under transnasal endoscopic control through the transoral adenoid curette. 
By this method, the main disadvantages of the traditional technique such as the damage to the torus tubarius or the pharyngeal muscles and the persistence of adenoid remnants could be avoided.

\subsubsection{Endoscopic suction diathermy following traditional curettage adenoidectomy} (Saxby et al., 2009)

All the adenoidectomies are performed under general anaesthesia with the patient appropriately positioned and draped. A Boyle-Davis mouth gag is used in order to hold the mouth open. Digital palpation of the palate is performed to assess adenoidal size, and to identify any eventual submucosal cleft. The first part of the surgical procedure consists in the removal of the adenoid tissue with sweeping movements of an appropriately sized adenoid curette. Haemostasis is achieved with a moist gauze swab left in place for several minutes. After removal, any eventual residual adenoid tissue is transnasally assessed using a $0^{\circ}$ scope. A Y-suction catheter is used to clear the field and allow assessment of the epipharynx. Ablation of residual tissue by electrocautery is achieved using a suction diathermy coagulator (Valleylab, Tyco Healthcare Group, Boulder, Colorado, USA) placed transorally under a transnasal endoscopic visualization. The suction diathermy is bent $90^{\circ}$, $2.5 \mathrm{~cm}$ from the distal end to get to the epipharynx through the mouth. Care should be taken to avoid a Eustachian injury by the diathermy tip. Thus, the removal of the residual adenoid tissue should proceed medially from anterior to posterior.

\subsubsection{Traditional curette and transnasal endoscopic adenoidectomy (transnasal straight microdebriders and transnasal endoscopic view) (Pagella et al., 2009)}

With the child under general anaesthesia via an orotracheal tube, a Crowe-Davis mouth gag is inserted; the patient is placed supine in the Rose position, then a conventional adenoidectomy with a Shambaugh adenotome and Shambaugh curette is performed. A catheter is passed through the nose to assure cessation of bleeding and removal of any clot, and the nasopharynx is inspected using a $0^{\circ}, 2.7 \mathrm{~mm}$ rigid fibre-optic endoscope with a video attachment.

In the presence of residual adenoid tissue still causing a significant obstruction of the nasopharynx, the patients undergo completion of adenoidectomy using a powered shaver. The shaver used is the XPS (Xomed Powered System by Medtronic, Jacksonville, FL) with a $2.9 \mathrm{~mm}$ Tricut straight blade and straight-through suction irrigation (Figure 10).

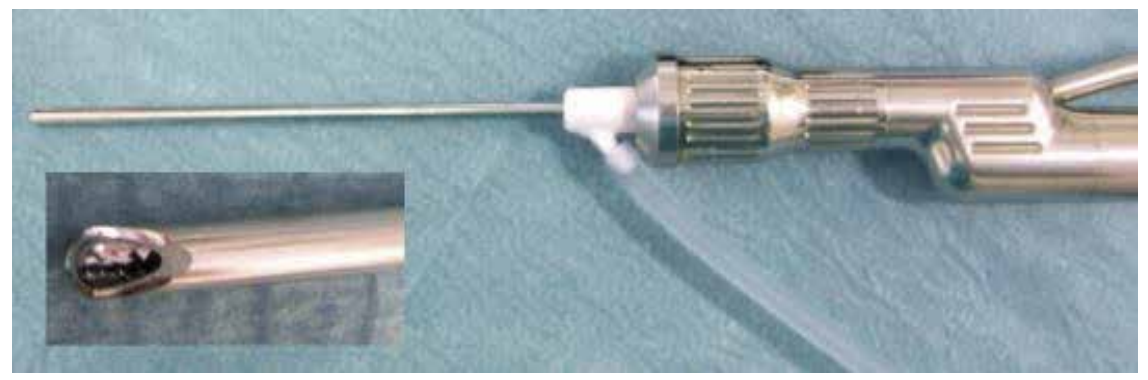

Fig. 10. The straight XPS shaver, as described above in the text.

The device should be set at $500 \mathrm{rpm}$ in the oscillating mode, with concomitant irrigation. Under constant endoscopic view the shaver cannula is passed through the nose with the suction switched off, so as to allow passage to the nasopharynx without traumatizing the 
nasal mucosa. The suction is then switched on and residual adenoid tissue is removed under endoscopic vision with care not to damage the torus tubarius (Figure 11 - 14). The cutting and aspirating action of the shaver removes both adenoid tissue and blood, providing a clear surgical field and keeping the oscillating cannula always under visual control. Once haemostasis is achieved by several saline solution irrigations, the equipment is removed and the child is then handed back to the anaesthetists for awakening and extubation.

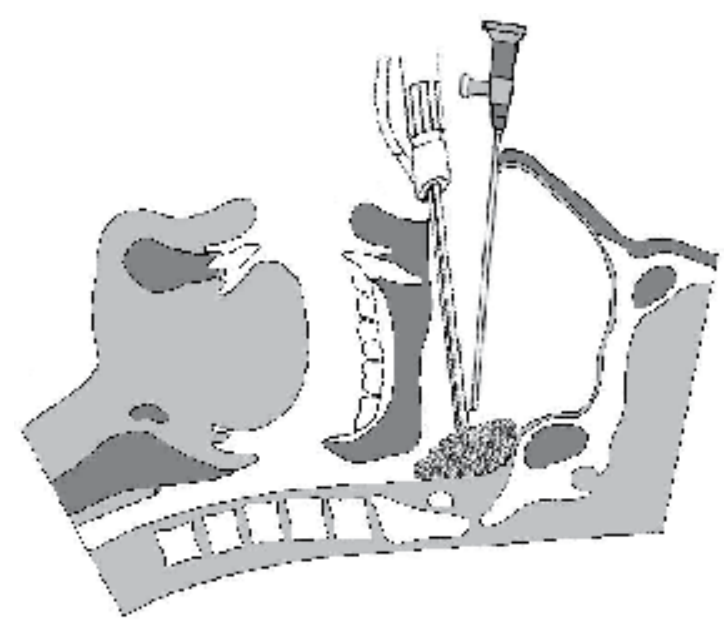

Fig. 11. Schematic representation of the traditional curette and transnasal endoscopic adenoidectomy.

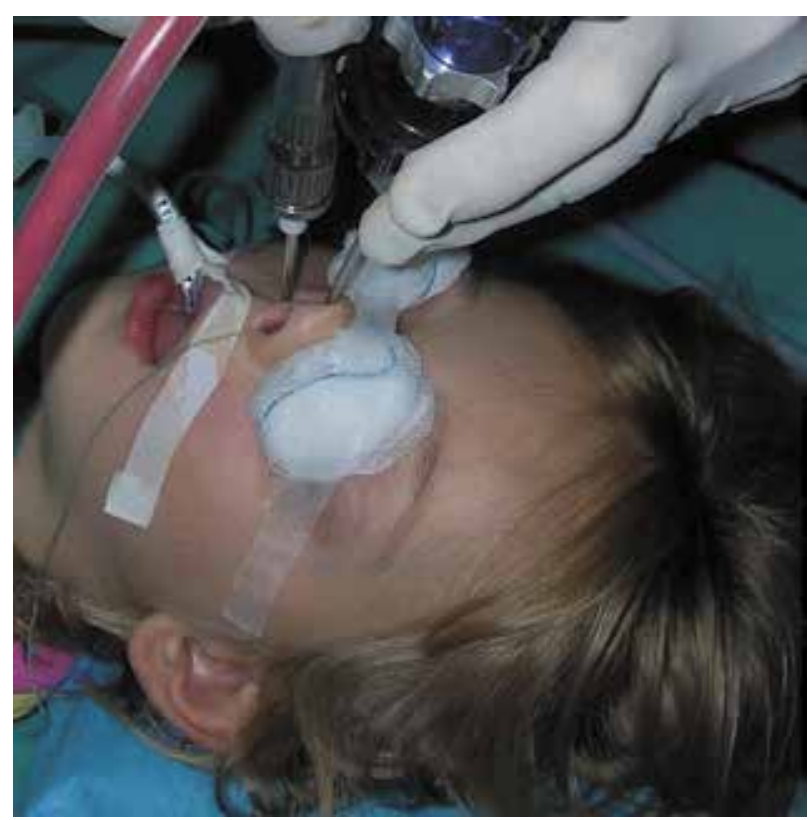

Fig. 12. External view of the microdebrider and the endoscope, both placed in the nasal cavity. 


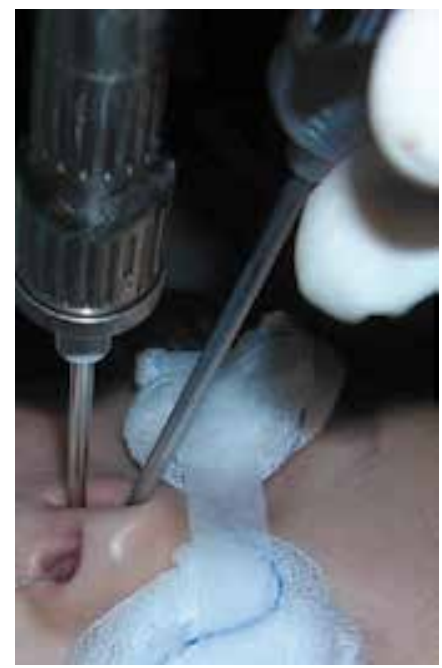

Fig. 13. Closed-detail of the relative placement of the two instruments into the right nostril.

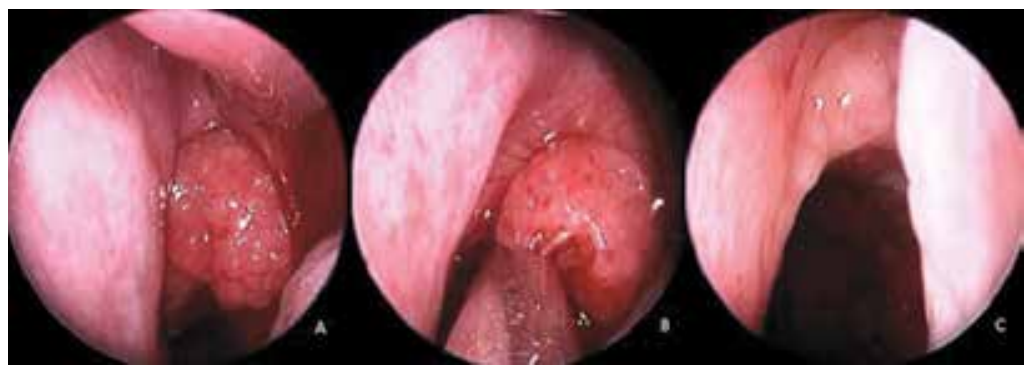

Fig. 14. Intraoperative sequential steps of the removal of adenoid remants after a traditional adenoidectomy. The scope is introduced in the left nasal fossa to permit a safe control of the entire surgical act. Lymphatic tissue still obstructing the nasopharynx (A); removal of the latter tissue with the straight shaver (B); the surgical field at the end of the procedure (C).

The value of a transnasal endoscopic view for assessing the complete adenoid removal at the end of a traditional adenoidectomy has been repeatedly underlined; this counts especially for the tissue placed superiorly in the nasopharynx and around the choanal sill but is effective as well for the control of active bleeding areas. (Buchinsky et al., 2000; Elluru et al., 2002; Havas \& Lowinger, 2002; Shin \& Hartnick, 2003; Stanislaw et al., 2000)

The use of the transnasal microdebrider assures a complete adenoidectomy and, in particular, a better control over the extent of the resection, especially around the choanal sill, the posterior nose and the torus tubarius. On the other hand, and in the presence of bulky/obstructing adenoids, a pure microdebrider adenoidectomy is time-consuming procedure; moreover, sometimes results as a difficult act because of the limited manoeuvrability of the instruments in the inferior nasopharynx. In our experience, in case of children with large adenoids, a conventional curettage and/or the usage of the adenotome removes rapidly the main tissue bulk. Then, the transnasally controlled power-assisted endonasal approach, permits an accurate residual tissue exeresis, a correct evaluation and 
effective treatment of active bleeding points, and a decreased traumatism of the region. By performing the combined adenoidectomy approach, there is an obvious increase in operative time when both curette and power-assisted techniques are used. However, in experienced hands, this increase is limited to some minutes. According to Koltai et al. (Koltai et al., 2002), initially it may appear that the power-assisted adenoidectomy is a more hemorrhagic operation than the traditional operation; this happens because the microdebrider removes small pieces of tissue with each oscillation, leaving a raw surface that bleeds during the rest of the procedure. However, when continuous suction is used, the blood is evacuated along with the excised tissue, leaving a clear and unobstructed view of the operating field. In our experience, there was no increased primary or secondary bleeding related to the use of the microdebrider. By contrast, we were certain that we had achieved a complete clearance of the rhinopharynx in every single patient.

\subsubsection{Transoral Endonasal-Controlled Combined Adenoidectomy (TECCA) (Pagella et} al., 2010)

As a first step a traditional transoral adenoidectomy is performed with a Shambaugh adenotome and Shambaugh curette. Secondarily, a $0^{\circ}, 2.7-\mathrm{mm}$, rigid fiber optic endoscope with a video attachment is introduced through the nostrils to inspect the nasopharynx and ensure a complete removal of the adenoid tissue. In the presence of residual adenoid tissue still obstructing the nasopharynx, the patient undergoes a completion of the adenoidectomy with the curved microdebrider. We use the $60^{\circ}$ curved, 4-mm Tricut blade and straightthrough suction irrigation microdebrider by XPS (Xomed Powered System by Medtronic, Jacksonville, FL). The device is set at $500 \mathrm{rpm}$ in the oscillating mode. Under endoscopic transnasal view the curved microdebrider, with the suction switched off to avoid oropharyngeal damages during the introduction, is advanced through the oral cavity and reaches the nasopharynx. The suction is then switched on and the residual adenoid tissue is removed under transnasal endoscopic vision, with care not to damage the torus tubarius or the pharyngeal muscles (Figure 15 - 17). The constant aspiration of the power-assisted instrument permits a complete removal of the adenoid tissue and a bloodless surgical area. Once haemostasis is achieved, and abundant irrigation of the field with saline solution is performed, the equipment is removed.

This new technique seems to be as safe and effective as the previous described one (transnasal microdebrider and transnasal endoscopic control). Moreover by performing the TECCA technique, some problems encountered during the latter procedure (Traditional curette and transnasal endoscopic adenoidectomy), as the contact between the scope and the debrider if both are passed transnasally, seem to be addressed; to us, the efficacy and safety of both procedures are similar. As stated before, we underline that we would rather not perform the entire procedure with the debrider so as not to significantly extend the total operative time; this is why we propose to perform the first step with standard adenoidectomy instruments. One possible limitation of this technique might be the higher price of the curved blades, as usually happens with the new equipment.

In conclusion, TECCA appears to be efficient in removing adenoid tissue that still obstructs the nasopharynx, in particular, when such remnants are situated in the superior part of the nasopharynx and/or the peritubaric region. Apparently, such procedure carries no additional risk compared to other techniques. Indeed, it permits a better maneuverability of the instruments in cases of narrow nasal spaces for a complete clearance of the nasopharyngeal area. 


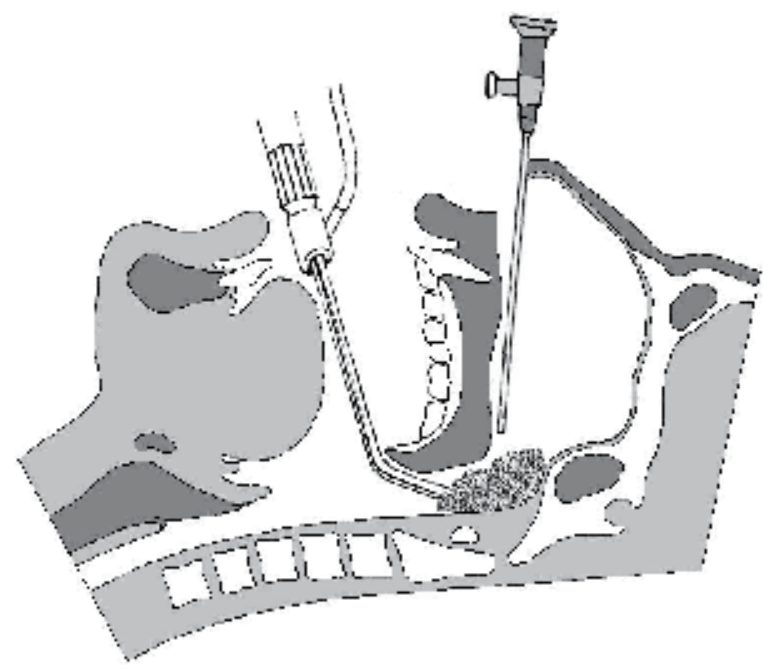

Fig. 15. Schematic representation of the second surgical step of the TECCA, as described in the text.

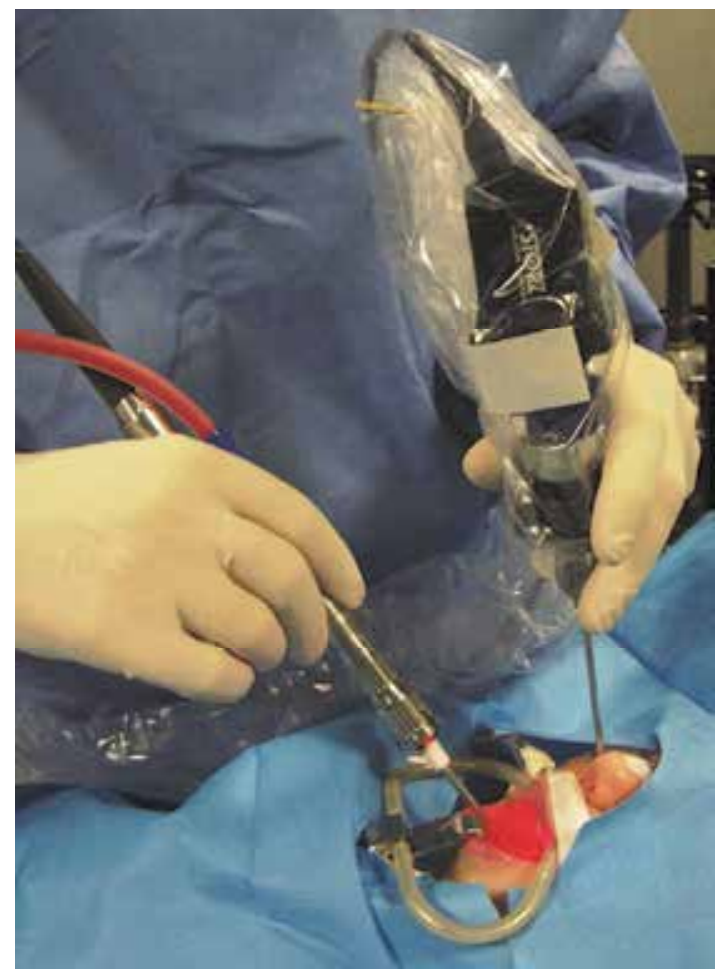

Fig. 16. External view of the second surgical step of a TECCA procedure. 


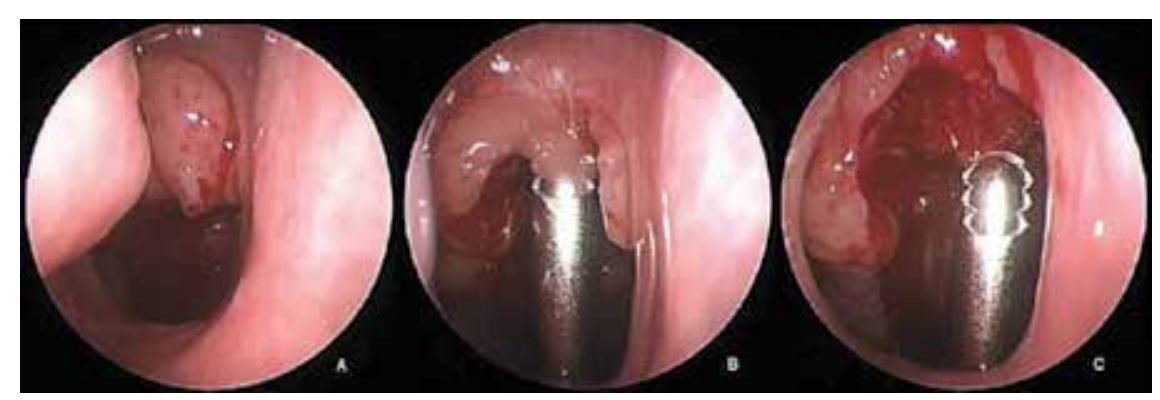

Fig. 17. Intraoperative sequential steps of residual adenoid removal by the TECCA technique. The scope is placed transnasally between the septum and the right inferior turbinate in order to control the transoral adenoidal clearance by the curved microdebrider. In $\mathrm{A}$ adenoid remnants at the end of a traditional adenoidectomy. In $\mathrm{B}$ and $\mathrm{C}$ remnant's exeresis through the transorally placed power-assisted instrument.

\section{Conclusion}

Since many years, adenoidectomy is one of the most common surgical procedures performed by Otolaryngologists in children. Historically, in the pre-endoscopic era, this procedure was performed blindly, without a direct visualization of the surgical field. Conventional "blind" adenoidectomy (adenotome or curette) may achieve the desired results in many patients; however, as many authors state, it frequently fails to obtain a complete tissue removal and, thus is less effective than direct/indirect visualization techniques. The indirect, mirror-controlled adenoidectomy was the first attempt to obtain a view of the nasopharynx during the operation. More recently, in the endoscopic era, the use of the endoscopes and other modern surgical equipment (p.e. microdebriders, suctiondiathermy) permitted the development of several endoscopic-assisted approaches; these innovative techniques offered the surgeon a clear, direct view of the rhinopharynx, enabling a complete and fine removal of the adenoid tissue, thus, avoiding an excessive and unnecessary trauma to the surrounding structures.

\section{References}

Al-Mazrou, KA; Al-Qahtani, A \& Al-Fayez AI. (2009). Effectiveness of transnasal endoscopic powered adenoidectomy in patients with choanal adenoids. International Journal of Pediatric Otorhinolaryngology, Vol.73, No.12, pp. 1650-1652.

Ark, N; Kurtaran, H; Ugur, KS; Yilmaz, T; Ozboduroglu, AA \& Mutlu, C. (2010). Comparison of adenoidectomy methods: examining with digital palpation vs. visualizing the placement of the curette. International Journal of Pediatric Otorhinolaryngology, Vol.74, No.6, pp. 649-651.

Baker, S. (1939). Fighting for life, pp. 140-141. The Macmillan Company. New York, NY.

Becker, SP; Roberts, N \& Coglianese, D. (1992). Endoscopic adenoidectomy or relief of serous otitis media. Laryngoscope, Vol.102, No.12 Pt 1, pp./1379-1384.

Brodsky, L. (1996). Adenoidectomy, In: Atlas of Head \& Neck Surgery-Otolaryngology, Bailey, BJ; Calhoun, KH; Coffey, AR \& Neely, JG, pp. 816-817, Lippincott-Raven, Philadelphia, PA. 
Bross-Soriano, D; Schimelmitz-Idl, J \& Arrieta-Gomez, JR. (2004), Endoscopic adenoidectomy: use or abuse of technology? Cirugia y Cirujianos, Vol.72, No.1, pp. 15-19, 21-22.

Buchinsky, FJ; Lowry, MA \& Isaacson, G. (2000). Do adenoids regrow after excision? Otolaryngology-Head and Neck Surgery, Vol.123, No.5, pp. 576-581.

Cannon, CR; Replogle, WH \& Schenk, MP. (1999). Endoscopic-assisted adenoidectomy. Otolaryngology-Head and Neck Surgery, Vol.121, No.6, pp.740-744.

Costantini, F; Salamanca, F; Amaina, T \& Zibordi, F. (2008). Videoendoscopic adenoidectomy with microdebrider. ACTA Otorhinolaryngologica Italica, Vol.28, No.1, pp.26-29.

Curtin, JM. (1987). The history of tonsil and adenoid surgery. Otolaryngologic Clinics of North America, Vol.20, No.2, pp. 415-419.

Discolo, CM; Younes, AA; Koltai, PJ. (2001). Current techniques of adenoidectomy. Operative Techniques in Otolaryngology-Head and Neck Surgery, Vol.12, No.4, pp. 199-203.

El-Badrawy, A \& Abdel-Aziz, M. (2009). Transoral Endoscopic Adenoidectomy. International Journal of Otolaryngology, 949315. Epub 2009 Jul 28.

Elluru, RG; Johnson, L; Myer, CM. (2002). Electrocautery adenoidectomy compared with curettage and power-assisted methods. Laryngoscope, Vol.112, No.8 Pt. 2 Suppl.100, pp. 23-25.

Ezzat, WF. (2010). Role of endoscopic nasal examination in reduction of nasopharyngeal adenoid recurrence rates. International Journal of Pediatric Otorhinolaryngology, Vol.74, No.4, pp. 404-406.

Havas, T \& Lowinger, D. (2002). Obstructive adenoid tissue: an indication for poweredshaver adenoidectomy. Archives of Otolaryngology-Head and Neck Surgery, Vol.128, No.7, pp. 789-791.

Hays, HM. (1924). Diseases of pharynx, nasopharynx and hypopharynx, In: Pediatrics, Abt IA, (3rd Ed.), pp. 217-218, Saunders WB, Philadelphia, PA.

Heras, HA \& Koltai, PJ. (1998). Safety of powered instrumentation for adenoidectomy. International Journal of Pediatric Otorhinolaryngology, Vol.44, No.2, pp. 149-153.

Huang, HM; Chao, MC; Chen, YL \& Hsiao, HR. (1998). A combined method of conventional and endoscopic adenoidectomy. Laryngoscope, Vol.108, No.7, pp. 1104-1106.

Jonas, NE; Sayed, R \& Prescott, CA. (2007). Prospective, randomized, single-blind, controlled study to compare two methods of performing adenoidectomy. International Journal of Pediatric Otorhinolaryngology, Vol.71, No.10, pp. 1555-1562.

Joshua, B; Bahar, G; Sulkes, J; Shpitzer, T \& Raveh, E. (2006). Adenoidectomy: long-term follow-up. Otolaryngology-Head and Neck Surgery, Vol.135, No.4, pp. 576-580.

Kaiser, AD. (1932). Children's tonsils in or out? pp. 2, 3, 8-10. JB Lippincott. Philadelphia, PA.

Koltai, PJ; Kalathia, AS; Stanislaw, P \& Heras, HA. (1997). Power-assisted adenoidectomy. Archives of Otolaryngology-Head and Neck Surgery, Vol.123, No.7, pp. 685-688.

Koltai, PJ; Chan, J \&Younes, A. (2002). Power-assisted adenoidectomy: total and partial resection. Laryngoscope, Vol.112, No.8 Pt.2 Suppl.100, pp. 29-31.

Kornblut, AD. (1987). A traditional approach to surgery of the tonsil and adenoids. Otolaryngologic Clinics of North America, Vol.20, No.2, pp. 349-363.

Kwok, P \& Hawke, M. (1987). The use of suction cautery in adenoidectomy. The Journal of Otolaryngology, Vol.16, No.1, pp. 49-50. 
Lo, S \& Rowe- Jones, J. (2006). How we do it: Transoral suction diathermy adenoid ablation under direct vision using a 45 degree endoscope. Clinical Otolaryngology, Vol.31, No.5, pp. 436-455.

Meyer, W. (1870). On adenoid vegetations in the nasopharyngeal cavity: their pathology, diagnosis and tretament. Medico-chirurgical Trans (London), Vol.53, pp. 191-216.

Monroy, A; Behar, P \& Brodsky, L. (2008). Revision adenoidectomy-a retrospective study, International Journal of Pediatric Otorhinolaryngology, Vol.72, No.5, pp. 565-570.

Owens, D; Jaramillo, M \& Saunders, M. (2005). Suction diathermy adenoid ablation. The Journal of Laryngology and Otology, Vol.119, No.1, pp. 34-35.

Pagella, F; Matti, E; Colombo, A; Giourgos, G \& Mira, E. (2009). How we do it: a combined method of traditional curette and power-assisted endoscopic adenoidectomy. Acta Otolaryngologica. Vol.129, No.5, pp. 556-559.

Pagella, F; Pusateri, A; Matti, E \& Giourgos, G. (2010). Transoral Endonasal-Controlled Combined Adenoidectomy (TECCA). Laryngoscope, Vol.120, No.10, pp. 2008-2010.

Paradise, JL. (1996). Tonsillectomy and adenoidectomy, In: Pediatric Otolaryngology, Blueston, CD; Stool SE; Kenna MA, (3rd Ed.), pp. 1054-1065, WB Saunders, Philadelphia, PA.

Parikh, SR; Coronel, M; Lee, JJ \& Brown, SM. (2006). Validation of a new grading system for endoscopic examination of adenoid hypertrophy. Otolaryngology-Head and Neck Surgery, Vol.135, No.5, pp. 684-687.

Parson, SP. (1996). Rhinologic uses of powered instrumentation in children beyond sinus surgery. Otolaryngologic Clinics of North America, Vol.29, No.1, pp. 105-114.

Paul of Aegina (A.D. 625-690). The seven books of Paulus Aegineta. (1847). Translated from the Greek by Francis Adams, Sydenham Society Instituted, London.

Regmi, D; Mathur, NN \& Bhattarai, M. (2011). Rigid endoscopic evaluation of conventional curettage adenoidectomy. The Journal of Laryngology and Otology, Vol.125, No.1, pp. 53-58.

Rodriguez, K; Murray, N \& Guarisco, JL. (2002). Power-assisted partial adenoidectomy. Laryngoscope, Vol.112, No.8 Pt.2 Suppl.100, pp. 26-28.

Saxby, AJ \& Chappel, CA. (2009). Residual adenoid tissue post-curettage: role of nasopharyngoscopy in adenoidectomy. ANZ Journal of Surgery, Vol.79, No.11, pp. 809-811.

Sherman, G. (1982). "How I do it"-head and neck and plastic surgery. A targeted problem and its solution. Innovative surgical procedure for adenoidectomy. Laryngoscope, Vol.92, No. 6 Pt. 1, pp. 700-701.

Shin, JJ \& Hartnick, CJ. (2003). Pediatric endoscopic transnasal adenoid ablation. The Annals of Otology, Rhinology, and Laryngology. Vol.112, No.6, pp. 511-514.

Skilbeck, CJ; Tweedie, DJ; Lloyd-Thomas, AR \& Albert, DM. (2007). Suction diathermy for adenoidectomy: complications and risk of recurrence. International Journal of Pediatric Otorhinolaryngology. Vol.71, No.6, pp. 917-920.

Stanislaw, P; Koltai, PJ \& Feustel, PJ. (2000). Comparison of power-assisted adenoidectomy vs adenoid curette adenoidectomy. Archives of Otolaryngology-Head and Neck Surgery. Vol.126, No.7, pp. 845-849.

Thornval, A. (1969). Wilhelm Meyer and the adenoids. Archives of Otolaryngology, Vol.90, No.3, pp. 383-386. 
Tolczynski, B. (1955). The recurrence of adenoids. Canadian Medical Association Journal, Vol.72, No.9, pp. 672-673.

Van Gelder, L. (1974). Open nasal speech following adenoidectomy and tonsillectomy. Journal of Communication Disorders, Vol.7, No.3, pp. 263-267.

Walker, P. (2001). Pediatric Adenoidectomy Under Vision Using Suction-Diathermy Ablation. Laryngoscope, Vol.111, No.12, pp. 2173-2177.

Walner, DL; Parker, NP \& Miller, RP. (2007). Past and present instrument use in pediatric adenotonsillectomy. Otolaryngology-Head and Neck Surgery, Vol.137, No.1, pp. 4953.

Wan, YM; Wong, KC \& Ma, KH. (2005). Endoscopic-guided adenoidectomy using a classic adenoid curette: a simple way to improve adenoidectomy. Hong Kong Medical Journal, Vol.11, No.1, pp. 42-44.

Wiatrak, BJ \& Wooley, AL. (2005). Pharyngitis and adeno tonsillar disease, In: Otolaryngology-head and neck surgery, Cummings, CW; Flint, PW; Harker, LA, et al., pp. 4150-4165, Mosby Inc., Philadelphia, PA.

Yanagisawa, E \& Weaver, E. (1997). Endoscopic adenoidectomy with the microdebrider. Ear Nose and Throat Journal, Vol.76, No.2, pp. 72-74.

Younis, RT \& Lazar, RH. (2002). History and current practice of tonsillectomy. Laryngoscope, Vol.112, No.8 Pt.2 Suppl.100, pp. 3-5. 


\section{Part 2}

Endoscopy of the Central Nervous System 



\title{
Endoscopy in Intracranial Pathology
}

\author{
Marwan Najjar and Ali Turkmani \\ American University of Beirut \\ Lebanon
}

\section{Introduction}

The use of endoscopy in intracranial pathology may be divided into two main subsets of procedures. The first subset entails the use of the endoscope in the management and treatment of intracranial intra-ventricular pathologies such as hydrocephalus, arachnoid cysts, and intra-ventricular tumors. The second includes trans-nasal and sinus endoscopy to manage mostly pituitary tumors, and other skull base pathologies, in addition to endoscope assisted microsurgery. This chapter, thus, will be divided into two parts to shed light on these two main endoscopic techniques used in intracranial pathologies.

\section{Intracranial intra-ventricular Endoscopy}

The first part of this chapter on Endoscopy for Intracranial Pathology will focus mainly on intracranial intra-ventricular neuroendoscopy. As the brain is a solid organ, neuroendoscopy is mostly limited to intra-ventricular endoscopy in the cerebrospinal fluid filled ventricular cavities. This is particularly facilitated in the setting of dilated ventricles or hydrocephalus. Obstructive hydrocephalus is actually one of the most common indications for endoscopic management in modern neurosurgical practice. In addition to management of hydrocephalus, a variety of intra-ventricular and other deep seated intracranial lesions may be approached endoscopically.

\subsection{History}

Endoscopic management of hydrocephalus was attempted as early as 1910 when VL L'Espinasse, a urologist, used the cystoscope to cauterize the choroid plexus (Hellwig et al, 2005). Dandy later refined this method and coined the terms ventriculoscope and ventriculoscopy for the instrument and technique. Mixter then, in 1923, performed the first successful endoscopic third ventriculostomy (ETV), and proposed ETV as a treatment option in occlusive hydrocephalus. Putnam later, in 1934 and then Scarff in 1936 reported on their series of patients treated with endoscopic coagulation of the choroid plexus using the special coagulation-endoscope. The mortality and morbidity of the choroid plexus coagulation procedures was high, and with the introduction of valved shunts in 1949, endoscopic management of hydrocephalus fell out of favor. With improved optics over the past two decades, and since the use of valved shunts is fraught with several known complications, endoscopic management of hydrocephalus regained intense interest. 


\subsection{Endoscopic third ventriculostomy for hydrocephalus}

Endoscopic third ventriculostomy (ETV) is emerging now as one of the preferred treatment modalities for obstructive hydrocephalus (Schroeder \& Niendorf, 2002).The conventional ventriculostomy is simply a ventriculo-subarachnoidal shunt through a minimally invasive endoscopic approach with excellent endoscopic exposure and handling. The progress in magnetic resonance imaging (MRI) and Cine-Phase Contrast MRI in particular offers a reliable method for pre- and post-operative assessment of the patients (Fukuhara et al, 1999; Schroeder et al, 2000).

The surgical technique entails entering the foramen of Monroe after cannulating the lateral ventricle via a coronal burr hole and then after inspecting the floor of the third ventricle, perforating the floor with a blunt stylet or instrument and enlarging the perforation using a fogarthy balloon catheter or the endoscopic forceps. Inspection of the pre-pontine cistern is then performed and obstructing membranes may be similarly opened. A rigid neuroendoscope is usually placed through the burr hole performed approximately $2-3 \mathrm{~cm}$ lateral to the midline and just anterior to the coronal suture. Although flexible fiberscopes have been used for ETV, they offer a considerably inferior image quality and there is usually difficulty with their orientation, guidance, and fixation (Kamikawa et al, 2001). Before surgery, the relationship between the floor of the third ventricle and the tip of basilar artery is carefully evaluated on the sagittal MRI to reduce the risk of injury to the basilar artery (Wilcock et al, 1997).

ETV is considered a simple, fast, and safe procedure (Schroeder et al, 2002). Obstructive hydrocephalus represents the most important indication for ETV (Jones et al, 1994). Shunt independence, is the ultimate measure of the successful outcome of ETV. ETV for obstructive hydrocephalus has a failure rate of $20-40 \%$ in various series (Siomin et al, 2002). The failure rate, however, is markedly reduced, if certain selection criteria are taken into consideration, and these include obstructive hydrocephalus, age more than one year, history free of meningitis and subarachnoid hemorrhage, normal ventricular anatomy, tectal gliomas, posterior fossa tumors, and pineal region tumors (Bargalló et al, 2005; Li et al, 2005; O Brien et al, 2006; Oi et al, 2001; Pople et al, 2001; Sainte-Rose et al, 2001; Wellons et al, 2002).

Patients with obstructive hydrocephalus secondary to idiopathic aqueductal stenosis, tectal gliomas, and third or fourth ventricular tumors seem to have the best outcome after ETV (figure 1a\&b) Communicating hydrocephalus secondary to meningitis or intraventricular or subarachnoid hemorrhage is associated with a higher failure rate (Siomin et al, 2002). Age seems also to be a very important factor, where infants less than 1 year of age have a higher failure rate, and in some series, under 6 months in particular. Studies have demonstrated a gradual decrease in ventricle size over months to years postoperatively, coinciding with clinical improvement (Buxton et al, 2002). The size of the ventricles is not a good predictor to use in the evaluation of the outcome within three months of surgery (Feng et al, 2004). In the early postoperative period, a decrease in the size of the ventricles is often minimal and not visible before three to four weeks. Cinephase contrast MRI may be used to determine the patency of the stoma and may be used in follow up and has been demonstrated to correlate with patency of the stoma intraoperatively, where minor flow appears to be an early sign of closure (Fukuhara et al, 1999). 


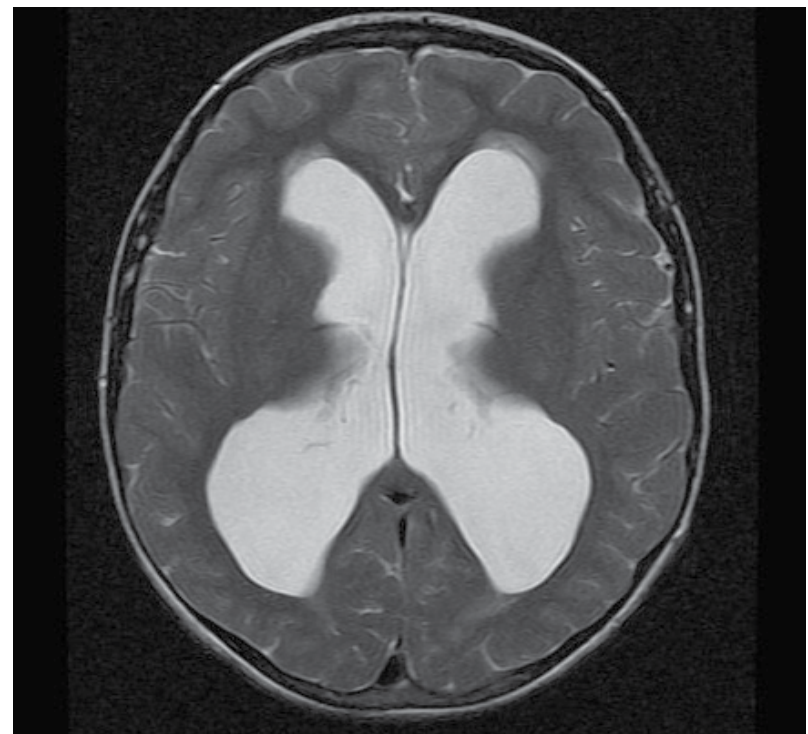

Fig. 1. a. Axial T2W MRI sequence demonstrating hydrocephalus secondary to tectal glioma. The child underwent endoscopic $3^{\text {rd }}$ ventriculostomy with good outcome.

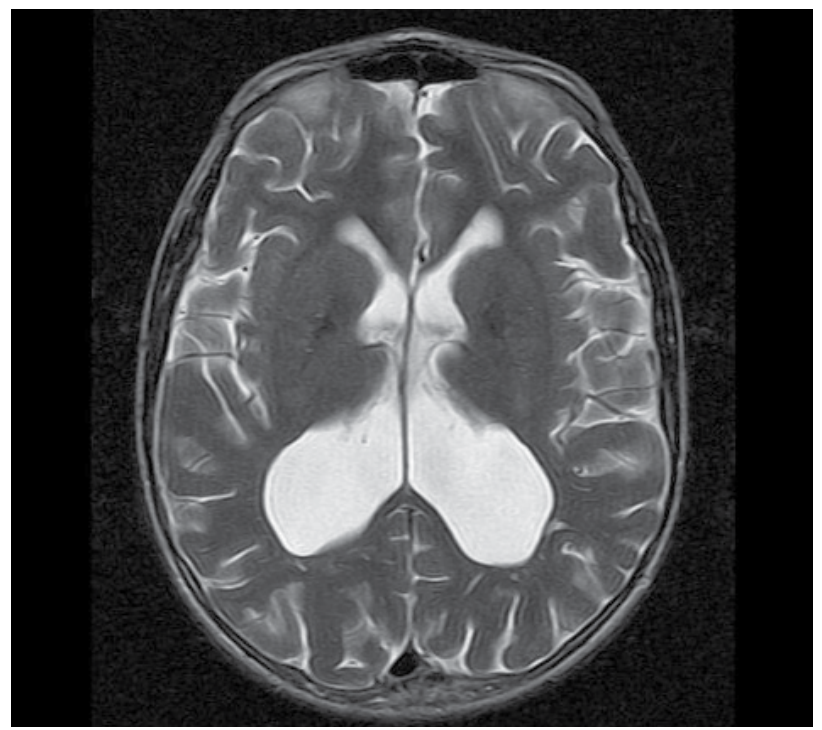

Fig. 1. b. Post operative axial T2W MRI sequence one year after surgery showing reduced ventricular size compared to the pre-operative examination.

A complication rate range of $0-20 \%$ is reported (Schroeder et al, 2002). The most serious complication of ETV is the injury to the basilar artery. The correct fenestration site is crucial for ETV success, which is recommended to be halfway between the infundibular recess and the mammillary bodies in the midline, just behind the dorsum sellae. Other complications reported are subdural hygroma (figure 2), CSF leakage, ventriculitis, diabetes incipidus, and third cranial nerve injury (Buxton et al, 2002; Fukuhara et al, 1999; Sainte-Rose et al, 2001). In 
one series of 193 ETVs done in 188 patients, permanent morbidities occurred in $1.6 \%$, and $7.8 \%$ had transient morbidities (Schroeder et al, 2002). There were two deaths (1\%). It was noted that during the course of the study, the complication rate dropped significantly, and no deaths or permanent morbidities occurred in the last 100 patients. In our unpublished series of $42 \mathrm{ETV}$ procedures done for various causes of hydrocephalus, we had no procedure related complications, other than stoma occlusion in 4 of the patients necessitating ventriculoperitoneal shunting. When done by an experienced endoscopist, and for the proper indications, we feel that ETV has a high success rate with a minimal chance of complications.

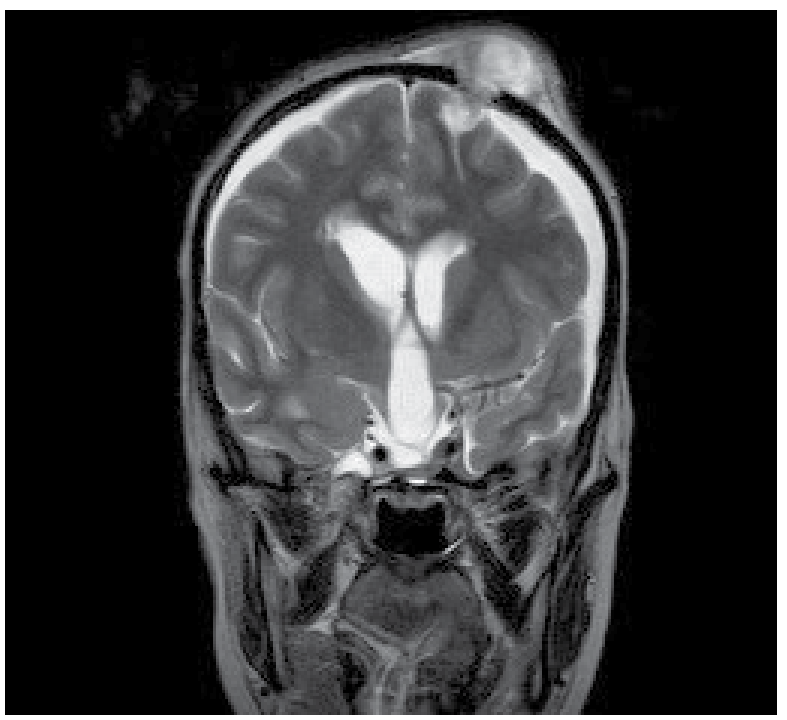

Fig. 2. Coronal T2W MRI sequence showing bilateral subdural hygromas and subcutaneous collection post endoscopic $3^{\text {rd }}$ ventriculostomy for hydrocephalus. The stoma later blocked and a shunt was placed.

Whereas endoscopic $3^{\text {rd }}$ ventriculostomy has a definite role in the management of $3^{\text {rd }}$ ventricular, pineal region, thalamic, and tectal lesions, allowing simultaneous treatment of hydrocephalus and biopsy of the lesion, the role of ETV as a routine preoperative measure to treat hydrocephalus in posterior fossa tumors is less defined. In one study where patients with hydrocephalus and posterior fossa tumors either received ETV before resective surgery or conventional management, ETV dropped the need for a shunt from $20 \%$ to $6 \%$ (SainteRose et al, 2001). Other authors confirm that ETV is an efficient procedure for controlling hydrocephalus associated with posterior fossa tumors, but find that the low rate of persistent hydrocephalus after tumor removal (9-12\%) does not justify adopting routine preoperative third ventriculostomies (Fritsch et al, 2005; Morelli et al, 2005). Despite being successful, the two ETV procedures done before microsurgical resection in our previously reported series do not provide sufficient evidence to support performing ETV as a routine preoperative procedure (Najjar et al, 2010).

\subsection{Endoscopic management of intra-ventricular lesions}

Intra-ventricular lesions are often deep seated intra-cranial pathologies that pose a diagnostic and therapeutic challenge. They are often associated with hydrocephalus, and dealt with via stereotactic or open biopsies along with a CSF diversion procedure such as 
ventriculo-peritoneal shunting. Endoscopy is quickly rising as a minimally invasive technique that is extremely helpful in the management of certain intraventricular lesions thru the ability to get pathological tissue diagnosis, CSF markers, and treat the accompanying hydrocephalus, all in the same procedure. As just mentioned, these patients often undergo at least 2 different procedures such as stereotactic biopsies, shunts, and even bilateral shunts. The endoscopic procedure may be the only procedure needed in many of these patients who harbor chemosensitive or radiosensitive tumors that do not need further removal surgery. In others, endoscopic resection of cystic or small intraventricular lesions may be the definitive surgical management.

Endoscopic tumor management was successful in up to $96 \%$ of the cases in one series where 23 out of 24 biopsies and 2 out of 2 resections were successful (Souweidane 2005). In another series of 34 patients with pineal region tumors, histological diagnosis was obtained in $94 \%$ of the cases (Pople et al, 2001). Definitive treatment was then designed for each tumor according to the diagnosis. In our reported series, 8 out of 9 endoscopic biopsies were successful (89\%), and the procedure was definitive in all the patients, where none required additional surgery (Najjar et al, 2010). Neuroendoscopic procedures, thus, have a great advantage in chemo- or radiosensitive tumors, such as germinomas, pineoblastomas, and primitive neuroectodermal tumors and are the preferential management option in most pineal region and posterior third ventricular tumors, since they guide further management whether microsurgical resection or other non-surgical therapies (Gangemi et al, 2001).

Some tumors may, on the other hand, be treated observantly. Certain thalamic gliomas that are beyond surgical resection may be observed, and others may be irradiated, depending on the grade of the tumor (Selvapandian 2006). An endoscopic biopsy, along with $3^{\text {rd }}$ ventriculostomy to treat associated hydrocephalus, may easily attain the diagnosis (figure 3). A biopsy is not even necessary in tectal gliomas, since these lesions are usually indolent and the mere treatment of hydrocephalus and endoscopic inspection of the benign mass are enough according to several authors (Li et al, 2005; Ternier et al, 2006; Wellons et al, 2002). We had 4 patients with small tectal lesions who were successfully treated with endoscopic $3^{\text {rd }}$ ventriculostomy, and had stable tumors and patent ventriculostomies through out the follow up period (Najjar et al, 2010).

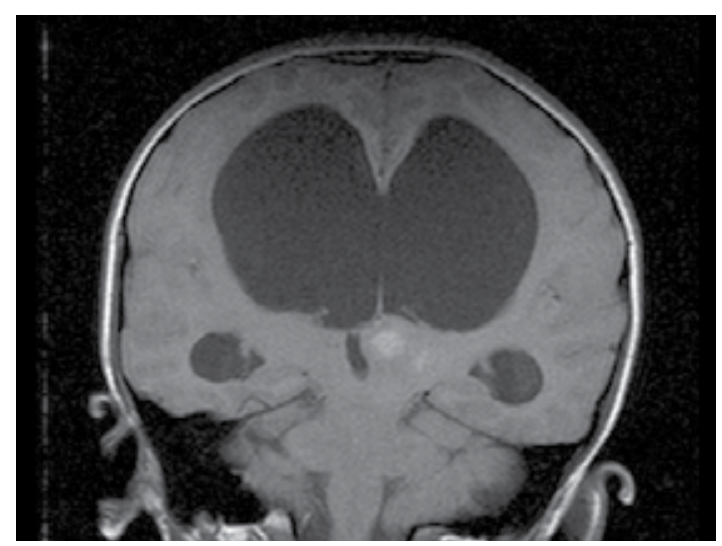

Fig. 3. Coronal T1W MRI sequence showing a small left thalamic lesion with hydrocephalus. The child underwent endoscopic $3^{\text {rd }}$ ventriculostomy and biopsy. Symptomatic hydrocephalus was thus treated and the low grade tumor proven at biopsy was stable and followed with imaging. 
Colloid cysts, simple pineal cysts, and small intraventricular tumors may be removed or debulked endoscopically. Colloid cyst endoscopic surgery is described widely in the literature and is becoming a popular surgical technique in the management of these lesions (Grondin et al, 2007; Horn et al, 2007; Schroeder \& Gaab 2002). Navigation assisted endoscopy may be needed in some of these patients, especially when there is no accompanying hydrocephalus (Souweidane 2005). Navigation could be also of great value in colloid cyst surgery, especially in selecting the entry point necessary for the optimal working angle (Schroeder \& Gaab 2002).

Intra-ventricular arachnoid cysts such as suprasellar cysts and quadrigeminal plate cysts are optimal targets for endoscopic surgical management (Greenfield 2005). The cyst is usually fenestrated at two points to communicate it with the ventricular system and the adjacent arachnoid cisterns (ventriculo-cysternal and cysto-arachnoid communication). The success rate is quite high, and the simple procedure helps avoid implanatation of a permanent cystoperitoneal shunting device in most patients. The more common temporal extraparenchymal arachnoid cysts, however, pose a more difficult endoscopic task. The anatomy is often more difficult and the fenestration site is close to critical structures. These temporal cysts may also be managed by microsurgical removal and/ or cysto-peritoneal shuting with similar results, and there is no consensus on their management (Tamburrini 2007).

Most patients with intraventricular lesions have associated symptomatic hydrocephalus. As mentioned earlier, endoscopic $3^{\text {rd }}$ ventriculostomy (ETV) has a high success rate in tumor related obstructive hydrocephalus with long term patency ranging from $70-90 \%$, and minimal complications. The ventriculostomy is usually done through the standard frontal burr hole, which may be adjusted to a more anterior position so the biopsy procedure can be done concomitantly (O'Brien 2006). Other authors advocate a 2 burr hole technique for optimal results, but we have found a single burr hole sufficient to perform the $3^{\text {rd }}$ ventriculostomy and access most lesions in the $3^{\text {rd }}$ and lateral ventricles to get a biopsy or perform a resection or debulking procedure. We also prefer to perform the ETV first, as preferred by most authorities, to avoid obscuring the operative field with blood-stained CSF after the biopsy. Smaller ventricles, presence of void signal on sagittal T2W MRI images, and presence of flow on cine-phase-contrast MR flow imaging, are helpful indicators of ventriculostomy patency, and when absent, close follow up is advised depending on the clinical condition (figure 4).

\subsection{Conclusion}

In summary, intra-ventricular neuroendoscopic techniques have rapidly become invaluable in the management of intra-ventricular brain lesions and hydrocephalus. In experienced hands, and after careful planning, biopsies can be taken safely, and adequately, outlining further management, especially in chemo and/or radio sensitive tumors. Other lesions may be resected, or debulked, and some may be observed. Cystic lesions, such as arachnoid cysts, may be resected or fenestrated, and the accompanying hydrocephalus is often treated in the same procedure, either with $3^{\text {rd }}$ ventriculostomy, or with shunting after fenestration of the septum pellucidum in patients with large $3^{\text {rd }}$ ventricular lesions. Thus, endoscopic procedures provide a minimally invasive approach to these pathologies, and might overcome complications that are usually associated with the conventional therapeutic strategies. These endoscopic procedures have simply become an indispensable part of our neurosurgical armamentarium available for the management of intra-ventricular pathologies. 


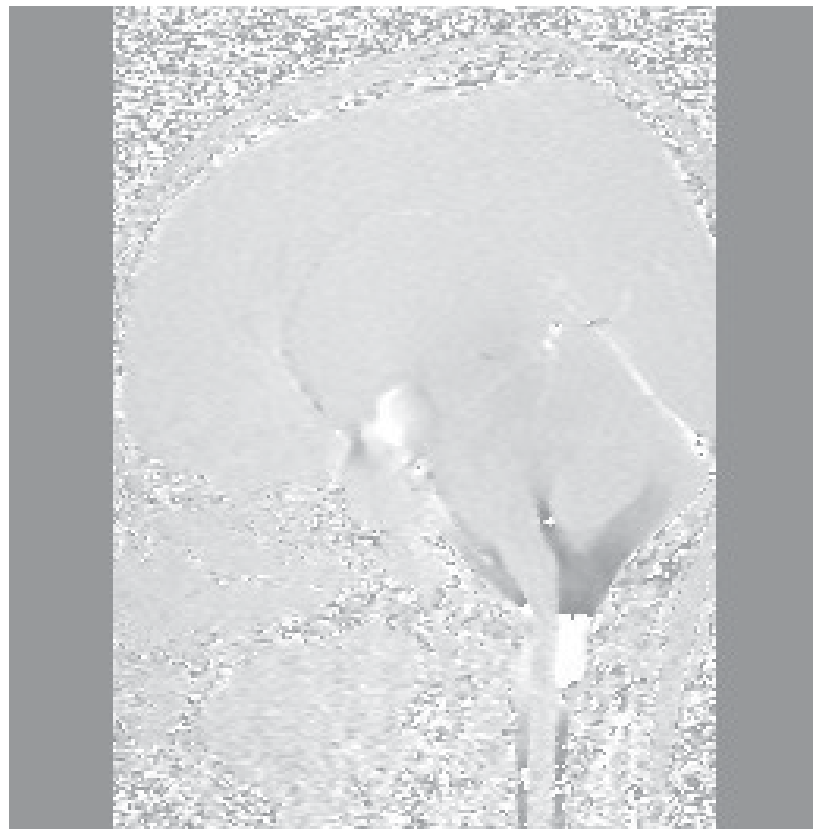

Fig. 4. Post-operative cine-phase contrast MR flow study for the child shown in figure 2 demonstrating good flow of CSF at the level of the ventriculostomy (central white signal).

\section{Endoscopic endonasal transsphenoidal \& extended skull base approaches}

The second part of this chapter deals with endoscopic skull base approaches, with special emphasis on their most common application: the endoscopic transphenoidal approach to sellar and pituitary tumors. This surgery has continuously evolved in the past century, and its history is a complex tale of development of innovative ideas coupled with periods of extensive surgical experimentation, striving to attain the best surgical results with minimal morbidity.

\subsection{Historical perspective}

The pituitary tumor resection performed by Canton and Paul, and via a temporal approach as advised by Homsley in 1893, is often sited as the first transcranial approach to pituitary tumors. Soon after, and in 1907, Schloffer attempted the first transsphenoidal surgery (Schloffer 1907). During that period, multiple variations of the inferior transnasal exposure were developed, and among the most popular were Cushing's sublabial route, and Hirsch's transnasal approach (Hirsch 1910). These approaches, however, and especially the latter, were sometimes extensive, and suffered significant technical difficulties, especially in the field of illumination. In 1956, Dott, and later Guiot, revived the interest in transsphenoidal pituitary surgery, and established its basic techniques. It was only till Hardy introduced the operating microscope in 1965, however, that the procedure gained wide acceptance, and became the work horse of pituitary surgery (Gandhi et al, 2009).

Variations of the sublabial transsphenoidal technique were then developed, with transnasal transseptal route, and other approaches aiming at a deeper mucosal incision to avoid nasal and dental complications. Despite the marked improvement in stereoscopic visualization 
afforded by microscopic surgery, deeper incisions were difficult to perform because of the narrow surgical field. In 1977, Apuzzo described the use of the endoscope as an adjunct to the microscope in tumors with extrasellar extension, to afford better visual control around the corners, and to help obtain a panoramic view in the depth of the field (Appuzo et al, 1997). This was an essential step in the development of the extended transsphenoidal approach, expanding the boundaries of the surgical field to include lesions extending to the cribriform plate anteriorly, the cavernous sinuses laterally, and the clivus and foramen magnum inferiorly.

The pure endoscopic technique was described in the 1990s, first by Jankowski in 1992, and then three years later by Jho and Carrau who are regarded as the pioneers of the pure endoscopic endonasal approach (Jho \& Carrau, 1997). In 1998, Cappabianca introduced the term functional endoscopic pituitary surgery (Cappabianca et al, 1999). The advantages of the pure endoscopic technique include the reduction of nasal complications, better visualization of the blind corners with angled telescopes, and the ability to change perspectives between close-up and panoramic views. The endoscopic technique has since spread and is being practiced in many centers around the world.

\subsection{Endoscopic endonasal approaches: Technical considerations}

The endoscopic endonasal transphenoidal approach can be divided into 3 phases: the nasal phase, the sphenoidal phase, and the sellar phase. In the nasal stage, the rigid endoscope is introduced along the floor of the nasal cavity reaching the choana which is a fundamental finding in this phase. Its medial aspect represents the vomer which confirms the midline of the approach, while its roof takes the shape of the sphenoid sinus's floor. Proceeding with the endoscope in a superior direction, the middle turbinate is encountered and dislocated laterally to ensure an adequate surgical pathway. Then, the endoscope can be angled along the spheno-ethmoid recess reaching the sphenoid ostium. This heralds the beginning of the next phase; the sphenoidal phase. After identifying the sphenoid ostium, the mucosa covering the ethmoid recess has to be cauterized to reduce possible annoying bleeding from the septal branches, exposing the whole anterior wall of the sphenoid sinus. The ostium is enlarged completing the anterior sphenoidectomy step (figure 5). Reviewing the anatomy of the sphenoid sinus septae on the pre-op CT scan helps understanding the sellar floor and the medial extent of the cavernous sinus. Preservation of the sphenoidal mucosa is important to ensure an adequate muco-ciliar transport which plays a major role in the physiology of the naso-sinusal ventilation (Kalushar 1997). Moreover, the proximity of the internal carotid artery (ICA) and the sphenoid sinus must be considered while removing the sphenoid sinus mucosa as the bone covering its anterior loop might be missing in 4-8\% (Fujii et al 1979). Now, the posterior wall of the sphenoid sinus with the sellar floor at its center is recognizable and we can fix the endoscope to its holder freeing the surgeon hands for the next step (figure 6). The sellar phase starts with opening of the sellar floor by means of microdrill or a kerrison's rongeur depending on its consistency and it may be extended as required by the specific pathology reaching the sphenoid planum above, the anterior limit of the cavernous sinus on the side or the clivus inferiorly. Then, the dura is incised and care should be applied not to reach the perisellar sinus or the ICA itself. Before removing the adenoma, the pituitary gland should be identified and preserved. Removal of macroadenomas should be performed sequentially starting laterally to avoid premature delivery of the redundant diaphragm into the operating field. After emptying the intrasellar adenoma, an angled endoscope may be advanced for a better inspection of the supra and retrosellar compartments. 


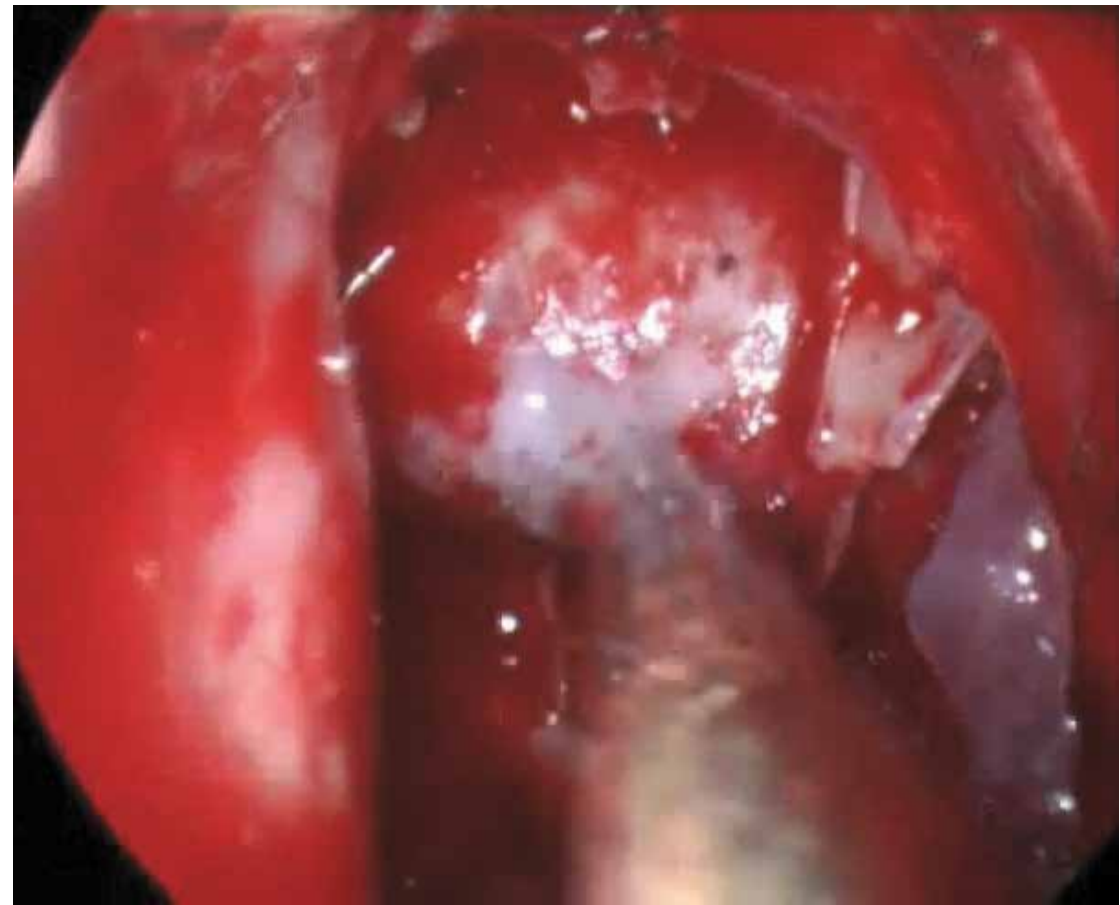

Fig. 5. Endoscopic view of the sellar floor during the sphenoidal phase

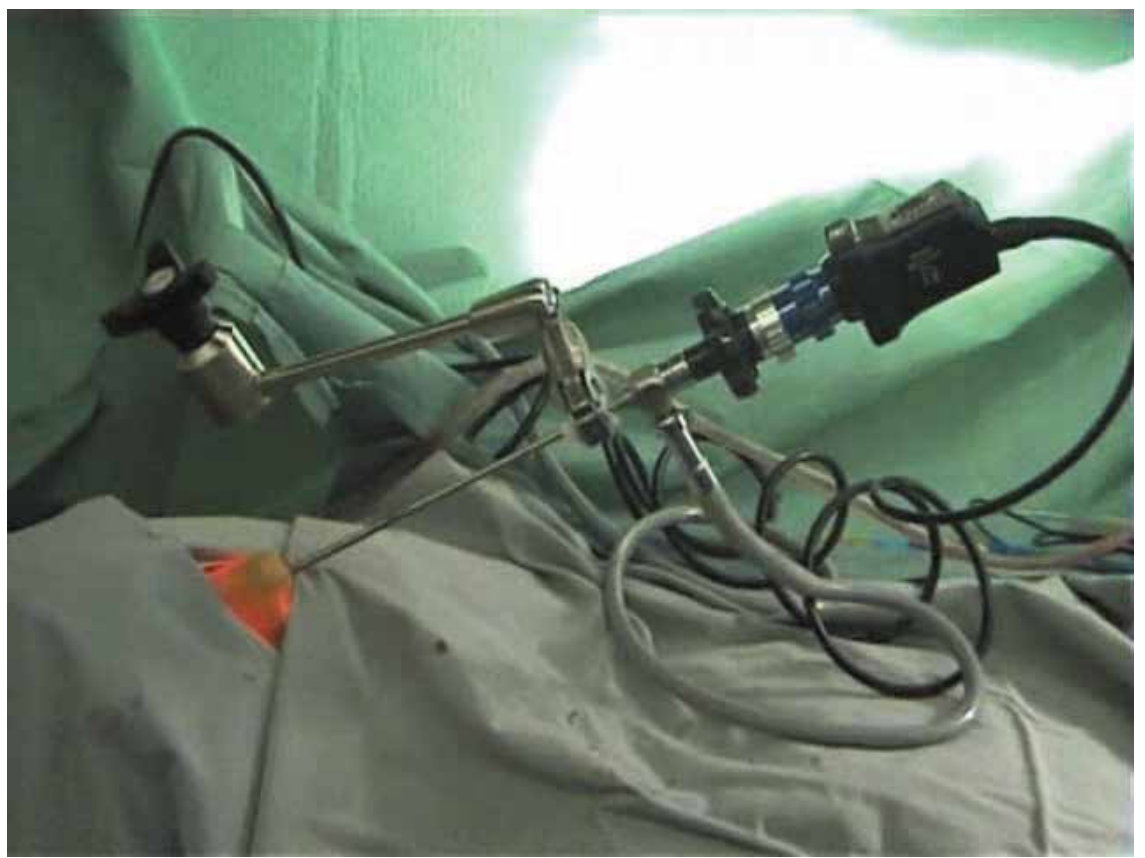

Fig. 6. Use of the endoscope holder in endoscopic transsphenoidal surgery for pituitary adenomas 
A variation of the technique described above entails using both nostrils and the dynamic process of the two surgeons working together without the endoscope holder (2 nostril, 4 hand technique). Although many centers have moved towards adopting this more dynamic technique, we feel that it entails more aggressive work in the nose, often not needed in the soft, easy to remove pituitary adenomas. This technique becomes necessary, however, for more extensive skull base pathology. With the recent revolution of skull base surgery, the endoscope is increasingly used for attacking skull base lesions from below. The same rules of endoscopic endonasal transphenoidal approach may be applied but in an extended fashion to treat a wide variety of pathologies involving the cribriform plate, the planum sphenoidale, the cavernous sinus, the sellar and parasellar compartments, the clivus, down to the foramen magnum and C2 level. The ethmoido-pterygo-sphenoidal approach allows a rapid, wide, extracerberal, and direct exposition of the skull base floor. Yet, CSF leak and the inability to cross nerves, represent major challenges that limit its widespread use. In a series of 16 craniopharyngiomas, Gardner reported high rates of preservation of endocrine function without sacrificing the extent of resection using the extended endoscopic approach (Gardner et al, 2008). A high rate of post-op CSF leak was noted (58\%) though, highlighting the importance and complexity of skull base reconstruction in these patients.

\subsection{Endoscopic endonasal surgery: Advantages}

The main advantages of the endoscopic technique may be divided into nasal and neurological (Har-El 2005). In addition to an excellent view of the bony and soft tissues in the nose, the endoscopic approach helps avoid several complications commonly seen in the transseptal microscopic approach, such as perforation, septal deformity, saddle nose deformity, nasal obstruction, and long term epistaxis and crusting. It also helps avoid dental complications seen at times after the sublabial microscopic approach including hypoesthesia or anesthesia of the incisor teeth (Har-El 2005). Recovery from an otolaryngologic aspect of the surgery is often rapid, with no swelling or oral wounds that contribute to postoperative pain and discomfort. Normal food intake is immediate, and hospital stay is shorter.

The neurological advantages are mostly related to superior visualization in the intrasellar cavity, especially when the angled telescopes are used to look for residual tumor in the blind corners and suprasellar area. When judging any new surgical technique, it must be compared with the current gold standard on the most important key indicators: oncological and endocrine outcomes, re-operation rates, and complications. In a meta-analysis on over 800 pts from studies published prior to 2006, both safety and efficacy of the endoscopic approach was demonstrated with high rates of gross total removal of tumors and normalization of endocrine function with improved vision (Tabaee et al, 2009a). In another study, an angled endoscope was used to evaluate 40 patients who underwent a microscopic resection of a pituitary tumor (Helal 1995). A large percentage $(40 \%)$ had residual tumor. The same result was reproduced by Jarrahy's assessment of the efficacy of endoscopy in pituitary adenoma resection (Jarrahy et al, 2000). Others authors, using a hybrid technique, have agreed that the endoscopic view is superior when compared to the microscopic view (Baussart et al, 2005). Dehdashti et al reported on the early surgical results in 200 patients who had the pure endoscopic endonasal approach for their pituitary adenomas and compared them with previous microsurgical series (Dehdashti et al, 2008). He found comparable rates of gross total resection at $98 \%$ for intrasellar tumors and $96 \%$ for tumors with suprasellar extention (figure 7a\&b). 


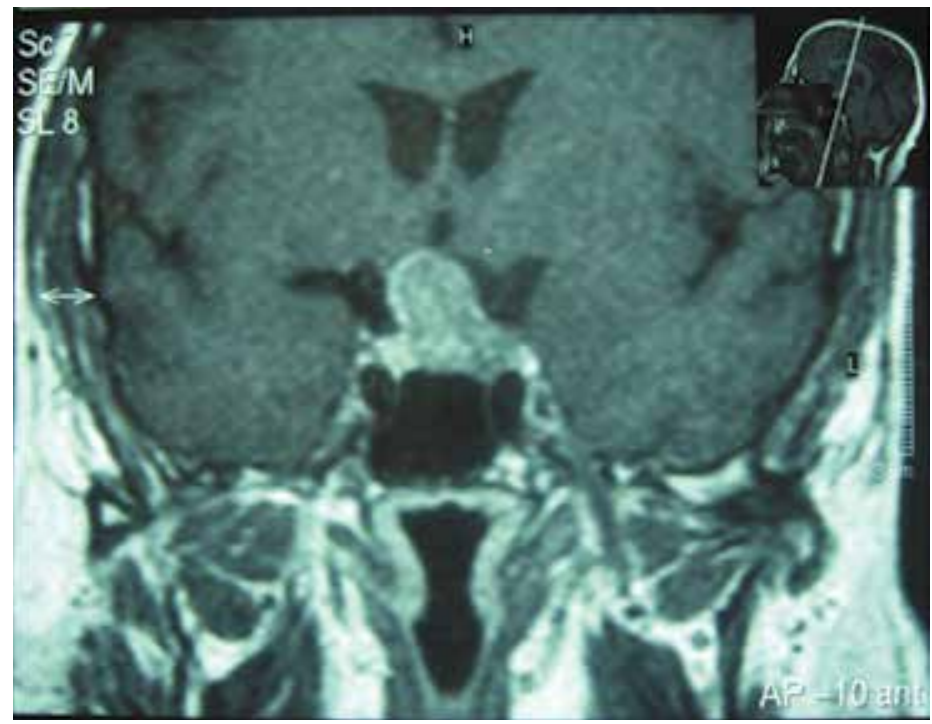

Fig. 7. a. Coronal T1W MRI sequence of the brain with gadolinium showing a large pituitary macroadenoma displacing the optic chiasm superiorly and the pituitary gland to the right side. The patient suffered visual field disturbances.

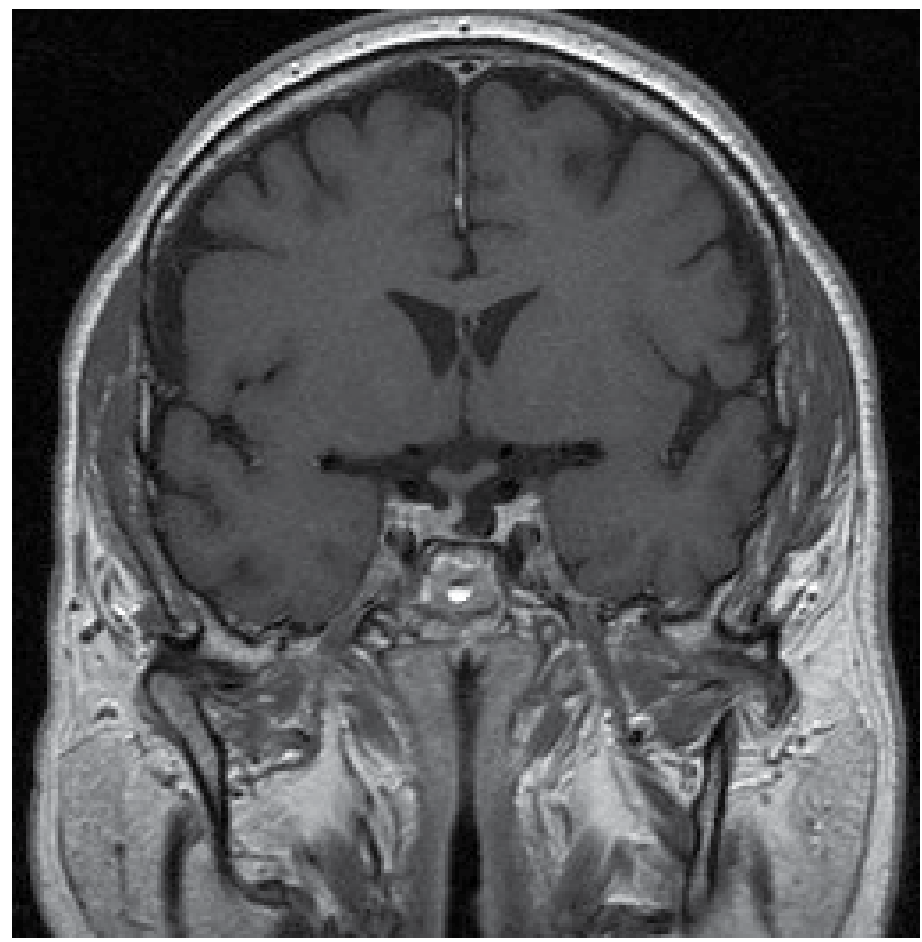

Fig. 7. b. Post-operative coronal T1W sequence of the brain with gadolinium for the same patient showing complete removal of the tumor. The patient's visual field defect resolved after surgery. 
A multitude of studies support excellent endocrinologic outcomes following endoscopic surgery for secreting pituitary adenomas. D'Haens et al assessed 120 patients with functioning adenomas in a retrospective study (D'Haens et al, 2009). The remission rate of hypersecretion was significantly better in the endoscopic group (63 vs 50\%). Kabil et al reported on 300 pts undergoing endoscopic resection with a similar cure rate of $87 \%$ in GH secreting adenomas, $86 \%$ in Cushing's disease and $80 \%$ in prolactinomas (Kabil et al, 2005). We have also noted a high cure rate in our endoscopic series of patients treated for Cushing's disease (figure 8a\&b). A 90\% biochemical cure in a purely endoscopic series of 21 patients was also reported in another series (Tabaee et al, 2009b). In patients with macroadenomas with visual field cuts, complete normalization of preoperative visual defect was noted in $50 \%$ of endoscopically approached tumors in one study, with an additional $39 \%$ having improvement (Dehdashti et al, 2008). Zhang et al achieved $70 \%$ recovery of preoperative visual defects in over 300 cases (Zhang et al, 2008). These reported rates of visual recovery in the endoscopic literature are slightly higher than those traditionally reported for microscopic approaches (Schaberg et al, 2010).

Length of stay has been shown to be decreased in multiple series (O'Malley et al, 2008). Patients were able to return home 2 days earlier on average (Higgins et al, 2008). We have had a similar experience in our patient series, where the average hospital stay was 2 or 3 days. In one review, the endoscopic group had a statistically significant reduction in their hospital stay: 3.4 vs 8.3 days (Neal et al, 2007). Similarly, a number of studies demonstrated an operative time that is significantly less on average by about 1hour (O'Malley et al, 2008).

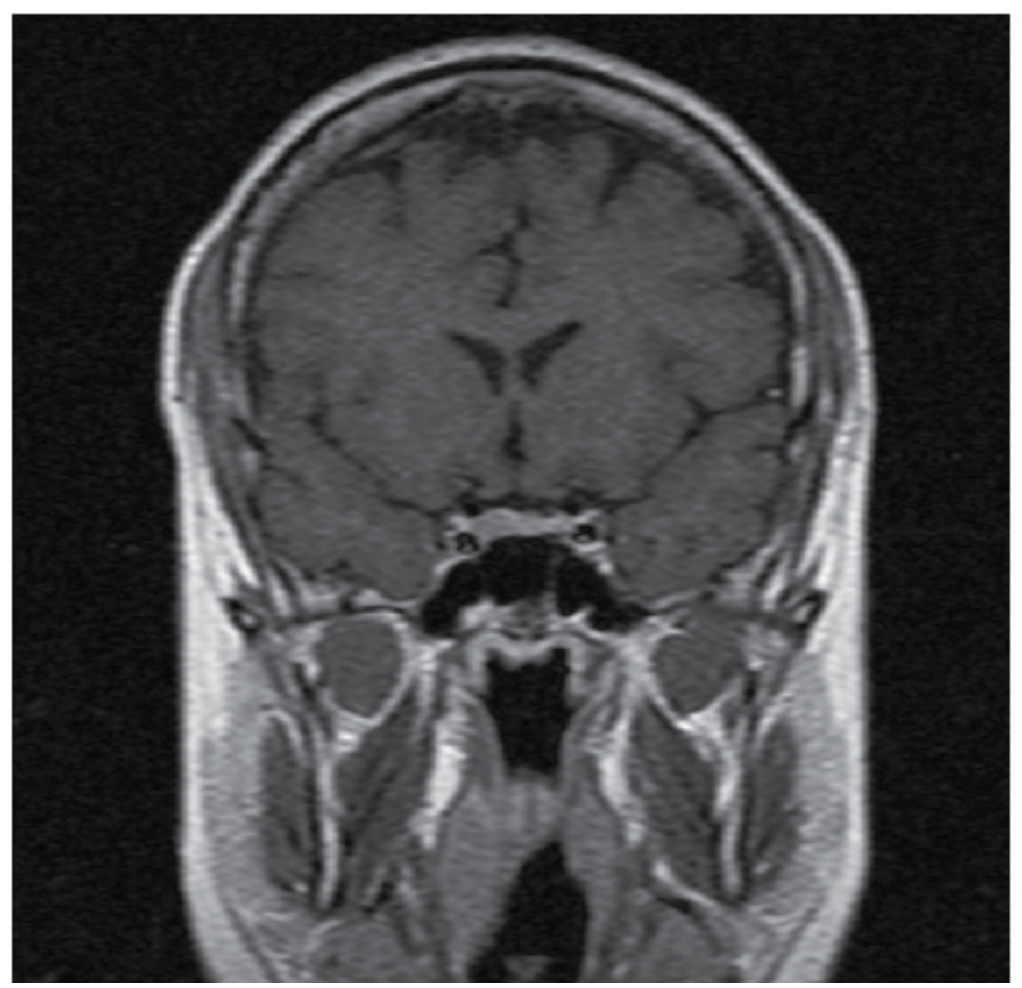

Fig. 8. a. Coronal T1W MRI sequence of the brain showing a hypointense lesion in the right sellar area indicating a pituitary microadenoma. The patient had Cushing's disease. 


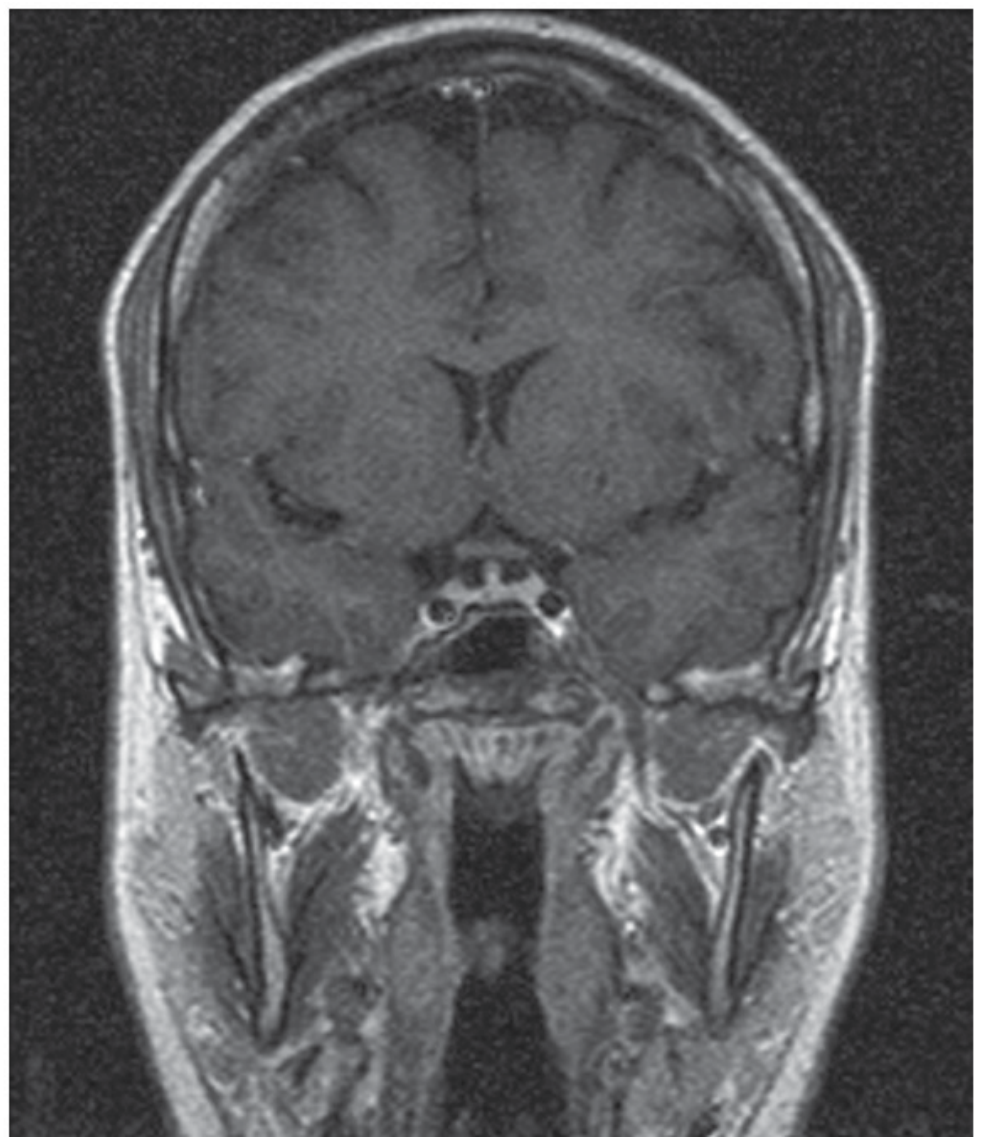

Fig. 8. b. Post-operative coronal T1W MRI sequence of the brain with gadolinium at 1 year after endoscopic removal of the adenoma indicating complete removal with no recurrence. The patient had endocrinological cure by blood testing as well.

\subsection{Complications and disadvantages}

The major complications, which are quite rare, are similar to those seen in the routine microscopic surgery and include CSF leak, vascular injuries, intracranial injury, endocrine abnormalities, meningitis, and death. The complications rates have been comparable between the two techniques. O'Malley et al reported on 50 pts, half of them undergoing microscopic approaches and half undergoing endoscopic approaches, and showed comparable complication rates between the two groups for both CSF leak and incidence of diabetes insipidis with a trend toward less diabetes incipidus (DI) in the endoscopic group (O'Malley et al, 2008) . Kabil et al demonstrated a 2\% CSF leak in a series of over 650 patients undergoing endoscopic resection (Kabil et al, 2005). Minor complications include septal perforation, trauma to external nose and sinusitis. The nasal complications are much less in the endoscopic approach when compared to microsurgical sublabial approaches as mentioned earlier.

The development of a new surgical technique often begets criticism due to the possibility of a learning curve. In modern medicine, there is a distinct advantage to a multidisciplinary team approach with otolaryngologists and neurosurgeons working together in the surgical management of these cases. Another controversy is the lack of stereoscopic vision .Yet, 
novel 3D endoscopy provides stereoscopic view and may help overcome this obstacle. The next step in the evolution of endoscopic transphenoidal surgery is linked to ongoing work utilizing intra-operative magnetic resonance imaging and robotics, and to the miniaturization of the optical systems in terms of chip-stick technology, and the cooperation between different technologies and industries (Cappabianca et al, 2008).

\subsection{Conclusion}

In summary, the endoscopic techniques used in anterior skull base surgery have caused a revolution in the management of pituitary tumors and several other lesions in the anterior skull base and clival regions. The approach is minimally invasive, and affords excellent visualization of deep structures. The endoscopic technique is moving to be the standard technique for pituitary tumors and other sellar lesions. Patients have much less nasal complaints, and stay less in the hospital. Most authorities report superior visualization of small tumor remnants in the corners, usually blind in microscopic surgery. For more extensive lesions of the anterior skull base and clivus on the other hand, the significant CSF leak rate, though reduced with recent nasal flap techniques, and significant nasal side effects seen in the more extensive extended endoscopic approaches, have stalled their widespread acceptance. The technology is continuously evolving, however, and much is to be expected in a very exciting and rapidly evolving field.

Note: All the figures listed are original figures.

\section{References}

Hellwig, D.; Grotenhuis, J.A.; Tirakotai W.; Riegel, T.; Schulte, D.M.; Bauer, B.L.; Bertalanffy, H. (2005). Endoscopic third ventriculostomy for obstructive hydrocephalus. Neurosurg Rev, Vol. 28, pp. 1-34

Schroeder, H \& Niendorf, W. (2002). Complications of endoscopic third ventriculostomy, J Neurosurg, Vol 96, No. 6, pp. 1032-1040

Fukuhara, T; Vorster, S; Ruggieri, P; Luciano, M. (1999). Third ventriculostomy patency: comparisons of findings at cine phase-contrast $\mathrm{MR}$ imaging and at direct exploration, American Journal of Neuroradiology, Vol. 20, No. 8, pp. 1560-1566

Schroeder, H; Schweim, C; schweim,K; Gaab, M. (2000). Analysis of aqueductal cerebrospinal fluid flow after endoscopic aqueductoplasty by using cine phasecontrast magnetic resonance imaging, J Neurosurg, Vol. 93, No. 2, pp. 237-244

Kamikawa, S; Inui, A; Tamaki, N; Kobayashi, N; Yamadori, T. (2001). Application of flexible neuroendoscopes to intracerebroventricular arachnoid cysts in children: use of videoscopes, Minim Invasive Neurosurg, Vol. 44, No. 4, pp. 186-189

Wilcock, D.J.; Jaspan, T; Worthington, B.S.; Punt, J. (1997). Neuro-endoscopic third ventriculostomy: evaluation with magnetic resonance imaging, Clinical Radiology, Vol. 52, No. 2, pp. 50-54

Schroeder, H.W.; Niendorf, W.R.; Gaab, M.R. (2002). Complications of endoscopic third ventriculostomy. J Neurosurg, Vol. 96, No.4 , pp.1032-1040

Jones, R.F.; Kwok, B.C.; Stening, W.A.; Vonau, M. (1994). The current status of endoscopic third ventriculostomy in the management of non-communicating hydrocephalus, Minim Invasive Neurosurg, Vol. 37, No. 1, pp. 28-36 
Siomin, V.; Cinalli, G.; Grotenhuis, A.; Golash, A.; Oi, S.; Kothbauer, K.; Weiner, H.; Roth, J.; Beni-Adani, L.; Pierre-Kahn, A.; Takahashi, Y.; Mallucci, C.; Abbott, R.; Wisoff, J.; Constantini, S. (2002). J Neurosurg, Vol. 97, No. 3, pp. 519-524

Bargalló, N.; Olondo, L.; Garcia, A.; Capurro, S.; Caral, L; Rumia, J. (2005). Functional analysis of third ventriculostomy patency by quantification of CSF stroke volume by using cine phase-contrast MR imaging, AJNR Am J Neuroradiol, Vol. 26, No. 10, pp. 2514-2521

Li, K.W.; Roonprapunt, C.; Lawson, H.C.; Abbot, I.R.; Wisoff, J.; Epstein, F.; Jallo, G.I. (2005). Endoscopic third ventriculostomy for hydrocephalus associated with tectal gliomas, Neurosurg Focus, Vol. 18, No. 6A, pp. E2

O Brien, D.F.; Hayhurst, C.; Pizer, B.; Malluci, C.L. (2006). Outcomes in patients undergoing single-trajectory endoscopic third ventriculostomy and endoscopic biopsy for midline tumors presentingwith obstructive hydrocephalus, J Neurosurg, Vol. 105, No. 3 Suppl, pp. 219-226

Oi, S.; Kamio, M.; Joki, T.; Abe, T. (2001). Neuroendoscopic anatomy and surgery in pineal region tumors: role of neuroendoscopic procedure in the "minimally-invasive preferential" management, J Neurooncol, vol. 54, No. 3, pp. 277-286

Pople, I.K.; Athanasiou, T.C.; Sandeman, D.R.; Coakham, H.B. (2001). The role of endoscopic biopsy and third venrticulostomy in the management of pineal region tumors, $\mathrm{Br} \mathrm{J}$ Neurosurg, Vol. 15, No. 4, pp. 305-311

Sainte-Rose, C.; Cinalli, G.; Roux, F.E.; Maixner, R.; Chumas, P.D.; Mansour, M.; Carpentier, A.; Bourgeois, M.; Zerah, M.; Pierre-Kahn, A.; Renier, D. (2001). Management of hydrocephalus in pediatric patients with posterior fossa tumors: the role of endoscopic third ventriculostomy, J Neurosurg, Vol, 95, No. 5, pp. 791-797

Wellons, J.C. 3rd; Tubbs, R.S.; Banks, J.T.; Grabb, B.; Blount, J.P.; Oakes, W.J.; Grabb, P.A. (2002). Long-term control of hydrocephalus via endoscopic third ventriculostomy in children with tectal plate gliomas, Neurosurgery, Vol. 51, No. 1, pp. 63-67

Buxton, N.; Turner, B.; Ramli, N.; Vloeberghs, M. (2002). Changes in third ventricular size with neuroendoscopic third ventriculostomy: a blinded study, J Neurol Neurosurg Psychiatry, Vol. 72, No. 3, pp. 385-387

Feng, H.; Huang, G.; Liao, X.; Fu, K.; Tan, H.; Pu, H. (2004). Endoscopic third ventriculostomy in the management of obstructive hydrocephalus: an outcome analysis, J Neurosurg, Vol. 100, No. 4, pp. 626-633

Fritsch, M.J.; Doerner, L.; Kienke, S.; Mehdorn, H.M. (2005). Hydrocephalus in children with posterior fossa tumors: role of endoscopic third ventriculostomy, J Neurosurg, Vol. 103, No. 1 ( Suppl), pp. 40-42

Morelli, D.; Pirotte, B.; Lubansu, A.; Detemmerman, D.; Aeby, A.; Fricx, C.; Berré, J.; David, P.; Brotchi, J. (2005). Persistent hydrocephalus after early surgical management of posterior fossa tumors in children: is routine preoperative endoscopic third ventriculostomy justified? J Neurosurg, Vol. 103, No. 3 ( Suppl), pp. 247-252

Najjar, M.W.; Azzm, N.I.; Baghdadi, T.S.; Turkmani, A.H.; Skaf, G. (2010). Endoscopy in the management of intra-ventricular lesions: preliminary experience in the Middle East, Clin Neurol Neurosurg, Vol. 112, No. 1, pp. 17-22 
Souweidane, M.M. (2005). Endoscopic management of pediatric brain tumors, Neurosurg Focus, Vol. 18, No. 6A, pp. E1

Gangemi, M.; Maiuri, F.; Colella, G.; Buonamassa, S. (2001). Endoscopic surgery for pineal region tumors, Minim Invasive Neurosurg, Vol. 44, pp. 70-73

Selvapandian, S. (2006). Endoscopic management of thalamic gliomas, Minim Invasive Neurosurg, Vol. 49, pp. 194-196

Ternier, J.; Wray, A.; Puget, S.; Bodaert, N.; Zerah, M.; Sainte-Rose, C. (2006). Tectal plate lesions in children. J Neurosurg, Vol. 104, No. 6 ( Suppl), pp. 369-376

Grondin, R.T.; Hader, W.; MacRae, M.E.; Hamilton, M.G. (2007). Endoscopic versus microsurgical resection of third ventricle colloid cysts, Can J Neurol Sci, Vol. 34, pp. 197-207

Horn, E.M.; Feiz-Erfan, I.; Bristol, R.E.; Lekovic, G.P.; Goslar, P.W.; Smith, K.A.; Nakaji, P; Spetzler, R.F. (2007). Treatment options for third ventricular colloid cysts: comparison of open versus endoscopic resection, Neurosurgery, Vol. 60, No. 4, pp. 613-618

Schroeder, H.W. \& Gaab, M.R. (2002). Endoscopic resection of colloid cysts, Neurosurgery, Vol. 51, No. 6, pp.1441-1445

Greenfield, J.P.; Souweidane, M.M. (2005). Endoscopic management of intracranial cysts, Neurosurg Focus, Vol. 19, No. 6, pp. E7

Tamburrini, G.; Dal Fabbro, M.; Di Rocco, C. (2008). Sylvian fissure arachnoid cysts: a survey on their diagnostic workout and practical management, Childs Nerv Syst, Vol. 24, No. 5, pp. 593-604

Schloffer, H. (1907). Erfolgreiche operation eines hypophysentumors auf nasalem wege, Wein Klin Wochenschr, Vol. 20, pp. 621-624

Hirsch, O. (1910). Demonstration eines nach einer neuen method operiten hypophysentumors, Verh Dtsch Ges Chir, Vol. 39, pp. 51-56

Gandhi, C.D..; Christiano, L.D..; Eloy, J.A..; Prestigiacomo, C.J..; Post, K.D. (2009). The historical evolution of transsphenoidal surgery : facilitation by technological advances, Neurosurg Focus, Vol. 27, No. 3, pp. E8

Apuzzo, M.L.J.; Heifetz, M.; Weiss, M.H.; Kurze, T. (1977). Neurosurgical endoscopy using the side-viewing telescope.technical note, J Neurosurg, Vol. 16, No. 3, pp. 398-400

Jho, H.D.; Carrau, R.L. (1997). Endoscopic endonasal transsphenoidal surgery: experience with 50 patients, J Neurosurg, Vol. 87, pp. 44-51

Cappabianca, P.; Alfieri, A.; Colao, A.; Ferone D.; Lombardi, G.; de Divitiis, E. (1999). Endoscopic endonasal transsphenoidal approach: an additional reason in support of surgery in the management of pituitary lesions, Skull Base Surgery, Vol. 9, No. 2, pp. 109-117

Kaluskar, S.K. (1997). Pre- and postoperative mucociliary clearance in functional endoscopic sinus surgery, Ear Nose Throat J, Vol. 76, No. 12, pp. 884-886

Fujii, K.; Chambers, S.M.; Rhoton, A.L.Jr. (1979). Neurovascular relationships of the sphenoid sinus. A microsurgical study, J Neurosurg, Vol. 50, No. 1, pp. 31-39

Gardner, P.A.; Kassam, A.B.; Snyderman, C.H.; Carrau, R.L.; Mintz, A.H.; Grahovac, S.; Stefko, S. (2008). Outcomes following endoscopic, expanded endonasal resection of suprasellar craniopharyngiomas: a case series, J Neurosurg, Vol. 109, No.1, pp. 6-16 
Har-El, G. (2005). Endoscopic transnasal transsphenoidal pituitary surgery-Comparison with the traditional sublabial transseptal approach, Otolaryngol Clin N Am, Vol. 38, pp. 723-735

Tabaee, A.; Anand, V.K.; Barròn, Y.; Hitzik, D.H.; Brown, S.M.; Kacker, A.; Mazumdar, M; Schwartz, T.H. (2009a).Endoscopic pituitary surgery: a systematic review and metaanalysis, J Neurosurg, Vol. 111, No. 3, pp. 545-554

Helal, M.Z. (1995). Combined micro-endoscopic trans-sphenoidal excisions of pituitary macroadenomas. Eur Arch Otorhinolaryngol, Vol. 252, No. 3, pp.186-189

Jarrahy, R.;Berci, G.;Shahinian, H.K. (2000). Assessment of the efficacy of endoscopy in pituitary adenoma resection, Arch Otolaryngol Head Neck Surg, Vol. 126, No. 12, pp. 1487-1490

Baussart ,B.; Aghakhani, N.; Portier, F.; Chanson, P.; Tadié, M.; Parker, F. (2005). Endoscopeassisted microsurgery for invasive endo-and suprasellar pituitary macroadenomas: a consecutive retrospective study with 13 patients, Neurochirurgie, Vol. 51, No.5, pp. $455-463$

Dehdashti, A.R.; Ganna, A.; Karabatsou, K.; Gentili, F. (2008). Pure endoscopic endonasal approach for pituitary adenomas: early surgical results in 200 patients and comparison with previous microsurgical series, Neurosurgery, Vol. 62, No. 5, pp. 1006-1015.

D'Haens, J.; Rompaey, K.V.; Stadnik, T.; Haentjens, P.; Poppe, K.; Velkeniers, B. (2009). Fully endoscopic transsphenoidal surgery for functioning pituitary adenomas: a retrospective comparison with traditional transsphenoidal microsurgery in the same institution, Surg Neurol, Vol. 72, No. 4, pp. 336-340.

Kabil, M.S.; Eby, J.B.; Shahinian, H.K. (2005). Fully endoscopic endonasal vs transseptal transphenoidal pituitary surgery, Minim Invasive Neurosurg, Vol. 48, No. 6, pp. 348-354

Tabaee, A.; Anand, V.K.; Barrón, Y.; Hitzik, D.H.; Brown, S.M.; Kacker, A.; Mazumdar, M; Schwartz, T.H. (2009b). Predictors of short-term outcomes following endoscopic pituitary surgery, Clin Neurol Neurosurg, Vol. 111, No. 2, pp. 119-122

Zhang, Y.; Wang, Z.; Liu, Y.; Zong, X.; Song, M.; Pei, A.; Zhao, P.; Zhang, P.; Piao, M. (2008). Endoscopic transsphenoidal treatment of pituitary adenomas, Neurol Res, Vol. 30, No. 6, pp. 581-586

Schaberg, M.R.; Anand, V.K.; Schwartz,T.H.; Cobb,W. (2010). Microscopic versus endoscopic transnasal pituitary surgery, Curr opin otolaryngol head neck surg, Vol. 18, No. 1, pp. 8-14

O'Malley, B.W. Jr.; Grady , M.S.; Gabel, B.C.; Cohen, M.A.; Heuer, G.G.; Pisapia, J.; Bohman, L.E.; Leibowitz, J.M. (2008). Comparison of endoscopic and microscopic removal of pituitary adenomas: single-surgeon experience and the learning curve, Neurosurg Focus, Vol. 25, No. 6, pp. E10

Higgins, T.S.; Courtemanche, C.; Karakla, D.; Starsnick, B.; Singh, R.V.; Koen, J.L.; Han, J.K. (2008).Analysis of transnasal endoscopic versus transseptal microscopic approach for excision of pituitary tumors, Am J Rhinol, Vol. 22, No. 6, pp. 649-652 
Neal, J.G.; Patel, S.J.; Kulbersh, J.S; Osguthorpe, J.D.; Schlosser, R.J. (2007). Comparison of techniques for transsphenoidal pituitary surgery, Am J Rhinol, Vol. 21, No. 2, pp. 203-206

Cappabianca, P.; Cavallo, L.M.; de Divitiis, O.; Solari, D.; Esposito, F.; Colao, A.(2008). Endoscopic pituitary surgery, Pituitary, Vol. 11, No. 4, pp. 385-390 


\title{
Technical and Clinical Evolution of Modern Neuroendoscopy
}

\author{
P. Grunert and J. Oertel \\ Johannes Gutenberg University \\ Germany
}

\section{Introduction}

Endoscopy plays an important part in the present minimally invasive neurosurgery. The concept, the indications and the technical development have a long history. We can distinguish an early period at the end of 19th and beginning of 20th century and an advanced period toward the end of the 20th century. Each of these periods can be further divided in a time of an obvious medical problem and first idea of possible solution, further in the next step a time of intensive technical improvements and finally the clinal application of endoscopes during surgical interventions. This historical development of endoscopy is true for all branches of surgical application. In this chapter it will described in the field of neurosurgery.

\section{History of neuroendoscopy}

\subsection{Early endoscopic period in the $19^{\text {th }}$ and beginning $20^{\text {th }}$ century 2.1.1 The problem and the first technical solution by Philipp Bozzini}

Originally the direct visual inspection of organs such as bladder, female genital organs or digestive tract through natural orifices was restricted to few centimetres with a speculum. To see deeper into the human cavities and to improve the visual quality, a better illumination was necessary.

The physician Philipp Bozzini constructed at the beginning of the 19th century an optical device which enabled to look deeper into the human cavities. Bozzini was born in Mainz Germany 1773 as a son of Italian immigrants (Doglietto et al 2005; Netzhat 2005; Shah 2002). He studied medicine in Mainz and Jena. After obtaining his decree he settled as a physician in Mainz and later in Frankfurt. He constructed a device equipped with a speculum and two chambers one for light conduction and a second for direct visual inspection. Candle light was used for the illumination of the cavity. The isolation between these two chambers was necessary to be not blinded during the inspection. Bozzini called this device "Lichtleiter" (illuminating conduction) (Fig. 1).

First experiments with this "Lichtleiter" were demonstrated in Vienna 1806/1807 in the Josephinum, a foundation of the emperor Joseph II as a college for training of surgeons for the Habsburg army. Bozzini performed there with his optical instrument experiments on cadavers in different orifices of the body. However the medical academy of the Josephinum had not recognized the diagnostic potential of his invention. They estimated the device as a mere curiosity and called the instrument laterna magica in corpore humano (magic lamp in human body). Unfortunately Bozzini died 1809 on typhus and his invention fell for long time into oblivion. 


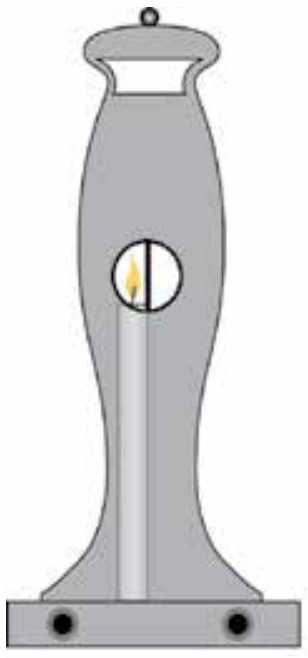

a

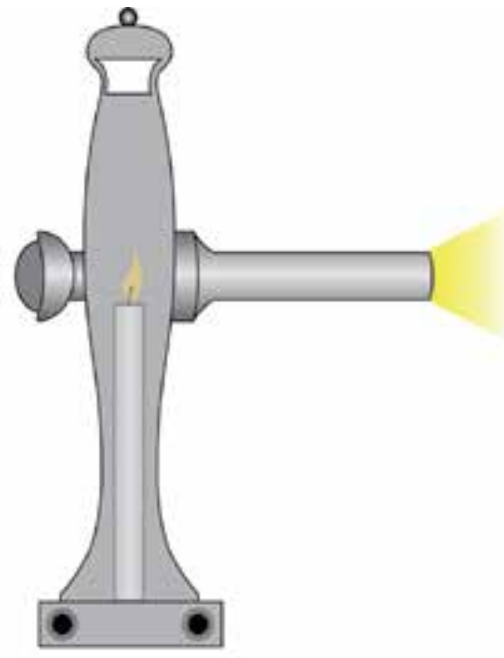

b

Fig. 1. Bozzini's "Lichtleiter". Front view(a). Notice the separated chambers for illumination and for observation. The black holes at the bottom are connected with the chamber and provide oxigen for the candle light.The opening on the top schould protect against overheating. Side view(b) with an attached speculum.

\subsubsection{Technical development of endoscopes}

50 years after the death of Bozzini his idea of illumination was again picked up. The French physician Antoine Jean Dèsormeaux constructed around 1853 a technically improved version of Bozzinis "Lichtleiter". He called this instrument an endoscope (Shah 2002). As the instrument of Bozzini the device was without optical lenses. The innovation in comparison to Bozzinis "Lichtleiter" consisted in the use of a single optical canal without separation of canals for light conductance and for observation. Additionally the candle light of the "Lichtleiter" was replaced by an external light source using gas oil, the light of which was reflected by means of a mirror from a side arm into the optic canal. 1865 he reported about the first clinical applications of this endoscope in the urethra and the bladder (Dèsormeau 1865). In the second half of the 19th century the endoscopes became increasingly important in urology and gynaecology.

Although having precursors in the second half of the 19th century such as Joseph Grünfeld in Vienna and Gustave Trouve in Paris, the development of the modern endoscopes is ascribed to Maximilian Nitze (1848-1912). Nitze organized partly in Berlin, partly in Dresden and partly in Vienna the development of each module of the endoscope the illumination, the optical system and the mechanical system. Under his surveillance the modules were set together to a functioning clinically applicable device. Therefore it is correct to speak of Nitze as the inventor of the modern endoscopy.

Nitze was born in Berlin 1848 (Doglietto et al 2005; Netzhat 2005; Verger-Kuhnke 2007). He studied medicine at different German universities and obtained his medical degree 1874. In 1875 he worked in the state hospital of Dresden. There in the department of gynaecology he saw the necessity to develop an optical device allowing better inspection of the female genital organs. The main idea of Nitze was to transfer the illumination source into the tip of 
the instrument and to use a system of lenses in imitation of a microscope. The optical problem solved by an order of Nitze Louis Charles Bénèche (Schultheiss \& Moll 2009). Bénèche was borne in Berlin as a son of reformed Hugenotts. He was specialized in construction of microscopes. Regarding endoscopes he was confronted with the problem of a long shaft in relation to a very small diameter and thus a very restricted and small view. His ingenious basic solution was to construct a lens system based on the principle of a Kepler telescope (Fig. 2b). In front of the lens system at the very tip was placed wide-angle lens to increase the viewing angle and to bundle the rays inside the shaft. The first lens of the optic system at the tip with a short focus distance f1 produced a real image inside the shaft close to the tip in an upside down position. This image was zoomed by a second lens positioned at the middle of the shaft with a focus distance $\mathrm{f} 2$ measuring half of the shaft length. By this optical construction at the proximal end of the endoscope a zoomed and virtual image in upside down position was created (Fig. 2). The mechanical realisation was difficult to be performed in Dresden and Nitze was referred to the Viennes instrument maker Joseph Leiter. For this reason Nitze quit in Dresden and moved to Vienna where he started to work in the surgical department of Leopold knight of Dittel, who was professor of surgery at the university of Vienna and highly interested in urology and endoscopy. There Nitze found the best presuppositions for his scientific work. Already 1879 he presented with Leiter the first cystoscope. Leiter published the prototype 1880 with the title "electro-

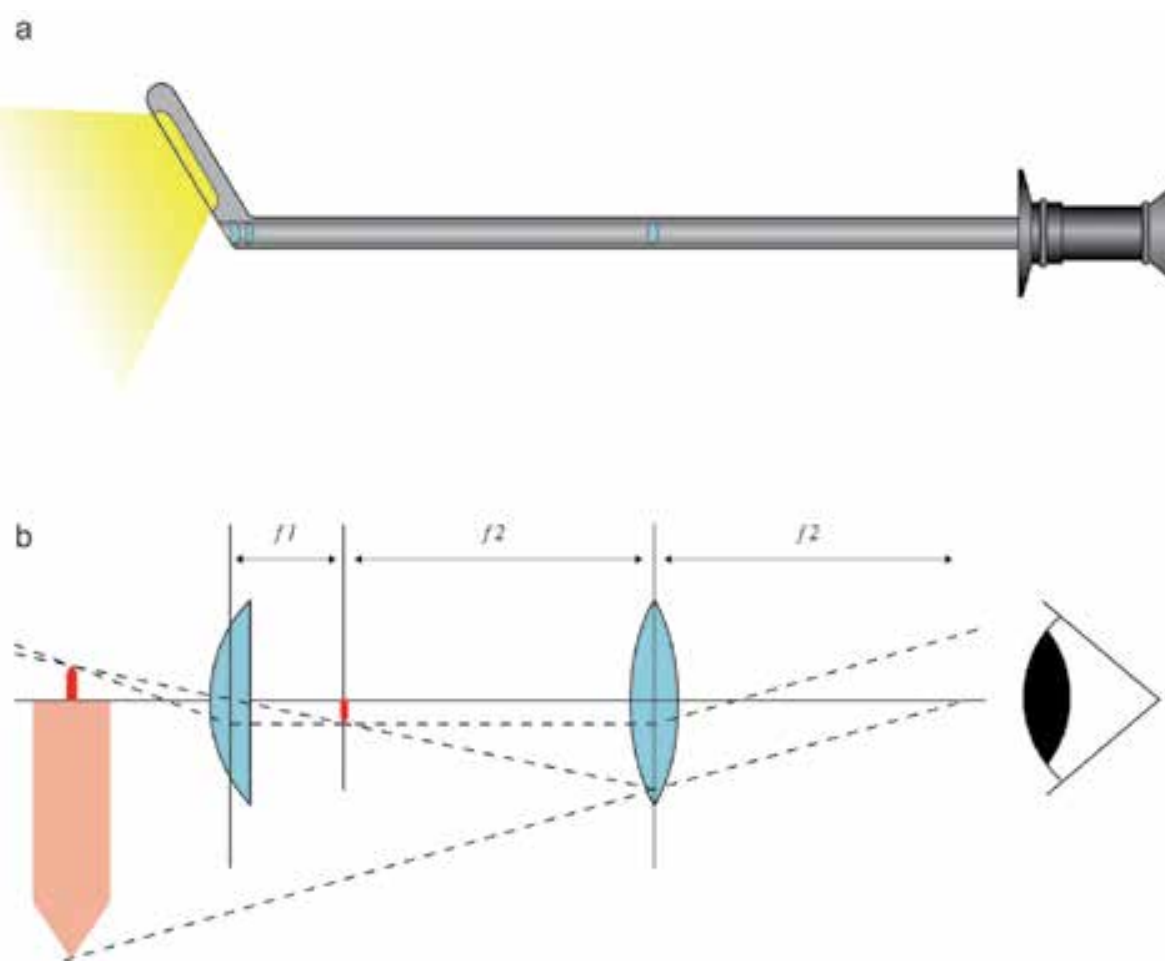

Fig. 2. Principle of Nitze endoscope. Mechanical construction of the cystoscope with lenses within the shaft and the illumination at the tip of the endoscope(a). Optic principle of Nitze endoscope constructed as a "Keppler" telescope producing an imaginary zoomed image in upside down position(b). 
endoscopic instruments description and instruction by M. Nitze and J Leiter". Using platinium wire as distal illumination source this prototype had to be equipped with an expensive cooling system to prevent burning (Leiter 1880). After invention of the incandescent electric light bulb 1878 by Thomas Alva Edison the platinium wire was replaced in the endoscope by an electric lamp, which needed no additional cooling system. This endoscope became known as "von Dittel-Leiter cystoscope". The experiences with endoscopic diagnosis and treatment of the bladder published Nitze 1889 as a text book "Lehrbuch der Kystoskopie. Technik und klinische Bedeutung"(text book of cystoscopy: Technique and clinical relevance) (Nitze 1889). The handling of the Nitze endoscopes needed experience and practice because the images were originally upside down. Only 1907 the Swiss Zeiss company developed a prism for the endoscope which reversed the picture again into the upside position.

\subsubsection{First clinical applications of endoscopy in neurosurgery}

Although the basic development of endoscopes was associated with Germany and Austria, the serial production at the beginning of $20^{\text {th }}$ century shifted to US where Reinhold Wappler (Shah 2002) a manufacturer for medical instruments who emigrated 1890 from Germany to the United States founded the American Cystoscope Makers Incorporation (ACMI). Thus at the beginning of the 20th century endoscopes mostly in form of Nitze type cystoscopes were available in the medical centres in the most American universities. Neurosurgery was in that time a new surgical field with great potential of innovation and technical improvement. Additionally at this time in the US neurosurgery much earlier than in Europe started to separate from the general surgery and was becoming an independent discipline with own development of instruments, new operative approaches and own medical training. This gave an additional push for neurosurgeons to apply new technologies in this promising surgical field. It is therefore not surprising that first endoscopic procedures in the brain started nearly simultaneously at different universities just in the United States in the 20s of the $20^{\text {th }}$ century. The first neuroendoscopic procedure was performed 1910 in the department of neurological surgery at the Northwest University, Chicago by the urologist Victor Darwin Lespinasse and the neurosurgeon Allen Buckner Kanavel, an innovative neurosurgeon who described also 1909 the infranasal transphenoidal approach to the sella turcica (Northwestern University, Dept. of History). With a cystoscope they coagualated the plexus chorioideus in two children with hydrocephalus. They had not continued with this type of procedure because one child died just after the operation and the other survived only 5 years.

Walter Edward Dandy is also associated with early neuroendoscopy (Hsu et al. 2009). Dandy was in the first half of $20^{\text {th }}$ century one of the most innovative neurosgeons making substantial contributions to all fields of neurosurgery. Dandy was a son of British immigrants. He attended the medical school at John Hopkins University in Baltimore, Maryland and passed the examinations 1910. From 1910-1911 he worked in Cushings Hunterian laboratory at the John Hopkins University. Among many other topics, Dandy studied there experimental hydrocephalus produced in dogs by obstructions of the aqueduct with a cotton piece introduce from the posterior fossa through the IV. ventricle (Aschoff 1999, Dandy \& Blackfan 1913, 1914). He published these results together with Blackfan 1913 as a short communication in JAMA (Dandy \& Blackfan 1913) and 1914 as a comprehensive basic work in A. J. dis. Child (Dandy \& Blackfan 1914). As consequence of their experimental study they distinguished between an obstructive and a communicating hydrocephalus. Based on the experimental work Dandy tried also to explain the so called "idiopathic" hydrocephalus of adults which corresponds mostly to the low pressure 
hydrocephalus in our present terminology (Dandy 1921). Due to their theoretical results on hydrocephalus the logical therapeutic approach for communicating hydrocephalus consisted in reduction of the CSF production by extirpation or coagulation the choroid plexus in the lateral ventricle (Dandy 1918) and for obstructive hydrocephalus in producing an artificial bypass from the ventricles into the subarachnoidal space. This was achieved anatomically by means of an open ventriculostomy perforating the lamina terminalis from a temporopolar approach (Aschoff et al 1999; King 2003). The disadvantage of this method consisted besides of the occasional necessity to sacrifice the optic nerve also in the frequent occurrence of hygromas (King 2003). To overcome this problem Dandy preferred since 1933 a more posterior extradural subtemporal approach (Aschoff et al 1999, Dandy 1933, King 2003). It is reported that Dandy performed also 2 endoscopic inspections in children with hydrocephalus. However he was not able to complete the operation endoscopically and he had to change to the open approach. Dandy called this endoscope due to the application in the human ventricles from this time on a "ventriculoscope". It is further reported that Dandy published the results of these two endoscopic operations in Johns Hopkins Hospital Bulletin 1922 in two papers "Cerebral ventriculoscopy" and "An operative procedure for hydrocephalus". These citations although often cited remain however dubious because a closer inspection of all publications of Dandy in John Hopkins Hosp. Bull shows, that in this journal were no publications concerning his neuroendoscopic work. Nevertheless the very thorough evaluation of Dandy's correspondence by Hsu (Hsu et al 2009) speaks in favour that Dandy was initially interested in the application of endoscopes in the ventricles and probably he performed also few endoscopic procedures in children with hydrocephalus but gave up very early this technique without publishing his experience on this topic.

Temple Fay and Francis Grant were other two neurosurgeons who tried endoscopically to treat hydrocephalus in children. Temple Fay born in Seattle worked as neurosurgeon at the Temple University Philadelphia. 1923 he published an endoscopic operation of a child with the title "Ventriculoscopy and intraventricular photography in internal hydrocephalus" (Fay \& Grant 1923) he treated one year ago together with Francis Grant, the later chairman of the neurosurgery at the Temple University. They described the operation and discussed the potential and the limitations of this method. However their endoscopic operation was not successful.

The most successful pioneer of neuroendoscopy was William Jason Mixter.

Mixter graduated at the Havard medical school and worked as surgeon in the Massachusetts general hospital. There he worked together with his father Samuel Jason Mixter, a recognized surgeon, who described the spinal dorsal stabilisation at the level C1/C2 as early as 1910. With his father William Mixter had 2 beds for treating also neurosurgical patients. Their neurosurgical results were so good, that a neurosurgical unit was established. From 1933-1946 William Mixter was there the chief of the department of neurosurgery. He is well known to the neurosurgery by describing 1934 a disc herniation as reason for sciatica. 1923 William Mixter published the first successful endoscopic ventriculocisternostomy, performed with an cystoscope by transventricular approach through the foramen of Monroe by perforating the floor of the third ventricle (Mixter 1923). Exactly this technique was the example for later endoscopic ventriculostomies in the modern advanced neuroendoscopy.

The only neurosurgeon who continued with neuroendoscopy in the treatment of hydrocephalus till the development of shunt systems was John E. Scarff.

Scarff, finished his study at John Hopkins University 1924 and worked in the Hunterian laboratory at John Hopkins University between 1925-1927. He operated there also with 
Dandy. Later working at the Columbia University in New York he continued with the neurosurgeon Byron Stookey to treat hydrocephalus as developed in the 20s (Scarff 1936, 1951, Scarff \& Stookey 1936) perforating the lamina terminalis and the floor of the third ventricle in patients with obstructive hydrocephalus.

\subsection{Advanced period of neuroendoscopy at the end of $20^{\text {th }}$ century \\ 2.2.1 Idea and motivation}

Although the shunt operations were very successful in the treatment of hydrocephalus of any origin, they had typical complications due to implanted material such as shunt infections and shunt obstructions. This draw back made necessary in the life of the patient often repeated operative revisions. One of the starting ideas of the modern neuroendoscopy was therefore to reintroduce the endoscopic methods instead of shunt implantations in the treatment of obstructive hydrocephalus with technically advanced instrumensts. The endoscopic procedure in form of the ventriculocysternostomy had the advantage of being a single procedure and being more physiological regarding the CSF circulation than the shunt systems using a valve with constant flow. Other motivation to apply endoscopy in neurosurgery was the general trend in the operative disciplines in the late $80^{\text {th }}$ toward a minimally invasive surgery.

\subsubsection{Technical development}

Present neuroendoscopy would not be possible without substantial improvements in image quality and endoscope flexibility. The major breakthrough in the endoscope technology and optical quality goes back to the work of Harold Horaz Hopkins (1918-1994), a professor of physics at the university of Reading, United Kingdom (Mc Combie \& Smith 1998). He have probably remained only a recognized physicist, if he had not been approached 1951 at a dinner party by a physician who complained about the poor image quality and rigidity of the endoscopes. Encouraged by the subsequent discussion Hopkins developed the idea of using glass fibres for image transmission. 1951 he assembled a bundle of glass fibres with a higher refractory index of the core than of the cladded coat to improve light transmission over a longer distance by total reflexion (Fig. 3).

1953 he and his fellow researcher produced a bundle of fibres with precisely the same order at the proximal and distal ends, leading to a coherent image. They published their results in Nature 1954 (Hopkins \& Kapany 1954). In the following years there was no interest from the industry in the technique and Hopkins could not continue his research. He sent letters about his invention and obtained a positive response only from Basil Hirschowitz, a gastroenterologist in Ann Arbor, US. He visited Hopkins and discussed the details of his work. After his return to US Hirschowitz continued the work of Hopkins. He improved the technique using a better permanent coating of the fibres. The prototype of this flexible fiberscope was presented for the first time at a meeting of gastroenterologists in Colorado Springs in 1957 (Hirschowitz et al 1958, McCombie \& Smith 1998). To improve the image quality the number of glass fibres increased up to 200000 . For this amount of fibres the preservation of the topological fibre arrangement at both ends of the fibre bundle was essential. 1975 Max Eppstein, a German scientist developed a system of isolation which preserved the alignment of the fibers and thus guaranteed an undistorted image during twisting of the endoscope (Eppstein 1975, 1980). The development of the flexible fiberscope was completed by implementation of an external cold light source and an adjustable tip of the endoscope. 


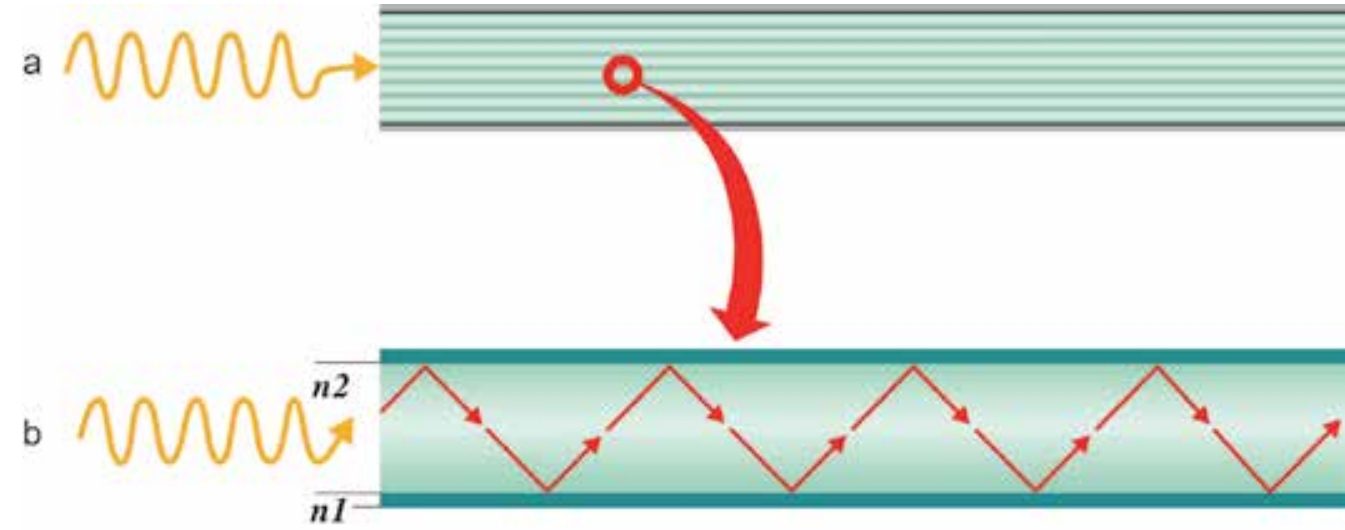

refraction: $n 1<n 2$

Fig. 3. Principle of Hopkins flexible glass fiberscope. Complete fibre bundle (a). Single fibre showing the propagation of the image over long distance inside the endoscope(b). This works by total reflection based on the lower refraction of the coating $\mathrm{n} 1$ in comparison to the glass fibre $\mathrm{n} 2$.

In his theoretical work as physicist Hopkins made substantial contributions to mathematical calculations of relay lenses aberrations and its possible compensation (Hopkins 1950, Hopkins \& Tiziani 1966). This theoretical knowledge was the presupposition for his main endoscopic invention the improvement of the image quality of the rigid endoscopes. His main idea was to replace the relay of lenses with long interspaces of air by rod glass lenses with small air gaps between the appropriate cut rod edges. This reversed the proportion of air and glass inside the shaft. The higher refractive index of glass in comparison with air improved the light transmission by a factor of 9 (Fig. 4).

a
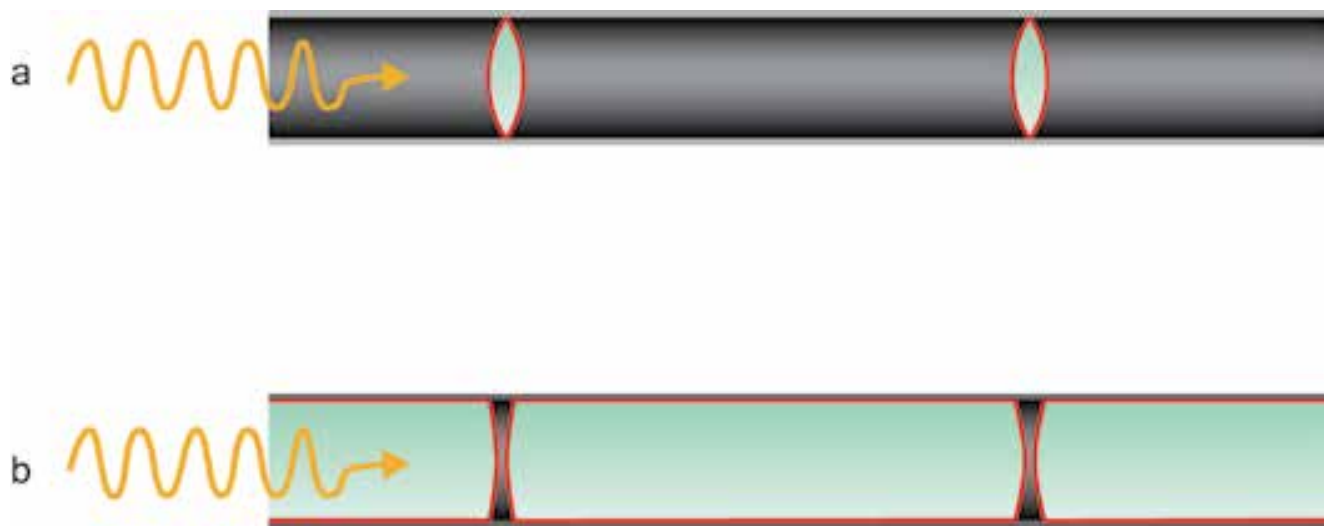

Fig. 4. Principle of rigid rod lens endoscope. Nitze optics with small lenses and long air interspaces between the lenses (a). Hopkins rigid rod lens endoscope (b) with reversed portion of glass to air in comparison to Nitze optic improving the optical quality and illumination by a factor of 9 . 
Additionally this increased the viewing angle and allowed simultaneously to decrease the diameter of the optics. Supported was the light transmission by an anti-reflection coating of the lenses. All this improvements resulted in an approximately 80 fold increase of illumination in comparison to the available standard rigid endoscopes of that time. The invention was made 1960 and the first prototype was presented 1961 during an urological meeting in Brazil (McCombie \& Smith 1998). It followed the same silence and ignorance. In 1964 Hopkins presented the same invention again during a lecture in Düsseldorf. This time, however, the potential of his invention was immediately recognized by the attending Karl Storz, the head of the Storz Company for optical instruments. A fruitful cooperation began. Storz contributed substantially by replacing the illumination source from the tip of the endoscope with a remote external device. This so-called "cold light" was originally a halogen light that was transported via separate glass fibres through the shaft of the endoscope to its tip. 1967 the first commercial product was introduced to the market. Today xenon light sources are used instead halogen, whose effective power was restricted to $250 \mathrm{~W}$ and caused a yellow image quality.

The technical development of rigid rod-lens endoscopes was completed by the introduction of video cameras for imaging to replace direct observation by surgeon looking through the endoscope. For this purpose light, miniature electronic cameras equipped with chargecoupled (CCDs) transforming the light into a digital signal contributed essentially to the miniaturization of the cameras, which could be directly connected to the endoscope oculars with a sterile cover. This set up originally assembled for urology, minimally invasive abdominal surgery and arthroscopy was commercially available at the end of the 1980s.

For optical reasons the application of endoscopy is restricted to cavities of the human body. In the intracranial space this are the ventricles and the epiarachnoid space. Therefore pathologies in the ventricles constituted also the first applications of this minimally invasive endoscopic technique.

\subsubsection{Clinical application}

In the late 80s several groups of neurosurgeons scattered around the world such as Patric Kelly (Kelly et al 1986), Kim Manwaring and Allan Cohen in US, Jaques Caemaert in Belgium (Caemeaert 1992), (Jones et al 1996). Christiane Sainte-Rose in France (Sainte-Rose 1992) and Nigel Jones and Charles Teo in Australia made positive experience with the ETV technique. Strong activity regarding the systematic endoscope related basic anatomical research, the adaptation of endoscopic instruments to neurosurgical requirements and the indications, applications and standards in the neuroendoscopy are associated with 5 centres on the European continent. Activity at these centres started nearly simultaneously and independently of each other around 1990. Notable neurosurgical centres were in Germany Dieter Hellwig and Bernhard Bauer in Marburg (Hellwig \& Bauer 1992, Hellwig et al 1997), Axel Perneczky, Peter Grunert, Nikolai Hopf and Klaus Resch in Mainz (Perneczky et al 1994, 1998) and Michael Gaab, Henry Schröder and Joachim Oertel in Greifswald (Gaab \& Schröder 1998, Oertel et al 2009). Further in Netherland Nijmegen Andre Grotenhuis a close friend of Perneczky and in Belgium Ghent Jaques Caemaert. This centres had a common endoscopic concept and a synchronous development regarding neuroendoscopic technical innovations and an increase in clinical experience with a similar number of surgical patients. Despite some different opinions on details, the activity of these centres can be summarized due to their lasting effect abroad as a common "continental" development of modern 
contemporary neuroendoscopy. Their knowledge on endoscopy spread over the world due to many practical international courses organized in each centre every year.

The first practical experience in neuroendoscopy was gathered during intraventricular third ventriculostomies for the treatment of obstructive hydrocephalus. This was also in the 90s the most frequent type of neuroendopic operations.

Most of the centres performed initially the endoscopic third ventriculostomies (ETV) by frame based stereotactic technique. This enabled the planning of a precise trajectory through the foramen of Monro to the floor of the third ventricle. Additionally for the long nose-heavy cystoscopes the stereotactic frame provided in that time an excellent stable holding of the endoscope and made possible for the surgeon to work with both hands simultaneously. A disadvantage of framebased endoscopy consisted in the restricted mechanical flexibility to change the position of the endoscope under visual endoscopic view. This was partially overcome by construction of a guiding block with a ball joint which allowed under $0^{\circ}$ a straight precise introduction of the endoscope and simultaneously after unlocking the endoscope in the block to have a free play in every direction under visual endoscopic view of $15^{\circ}$ (Fig. 5).

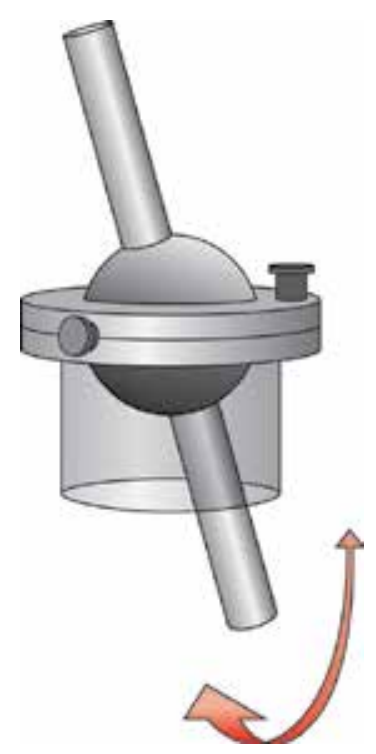

Fig. 5. Guiding block with ball joint. The ball joint allowed movement of the endoscope in any direction in a range of $15^{\circ}$. The upper metallic disc with the screws at the guiding block provided a fixation of the endoscope.

The stereotactic endoscopic third ventriculostomy was performed using CT images. With the CT adapted localisation frame the coordinates of two target points were calculated one at the level of the foramen of Monro and a second few slices below at the level of the floor of the third ventricle. Since in transversal CT slices the floor of the third ventricle was in general not visible the slice at the upper level of dorsum sellae was chosen for calculation. To protect the important surrounding anatomical structures the optimal target point was in the midline immediately behind the dorsum sellae and in front of the tip of the basilar artery (Fig. 6). 

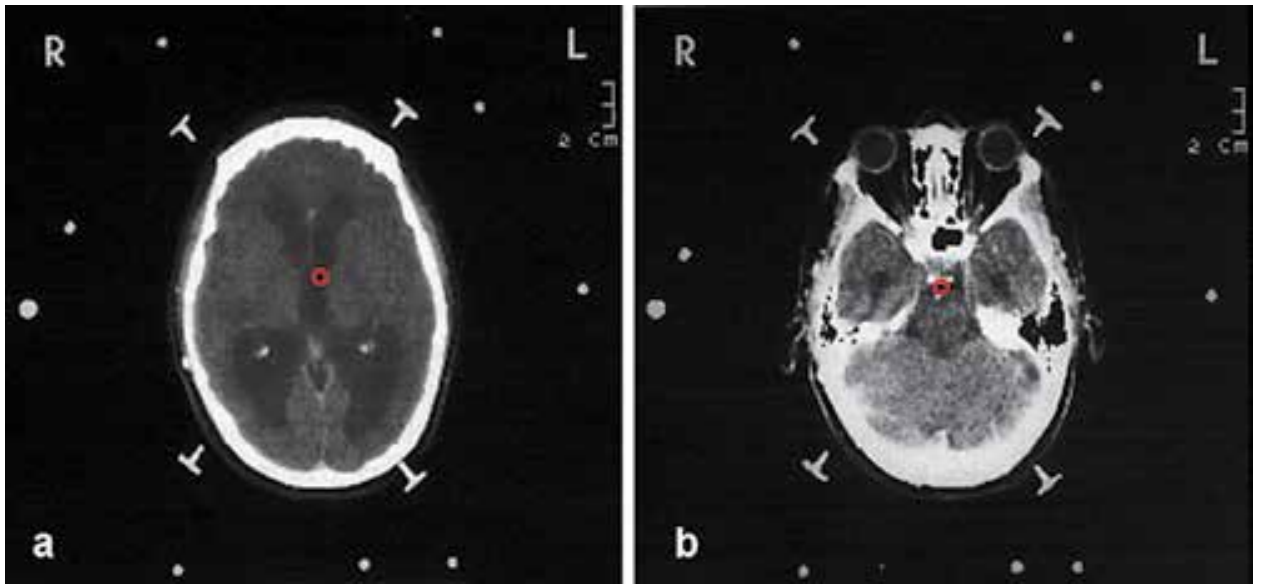

Fig. 6. CT-based stereotactic calculation of two target points for the ventriculostomy.

Calculation of the foramen of Monro visualized by a circle (a) and the target at the floor of the third ventricle between the dorsum sellae and the basilar artery in the midline (b).

This CT -based calculation constituted the planed area for perforation. The final area for perforation was decided during the endoscopic procedure respecting the directly visible anatomical structures at the floor of the third ventricle. These two calculated target points one at the level of the foramen Monro and the second behind the dorsum sellae defined geometrically a line in the space which corresponded to the optimal trajectory (Fig. 7).

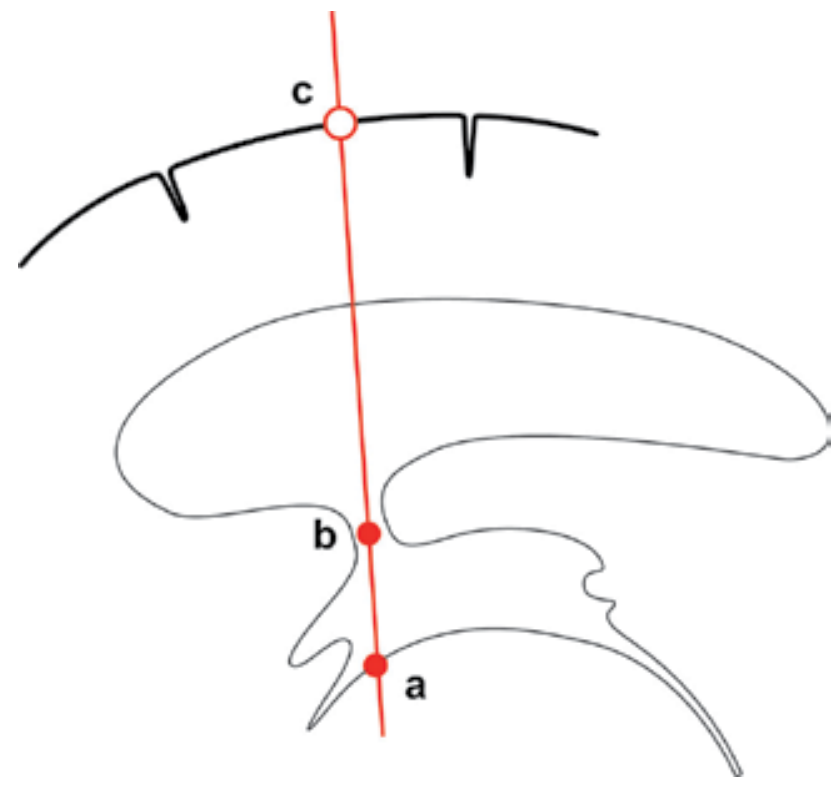

Fig. 7. Principle of stereotactic ventriculostomy. The coordinates of two points in the space were calculated, (a) at the level of the floor of the third ventricle and (b) at the level of the foramen of Monro. These two points defined a straight line in the space and thus implicitly also the trepanation point respectively the point (c) where the cortex was perforated. 
Additionally these two target points determined implicitly the trepanation point. In most of the cases area thus defined trepanation point was within an area $1 \mathrm{~cm}$ before till $1 \mathrm{~cm}$ behind the coronar suture in the anterior posterior direction and $1.5 \mathrm{~cm}-3 \mathrm{~cm}$ laterally from the midline. For these stereotactic procedures originally a $6 \mathrm{~mm}$ diameter rigid Wolff cystoscope (Fig. 8) with four channels one $1.5 \mathrm{~mm}$ diameter working channel $2.3 \mathrm{~mm}$ optical channel, and two channels for rinsing were used. Because of their much better optical quality the rigid endoscopes were superior for neurosurgical purposes in the ventricles than the flexible fibrescopes.

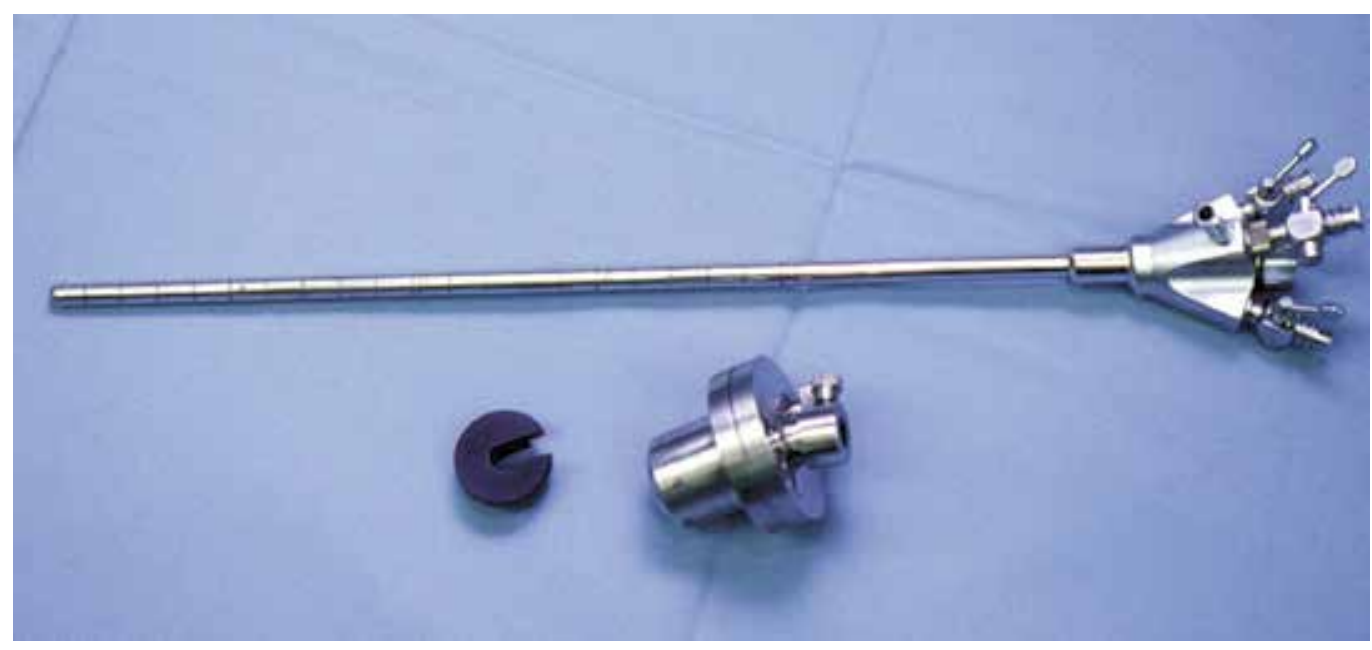

Fig. 8. First stereotactic endoscopic equipment in Mainz. Wolff $6 \mathrm{~mm}$ cystoscope with 4 channels at the top. At the bottom: guiding block with the ball joint (right side) and a plastic disc for adjusting the endoscope in a coaxial direction of $0^{\circ}$.

After stereotactic calculation of the trajectory the head of the patient in the frame was fixed in the operating theatre by Mayfield adapter to the operating table in an inclination of $20^{\circ}-$ $30^{\circ}$. By this inclination the burr-hole was at the top and CSF leakage from the lateral ventricle during the endoscopy was minimal. This precaution guaranteed that the endoscope was advanced in the ventricles entirely within CSF and disturbing optical artefacts in form of mirror images could be avoided. After setting a burr- hole, opening of the dura mater a corticotomy was performed and slightly dilated on the surface to let easily introduce the endoscope into the brain substance. In the depth of $4.5 \mathrm{~cm}-5 \mathrm{~cm}$ the endoscope was fixed the trocar removed and the optic introduced. Usually at this level the endoscope tip was inside the lateral ventricle and a view of the lateral ventricle centred on the foramen of Monro was possible (Fig. 9).

Inside the lateral ventricle under visual control the endoscope was advanced to the foramen of Monro. To prevent injury during the passage through the foramen of Monro it was essential that the optical and mechanical axes coincided. For this reason during introduction of the endoscope the $0^{\circ}$ optics was always used. At the level of the foramen the anatomical structures of the fornix and the deep intracerebral veins had to be respected. In the third ventricle the perforation was performed in front of the mamillary bodies and behind the dorsum sellae and the infundibular recessus (Fig. 10). 


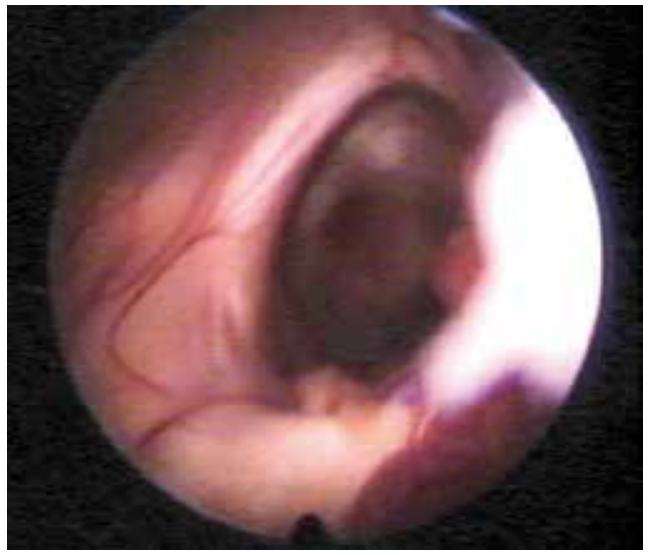

Fig. 9. Foramen of Monro during first endoscopic ventriculostomy in Mainz 1992.

Endoscopic view of the right foramen of Monro confined medially by the fornix. The white spot in the image corresponds to a piece of ependyma wall sticking on the optics of the endoscope. It covers partialy the choroid plexus and the thalamostriatal vein. The floor of the third ventricle is visible through the foramen of Monro with a red vascularized spot corresponding to the infundibular recessus.
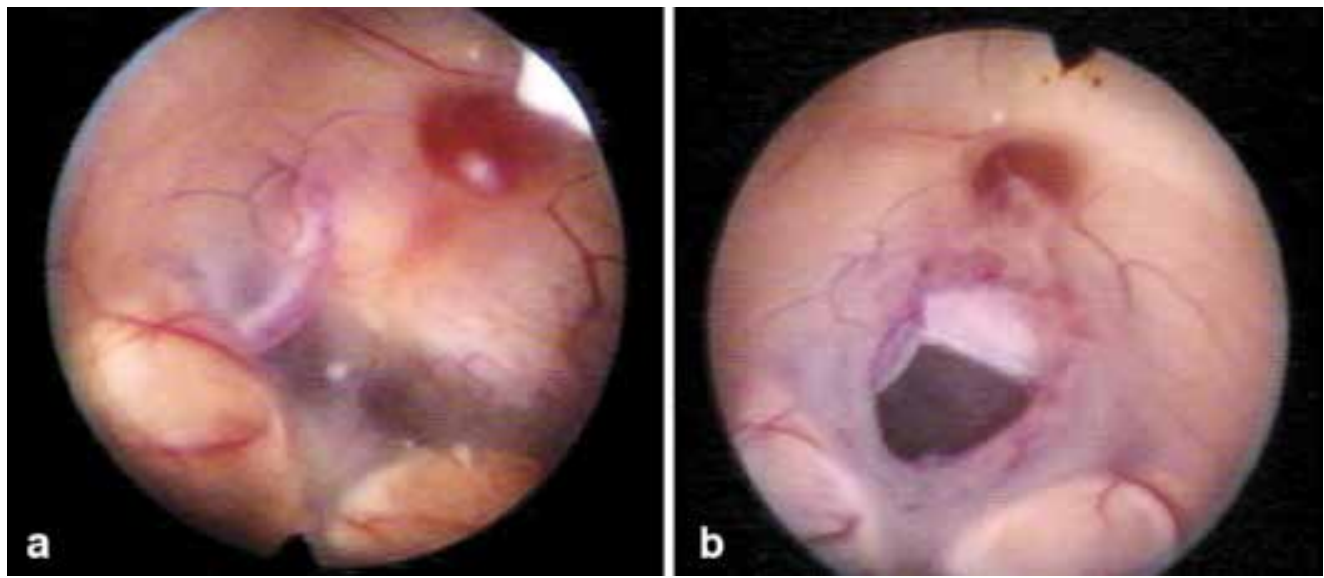

Fig. 10. Endoscopic view of the floor of the third ventricle during first ventriculostomy in Mainz 1992. Anatomy before perforation (a). Notice the mamillary bodies, and the posterior communicating artery. The red spot is the recessus infudibularis. Floor of the third ventricle after perforation (b). Notice the perforation between the dorsum sellae and in front of the mamillary bodies.

In patients with long lasting hydrocephalus the floor of the third ventricle was often thinned out to a translucent membrane. In these cases the whole anatomical structures laterally and under the floor of the third ventricle were visible including the basilar artery, the posterior communicating arteries and the occulomotor nerves. The perforation was performed bluntly with a Fogarty catheter or a forceps and dilated in steps with a balloon catheter till a diameter between $5 \mathrm{~mm}-7 \mathrm{~mm}$ was reached. 
The ventriculocysternostomies were very effective in the treatment of obstructive hydrocephalus. We performed the first 40 patients with the stereotactic framebased technique and the consecutive with free hand technique. From 150 evaluated patients operated between 1992-2000 best results with a long lasting success rate of $84 \%$ were obtained in adults with benign aqueduct stenosis followed by adults with a tumour obstructing the aqueduct with a success rate of $81.6 \%$. Infants had less good outcome. A closure of the stoma was a rare event. In these patients we performed a reventriculostmy and were successful in $75 \%$ of the patients. Patients who had no profit of the ventriculostomy or worsened obtained a ventriculo-peritoneal shunt.

Comprehensive survey of the techniques and results of the third ventriculostomy in obstructive hydrocephalus can be found in the excellent review article of Hellwig published in the Neurosurgical Review (Hellwig et al 2005).

This above mentioned basic endoscopic technique with slight modifications was applied in the $90^{\text {th }}$ for pellucidotomies in cases of unilateral dilatation of the lateral ventricle. Further the endoscopic technique was used for opening of cysts inside the ventricle or in the ventricle wall including the so called "captured fourth ventricle" in the posterior fossa. To prevent a secondary closure of the perforation for this last type of pathology a catheter was implanted through the aqueduct into the cyst in the fourth ventricle connecting the fourth ventricle with the third and lateral ventricles. Additionally in selected patients with thin good accessible membrane in the aqueduct an aqueductoplasty by opening and dilatation of the membrane inside the aqueduct was performed. However the complication rate of the aqueductoplasty was due to the nuclei and pathways in the midbrain immediately around the aqueduct higher than for the ventriculostomies. With higher experience and improved technology for hemostasis intraventricula lesions such as colloid cysts and intraventricular tumours were also removed or biopsied by minimally invasive endoscopic technique.

Hopf adapted the neuronavigation systems to endoscopes and the endoscopes could be used as apointers for planning the trajectory and for additional orientation during surgery (Hopf et al 1999). The second generation of endoscopes were already adapted to the neurosurgical requirements they were shorter and not so nose-heavy. This improved the handling of the endoscope by one person. With greater experience the frame based stereotactic technique was replaced by a free hand technique supported by holding arms. These were at the beginning two Leila retractor arms and later special arm holders which were attached to the operating table. The development of the endoscopes was pushed on ahead by the Storz, Wolff and Aesculap companies.

At the beginning of 90s Norbert Hüwel from Mainz used flexible endoscopes during syringostomies in the treatment of syringomyelia. He introduced the flexible endoscope into the syrinx cavity and fenestrated the membranes inside the syrinx in addition to the implantation of a syringo-subarachnoidal tube. However in patients with a syringomyelia due to Arnold Chiari II malformation the resection of the cerebellar tonsil with the aim to improve the CSF flow at the craniospinal level was superior to the endoscopic treatment.

In this time in Mainz Perneczky integrated the endoscope during microvascular procedures and resections of tumours to control the position of the aneurysma clip or with high degree optics "around the corner" to control the completeness of the tumour resection at the skull base. The idea was to use the endoscope during microsurgical interventions at a particular stage of the operation as a tool similar as any other surgical instrument such as the scalpel the suction or the drill. By means of the endoscope the illumination was transported into the 
depth of the operating area and complemented the view from the perspective of the microscope. By the endoscope more anatomical details could be distinguished. This proved to be valuable for instance during microvascular decompressions and all types of skull base surgery. Perneczky coined for this type of operations the term "endoscope assisted microsurgery"'(Perneczky et al 1994, Pernecky \& Fries 1998).

This idea of transportation of the illumination by means of an endoscope into the depth of the operating area was quickly picked up by neurosurgeons in the US and applied for transnasal pituitary surgery by Hae-Dong Jho (Jho 1996a, 1996b) 1996 in the endoscopic department of the centre for minimally invasive neurosurgery in Pittsburg. In Europe this type of endoscopic pituitary surgery was introduced 1998 by Cappabianca in Naples (Cappabianca et al 1998). By this minimally invasive endoscopic approach Amin Kassam (Kassam et al 2010) described removal also of lesions at the frontal skull base and clivus. In Mainz endoscopic pituitary and skull base surgery was introduced 2003 by the pupils of Perneczky, Robert Reisch and Patra Charalampaki. After both left Mainz, this work was continued by Ali Ayyad and Jens Conrad. Meanwhile the scope of endoscopic neurosurgical operations includes also spinal and periferal nerve surgery. Joachim Oertel coming from Hannover, where he was working with Gaab, introduced 2009 the spinal endoscopy also in Mainz.

\section{Conclusion}

From the historical point of view we can distinguish two periods in neuroendoscopy. An early period at the beginning of the modern neurosurgery in the 20 s of the $20^{\text {th }}$ century and an advanced period since the late $80 \mathrm{~s}$ of the $20^{\text {th }}$ century. In each period the clinical application was proceeded, accompanied and closely related to the technical development of the appropriate endoscopes. We can summarize this early neuroendoscopy period as a time of the first trials. They took place at different universities in the United States by pioneers of the modern neurosurgery. They recognized already in that time the potential use of the neuroendoscopy in the ventricles, but they gave up this technique after few trials due to technical and optical limitations of the available endoscopes. In contrary to the early period of neuroendoscopy the modern endoscopes fulfil all requirements and expectations regarding optical quality, safety and flexibility necessary also for neurosurgical applications. Therefore in this advanced period of neuroendoscopy the endoscopes became an accepted and permanent tool during neurosurgical interventions in a variety of neurosurgical fields.

\section{Acknowledgment}

The authors thank Stefan Kindel for his accurate, detailed and likewise artistically attractive illustrations.

\section{References}

Aschoff, A.; Kremer, P.; Hashemi, B. \& Kunze S. (1999). The scientific history of hydrocephalus and its treatment. Neurosurg. Review 22: 67-93

Cappabianca, P.; Alfieri, A. \& Devitiis, E. (1998). Endoscopic endonasal transspenoidal approach to the sella: towards functional endoscopic pituitari surgery (FEPS) Minim invas neurosurg. 41: 66-73 
Caemaert, J.; Abdullah, J.; Calliaw, L.; Carton, D.; Dhooge, C. \& Coster, R. (1992). Endoscopic treatment of suprasellar arachnoidal cysts. Acta neurochirurgica (Wien) 119: 68-73

Dandy, WE. \& Blackfan, KD. (1913). An experimental and clinical study on internal hydrocephalus. JAMA. 61: 2216-2217

Dandy, WE. \& Blackfan, KD. (1914). Internal hydrocephalus. An experimental clinical and pathological study. Am J. Dis Child 8: 406-482

Dandy, WE. (1918). Extirpation of the choroid plexus of the lateral ventricles in communicating hydrocephalus. Ann. Surg. 37: 569-579

Dandy, WE. (1921). The cause of so called idiopathic hydrocephalus Johns Hopkins Hosp. Bull. 32: 67-74

Dandy, WE. (1932). Practice of surgery, vol XII, chapter 1, (Dean Lewis ed.), WF Prior Company, Hagerstown

Dèsormeaux, AJ. (1865). De l'endoscope et de ses applications au diagnostique et au traitment de affections de l'urethre et de la vessie, JB Baillier, Paris

Doglietto, F.; Prevello, DM.; Jane, JA.; Han, J. \& Laws ER. (2005). A brief history of endoscopic transsphenoidal surgery - from Philipp Bozzini to the first world congress of endoscopic skull base surgery. JNS Neurosurgical focus: vol 19(6) DOI: 10.3171/foc. 2005.19.6.4

Eppstein, M. \& Marhic, ME. (1975) Fiber optic laser illumination.Proc IEEE 63: 727

Eppstein,M. (1980). Developments of optical instrumentation. Science 210:280-285

Fay, T. \& Grant, FC. (1923). Ventriculoscopy and intraventricular photography in internal hydrocephalus. JAMA 80:461-463

Gaab, MR. \& Schröder, HW. (1998). Neuroendoscopic approach to intraventricular lesions. J. Neurosurg. 88: 496-505

Hellwig, D.; Grotenhuis, JA.; Tirakotai, W.; Riegel, T.; Schulte, DM.; Bauer, BL. \& Bertalanffy, H. (2005). Endoscopic third ventriculostomy for obstructive hydrocephalus. Neurosurg. Rev. 28: 1-34

Hellwig, D. \& Bauer, BL. (1992). Minimally invasive neurosurgery by means of ultrathin endoscope. Acta neurochir Suppl. (Wien) 54: 63-68

Hellwig, D.; Benes, L.; Bertalanffy, H.\&Bauer, BL. (1997). Endoscopic stereotaxy - an eight year experience. Stereotact. Funct. Neurosurg. 68: 90-97

Hirschowitz, BI.; Curtis, LE.; Peters, CW. \& Pollard,HP. (1958). Demonstration of a new gastroscope the "fiberscope". Gastroenterology 35: 50-53

Hopf, N.; Grunert, P.; Darabi, K.; Busert, C. \& Bettag, M. (1999). Frameless neuronavigation applied to endoscopic surgery. Minim. Invas Neurosurg. 42: 187-193

Hopkins, HH. (1950). Wave theory of aberrations. Oxford university press.

Hopkins, HH. \& Kapany, NS. (1954). A flexible fiberscope using static scanning. Nature 1954; 173:39-41

Hopkins, HH. \& Tiziani, HJ. (1966). A theoretical and experimental study of lens centring errors and their influence on image quality. Brit J. Appl. Phys. 17: 33-55

Huewel, N.; Perneczky, A.; Urban V.\& Fries G. (1992). Neuroendoscopic technique for the operative treatment of septated syringomyelia. Acta Neurochir. Suppl. 54:59-62 Walter Dandy and neuroendoscopy. Historical vignette. JNS Neurosurgical Focus (3) DOI: 10.3171/2009.1. PEDS08342 
Jho, HD. \& Carau, RL. (1996). Endoscopy assisted transshenoidal surgery of pituitary adenoma. Technical note. Acta neurochirurgica. 138:1416-1425

Jones, RFC.; Stenting WA.\& Brydon M.(1990). Endoscopic third ventriculostomy. Neurosurgery 26: 86-92

Kassam, AB.; Prevedello, DM.; Carau, RL.; Suyderman, CH.; Thomas, A. \& Horowitz, MB. (2010). Endoscopic endonasal skullbase surgery. Analysis of complications in the author's initial 800 patients. J. Neurosurgery. PMID 21166570

Kelly, PJ.; Goerss, SJ.; Kall, BA. \& Kispert, DB. (1986). Computed tomography based stereotactic third ventriculostomy: technical note. Neurosurgery. 18: 791-794

King, RB. (2003). Third ventriculostomy for internal hydrocephalus complicated by unrecognized subdural hygroma and hematoma: a case report of a patient treated by Walter Dandy. JNS. DOI: 10.3171/jns 2003.985.1136

Leiter, J. (1880). Elektro-Endoskopische Instrumente. Beschreibung und Instruction durch M. Nitze und J. Leiter. Wilhelm Braunmüller und Söhne Wien.

Mc Combie, CW. \& Smith, J. (1998). Harold Horaz Hopkins. 6. December 1918-22. October 1994. Biographical memories fellows of the royal sociaty. 44: 239-252

WJ Mixter, WJ. (1923). Ventriculoscopy and puncture of the floor of the third ventricle. Boston med Surg J. 188: 277-278

Netzhat, C. (2005). Bozzini the beginning of early modern endoscopy, chapter 6 (a), the era Nitzechapter chapter 11(b) History of Endoscopy, http//laparoscopy.blogs.com /endoscopyhistory

Nitze, M. (1889). Lehrbuch der Kystoskopie. Technik und klinische Bedeutung. Von Bergmann Verlag, Wiesbaden

Northwestern university Feinberg school of medicine. Dept. of history. http//www. feinberg.northwestern.edu/neurosurgery/residency/history

Oertel, JM.; Baldauf, J.; Schröder, HW. \& Gaab, MR. (2009). Endoscopic options in children: experience with 134 procedures J. Neurosurg. ( Suppl. Pädiatr.) 3:81-89

Perneczky, A.; Cohen, A.; George, B. \& Kanno, T. (1994). Editorial. Minimal. Invas. Neurosurgery. 37:1

Perneczky, A. \& Fries, G. (1998). Endoscope-assisted brain surgery Part I. Evolution, basic concept and current technique. Neurosurgery. 42: 557-569

Sainte- Rose, C. (1992). Third ventriculostomy. Neuroendoscopy, Manwaring HK.\& Crone KR.eds, Neuroendoscopy, Mary Ann Lieber, New York

Scarff, JE. (1936). Endoscopic treatment of hydrocephalus: description of the ventriculoscope and primary report of cases. Arch. Neurol.Psychiatry 35: 853-861

Scarff, JE.\& B. Stookey, B. (1936). Treatment of obstructive hydrocephalus by third ventriculostomy. Report of two cases. Arch Neur. 36: 1400-1411

Scarff, JE. (1951). Treatment of obstructive hydrocephalus by puncture of lamina terminalis at the floor of the third ventricle. J. Neurosurg. 8: 204-213

Shah. J. (2002). Endoscopy through the ages. BJU International. 89: 645-652

Schultheiss, D.\& Moll, F. (2009). Die Geschichte der Urologie in Dresden (The history of urology in Dresden), Springer Verlag, ISBN 9783642035937, Berlin

Verger-Kuhnke, AB.; Reuter, MA. \& Beccaria,ML. (2007). Biography of Maximilian Nitze (1848-1906) and his contribution to urology. Acta Urol. Esp. 31: 697-704 


\title{
Minimally Invasive Endoscopic and Endoscopy-Assisted Microsurgery of Vestibular Schwannoma
}

\author{
Betka Jan et al.* \\ Charles University in Prague, $1^{\text {st }}$ Faculty of Medicine, \\ Department of Otorhinolaryngology and Head and \\ Neck Surgery, Faculty Hospital Motol, Prague, \\ Czech Republic
}

\section{Introduction}

Endoscopic techniques have revolutionized the practice of surgery in a number of specialities. Endoscopes have the ability to provide high magnification and illumination of the operative field, as well as the possibility to look around the corner past obstructing tissues and structures, thus allowing for more radical and safer surgeries. With several clinical applications, smaller incisions and surgical approaches are now possible, resulting in decreased postoperative pain, faster rehabilitation, better cosmetic results, and shorter hospitalization.

Similar to other specialities the endoscope represents an established component of the contemporary skull base surgery armamentarium. Its application in various skull base, otologic and neurosurgical procedures, either as a sole visualizing tool or as an adjunct to the microscope, is constantly expanding. The aim of this chapter is to review the application of endoscopic techniques in the treatment of vestibular schwannoma.

\section{Vestibular schwannoma}

Vestibular schwannomas are benign, slow-growing tumors which arise from the sheath of the eighth cranial nerve. The transition zone (Obersteiner-Redlich zone) of the superior or inferior vestibular nerves where the covering myelin changes from a central to a peripheral type or vestibular ganglion are estimated to be the site of pathologic proliferation of Schwann cells leading to tumor (Tallan et al. 1993, Zverina, 2010). Thus acoustic neuroma or

\footnotetext{
* Chovanec Martin ${ }^{1,2}$, Zverina Eduard ${ }^{1,3}$, Profant Oliver ${ }^{1,4}$, Lukes Petr $^{1}$, Skrivan Jiri ${ }^{1}$, Kluh Jan ${ }^{1}$ and Fik Zdenek ${ }^{1,2}$

${ }^{1}$ Charles University in Prague, $1^{\text {st }}$ Faculty of Medicine, Department of Otorhinolaryngology and Head and Neck Surgery, Faculty Hospital Motol, Prague, Czech Republic

${ }^{2}$ Charles University in Prague, $1^{\text {st }}$ Faculty of Medicine, Institute of Anatomy, Prague, Czech Republic ${ }^{3}$ Charles University in Prague, $3^{\text {rd }}$ Faculty of Medicine, Department of Neurosurgery, Faculty Hospital Kralovske Vinohrady, Prague, Czech Republic

${ }^{4}$ Department of Auditory Neurosciene, Institute of Experimental Medicine AS CR, Prague, Czech Republic
} 
neurinoma represent misnomers. The proportion of tumors arising from each of the two vestibular nerves superior or inferior has been reported to be equal (Clemis et al. 1986). There are two distinct clinical presentations for vestibular schwannomas: Sporadic unilateral tumor is the most common form occurring in $95 \%$ of cases, and the rare hereditary bilateral tumors which are typically a sign of neurofibromatosis type 2 .

Vestibular schwannomas have an incidence of 10 to 15 per million per year and represent the most common tumor in neurotology (Myrseth et al. 2007, Zverina, 2010). These tumors account for approximately $10 \%$ of all intracranial and 80 to $95 \%$ of cerebellopontine angle tumors (Springborg et al. 2008). Most commonly vestibular schwannomas occur during the fourth and fifth decades of life and with slightly higher incidence among females than males. The reported increasing incidence along with the higher prevalence of smaller tumors being diagnosed during the last decades probably reflect improvements in diagnostic methods and growing awareness of both patients and specialists rather than a true increase in tumor incidence (Stangerup et al. 2010).

The diagnosis is either made or suspected based on clinical symptoms at presentation, audiometric testing, or imaging methods. Symptoms and signs associated with vestibular schwannomas have been known for over 150 years and are due to direct tumor compression, invasion or vascular compromise of the surrounding structures (Betka et al. 2008).

Hearing loss is the most common and also most frequent initial symptom and reason for patients to seek medical care. Typically, patients will develop a slowly (over months or years) progressive hearing loss. Despite not being specific, progressive sensorineural hearing loss at the high frequencies is the most common finding. Sudden onset of hearing loss is found in 12-22\% of patients (Myrseth et al. 2007). Normal hearing is reported in about $5 \%$ of patients. Tinnitus is the second most frequent symptom of vestibular schwannoma. Its incidence usually coincides with hearing loss. Tinnitus may be constant or fluctuating in quality and quantity. Rarely is tinnitus the only symptom. Hearing loss indicates either damage of the cochlear nerve or occlusion of the labyrinthine artery. The origin of tinnitus is uncertain as it can be present even in the deaf ear. In conclusion, any unilateral or asymmetric sensorineural hearing loss and unilateral tinnitus deserve attention (Betka et al. 2008).

Vestibular symptoms are found in $40-60 \%$ of patients who may report a combination of vertigo, dizziness and unsteadiness. Balance problems are minor in the majority of patients probably reflecting very slow destruction of the vestibular nerve during the tumor growth however, once the tumor becomes expansive symptoms tend to worsen. Dysequilibrium is the most common vestibular symptom. Initially it is due to reduced or abolished unilateral peripheral vestibular function and incomplete compensation due to the tumor. It tends to be constant in nature, progressive in severity and aggravated by head movements. True vertigo, occurring in about $5-10 \%$ of cases, typically occurs early in the history of the tumor growth and may last for several days and weeks before resolving due to compensation of the acute peripheral vestibular function loss (Betka et al. 2008). Persistent vertigo is associated with a significant reduction in quality of life among vestibular schwannoma patients (Myrseth et al. 2007, Saman et al. 2009). In patients with large tumors, dysmetria and ataxia due to cerebellar and brainstem compression are common signs.

Headache is recalled by $10-30 \%$ of patients. It is typically described as a fullness or pressure in the ear or retroauricular region ipsilateral to the tumor. Trigeminal nerve compression 
and stretching may manifest as a paresthesia, hypesthesia or neuralgia occurring in about 5$10 \%$ of cases. Clinically apparent facial nerve dysfunction is a relatively rare finding as facial nerve tolerates a large degree of compression, stretching and distortion (Betka et al. 2008).

The growth rate of vestibular schwannoma varies and can be categorized into one of three patterns: slow (1-2 mm/year), medium (2-4 mm/year), and fast (1-2 cm/year). The majority of tumors grow slowly and in some cases spontaneous regression was reported (Zverina et al. 2010).

If not treated, vestibular schwannomas can reach a remarkable size causing severe compression of the brainstem and cerebellum eventually leading to hydrocephalus and intracranial hypertension (Zverina, 2010). However, many of these tumors fail to become symptomatic during a patient's life (Betka et al. 2008).

There are three treatment options for patients with a vestibular schwannoma: observation, radiosurgery/radiotherapy, and microsurgery.

The concept of observation (wait and scan) is based on two main principles. First, a high proportion of vestibular schwannomas do not grow or grow very slowly following the diagnosis. Second, there is little evidence that active treatment of a non-growing lesion is beneficial to the patient, but it is well documented that active treatment may cause additional complaints and decrease quality of life (Martin et al. 2001, Myrseth et al. 2006, Cheng et al. 2009, Myrseth et al. 2009). Therefore, in spite of increasing hearing loss in many patients, the conservative approach may be a reasonable option for patients with small and medium sized tumours (Yamakami et al. 2003, Sandooram et al. 2004, Zverina, 2010, Sughrue et al. 2011). For the success of observation close follow up and patient's compliance are crucial. The failure rate (e.g. continuous growth or new and progressive symptoms) that need conversion to active treatment is reported to be 15-50\% (Wackym, 2005, Myrseth et al. 2007, Betka et al. 2008).

Although there are enthusiastic supporters for both arms of active treatment (e.g. radiosurgery/radiotherapy and microsurgery), the data on which to base an objective opinion are incomplete (Yamakami et al. 2003, Myrseth et al., 2007, Myrseth et al. 2009, Zverina, 2010). Radiosurgery refers to the delivery of a therapeutic radiation dose in a single fraction while radiotherapy represents the delivery of a therapeutic radiation in multiple fractions. Radiosurgery represented by the Leksell's gamma knife surgery (GKS) represents the treatment strategy with the biological chance of achieving permanent tumor control for late-responding and slowly proliferating tumors as vestibular schwannoma (Linskey, 2000). Other methods of stereotactic radiosurgery such as linear accelerator (LINAC) and cyber knife, and fractionated radiotherapy are employed for treatment of vestibular schwannomas with similar results (Wackym, 2005, Sakamoto et al. 2009). Radiosurgery does not attempt to eliminate the tumor or cure patients thus it is difficult to compare with microsurgery. Ideal results of radiosurgery are control/no further growth of tumor, preservation of facial nerve function and hearing without any complications. This form of treatment is not appropriate for patients with tumors larger than $3 \mathrm{~cm}$ in diameter, as radiosurgery is problematic in larger tumors. Indications in patients with intrameatal tumors, tumors indenting the brainstem and patients with balance problems is also speculative (Wackym, 2005, Zverina, 2010, Cakrt et al. 2010).

The majority of centers report tumor control rates between 90 and 100\%, but few report observation periods longer than five years (Myrseth et al. 2007). Useful hearing preservation is reported in about half of cases approaching 3-4 years after radiosurgical treatment (Yang 
et al., 2010). The effect on tinnitus and vertigo is largely unknown (Wackym et al. 2008, Gerosa et al. 2010). Post-irradiation facial and trigeminal nerve dysfunction decreased to less than $5 \%$ as the treatment dose to the tumor margin has been gradually lowered during the last decades thus making the treatment very attractive for the patients and physicians (Myrseth et al. 2007, Myrseth et al. 2009). Hydrocephalus after stereoradiosurgical treatment is reported in $2-4 \%$ of patients. The mechanism of its development remains controversial. Frequent monitoring and early intervention is highly recommended to prevent irreversible cerebral damage (Myrseth et al. 2007). Induction of secondary neoplasia and malignant transformation of vestibular schwannoma has been reported rarely (Zverina, 2010).

Despite the fact that published data are difficult to interpret, mainly due to lack of long-term follow up, changing treatment protocols, lack of control groups, limited understanding of the natural history of treated tumors, insufficient stratification of patients and incomplete reporting results, there is little debate that radiosurgery has the ability to achieve tumor control with short-term low complication rates and a shorter hospital stay, and its role in the treatment of vestibular schwannoma is expanding (Wackym, 2005, Myrseth et al. 2007).

The objectives of surgical management are radical resection of the tumor, preservation of facial nerve function, conservation of hearing, and preservation of neighbouring neurovascular structures. Surgical treatment is technically challenging with gradual learning curve (Roser \& Tatagiba, 2010). In many contemporary centres, vestibular schwannoma patients are operated on by a multidisciplinary team consisting of neurootologist and neurosurgeon. Ideally, it is a true team with each participant capable of performing the entire operation (Skrivan et al. 2004, Myrseth et al. 2007). This unique cooperation among the two disciplines has allowed each to bring the best of their speciality to the management of vestibular schwannoma (Zverina et al. 2010).

Three different approaches (e.g. translabyrinthine, middle fossa and retrosigmoid) were developed for the microsurgical treatment of vestibular schwannoma. Debate continues as to which of the three approaches is best suited for large and for small tumors, and which technique allows for best results. Several articles have eloquently outlined the advantages and disadvantages of each approach (Colleti \& Fiorino, 2005, Wackym, 2005, Myrseth et al. 2007, Betka et al. 2008, Sughrue et al. 2010a, Sughrue et al. 2010b). The translabyrinthine and the retrosigmoid approaches can be used for all tumor sizes, whereas the middle fossa approach is useful only for removal of intrameatal tumors. Hearing preservation can be achieved only through the retrosigmoid and middle fossa approaches. The approach is chosen based on criteria such as tumor size, internal auditory canal extension, preoperative hearing level, and the surgeon's experience. Many teams employ all three approaches in an effort to take advantage of each of them and to achieve the best outcome for each patient. Radical tumor removal can be achieved in the majority of cases, and there is a seldom role for partial resection (e.g. preservation of functional neural structures, advanced patient age, hearing status in the contralateral ear).

The morbidity associated with vestibular schwannoma surgery has declined steadily since the first successful operation was performed more than 100 years ago. The development of the surgical microscope in the 1960s revolutionized the field of its treatment.

Recent results following microsurgical removal of vestibular schwannomas are in general excellent although mortality and morbidity are not completely eliminated. Today most large series report mortality rates of 1\% or less (Yamakami et al. 2003, Myrseth et al. 2007, Zverina et al. 2010). Facial nerve injury, hearing loss, postoperative balance problems, cerebrospinal fluid leak and headache are among the main risks of surgical treatment of vestibular schwannoma. 
With the routine use of intraoperative neuromonitoring anatomic preservation of facial nerve in the large series from the centers ranges from 90\% to 100\% (Wackym, 2005, Myrseth et al. 2007). Even if facial nerve is anatomically and physiologically intact after tumor resection, paresis may develop during the early postoperative period with improvement of function gradually over the next months. The single most important predictive factor of facial nerve preservation is the size of the treated tumor. In the group of surgically treated small and medium size tumors normal function of the facial nerve can be achieved in the vast majority of cases. Immediate postoperative normal function was shown to be better in cases of retrosigmoid and translabyrinthine approaches (Colleti \& Fiorino, 2005). In the group of large tumors immediate postoperative normal facial nerve function can be achieved in about $50 \%$ of cases treated by the experienced team with subsequent improvement to normal or near normal (House Brackmann grade I and II) in about 60 to $80 \%$ of cases (Magnan et al. 2002, Zverina, 2010). Tumor adherence to the facial nerve, intraoperative monitoring and age represent also important prognostic markers of facial nerve preservation (Sughrue et al. 2010b).

Type of surgical approach, tumor size, younger age of the patient, and the use of intraoperative monitoring have all been implicated as predictive factors of hearing preservation. Beside surgical experience and skill, the chance for hearing preservation is influenced by the proper identification of the cochlear nerve and tumor adhesion to the cochlear nerve. Hearing preservation in patients with large tumors is difficult and less likely to succeed compared with small or medium-sized tumors. Reported hearing preservation in the case of tumors $>2 \mathrm{~cm}$ ranges from 5 to $53 \%$ (Wanibuchi et al. 2009). The success rate in the group of small tumors is significantly better ranging from 17 to $100 \%$ in some series. In a recent review of results of hearing preservation after microsurgical resection the hearing preservation rate was $64 \%$ in tumors $<1 \mathrm{~cm}$ and $61 \%$ in tumors $1-1.5 \mathrm{~cm}$. The results of this multivariate analysis have shown the middle fossa approach to be more effective for preservation of hearing. The increased risk of surgically related hearing loss with retrosigmoid approach possibly reflect the fact that in this approach the cochlear nerve usually presents on the microscopically blind anterior side of tumor, and is encountered late, while the middle fossa approach permits earlier identification of the nerve, which can subsequently be safely avoided during tumor resection (Sughrue et al. 2011).

Following surgery, patients may develop balance problems, but eventually most recover well. Vestibular compensation after tumor removal and vestibular nerve sectioning can take weeks to months. Large tumor size, postoperative rehabilitation, central vestibular pathology and cerebellar dysfunction, surgical approach, impairment of vision a depression are among the main factors that influence the eventual level of compensation and the time taken to achieve this (Saman et al. 2009, Cakrt et al. 2010).

Some pain is expected in most patients immediately after surgery. Headache that persists weeks to months after surgery is not a major problem in the majority of patients but in some cases it can be incapacitating and decreasing quality of life. The reported incidence of postoperative headache has ranged from $0 \%$ to $73 \%$ depending on the type of surgical approach, technique used, and interval since surgery. The retrosigmoid approach was associated with higher incidence of debilitating persistent postoperative headache when compared with the translabyrinthine or middle fossa approaches. There is evidence indicating that a standard craniectomy may be associated with significant postoperative headache, which may be reduced with minimal bone removal during craniectomy (Magnan et al. 2002, Ryzenman et al. 2005, Wackym et al. 2005, Myrseth et al. 2007). 
Recurrence rates after radical tumor removal, regardless of the type of approach used is less than $2 \%$. There are compelling reasons to conclude that the recurrence rate is higher if hearing preservation approaches are employed reflecting possibly incomplete tumor removal, especially at the fundus of the internal auditory canal (Yamakami et al., 2003, Zverina, 2010).

Cerebrospinal fluid leak is the most common postoperative complication with the rate of around 10 to $20 \%$ of patients. It is associated with a higher risk of meningitis and must therefore be recognized and treated promptly. According to some works leakage after retrosigmoid or middle fossa approaches are more likely to require surgical intervention than a leak after a translabyrinthine approach. The majority of leaks are a result of poor intraoperative identification of exposed air cells within the temporal bone (Wackym et al. 1999, Zverina, 2010)

\section{Historical perspective of endoscopic surgery of the cerebellopontine angle}

Development of endoscopic surgery of vestibular schwannoma parallels application of endoscopes for other procedures in the cerebellopontine angle (e.g. diagnostic cisternoscopy, vestibular neurectomy, microvascular decompression) thus it must be understood in its broader context.

Urologist L'Espinase through use of a cystoscope attempted fulguration of the choroid plexus bilaterally in two infants with hydrocephalus in 1910 (Ozluoglu \& Akbasak, 1996, as cited in Davis 1936). Doyen, who reported an endoscopic approach to the fifth cranial nerve by means of a retrosigmoid craniectomy in 1917, is credited with being the first to use an endoscope for the cerebellopontine angle surgery. He also developed instruments for the endoscopic division of the sensory roots of the nerve (Doyen, 1917).

In the late 1940s and early 1950s, the development of a coherent fiber optic bundles for image transmission, and an incoherent bundle for light transmission by Hopkins greatly aided the improvement of endoscopes that are used in modern surgical practice (Prott, 1974, Griffith, 1975, Mer et al. 1967). Later on the endoscopic anatomy of the cerebellopontine angle via translabyrinthine end retrosigmoid approach was described in detail by Prott and Opel (Prott, 1974, Opel, 1974, 1981, O’Donough et al. 1993).

Bremond and co-workers were the first reported minimally invasive retrosigmoid approach to treat the cerebellopontine angle pathologies. The authors had established that the procedure requires only a small retroauricular incison and craniectomy and the risk of the this appears to be minimal (Bremond et al. 1974, Bremond et al. 1975). Similary Fukushima used the endoscope to explore the cerebellopontine angle, cisterna magna, C1-C2 space and Meckel's cave from small burr hole and stated that cisternoscopy could be useful for the differential diagnosis of small tumors, vascular abnormalities or neurovascular compression syndromes (Fukushima, 1978).

In the 1990s few teams supported the employment of endoscopy during the retrosigmoid or retrosigmoid-retrolabyrinthine microsurgery of vestibular schwannoma. Endoscope was shown to be an ideal adjunct to hearing-preserving microsurgery of vestibular schwannomas, enabling direct control of the lateral aspects of the internal auditory canal, intrameatal portion of tumor and nerves while decreasing the rate of potential complications (Magnan et al. 1993, McKennan 1993, Magnan et al. 1994, Rosenberg et al. 1994, Tatagiba et al. 1996, Goksu et al. 1999, King \& Wackym, 1999, Wackym et al. 1999). Benefits of the endoscope during microsurgical vestibular neurectomy to treat intractable vertigo (e.g. 
Meniere's disease) were described at the same time. It was shown that endoscopy allows improved identification of the nervus intermedius, facial, cochlear, and vestibular nerves and adjacent neurovascular relationships without the need for significant brain retraction. In addition, endoscope was shown to be beneficiary for identification of the cleavage plane between the cochlear and vestibular nerves.

Endoscopy was also evaluated for the placement of an auditory brainstem implant via the translabyrinthine, retrosigmoid, and middle cranial fossa approaches. Authors stated that endoscopy provided superior visualization of the lateral recess of the fourth ventricle than the operating microscope with all approaches and recommended the retrosigmoid approach as it provides the best view of the implantation site and the easiest angle for placement of the neuroprosthesis (Friedland \& Wackym, 1999).

Endoscopic technique and assistance during microvascular decompression of the facial nerve for hemifacial spasm, trigeminal nerve for trigeminal neuralgia, glossopharyngeal nerve for glossopharyngeal neuralgia, and cochlear nerve for incapacitating tinnitus were shown to add additional accuracy rate in identifying nerve-vessel conflicts and even revealed a significant number of persistent nerve compression in despite negative binocular microscopic evidence (Magnan et al. 1993, Magnan et al. 1997, Jarrahy et al. 2000, Badr-El-Dine et al. 2002, El-Garem et al. 2002, Miyazaki et al. 2005, Chen et al. 2008, Guevarra et al. 2008).

Growing experience with endoscopic control and assistance for microsurgical management of other cerebellopontine angle pathologies led to a broader application of these techniques for tumor treatment including vestibular schwannomas, meningiomas and epidermoids (Magnan et al. 1993, King \& Wackym, 1999, Wackym et al. 1999, Magnan et al. 2002, Schroeder et al. 2004, Hori et al. 2006, de Vitiis et al. 2007). Progressive implementation of rigid endoscopy in cerebellopontine angle surgery, has revealed it to be an equal if not superior imaging tool in this anatomic region. Cerebellopontine angle endoscopy was no longer seen as a risky procedure (Ozluoglu \& Akbasak, 1996, Fries \& Pernecki, 1998, Pernecki \& Fries, 1998, Wackym et al. 1998, Miyazaki et al. 2005, Koval, 2009). Although its use has been described as an adjunctive imaging modality in many surgical procedures the use of endoscopy as the sole means of intra-operative imaging in this setting has not been reported at that time.

Microvascular decompressions were the first fully endoscopic procedures reported (Eby et al. 2001, Jarrahy et al. 2002a, Jarrahy et al. 2002b, Kabil et al. 2005, Cheng et al. 2008). The first report of fully endoscopic resection of vestibular schwannoma was by Shainian and coworkers in 2004 (Shahinian et al. 2004). Kabil and Shahinian in 2006 have presented 112 fully endoscopic procedures performed via $1.5 \mathrm{~cm}$ retrosigmoid craniotomy with excellent results. $95 \%$ tumors were completely removed. Subtotal removal was performed in $5 \%$ of patients in an attempt to preserve their hearing. Anatomical preservation of the facial nerve was achieved in all patients and of the cochlear nerve in $82 \%$ of hearing ears. Some or serviceable hearing was preserved in $58 \%$ of preoperative cases. There were no major neurological complications (Kabil \& Shahinian, 2006).

Recently, the first high-definition (HD) cameras designed for endoscopic surgery have been developed. They provide the HD Television (HDTV) image format that provides an image with improved color fidelity and enhanced image resolution that is comparable to the image obtained when looking through the microscope. Their application in the cerebellopontine angle surgery is promising. Comparing the standard three-chip camera with a HD three- 
chip camera for video-assisted vestibular schwannoma surgery and microvascular decompression has provided a more detailed image with improved resolution of tissue and vasculature of the tumor, nerves and brainstem (Schroeder and Nehlsen, 2010).

\section{Rationale for endoscopic surgery of the vestibular schwannoma}

The main goals in vestibular schwannoma surgery are complete tumor removal with preservation of neurovascular structures and their function (e.g. facial nerve, hearing), and minimization of the sequelae of surgery and its complications (e.g. persistent balance problems, headache, cerebrospinal fluid leaks). The development of the surgical microscope in the 1960s revolutionized the field of both otology and neurosurgery as it provided accurate and detailed imaging of very restricted spaces, with simultaneous possibility of bimanual dissection. Despite the fact that endoscopic technique is applicable for each type of vestibular schwannoma surgical approach its benefits were predominantly shown to overcome some of the main disadvantages of the microsurgical retrosigmoid approach (Low 1999a).

Compared with the surgical microscope, endoscopes provide a wide angle of view with superb illumination in the depth and an increased depth of focus even with high magnification. Employment of endoscopic technique allows unobstructed visualization of all critical neurovascular structures of the cerebellopontine angle with simultaneous reduction in the craniotomy size, thus eliminating or at least reducing the need for retraction of the cerebellum. Both factors might reduce the number of complications resulting from dissection in this region (Magnan et al. 2002, Goksu et al. 2006).

Furthermore, angled optics expand the lateral boundaries of the microsurgical view. Endoscopes with angles of $30^{\circ}, 45^{\circ}$ and $70^{\circ}$ can be used for early identification of the relationship between the tumor and neighboring structures in the cisternal part of dissection. The early identification of the brainstem and neurovascular structures (e.g. early identification of the position of the facial nerve to tumor or inspection of relationship between the tumor and brain or vessels) in the case of large tumors may be important in order to plan subsequent surgical steps (Fries \& Pernecki 1998, Gerganov et al. 2009).

Using the surgical microscope with its direct forward view, it is virtually impossible to look around the corner due to the oblique angle of the internal auditory canal in relation to the trajectory of the dissection. Inability to completely visualize the lateral extent of the tumor as well as incomplete visualization of the exposed air cells are among the main disadvantages of the retrosigmoid approach. Excessive drilling of the petrous bone that is necessary in tumors extending to the lateral parts and fundus of internal auditory canal needed to achieve safe and radical tumor removal, might hamper the chance for hearing preservation as a consequence of injury of the posterior semicircular canal or labyrinth (Koval et al. 1993, Low 1999b, Goksu et al. 2005). Simultaneously, extensive drilling of the posterior meatal wall with improper sealing of the opened pneumatic system is associated with increased risk of cerebrospinal fluid leak (Valtonen et al. 1997). All these disadvantages can be alleviated by angled endoscopes that can be used for both inspection and visualization of the tumor and neurovascular structures during dissection within the internal auditory canal. Moreover, after completion of tumor removal the endoscope can be used to inspect the fundus for residual tumor and integrity of labyrinthine artery, facial and cochlear nerve. Similarly endoscopes are used for visualization of the drilled-out portion of the petrous bone for opened air cells that might need sealing. Some authors report that the risk of 
cerebrospinal fluid leaks have been lowered or even completely avoided (Valtonen et al. 1997, Wackym et al. 1999, Wackym et al. 2002, Gerganov et al. 2010). Under specific anatomical conditions angled endoscopes can be beneficial to identify and manage high jugular bulb (Betka et al. 2010). Minimally-invasive approach with limited bony removal is advantageous for the case of eventual Bone Anchored Hearing Aid implantation in case of single sided deafness rehabilitation.

Endoscopic approach for vestibular schwannoma surgery has some potential disadvantages as well. The two-dimensional view obtained by the endoscope instead of the threedimensional view of the microscope represents an often cited disadvantage. Compared to the static imaging obtained by the surgical microscope, endoscopes of varying diameter, length and angulation allow surgeons to dynamically rotate and alter their perspective of the surgical field to compensate for the two-dimensional view. This amounts to a greater appreciation of the three-dimensional relationships between the tumor and the surrounding structures (Kabil \& Shahinian, 2006). Furthermore novel binocular three-dimensional endoscopical technique during skull base surgery facilitating depth perception has been described recently.

Because angled endoscopes have a sharp front edge compared with a $0^{\circ}$ endoscope, the surgeon cannot see the insertion trajectory directly. It is necessary to keep in mind that there could be a risk of damaging the neurovascular structures (Hori et al., 2006). Cerebellum can be protected during the procedure by covering it with a neurosurgical cotton, piece of Penrose drain or artificial dura. However working with $30^{\circ}$ endoscopes has been repeatedly shown to be safe (Magnan et al. 2002, Goksu et al. 2005, Miyazaki et al. 2005). Risk of injury with insertion of $45^{\circ}$ and $70^{\circ}$ endoscopes is probably higher, but this can best alleviated by microscopic control. Complications due to inadvertent injury of healthy tissues have not been reported reflecting judicious use of the instruments.

As the delicate bimanual dissection is crucial for safe management of neurovascular structures of the cerebellopontine angle, a significant number of experts always struggled against the possibility of compromising one of their hands in endoscopic handling. However this potential problem was solved with introduction of either rigid pneumatic holding arms for the endoscope or freehand endoscopic technique with the endoscope being moved and held by a second surgeon. Both techniques were shown to be safe at allowing bimanual surgical dissection (Eby et al. 2001, Jarrahy et al. 2002a, Jarrahy et al. 2002b, Schroeder et al. 2004, Kabil \& Shahinian, 2006, de Vitiis et al. 2007). Another important step forward was the introduction of an irrigation system, eliminating the time consuming and unsafe practice of removing and reinserting the endoscope.

Among the main drawbacks of endoscopic surgery of vestibular schwannoma is the risk of injury of very sensitive and critical structures of the cerebellopontine angle as the endoscope does not see the instrument used before it passes in front of the lens. Thus it is mandatory to achieve synchronized, in-and-out movements of the endoscope together with the instruments (Jarrahy et al. 2002a, Jarrahy et al. 2002b, Kabil \& Shahinian, 2006, de Vitiis et al. 2007). Using the video-endoscopy assisted microsurgical technique the handling of instruments is controlled by the microscope before coming in front of the lens of the endoscope (Magnan et al. 2002, Miyazaki et al. 2005, Betka et al. 2010, Gerganov et al. 2010).

Another potential risk of the endoscopic technique is possibility of heat injury from prolonged use of the endoscope too close to the cranial nerves and other brain structures (Betka et al. 2010, Gerganov et al. 2010). Thus it is of the utmost importance to irrigate the operating field regularly, because the tip of the endoscope may become very hot (Wackym 
et al. 2002, Magnan et al. 2002, Goksu e al. 2005, Hori et al. 2006, Betka et al. 2010, Gerganov et al. 2010).

\section{Endoscopic anatomy of the cerebellopontine angle}

O'Donoghue and O'Flynn divided the CPA area into four levels on the basis of neuroendoscopic inspection of 10 fresh cadaver heads (O'Donoghue \& O'Flynn, 1993). Level 1 contains the trigeminal and abducens nerves, Meckel's cave, superior cerebellar artery and superior petrosal vein. Level 2 contains the acousticofacial bundle and anterior inferior cerebellar artery. Level 3 contains the lower cranial and posterior inferior cerebellar artery. Level 4 contains the lower medulla, spinal cord, spinal root of the accessory nerve and hypoglossal nerve.

The majority of authors rather divide the region of cerebellopontine angle into three neurovascular complexes with no distinct borders (Rhoton, 2000a, Yuguang et al. 2005). The upper complex is related to the superior cerebellar artery, a middle complex related to the anterior inferior cerebellar artery and a lower complex related to the posterior inferior cerebellar artery (Fig.1). Each neurovascular complex includes one of the three parts of the brainstem (midbrain, pons, medulla), one of the three surfaces of the cerebellum (e.g. tentorial, petrosal and occipital), one of the three cerebellar peduncles, and one of the three major fissures between the cerebellum and the brainstem. In addition, each neurovascular complex contains a group of cranial nerves.
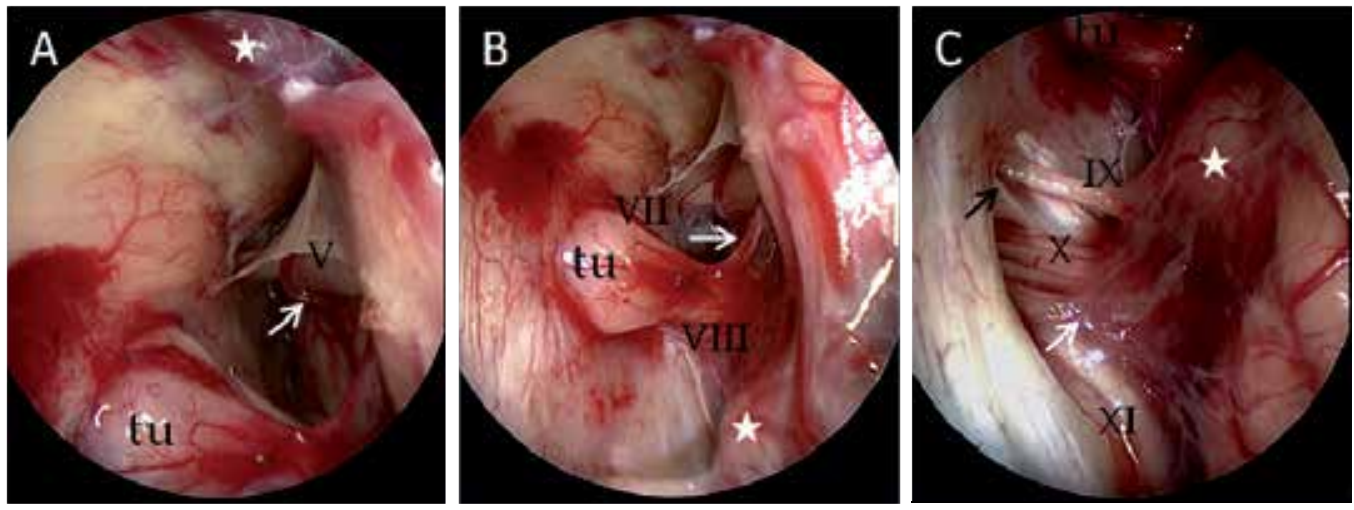

Fig. 1. Endoscopic anatomy of the cerebellopontine angle (left side). A: Upper neurovascular complex with the superior cerebellar atery (arrow) over the trigeminal nerve (V) and superior petrosal vein (asterisk); B: Middle neurovascular complex with the anterior inferior cerebellar atery (arrow), bundle of the facial (VII) and vestibulocochlear (VIII) nerves is distorted by the tumor (tu), floculus (asterisk); C: Lower neurovascular complex with the posterior inferior cerebellar atery (white arrow) over the lower cranial nerves. The spinal accessory fibers (IX) enter the jugular foramen bellow the cranial fibers of the eleventh cranial nerve that is in contact with the vagus nerve $(X)$. Glossopharyngeal nerve at the level of the jugular foramen enters the glossopharyngeal meatus, that is separated by a dural septum (black arrow) from the vagal meatus.

The upper neurovascular complex includes the superior cerebellar artery, midbrain, cerebellomesencephalic fissure, superior cerebellar peduncle, tentorial surface of the 
cerebellum, and the oculomotor, trochlear, and trigeminal nerves (Fig.1) (Rhoton, 2000a, Cappabianca et al. 2002, Yuguang et al. 2005).

The superior cerebellar artery (SCA) arises in front of the midbrain, usually from the basilar artery near its apex. The artery passes below the oculomotor nerve, but may infrequently arise from the proximal posterior cerebral artery and pass above the oculomotor nerve. Close to its origin superior cerebellar artery encircles the brainstem near the pontomesencephalic junction, passing below the trochlear nerve and above the trigeminal nerve. Its proximal portion courses medial to the free edge of the tentorium cerebelli, and its distal part passes below the tentorium. After passing above the trigeminal nerve, it reaches the cerebellomesencephalic fissure, where it runs on the superior cerebellar peduncle and terminates by supplying the tentorial surface of the cerebellum (Rhoton, 2000a, Rhoton 2000b, Rhoton, 2000c).

The trigeminal nerve joins the brainstem about halfway between the lower and upper borders of the pons. Junction of the sensory rootlets of the nerve (e.g. main cone) with the brainstem is frequently obscured by little projection of cerebellum. There may be aberrant sensory rootlets that penetrate the pons outside the main sensory cone. These are typically rostral to the main cone and related to the first division of the nerve. Motor rootlets arise even more rostral to the main sensory cone. The trigeminal nerve runs obliquely upward from the lateral part of the pons toward the petrous apex. Fibres of the third-division remain caudolateral while the fibres of the first-division remain rostromedial during the course from the pons to the trigeminal ganglion. The fifth nerve exits the posterior fossa to enter the middle cranial fossa by passing forward and beneath the tentorial attachment to enter Meckel's cave, which sits in the trigeminal impression on the upper surface of the petrous part of the temporal bone (Rhoton, 2000a).

The superior petrosal vein (SPV) is one of the most frequently encountered veins during vestibular schwannoma surgery. SPV may be formed by the terminal segment of a single vein or by the common stem formed by the union of several veins that empty into the superior petrosal sinus. Tributaries of the SPV are the transverse pontine and pontotrigeminal veins, the veins of the cerebellopontine fissure and the middle cerebellar peduncle, and the common stem of the veins draining the lateral part of the cerebellar hemisphere (Rhoton, 2000a, Rhoton 2000d, Ebner et al. 2009, Koerbel et al. 2009).

Understanding the anatomy of the middle neurovascular complex is crucial for the surgical treatment of vestibular schwannoma (Fig.1) (Rhoton, 2000a, Magnan et al. 2002, Miyazaki et al. 2005). The middle complex includes the anterior inferior cerebellar artery, pons, middle cerebellar peduncle, cerebellopontine fissure, petrosal surface of the cerebellum, and the abducens, facial, and vestibulocochlear nerves (Rhoton, 2000a, Cappabianca et al. 2002, Yuguang et al. 2005).

The anterior inferior cerebellar artery (AICA) arises at the pontine level from the basilar artery, usually as a single trunk. Its origin can be bifurcated or even triplicated. From its origin AICA courses backward around the pons being in contact with the abducens nerve, and proceeds to the cerebellopontine angle where it is related to the facial and vestibulocochlear nerves. After coursing near and sending branches to the facial and vestibulocochlear nerves entering the internal auditory canal and to the choroid plexus protruding from the foramen of Luschka, it passes around the flocculus on the middle cerebellar peduncle to supply the lips of the cerebellopontine fissure and the petrosal surface of the cerebellum. AICA commonly bifurcates near the seventh-eighth nerve 
complex to form a rostral and a caudal trunk. The rostral trunk sends its branches laterally along the middle cerebellar peduncle to the superior lip of the cerebellopontine fissure and the adjoining part of the petrosal surface, and the caudal trunk supplies the inferior part of the petrosal surface, including a part of the flocculus and the choroid plexus. The AICA gives rise to perforating arteries to the brainstem, choroidal branches to the tela and choroid plexus, and the nerve-related arteries, including the labyrinthine, recurrent perforating, and subarcuate arteries (Rhoton, 2000a, Rhoton 2000b).

The facial nerve arises from the brainstem near the lateral end of the pontomedullary sulcus 1 to $2 \mathrm{~mm}$ anterior to the point at which the vestibulocochlear nerve joins the brainstem at the lateral end of the sulcus. The interval between the seventh and eighth nerves is greatest at the level of the pontomedullary sulcus and decreases as these nerves approach the porus of internal auditory canal.

Glossopharyngeal nerve, foramen of Luschka and flocculus can be used as anatomical landmarks to help with identification of the facial nerve at the level of brainstem. The point of origin of the facial nerve from the brainstem is 2 to $3 \mathrm{~mm}$ above the most rostral rootlet of the glossopharyngeal nerve. The foramen of Luschka, the sleevelike lateral recess of the fourth ventricle, is situated at the lateral margin of the pontomedullary sulcus, just behind the junction of the glossopharyngeal nerve with the brainstem, and immediately posteroinferior to the junction of the facial and vestibulocochlear nerves with the brainstem. The foramen itself is infrequently well visualized but tuft of the choroid plexus protruding out of the foramen of Luschka over the posterior surface of the glossopharyngeal and vagus nerves, just inferior to the junction of the facial and vestibulocochlear nerves with the brainstem can be well identified (Friedland \& Wackym, 1999, Rhoton, 2000a, Koval \& Krempaska, 2009). Flocculus, projects from the margin of the lateral recess and foramen of Luschka into the cerebellopontine angle, just posterior to facial and vestibulocochlear nerves.

Proper identification of the foramen of Luschka represent a crucial step in successful placement of the auditory brainstem neuroprosthesis to rehabilitate deafness in the cases of bilateral cochlear nerve loss. Choroid plexus, glossopharyngeal nerve and possibly stump of the eighth nerve represent key anatomical landmarks of the foramen of Luschka (Colleti et al. 2000, Skrivan et al. 2003, Brito Neto et al. 2005).

As stated earlier the facial and vestibulocochlear nerve are in close contact with AICA at the level of the brainstem. In most cases, the AICA passes below the nerves as it encircles the brainstem, but it also may pass above or between the nerves (Rhoton, 2000a, Rhoton, 2000c). Both nerves show relationship with the vein of the pontomedullary sulcus, the veins of the cerebellomedullary fissure, middle cerebellar peduncle, and cerebellopontine fissure veins on the side of the brainstem (Rhoton, 2000a, Rhoton 2000d).

As the seventh-eigth nerve complex runs from the brainstem forward and laterally to the internal auditory canal they usually display contact with the loop of AICA which reaches the porus or protrudes into the canal in almost half of the cases. During the cisternal course the facial nerve is anterior, cochlear portion is inferior and posterior and vestibular portion is superior and posterior. As the nerves approach the porus of the internal auditory canal facial and cochlear portion of the eigth cranial nerve are anterior, and vestibular portion is posterior and lateral. The position of the nerves is most constant in the lateral portion of the internal auditory canal, which is divided into a superior and an inferior portion by the transverse or falciform crest. The facial and the superior vestibular nerves are superior to the 
transverse crest. The facial nerve is anterior to the superior vestibular nerve and is separated from it at the lateral end of the meatus by the vertical crest or "Bill's bar". The cochlear and inferior vestibular nerves are located below the transverse crest with the cochlear nerve located anteriorly and inferior vestibular nerve posteriorly. Because vestibular schwannoma arise in the posteriorly placed vestibular nerves, they usually displace the facial nerve anteriorly and cochlear nerves inferiorly and anteriorly (Rhoton, 2000a, Magnan et al. 2002, Miyazaki et al. 2005).

The nervus intermedius is usually joined to the ventral surface of the vestibulocochlear nerve a few millimetres adjacent to the brainstem, then has a free segment in the cisternal part as it courses to join the facial motor root.

Labyrinthine artery (or arteries) accompanying the nerves is usually a branch of premeatal segment of AICA, but it can arise from the meatal or postmeatal portion of AICA, basilar artery or posterior inferior cerebellar artery (Rhoton, 2000a, Rhoton 2000c). Injury of the internal auditory artery can hamper hearing preservation during vestibular schwannoma removal.

Looking on the posterior petrosal face we can identify the subarcuate fossa that is enetered by the subarcuate artery. It is located superolateral to the porus of internal auditory canal.

Other important structures are the endolymphatic duct and sac, situated inferolateral to the internal auditory canal. It should be preserved when elevating the dura and opening the canal if there is the possibility of preserving hearing.

The lower neurovascular complex is related to the posterior inferior cerebellar artery, and includes the medulla, inferior cerebellar peduncle, cerebellomedullary fissure, suboccipital surface of the cerebellum, and the glossopharyngeal, vagus, spinal accessory, and hypoglossal nerves (Fig.1) (Rhoton, 2000a, Cappabianca et al. 2002, Yuguang et al. 2005).

The posterior inferior cerebellar artery (PICA) arises from the vertebral artery at the medullary level, encircles the medulla, passing in relationship to the lower cranial and hypoglossal nerves to reach the surface of the inferior cerebellar peduncle, where it dips into the cerebellomedullary fissure and terminates by supplying the suboccipital surface of the cerebellum (Rhoton 2000c).

The glossopharyngeal nerve arises as one or rarely two rootlets from the upper medulla, posterior to the olive in the post-olivary sulcus, just caudal to the origin of the facial nerve. It courses ventral to the choroid plexus protruding from the foramen of Luschka on its way to the jugular foramen. The smaller ventral rootlet has been demonstrated to be motor and the larger dorsal rootlet to be sensory. The glossopharyngeal nerve enters the dural subcompartment of the jugular foramen called the glossopharyngeal meatus (Rhoton, 2000a).

The vagus nerve arises below the glossopharyngeal nerve in the post-olivary sulcus as tightly packed rootlets posterior to the superior third of the olive. The most rostral vagal fibers arise adjacent to the glossopharyngeal rootlets. The vagus nerve is composed of multiple combinations of large and small rootlets that pass ventral to the choroid plexus protruding from the foramen of Luschka on its way to the jugular foramen. The vagal rootlets enter the dural subcompartment of the jugular foramen called the vagal meatus. It is inferior to the glossopharyngeal meatus from which it is separated by a dural septum. The vagus nerve is joined by the accessory nerve as it enters the dura (Rhoton, 2000a).

The accessory nerve arises as a widely separated series of rootlets that originated from the medulla at the level of the lower two-thirds of the olive and from the upper cervical cord. 
The cranial rootlets arise as a line of rootlets just caudal to the vagal fibers in the post-olivary sulcus. These are more properly regarded as inferior vagal rootlets, since they arise from vagal nuclei. It may be difficult to separate the lower vagal fibers from the upper accessory rootlets because the vagal and cranial accessory fibers usually enter the vagal meatus as a single bundle. The upper rootlets of the spinal portion of the accessory nerve originate several millimeters caudal to the lowest cranial accessory fibers and either course to join the cranial accessory bundle or enter the lower border of the vagal meatus separate from the cranial accessory rootlets. The spinal accessory fibers pass superolateral from their origin to reach the jugular foramen. Although the cranial and spinal portion of the accessory nerve most frequently enter the vagal meatus together, they may infrequently be separated by a dural septum (Rhoton, 2000a).

The rootlets of the hypoglossal nerve arise from the medulla along the anterior margin of the lower two-thirds of the olive in the preolivary sulcus. The rootlets course anterolateral through the subarachnoid space and pass behind the vertebral artery to reach the hypoglossal canal. Before entering the canal, the rootlets collect into two bundles, and in some cases, the canal is even divided by a bony septum that separates the two bundles (Rhoton, 2000a).

\section{Technique of minimally invasive endoscopic and endoscopy-assisted microsurgery of vestibular schwannoma}

In its basic aspects the surgery proceeds in a similar fashion as an neurotological procedure. General endotracheal anesthesia is administered, profound and balanced with paralysis avoided. Before durotomy, both controlled hypotension and assisted hyperventilation to obtain a pCO2 of about $25 \mathrm{~mm} \mathrm{Hg}$ are crucial to lower the intracranial pressure and to help spontaneous cerebellar retraction. Bolus of corticoids at the same moment can be beneficial. Mannitol infusions and lumbar drainage are not needed (Miyazaki et al. 2005, Goksu et al. 2006, Betka et al. 2010). If experience shows that the surgery will take more than 3 hours a Foley catheter is placed. A second generation cephalosporin is used for perioperative prophylaxis.

The patient is placed in supine position with the head rotated away from the ipsilateral ear. Patient positioning is crucial, making sure that the shoulder does not restrict access to the surgical field and proper manipulation with instruments. Fixation of the skull is usually not necessary, fastening of the contralateral forehead with a gel sheet is adequate. Another option is to secure the head in a Mayfield 3-pin head clamp. The table is positioned so that the head is elevated above the level of heart.

For the approach the area to be shaved, only 2 to $3 \mathrm{~cm}$ behind the ear is sufficient. The landmarks for skin incison are Frankfurt line joining the outer canthus to the superior part of the external auditory meatus, and a line along the posterior margin on the mastoid. Placement of a minicraniotomy can be marked as a $2 \mathrm{~cm}$ circle below and behind from the point where the two lines cross. The incision is outlined as an anterior concave line of 3 to $6 \mathrm{~cm}$ in length arching from the tip of the mastoid at a position one finger's width back of the oblique line by passing the backside of the keyhole (Fig.2). Placement of the incision is infiltrated with 1: 200000 Epinephrine. The skin flap is elevated with sharp dissection of the temporalis fascia and the pericranium ending at the level of the external auditory canal. Using the cautery knife and raspatory nuchal 
muscles are detached from the mastoid and occipital bone backwards and a self retaining retractor is placed in position.
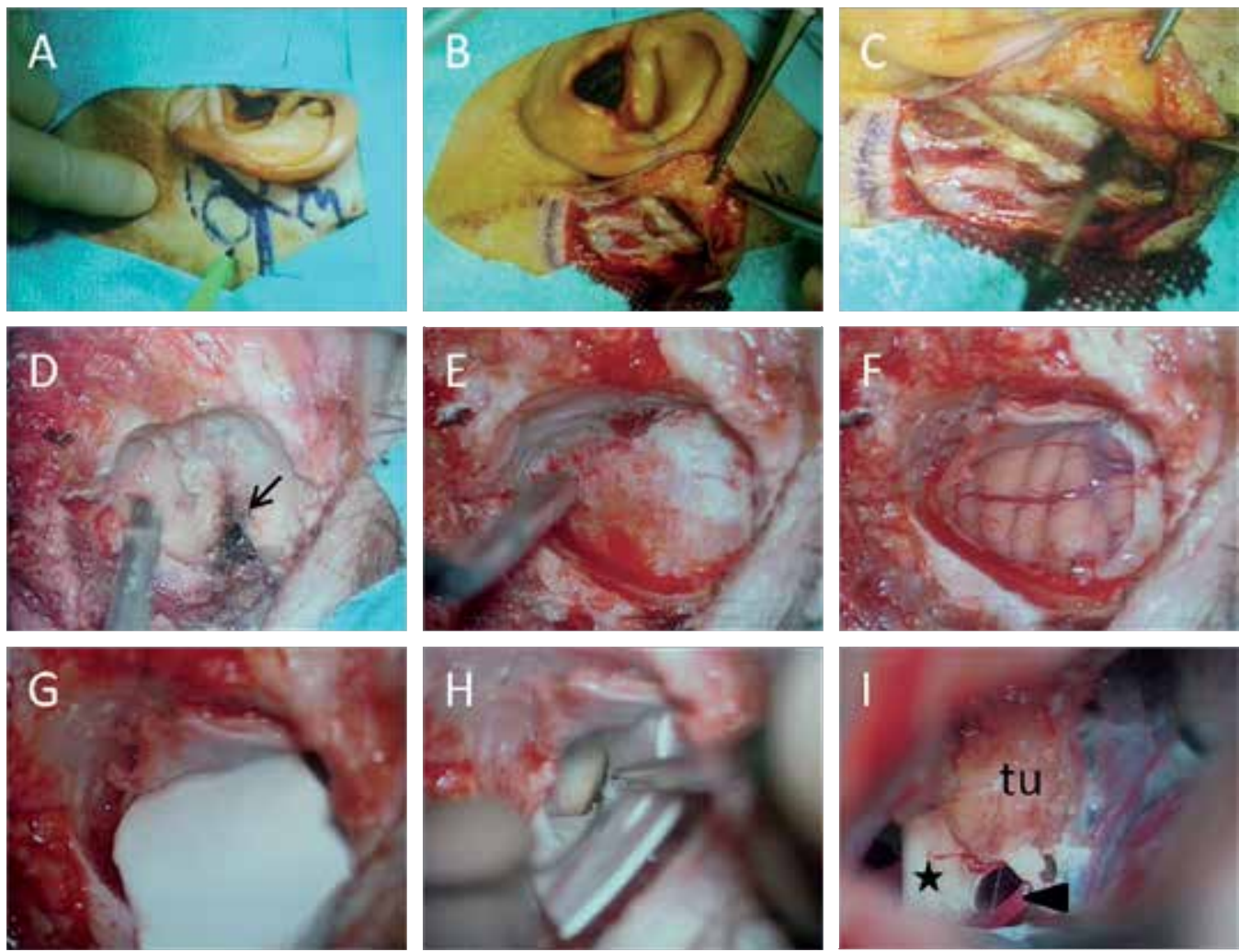

Fig. 2. Surgical steps of the minimally invasive retrosigmoid approach for vestibular schwannoma (left side). A: landmarks for skin incison (Frankfurt line and a line along the posterior margin on the mastoid) and place of minicraniotomy (circle) are outlined, B: elevation of the skin flap, C: detachment of the muscles, D: drilling is centered on the emissary vein (arrow), E: minicraniotomy is finished, it is centered on the confluence of sigmoid and transverse sinuses, F: anteriorly based dural flap is elevated and fixated with the suture, G and H: sheet of artificial dura is placed over the cerebellum, the cisterna is opened and cerebrospinal fluid is let to escape and the surgeon waits for the spontaneous cerebellar retraction to occur, $\mathrm{H}$ : once adequate cerebellar relaxation has been achieved no brain retractors are needed and dissection of tumor (tu) can proceed (asterisk denotes the seven-eight nerve bundle, arrowhead points on anterior inferior cerebellar artery).

Once adequate exposure of bone is achieved the keyhole craniotomy is performed (Magnan et al. 2002, Miyazaki et al. 2005). Using the cutting burr the drilling starts with the mastoid emissary vein in the centre as the landmark. Asterion represents another anatomic landmark for craniotomy placement as its helps to localize the confluence of sigmoid and transverse sinuses (Rhoton, 2000a, Rhoton, 2000b, Kabil \& Shahinian, 2006). All the bone dust is collected to prepare a pate that is solidified with fibrin glue at the end of surgery and used for craniotomy closure. In order to prevent cerebrospinal fluid leakage, all opened mastoid 
air cells are obliterated with the bone wax. If not used from the beginning it is suggested to use the operating microscope when the dura and posterior margin of sigmoid sinus is approached. It is even safer to use the diamante at this stage of drilling.
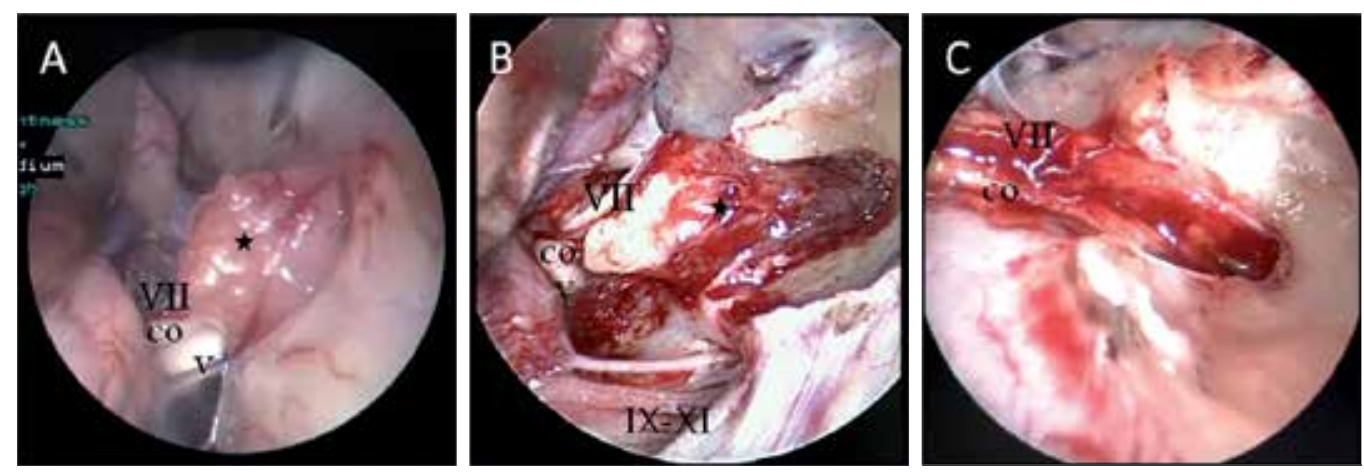

Fig. 3. Minimally invasive endoscopy-assisted removal of small (grade II) vestibular schwannoma (right side). A: anatomic mapping after opening the cisterna (VII: facial nerve, v: vestibular nerves, labyrinthine artery is in between the facial and vestibular nerves), B: internal auditory canal is opened and vestibular nerves sectioned and tumor (asterisk) is exposed, C: control of radicality of resection and integrity of facial and cochlear (co) nerves.
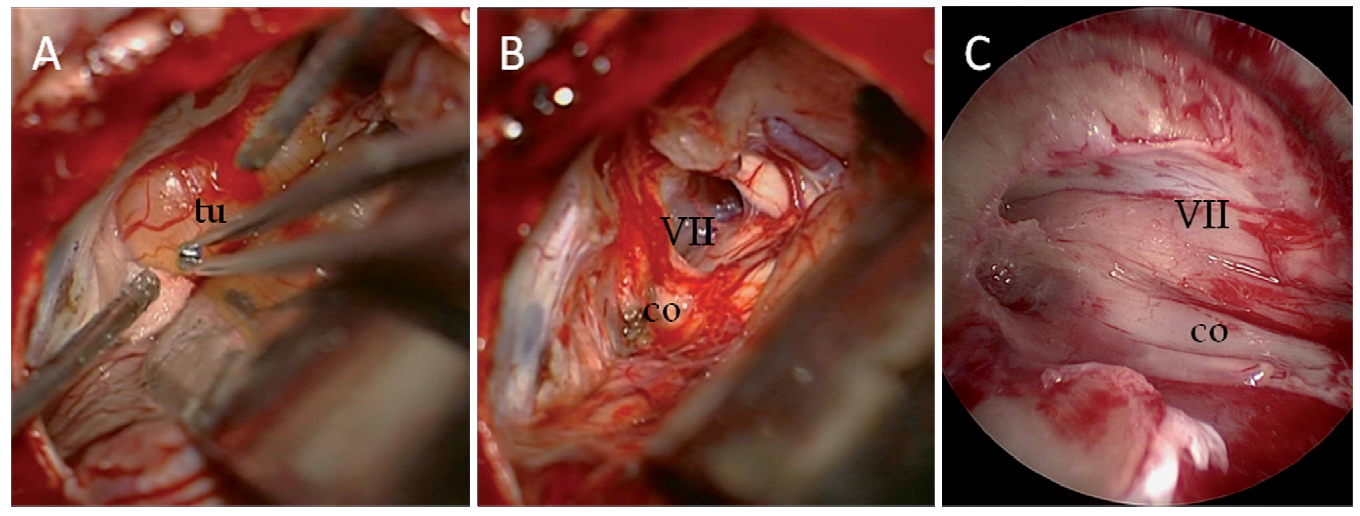

Fig. 4. Minimally invasive endoscopy-assisted removal of large (grade IV) vestibular schwannoma (left side) with preservation of facial and cochlear nerve including preoperatively useful hearing. A: anatomic mapping after opening the cistern, B: cisternal portion of tumor was removed with preservation of cochlear (co) and facial nerve (VII), C: C: control of radicality of resection and integrity of facial and cochlear (co) nerves.

After adequate exposure of the posterior fossa and hemostasis the dura can be incised. An anterior based U-shaped flap is appropriate as its fixation with stay suture helps to retract the sigmoid sinus to gain better access to the cerebellopontine angle. While suctioning of the cerebrospinal fluid, wait for spontaneous cerebellar retraction. Fine neurosurgical cotton $1.5 \mathrm{~cm}$ in width and $5 \mathrm{~cm}$ in length is placed over the cerebellum to protect it from injury. Cerebellum can be gently pressed by the mid portion of the suction that is gently advanced into the safe corridor between the hemisphere and posterior aspect of the 
petrous bone until the cerebellopontine cistern is opened at the level of lower cranial nerves. Once adequate cerebellar relaxation has been achieved it is usually not necessary to insert any brain retractor. The endoscopes used are mainly $0^{\circ}$ and $30^{\circ}$ angled rigid endoscopes of standard 2.7 and $4 \mathrm{~mm}$ in diameter and 6,11 and $14 \mathrm{~cm}$ in length according to the situation. If needed $45^{\circ}$ and $70^{\circ}$ angled rigid endoscopes are used. An irrigation sheath attached to the endoscope clears blood and debris from the lens, eliminating the time consuming and unsafe practice of removing and reinserting the endoscope. A pneumatic holding arm is preferred to secure the endoscope in position, allowing bimanual surgical dissection. Other option is freehand endoscopic technique with an endoscope being moved and held freehand by a second surgeon, with the first surgeon using two other instruments inside the surgical field.

The endoscope and the optical fiber cable used are completely sterilized by autoclaving. By covering the camera cable and the camera adaptor with a sterilized polyethylene cover, endoscopes can be easily exchanged under clean handling conditions. To maintain asepsis, the endoscopic examination is followed on a monitor.
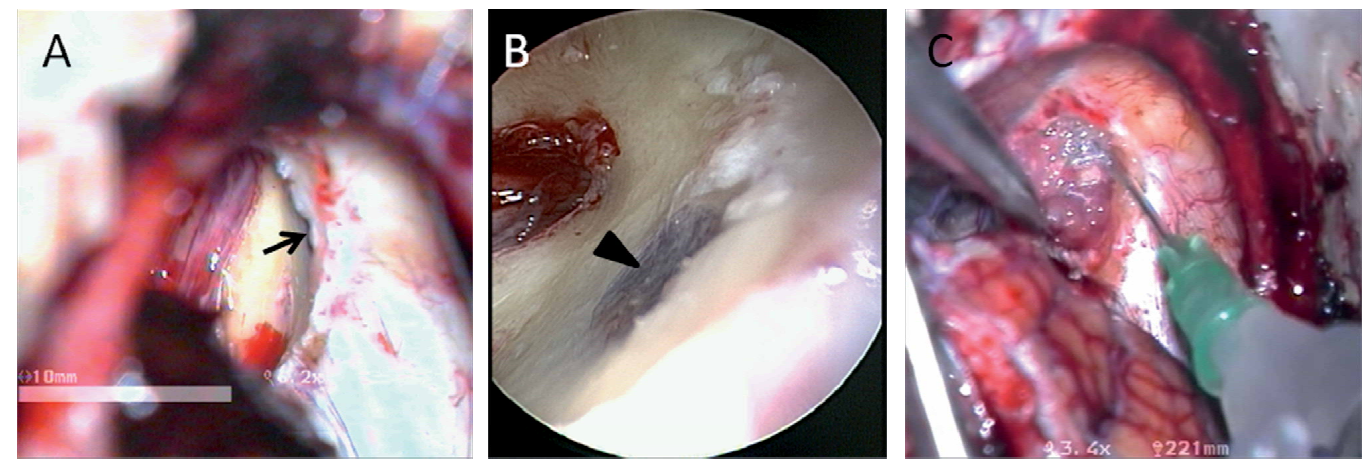

Fig. 5. Endoscopic identification of opened petrous bone air cells. A: with surgical microscope opened air cells might be not visualized with risk for cerebrospinal fluid leakage (position identified by arrow), B: endoscopic view with identification (arrowhead) of opened air cells after drilling of posterior meatal wall, C: sealing of the opened air cells with piece of muscle and tissue glue.

Upon entering the cerebellopontine angle a preliminary survey of the actual neurovascular anatomy including the trigeminal, facial, and lower cranial nerves, as well as the vascular structures is performed (Fig.3 and 4). Neuromonitoring of the facial nerve and hearing is a standard during vestibular schwannoma surgery. Function of the facial nerve stimulator can be checked with stimulation of the accessory nerve rootlets.

Once all the critical structures are identified tumor dissection can take place. The dura overlying the petrous bone posterior to internal auditory canal is cauterized and incised and a diamond bur is used to open the IAC, following the tumor extent laterally within the canal.

No matter whether microsurgical or endoscopic technique is used the basic surgical concept is always to debulk tumor in order to relieve the pressure on the surrounding neurovascular 
structures. Microdissecting instruments as well as the cavitron ultrasonic aspirator (CUSA) can be used to accomplish this step. Having completed debulking it is easier to appreciate the full anatomical course of the cranial nerves and vessels and to protect them from potential damage. During dissection the most adherent points between the tumor and nerves are recognized and addressed last.
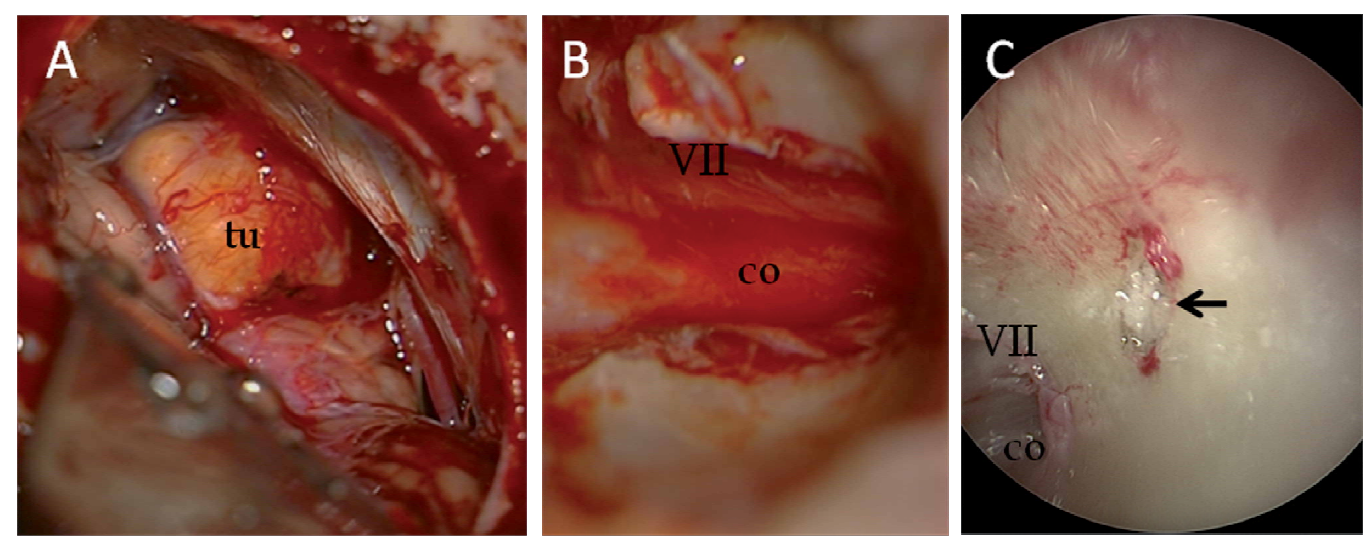

Fig. 6. Minimally invasive endoscopy-assisted removal of large (grade IV) vestibular schwannoma (right side) with preservation of facial and cochlear nerve but "unexplainable" loss of evoked auditory brainstem response during the internal auditory canal opening. A: view of the tumor (tu) after opening the cistern, B:endoscopic control of radicality of resection and integrity of facial (VII) and cochlear (co) nerves, C: loss of hearing was explained with identification of the opened posterior semicircular canal (arrow).

If endoscopic technique is preferred the dissection in the region of the cerebellopontine angle is guided by a $0^{\circ}$ endoscope in much the same manner as the microsurgical procedure. Tumor dissection within the internal auditory canal is guided preferentially by the $30^{\circ}$ angled endoscope, allowing complete visualization of the lateral extent of the tumor as it is separated from the facial and cochlear nerves. The endoscopes are inserted in the upper part of approach, perpendicular to the sigmoid sinus, or in the angle between the petrous bone and tentorium, while the surgical instruments are inserted below it, along the cerebellar surface. Such position permits both inspection of the relationship between the tumor and critical neurovascular structures, and continuous control of surgical manoeuvres of the instruments, which constantly remain under direct endoscopic view.

Once tumor dissection is completed endoscopic inspection of fundus of the internal auditory canal for residual tumor, integrity of cochlear and facial nerves and meticulous inspection of drilled-out portion of the petrous bone for open air cells that need sealing is performed (Fig.3, 4, 5, 6 and 7). The facial nerve is once again stimulated to confirm its function. Small pieces of fat or muscle together with fibrin glue are used to seal the opened internal auditory canal and petrous bone air cells. Cisterns are repeatedly washed. The dura is reapproximated and sutured to provide a watertight closure.

Bony pate is used to replace the drilled bone at the craniotomy site. Soft tissues of the scalp are closed in anatomical layers without the use of any drains. Following the surgery the 
patients are typically transferred to intensive care unit for overnight monitoring. Early vestibular rehabilitation is supported postoperatively.
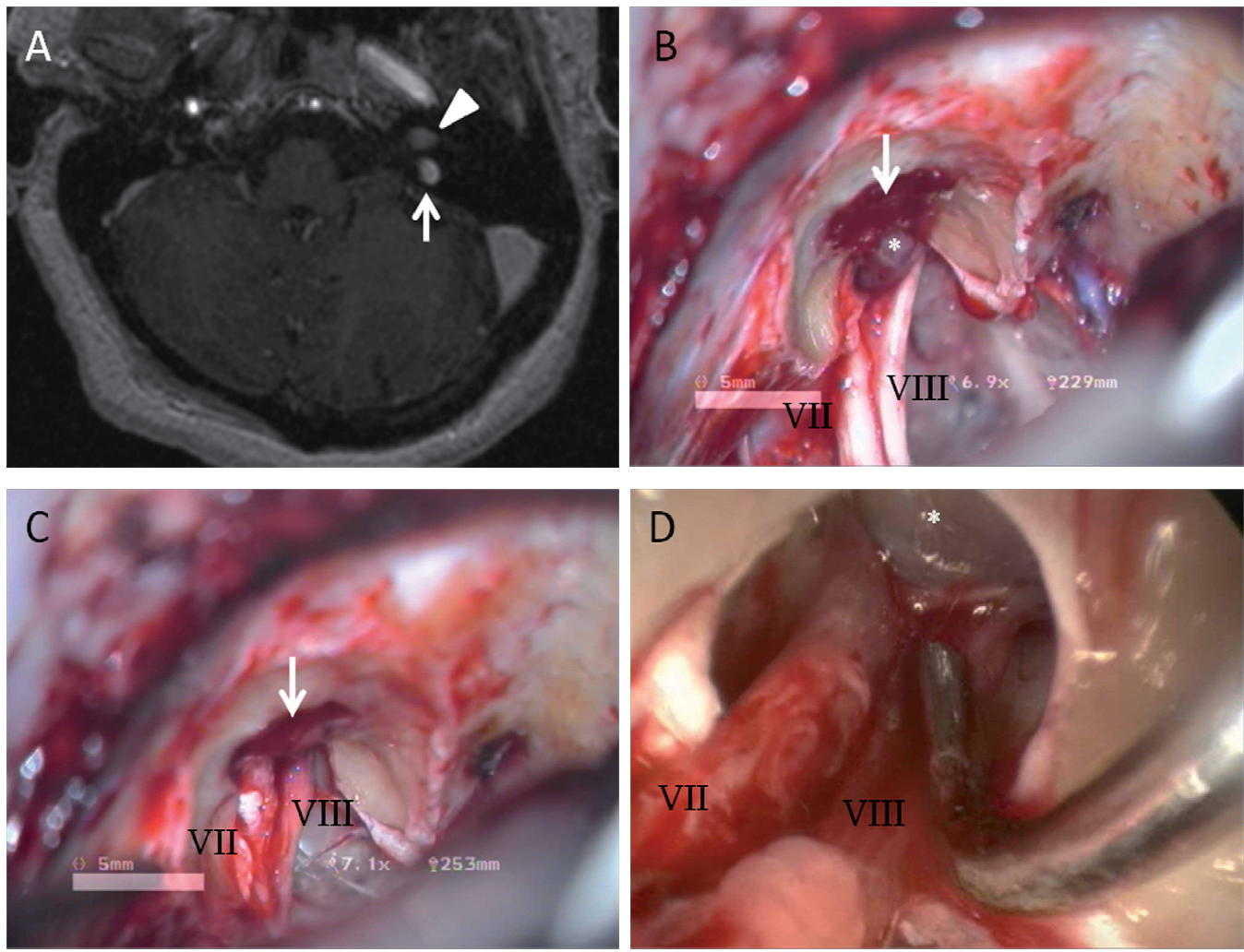

Fig. 7. Minimally invasive endoscopy-assisted removal of intrameatal (grade I) vestibular schwannoma (left side) in case of high jugular bulb. A: MRI showing the high jugular bulb (arrow) being positioned posterior to the internal auditory canal and tumor (arrowhead), B: after drilling the posterior meatal wall high jugular bulb blocks the approach to the medial portion of the tumor, facial (VII) and vestibulocochlear nerve (VIII) can be seen, C: jugular bulb was compressed and major part of the tumor was removed, D: endoscope is used to control tumor removal in the region of fundus of the internal auditory canal.

\section{Conclusions}

The recent technological advances provided high-quality endoscopes of small diameters and varying lengths and angles, irrigation sheaths for cleaning the lenses inside the operative field, endoscope holders that allow the surgeon to continue using their bimanual microsurgical skills and sophisticated instruments suitable for endoscopic microsurgery. These improvements permit us to use the endoscope, with its wide panoramic, multiangled, and close-up view of the anatomic structures, to ideally express its best properties in deepseated and narrow space-located lesions of the skull base. 
The introduction of endoscopic technique into the repertoire of the surgical methods of treatment of the cerebellopontine angle pathologies has proved to be useful. Although only limited surgical series concerning the employment of the endoscopic techniques for the removal of vestibular schwannomas have been reported recently, it seems clear that the endoscopes offer some advantages over the conventional microsurgical technique either in reducing the invasiveness or in allowing safer and more radical tumor removal, thus helping to lower the complication rate and improving the results. Nevertheless, good equipment and special training are absolutely necessary for attainment of optimal results.

\section{Acknowledgements}

This work was supported by grants of the Internal Grant Agency of the Ministry of Health of the Czech Republic NS/9909-4, NT/12459-5, NT/11543-6 and Charles University project for support of Specific University Research No. 262510. Authors are grateful to Daniel Kameny M.D. for reading the manuscript.

\section{References}

Badr-El-Dine M, El-Garem HF, Talaat AM, Magnan J (2002). Endoscopically assisted minimally invasive microvascular decompression of hemifacial spasm. Otol Neurotol 23:122-128.

Betka J, Zverina E, Lisy J, Chovanec M, Kluh J, Kraus J (2008). Vestibular schwannoma. Otorinolaryngol Phoniatr (Prague) 57:221-225.

Betka J, Zverina E, Chovanec M, Kluh J, Lukes P, Kraus J, Lisy J (2009). Minimally invasive endoscopic and endoscopy-assisted microsurgery of vestibular schwannoma. Endoskopie 18:67-71.

Bremond G, Garcin M, Magnan J, Bonnaud G (1974). L'abord a minima de l'espace pontocerebelleux. Cah ORL 19:443-460.

Bremond G, Garcin M (1975). Microsurgical approach to the cerebellopontine angle. J Laryngol Otol 89:237-248.

Brito Neto RV, Bento RF, Yasuda A, Ribas GC, Rodrigues AJ Jr (2005). Anatomical references in auditory brainstem implant surgery. Braz J Otorhinolaryngol 71:282286.

Cakrt O, Chovanec M, Funda T, Kalitova P, Betka J, Zverina E, Kolar P, Jerabek J (2010). Exercise with visual feedback improves postural stability after vestibular schwannoma surgery. Eur Arch Otorhinolaryngol 267:1355-1360.

Cappabianca P, Cavallo LM, Esposito F, de Divitiis E, Tschabitscher M (2002). Endoscopic examination of the cerebellar pontine angle. Clin Neurol Neurosurg 104:387-391.

Chen MJ, Zhang WJ, Yang C, Wu YQ, Zhang ZY, Wang Y (2008). Endoscopic neurovascular perspective in microvascular decompression of trigeminal neuralgia. J Craniomaxillofac Surg 36:456-461.

Cheng WY, Chao SC, Shen CC (2008). Endoscopic microvascular decompression of the hemifacial spasm. Surg Neurol 70 Suppl 1:40-46. 
Cheng S, Naidoo Y, da Cruz M, Dexter M (2009). Quality of life in postoperative vestibular schwannoma patients. Laryngoscope 119:2252-2257.

Clemis JD, Ballad WJ, Baggot PJ, Lyon ST (1986). Relative frequency of inferior vestibular schwannoma. Arch Otolaryngol Head Neck Surg 112:190-194.

Colletti V, Fiorino FG, Carner M, Giarbini N, Sacchetto L, Cumer G (2000). Advantages of the retrosigmoid approach in auditory brain stem implantation. Skull Base Surg 10:165-170.

Colletti V, Fiorino F (2005). Is the middle fossa approach the treatment of choice for intracanalicular vestibular schwannoma? Otolaryngol Head Neck Surg 132:459466.

de Divitiis O, Cavallo LM, Dal Fabbro M, Elefante A, Cappabianca P (2007). Freehand dynamic endoscopic resection of an epidermoid tumor of the cerebellopontine angle: technical case report. Neurosurgery 61:239-240.

Doyen E (1917). In: Surgical therapeutics and operative techniques, vol. 1., 599-602, Balliere, Tindall and Cox, London.

Ebner FH, Roser F, Shiozawa T, Ruetschlin S, Kirschniak A, Koerbel A, Tatagiba M (2009). Petrosal vein occlusion in cerebello-pontine angle tumour surgery: an anatomical study of alternative draining pathways. Eur J Surg Oncol 35:552-556.

Eby JB, Cha ST, Shahinian HK (2001). Fully endoscopic vascular decompression of the facial nerve for hemifacial spasm. Skull Base 11:189-197.

El-Garem HF, Badr-El-Dine M, Talaat AM, Magnan J (2002). Endoscopy as a tool in minimally invasive trigeminal neuralgia surgery. Otol Neurotol 23:132-135.

Friedland DR, Wackym PA (1999). Evaluation of surgical approaches to endoscopic auditory brainstem implantation. Laryngoscope 109:175-180.

Fries G, Perneczky A (1998). Endoscope-assisted brain surgery: Part 2-analysis of 380 procedures. Neurosurgery 42:226-232.

Fukushima T (1978). Endoscopy of Meckel's cave, cisterna magna and cerebellopontine angle. Technical note. J Neurosurg 48:302-306.

Gerganov VM, Giordano M, Herold C, Samii A, Samii M (2010). An electrophysiological study on the safety of the endoscope-assisted microsurgical removal of vestibular schwannomas. Eur J Surg Oncol 36:422-427.

Gerosa M, Mesiano N, Longhi M, De Simone A, Foroni R, Verlicchi A, Zanotti B, Nicolato A (2010). Gamma Knife surgery in vestibular schwannomas: impact on the anterior and posterior labyrinth. J Neurosurg 113 Suppl:128-135.

Goksu N, Bayazit Y, Kemaloglu Y (1999). Endoscopy of the posterior fossa and dissection of acoustic neuroma. J Neurosurg 91:776-780.

Goksu N, Yilmaz M, Bayramoglu I, Aydil U, Bayazit YA (2005). Evaluation of the results of endoscope-assisted acoustic neuroma surgery through posterior fossa approach. ORL J Otorhinolaryngol Relat Spec 67:87-91.

Goksu N, Bayazit YA, Bayramoglu I, Isik B, Yilmaz M, Kurtipek O, Uygur K (2006). Surgical exposure in retrosigmoid approach: do we need cerebellar retractors? Surg Neurol 65:631-634.

Griffith HB (1975). Technique of fontanelle and presutural ventriculoscopy and endoscopic ventricular surgery in infants. Childs Brain 1:359-363. 
Guevara N, Deveze A, Buza V, Laffont B, Magnan J (2008). Microvascular decompression of cochlear nerve for tinnitus incapacity: pre-surgical data, surgical analyses and long-term follow-up of 15 patients. Eur Arch Otorhinolaryngol 265:397-401.

Hori T, Okada Y, Maruyama T, Chernov M, Attia W (2006). Endoscope-controlled removal of intrameatal vestibular schwannomas. Minim Invasive Neurosurg 49:25-29.

Jarrahy R, Young J, Berci G, Shahinian HK (1999). Endoscopic skull base surgery II: a new animal model for surgery of the posterior fossa. J Invest Surg 12:335339.

Jarrahy R, Berci G, Shahinian HK (2000). Endoscope-assisted microvascular decompression of the trigeminal nerve. Otolaryngol Head Neck Surg 123:218-223.

Jarrahy R, Cha ST, Eby JB, Berci G, Shahinian HK (2002a). Fully endoscopic vascular decompression of the glossopharyngeal nerve. J Craniofac Surg. 13:9095.

Jarrahy R, Eby JB, Cha ST, Shahinian HK (2002b). Fully endoscopic vascular decompression of the trigeminal nerve. Minim Invasive Neurosurg 45:32-35.

Kabil MS, Eby JB, Shahinian HK (2005). Endoscopic vascular decompression versus microvascular decompression of the trigeminal nerve. Minim Invasive Neurosurg 48:207-212.

Kabil MS, Shahinian HK (2006). A series of 112 fully endoscopic resections of vestibular schwannomas. Minim Invasive Neurosurg 49:362-368.

Koval J, Molcan M, Bowdler AD, Sterkers JM (1993). Retrosigmoid transmeatal approach: an anatomic study of an approach used for preservation of hearing in acoustic neuroma surgery and vestibular neurotomy. Skull Base Surg 3:16-21.

Koval J, Krempaska S, Kaliarik L (2009). Minimal retrosigmoidal approach with applied endoscopy. Otorinolaryngol Phoniatr (Prague) 58:8-14.

Koval J, Krempaska S (2009). Anatomic logic of the foramen Luschka in neurootologic surgery. Otolaryngol Pol 63:398-402.

King WA, Wackym PA (1999). Endoscope assisted Surgery for acoustic neuromas Vestibular Schwannomas): early experience using the rigid hopkins telescope. Neurosurgery 44:1095-1102.

Koerbel A, Gharabaghi A, Safavi-Abbasi S, Samii A, Ebner FH, Samii M, Tatagiba M (2009). Venous complications following petrosal vein sectioning in surgery of petrous apex meningiomas. Eur J Surg Oncol 35:773-779.

Linskey ME (2000). Stereotactic radiosurgery versus stereotactic radiotherapy for patients with vestibular schwannoma: a Leksell Gamma Knife Society 2000 debate. J Neurosurg 93 Suppl 3:90-95.

Low WK (1999a). Middle cranial fossa approach to the internal auditory Meatus:A Chinese temporal bone study. ORL J Otorhinolaryngol Relat Spec 61:142145.

Low WK (1999b). Enhancing hearing preservation in endoscopic-assisted excision of acoustic neuroma via the retrosigmoid approach. J Laryngol Otol 113:973977. 
Magnan J, Chays A, Caces F, Lepetre C, Cohen JM, Belus JF, Bruzzo M (1993). Contribution of endoscopy of the cerebellopontine angle by retrosigmoid approach. Neuroma and neuro-vascular de compression. Ann Otolaryngol Chir Cervicofac 110:259-265.

Magnan J, Chays A, Lepetre C, Pencroffi E, Locatelli P (1994). Endoscopy of the cerebellopontine angle. Am J Otol 15:366-370.

Magnan J, Caces F, Locatelli P, Chays A (1997). Hemifacial spasm: endoscopic vascular decompression. Otolaryngol Head Neck Surg 117:308-314.

Magnan J, Barbieri M, Mora R, Murphy S, Meller R, Bruzzo M, Chays A (2002). Retrosigmoid approach for small and medium-sized acoustic neuromas. Otol Neurotol 23:141-145.

Martin HC, Sethi J, Lang D, Neil-Dwyer G, Lutman ME, Yardley L (2001). Patient-assessed outcomes after excision of acoustic neuroma: postoperative symptoms and quality of life. J Neurosurg 94:211-216.

McKennan K (1993). Endoscopy of the internal auditory canal during hearing conservation acoustic neuroma surgery. Am J Otol 14:259-262.

Mer SB, Derbyshire AJ, Brushenko A, Pontarelli DA (1967). Fiberoptic endotoscopes for examining the middle ear. Arch Otolaryngol 85: 387-393.

Miyazaki H, Deveze A, Magnan J (2005). Neuro-otologic surgery through minimally invasive retrosigmoid approach: endoscope assisted microvascular decompression, vestibular neurotomy, and tumor removal. Laryngoscope 15:1612-1617.

Myrseth E, Møller P, Wentzel-Larsen T, Goplen F, Lund-Johansen M (2006). Untreated vestibular schwannomas: vertigo is a powerful predictor for health-related quality of life. Neurosurgery 59:67-76.

Myrseth E, Pedersen PH, Møller P, Lund-Johansen M (2007). Treatment of vestibular schwannomas. Why, when and how? Acta Neurochir (Wien) 149:647-660.

Myrseth E, Møller P, Pedersen PH, Lund-Johansen M (2009). Vestibular schwannoma: surgery or gamma knife radiosurgery? A prospective, nonrandomized study. Neurosurgery 64:654-661.

O'Donoghue GM, O'Flynn P (1993). Endoscopic anatomy of the cerebellopontine angle. Am J Otol 14:122-125.

Oppel F (1978). Endoscopy of the cerebello-pontine angle: its diagnostic and therapeutic possibilities. In: Advances and Technical Standards in Neurosurgery, vol. 5, 269-275, Springer-Verlag, ISBN 0387814418, Berlin.

Oppel F (1981). Indications and operative technique for endoscopy of the cerebellopontine angle. In: The Cranial Nerves, Samii M, Jannetta P, 429-437, Springer-Verlag, ISBN 0387106200, Berlin.

Ozluoglu LN, Akbasak A (1996). Video endoscopy-assisted vestibular neurectomy: a new approach to the eighth cranial nerve. Skull Base Surg 6:215-219.

Perneczky A, Fries G (1998). Endoscope-assisted brain surgery: part 1--evolution, basic concept, and current technique. Neurosurgery 42:219-224.

Prott W (1974). Cisternoscopy endoscopy of the cerebello-pontine angle. Acta Neurochir 31:105-113. 
Putnam TJ (1942). The surgical treatment of infantile hydrocephalus. Surg Gynecol Obstet 76:171-182.

Rhoton AL Jr (2000a). The cerebellopontine angle and posterior fossa cranial nerves by the retrosigmoid approach. Neurosurgery 47 Suppl 3:93-129.

Rhoton AL Jr (2000b). The temporal bone and transtemporal approaches. Neurosurgery 47 Suppl 3: 211-265.

Rhoton AL Jr (2000c). The cerebellar arteries. Neurosurgery 47 Suppl 3: 29-68.

Rhoton AL Jr (2000d). The posterior fossa veins. Neurosurgery 47 Suppl 3:69-92.

Rosenberg SI, Silverstein H, Willcox TO, Gordon MA (1994). Endoscopy in otology and neurootology. Am J Otol 15:168-172.

Roser F, Tatagiba MS (2010). The first 50s: can we achieve acceptable results in vestibular schwannoma surgery from the beginning? Acta Neurochir (Wien) 152:1359-1365.

Ryzenman JM, Pensak ML, Tew JM Jr (2005). Headache: a quality of life analysis in a cohort of 1,657 patients undergoing acoustic neuroma surgery, results from the acoustic neuroma association. Laryngoscope 115:703-711.

Sakamoto GT, Blevins N, Gibbs IC (2009). Cyberknife radiotherapy for vestibular schwannoma. Otolaryngol Clin North Am 42:665-675.

Saman Y, Bamiou DE, Gleeson M (2009). A contemporary review of balance dysfunction following vestibular schwannoma surgery. Laryngoscope 119:2085-93.

Samii M, Matthies C (1997). Management of 1000 vestibular schwannomas (acoustic neuromas): the facial nerve-preservation and restitution of function. Neurosurgery 40:684-694.

Sandooram D, Grunfeld EA, McKinney C, Gleeson MJ (2004). Quality of life following microsurgery, radiosurgery and conservative management for unilateral vestibular schwannoma. Clin Otolaryngol Allied Sci 29:621-627.

Schroeder HW, Oertel J, Gaab MR (2004). Endoscope-assisted microsurgical resection of epidermoid tumors of the cerebellopontine angle. J Neurosurg 101:227232.

Schroeder HW, Nehlsen M (2009). Value of high-definition imaging in neuroendoscopy. Neurosurg Rev 32:303-308.

Shahinian HK, Eby JB, Ocon M (2004). Fully endoscopic excision of vestibular schwannomas. Minim Invasive Neurosurg 47:329-332.

Skrivan J, Zverina E, Betka J, Svetlik M, Kluh J, Sollmann WP, Kraus J, Belsan T, Tichy T, Sedlak S, Topol M (2003). Use of the auditory brainstem neuroprosthesis in the Czech Republic. Cas Lek Cesk 142:29-33.

Skrivan J, Zverina E, Betka J, Kluh J, Kraus J (2004). Our surgical experience with large vestibular schwannomas. Otolaryngol Pol 58:69-72.

Springborg JB, Poulsgaard L, Thomsen J (2008). Nonvestibular schwannoma tumors in the cerebellopontine angle: a structured approach and management guidelines. Skull Base 18:217-227.

Stangerup SE, Tos M, Thomsen J, Caye-Thomasen P (2010). True incidence of vestibular schwannoma? Neurosurgery 67:1335-1340. 
Sughrue ME, Yang I, Aranda D, Kane AJ, Parsa AT (2010a). Hearing preservation rates after microsurgical resection of vestibular schwannoma. J Clin Neurosci 17:11261129.

Sughrue ME, Yang I, Rutkowski MJ, Aranda D, Parsa AT (2010b). Preservation of facial nerve function after resection of vestibular schwannoma. Br J Neurosurg 24):666671.

Sughrue ME, Kane AJ, Kaur R, Barry JJ, Rutkowski MJ, Pitts LH, Cheung SW, Parsa AT (2011). A prospective study of hearing preservation in untreated vestibular schwannomas. J Neurosurg 114:381-385.

Tallan EM, Harner SG, Beatty CW, Scheithauer BW, Parisi JE (1993). Does the distribution of Schwann cells correlate with the observed occurrence of acoustic neuromas? Am J Otol 14:131-134.

Tatagiba M, Matthies C, Samii M (1996). Microendoscopy of the internal auditory canal in vestibular schwannoma surgery. Neurosurgery 38:737-740.

Valtonen HJ, Poe DS, Heilman CB, Tarlov EC (1997). Endoscopically assisted prevention of cerebrospinal fluid leak in suboccipital acoustic neuroma surgery. Am J Otol 18:381-85.

Wackym PA, King WA, Barker FG, Poe DS (1998). Endoscope-assisted vestibular neurectomy. Laryngoscope 108:1787-1793.

Wackym PA, King WA, Poe DS, Meyer GA, Ojemann RG, Barker FG, Walsh PR, Staecker H (1999). Adjunctive use of endoscopy during acoustic neuroma surgery. Laryngoscope 109:1193-1201.

Wackym PA, King WA, Meyer GA, Poe DS (2002). Endoscopy in neuro-otologic surgery. Otolaryngol Clin North Am 35:297-323.

Wackym PA (2005). Stereotactic radiosurgery, microsurgery, and expectant management of acoustic neuroma: basis for informed consent. Otolaryngol Clin North Am 38:653670.

Wackym PA, Hannley MT, Runge-Samuelson CL, Jensen J, Zhu YR (2008). Gamma Knife surgery of vestibular schwannomas: longitudinal changes in vestibular function and measurement of the Dizziness Handicap Inventory. J Neurosurg 109 Suppl:137-143.

Wanibuchi M, Fukushima T, McElveen JT Jr, Friedman AH (2009). Hearing preservation in surgery for large vestibular schwannomas. J Neurosurg 111:845-854.

Yamakami I, Uchino Y, Kobayashi E, Yamaura A (2003). Conservative management, gamma-knife radiosurgery, and microsurgery for acoustic neurinomas: a systematic review of outcome and risk of three therapeutic options. Neurol Res 25:682-690.

Yang I, Sughrue ME, Han SJ, Aranda D, Pitts LH, Cheung SW, Parsa AT (2010). A comprehensive analysis of hearing preservation after radiosurgery for vestibular schwannoma. J Neurosurg 112:851-859.

Yuguang L, Chengyuan W, Meng L, Shugan Z, Wandong S, Gang L, Xingang L (2005). Neuroendoscopic anatomy and surgery of the cerebellopontine angle. J Clin Neurosci 12:256-260. 
Zverina E (2010). Acoustic neuroma--vestibular schwannoma--personal experience of up-todate management. Cas Lek Cesk 149:269-276. 


\section{Part 3}

Endoscopy in Ophtalmology 



\title{
Ocular Endoscopy
}

\author{
Durval Moraes de Carvalho', Francisco Eduardo Lima² \\ and Durval Moraes de Carvalho Jr ${ }^{3}$ \\ ${ }^{1,2}$ Centro Brasileiro de Cirurgia de Olhos \\ ¿Universidade Federal de Goiás - UFG \\ ${ }^{3}$ Centro Brasileiro da Visão \\ Brazil
}

\section{Introduction}

The ocular endoscopy is available to the ophthalmology since the early 90 's, however, until now there has not been consolidated as an essential tool to the world's ophthalmology (Uram, 1992b). The intraocular view is usually possible through adequate light and lens devices, due to the transparency of the part. These tools are practical and they may be also economical; in the cases which the cornea, anterior chamber, crystalline and vitreous are confusing the transparency, the ophthalmology has other resources using ultrasound and $x$ ray devices which enable the insight of the intraocular problems.

Nowadays the ocular endoscopy is an important resource to ophthalmology practice. Besides the sporadic use of it to the intraocular visualization, there are two other procedures in which it is essential: (a) In glaucoma surgery, Endoscopic cyclophotocoagulation (ECP) (Chen et al., 1997; Lima, 2000; Uram, 1992a; Lima, 1997). (b) In the implantation of intraocular lenses (IOL) in eyes without a capsular support (Scleral fixation) (Carvalho et al., 1996).

\section{Glaucoma surgery using endoscopy: The Carvalho-Lima technique}

The Glaucoma is an illness of the optical nerve which is irreversible and the intraocular pressure (IOP) is the most frequent cause of it. The primary open-angle glaucoma (POAG) is far the most common type of glaucoma in clinical practice, initially being treated with eye drops. For the cases in which the glaucoma surgery is indicated there are mainly two approaches:

One of them which is classical in all the world is the trying to increase the drainage of aqueous flow, the so called filtering surgeries. The main complications associated with filtering surgeries are due either to scaring process or overfiltration.

The other surgical approach to treat glaucoma is based on diminishing the aqueous production, and have the IOP reduced. Those are the cyclodestructive procedures. These procedures were usually performed by a transscleral route, either by freezing the ciliary body (cyclocryotherapy) or by coagulating the ciliary body with a laser source. Because the surgeon is not able to see the targets being treated, adjacent tissues may be damaged during 
this process, which may contribute to a relatively high rate of complications, such as pain, visual acuity reduction, inflammation, hypotony and phthisis bulbi.

Recently, a new device (Fig. 1) that combines a laser source (Uram, 1992b), endoscopic probe (Fig. 2) and an illumination beam in the same probe has been developed. This instrument has the unique ability of simultaneous visualization and treatment of the ciliary body through a pars plana or anterior segment approach, or even combined with a cataract extraction. Additionally, some authors (Chen et al., 1997; Lima, 2000; Uram, 1992a; Lima, 1997) have demonstrated the safety and efficacy of the endocyclophotocoagulation (ECP) for treatment of refractory glaucoma.

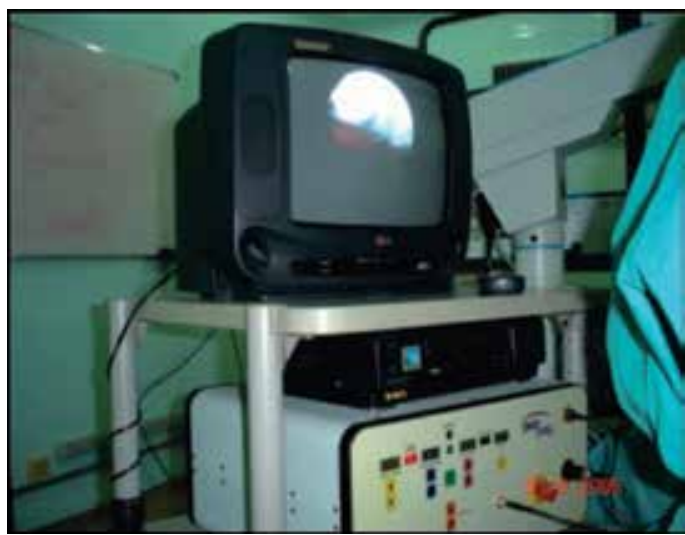

Fig. 1. Ocular endoscopy device; LASER (MicroProbe, ENDOOPTIKS, Little Silver, NJ, USA).

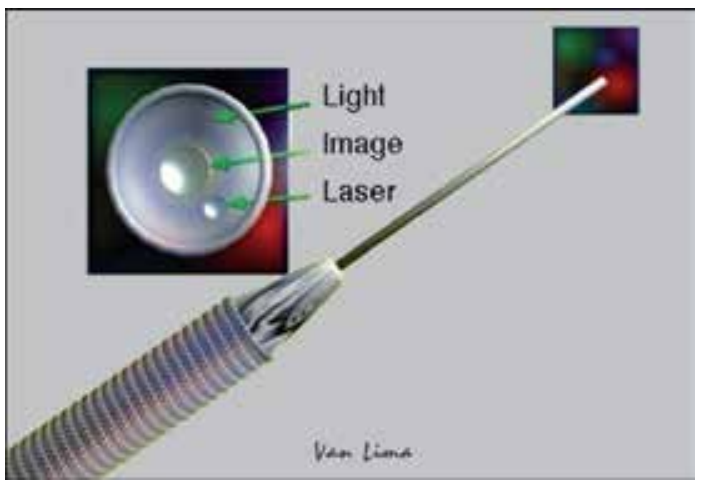

Fig. 2. The ECP probe showing its layers of optic fiber responsible for the light, videoendoscopy and LASER.

The ECP was done with a commercially available device (MicroProbe, ENDOOPTIKS, Little Silver, NJ, USA) with an endoscope with a 110-degree field of view and a focal distance of $2 \mathrm{~mm}$, camera and an $810 \mathrm{~nm}$ wavelength diode laser source with maximum power of $1.2 \mathrm{~W}$. In most of the cases the laser power used is $0.5 \mathrm{~W}$, continuous mode for approximately 2 seconds to produce both whitening and shrinkage of the ciliary processes. Laser power and/or duration is decreased if a "pop" is heard. 
In phakic eyes the Carvalho-Lima's technique is indicated.

Carvalho-Lima's technique consists of phacoemulsification combined with ECP. ECP is acompplished through the bag and before the intraocular lens implantation (Fig. 3).

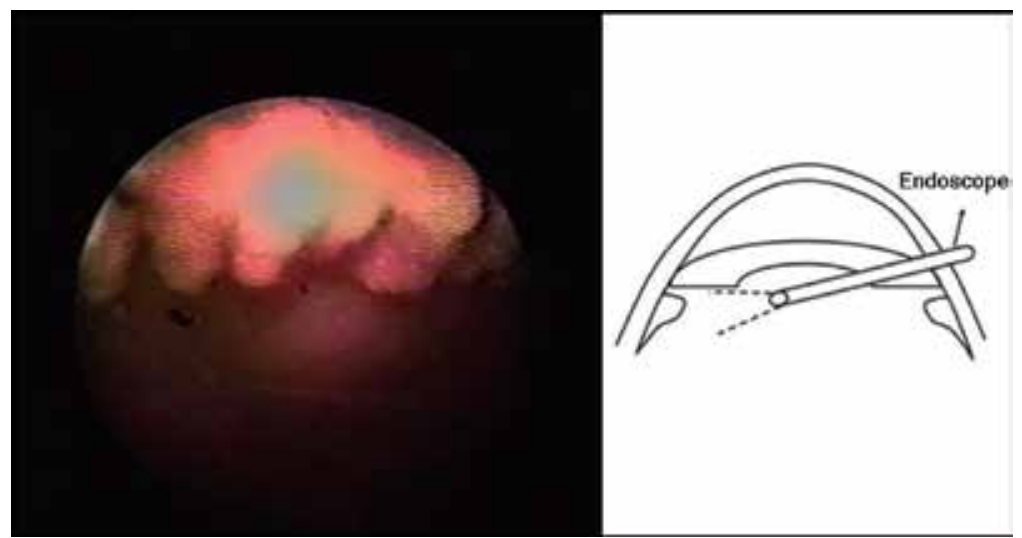

Fig. 3. Photocoagulation of the most bulging part of the ciliary process.

External scleral depression is performed to facilitate the photocoagulation of the entire ciliary process, including the valleys between the the crests of the pars plicata and the anterior third of the pars plana. Additionally, external scleral depression is usefull to guide the surgeon on how much of the ciliary process has been photocoagulated. This technique won a prize in the movies festival of ASCRS in 1999 (Fig. 4).

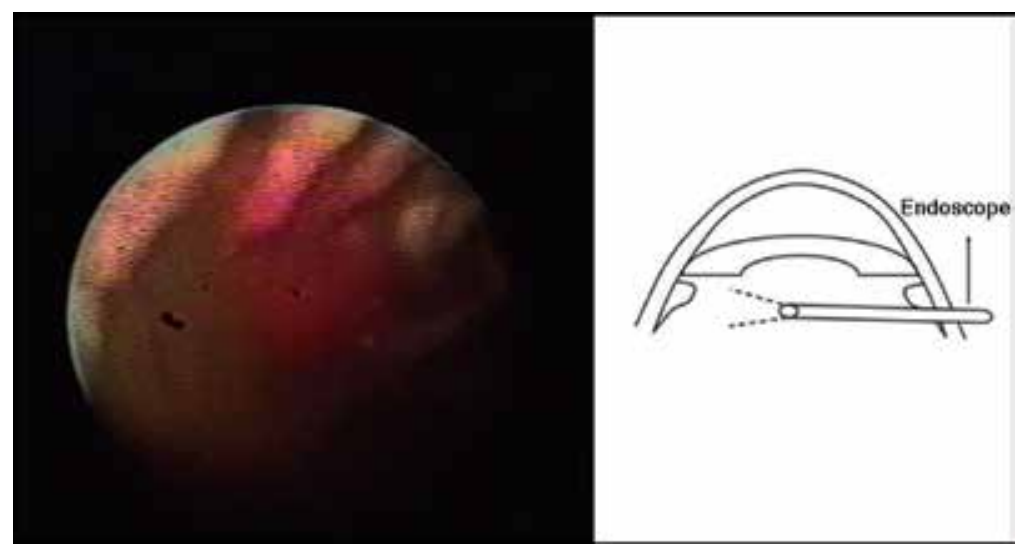

Fig. 4. Photocoagulation of the tail of the ciliary process with scleral depression.

In pseudophakic eyes the procedure is performed by a superior temporal pars plana incision, $3.5 \mathrm{~mm}$ from the limbus after anterior vitrectomy.

In all eyes ECP is done to $210^{\circ}$ of the ciliary body, corresponding to 2 to 9 hours in the right eye and from 3 to 10 hours in the left eye, including the anterior third of the pars plana. Subconjunctival injection of tobramycin and dexamethasone is performed after each procedure. Additionally, $0.1 \mathrm{ml}$ of dexamethasone was injected in the anterior chamber. Topical antibiotics, corticosteroids and atropine were prescribed post-operatively and tapered as the intraocular inflammation decreased. 
It is has been observed that when the laser was used in the pars plana just behind the ciliary processes the hypotensive effect was greater. Would pars plana also be a secretor of aqueous? An experimental work done by Dr Durval M. Carvalho JR, MD (Carvalho Jr, 2002) in 2002 was to make it clear most of our doubts. The photocoagulation caused by the ECP causes three different tissue reactions, which varies according to the region of the ciliary body (Fig. 5 and 6), the intensity and time exposed to the laser. The first reaction observed is the whitening of the surface and if the laser exposition continues there is a shrinkage of the ciliary process. If the exposition is excessive, the energy is stored inside the ciliary process and it causes its explosion, emitting a short sound, possible to be heard (pop). The pop indicates overtreatment and is associated with hemorrhage, pigment dispersion and inflammatory reaction. Even though the explosion guarantees the destruction of the ciliary process, it must be avoided so as to cause less side effects.

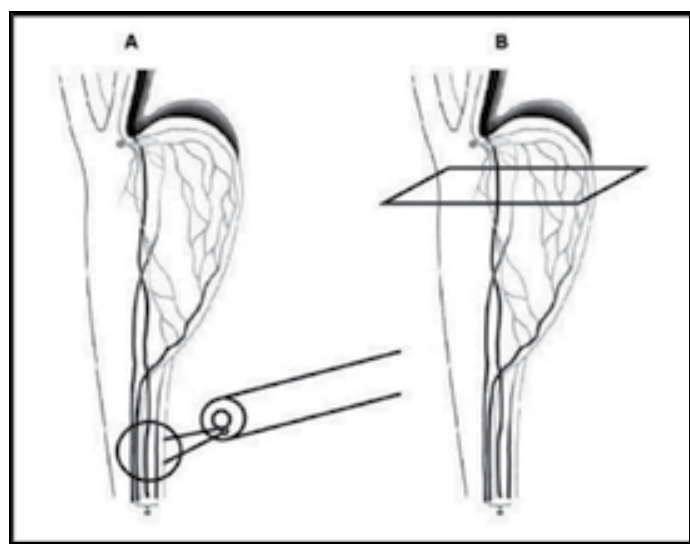

Fig. 5. Photocoagulation of the pars plana.

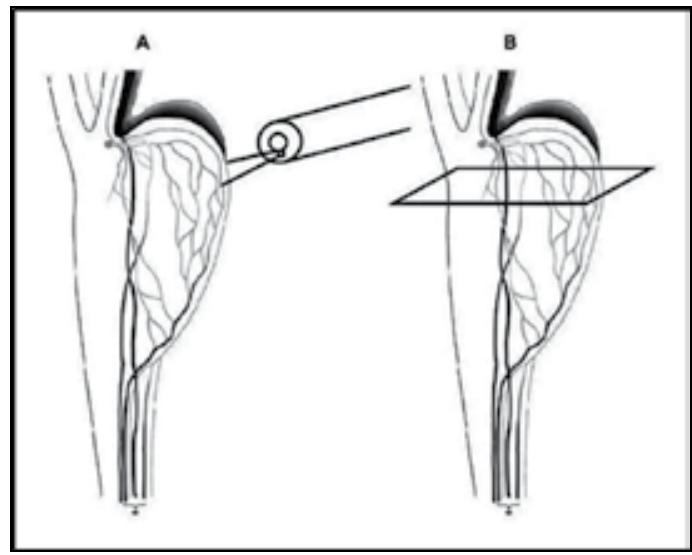

Fig. 6. Photocoagulation of the pars plicata.

In an experimental study (Carvalho Jr, 2002) evaluated the effect of the ECP in the different parts of the ciliary body in rabbit eyes. In the hystopathological analyses (Fig. 7) it was observed an indirect effect of the laser in the microcirculation of the ciliary body and not only the direct lesion to the epithelium. One of the most elucidative founds was the presence 
of vascular congestion of all the ciliary process when only the pars plana was photocoagulatedm suggesting strong suspect of a coagulative trombosis caused by the laser (Fig. 8).

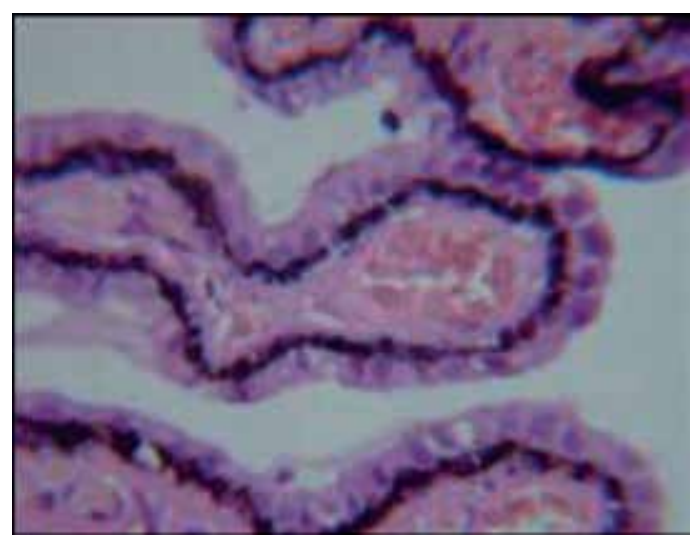

Fig. 7. Hystopathological photograph of a ciliary process of the eye of a rabbit without structure alterations.

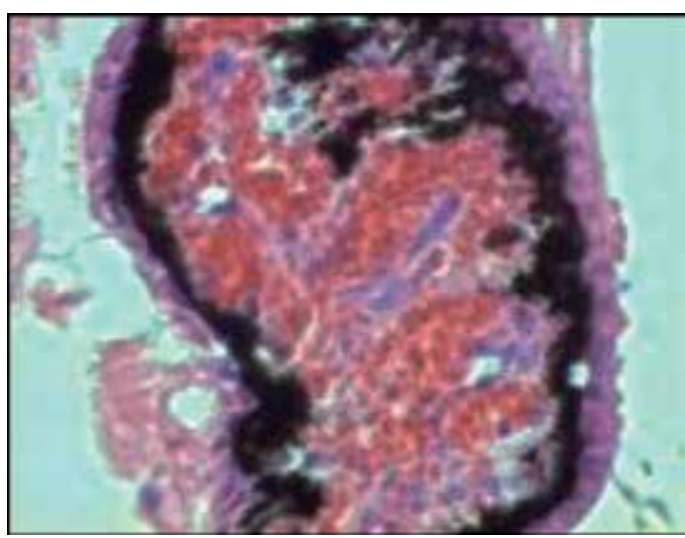

Fig. 8. Ciliary process enlarged with a vascular congestion after recent photocoagulation of the pars plana region.

So these findings may explain in part the variation of results according to the extension to be treated. The applications only in the part of the head (anterior) of the ciliary process can not achieve all the venule arcades, justifying this way an effect bellow the expected, and also explain the eyes treated $360^{\circ}$ not to evolute to a bulbar atrophy. On the other hand the treatment only of the pars plana region reaches a higher hypotensor effect, justifying some cases of transcleral cyclophotocoagulation being effective achieving only the pars plana region. The practical application of this found comes to help with the interest of treating a shorter extension of the ciliary body and obtain higher effectivity reducing the IOP, reducing the risk of complications such as the bulbar atrophy. For this the application must be selective and assisted where the endoscopy showed superior itself, although this is an invasive procedure. 
Lima and other authors, (Lima et al., 1997; Lima et al., 2004) obtained a clinical evidence in cases of refractory glaucoma treated by ECP, being photocoagulated all the ciliary process including its most posterior portion with the help of a scleral depression in a shorter extension. The reduction of the IOP to acceptable levels and its maintenance as time passes, and also the shorter risk of hypotony and bulbar atrophy has increased the confidence in the technique, that seems to be successesful to other indications and not only as the last option. Beyond the treatment of glaucoma and cataract combined, the main indications to ECP are:

- $\quad$ Eyes submitted to, at least, a filtration surgery with antimetabolics;

- As a primary procedure in eyes with glaucoma associated to a penetrating keratoplasty;

- As a primary procedure in neovascular glaucoma;

- Eyes submitted to drainage implant surgery;

- Eyes with the conjunctive attached and healed after the cataract surgery, retine surgeries, burn, traumas, etc.;

- Eyes with a congenital glaucoma after goniotomy and or trabeculotoctomy and a trabeculectomy.

Lima and other authors (Lima et al., 2004) have developed a prospective study to compare endoscopic cyclophotocoagulation (ECP) and the Ahmed drainage implant in the treatment of refractory glaucoma.

Sixty-eight eyes of 68 patients with refractory glaucoma were prospectively assigned to either ECP or Ahmed tube shunt implantation. All procedures were performed by a single surgeon. Eyes that were included were pseudophakic with a history of at least one trabeculectomy with antimetabolite, an intraocular pressure (IOP) equal to or above $35 \mathrm{mmHg}$ on maximum tolerated medical therapy, and a visual acuity better than light perception.

Exclusion criteria included eyes that had had previous glaucoma drainage device implantation or a cyclodestructive procedure. Success was defined as an IOP more than 6 $\mathrm{mmHg}$ and less than $21 \mathrm{mmHg}$, with or without topical anti-hypertensive therapy.

The mean follow-up was $19.82 \pm 8.35$ months and $21.29 \pm 6.42$ months, for the Ahmed and ECP groups, respectively $(\mathrm{p}=0.4)$. The pre-operative IOP, $41.32 \pm 3.03 \mathrm{mmHg}$ (Ahmed) and $41.61 \pm 3.42 \mathrm{mmHg}(\mathrm{ECP})(\mathrm{p}=0.5)$, and the mean post-operative IOP, at 24 months followup, $14.73 \pm 6.44 \mathrm{mmHg}$ (Ahmed) and $14.07 \pm 7.21 \mathrm{mmHg}(\mathrm{ECP})(\mathrm{p}=0.7)$, were significantly different from baseline in both groups $(\mathrm{p}<0.001)$. Kaplan-Meier survival curve analysis showed a probability of success at 24 months of $70.59 \%$ and $73.53 \%$ for the Ahmed and ECP groups respectively $(\mathrm{p}=0.7)$.

Complications included choroidal detachment (Ahmed 17.64\%, ECP 2.94\%), shallow anterior chamber (Ahmed 17.64\%, ECP 0.0\%) and hyphema (Ahmed 14.7\%, ECP 17.64\%).

In conclusion there was no difference in the success rate between the Ahmed Glaucoma Valve and ECP in refractory glaucoma. The eyes that underwent Ahmed tube shunt implantation had more complications than those treated with ECP.

A recent study (Lima et al., 2010) was designed to evaluate the safety and efficacy of phacoemulsification and endoscopic cyclophotocoagulation (ECP) as a primary surgical treatment for glaucoma and cataract.

Three hundred sixty-eight eyes from 243 patients with primary open-angle glaucoma and cataract that underwent an uncomplicated surgery from October 1998 to December 2006 with at least 2 years of follow-up were retrospectively enrolled. The patients were excluded if presented with a previous ocular history of any intraocular surgery or glaucoma laser treatment. Qualified success was defined as $5 \mathrm{mmHg}<$ intraocular pressure (IOP) $<21$ $\mathrm{mmHg}$ with or without topical antiglaucomatous drugs, and complete success as the same 
IOP levels without therapy at all timepoints. Additionally, the needed of any further glaucoma surgery was defined as failure.

The mean follow-up was $35.15 \pm 8.14$ months. The IOP pre-operatively $(23.07 \pm 5.52 \mathrm{mmHg})$ was significantly greater than in the first day post-operatively $(13.14 \pm 6.09 \mathrm{mmHg})$, and months $1(11.03 \pm 2.59 \mathrm{mmHg}), 6(12.33 \pm 3.01 \mathrm{mmHg}), 12(12.19 \pm 2.19 \mathrm{mmHg}), 24(12.14 \pm$ $2.89 \mathrm{mmHg})$ and in the last appointment $(12.29 \pm 2.44 \mathrm{mmHg})(\mathrm{p}<0.001$ in all timepoints $)$. The number of medications pre-operatively $(1.44 \pm 0.97)$ decreased $(0.37 \pm 0.74)(p<0,001)$. Furthermore, there was significantly improvement in the LogMar visual acuity $(p=0.01)$. $334(90.76 \%)$ eyes achieved qualified success, and 205 (55.7\%), complete success.

Complications included immediate post-operative IOP spike $14.4 \%$ (53/368), post-operative fibrin exudates in anterior chamber $7.06 \%$ (26/368), cystoid macular edema $4.34 \%(16 / 368)$, transitory hypotony $2.17 \%$ (8/368), iris bombé $1.08 \%(4 / 368)$.

Phacoemulsification associated with Endoscopic Cyclophotocoagulation is safe and effective as a primary procedure for combined glaucoma and cataract.

There is no gold standard for surgical treatment of glaucoma. All the available techiniques are unpredictable as far as the results and complications. Because of the direct approach to the ciliary body, the endoscopic cyclophotocoagulation (ECP) may be a reasonable option in these eyes.

\section{Endoscopy in scleral fixation}

In the past, cataract surgery just removed the opacified natural lens, living the patient without any substitute lens (aphakia), forced wear glasses with a very high degree. The history began to change in the war of 1945 when fragments of a transparent substance from the airplanes got incrusted in the eye of one of the pilots and it did not caused any reaction; based on this Dr. Nicholas Harold Ridley was able to have a lens of this material which PMMA (polimethilmetracrilate) made and implanted it in the eye of a human; this was the first experience of a lens implantation inside the eye; since then, the technological evolution so as to substitute the glasses by intraocular lens (IOL) has got no limits. There has been a great advance in the industries that make lens of different materials and functions, of equipments that make it easy the surgeries; surgical techniques more and more efficient and surgeons each time more able. Due to these concepts nobody else wants to wear glasses. Even all of this advanced technology, there are complicated cases of cataract surgery that intraocular lens support is lost. Not implant the intraocular lens is a great complication, making necessary use postoperatively glasses with high degrees from 12 to 13 diopters, causing a poor quality of vision. How to implant a intraocular lens in the eye which does not have the capsule to support it anymore? So as to answer these questions we brought togheter our experience with others surgeons experts in different techniques and wrote a book: "Cataract Surgery - Fixation and Secondary Implants" edited by Elsevier in Portuguese (by now). Either in the literature and also in the ophthalmological practice we noticed that we are able to gather the techniques of lens fixation in eyes without a capsular support in the following groups: Anterior Chamber Intraocular Lens; Fixating the lens at the Iris; Fixating the lens at the sclera.

\subsection{Anterior chamber intraocular lens}

Anterior Chamber Intraocular Lens is a intraocular lens adapted to the anterior segment and implanted at anterior chamber angle, transition between the corneal and iris. The ease of 
implantation is its advantage and the complications are related to corneal decompensation and iris inflammation.

\subsection{Fixation of the lens in the iris}

Fixation of the lens in the iris has the advantage of using most of the time the same intraocular lens of a regular cataract surgery. Its complications are most related to deviation of the pupil, iris inflammation, subluxation of the lens to fundus of the eye. This technique depends on good condition of the iris are therefore not for all cases.

\subsection{Scleral fixation with endoscopy}

We preferred the technique of fixation of the lens at the sclera because it can be applied in most of cases and intraocular lens stay closer to original position of the natural lens. Most of its complications like decentration of lens and cistic macular edema are related to incorrect positioning of the lens. These complications can be avoided by choosing the correct tecnique, its training and application of endoscopic fidings. Part of scleral fixation works behind the iris, exactly where the haptics of the lens are fixated at scleral wall. The haptics are tightened by unabsorbable suture, fixating them in the scleral with buried stitches. The difficulty is that the ideal place for positioning and fixating the lens is just behind the iris, on blind area. Many decentrations of lens had unknown causes, and could not be repaired due to lack of visualization.

With the advent of the endoscope was first presented by Leon and al. in 1991, allowed us expose the blind region. With the technological advance of the ocular endoscopy in the 90's it was supposed to be given a solution to this problem to fixate an intra-ocular lens because with the endoscopy it is possible to have a visualization of the region behind the iris. With endoscopy we find the answer of all decentrations of the lens. However we have this visualization difficulty during the execution of techniques of sclera fixation. We observed that to use the ocular endoscopy in the surgery of sclera fixation it is essential that our technique is used: Finger Crafted Technique (Carvalho et al., 2009). Since we started to work with ocular endoscopy (1996), we perform the surgeries of scleral fixation with total visualization of the ciliary body (behind the iris) at the moment of the line passage and we know exactly where the loop of the lens will be positioned. We have observed that the surgeons have had difficulties on the use of the endoscopy to this procedure recently, not because of the device but due to the surgery technique. Using the Finger Crafted Technique the surgeon handles the needle with the fingers and this permits total security and control to the movement in which the line is passed. In this technique, the other hand gets free enabling the use of the Endoscope easily and with practice. In my conclusion, the scleral fixation is not yet a reproductive technique, standardized and safe, not because of lack of the endoscopy, but because of the non-use of a technique that enable the use of the device with practice similar to what happens when the Finger Crafted Technique is used.

\subsection{Surgical technique 3.4.1 Materials}

The materials used in this surgical technique include a 30.5-gauge needle that is often used for insulin administration as a guide needle. Suture used in this technique is a 9-0 prolene suture with 2 needles of $0.65 \mathrm{~mm}$. A Blumental type of anterior maintainer is used to keep the anterior chamber from collapsing. A hook with a $\mathrm{T}$ tip is used as needed during the surgery. The endoscope provides direct visualization of the ciliary sulcus and aids in good positioning of the sutures and the IOL haptics (MicroProbe; Endooptiks, Little Silver, NJ). 
In all the other techniques which the needle is held by a needle holder and not by the fingers, at the moment of transfixation of the sclera, it is almost impossible or at least very hard to visualize with the endoscope and pass the line in the desirable local simultaneously. When the needle is handled with a needle holder, in all the moments, the other hand is needed to replace the needle on the needle holder and it is not possible to handle the endoscope. The details of this technique are complete in the link: Sclera Fixation of Posterior Chamber Lens Implant With Ocular Endoscopy= The Finger-Crafted Technique and Embedded Sclera Suture (Carvalho, D.M. \& D.M. Jr., 2007).

\subsubsection{Method}

Together with our book about sclera fixation, there is a DVD with the videos of each surgical technique. Due to the great diversity of cases that need the sclera fixation, we thought it would be better to gather the cases in 14 situations and so 14 videos. As an example: The eyes that are being operated from the cataract and it was not possible to implant the lens at that moment, and the surgeon decides to wait so as to implant a lens in a second oportunity, in these cases the surgical strategy to make the sclera fixation is completely different for a case that comes with an intraocular lens sprained for the vitreous cavity, as it is different to progam the sclera fixation in the eyes of people that are carriers of a crystalline bad formation syndrome such as the Marfan or Marchesane etc.

We will describe the Finger Crafted Technique when applicated in the surgery of an eye with a good vision potential and a carrier patient of a sprained lens for the vitreous. Let's imagine a patient that has already been operated of cataract for a while,and scratching the eye,the lens were sprained to the macula. Even having restriction in a case like this, there are several strategic possibilities. Let's supose it is a right eye, so we will observe first, what its ocular pressure is. The lens is next to the pupil or it is gathered to the retine? Suposing the ocular pressure over $25 \mathrm{~mm} / \mathrm{hg}$ and the lens next to the pupil we could program:

\subsubsection{Preparing the eye to an sclera fixation}

With the pupil dilated by eyedrops we make an incision in the conjunctiva in both sides of the eye, next to the EIXO of 3 and 9 hs. The conjunctiva is then folded in 2 equidistant points and the surgeon then performs a $1.5 \mathrm{~mm}$ sclerotomy perpendicular to the limbus, starting at $0.5 \mathrm{~mm}$. This sclerotomy should go down almost to the point of reaching the choroid.

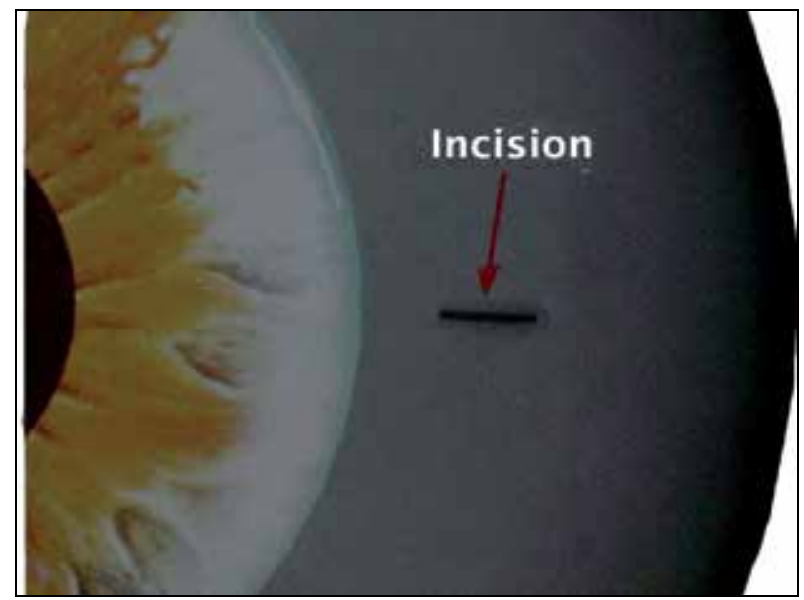

Fig. 9. Scleral Incision. 
While preparing the surgery table, the assistent surgeon can hand the surgeon the 9-0 prolene suture material, already cut in half, in the 30.5 gauge guide needle (insulin needle). The surgeon will hold the body of the needle with one hand and Mcpherson forceps are used to pass the threat through the bevel until the other end comes out of the base of the needle. This thread is then pulled until it is the same size as the portion not inserted in the needle.

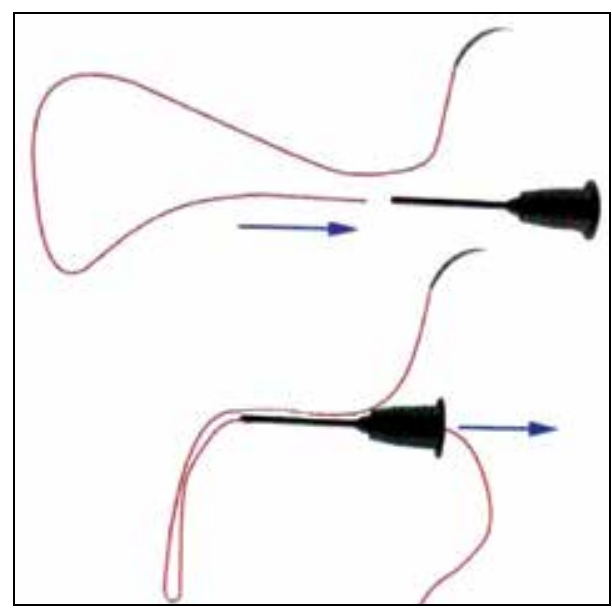

Fig. 10. Preparing the needle and bended needle prepared.

Using his fingers, the surgeon bends the tip of the needle to make it more ergonomic, pushing the eyelids away when the needle is introduced in the eye (fig. 10).

In the temporal limbus, around 7hs we perform a parecentese to the implantation to the chamber maintainer which keeps conected to a balanced salt solution, in a height of $10 \mathrm{~cm}$ over the eye level so as to keep it with a low pressure. Superior perictomy, opening the conjunctiva in an extension of $6 \mathrm{~mm}$. With the sclera exposed next to the superior limbus an incision is made on the frown (incision as inverted smile) also of $6 \mathrm{~mm}$ and it is made a

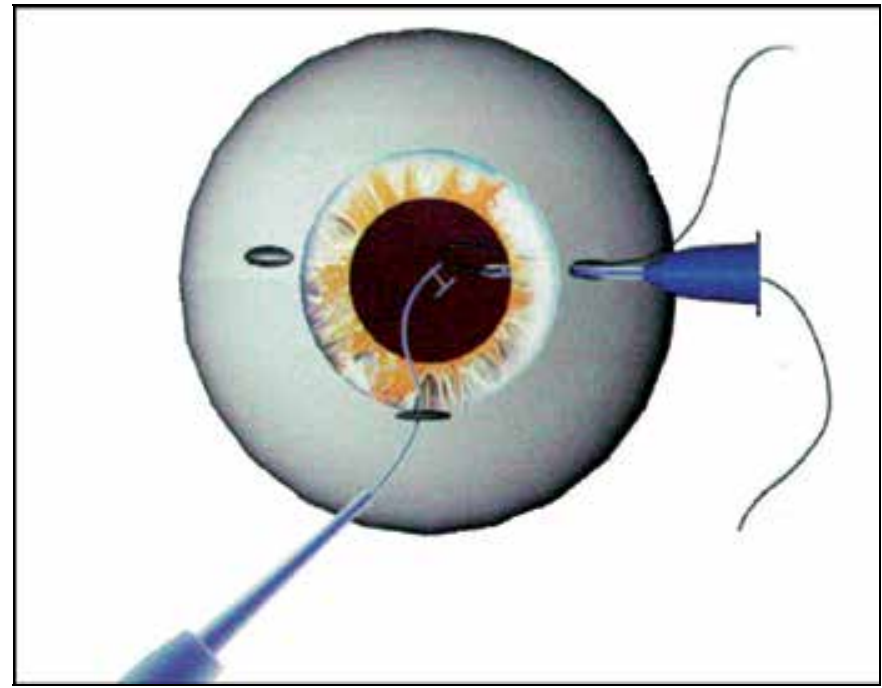

Fig. 11. Needle introduction. 
sclera delamination until we get to the cornea. In this phase, the penetration in the anterior chamber must be only of 2 to $2.50 \mathrm{~mm}$ in the middle of the incision even though the dilamination is of $6 \mathrm{~mm}$; This dilamination typical of the incisions used in the cataract surgeries for extracapsulars.

The surgeon introduces the endoscope through the main incision while, with the other hand, he introduces the previously threaded needle for the sclerotomy, which will be monitored as it penetrates the ciliary sulcus. Using the saline solution, placed about $80 \mathrm{~cm}$ above the eye surface, connected by the anterior chamber maintainer.

Once the tip of the needle is inserted, the solution is lowered to $10 \mathrm{~cm}$, the endoscope is removed and the needle is presented until it extends beyond the middle portion of the pupil. It is then retracted to loosen the loop of the thread which is found at the end, forming a loose loop. The other hand captures this loop with a hook, removing it from the eye.

The same maneuver is performed on the other side. Therefore, the main incision should be equidistant from the sclerotomy sites, to facilitate the view with the endoscope.

With the prolenes made apparently by the main incision, the incision is increased to the 6 $\mathrm{mm}$ completing the incision of the delamination previously elaborated.

The folded prolene (loops) are positioned on the side of the incision of the sclera delamination so as not to bother the lens to be taken away (fig. 12).

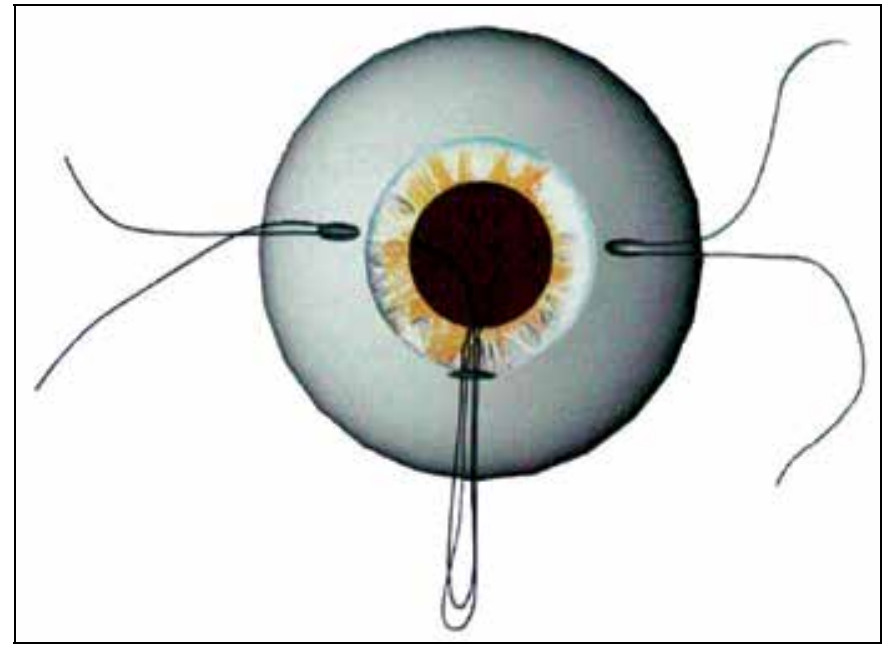

Fig. 12. Position of sutures after its introduction.

\subsubsection{Correcting the decentred IOL}

It is made one more parecentese of $1.50 \mathrm{~mm}$ in $2 \mathrm{hs}$ and it is introduced by it a hook with a straight holder so as to reach the haptic of the sprained lens in the vitreous. The lens is hold by the hook and it is moved softly and so as to avoid vitreous tractions in the moment of taking the lens we introduce the vitreofagus by the temporal sclerectomy, it is procedure one vitrectomy so as to totally set free the lens.

The lens is brought and kept in the anterior chamber by the hook and with the introduction of another hook by the main incision the function is passed to this new one which pulls and make it exterior the loop of the lens with the help of a spatula until it is taken from the eye (fig. 13). 


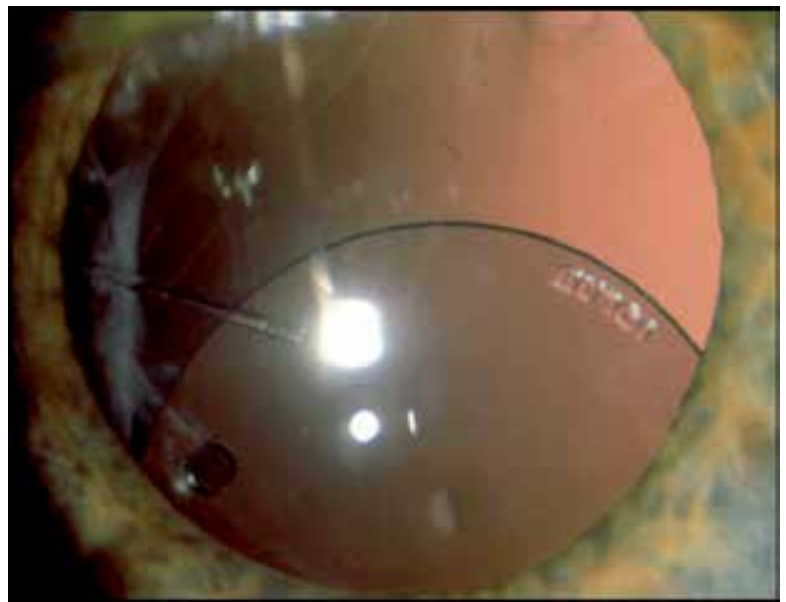

Fig. 13. Intraocular lens decentred.

\subsubsection{Implanting the IOL}

The chosen foldable, acrylic, monofocal or multifocal IOL in three pieces is placed on a support over the cornea, to prevent contamination.

Using those prolenes that were resting on the side of the main incision the first haptic is tied with three simple knots and so the lens is twisted and the second loop is also tied the same way (fig. 14).

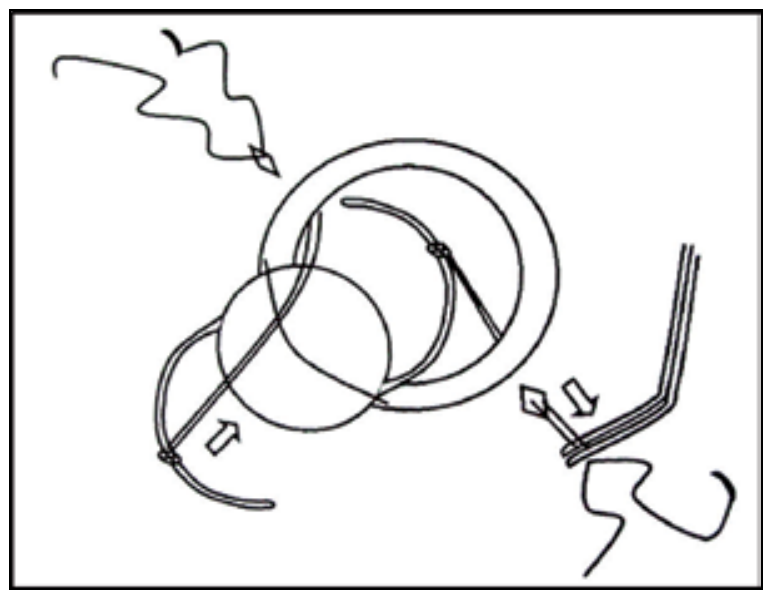

Fig. 14. Introducing lens attached to the prolene.

The anterior chamber is filled with viscoelastic, better if cohesive. In this moment it is closed the entrance of the balanced solution.

Carefully, with the MacPherson forceps the lens is introduced in the eye. In the same moment that it is being introduced, the prolenes that go out from the sclerectomy are pulled and held in the first haptic, so as to direct it to the ciliary sulcus.

The first haptic, positioned, the auxiliar will keep it in the same position holding the prolenes while the surgeon introduces and put in position the second haptic, using a forceps 
and the holding that keeps it. In this moment sometimes opening the infusion helps in the position of the iol.

After the IOL is positioned, the embedded Sclera Stitch is then put in place with the 2 prolenes from each sclerotomy, which came from the haptics. They are part of the same thread, and one end has been inserted into a needle while the other hasn't . The needle is then used to place a stitch outwards, on one of the borders, and then an inward stitch, on the other border. At the end, then, the embedded bared stitch is then placed in each sclerotomy. By tying the three single knots, the sclerotomy will be closed, thereby embedding the knots and fixating the IOL. After the prolenes are cut close to the sclera, the knots will tend to go deeper into the scleral incision and the tips will remain inside the sulcus (fig. 15).

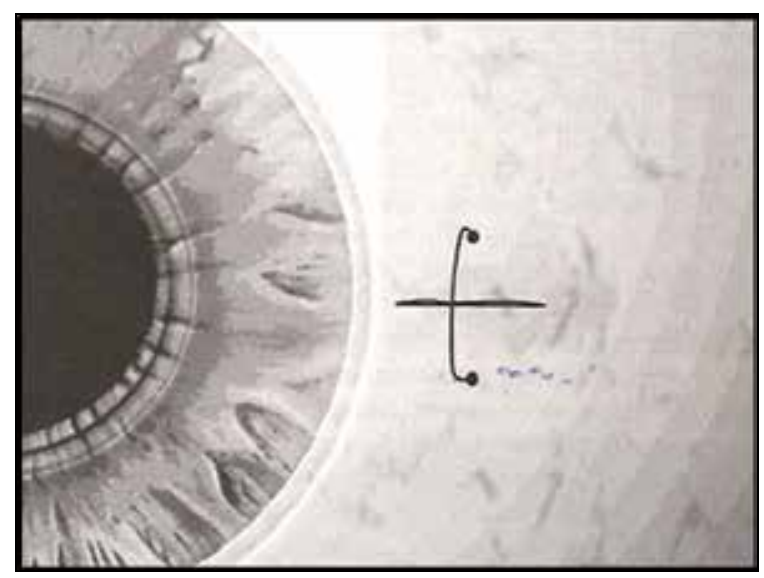

Fig. 15. Final Result of Buried Scleral Suture.

\subsubsection{Endocyclophotocoagulation}

As in this case the ocular pressure is high and, normally it tends to increase with the surgery, it was planned the endocyclophotocoagulation. When we notice that the lens is well stable in the eye we do not use its visualization by the endoscopy.

When the aim is only the photocoagulation:

The endoscope probe is introduced by the temporal sclerectomy and we perform the photocoagulation as it was already described before, reaching all the possible area of the ciliary body, when we use only an sclera incision. Generally we reach over 220 degrees.

After the endocyclophotocoagulation we take out the probe and introduces the vitreofagus through the same incision trying to take out the vitreous that may be held by the loops and that might get closed in the incisions. During the use of the vitreofagus it is necessary that the vial heigh is higher so as to balance the ocular pressure during the aspiration. At the end so as to avoid that the beams stay in the sclerectomy, we reduce the heigh of the vial before we take the vitreofagus and we also apirate the incision very well.

The sclerectomy is closed, the chamber mainteiner is taken, two or three stitches are made in the main incision so as to avoid the astigmatism, the conjunctiva is closed with absorbable prolenes.

The post operatory period is as simple as the cataract surgery post operatory where we generally use antibiotic eyedrops for 8 days, predinisolone eyedrops for 15 days and non hormonal antinflammatory eyedrops for for 30 days apllyed 4 times a day. 


\subsubsection{Discussion}

The videoendoscopy has a bunch of advantages for the sclera fixation. Before this videoendoscopy the patient and the surgeon used to have some complicationsm such as glaucoma, cystic macular edema, hemorrhage, descentration, IOL inclination without resourses for na immediate clear up.

The ocular endoscopy may help us in the implantation procedures of intraocular lens in eyes without a capsular support in several ways. For example:

\subsubsection{Diagnosis}

a. Inspect the retroirian region to identify vitreous beams, SINEQUIAS, and if the loop is positioned correctly in the recess.

b. Inspect the posterior pole about the retinian hemorrhagesm crystalline material, sprained IOL and others.

\subsubsection{Procedure assistence}

a. Monitoring the entrance of the needle by the sulcus;

b. Monitoring the vitreofagus point in the retroirian space and posterior pole;

c. Auxiliating in the repositioning of the sprained IOL;

d. Auxiliating in the needle penetration, as a support.

\subsubsection{Treatment}

a. Photocoagulate hemorrhage caused by the needle penetration;

b. Photocoagulate the ciliary processes for the treatment of associated glaucoma;

c. Photocoagulate the retine in the presence of associated retinian hemorrhages.

The penetration of the needle through the sclera monitored by the endoscope makes sure the thread fixation in the ciliary sulcus. It is a practical maneuver and it requires the pressurized eye through a maintainer and a protection with viscoelastic in the anterior chamber.As soon as the needle starts to be transfixated in the sclera,the surgeon stops looking through the microscope and follows through the screen the endoscope image. It is observed the showing of the identation through the tip of the needle, allowing its redirection to the ciliary sulcus if it shows in a wrong place.

This technique, besides making sure the correct position of the thread in the ciliary sulcus, it permits the photocoagulation of the hemorrhages that may appear due to the needle penetration, observed more frequently, in hypotensive eyes or with na inflammatory reaction sign.

Another resourse that this technique propotioned is the possibilty of impeding the uveal dislocation, pressing on the sclera the optic fiber itself, working as an opposite point and making it easier the passage of the needle.

With the use of the endoscopy in the sclera fixation it increased the precise to get right the ciliary sulcus during the needle perforation from outside to inside directed by the identation.

After implanting the lens it is possible to perform a review introducing behind the iris the endoscope probe to make sure that the lens is in the correct position. In the presence of descentration or inclination of the IOL in general we find one of the following alterations: Vitreous beam mixed with the haptic and the thread, the inadequate position in the introduction of the IOL, cortical mass lefts promoting kind of a gear over the haptic, fibrosis, rest of capsule gathered in the iris, dislocation of the knot on the holder and others. 
When the haptic, of a sprained IOL is on the periferic area, positioned in the vitreous basis, it has its access more difficult and for many times being the cause of the vitreous traction and retine torns, even though specific lens for the vitrectomy surgeries are used. The endoscopy may offer a better angule of vision in the retroirian region, with the advantage of dispersating the sclera depression or the exaggerated twisting of the eye.

The third function of the URAM E2 is the application of laser using the endoscopy, very useful in the last cases of sclera fixation, that frequently are associated to the glaucoma and being worse when the conjunctive is already manipulated.

The association of the videoendoscopy with the photocoagulation brought for us practice for the cases of afacic with glaucoma. It is frequent the surgical reintervention in the eyes which have already being operated due to the cataract and they turn to have glaucoma, mainly the ones that showed a higher ocular pressure. Before the endocyclophotocoagulation we used to avoid the indication for sclera fixation in these cases because we knew that if the glaucoma showed up the consequences would be very hard.

\section{References}

Althaus, C.; Sundmacher, R. (1993). Intraoperative intraocular endoscopy in transscleral suture fixation of posterior chamber lenses: consequences for suture technique, implantation procedure, and choice of PCL design. Refract Corneal Surg. 9(5):333-9.

Busacca, A. (1955)Physiology of the ciliary muscle as studied by gonioscopy. Ann Ocul.188(1):1-19. Paris.

Burian, H.M.; Allen, L. (1955). Mechanical changes during accommodation observed by gonioscopy. AMA Arch Ophthalmol. 54(1):66-72.

Carvalho, D.M.; Paranhos, F.R.L. (1993). Implante secondário de LIO de câmara posterior: fixação escleral. Rev Bras Oftalmol. 52(1):17-22.

Carvalho, D.; Lima, F.E.; Degani, M.I. (1996). Endoscopia Ocular e Fixaçao de Lente IntraOcular. Revista Brasileira de Oftalmologia 55. (4):285-7.

Carvalho, D.M. \& D.M. Jr. (2007). Fixação escleral: técnica de agulha guia (finger crafted technique) com ponto escleral sepultado. Cirurgia de catarata: fixação e implantes secundários. (3):19-47. Elsevier Editora Ltda.

Carvalho Jr, D.M. (2002). Ciclofotocoagulação endoscópica: estudo experimental com aplicação de laser diodo nas diferentes porções do corpo ciliar em olhos de coelhos pigmentados. Tese de Doutorado. Universidade de São Paulo. 70p. São Paulo, Brasil.

Chen, J.; Cohn, R.A.; Lin, S.C.; et al. (1997). Endoscopic photocoagulation of the ciliary body for treatment of refractory glaucomas. Am J Ophthalmol. 124(6):787-96.

Funk, R.; Rohen, J.W. (1987). SEM studies on the functional morphology of the rabbit ciliary process vasculature. Exp Eye Res. 45(4):579-95.

Funk, R.; Rohen, J.W. (1987). Intraocular microendoscopy of the ciliary-process vasculature in albino rabbits: effects of vasoactive agents. Exp Eye Res. 45(4):597-606.

Funk, R.; Rohen, J.W. (1988). Experimental studies on the functional morphology of the vascular system of the anterior eye segment in rabbits and primates. Fortschr Ophthalmol. 85(2):170-6.

Funk, R.; Rohen, J.W. (1988). Reactions of efferent venous segments in the ciliary process vasculature of albino rabbits. Exp Eye Res. 46(1):95-104. 
Funk, R.; Rohen, J.W. (1988). SEM studies of the functional morphology of the ciliary process vasculature in the cynomolgus monkey: reactions after application of epinephrine. Exp Eye Res. 47(4):653-63.

Funk, R.; Rohen, J.W. (1989). Microendoscopy of the anterior segment vasculature in the rabbit eye. Ophthalmic Res. 21(1):8-17.

Leon, C.S.; Leon, J.A. (1991). Microendoscopic ocular surgery: a new intraoperative, diagnostic and therapeutic strategy. Part I: Endoscopic equipment/methodology applied to cataract surgery with intraocular lens implantation. J Cataract Refract Surg. 17(5):568-72.

Leon, C.S.; Leon, J.A. (1991). Microendoscopic ocular surgery: a new intraoperative, diagnostic and therapeutic strategy. Part II: Preliminary results from the study of glaucomatous eyes. J Cataract Refract Surg. 17(5):573-6.

Leon, J.A.; Leon, C.S.; Aron-Rosa, D.; et al. (2000). Endoscopic technique for suturing posterior chamber intraocular lenses. J Cataract Refract Surg. 26(5):644-9.

Lima, F.E.; Carvalho, D.M.; Beniz, J.; Avila, M. (1997). Ciclofotocoagulaçäo endoscópica em glaucomas refratários. Rev. bras. oftalmol 56. (6):387-93.

Lima, F.E.; Carvalho, D.M.; Beniz, J.; Ávila, M. (1997). Ciclofotocoagulação Endoscópica em Glaucomas Refratários. Rev Bras Oft 56. (6):397-406.

Lima, F.E.; Costa, V. (2000). Management of complex glaucoma: Tube shunt versus cyclodestructive procedure. Glaucoma Perspectives in Practice - Issue 2.3.

Lima, F.E.; Magacho, L; Carvalho, D.M.; Susanna, R.; Ávila, M.P. (2004). A Prospective, Comparative Study between Endoscopic Cyclophotocoagulation and the Ahmed Drainage Implant in Refractory Glaucoma. Journal of Glaucoma 13(3): 233-237.

Lima, F.E.; Carvalho, D.M.; Ávila, M.P. (2010). Phacoemulsification and endoscopic cyclophotocoagulation as primary surgical procedure in coexisting cataract and glaucoma. Arquivos Brasileiros de Oftalmologia. 73(5).

Malbran, E. S. \& E. Jr.; Negri, I. (1986). Lens guide suture for transport and fixation in secondary IOL implantation after intracapsular extraction. Int Ophthalmol. 9 (2-3): 151-160.

Mizuno, K.; Asaoka, M. (1976). Cycloscopy and fluorescein cycloscopy. Invest Ophthalmol.15(7):561-4.

Mizuno, K.; Asaoka, M.; Muroi, S. (1977). Cycloscopy and fluorescein cycloscopy of the ciliary process. Am J Ophthalmol. 84(4):487-95.

Pavlin, C.J.; Rootman, D.; Arshinoff, S.; et al. (1993). Determination of haptic position of transsclerally fixated posterior chamber intraocular lenses by ultrasound biomicroscopy. J Cataract Refract Surg. 19(5):573-7.

Pietrabissa, A.; Scarcello, E.; Carobbi, A.; Mosca, F. (1994 ). Three-dimensional versus twodimensional video system for the trained endoscopic surgeon and the beginner. Endosc Surg Allied Technol. 2(6):315-7.

Scheie, H.G. (1953). Gonioscopy in the diagnosis of tumors of the iris and ciliary body, with emphasis on intraepithelial cysts. Trans Am Ophthalmol Soc. 51:313-31.

Uram, M. (1992a). Ophthalmic laser microendoscope ciliary process ablation in the management of neovascular glaucoma. Ophthalmology 99. (12):1823-8.

Uram, M. (1992b). Ophthalmic laser microendoscope endophotocoagulation. Ophthalmology 99. (12):1829-32.

Zarbin, M.A. et al. (1988). Endolaser treatment of the ciliary body for severe glaucoma. Ophthalmology 95. 1639. 


\section{Part 4}

\section{Digestive Endoscopy}





\title{
Laparoscopy in Diagnosis and Treatment of Small Bowel Diseases
}

\author{
Coco Claudio, Rizzo Gianluca, Verbo Alessandro, \\ Mattana Claudio, Pafundi Donato Paolo and Manno Alberto \\ Catholic University of the Sacred Hearth \\ Department of Surgical Sciences Rome \\ Italy
}

\section{Introduction}

Laparoscopy is defined as the technique of examining the abdominal cavity and its contents by creating a pneumoperitoneum. The first description of a laparoscopic approach goes back to 1901, by Kelling, who showed on a dog model that it was possible to look inside the abdomen by introducing a cystoscope after high-pressure insufflation (Kelling, 1901). The development of the technique over the next 50 years, passed through the creation of specific instruments that made easier accurate and complete examination of the peritoneal cavity, such as the Verres needle, first described to create pneumothorax for treating tuberculosis but successfully used in 1937 for the induction of the pneumoperitoneum (Veress, 1938) and the Hasson trocar (Hasson, 1974). In September 1985 Erich Muhe performed the first laparoscopic cholecystectomy in humans (Litynski, 1998). Muhe used a Veress needle to create a pneumoperitoneum, introduced a laparoscope through the umbilicus, and completed laparoscopic cholecystectomy in 2 hours. The technique was presented at the Annual Congress of the German Surgical Society held in Munich on April 1986, and rapidly became the gold standard in the treatment of symptomatic gallstones. Fast and worldwide success of laparoscopic cholecistectomy was based on the analysis of results in terms of hospitalization, bowel function resumption, wound-related complications and return to daily activities, which were much more satisfying as compared to those obtained with the open technique, thus causing rapid acceptance by surgeons and increasing demand by patients. The laparoscopic approach was widely applied in abdominal surgery, for the treatment of a great number of benign disease, from MRGE to hernia, and even to procedures in which dissection and extraction of solid organs was contemplated, as safe and easy techniques rapidly developed. This affected transplantation surgery in the way that people who accept to donate the kidney rapidly increased after the diffusion of the laparoscopic approach, because of the decreased morbidity of the operation. Although first met with skepticism, laparoscopy have been applied to malignancies, especially to colon cancer. Many multicenter prospective randomized trials comparing laparoscopic and open technique in colon cancer surgery, unequivocally demonstrated the same favorable short term results of laparoscopic colectomy shown when this approach was adopted for other benign diseases (less intra-operative bleeding, less post-operative pain, morbidity and 
immunological stress, early bowel movement, shorter hospital stay, early return to daily activities and better cosmetic results). The laparoscopic technique demonstrated also effective considering some pathological parameters of oncological radicality, as number of lymph node removed and cancer-free margins. Non differences about long-term results, in terms of incidence of recurrence and overall survival, were shown between open and laparoscopic approach in any prospective randomized trial (Nelson et al., 2004), Another area where laparoscopy found a place in the last two decades is bariatric surgery. This, in part, is because of the results of the laparoscopic Roux-en-Y gastric bypass procedure, especially the minimally invasive surgical benefits and the resolution of obesity-related comorbidities (Robinson et al., 2004). The Lap-Band (BioEnterics Lap-Band System; Inamed Health, Santa Barbara, CA) also has become a popular minimally invasive tool with less morbidity than the gastric bypass. Despite the improvement in outcomes with laparoscopy, the technique has limitations. The video images are projected in a 2-dimensional plane. The stability, focus and tilt depends on camera operator, and the ability to follow the natural movement of the surgeon's eyes is limited. The use of trocars anchored to the abdominal wall limits the range of motion of the long straight instruments and often induces awkward ergonomics (Ballantyne, 2002). This conditions, combined with the counteracting vectors generated by the abdominal wall (which require force to overcome), can lead to surgeon fatigue or, worse, neurapraxia. Another problem of laparoscopic technique is represented by the learning curve that must be substantial, especially for more complex procedures (Berguer, 1998; Ehrmantraut \& Sardi, 1997).

\section{General considerations about diagnostic and therapeutic potential of laparoscopy in small bowel diseases}

Small bowel diseases are rare and difficult to identify with traditional diagnostic tools. Diagnosis is often late, based on the appearance of occult rectal bleeding, occlusive syndrome or, more rarely, intestinal perforation. The exact identification and location of small bowel lesions were made easier in recent years by the development of more detailed diagnostic tools, such as double balloon enteroscopy and videocapsula (Gerson, 2009). The laparoscopic approach to the small bowel diseases may include resection or not. Well recognized examples of the first group are benign or malignant tumours; inflammatory bowel disease; Meckel's diverticulum; bleeding small bowel angiodysplasia; small bowel ischemia and stricture (postradiation, postischemic, etc). Non resectional laparoscopic small bowel procedures include laparoscopic enterolysis for acute small bowel obstruction, diagnostic laparoscopy for possible ischemic disease and laparoscopic palliative enteroenterostomy for bypassing obstructing nonresectable tumors. When dealing with small bowel pathologies, performing laparoscopy may be extremely challenging, as a consequence of the technical difficulties in the mobilization of the intestinal loops, especially if dilated as a consequence of an occlusion, or in identifying and localizing the lesions. Many difficulties were recently overcome with the help of technological development of instrumentation, such as the laparoscopic model of Ultracision ${ }^{\circledR}$, Harmonic scalpel ${ }^{\circledR}$ or Ligasure ${ }^{\circledR}$, so that nowadays laparoscopic diagnosis and treatment of small bowel diseases are to be considered feasible (Carrasco Rojas, 2004), although not easy to perform. In addition, endoscopic tattooing of the lesion, can make location of tumors easier. 


\section{Small bowel neoplasms}

Small bowel neoplasms represent $0.3 \%$ of all tumors, fewer than $2 \%$ of all gastrointestinal malignances, with an age-adjusted incidence of 1 per 100,000 and a prevalence of $0.6 \%$. Approximately, almost forty different histological types of both benign and malignant tumors have been identified (Neugut et al., 1998). Seventy five percent of tumors are benign at histologic diagnosis by biopsy and include leiomyomas, adenomas, lipomas and hamartomas. Malignant neoplasms, frequently symptomatic, include adenocarcinomas, carcinoids and lymphomas. Stromal tumors are considered as tumors with variable malignant power. Other type of small bowel malignant neoplasms are metastatic diseases by malignant melanoma, bronchogenic tumors, breast cancer and intrabdominal cancers. Surgery, is considered the first line therapy for most of the small bowel neoplasm, especially malignant and complicated benign tumors (Gill et al., 2001; Coco et al. 2010). Laparoscopic surgery represents a valid and feasible approach for the treatment of these neoplasms.

\subsection{Benign neoplasms}

Benign neoplasms are usually asymptomatic and only incidentally discovered, when complicated by obstruction or hemorrhage (more frequently occult). Despite the term "benign", exists a risk of malignant change for adenomas (malignant changes at presentation over $40 \%$, expecially in large adenomas with villous component or atypia,) and leyomiomas (risk for malignancy related to the tumor size and number of mitosis); because of their potential to undergo malignant transformation, these neoplasms should be removed (Witteman et al., 1993). Lipoma, hemangioma, Bunner's gland hamartoma and intestinal nodular lymphoid hyperplasia have no risk of malignant evolution and indication for surgery is limited to symptomatic lesions (intussusceptions, obstruption, bleeding) (Morgan et al., 2000). These neoplasm are often multiple; a carefully inspection of the entire small bowel is recommended before the treatment. Surgical options are different: endoscopic treatment (endoscopic polypectomy or mucosectomy especially for benign neoplasms of duodenum or proximal jejunum), excision via enterotomy (especially for small lesions) and small bowel segmentary resection. In the last two cases the laparoscopic approach is advisable not only becasuse the resection is safe and effective but also becasuse the mandatory examination of the entire small bowel can be performed acording to a miniinvasive approach which consent to avoid laparotomy for treating benign diseases.

\subsection{Malignant neoplasms}

A recent epidemiologic study concerning small bowel malignant neoplasm, conducted in the United States on 67843 patients from 1973 to 2005 by Bilimoria et al (Bilimoria et al 2009), showed an overall increase, in the last thirty years, of small bowel cancer (22.7 cases per million in 2004). In particular, the proportion of patients with carcinoid tumors increased significantly (from $27.5 \%$ to $44.3 \%$ ) whereas the proportion of patients with adenocarcinoma decreased (from $42.1 \%$ to $32.6 \%$ ). However, incidence rates is low and similar for both men and women before the age of 40 . In the last 30 years, there is a parallel increase between 40 and $55 \mathrm{yrs}$ in both sexes and a more rapidly growth in men than in women. The sites at major risk for malignant neoplasm are duodenum, for adenocarcinoma, and ileum, for carcinoids and lymphomas (Lepage et al., 2006). Treatment modality and oncologic outcome differs considering the various histological types. 


\subsubsection{Small bowel carcinoid}

Small bowel, especially terminal ileum, represents the most frequent location of neuroendocrine tumors (Fig. 1) in the gastrointestinal tract (among 30\%). Peak incidence is between the $6^{\text {th }}$ and $7^{\text {th }}$ decades of life. Clinical manifestations are vague or absent, and tumors are often incidentally detected at the time of surgery for other gastrointestinal diseases or during exploration for liver metastases. In approximately $20 \%$ of cases these neoplasms secrete bioactive mediators and give rise to the characteristic "carcinoid syndrome" (intermittent abdominal cramps, diarrhea, flushing, bronchospasm and cyanosis) (Kulke \& Meyer, 1999).

Nodal metastases after carcinoids are frequent (over $40 \%$ of cases) with no relations to tumor's dimensions, whereas liver metastases are usually associated to tumors $>2 \mathrm{~cm}$ in diameter (over $60 \%$ of cases). Resection of primary tumor with associated extensive mesenteric lymphadenectomy is appropriate, even in the presence of liver metastases. If diagnosis of intestinal carcinoid tumor is made after a limited resection of a small lesion, further surgery for extensive mesenteric lymphadenectomy is to be considered (Sutton et al., 2003). The indications for potentially curative liver resection are similar to those applicable to metastatic colorectal cancer. Disease unsuitable for partial hepatectomy unresponsive to alternative therapies, producing life-threatening complications and carcinoids with low proliferation index could be considered for liver transplantation (Yao et al., 2001). After radical resection of carcinoid tumors the 5yr-OS is good, with an OS rate of $70-80 \%$ in case of localized disease, $60-75 \%$ in case of nodal involvement and 30 $50 \%$ in case of liver metastases. In patients with liver metastases who underwent to hepatectomy or liver transplantation 5y-OS is respectively $70-80 \%$ and $60-70 \%$ (Shebani et al., 1999).

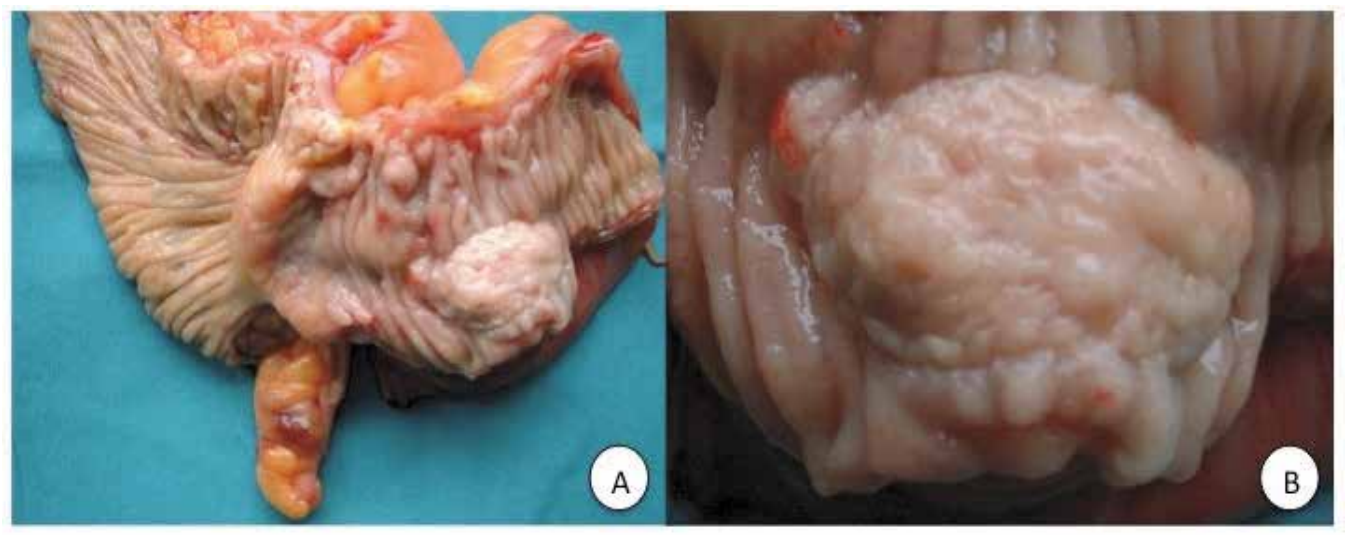

Fig. 1. A,B - Small bowel carcinoid

\subsubsection{Small bowel adenocarcinoma}

Adenocarcinoma (Fig. 2) represents the commonest histological type of small bowel tumors in the western world (30-50\% of small bowel malignant tumors). Duodenum and jejunum are the most frequent location sites. Peak incidence is in the 7th decade of life and there is a male preponderance. Adenomas, either single or multiple as expression of multiple polyposis syndromes are to be considered lesions at risk of developing 
malignancies. Small bowel adenocarcinomas, because of the presence of lymphatic tissue in small intestinal mucosa, early metastasize to regional lymph node. The most common symptoms at presentation are obstruction, bleeding, jaundice and weight loss. Surgical radical resection followed by adjuvant chemotherapy represent the therapy of choice (Neugut et al., 1997). For jejunal and ileal tumors, curative resection (R0) is to be intended as complete removal of the neoplastic mass with macro- and microscopically clear margin and regional lymphnode dissection. If infiltration of continuous organs is detected, en bloc resection is indicated as well as right colectomy should be considered in case of distal ileal lesion, to obtain a complete nodal dissection. For duodenal tumors located in the II or III duodenal portions, duodenopacreatectomy is indicated, while for IV portion lesions, pancreas-preserving segmental resection will be the treatment of choice; in both cases clear resection margins are mandatory to obtain satisfing long term results. For locally advanced unresectable or metastatic adenocarcinoma a palliative treatment should be considered to avoid complication as obstruction (by-pass or stent) or bleeding (limited resection of the bleeding mass). In case of single hepatic metastasis, the role of liver resection is unknown (Hutchins et al., 2001). Despite radical resection, the 5yr-OS rate is low. In a large landmark study conducted by the American College of Surgeons Commission on 5,000 small bowel adenocarcinomas, the overall 5-year disease-specific survival was $30.5 \%$, with a median survival of 19.7 months (Howe et al., 1999). Survival was lower in patients with duodenal tumors and in those over 75 years old, also because many surgeons are reluctant to perform radical resection in these cases (North \& Pack, 2000).
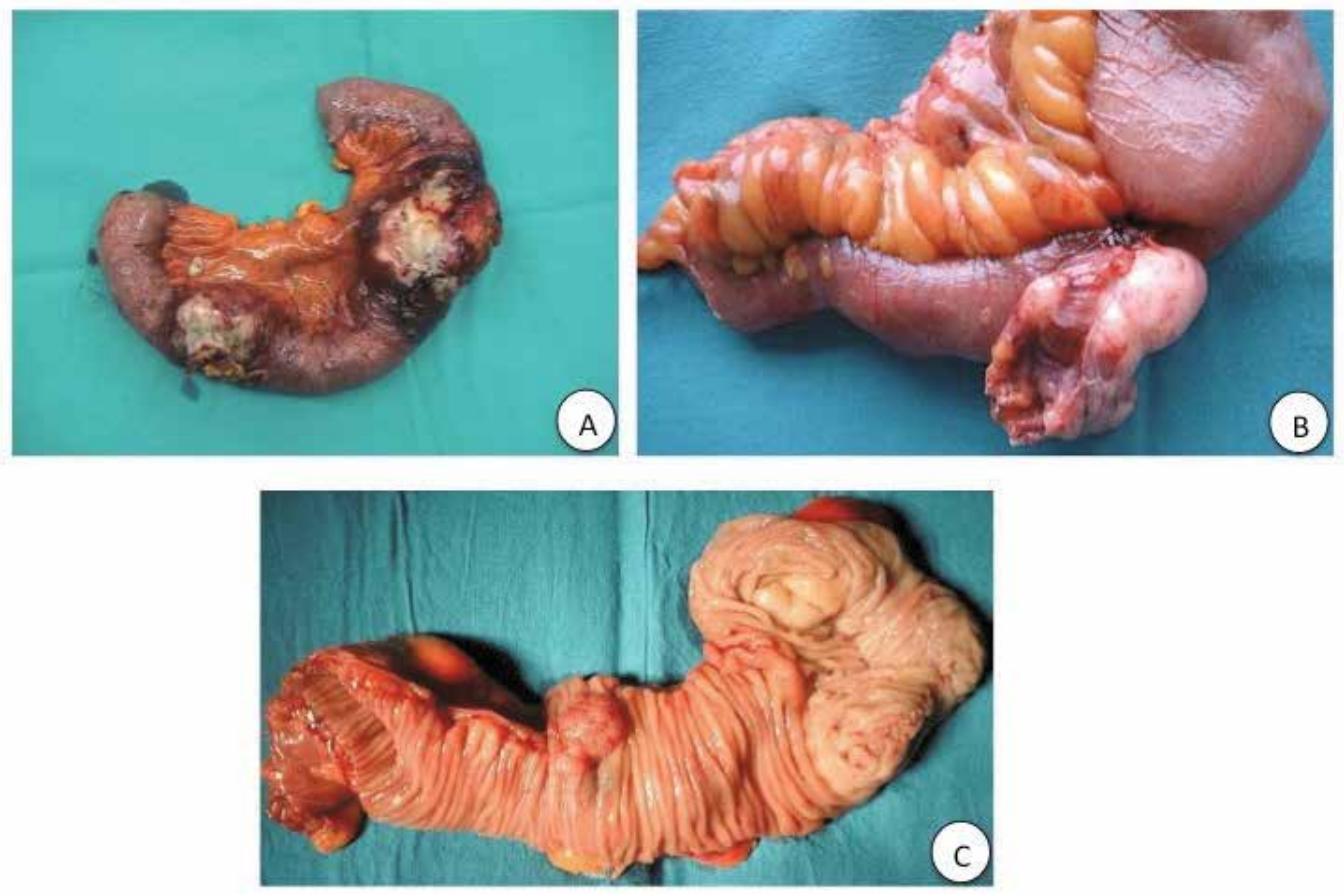

Fig. 2. A, B, C - Small bowel adenocarcinomas 


\subsubsection{Small bowel lymphoma}

Small bowel lymphoma (Fig. 3) can be primary or secondary. Primary lymphoma accounts for $15 \%$ to $20 \%$ of all malignant small bowel tumors and ileum represents the most common location site. The usual clinical presentation of gastointestinal lymphoma includes intermittent abdominal pain, fatigue, diarrhea, weight loss, and, occasionally, fever; less commonly, gastrointestinal bleeding, obstruction, or even perforation (up to 25\%). Chemoradiation is the therapy of choice for these neoplasms. In a clinical setting in which palpable adenopathy and hepatosplenomegaly are absent, with no evidence of disease on chest CT, diagnosis of primary intestinal lymphomas requires histologic confirmation. Only in this case, surgical exploration and resection of involved segments with regional lymph node dissection is requested to confirm diagnosis of lymphoma. Surgical treatment is required also in cases of complications as obstruction, bleeding and perforation. The overall prognosis of the more advanced stages of primary small intestinal lymphoma is poor, with an expected 5-year survival of $25 \%$ to $30 \%$ (Crump et al., 1999).
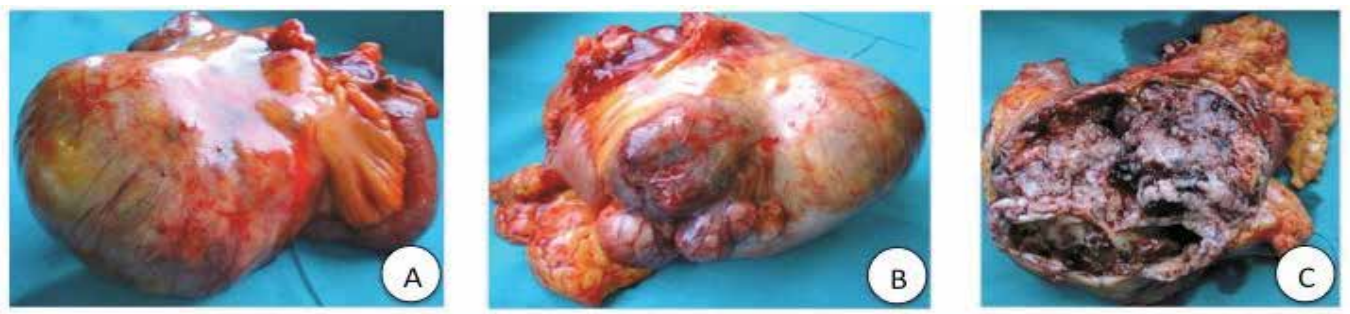

Fig. 3. A, B, C - Small bowel lymphomas

\subsubsection{Small bowel stromal tumor}

Stromal tumors account $<1 \%$ of all gastrointestinal tumors. GISTs (Gastro Intestinal Stromal Tumors) represents the most common stromal tumors with malignant power of the gastrointestinal tract (over 90\%) (Fig. 4). Many GISTs are discovered incidentally. When exist, symptoms of GISTs are obstruction, hemorrhage or, rarely, peritonitis. Malignant power of GIST depend by mitotic index and tumor size. Surgical complete gross resection with an intact pseudocapsule (non-disruptive techniques) and negative macroscopic margins (R0 or "R1" resection) is the definitive treatment for primary GISTs without evidence of peritoneal seeding or metastasis. En bloc resection is requested in case of infiltration of continuous organs (Demetri et al., 2004). Because GISTs rarely metastasize to lymph nodes, routine lymph node dissection is not warranted except when there is evidence of gross nodal involvement (Blay et al, 2005). In advanced cases surgery alone is not curative. Resection of intraperitoneal metastases should be considered if they are prone to intralesional bleeding, which may result in severe blood loss, peritonitis, and interference with Imatinib therapy but most metastatic lesions from GIST, particularly those to the liver, are multifocal, diffuse, and technically difficult to resect (Everett \& Gutman, 2008). The 5-year survival rate after the surgical resection of GIST was $43-95 \%$ in the pre-Imatinib era variable from $95 \%$ for low-risk GISTs to $0 \%-30 \%$ for high-risk GISTs. After the introduction of molecular targeted therapy with Imatinib and Sunitinib improvement in survival seems to be granted, but most prospective randomized studies are needed. 

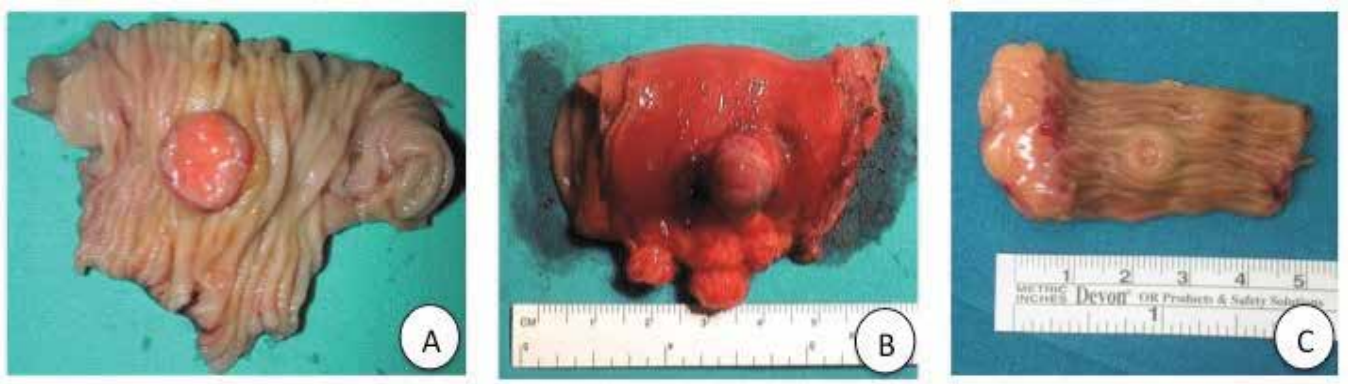

Fig. 4. A, B, C - Small bowel GISTs

\subsubsection{Metastatic neoplasms}

Secondary neoplastic involvement of the small intestine is more frequent than primary lesions. Primary tumors of colon, ovary, uterus, and stomach usually involve the small bowel, either by direct invasion or by intraperitoneal spread, whereas primaries of breast, lung, and melanoma, the malignancy which more frequently metastasize to the bowel, spread hematogenously. (Fig. 5). Surgical resection does not improve prognosis but is sometimes requested in case of complications.
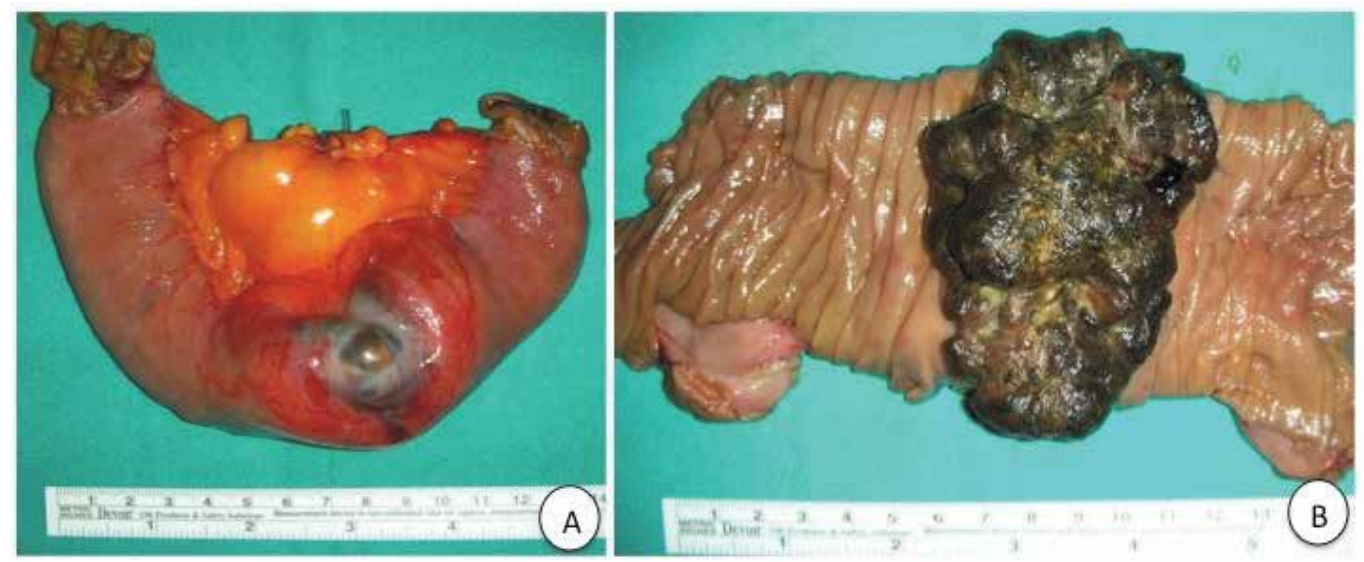

Fig. 5. A, B - Small bowel metastatic melanoma

\subsubsection{Role of laparoscopic surgery}

Among small bowel tumors, the mini-invasive approach is clearly accepted only for GISTs. The 2004 NCCN Task Force Report generally discouraged laparoscopic or laparoscopyassisted resection for GIST, limiting its use for tumors smaller than $2 \mathrm{~cm}$ at low risk of intraoperative rupture (Demetri et al., 2004). However, two years later, Novitsky et al. (Novitsky et al., 2006) analyzing the results of their series of 50 laparoscopically operated GISTs of mean size of $4.4 \mathrm{~cm}$ (range, 1.0-8.5 cm), showed that efficacy and recurrence rate were similar or even better than what reported in historical open-surgery control series, and suggested a revision of the 2004 NCCN guidelines. So the 2007 update stated that laparoscopic resection was acceptable for tumors measuring up to $5 \mathrm{~cm}$ in diameter and that tumors larger than $5 \mathrm{~cm}$ might be resectable by hand-assisted laparoscopic techniques 
(Demetri et al., 2004). Although there are no guidelines to state the feasibility of laparoscopic surgery for carcinoid, lymphoma, adenocarcinoma and other malignancies of the small intestine, we believe that this technique is feasible in these cases, especially with the help of the same useful devices used in laparoscopic colorectal cancer surgery (sterile drape ${ }^{\circledR}$, etc.).

\section{Small bowel Crohn's disease}

Crohn's disease (CD) is a common chronic inflammatory disease usually characterized by patchy, whole thickness, granulomatous lesions, that can affect any part of the gastrointestinal tract (Fig. 6). The incidence of CD is 5-10 per 100000 per year with a prevalence of 50-100 per 100000 (Carter et al., 2004). Clinical patterns include combined small and large intestinal pattern ( $26 \%$ to $48 \%)$, small intestine only pattern (11\% to $48 \%$ ) and colon only pattern (19\% to 51\%) (Munkholm \& Binder, 2004). Involvement of terminal ileum and colon is the most common pattern (55\%), while involvement of mouth, oesophagus, stomach and duodenum, is uncommon and rarely occurs without concurrent disease activity in the small bowel and/or colon (Thoreson \& Cullen, 2007). Patients with small bowel CD commonly present with an acute symptomatic picture, characterized by abdominal cramps, diarrohea, malaise and loss of weight that is primarily managed using steroids, immunomodulators (Azathioprine, Mercaptopurine, Methotrexate) or biological therapy (anti-TNF agents) (Travis et al, 2006). Surgical treatment is required in approximately 70 percent of patients for failed medical therapy, recurrent intestinal obstruction, malnutrition and for septic complications (free perforation, abscess). Reoperations are required in 70 to 90 percent of all patients and multiple procedures in more than 30 percent (Duepree et al, 2002). Resection and anastomosis is indicated for short segment with multiple strictures or active disease, diseased bowel with fistula, abscess or phlegmon. Strictureplasty is a safe and effective alternative to bowel resection as multiple ones are often required for the same patient, with high risk of a short bowel syndrome.

Laparoscopy has gained wide acceptance in gastrointestinal surgery with potential advantages in early post-operative outcome and cosmesis (Duepree et al, 2002; Dunker et al, 1998; Milsom et al., 1993; Reissman et al, 1996; Albaz et al., 2000) and its use is accepted in benign and malignant colorectal diseases. The first laparoscopic intestinal resection for CD was reported by J. Milsom in 1993 (Milsom et al., 1993). Laparoscopic surgery offers additional advantage of smaller abdominal fascial wounds, low incidence of hernias, and decreased rate of adhesive small-bowel obstruction (Albaz et al., 2000) than conventional surgery. The main concerns about laparoscopic approach to small bowel CD are: missing occult segments of disease and critical proximal strictures due to absence of tactile sensitivity; earlier recurrence due to possible reduced immune response, technical difficulties due to fragile inflamed bowel and mesentery and the presence of adhesions, fistulas, and abscesses (Uchikoshi et al., 2004; Lowney et al., 2005). A Cochrane review about the role of laparoscopic surgery in CD was recently published (Bobby et al., 2011). Two randomized controlled trials (Maartense et al., 2006; Milsom et al., 2001) comparing laparoscopic and open surgery for small bowel CD were identified for a total of 120 patients. About post-operative morbidity less patients in the laparoscopic group $(2 / 61 ; 3.27 \%)$ suffered wound infection compared to the open group $(9 / 59 ; 15.25 \%)$ but the difference was not statistically significant $(\mathrm{p}=0.23)$. There was no significant difference in the incidence of other postoperative complications (postoperative pneumonia, prolonged postoperative ileus 
and urinary infections). The incidence of anastomotic leak, intra abdominal abscess and 30 -day reoperation rates were comparable. The operation time was shorter in open surgery and the amount of intra operative blood loss was lesser in the open group (133 +/- $70 \mathrm{ml} /$ case) compared to laparoscopic group $(173$ +/- $123 \mathrm{ml} / \mathrm{case})$ although the difference was not statistically significant $[\mathrm{P}=0.25]$. There was no significant difference in postoperatve pain as defined by the amount of opioids requested by patients. Hospital stay was shorter in laparoscopic group compared to open group but the difference was not statistically significant $[\mathrm{P}=0.90]$. Conversion rates were similar in both the trials [ 3 out of 30 in Maartense 2006 and 2 out of 33 in Milsom 2001]. There was no significant difference in the reoperation rates for disease recurrence. Laparoscopic surgery for abdominal conditions is known to have associated with lesser incidence of adhesions and incisional hernias. Better cosmesis and body image obtained with the laparoscopic approach are well establisehd and are particularly relevant dealing with Crohn's disease, because of the young age of patients. Quality of Life (QoL) was not evaluated in this Cochrane review (Bobby et al., 2001), although a randomized control trial (Eshuis et al., 2010) reported similar QoL in both groups.

In conclusion, despite there are no potential benefits of laparoscopic surgery over open surgery, this approach for small bowel CD is feasible and as safe as the pen one, but with better cosmetic results and short-term post-operative outcome.
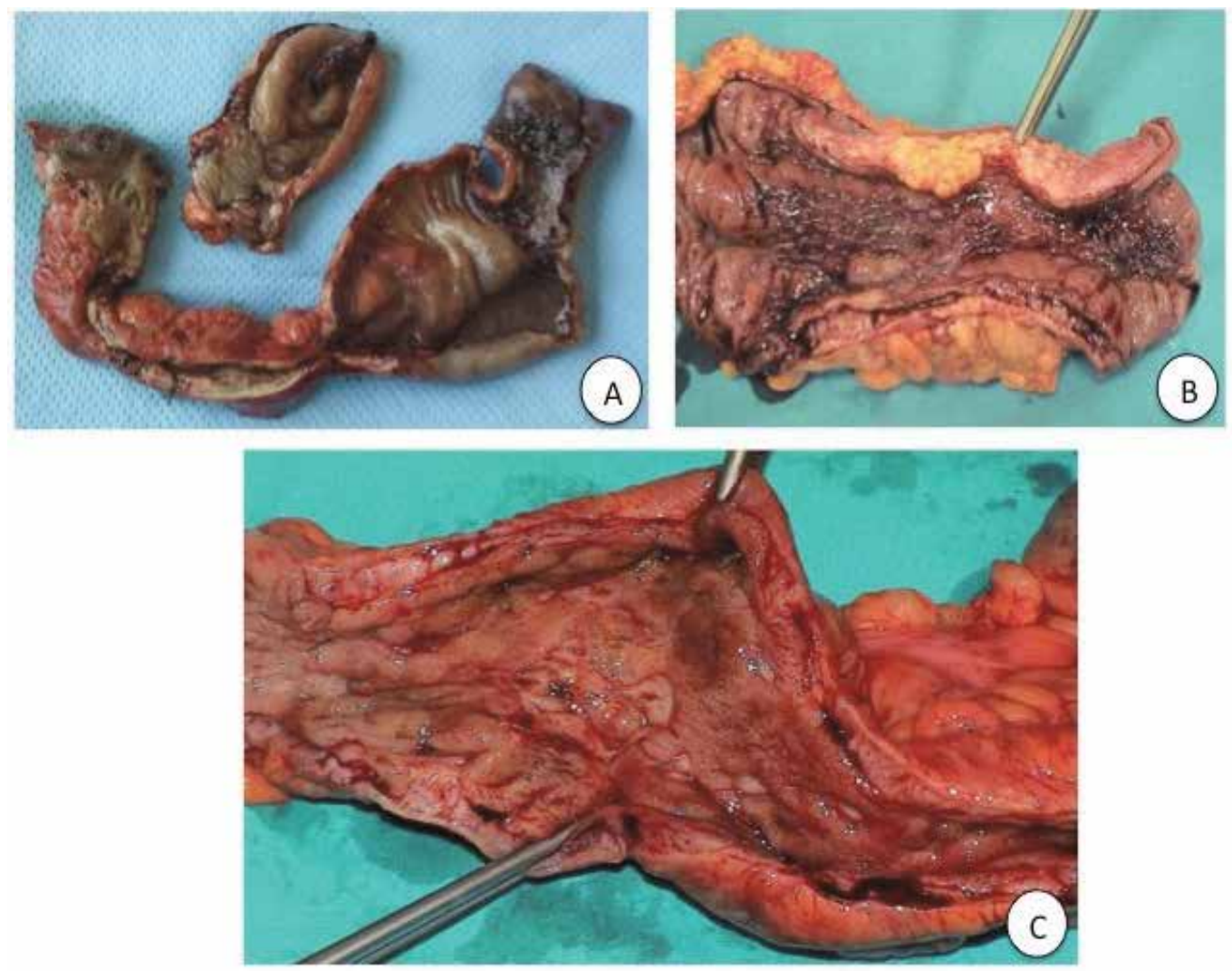

Fig. 6. A, B, C - Small bowel Crohn's Disease 


\section{Meckel's diverticulum}

Meckel's diverticulum is the most common congenital malformation of the gastrointestinal tract (incidence of between $0.6 \%$ and $4 \%$ ) due to persistence of the congenital vitellointestinal duct. It is a true diverticulum, typically located on anti-mesenteric border, $60 \mathrm{~cm}$ from the ileo-caecal junction, and contains all three coats of intestinal wall with its separate blood supply from the vitelline artery. In the fetal life, the omphalo-mesenteric duct connects the yolk sac to the intestinal tract and usually it obliterates in the 5th to 7th week of life. If obliteration fails, the congenital anomalies develop, leading to residual fibrous cords, umbilical sinus, omphalo-mesenteric fistula, enterocyst and, most commonly, Meckel's diverticulum. The range of incidence of complications due to Meckel's diverticulum is 4\%$16 \%$. Its occurrence in males and females is equal, but incidence of complications is three to four times greater in males. The risk of complications decreases with increasing age, with no other predictive factors. Bleeding from Meckel's diverticulum due to ectopic gastric mucosa is the most common clinical presentation, especially in younger patients. The main mechanism of bleeding is the acid secretion from ectopic mucosa, leading to ulceration of adjacent ileal mucosa. It is possible that the recurrent intussusception may cause trauma, inflammation, mucosal erosion and bleeding. Others complications in adults include: obstruction, intussusceptions, ulceration and, rarely, vescico-diverticular fistulae and tumours (Heinicke et al., 1997; Wong et al., 2005; Tan et al., 2005; Puliglandla et al., 2001).

Due to the rarity of cases in adults, it is still misdiagnosed preoperatively. Preoperative diagnosis of symptomatic Meckel's diverticulum is difficult; so, it is imperative to differentiate Meckel's diverticulum from other surgical conditions. This is particularly true in patients presenting with symptoms other than bleeding. In a study by Higachi et al. about 776 patients affected by Meckel's diverticulum, a correct preoperative diagnosis was made in $88 \%$ of those presenting with bleeding but only in $11 \%$ of cases when other symptoms were reported (Higaki et al., 2001). In doubtful cases, laparoscopy is an excellent diagnostic tool (Shalaby et al., 2005). However, technetium-99m pertechnate scan is the most common and accurate non-invasive investigation performed for these cases. In children, it has a sensitivity of $80 \%-90 \%$, a specificity of $95 \%$ and an accuracy of $90 \%$ (Kong et al., 1993) but in adults it is less reliable, with a sensitivity of $62.5 \%$, a specificity of $9 \%$ and an accuracy of $46 \%$ (Lin et al., 2002). As the technetium-99m pertechnate scan is specific to ectopic gastric mucosa and not to Meckel's diverticulum, it may be positive in gut duplication cysts with ectopic gastric mucosa (Kumar et al., 2005). The false negative scans may be due to the rapid dilution of radioactive secondary to fast bleeding from the ectopic mucosa, impaired vascular supply or insufficient gastric mucosa. The false negatives are also more common in patients presenting with other symptoms than bleeding. Other diagnostic method suggested to supplement the Meckel's scan is angiography but it is usually negative unless the bleeding rate is $40.5 \mathrm{~mL} / \mathrm{min}$. The treatment of choice for symptomatic Meckel's diverticulum is surgical resection. It can be achieved either by diverticulectomy or by segmental bowel resection, especially when there is palpable ectopic tissue at the junction between diverticulum and intestinal wall, intestinal ischaemia or perforation. It has long been stated that the risk of developing complications following the incidental removal of Meckel's diverticulum can offset the potential benefits of this procedure (Leijonmarck et al., 1996) and the subject is still object of debate. Opponents to incidental diverticulectomy often cite Soltero and Bill (Soltero \& Bill, 1976) who, in 1976, estimated that the life-time risk of complications from an untreated MD was $4.2 \%$, decreasing this risk to zero with increasing 
age, and so incidental diverticulectomy was not advisable. Twenty years later, the results of a large population-based study in Olmsted County, Minnesota, provided data in support of prophylactic diverticulectomy (Cullen et al., 1994). This study reported a $6.4 \%$ cumulative rate of developing complications from untreated MD that required surgery over a life-time, especially in male patients up to 80 years of age. Diverticulectomy for complicated MD carried an operative mortality and morbidity of $2 \%$ and $12 \%$, and a cumulative risk of long term complications of $7 \%$. The corresponding rates for incidental diverticulectomy are $1 \%$, $2 \%$ and $2 \%$, respectively (Cullen et al. 1994). A subsequent report from the Mayo Clinic recommended MD resection only in male patients younger than 50 years of age, when the diverticulum length is greater than $2 \mathrm{~cm}$, or when abnormal features are detected within the diverticulum: carcinoid tumors was found in $2.2 \%$ of the symptomatic patients and in $2.1 \%$ of the asymptomatic ones in this series (Park et al., 2005). More recently, however, a systematic review of the English literature on this subject shown that there is no compelling evidence to support prophylactic resection (Zani et al., 2008). In fact, resection of incidentally detected MD has a significantly higher early complication rate than that potentially occurring leaving the diverticulum in situ $(5.3 \%$ vs $1.3 \%, P<0.0001)$ (Cullen et al., 1994). With the advent of gastrointestinal stapling devices, excision has become safer, faster, and more efficient. Another advantage of stapling is that it closes the bowel lumen as it cuts, thereby completely reducing the chance of peritoneal contamination. The contraindications for stapler excision is a very broad-based or too short diverticula, because in these cases, the risk of including too much of the ileum during stapling or leaving behind part of the diverticulum is high. Another way to perform the excision is to exteriorize the diverticulum throgh a mini-laparotomy, resect it by stapler and close the enterotomy. It is of crucial importance that the direction of the staple line lies perpendicular to the longitudinal axis of the ileum so that the bowel lumen will not be compromised while the stapler is positioned at the base of the diverticulum. The reticulating head of the stapler is invaluable in these situations because it can be maneuvered precisely at the base of the diverticulum.

Laparoscopy was succesfully used to diagnose and treat patients with MD complicated by small bowel obstruction or bleeding caused by occult heterotopic gastric mucosa (Sanders, 1995; Rivas et al., 2003). Successful resection of a Meckel's diverticulum can also be accomplished through laparoscopy, using endostapling devices. The advantages and benefits of minimal access surgery can be truly appreciated in children with symptomatic Meckel's diverticulum. A recent study demonstrate that laparoscopic stapler resection of asymptomatic diverticulum during surgery for unrelated disease has been shown to produce no added morbidity (Ruh et al., 2010).

\section{Small bowel angiodysplasia and management of obscure-occult gastrointestinal bleeding}

Angiodysplasia (Fig. 7) is a vascular malformation that can be located in all gastrointestinal tract. When symptomatic, it causes gastrointestinal bleeding, frequently obscure and occult, and anemia. Small bowel cases are often difficult to localize because traditional endoscopic tools (EGDS and colonoscopy) are not helpful. (Sass et al., 2004; Bodner et al., 2005; Martinez-Ares et al., 2004). Small intestine enteroscopy (double-balloon enteroscopy) is the most specific method for diagnosis but its application is limited because it is a timeconsuming procedure, causes great discomfort to the patient, is often complicated by bleeding and perforation and has high false positive rate (Nguyen et al., 2005; Keuchel \& 
Hagenmuller, 2005; Warneke et al., 2004; Hartmann et al., 2005; Ell et al., 2002; Jones et al., 2005; Lewis \& Swain, 2002). Capsule endoscope is a valid diagnostic tool but histological diagnosis through biopsy and cannot be achieved (Hartmann et al., 2005; Ell et al., 2002; Jones et al., 2005; Lewis \& Swain, 2002). During the active stage of small intestinal bleeding, selective angiography can find the contrast medium flowing from the lesion into the intestinal tract, showing local shadow with a slightly high density, and concomitant embolic treatment can be performed (Yamaguchi et al., 2003). Scintigraphy with 99mTc-sestamibi marks the eritrocytes and is sensitive to mild intestinal bleeding, while it has no diagnostic value in the resting phase of bleeding or when it is less than $0.05 \mathrm{~mL} / \mathrm{min}$ (Rerksuppaphol et al., 2004). So, diagnosis of massive obscure gastro-intestinal bleeding is usually made by laparotomy, which is invasive with a false positive rate of $5 \%$. Laparoscopy can clearly, directly and conveniently observe the whole intestinal serosa and mesentery (Ell et al., 2002; Rerksuppaphol et al., 2004; Lee et al., 2000; Abbas et al., 2001; Loh \& Munro, 2003; Kok et al., 1998) so that many authors agree that it is a very promising tool in the diagnosis and treatment of acute massive small intestinal bleeding and can be used as a routine method (Ell et al., 2002; Rerksuppaphol et al., 2004; Abbas et al., 2001; Kok et al., 1998).

Laparoscopic exploration of small intestinal hemangiomas or vascular deformity should be extremely careful. The intestinal wall should be carefully explored for local prominence, pitting, overlapping and abnormal mensentery. The suspected bleeding segment should be palpated carefully with clamps to feel its hardness, flexibility, and activity. In case of active massive bleeding, intestinal peristalsis is active and blood often accumulates in the distal segment which is dark blue under laparoscope. In cases in which the bleeding site is not individuated by laparoscopy, perioperative enteroscopy generally allow to reach the goal (Ell et al., 2002; Lee et al., 2000; Loh \& Munro, 2003; Kok et al., 1998).

After the bleeding site was found by laparoscopy, laparoscopy-assisted bowel resection and enteroanastomosis were performed, by exploratory incision about $5 \mathrm{~cm}$ in length at the umbilicus level on the midline. The resected part of the small intestine should be $5 \mathrm{~cm}$ longer than the bleeding site that may result in a fast and reliable excision with light contaminations in the abdominal cavity. Enterectomy and enteroanastomosis can be performed, sometimes, with laparoscopic technique (Rerksuppaphol et al., 2004; Lee et al., 2000; Abbas et al., 2001; Loh \& Munro, 2003; Kok et al., 1998).

In conclusion, laparoscopy in diagnosis and treatment of massive small intestinal bleeding is a minimally invasive procedure with potentially grants less pain, short recovery time and definite therapeutic efficacy than open approach. Randomized studies are necessary.
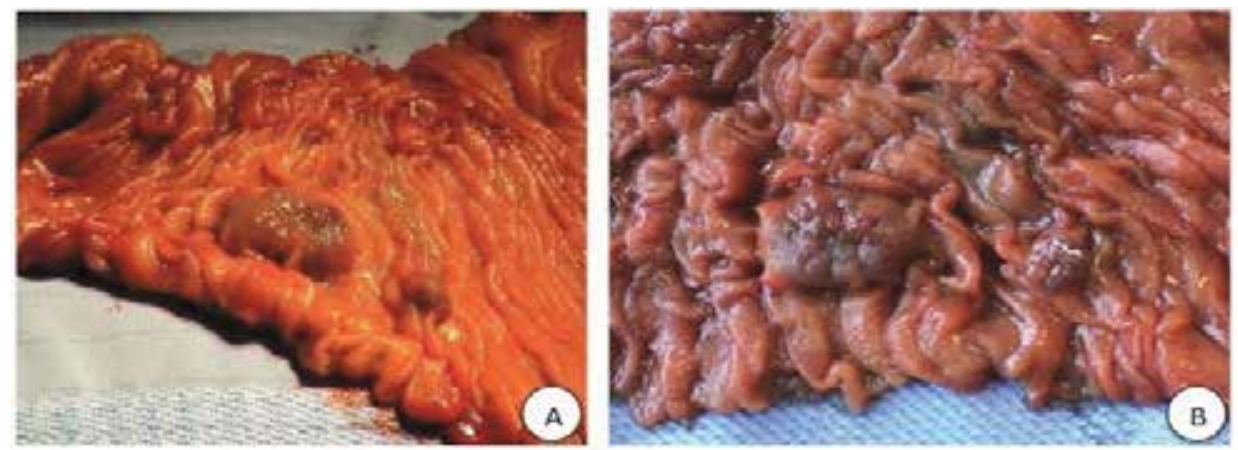

Fig. 7. A, B - Small bowel angiodysplasia 


\section{Other disease}

Several other rare diseases which can cause obstruction (internal hernia, small bowel bezoar, intussusception, pseudo-obstruction) and bleeding (arteriovenous malformations, Delafoy's ulcer) are described in literature. In many of these cases laparoscopic surgery could represents a safe alternative to traditional open approach.

\section{Adhesions and small bowel obstruction}

Adhesions represent the most frequent cause of small bowel obstruction (SBO). Foster et al. in 2005, reported that in 1997 , the $85 \%$ of the 32,583 unscheduled admissions for SBO in California were secondary to adhesions (Foster et al., 2006). In a recent Canadian study, of 552 patients admitted for SBO, 74\% were secondary to adhesions (Miller et al., 2000). Treating adhesive SBO by surgery could seem a paradox because laparotomy is the most common cause of adhesive SBO. The cumulative recurrence rate of SBO after one open adhesiolysis for SBO is $7 \%$ at 1 year, $18 \%$ at 10 years, and $29 \%$ at 25 years. In patients who underwent a second open laparotomy for SBOs, recurrence rate is higher: $17 \%$ at 1 year, $32 \%$ at 10 years, and $40 \%$ at 20 years (Fevang et al., 2004). In the management of SBO conservative management is unanimously accepted in the absence of signs of strangulation for a period varying from 12 hours to 5 days (Seror et al., 1993). CT scan is considered the ideal diagnostic tool to detect complicated SBO as it can show not only site, level and cause of obstruction but also sign of strangulation (Mak et al., 2006). About treatment of SBO two most large studies were performed. In the Canadian study by Foster et al (Foster et al., 2006) on 35,000 people admitted to the hospital for SBO, 75\% were managed successfully conservatively with a 1-year mortality of $23 \%$ and $81 \%$ of surviving patients had no additional SBO readmissions over the subsequent 5 years. Small bowel obstruction was initially considered a condition not suitable for laparoscopy, as a consequence of the limited view of the abdominal cavity due to the dilated bowel, with high risk of accidental enterotomies. As surgeon's experience increased and better technological devices were produced, laparoscopic treatment of SBO became possible and it was rapidly evident that, in experienced hands, it could be a viable alternative to laparotomy as it allowed to decrease potential additional adhesions, together with the well known advantages of this approach. Selection criteria for laparoscopy (Duh, 1998) may be helpful: proximal obstruction, partial obstruction, anticipated single band, localized distension on radiography, no sepsis, and mild abdominal distension. A review published in 2007 show that laparoscopic management of SBO was successful in $66 \%$ of patients with a conversion rate of 33.5\% (Ghosheh \& Salameh, 2007) mostly due to dense adhesions (28\%) followed by the need for bowel resection (23\%) for injury, ischemia, gangrene, and other causes. The rate of success was significantly higher $(\mathrm{p}<0.001)$ in patients operated in the first $24 \mathrm{~h}$ and in patients with bands $(54 \%)$, than in those with matted adhesions (31\%). A recent review reported a morbidity rate of $15.5 \%$ and a mortality rate of $1.5 \%$ (Ghosheh \& Salameh, 2007). Operative time longer than 120 minutes, intraoperative perforation, bowel necrosis, and conversion to laparotomy were significant predictors of post- operative morbidity (Strickland et al., 1999). Several animal studies (Riesman et al., 1996) supporting the hypothesis that laparoscopy leads to a decreased rate of adhesion formation as compared to laparotomy. This should be the main rationale to propose laparoscopy, rather than immediately recognizable benefits. It is not 
really clear, however, if laparoscopic adhesiolysis for SBO would lead to a decrease in recurrence rate. Ghosheh and Salameh (Ghosheh \& Salameh, 2007) reported an early recurrence of SBO in $22(2.1 \%)$ of 1,061 patients. However, no conclusion can be drawn regarding the true rate of recurrence of SBO since adequate follow-up is lacking in most of the published studies.

\section{Surgical techniques}

\subsection{General principles}

Mechanical bowel preparation antibiotic prophilaxis before laparoscopic surgery of the small bowel are the standard practice. Nasogastric tube and urinary catheter are commonly used, especially the last one as it is extremely useful in obtaining more space in the surgical field and decreasing chances of accidental injury by keeping the bladder empty during the procedure. The operation is performed under general anesthesia. The patient is positioned supine with tucked arms opened legs. The surgeon should stand facing the lesion: between the legs (our preferred position), on patient's right side, for lesions involving the proximal small bowel, or on the left side, for lesions involving the terminal ileum. The camera operator stands on surgeon's right side, if he is positioned between the legs of the patient or on the same side, if the surgeon is positioned laterally to the patient. The assistant stands on the opposite side of the operator. The surgeon should stand in line with the view of the laparoscope, with comfortable handling of ports and instruments with each hand. The monitor should be in front of the surgeon and facing the line of view of the telescope. The first trocar, used to introduce the laparoscope, should be placed in the umbilical region. Despite continuous evolution of both laparoscopic instruments and techniques, injuries to the intraabdominal structures are still a common complication of laparoscopy. Many of these injuries are related to the blind placement of the Veress needle or sharp first trocar into the abdomen,when performing the technique referred to as "closed" laparoscopy. Open laparoscopy, where the peritoneal cavity is opened before placing a blunt trocar into the abdomen, was then proposed and widely adopted, with remarkable success in avoiding major vessel injuries but not bowel ones. In response "optical-access" trocars were developed. These trocars were designed to decrease the risk of injury to intrabdominal structures by allowing the surgeon to visualize abdominal wall layers during placement. Two "optical-access" trocar systems are available: the first one uses a blade that strikes the fascia and peritoneum under laparoscopic visualization (Visiport, United States Surgical, Norwalk, CT), and the other one has a conical clear tip that is rotated under laparoscopic vision as it penetrates the fascia and peritoneum (Optiview, Ethicon Endo-Surgery, Cincinnati, $\mathrm{OH}$ ). An angled (30- or 45-degree) camera gives the best view of the small bowel mesentery and is much preferred over a 0-degree scope. Additional trocars (5 mm or 10-12 $\mathrm{mm}$, depending on instruments that will be used) are placed in the left and in the right abdomen, just below the level of the umbilicus. Other essential equipment includes atraumatic graspers for safely handling the bowel, laparoscopic scissors with attachment to monopolar cautery and laparoscopic intestinal staplers, both linear dividing (gastrointestinal anastomosis [GIA]-type) and linear closing (TA-type). Mesenteric section may be accomplished by using a combination of vascular endoscopic staplers, clips, or Ultracision Harmonic Scalpel® or Ligasure ${ }^{\circledR}$ which allow a proper dissection with minimal blood loss. 

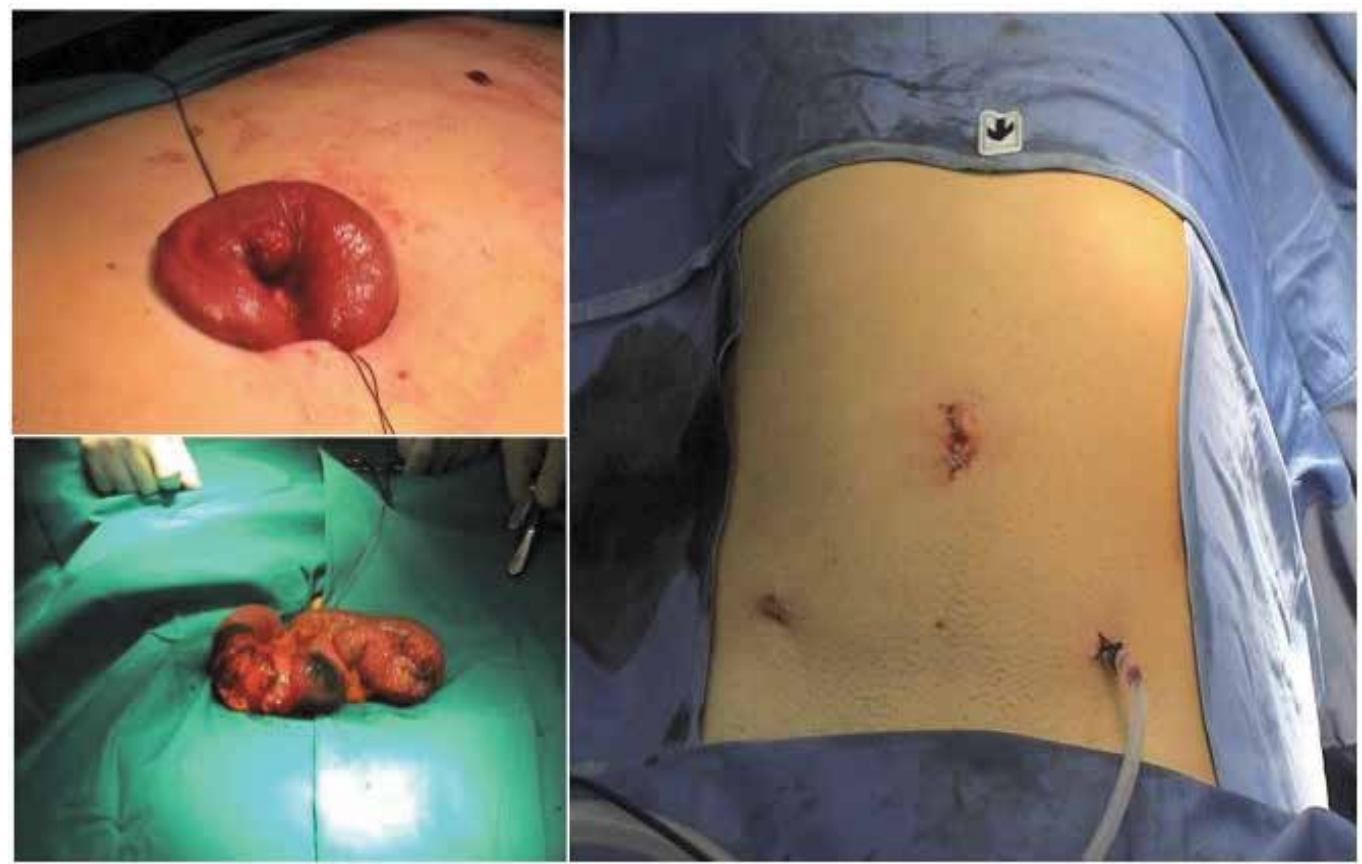

Fig. 8. Technical aspects of small bowel laparoscopic resection

Because of the potential for multifocal or unsuspected lesions, a careful exploration of the abdomen and all small bowel (from Treitz to ileo-cecal valve) is performed as the first step of the procedure, to exactly define the site and the extent of disease. Putting the patient on Trendelenburg position, the surgeon locates and grasps the transverse colon and maintains upward traction; then he changes the patient's position to reverse Trendelenburg, to slip down the small intestine away from the transverse colon, allowing identification of the Treitz ligament. The surgeon runs the small intestine between a pair of atraumatic clamps and identifies the segment that has to be resected. The location of the lesion will be more simple if previous spotting (during enteroscopy) has been performed. Adhesiolysis is often necessary. After identification and mobility evaluation of the small bowel tract involved by disease, a $4 \mathrm{~cm}$ midline peri-umbilical incision is performed, pneumoperitoneum is evacuated and the small bowel loop affected by disease, is pulled out from the abdominal cavity together with its mesentery (Fig. 8). Using a wound protection is mandatory if neoplasm is suspected. A V-shaped incision is performed on mesentery related to the small bowel loop affected by disease, using standard technique, and ligation of vascular pedicle is performed. Wide mesenteric excision is appropriate only when treating malignant lesions. The bowel is divided extracorporeally, by using an intestinal stapler. A mechanical or handsewn side-to-side or end-to-end anastomosis is usually performed extracorporeally. After performing the anastomosis, the mesenteric defect is closed (if possible) and the bowel is reintroduced into the abdominal cavity. After closure of the small incision and the reestablishment of the pneumoperitoneum, the surgeon must confirm hemostasis, and control the anastomosis. A para-anastomotic drainage is positioned using a trocar incision (Fig. 8). Mesenteric vessels ligation, bowel resection and anastomosis could also be performed intracorporeally. 


\subsection{Surgical techniques for small bowel resection for Crohn's disease}

Under general anesthesia, the patient is placed in a supine position with both arms tucked at his or her sides. Three 5/10/12 mm trocars respectively, a $30^{\circ}$ angled laparoscope and an intra-abdominal insufflation of $12 \mathrm{mmHg}$ are used. The pneumoperitoneurn is established following open Hasson trocar placement through an infraumbilical incision. Under laparoscopic vision, the other two trocars are placed in the patient's left flank, lateral to the rectus sheath. After a careful exploration of the abdomen to assess the site and extent of disease, the right colon, terminal ileum, and the inflammatory mass are fully mobilized, mainly with blunt dissection from the lateral to medial direction. The hepatic flexure is mobilized in most cases. No attempt should be made to transect the thick mesentery at this time. The right colon and the terminal ileum are extracted through a small $(5-7 \mathrm{~cm})$ right lower quadrant incision, using Pfannestiel or a midline incision. The terminal ileum and the cecum are transected with a linear stapler. An hand sewn side-to-side anastomosis is performed extracorporeally. In very simple cases, the ileocolic vascular pedicle is transected first with a $30-\mathrm{mm}$ vascular stapler; transection and formation of the ileo-colic anastomosis are then performed intracorporeally. Mobilization of ascending colon is required if neoplasm is located in terminal ileum.

\subsection{Surgical techniques for the treatment of adhesions}

The patient should be secured with a bean bag, and a modified lithotomy position should be considered for posssible intraoperative endoscopy. Two monitors are ideal for this surgery. The site of first trocar placement should be carefully planned away from existing scars. The $30^{\circ}$ scope provides excellent visualization. The pneumoperitoneum should never exceed 15 mmHg. The bowel should be examined for perforation and signs of ischemia. Free fluid should be aspirated and sent for gram staining, amylase, bilirubin, and culture. To facilitate exposure, table tilt, and external manual compression of the abdominal wall can be used. Second and third ports can be added to avoid excessive tenting of the abdominal wall. One of the most dreaded complications in laparoscopic management of small bowel obstruction is enterotomy. In a single comparative study (Wullstein \& Gross, 2003), the risk of perforation was $27 \%$ in the laparoscopic group which was clearly higher than in the open group. The real concern, however, is that the bowel injury may be missed at the time of operation, with potential devastating consequences. The risk of bowel injury can be diminished by following good surgical technique. Bowel graspers with non-locking handles should be used gently to run the bowel. Exposure can be achieved by pushing with closed instruments rather than by grasping. The small bowel should be inspected in a retrograde fashion beginning from the caecum and decompressed bowel until the point of transition is identified. Energy-based devices should be avoided to divide adhesions. There should be a low threshold for conversion and it is not to be considered as a sign of failure but just a good clinical judgement. Conversion is not correlated to the number of previous surgeries (Navez et al., 1998). However, it may be predicted by bowel distension over $4 \mathrm{~cm}$, a documented history of dense adhesions, and the presence of complete distal obstruction (Navez et al., 1998).

\section{Conclusions}

Small bowel diseases are rare and difficult to identify because diagnosis is late and often made on the base of the evidence of their complications. Small bowel laparoscopic surgery is extremely challenging, due to technical difficulties during mobilization of the intestinal 
loops, especially if dilated as a consequence of an occlusion. Nevertheless, after an adequate learning curve and with the help of small bowel endoscopic diagnostic tools, laparoscopic surgery of the small bowel can be considered as a feasible, safe and extremely accurate diagnostic and therapeutic choice for several small bowel diseases.

\section{References}

Abbas MA., Al-Kandari M. \& Dashti FM. (2001). Laparoscopic-assisted resection of bleeding jejunal leiomyoma. Surg Endosc, 15, 1359-1362.

Albaz O., Iroatulam AJN., Nessim A., Weiss EG., Nogueras JJ. \& Wexer SD. (2000). Comparision of laparoscopic assisted and conventional ileo-colic resection for Crohn's disease. Eur J Surg, 166, 213-217.

Ballantyne GH. (2002). The pitfalls of laparoscopic surgery: challenges for robotics and telerobotic surgery. Surg Laparosc Endosc Percutan Tech, 12, 1-5.

Berguer R. (1998). Surgical technology and the ergonomics of laparoscopic instruments. Surg Endosc, 12, 458-62

Bilimoria KY., Bentrem DJ. \& Wayne JD. (2009). Small bowel cancer in United States. Ann Surg, 249, 63-71

Blay JY.; Bonvalot S. \& Casali P. (2005). GIST consensus meeting panelists. Consensus meeting for the management of gastrointestinal stromal tumors. Report of the GIST Consensus Conference of 20-21 March 2004, under the auspices of ESMO. Ann Oncol, 16, 566-578

Bobby VMD., McKay D. \& Gardiner K. (2011). Laparoscopic versus Open surgery for small bowel Crohn's disease. Cochrane Database of Systematic Reviews, 1, CD006956

Bodner J.; Chemelli A.; Zelger B. \& Kafka R. (2005). Bleeding Meckel's diverticulum. J Am Coll Surg, 200: 631

Carrasco Rojas (2004). Minimally invasive surgery of small intestine. Rev Gastroenterol Mex, 69, Suppl1, 51-7

Carter MJ., Lobo AJ. \& Travis SPL. (2004). Guidelines for the management of inflammatory bowel disease in adults. Gut, 53, 1-16.

Coco C, Rizzo G, Manno A, Mattana C \& Verbo A. (2010). Surgical treatment of small bowel neoplasms. Eur Rev Med Pharmacol Sci., 14, 327-33.

Crump M., Gospodarowicz M. \& Shepherd FA. (1999). Lymphoma of the gastrointestinal tract. Semin Oncol, 26, 324-7

Cullen JJ., Kelly KA., Moir CR., Hodge DO., Zinsmeister AR. \& Melton LJ 3rd. Surgical management of Meckel's diverticulum. An epidemiologic, population-based study. Ann Surg, 220, 564-568; discussion 568-569

Demetri GD., Benjamin R. \& Blanke CD. (2004). Optimal management of patients with gastrointestinal stromal tumors. Expansion and update of NCCN Clinical Practice Guidelines. J Natl Compr Care Netw, 2, 51-526

Duepree H-J., Senagore AJ., Delaney CP., Brady KM. \& Fazio VW. (2002). Advantages of laparoscopic resection for ileocecal Crohn's disease. Dis Colon Rectum, 45, 605-610.

Duh QY. (1998). Small bowel obstruction. Endosurgery. Churchill Livingstone, New York, 425-431 
Dunker MS., Stiggelbout AM., Van Hogezand RA., Ringers J., Griffioen G. \& Bemelman WA. (1998). Quality of life, cosmesis and body image after laparoscopic-assisted and open ileocolic resection for Crohn's disease. Surg Endosc, 12, 1334-40.

Ehrmantraut W. \& Sardi A. (1997). Laparoscopy-assisted small bowel resection. Am Surg, 996-1001

Ell C, Remke S, May A, Helou L, Henrich R. \& Mayer G (2002). The first prospective controlled trial comparing wireless capsule endoscopy with push enteroscopy in chronic gastrointestinal bleeding. Endoscopy, 34, 685-689

Eshuis EJ, Slors JFM, Stokkers PCF, Sprangers MAG, Ubbink DT, Cuesta MA, Pierik EGJM. \& Bemelman WA. (2010). Long-term outcomes following laparoscopically assisted versus open ileo-colic resection for Crohn's disease. BJS, 97, 563-568.

Everett M. \& Gutman H. (2008). Surgical management of gastrointestinal stromal tumors: analysis of outcome with respect to surgical margins and technique. J Surg Oncol, 98, 588-93

Fevang B-TS, Fevang J \& Lie SA. (2004). Long-term prognosis after operation for adhesive small bowel obstruction. Ann Surg, 240, 193-201

Foster NM., McGory ML., Zingmond DS. \& Ko CY. (2006). Small bowel obstruction: a population-based appraisal. J Am Coll Surg, 203, 170-176

Gerson LB. (2009). Capsule endoscopy and deep enteroscopy: indications for the practicing clinician. Gastroenterology, 137, 1197-201

Ghosheh B. \& Salameh JR. (2007). Laparoscopic approach to acute small bowel obstruction: review of 1061 cases. Surg Endosc, 21, 1945-1949

Gill SS., Heuman DM. \& Mihas A.(2001). Small intestinal neoplasm. J Clin Gastroenterol, 33, 267-82

Hartmann D, Schmidt H, Bolz G, Schilling D, Kinzel F, Eick- hoff A, Huschner W, Moller K, Jakobs R, Reitzig P, Weickert U, Gellert K, Schultz H, Guenther K, Hollerbuhl H, Schoenleben K, Schulz HJ. \& Riemann JF. (2005). A prospective two-center study comparing wireless capsule endoscopy with intraoperative enteroscopy in patients with obscure GI bleeding. Gastrointest Endosc, 61, 826-832

Hasson H. (1974). Open laparoscopy: a report of 150 cases. J Reprod Med, 12, 234-238

Heinicke JM., Tedaldi R. \& Muller C. (1997). An unusual manifestation of Meckel's diverticulum: bleeding and perforation - a case report. Swiss Surg, 3, 97-9

Higaki S., Saito Y. \& Akazawa A. (2001). Bleeding Meckel's diverticulum in an adult. Hepatogastroenterology, 48, 1628-30

Howe JR., Karnell LH., Menck HR. \& Scott-Conner C. (1999). The American College of Surgeons Commission on Cancer and the American Cancer Society. Adenocarcinoma of the small bowel: review of the National Cancer Data Base, 1985-1995. Cancer, 86, 2693-706

Hutchins RR., Bani Hani A., Kojodjojo P., Ho R. \& Snooks SJ. (2001) Adenocarcinoma of the small bowel. ANZ J Surg, 71, 428-37

Jones BH., Fleischer DE., Sharma VK., Heigh RI., Shiff AD., Hernandez JL. \& Leighton JA. (2005) Yield of repeat wireless video capsule endoscopy in patients with obscure gastrointestinal bleeding. Am J Gastroenterol, 100, 1058-1064 
Kelling G. (1901). Die Tamponade der Speiseroehre und des magens mit beigsamen instrumenten. Verdhandlungen der Gesellschaft Deutscher Naturtorscher und Aerzte. Vogel verlag Leipzig, 73, 117-119

Keuchel M. \& Hagenmuller F. (2005). Small bowel endoscopy. Endoscopy, 37, 122-132

Kok KY., Mathew VV. \& Yapp SK (1998). Laparoscopic-assisted small bowel resection for a bleeding leiomyoma. Surg Endosc, 12, 995-996

Kong MS., Chen CY., Tzen KY., Huang MJ., Wang KL. \& Lin JN. (1993). Technetium-99m pertechnetate scan for ectopic gastric mucosa in children with gastrointestinal bleeding. J Formos Med Assoc, 92, 717-20

Kulke MH. \& Meyer RJ. (1999). Carcinoid tumors. N Engl J Med, 340, 858-68

Kumar R., Tripathi M., Chandrashekar N. Agarwala S, Kumar A, Dasan JB \& Malhotra A. (2005). Diagnosis of ectopic gastric mucosa using 99Tcm-pertechnetate: spectrum of scintigraphic findings. Br J Radiol; 78:714-20

Lee KH., Yeung CK., Tam YH., Ng WT. \& Yip KF. (2000). Laparascopy for definitive diagnosis and treatment of gastrointestinal bleeding of obscure origin in children. $J$ Pediatr Surg, 35, 1291-1293

Leijonmarck CE., Bonman-Sandelin K., Frisell J. \& Raf L. (1986). Meckel's diverticulum in the adult. Br J Surg, 73, 146-149

Lepage C., Bouvier AM., Manfredi S. Dancourt V. \& Faivre J. (2006). Incidence and management of primary malignant small bowel cancer: a well-defined French population study. Am J Gastroenterol, 101, 2826-32

Lewis BS. \& Swain P. (2002). Capsule endoscopy in the evaluation of patients with suspected small intestinal bleeding: Results of a pilot study. Gastrointest Endosc, 56, 349-353

Lin S., Suhocki PV., Ludwig KA. \& Shetzline MA. (2002). Gastrointestinal bleeding in adult patients with Meckel's diverticulum: the role of technetium $99 \mathrm{~m}$ pertechnetate scan. South Med J, 95, 1338-41

Litynski GS. (1998). Erich Muhe and the rejection of laparoscopic cholecystectomy: a surgeon ahead of his time. J Soc Laparosc Surg, 2, 341-346

Loh DL. \& Munro FD. (2003). The role of laparoscopy in the management of lower gastrointestinal bleeding. Pediatr Surg Int, 19, 266-267

Lowney JK., Deitz DW., Birnbaum EH., Kodner IJ., Mutch MG. \& Fleshman JW. (2005). Is there any difference in recurrence rates in laparoscopic ileo-colic resection for Crohn's disease compared with conventional surgery? Dis Colon Rectum, 49, 58-63.

Maartense S., Dunker MS., Slors JFM., Cuesta MA., Pierik EGJM., Gouma DJ., Hommes DW., Sprangers MA. \& Bemelman WA. (2006). Laparoscopic-Assisted Versus Open ileo-colic Resection for Crohn's Disease. Ann Surg, 243, 143-149.

Mak SY., Roach SC. \& Sukumar SA. (2006). Small bowel obstruction: computed tomography features and pitfalls. Curr Probl Diagn Radiol, 35, 65-74

Martinez-Ares D., Gonzalez-Conde B., Yanez J., Estevez E., Arnal F, Lorenzo J., Diz-Lois MT. \& Vazquez-Iglesias JL. (2004). Jejunal leiomyosarcoma, a rare cause of obscure gastrointestinal bleeding diagnosed by wireless capsule endoscopy. Surg Endosc, $18,554-556$

Miller G., Boman J., Shrier I. \& Gordon PH. (2000). Etiology of SBO. Am J Surg, 180, 33-36 
Miller G., Boman J., Shrier I. \& Gordon PH. (2000). Natural history of patients with adhesive small bowel obstruction. Br J Surg, 87, 1240-1247

Milsom JW., Hammerhofer KA., Bohm B., Marcello P., Elson P. \& Fazio VW. (2001). Prospective randomised trial comparing laparoscopic vs conventional surgery for refractory ileocolic Crohn's disease. Dis Colon Rectum, 44, 1-9.

Milsom JW., Lavery IC., Bohm B. \& Fazio VW. (1993). Laparoscopically assisted ileocolectomy in Crohn's disease. Surg Laparosc Endosc, 3, 77-80.

Morgan DR., Mylankal K, Barghouti N. \& Dixon MF. (2000). Small bowel haemangioma with local lymph node involvement presenting as intussusception. J Clin Pathol, 53, 552-3

Munkholm P. \& Binder V. (2004). Clinical features and natural history of Crohn's disease. Kirsner's inflammatory bowel diseases. 6th Edition; 289-300.

Navez B., Arimont JM \& Guiot P. (1998) Laparoscopic approach in acute small bowel obstruction. A review of 68 patients. Hepatogastroenterology, 45, 2146-2150

Nelson H., Sargent DJ. \& Wieand HS. (2004). A comparison of laparoscopically assisted and open colectomy for colon cancer. N Engl J Med, 350, 2050-9

Neugut AI., Jacobson JS., Suh S. Mukherjee R. \& Arber N. (1998). The epidemiology of cancer of the small bowel. Cancer Epidemiol Biomarkers Prev, 7, 243-51

Neugut AI., Marvin MR., Rella VA. \& Chabot JA. (1997). An overview of adenocarcinoma of the small intestine. Oncology, 11, 529

Nguyen NQ., Rayner CK. \& Schoeman MN. (2005). Push enteroscopy alters management in a majority of patients with obscure gastrointestinal bleeding. J Gastrointestinal Hepatol, 20, 716-721

North JH. \& Pack MS. (2000). Malignant tumors of the small intestine: a review of 114 cases. Am Surg, 66, 46-51

Novitsky YW., Kercher KW., Sing RF. \& Heniford BT. (2006). Long-term outcomes of laparoscopic resection of gastric gastrointestinal stromal tumors. Ann Surg, 243, 738-745

Park JJ., Wolff BG., Tollefson MK., Walsh EE. \& Larson DR. Meckel diverticulum: the Mayo Clinic experience with 1476 patients. Ann Surg; 241: 529-533

Puligandla PS., Becker L., Driman D., Prokopiw I., Taves D. \& Davies ET. (2001). Inverted Meckel's diverticulum presenting as chronic anemia: case report and literature review. Can J Surg, 44, 458-9

Reissman P., Salky BA., Pfeifer J., Edye M., Jagelman DG. \& Wexner SD. (1996). Laparoscopic surgery in the management of inflammatory bowel disease. Am J Surg, 171, 47-51.

Rerksuppaphol S., Hutson JM. \& Oliver MR. (2004). Ranitidine-enhanced 99mtechnetium pertechnetate imaging in children improves the sensitivity of identifying heterotopic gastric mucosa in Meckel's diverticulum. Pediatr. Surg Int, 20, 323-325

Reissman P., Teoh TA. \& Skinner K. (1996). Adhesion formation after laparoscopic anterior resection in a porcine model: a pilot study. Surg Laparosc Endosc, 6, 136-139

Rivas H., Cacchione RN. \& Allen JW. (2003). Laparoscopic management of Meckel's diverticulum in adults. Surg Endosc, 17, 620-622

Robinson TN. \& Stiegmann GV. (2004). Minimally invasive surgery. Endocopy, 36, 48-51 
Ruh J., Paul A., Dirsch O., Kaun M. \& Broelsch CE. (2010) Laparoscopic resection of perforated Meckel's diverticulum in a patient with clinical symptoms of acute appendicitis. Surg Endosc, 4207-14.

Sanders LE. (1995). Laparoscopic treatment of Meckel's diverticulum. Obstruction and bleeding managed with minimal morbidity. Surg Endosc, 9, 724-727

Sass DA., Chopra KB., Finkelstein SD. \& Schauer PR. (2004). Jejunal gastrointestinal stromal tumor: a cause of obscure gastrointestinal bleeding. Arch Pathol Lab Med, 128, 214217

Seror D., Feigin E., Szold A., Allweis TM., Carmon M., Nissan S. \& Freund HR. (1993). How conservatively can postoperative small bowel obstruction be treated? Am J Surg, $165,121-125$

Shalaby RY., Soliman SM., Fawy M. \& Samaha A. (2005). Laparoscopic management of Meckel's diverticulum in children. J Pediatr Surg, 40, 62-7

Shebani KO., Souba WW., Finkelstein DM., Stark PC., Elgadi KM., Tanabe KK. \& Ott MJ. ( 1999). Prognosis and survival in patients with gastrointestinal tract carcinoid tumors. Annals of Surgery, 229, 815-823

Soltero MJ. \& Bill AH. (1976). The natural history of Meckel's Diverticulum and its relation to incidental removal. A study of 202 cases of diseased Meckel's Diverticulum found in King County, Washington, over a fifteen year period. Am J Surg, 132, 168173

Strickland P., Lourie DJ., Suddleson EA., Blitz JB. \& Stain SC. (1999). Is laparoscopy safe and effective treatment of acute small bowel obstruction? Surg Endosc, 13, 695-698

Sutton R., Doran HE., Williams EM., Vora J., Vinjamuri S., Evans J., Campbell F., Raraty MG., Ghaneh P., Hartley M., Poston GJ. \& Neoptolemos JP. (2003). Surgery for midgut carcinoid. Endocrine-Related Cancer, 10, 469-81

Tan YM. \& Zheng ZX. (2005). Recurrent torsion of a giant Meckel's diverticulum. Dig Dis Sci, 50, 1285-7

Thoreson R. \& Cullen JJ. (2007). Pathophysiology of Inflammatory Bowel disease: An overview. Surg Clin N Am, 87, 575-585.

Travis SP., Stange EF., Lémann M., Oresland T., Chowers Y., Forbes A., D’Haens G., Kitis G., Cortot A., Prantera C., Marteau P., Colombel JF., Gionchetti P., Bouhnik Y., Tiret E., Kroesen J., Starlinger M. \& Mortensen NJ. (2006). European Crohn's and Colitis Organisation. European evidence based consensus on the diagnosis and management of Crohn's disease: current management. Gut, 55 (Suppl 1), i16-35.

Uchikoshi F., Ito T., Nezu R., Tanemura M., Kai Y., Mizushima T., Nakajima K., Tamagawa H., Matsuda C. \& Matsuda (2004). H. Advantages of laparoscopic-assisted surgery for recurrent Crohn disease. Surg Endosc, 18, 1675-1679.

Veress J. (1938). Neues Instrument Zur Ausfuhrung von brustoder Bachpunktionen und Pneumothoraybehundlung. Deutsch Med Wochenschr, 64, 1480-148

Warneke RM., Walser E., Faruqi S., Jafri S., Bhutani MS. \& Raju GS. (2004). Cap-assisted endoclip placement for recurrent ulcer hemorrhage after repeatedly unsuccessful endoscopic treatment and angiographic embolization: case report. Gastrointest Endosc, 60, 309-312 
Witteman BJ., Janssens AR., Griffioen G. \& Lamers CB. (1993). Villous tumours of the duodenum. An analysis of the literature with emphasis on malignant transformation. Neth J Med, 42, 5-11

Wong JH., Suhaili DN. \& Kok KY. (2005). Fish bone perforation of Meckel's diverticulum: a rare event? Asian J Surg, 28, 295-6

Wullstein C. \& Gross E. (2003). Laparoscopic compared with conventional treatment of acute adhesive small bowel obstruction. Br J Surg, 90, 1147-1151

Yamaguchi T. \& Yoshikawa K. (2003), Enhanced CT for initial localization of active lower gastrointestinal bleeding. Abdom Imaging, 28, 634-636

Yao KA., Talamonti MS., Nemcek A., Angelos P., Chrisman H., Skarda J., Benson AB., Rao S. \& Joehl RJ. (2001). Indications and results of liver resection and hepatic chemoembolization for metastatic gastrointestinal neuroendocrine tumors. Surgery, 130, 677-685

Zani A., Eaton S., Rees CM. \& Pierro A. ( 2008). Incidentally detected Meckel diverticulum: to resect or not to resect? Ann Surg, 247, 276-281 


\title{
Surgical Treatment of Gastroesophageal Reflux Disease
}

\author{
Filippo Tosato1, Salvatore Marano1, \\ Stefano Mattacchione ${ }^{1}$, Barbara Luongo ${ }^{1}$, \\ Giulia Paltrinieri ${ }^{1}$, Valentina Mingarelli ${ }^{1}$ \\ and Leoluca Vasapollo ${ }^{2}$ \\ ${ }^{1}$ Referral Center for the Surgical Treatment \\ of Gastroesophageal Reflux Diseases, \\ "Sapienza" University of Rome \\ 2Sandro Pertini Hospital, Rome \\ Italy
}

\section{Introduction}

Gastro-esophageal reflux disease (GERD) is "a condition which develops when the reflux of stomach contents causes troublesome symptoms (i.e., at least two heartburn episodes per week) and/or complications" (Vakil et al 2006) and represents one of the fastest growing disease affecting the alimentary tract. Recently studies on the epidemiology of GERD demonstrating that GERD is a highly prevalent disorder with $10-20 \%$ of individuals affected in western civilization (Dent et al 2005). When GERD is defined as twice weekly reflux over several months, $10-20 \%$ of individuals in Western civilization are affected, which is significantly higher than in Asian population (5\%) (Bonatti et al 2008). In a large prospective American cohort study is reported that $25 \%$ of investigated individuals experienced nocturnal reflux symptoms (Fass et al 2005). A recent population-based study demonstrated the prevalence of reflux symptoms to be $44 \%$ with $24 \%$ of individuals experiencing symptoms for two days or more per week. The prevalence of oesophagitis and Barrett's oesophagus was $12 \%$ and $1.3 \%$ respectively, irrespective of symptoms (Zagari et al 2008).Thirty-three per cent of individuals with oesophagitis and $46 \%$ with Barrett's oesophagus were asymptomatic. Severe GERD can lead to potentially avoidable complications including severe oesophagitis with scarring and stricture formation, Barrett's esophagus and adenocarcinoma.When symptoms become frequent and severe enough to require regular medication, there is a significant impact on quality of life (Ware et al 1992).(QOL)

\section{Pathophysiology}

GERD results from failure of the reflux barrier. This barrier has three components: (1) an intra-abdominal esophagus of adequate length, (2) an extrinsic sphincter, the esophageal hiatus, and (3) an intrinsic sphincter, the lower esophageal sphincter (Bloom et al 2009). 
Relaxation of the LES and crura are normal physiological processes occurring during swallowing and also during gas venting. Relaxations not initiated by a swallow are known as transient lower esophageal relaxations (TLESRs). Abnormal TLESRs have a greater crosssectional area at the gastro-oesophageal junction resulting in the reflux of gastric fluid in addition to gas. TLESRs probably account for $90 \%$ of reflux episodes. The TLESR reflex is initiated by tension receptors in the stomach and mediated by a vagovagal pathway via the brainstem leading to simultaneous relaxation of the crura, LES and inhibition of peristalsis (Pandolfino et al 2003). These responses are inhibited by gammaaminobutyric acid-B (GABA-B) receptor agonists, and thus may constitute a future therapeutic target. Hiatus hernias appear to increase the magnitude of reflux during TLESRs. Transient lower oesophageal relaxations appear to be less significant in more severe reflux esophagitis. A hypotensive LES, which allows high pressure gradients across the diaphragm, is probably responsible for more severe esophagitis (Barham et al 1995). Reflux occurs more frequently when the pressure in the LES is $<10 \mathrm{~mm} \mathrm{Hg}$, and free reflux only occursif the LES pressure is $<4 \mathrm{~mm} \mathrm{Hg}$ (Kahrilas et al 1986; Dodds et al 1982). Factors which relax the LES, such as caffeine, fat, smoking, drugs (calcium channel antagonists and nitrates) and gastric distention, will increase the likelihood of reflux. The association of increased body mass index and GERD remains unclear (Hampel et al 2005; Pandolfino et al 2006). A recent study failed to demonstrate an increased risk of reflux symptoms or esophagitis in obese individuals. Acid in the distal oesophagus has been shown to be neutralised by saliva. Therefore any processes reducing saliva production results in a delay in acid neutralisation. Nocturnal reflux episodes are prolonged due to depressed salivation. Acid is cleared by oesophageal peristalsis. Therefore impaired distal esophageal peristalsis results in prolonged acid exposure to acid reflux episodes. This is apparent in both hiatus hernias and ulcerative oesophagitis where there is peristaltic dysfunction (Kahrilas et al 2006, Johnson et al 1980).

\section{Simptomatology}

Typical GERD symptoms - heartburn and regurgitation-reflect dysfunction of the reflux barrier.

Dysphagia is a third, less specific, GERD symptom. It may be caused by GERD itself or stricture complicating GERD. GERD-related dysphagia must be differentiated from functional and mechanical dysphagia resulting from multiple other diseases that cause symptomatic esophageal obstruction. Atypical GERD symptoms are cough, asthma, laryngitis, sore throat, chest pain, abdominal pain, and bloating. These symptoms in the absence of typical GERD symptoms point to diseases other than GERD. Careful investigation of alternative causes of atypical symptoms is necessary. Sophisticated testing, including impedance/pH monitoring, must be performed if GERD is believed to be the cause of atypical symptoms and surgery is being considered (Bajbouj et al 2007; Fornari et al 2007). Comparing international literature with our case studies we can confirm that number of patients with atypical symptoms referring to our Referral Center for the Surgical Treatment of Gastroesophageal Reflux Diseases is growing. In fact 644 patients (30.7\%), who underwent to endoscopic exams, showed typical and atypical symptoms (heartburn, pyrosis, regurgitation, asthma, laryngospasm, pulmonary fibrosis), and 96 patients (4.6\%) had only atypical symptoms. 
Reflux esophagitis is defined as reflux causing inflammation or ulceration of the esophagus. Attempts have been made to classify the extent of damage, of which the Los Angeles classification is now the most commonly used:

Grade Extent of esophageal inflammation

A Mucosal breaks $<5 \mathrm{~mm}$ not extending between folds

B Mucosal breaks $>5 \mathrm{~mm}$ not extending between folds

C Mucosal breaks extending between folds

D Mucosal breaks extending between $>2$ folds

involving $>75 \%$ of the circumference

\section{Diagnosis}

The signs and symptoms are insufficient to establish a conclusive diagnosis of GERD, regardless of their frequency and intensity, resulting in a diagnostic certainty of around $40 \%$. Endoscopy is not usually performed in young adults patients with typical history of GERD since it does not alter the clinical evolution when compared to the empiric treatment. In patients with non-erosive GERD, the use of the symptom score (moderate or severe) allows a diagnostic certainty of up to $40 \%$ of the cases. In these cases, the upper digestive endoscopy (UDE) does not alter the clinical evolution, when compared to the empiric treatment. It is interesting to remember that, in cases of erosive GERD with typical symptoms, however, the UDE improves the diagnostic accuracy and also establishes a differential diagnosis with other diseases, such as cancer. The 24-hour $\mathrm{pH}$-metry is the most important resource for a definite diagnosis of acid reflux, which constitutes most of reflux episodes, establishing or ruling out the diagnosis with a $90 \%$ and $95 \%$ certainty, respectively. Actually the acid component of the gastric refluxate is responsible for most of the symptoms and pathology associated with GERD, however, other components, such as bile, may also contribute (known as nonacidreflux). Quantification of reflux can be achieved either by measuring acid exposure to the distal esophagus ( $\mathrm{pH}$ studies) or movement of liquid in the distal esophagus (impedance studies). Combined impedance and $\mathrm{pH}$ measurement characterizes all acid and non-acid reflux episodes.

Nearly $70 \%$ of patients with heartburn do not have evidence of erosive changes on endoscopy. Of these, a proportion have increased acid reflux on 24-hour $\mathrm{pH}$ monitoring and are classified as having non-erosive reflux disease (NERD) (Jones et al 1995).

In patients with atypical manifestations, the conventional esophageal $\mathrm{pH}$-metry contributes little to the diagnosis of GERD. The current available evidence does not support the routine use of proximal $\mathrm{pH}$ monitoring. In patients with atypical manifestations, the impedance-pHmetry substantially contributes to the diagnosis of GERD. In patients undergoing prolonged treatment with PPI, the histological esophageal alterations can remain practically unaltered, regardless of the occurrence or not of symptoms and signs. On the other hand, the histological alterations accompany the degree of severity of the esophagitis. Therefore, the evaluation of the histological signs increases the diagnostic probability of GERD. The observation of the dimensions of the distal esophagus intercellular space increases the probability of diagnostic certainty and also allows the analysis of the therapeutic response. Esophageal biopsies in patients with suspected GERD for the analysis of basal cell proliferation allow, in absence of the latter, ruling out the diagnosis or active disease. The isolated presence of the basal layer proliferation, however, has little diagnostic value. Although the basal cell thickness allows the analysis of the therapeutic response, it is not 
correlated with the clinical response. The presence of reflux symptoms in asthmatic patients results in a small increase in the probability of diagnostic certainty. In asthmatic patients with reflux symptoms, the normal $\mathrm{pH}$-metry can predict the absence of therapeutic response with PPI. A significant number of patients with asthma (57\%) also present gastroesophageal reflux.GERD may also produce esophageal injury. Esophagogastroduodenoscopy (EGD) with biopsy has replaced the upright air-contrast phase of the barium esophagram for mucosa evaluation. EGD and biopsy both diagnose and assess esophageal injury by visual and histopathologic mucosal examination. Visual assessment of esophageal injury is graded using the Los Angeles classification. Histopathologic findings, although nonspecific, are confirmatory in the clinical setting of GERD. The finding of specialized columnar epithelium (Barrett esophagus) in the tubular esophagus is secondary to GERD. In the absence of dysplasia, surveillance esophagoscopy and biopsy are required in patients who have Barrett esophagus, regardless of therapy (medical or surgical).

If dysphagia is the predominant symptom and the diagnosis is in question, the examination should start as a timed barium esophagram.

Esophageal manometry excludes unsuspected motility disorders or motility disorders masquerading as GERD, confirms adequate esophageal peristalsis for GERD surgery, and quantifies preoperative resting pressure and relaxation of the lower esophageal sphincter for later comparison. It must be considered in all surgical patients when clinically or phmetrically a motility disorder is suspected.

\section{Management}

The aim of GERD treatment is to effectively control symptoms and prevent GERDassociated complications. As with many conditions, adopting a stepped approach to treatment helps tailor disease severity to treatment regimen.

\subsection{Lifestyle changes}

Simple manoeuvres may have a marked effect on symptoms. These are outlined below:

- dietary changes. Some substances influence oesophageal physiology favouring increased acid reflux. These include fat, caffeine and alcohol

- $\quad$ avoiding late meals. Acid reflux episodes are prolonged when asleep as a result of both gravity, and also reduced peristalsis and acid clearance. Nocturnal reflux can therefore be minimised by consuming small meals long before sleep

- although the association of obesity and GERD is unclear, there does appear to be an association with esophageal adenocarcinoma and many other well-known diseases.11 On this basis weight loss is suggested as part of GERD management.

\subsection{Antacids/alginate combinations}

Antacids consist of calcium carbonate, magnesium and aluminum salts in various compounds or combinations. The effect of antacids is due to partial neutralization of gastric hydrochloric acid and inhibition of the proteolytic enzyme, pepsin. Alginate mechanism of action is due to the formation of a gel in the presence of gastric acid. Alginate-based reforming formulations usually contain sodium or potassium bicarbonate; in the presence of gastric acid, the bicarbonate is converted to carbon dioxide, which becomes entrapped within the gel precipitate, converting it into a foam which floats on the surface of the gastric 
contents, much like a raft on water. Both in vitro and in vivo studies have demonstrated that alginate-based rafts can entrap carbon dioxide, as well as antacid components contained in some formulations, thus providing a relatively $\mathrm{pH}$ neutral barrier. Antacids and alginates have been shown to improve reflux symptoms, however, they do not heal oesophagitis (Stanciu et 1974). They are indicated for very mild symptoms, where step-up treatment is not necessary. There is no role for antacids/alginates in the maintenance of GERD.

Despite the development of potent medications for the treatment of GERD, antacids remain a mainstay of treatment. Antacids neutralize the acid in the stomach so that there is no acid to reflux. The problem with antacids is that their action is brief. They are emptied from the empty stomach quickly, in less than an hour, and the acid then re-accumulates. The best way to take antacids, therefore, is approximately one hour after meals or just before the symptoms of reflux begin after a meal. Since the food from meals slows the emptying from the stomach, an antacid taken after a meal stays in the stomach longer and is effective longer. For the same reason, a second dose of antacids approximately two hours after a meal takes advantage of the continuing post-meal slower emptying of the stomach and replenishes the acid-neutralizing capacity within the stomach.

Antacids may be aluminum, magnesium, or calcium based. Calcium-based antacids (usually calcium carbonate), unlike other antacids, stimulate the release of gastrin from the stomach and duodenum. Gastrin is the hormone that is primarily responsible for the stimulation of acid secretion by the stomach. Therefore, the secretion of acid rebounds after the direct acidneutralizing effect of the calcium carbonate is exhausted. The rebound is due to the release of gastrin, which results in an overproduction of acid. Theoretically at least, this increased acid is not good for GERD.

Acid rebound, however, has not been shown to be clinically important. That is, treatment with calcium carbonate has not been shown to be less effective or safe than treatment with antacids not containing calcium carbonate. Nevertheless, the phenomenon of acid rebound is theoretically harmful. In practice, therefore, calcium-containing antacids such as Tums and Rolaids are not recommended. The occasional use of these calcium carbonatecontaining antacids, however, is not believed to be harmful. The advantages of calcium carbonate-containing antacids are their low cost, the calcium they add to the diet, and their convenience as compared to liquids.

Aluminum-containing antacids have a tendency to cause constipation, while magnesiumcontaining antacids tend to cause diarrhea. If diarrhea or constipation becomes a problem, it may be necessary to switch antacids or alternately use antacids containing aluminum and magnesium.

\subsection{Histamine antagonists}

Although antacids can neutralize acid, they do so for only a short period of time. For substantial neutralization of acid throughout the day, antacids would need to be given frequently, at least every hour. The first medication developed for more effective and convenient treatment of acid-related diseases, including GERD, was a histamine antagonist, specifically cimetidine. $\mathrm{H} 2$ antagonists are very good for relieving the symptoms of GERD, particularly heartburn. However, they are not very good for healing the inflammation (esophagitis) that may accompany GERD. In fact, they are used primarily for the treatment of heartburn in GERD that is not associated with inflammation or complications, such as erosions or ulcers, strictures, or Barrett's esophagus. Four 
different $\mathrm{H} 2$ antagonists are available by prescription, including cimetidine, ranitidine, nizatidine, and famotidine. All four are also available over-the-counter (OTC), without the need for a prescription. However, the OTC dosages are lower than those available by prescription. Histamine is an important chemical because it stimulates acid production by the stomach. Released within the wall of the stomach, histamine attaches to receptors (binders) on the stomach's acid-producing cells and stimulates the cells to produce acid. Histamine antagonists work by blocking the receptor for histamine and thereby preventing histamine from stimulating the acid-producing cells. (Histamine antagonists are referred to as $\mathrm{H} 2$ antagonists because the specific receptor they block is the histamine type 2 receptor.) Because histamine is particularly important for the stimulation of acid after meals, $\mathrm{H} 2$ antagonists are best taken 30 minutes before meals. The reason for this timing is so that the $\mathrm{H} 2$ antagonists will be at peak levels in the body after the meal when the stomach is actively producing acid. $\mathrm{H} 2$ antagonists also can be taken at bedtime to suppress nighttime production of acid.

\subsection{Proton pump inhibitors}

The second type of drug developed specifically for acid-related diseases, such as GERD, was a proton pump inhibitor (PPI), specifically, omeprazole. A PPI blocks the secretion of acid into the stomach by the acid-secreting cells. Proton pump inhibitors (PPIs) bind to enzymes in the stomach membrane that produce hydrochloric acid. PPIs reduce levels of stomach acid, and are commonly used to reduce acid reflux symptoms, heal ulcers, and treat gastroesophageal reflux disease (GERD).The advantage of a PPI over an H2 antagonist is that the PPI shuts off acid production more completely and for a longer period of time. Not only is the PPI good for treating the symptom of heartburn, but it also is good for protecting the esophagus from acid so that esophageal inflammation can heal. PPIs are used when $\mathrm{H} 2$ antagonists do not relieve symptoms adequately or when complications of GERD such as erosions or ulcers, strictures, or Barrett's esophagus exist. Five different PPIs are approved for the treatment of GERD, including omeprazole, lansoprazole, rabeprazole, pantoprazole, and esomeprazole. A fifth PPI product consists of a combination of omeprazole and sodium bicarbonate. PPIs are best taken an hour before meals. The reason for this timing is that the PPIs work best when the stomach is most actively producing acid, which occurs after meals. If the PPI is taken before the meal, it is at peak levels in the body after the meal when the acid is being made. Esomeprazole $20 / 40 \mathrm{mg} /$ day, lansoprazole $30 \mathrm{mg} /$ day, omeprazole $20 / 40 \mathrm{mg}$ /day, pantoprazole $40 \mathrm{mg} /$ day and rabeprazole $20 \mathrm{mg} /$ day are equivalent in the treatment of patients with erosive and non-erosive GERD. The report of the occurrence of neoplasia with the chronic use of PPI is not supported by evidence. The gastric mucosa, however, is altered in these conditions (chronic gastritis, atrophy and polyps of fundic glands). The prevalence of gastric atrophy signs increases along the years, mainly when $H$. pylori is present.

At the primary care level, PPI or a combination of alginate-antacid and acid suppressive therapy can be administered at the discretion of the physician, as combination therapy, which may potentially be more beneficial than acid suppressive therapy alone. Similarly, patients who fail full-dose PPIs, plus/minus adjuvant therapies, may benefit from step-up therapy to twice daily PPIs even if there is no difference in randomized studies regarding the clinical response to the treatment with PPI taken as two daily doses, when compared to a single daily dose. 
When the PPI were used in full dose (esomeprazole: $20 \mathrm{mg} /$ day and $40 \mathrm{mg} /$ day; lansoprazole: $15 \mathrm{mg} /$ day and $30 \mathrm{mg} /$ day; omeprazole: $40 \mathrm{mg} /$ day; pantoprazole 20 $\mathrm{mg}$ /day and $40 \mathrm{mg} /$ day; rabeprazole: $10 \mathrm{mg}$ /day and $20 \mathrm{mg}$ /day), no statistical difference was observed between 4 and 8 weeks of treatment. Nevertheless, in cases of therapeutic failure, the time of treatment can be extended from 4 to 8 weeks, as although no significant difference was observed between the two periods, the number of satisfactory responses is higher after 8 than after 4 weeks.

\subsection{Pro-motility drugs}

Pro-motility drugs work by stimulating the muscles of the gastrointestinal tract, including the esophagus, stomach, small intestine, and/or colon. One pro-motility drug, metoclopramide, is approved for GERD. Pro-motility drugs increase the pressure in the lower esophageal sphincter and strengthen the contractions (peristalsis) of the esophagus. Both effects would be expected to reduce reflux of acid. However, these effects on the sphincter and esophagus are small. Therefore, it is believed that the primary effect of metoclopramide may be to speed up emptying of the stomach, which also would be expected to reduce reflux. Pro-motility drugs are most effective when taken 30 minutes before meals and again at bedtime. They are not very effective for treating either the symptoms or complications of GERD. Therefore, the pro-motility agents are reserved either for patients who do not respond to other treatments or are added to enhance other treatments for GERD.

\subsection{Foam barriers}

Foam barriers provide a unique form of treatment for GERD. Foam barriers are tablets that are composed of an antacid and a foaming agent. As the tablet disintegrates and reaches the stomach, it turns into foam that floats on the top of the liquid contents of the stomach. The foam forms a physical barrier to the reflux of liquid. At the same time, the antacid bound to the foam neutralizes acid that comes in contact with the foam. The tablets are best taken after meals (when the stomach is distended) and when lying down, both times when reflux is more likely to occur. Foam barriers are not often used as the first or only treatment for GERD. Rather, they are added to other drugs for GERD when the other drugs are not adequately effective in relieving symptoms. There is only one foam barrier, which is a combination of aluminum hydroxide gel, magnesium trisilicate, and alginate.

\section{Surgery}

When the diagnosis of reflux is objectively confirmed, surgical therapy should be considered in individuals who (Rice et al 2008):

1. have failed medical management (inadequate symptom control, severe regurgitation not controlled with acidsuppression, or medication side effects)

OR

2. opt for surgery despite successful medical management (due to quality of life considerations, lifelong need for medication intake, expense of medications, etc.)

OR

3. have complications of GERD (e.g., Barrett's esophagus, peptic stricture) (Spechler et 1996; Lagergren et al 1999) 
OR

4. have extra-esophageal manifestations (asthma, hoarseness, cough, chest pain, aspiration) (Rakita et al 2006; Oelshlager et al 2002).

The coexistence of Barrett's esophagus with gastroesophageal reflux symptoms is considered by many a clear indication for antireflux surgery (Oelshlager et al 2002). Surgical intervention for asymptomatic Barrett's esophagus is more controversial, however. While the metaplastic changes of Barrett's have been reported to regress to a greater degree in the post-surgical population compared with medically treated patients, to date there is no demonstrable improvement in esophageal adenocarcinoma rates (Rossi et al 2006; Chang et al 2007).

Today there is increased tendency worldwide to utilize surgery in the earlier stages of the disease (Spechler et al 2001). This change in clinical practice is mainly due to advancements in surgical technique, the increased patient satisfaction by laparoscopy, and the increased awareness of the impairment in quality of life of patients who are not efficiently treated. Moreover, the increasing enthusiasm of patients and surgeons for minimally invasive surgery has led to the wider application of laparoscopy in the management of GERD in many institutes worldwide.

The large success of laparoscopic surgery as an effective treatment of gastroesophageal reflux disease, has established minimal invasive surgery as the gold standard in the surgical treatment of this condition.

The guidelines from American Society for Gastrointestinal and Endoscopic Surgeons (SAGES) claim that surgery in GERD is curative in $85-93 \%$ of cases and suggest that the procedure may be appropriate in patients who have failed medical management, decide for surgery despite successful medical management, have complication of GERD, have medical complications attributable to a large hiatal hernia, or have "atypical" symptoms and reflux documented on $24 \mathrm{~h} \mathrm{pH}$ monitoring (SAGES Guidelines 2010).

Antireflux surgery has been shown to be very effective in relieving symptoms in $88-95 \%$ of patients, with excellent patients satisfaction, both in short and long term studies (Laffularde et al 2001; McKenzie et al 1996).

There are several trials favoring the clinical outcome of laparoscopic antireflux surgery compared to long-term PPI therapy. A large randomized clinical trial from the UK has shown significantly better physiological control of reflux in patients having undergone laparoscopic Nissen fundoplication than patients under maintenance PPI therapy (Laffularde et al 2001).

A randomized trial with 5-year follow-up, demonstrated that antireflux surgery is more effective than proton pomp inhibitor (PPI) drugs in controlling GERD symptoms (Laffularde et al 2001; Lundell et al 2001).

At 7-years follow-up Lundell et all reported the results of a randomized controlled trial of patients with esophagitis treated with omeprazole or surgery. The two treatments were similar regarding the incidence of recurrent esophagitis $(10.3 \%$ omeprazole versus $11.8 \%$ antireflux surgery). In addition the two therapies appeared to be equivalent in healing esophageal mucosa (Lundell et al 2007).

Another randomized trial, with 10 year follow-up, evaluating the effectiveness of medical therapy (omeprazole) versus antireflux surgery found that patients who underwent surgery had improved symptoms' relief when compared to the medically treated group (Spechler et al 2001). 
After 1991 when Dallemagne et al (Dallemagne et al 1991)performed the first laparoscopic Nissen Fundoplication, this technique was preferred to open procedures to result in lower morbidity and mortality, shorter hospital stay, faster recovery and less postoperative pain.

In a review by Catarci et al, laparoscopic fundoplication was as effective as its open counterpart with reduced morbidity, shorter hospital stay, and recovery but without any significant difference in early functional results and outcome (Catarci et al 2004).

Salminen et al in a recent randomized controlled trial, with 11-year follow-up, compared laparoscopic approach and conventional Nissen Fundoplication and concluded that the open and laparoscopic approaches for the Nissen fundoplication have similar long-term subjective symptomatic outcome despite the significantly higher evidence of incisional hernia and defective fundic wraps at endoscopy in the open group (Salminen et al 2007).

In a recent review Peters and colleagues compared 503 laparoscopic anti-reflux surgery and 533 open anti-reflux surgery. In this meta-analysis the authors conclude that laparoscopic anti-reflux surgery enables a faster convalescence and return to productive activity, with a reduced risk of complications and a similar treatment outcome compared with that of the open approach (Peters et al 2009).

The surgical management of GERD has been difficult to study scientifically because of the significant variation in the surgical techniques used.

The LOTUS trial (Attwood et al 2008)was designed to identify a methodology for standardization of the surgical technique and to measure the effectiveness of this standardization. This study has shown that surgeons are able to standardize their work for the purpose of measuring the outcome of an operative procedure within the context of a randomized trial.

Several series have shown the early outcomes of laparoscopic Nissen fundoplication to be excellent, with minimal morbidity and mortality, marked reduction in distal esophageal acid exposure, and symptom control for the overwhelming majority of patients. There have been multiple series with 5-year outcomes following Laparoscopic Nissen Fundoplication (Table 1). Heartburn was controlled in approximately $90 \%$ of patients, the revision rate was between 1 and $17 \%$, and $86 \%-92 \%$ of patients that were off antireflux medications.

In the German experience, in 164 hospitals performing laparoscopic antireflux surgery and 2053 operation were reported in 1999. In 65\% of the cases the surgical procedures were total fundoplication, in $31 \%$ partial fundoplication and in $4 \%$ other techniques. In total fundoplication there were 5 different techniques, in partial plication 5 and in the other group 3 (Huttl et al 2005).

Actually in laparoscopic antireflux surgery there are two debated questions today: if partial or total fundusplication must be performed and if this last is preferred division of short gastric vessels must be routinely performed or not.

\subsection{Partial versus total fundoplcation}

The total fundoplication involves a 360-degree wrap of the gastric fundus around the esophagus and is the most commonly performed anti-reflux operation. Total wrap (Nissen) supports and acknowledge the fact that the wrap needs to be "floppy" to minimize postoperative dysphagia (DeMeester et al 1986). It should also be noted that a floppy Nissen fundoplication is safe and effective in patients suffering from a defective esophageal peristalsis. Finally, proponents of the Nissen note a decreased effectiveness of a partial Fundoplication in controlling reflux symptoms. 


\begin{tabular}{|l|c|c|c|c|c|}
\hline Series & FU (years) & N (\%FU) & $\%$ Hb Relief (0-1) & Revision (\%) & $\begin{array}{c}\text { Off PPI+H2-A } \\
(\%)\end{array}$ \\
\hline $\begin{array}{l}\text { Morgenthal et al. 2007 } \\
\text { (Emory, GA, USA) }\end{array}$ & 11,0 & $166(59)$ & 89 & 10,8 & 70 \\
\hline $\begin{array}{l}\text { Dallemagne et al. 2006 } \\
\text { (Belgium) }\end{array}$ & 10,3 & $45(66)$ & 96 & 1,4 & $92^{*}$ \\
\hline $\begin{array}{l}\text { Kelly et al. 2007 } \\
\text { (Australia) }\end{array}$ & 10 & $226(90)$ & 84 & 17 & 79 \\
\hline $\begin{array}{l}\text { Zaninotto et al. 2007 } \\
\text { (Italy) }\end{array}$ & 8,1 & $138(95)$ & - & 9,4 & 79 \\
\hline $\begin{array}{l}\text { Bammer et al. 2001 (mayo, } \\
\text { FL, USA) }\end{array}$ & 6,4 & $171(59)$ & 94 & 1,0 & $86^{*}$ \\
\hline $\begin{array}{l}\text { Laffularde et al. 2001 } \\
\text { (Australia) }\end{array}$ & 6 & $166(93)$ & 87 & 14,2 & 89 \\
\hline $\begin{array}{l}\text { Oelschlager et al 2008 } \\
\text { (Seattle, WA, USA) }\end{array}$ & 5,8 & $288(65)$ & - & 3 & 88 \\
\hline $\begin{array}{l}\text { Anvari, Allen 2003 } \\
\text { (Ontario, Canada) }\end{array}$ & 5 & $181(48)$ & - & 3,6 & 86 \\
\hline $\begin{array}{l}\text { Booth et al. 2002 } \\
\text { (England) }\end{array}$ & 4 & $140(78)$ & 90 & 6,3 & \\
\hline
\end{tabular}

$\mathrm{FU}=$ follow-up; $\mathrm{HB}=$ heartburn; $0-1=$ none-mild; *Does not include patients on $\mathrm{H} 2-\mathrm{A}$

Table 1. Laparoscopic $360^{\circ}$ Fundoplication Series

Two partial fundoplications are commonly performed, the Dor and Toupet fundoplication (anterior and posterior wrap). Of these two partial wraps, the Toupet is the most commonly performed partial Fundoplication (Roberts et al 2006).

The Nissen procedure finds excellent symptomatic control but is reported to also have a high postoperative dysphagia rate and specific side effects such as inability to belch and vomit, and gas bloat syndrome (Sgromo et al 2008; Watson et al 2001).

The Toupet procedure is thought to produce less postoperative side effects than the Nissen procedure but studies have indicated that the recurrence rate of reflux symptoms may be higher after this procedure (Yobe et al 1997; Fernando et al 2002).

Horvath et al defines in a study the preoperative risk factors that predispose patients to failure. Data from 48 patients with objective follow-up performed as a part of a prospective long-term outcome project: 24-h pH-monitoring, manometry, esophagogastroduodenoscopy at 6 months, 3 years and 6 years, was analyzed (Horvath et al 1999). Patients in whom testing was completed were divided into two groups based on their results: those whose procedures failed $(n=22)$ and those who had a successful outcome after a Toupet fundoplication $(n=26)$. Surgical failure was defined as an abnormal postoperative De Meester score, with or without symptomatic recurrence. The authors shows that age, sex, primary and secondary presenting symptoms, presence of hiatal hernia, and esophageal dysmotility were not predictive of failure following a Toupet procedure and the numbers of years of GERD symptoms before surgery also did not predict failure after Toupet fundoplication. Horvath and colleagues concluded that the laparoscopic Toupet fundoplication appears to provide a weaker antireflux barrier than the Nissen procedure and is probably an insufficient procedure for patients with severe GERD. It may also 
predispose patients to postoperative mediastinal warp hernation. Independent preoperative predictors of failure after Toupet were:

- $\quad$ LES pressure $<5 \mathrm{mmHg}$ an preoperative manometry,

- Distal esophageal aperistaltic segment,

- Biopsy proved Barrett's metaplasia,

- Presence of stricture,

- Grade III or IV esophagitis an endoscopy,

- $\quad$ Preoperative De Meester score $>50$.

Guerin et al in a recent randomized trial with a 3 years follow-up, compared Nissen versus Toupet Fundoplication. Both groups in this study presented comparable preoperative findings (Guerin et al 2007). The Toupet procedure presented a higher level of invaliding functional symptoms in the immediate postoperative period, and the difference with the Nissen Fundoplication group was statistically significant for hyperflautolence, solids dysphagia and incapacity to belch. But at 1 and 3 years follow-up there wasn't any statistically significant difference of invalidating symptoms and the satisfaction level remained high. The authors concluded that the choice of the technique did not seem to be determined by the preoperative investigations, except when it diagnosticated a brachy esophagus and they also confirmed that laparoscopic Nissen or Toupet Fundoplication provides a high level of patients satisfaction despite invalidating side effects during the first postoperative year.

Strate et al compared laparoscopic Toupet and Nissen Fundoplication in 200 patients with esophageal motility disorders within 2 years follow-up (Strate et al 2008). The authors led to the following conclusion:

- Esophageal motility disorder does not effect postoperative clinical outcome and requires no tailoring of surgical management;

- The Toupet procedure is more effective in reflux control;

- Postoperative dysphagia is significantly higher after Nissen Fundoplication;

- Toupet Fundoplication reduces the rate of reoperation due to mechanical failure.

For Strate and colleagues the Toupet Fundoplication seems to be the better operative procedure for patients suffering from GERD.

Another similar randomized clinical trial by Booth and colleagues compared the two procedures in 127 patients considering preoperative esophageal manometry after 1 year follow-up (Booth et al 2008). In the study there was no significant difference in the prevalence or severity of symptoms after surgery between the Nissen group and Toupet group except for a greater prevalence of dysphagia and chest pain when eating in the Nissen group and there were no postoperative differences in the prevalence or severity of symptoms between the effective and ineffective motility groups, other than an increased severity score for flatus in the effective motility group 6 months after surgery. At 1 year follow-up there were no significant differences in Visick score between Nissen and Toupet group. The authors provides no clear conclusions regarding the efficacy and durability of Nissen compared with Toupet Fundoplication in controlling the reflux symptoms of heartburn and regurgitation, although there are greater postoperative acid exposure time and more $\mathrm{pH}$ failures in the Toupet group.

Sgromo et al compared the long-term outcome of Nissen and Toupet fundoplication by evaluating symptoms and quality of life at 7 years follow-up (Sgromo et al 2008). The authors concluded that long-term satisfaction, general symptoms score and quality of life 
were equivalent after laparoscopic Nissen or Toupet fundoplication, despite, a significantly increased prevalence of persistent heartburn after laparoscopic Toupet fundoplication.

Kamolz et al used the Gastrointestinal Quality of Life Index (GIQLI) for evaluating Nissen laparoscopic fundoplication versus Toupet procedure (Kamolz et al 2002). At 3 years and 5 years follow-up, the analysis of quality of life data showed that the GIQLI score remained stable in comparison with the 1 year follow-up data. Patients satisfaction with surgery was rated as "excellent" or "good" in $97.7 \%$ of patients. There were no significant differences between the 2 groups. The authors concluded that quality of life scores for both surgical groups were almost equal and postoperative outcome were comparable to values in healthy controls.

In a similar retrospective study, Zugel et al compared the results of 122 Toupet and 40 Nissen laparoscopic fundoplication in terms of patients satisfaction at 19 months follow-up. Both groups offered effective therapy for reflux, with more than $90 \%$ patient satisfaction (Zugel et al 2002). The authors concluded that both surgical techniques were effective in the treatment of GERD.

Laws et al used a randomized prospective study to compare the Nissen fundoplication versus the Toupet fundoplication for GERD (Laws et al 1997). At 27.2 months follow-up, postoperative symptomatology was judged using a modified Visick scale using the following grades in 38 patients:

- I no symptoms,

- $\quad$ II minimal symptoms, no lifestyle changes, no need to see a doctor,

- III significant symptoms requiring lifestyle changes of doctor's help,

- IV debilitating symptoms or reoperation.

There were no grade IV's. At follow-up, Visick score after the Nissen wrap were I-13, II-8, III-2 and after the Toupet procedure were I-12 and II-3. The authors concluded that a partial or complete wrap after division of the short gastric vessels offers effective therapy for reflux esophagitis with $>90 \%$ patients satisfaction.

Farrell and colleagues compared the effectiveness and durability of Toupet (79 pts) and Nissen (59 pts) procedures as a function of preoperative esophageal motility (Farrell et al 2000). Patients scored heartburn, regurgitation and dysphagia preoperatively, and at 6 weeks and 1 years, using 0 to 3 scale. At 6 weeks after operation, heartburn and regurgitation were similarly improved in both groups, but dysphagia was more prevalent among Nissen patients. After 1 year, heartburn and regurgitation were re-emerging in Toupet patients, and dysphagia was again similar between groups. Patients with impaired motility who have Nissen fundoplication are no more likely to suffer persistent dysphagia than their counterparts who have Toupet fundoplication. But, patients with normal motility are more likely to develop symptoms recurrence after Toupet fundoplication than Nissen procedure, with no distinction in dysphagia rates. The authors concluded that Toupet patients suffer more heartburn recurrence than Nissen patients, with similar dysphagia.

Fernando et al compared Toupet procedure versus Nissen fundoplication (Fernando et al 2002). Extended outcome and quality of life measurement (SF36 and HRQOL) were available for 142 patients at a mean follow-up of 19.7 months. Since there was a potential bias with a greater proportion of esophageal dysmotility with Toupet patients, further analysis was performed by dividing patients into four groups: group 1, Nissen patients with decreased motility; group 2, Toupet patients with decreased motility; group 3, Nissen patients with normal motility; and group 4, Toupet patients with normal motility. 
Comparison were made as follows: group 1 versus group 2, group 3 versus group 4, and group decreased motility versus group normal motility. There were no significant differences between Nissen and Toupet groups except for a higher incidence of dysphagia in the Toupet group. Resumption of proton pump inhibitors was required in $20 \%$ Nissen patients compared to $38 \%$ Toupet patients $(\mathrm{p}<0.05)$. Only $7 \%$ in the Nissen group were dissatisfied with their surgery compared to $21 \%$ patients in the Toupet group $(p<0.05)$. The SF36 scores were similar in all quality of life domains except for the domain of physical function, where better scores were seen in the Nissen group compared to the Toupet group $(\mathrm{p}<0.05)$. Comparison of group 1 versus group 2 revealed no significant difference in SF36 and HRQOL scores, symptoms, and medication use. Comparison of group 3 versus 4 demonstrated slightly worse HRQOL scores in the Toupet group and poorer scores $(\mathrm{p}<0.05)$ in the SF36 domains of vitality and mental health for the Toupet patients. The Toupet patients with normal motility also complained of more dysphagia and waterbrash $(\mathrm{p}<0.05)$. Comparison was also made between patients with impaired motility and those with normal motility. There were no differences in SF36 scores, HRQOL score, symptoms and medication use. This analysis supports the hypothesis that the differences seen were related to differences in esophageal motility. Surprisingly, they found more dysphagia in the Toupet group. These differences was not seen when they were analyzing the results of patients in the impaired motility groups but was present in patients with normal motility. The reason for this is unclear but may be related to a greater degree of recurrent esophagitis because of reflux in Toupet patients.

Dallemagne and colleagues compared laparoscopic Nissen fundoplication versus Toupet procedure at 5 years and 10 years follow-up (Dallemagne et al 2006). At 5 years follow-up, 58 Nissen patients and 28 Toupet patients completed the study, and at 10 years 49 Nissen and 20 Toupet patients completed the follow-up. At 5 years the heartburn was relieved in $98 \%$ of the Nissen group and $86 \%$ of the Toupet group. There was no significant difference between the two groups. Ten years after surgery the heartburn still was controlled in $96 \%$ of Nissen patients and $90 \%$ of Toupet group. Also in this case there was no significant difference between the two groups. Kaplan-Meier estimates of recurrence-free proportion were evaluated and control of reflux was still obtained in $93.3 \%$ of the Nissen groups and $81.8 \%$ of Toupet patients at 10 years $(p=0.17)$. There was no significant difference in the incidence of side effects between partial and total fundoplication. The incidence of postoperative dysphagia in patients with preoperative impaired esophageal motility was not different after partial or total fundoplication. Dallemagne and colleagues concluded that Nissen patients have better results than Toupet patients, although the differences was not statistically significant and they observed more recurrences after partial fundoplication than after Nissen fundoplication.

Fein et al compared partial fundoplication versus total fundoplication at 10 years follow-up. 88 patients received a Nissen procedure and 10 a Toupet fundoplication (Fein et al 2008). Follow-up of the patients included disease-related questionnaire and GastroIntestinal Quality of Life Index (GIQLI). Positive pH score were $21 \%$ in Nissen group and $56 \%$ in Toupet patients. The heartburn was present in $29.7 \%$ of Nissen groups and $12.5 \%$ of Toupet patients, regurgitation in $15.1 \%$ of the Nissen group and $10 \%$ of the Toupet group, dysphagia in $30.6 \%$ of the Nissen group and $28.6 \%$ of the Toupet group. Patients who had undergone Toupet fundoplication (43\%) took proton pump inhibitors significantly more often than patients who underwent Nissen fundoplication (14\%). None of the differences 
regarding the various procedures were significant about the GIQLI. In the observational scores, Nissen fundoplication appeared to control reflux better than partial fundoplication. In conclusion, despite the difficulties in comparing the result of single experiences of partial and total placation almost all combined experiences reported a good clinical and instrumental result for Toupet and Nissen procedures with light preference of Nissen for a better reflux control with similar others side effects.

\subsection{Division versus non division of the short gastric vessels}

The total fundoplication wrap achieves very effective control of reflux, although it can be followed by some troublesome side effects, such as dysphagia, gas bloat and inability to belch. To minimize the risk of developing these side effects, Nissen's procedure has been modified in a variety of ways concerning the length of the placation (short: $2 \mathrm{~cm}$, long: 3-4 $\mathrm{cm}$ ), its contention's degree (tight or floppy), the fixation of posterior wrap to the diaphragm or the esophagus, the routine iatal repair and the routine division of the first two or three short gastric vessels (true, Nissen) or it's preservation (Rossetti variation). There is a general agreement in literature to prefer a short floppy placation with no routine iatal repair and no wrao fixation. A still debated question is the significance of short gastric vessels division (deMeester et al 1986). It has been claimed that this step is followed by a lower risk of dysphagia, gas bloat and other side effects (deMeester et al 1986; Donehaue et al 1985) However, some surgeons claim that an equally good outcome can be achieved without dividing these vessels (Rossetti et al 1977; Watson et al 1997; Watson et al 1995; Anvari et al 1996) and this evidence is confirmed by randomized controlled trial.

Chryos et al in a prospective randomized trial compared Nissen (with division gastric vessels) to Nissen-Rossetti (without division gastric vessels) technique after 12 months follow-up (Chryos et al 2001). The authors concluded that division of short gastric vessels while performing laparoscopic Nissen fundoplication does not improve clinical outcome and laboratory finding in patients with GERD, and, at the same time, is associated with prolongation of the operating time and increased incidence of postoperative gas bloat syndrome.

In a recent multicentric trial of 1340 patients, Pessaux et al, concluded that the division of short gastric vessels did not improve clinical outcome after 2 or 5 years follow-up and increased the incidence of gas discomfort (Pessaux et al 2005).

In a recent randomized controlled trial Yang, Watson and colleagues reported the clinical outcome at 10 years follow-up (Yang et al 2008). The surgeons of this study have just reported the 6 months and 5 years outcome from this trial in previous publications $\left(\mathrm{O}^{\prime}\right.$ Boyle et al 2002; Watson et al 1997).

The authors confirm what many other papers claim: the division of the short gastric vessels does not influence the clinical outcome (Luostarinen et al 1999; Blomqvist et al 2000).

At 10 years follow-up, the authors concluded that there were no significant differences between the 88 patients that completed the study for either incidence or severity of dysphagia, heartburn, or overall satisfaction. These outcomes were identical to the outcomes from the earlier follow-up.

In a study of 138 patients, Sato et al analyzed the effect of short gastric vessels division on postoperative dysphagia (Sato et al 2002). They reported that laparoscopic Nissen fundoplication with or without division of short gastric vessels achieved a similar outcome. Their research suggested that patient selection and accurate construction of the fundoplication were the most important factors in minimizing postoperative dysphagia. 
Mardani, Lundell and colleagues designed a randomized controlled trial to determine the long-term results of total Nissen Fundoplication with or without division of short gastric vessels in a 10 years follow-up (Mardani et al 2009). They reported that mechanical sideeffects remain a problem following construction of a total wrap, and are perhaps of even greater concern when the operation is performed laparoscopically. There is a widely held view among surgeons that a wrap should be short end tension free (deMeester et al 1986; Rossetti et al 1977). However, the optimal length of total wrap to minimize subsequent obstructive complaints remains to be clarified in Mardani and colleagues randomized trial, in fact it may sometimes be necessary to divide some of the short gastric vessels in order to construct a tension free wrap, but this cannot be recommended in routine surgical practice (Mardani et al 2009). In a precedent report the same authors have reported subtle manometric differences between the two study groups, offering a physiologic background to a potential difference in functional outcome (Engstrom et al 2004), but it appears that these differences in lower esophageal sphincter response to gastric distension with air do not translate into important and clinically relevant functional correlates.

At 10 years follow-up, the authors concluded that with total Fundoplication it makes no difference whether the fundus is mobilized or not and that both types of repair provide long-lasting control of reflux (Mardani et al 2009).

Leggett et al. compared laparoscopic Nissen Fundoplication and Rossetti's modification in 239 patients, follow-up for the Rossetti group (138 patients) extends from 36 to 82 months and that for the Nissen group (101 patients) from 17 to 35 months (Leggett et al 2000). All patients experienced relief from symptomatic gastroesophageal reflux, whether they received the Rossetti modification or the Nissen Fundoplication. In their series, they found no statistically significant differences in intraoperative, postoperative, or overall complications between the two procedures. Prolonged postoperative dysphagia requiring dilation was significantly higher in the Rossetti group than in the Nissen group. But the percentage of patients requiring dilatation in both groups was higher in the first 20 than in the last 20 cases, and the authors state that with experience, surgeons become better able to judge the tightness of the crural closure, the size of posterior window, and the looseness of the wrap.

Table 2 shows of results of a different series that compared total fundoplication with or without division short gastric vessels.

In another prospectively randomized trial Kosek and colleagues compared the clinical and functional results after total fundoplication with or without division of short gastric vessels after five years follow-up (Kosek et al 2009). During long-term follow-up median DeMeester score decreased without statistically significant differences between the two groups and Gastrointestinal Quality of Life and patient satisfaction were similar in both group. The authors concluded that in their patient population division of the short gastric vessels during Nissen fundoplication that it has no statistically significant influence on clinical or functional outcome during a 5-year follow-up period. They therefore do not recommend routine division of the short gastric vessels in the course of total fundoplication. The intraoperative decision to divide the vasa gastricae breves may be made in some patients to obtain a tension-free fundoplication.

Finally in spite that many authors still routinely employ Nissen technique to perform a 360 degree short fundoplication there is no evidence support this position according to randomized study.

Rossetti variation (without section of short gastric vessels) can be "short e floppy" as well according the surgeon's experience and the use of an endoesophageal bougie of $54-60 \mathrm{Fr}$. 


\begin{tabular}{|c|c|c|c|c|c|c|c|c|}
\hline & \multicolumn{2}{|c|}{$\begin{array}{l}\text { Crysos et al. - } \\
12 \text { months FU * }\end{array}$} & \multicolumn{2}{|c|}{$\begin{array}{c}\text { Pessaux et al. - } \\
5 \text { years FU * }\end{array}$} & \multicolumn{2}{|c|}{$\begin{array}{l}\text { Yang et al. - } \\
10 \text { years FU }\end{array}$} & \multicolumn{2}{|c|}{$\begin{array}{l}\text { Mardani et al. - } \\
10 \text { years FU ** }\end{array}$} \\
\hline & $\begin{array}{c}\text { Not } \\
\text { Divided } \\
32 \mathrm{pt}\end{array}$ & $\begin{array}{c}\text { Divided } \\
24 \mathrm{pt}\end{array}$ & $\begin{array}{c}\text { Not } \\
\text { Divided } \\
404 \text { pt }\end{array}$ & $\begin{array}{c}\text { Divided } \\
305 \mathrm{pt}\end{array}$ & \begin{tabular}{|c|} 
Not \\
divided \\
$44 \mathrm{pt}$ \\
$(\%)$
\end{tabular} & $\begin{array}{c}\text { Divided } \\
44 \text { pt }(\%)\end{array}$ & $\mid \begin{array}{c}\text { Not Divided } \\
31 \mathrm{pt}\end{array}$ & $\begin{array}{c}\text { Divided } \\
42 \mathrm{pt}\end{array}$ \\
\hline Reccurence & - & - & $35(8,7)$ & $\begin{array}{c}40 \\
(13,1)\end{array}$ & - & - & - & - \\
\hline Heartburn & 0 & $1(4)$ & - & - & 18 & 11 & $1,9(1,4)$ & $1,4(0,7)$ \\
\hline Chest pain & $1(3)$ & 0 & $1(0,25)$ & 0 & - & - & - & - \\
\hline Regurgitation & 0 & 0 & - & - & 17 & 9 & - & - \\
\hline Respiratory & $2(16)$ & $1(4)$ & $2(0,5)$ & $2(0,6)$ & 20 & 24 & - & - \\
\hline Dysphagia & $5(16)$ & $4(17)$ & $26(6,4)$ & $28(9,2)$ & & & $2,4(1,6)$ & $2,0(1,5)$ \\
\hline Solids & 5 & 4 & - & - & 44 & 59 & - & - \\
\hline Liquids & 0 & 0 & - & - & 14 & 14 & - & - \\
\hline $\begin{array}{l}\text { Gas-bloating } \\
\text { Syndrome }\end{array}$ & $6(19)$ & $9(38)$ & $44(10,9$ & $26(8,5)$ & 32 & 41 & - & - \\
\hline Diarrhea & - & - & $3(0,7)$ & $7(2,3)$ & - & - & $2,6(1,6)$ & $2,4(1,5)$ \\
\hline Indigestion & - & - & $3(0,7)$ & $6(2)$ & - & - & $3,1(1,5)$ & $3,4(1,2)$ \\
\hline Constipation & - & - & $1(0,25)$ & 0 & - & - & $2,3(1,2)$ & $2,4(1,3)$ \\
\hline $\begin{array}{l}\text { Abdominal } \\
\text { pain }\end{array}$ & - & - & $4(1)$ & $7(2,3)$ & - & - & $2,8(1,4)$ & $2,5(1,2)$ \\
\hline Hiccup & - & - & $2(0,5)$ & $2(0,6)$ & - & - & - & - \\
\hline Flatulence & - & - & $8(2)$ & $12(4)$ & - & - & - & - \\
\hline Nausea & - & - & 0 & $1(0,3)$ & 10 & 21 & - & - \\
\hline
\end{tabular}

$\mathrm{Pt}=$ patients; ${ }^{*}=$ Numbers in parentheses represent percentages. ${ }^{* *}=$ Values are mean (s.e.m.). In all series no significant differences demonstrated between trial groups.

Table 2. Laparoscopic Total fundoplication: not divided (Nissen-Rossetti procedure) vs divided (Nissen procedure) short gastric vessels

In the few cases in which a "floppy" placation is not feasible than short gastric vessels must be divided and there a Nissen procedure performed.

In conclusion, Laparoscopic fundoplication is an established treatment for symptomatic gastro esophageal reflux disease and must be considered now days as the "gold standard" surgical procedure, tailoring a only marginal role to open surgery. It effectively controls heartburn and regurgitation, but it can be associated with unwanted effects, principally postoperative dysphagia, postprandial fullness, inability to belch or vomit and increased passage of flatus (tab. 2).

The choice of a total (360 degree) or a partial (270 degree) placation is difficult and not supported with clear evidence both in randomized and non randomized series, even if a better control of reflux symptoms in long follow-up studies gave a reason of a much a larger experience of 360 degree plication performed by reflux surgeon.

Total fundoplication is than the most performed operation for surgical treatment of GERD. Division of short gastric vessels (Nissen) or their preservation (Rossetti variation) is the last debated point. Randomized and non-randomized studies seem to point out in a precise way 
that a division of short gastric vessels is unnecessary to perform a "short and floppy" placation: the two main objectives to achieve is to prevent post operative dysphagia.

\section{Barrett's esophagus}

Barrett's esophagus is an acquired abnormality that is characterized grossly by an upward displacement of the squamo-columnar junction, with replacement of the typical whitish smooth esophageal mucosa by a velvety, reddish mucosa (Oelschlager et 2003). The columnar-lined esophagus was described by Norman Barrett in 1950 (Barrett 1950), reported to be associated with gastroesophageal reflux disease in 1953 (Allison et al 1953) and convincingly linked with oesophageal adenocarcinoma in 1975 (Naef et al 1975). The paradigm is that Barrett's esophagus arises as a complication of symptomatic gastroesophageal reflux disease and predisposes to esophageal adenocarcinoma.

BE is detected in approximately $6-12 \%$ of patients with GERD (Winters et al 1987, Cameron et al 1992). At present, BE is the most common cause of esophageal adenocarcinoma, a deadly malignancy with a frequency that has been rising strikingly in Western countries and a mortality rate that still exceeds $80 \%$ (Parker et 1997). In the USA, the incidence of esophageal adenocarcinoma has increased more than sixfold over the past three decades (Pohl et al 2005). The absolute risk of patients with BE for developing cancer is approximately $0.5 \%$ per year (Hirota et al 1999).

The metaplastic mucosa was confirmed by biopsy of the tubular esophagus during endoscopy.

Controversy over criteria for diagnosis of BE primarily concerns whether intestinal metaplasia (IM) is required for a diagnosis of BE. In the USA, BE has been defined by the Parameters Committee of the American College of Gastroenterology as the metaplastic replacement of any length of the esophageal epithelium that can be recognized at endoscopy and that is confirmed by biopsy to have specialized intestinal metaplasia, defined by the presence of goblet cells (Wang et al 2008). The vast majority of adenocarcinomas of the esophagus are accompanied by IM in multiple studies (Paraf et al 1995; Cameron et al 1995, Smith et al 1984). Therefore, it has been believed that esophageal adenocarcinoma arises in intestinal type mucosa with goblet cells within a columnar-lined esophagus (CLE).

CLE can involve any of three types of epithelium: fundic (gastric), cardial (junctional), and specialized intestinal metaplasia.

The British Society of Gastroenterology does not require confirmation of intestinal metaplasia in biopsies from the esophagus to establish this diagnosis (Gastroenterology TBSO 2005; Playford et al 2006.

In the concept of these guidelines, the presence of IM is thought to be less important for the diagnosis of BE than the presence of a proper esophageal gland, squamous island, and/or double muscularis mucosa (Takubo et al 1991; Takubo et al 1995; Long et al 1999). The most important rationale behind this view is related to the high rate of sampling errors at index endoscopy. Repeated endoscopy and biopsy are often necessary to confidently detect or exclude the presence of IM. Based on a recent retrospective study, an estimated eight biopsies are necessary for an adequate assessment of the presence of intestinal metaplasia (Harrison et al 2007). Furthermore, a previous study from the UK National Barrett's Oesophagus Registry (UKBOR) has not only confirmed this, but has demonstrated a similar neoplastic risk in patients with columnar metaplasia with and without demonstrable intestinal metaplasia (Vaezi et al 1996; Lieberman et al 1997; Locke et 2003; Smpliner et al 
2002; Watson et al 2005; Shepherd et al 2003; Gatenby et al 2008). The relative risk of adenocarcinoma development in patients with columnar-lined esophagus has been estimated at 5-125 fold higher than of control populations (Van der Veen et al 1989; Bartelsman et al 1992; Iftikar et al 1992; Solaymani-Dodaran et 2004; Anderson et al 2003) with the overall annual adenocarcinoma risk in columnar-lined esophagus at $0.69 \%$ (range 0-3.6\%) per annum (Gatenby et al 2008).

Like the British Society of Gastroenterology, the Japan Esophageal Society defines BE as a CLE with at least one of the following: a proper esophageal gland, squamous island, or double muscularis mucosae (Japan esophageal Society 2009). In a recent review of 141 cases, Takubo et al. demonstrated that more than $70 \%$ of primary esophageal adenocarcinomas were adjacent to cardiac and/or fundic rather than intestinal type mucosa with goblet cells (Takubo et al 2009). This suggests BE might be better defined as the presence of metaplastic columnar-lined esophagus with or without goblet cells, which is in accordance with the British and Japanese definition of BE. As there are still few data on the risk of esophageal adenocarcinoma in CLE lacking IM.

Controversy has surrounded the most appropriate means of reflux control in patients with CLE. While pharmacological acid suppression is the least invasive and most suitable for elderly patients and those with comorbidity, the high incidence of hiatal hernia, lower esophageal sphincter failure, peristaltic impairment, and reflux of duodenal juice renders proton pump inhibitor (PPI) therapy less effective in columnar-lined esophagus than in less severe reflux disease, with up to $40 \%$ still demonstrating pathological acid exposure after receiving up to $80 \mathrm{mg}$ per day of omeprazole (Lundell et al 2001; Katzka et al 1994; Sampliner et al 1994; Ouatu-Lascar et al 1998; Sharma et al 1997). Several series have suggested that fundoplication, by virtue of its ability to correct hiatal hernia, lower esophageal sphincter failure, and reflux of duodenal juice, confers some protection against adenocarcinoma development (Wassnaar et al 2010).

In conclusion BE and CLE may be considered as a synonym.

Progression of BE in this paper is defined as a change in histological findings on biopsy from CLE to any form of dysplasia or an increase in grade of dysplasia. Development of adenocarcinoma is also considered progression of disease. Regression is defined as change from high-grade dysplasia (HGD) to low-grade dysplasia (LGD) or no dysplasia, change from LGD to metaplasia or loss of metaplasia, and change from CLE to complete loss of metaplasia. Shortening of the segment or development of squamous cell islands, although considered by some as regression, usually is not accurately measured and reported, and is therefore, not considered regression in our report. Long-segment BE (LSBE) is defined as $>3$ $\mathrm{cm}$, short segment BE (SSBE) is defined as a length $1-3 \mathrm{~cm}$ seen at endoscopy and confirmed by biopsy, ultra-short segment Barrett Esophagus $<1 \mathrm{~cm}$.

The goal of treatment of columnar-lined esophagus is to prevent non-neoplastic complications and development of dysplasia and adenocarcinoma by control of gastroesophageal reflux while maintaining a healed mucosa (Sampliner et al 2002).

Patients with columnar-lined esophagus are among those with the most severe gastroesophageal reflux disease (Winters et al 1987; Avidan et al 2002; Liebermann et al 1997; Locke et al 2003, Csendes et al 2002) and adequate control of reflux is difficult (Katzka et al 1994; Ouatu-Lascar et al 1998; Sharma et al 1997). Medical therapy does not prevent biliary reflux into the esophagus (Vaezi et 1996; Manifold et al 2000), and only surgical 
correction of the defective gastroesophageal sphincter can abolish this (Parilla et al 2003; Watson et al 1997; Zaninotto et al 2002).

Three recent studies have investigated the effect of PPI treatment on the risk of progression of BE to dysplasia or adenocarcinoma (Cooper et al 2006; Hillman et al 2004; Nguyen et al 2009). The results of these controlled studies suggest a protective effect of PPIs in limiting the progression of $\mathrm{BE}$, but they do not eliminate the risk of developing $\mathrm{AC}$.

In the study by Hillman et al, patients were stratified according to delay in starting PPI therapy after the diagnosis of BE was established (Hillman et al 2004). Patients who delayed PPI therapy for $\geq 2$ years after being diagnosed with BE had 5.6 times higher risk of developing low grade dysplasia than patients who used PPI within the first year after diagnosis. Furthermore, patients with $\mathrm{BE}$ had up to a 20 times higher risk of developing high grade dysplasia or adenocarcinoma when PPI therapy was delayed for 2 years after diagnosis of BE. Although this suggests a substantial protective effect, the absolute risk of developing high grade dysplasia or adenocarcinoma was low, 3\%, at a median follow-up of 4.7 years. The small rate of progression of BE makes it very difficult to show a difference between treatments.

In another study, Cooper et al considered 188 patients with IM who were treated with a PPI, the risk of developing low grade dysplasia within 5 years of the diagnosis of BE was around $2.5 \%$, and the risk of high grade dysplasia or adenocarcinoma was around $2 \%$ while taking PPI therapy (Cooper et al 2006).

However, when following patients for $>5$ years, Nguyen et al recently have found a much higher risk of developing adenocarcinoma (Nguyen et al 2009). They have studied 344 patients diagnosed with BE without dysplasia, with a mean follow-up of 7.6 years. They found that the chance of developing HGD or adenocarcinoma was 7.4\%. Moreover, this risk was even higher when not taking PPIs (14.2\%).

The hypothesis that surgery is superior to medical therapy comes from the assumption that surgery provides better control of GERD than do PPIs, and this should translate into lower progression rates. There have been very few studies comparing medical and surgical therapy.

Gatenby et al published the results of their review of a cohort of 738 patients with BE (Gatenby et al 2009). They compared 41 patients with anti-reflux surgery to 551 treated medically with PPIs, 42 patients treated with $\mathrm{H} 2$ receptor antagonists (H2RAs), 95 patients treated with H2RA followed by PPI and 9 patients with treatment. After a followup of 5 years after medical therapy and 6 years after surgical therapy, there was however a trend toward antireflux surgery being more protective. No patients in the antireflux group developed HGD or AC as compared to $4.3 \%$ in the all-medical therapies group $(\mathrm{P}=$ 0.13). There were not enough patients in the surgical arm to determine if this was a significant difference.

Parrilla et al have published the only randomized study comparing 43 patient treated with medical treatment and 58 with antireflux surgery (Parrilla et al 2003). In that study, 101 patients with BE were treated between 1982 and 2000. Medical treatment consisted of H2RA treatment initially and then omeprazole from 1992 onward. Surgery was performed through laparotomy with Nissen fundoplication in 56 patients and a Collis-Nissen procedure in the other two because of short esophagus. All patients had annual clinical, endoscopic and histological follow-up, and patients who had an operation also had a $\mathrm{pH}$ study and manometry at 1 year postoperatively and every 5 years thereafter, or if they presented with 
recurrent GERD symptoms. Mean follow-up was 6 years for the medical therapy group and 7 years for the surgical group. Progression of BE to any dysplasia was found in eight patients $(19 \%)$ in the medical treatment group and in three in the surgical group (5\%). Two patients in each group progressed to adenocarcinoma, which was confirmed after esophageal resection. Although differences in progression rates between the two groups were not significant according to the authors, when a sub-analysis was performed including only patients in the surgical arm with normal $\mathrm{pH}$, the progression rate dropped to $2 \%$, which was a significantly lower chance of progression of disease than in the medical group. The hypothesis that surgery is superior to medical therapy comes from the assumption that surgery provides better control of GERD than do PPIs, and this should translate into lower progression rates. The control of reflux is essential in preventing progression of disease, is backed up by the fact that, in most studies, the patients with progression after surgical treatment seem to have recurrent reflux. This observation, that control of reflux is essential in preventing progression of disease, is backed up by the fact that, in most studies, the patients with progression after surgical treatment seem to have recurrent reflux (Oelschlager et al 2003; O'Riordan et al 2004; Biertho et al 2007; Lagergren et al 2007; Csendes et al 2004).

Hofstetter et al have published the study with the longest follow-up (Hofstetter et al 2001). They showed results for a series of 97 patients, with complete endoscopic follow-up in 79, at a median of 5 years. No patients developed HGD or adenocarcinoma, but four had progression of metaplasia to LGD (5\%).

Bowers et al, have reported a similar series with a mean follow-up of 4.6 years (Bowers et al 2002). Their 104 patients underwent open or laparoscopic fundoplication. Of these, 64 patients had endoscopic follow-up with biopsy. None of the patients developed HGD or adenocarcinoma. Only one patient had progression to LGD (1.5\%).

Wassenaar and Oelschlager in a recent review are summarized the result of 11 publications on surgical treatment for BE that included results on prevention of progression, as well as regression of metaplasia or dysplasia (Wassenaar et al 2010). A total of 551 patients were considered with a median follow up of 3.6 years. The progression rate of metaplasia to dysplasia or adenocarcinoma was $3.4 \%$ and $0.7 \%$ respectively and the regression rate was $30.5 \%$.

Kamolz and colleagues evaluated and compared quality of life data before and after laparoscopic antireflux surgery in GERD patients with and without BE (Kamolz et al 2003). The authors concluded that non-BE patients undergoing laparoscopic antireflux surgery achieved a better quality of life improvement than those patients with BE. The authors compared QoL data of both groups to the mean value of general population. This means that laparoscopic antireflux surgery is able to improve QoL significantly in all GERD patients, with and without BE.

In Conclusion, surgical treatment is able only to control acid and biliopancreatic refluxate, with an improvement of quality of life. A very important point of view is the efficacy of antireflux barrier, infact after surgical treatment, there is also still progression of disease although the risk seems to become very small when this treatment is successful.

The complexity of assessment and management of CLE require a multidisciplinary approach, in regard of diagnosis and strategies of treatment. This is particularly true for surgical therapy, which has to be effective and long lasting; therefore, it should be preferably performed by experienced surgical teams. 


\section{Quality of life after antireflux surgery}

The large success of laparoscopic surgery as an effective treatment of gastroesophageal reflux disease, has established minimal invasive surgery as the gold standard in surgical treatment of this condition. Among antireflux procedures, laparoscopic total fundoplication is the most commonly used, providing excellent symptom relief (Watson et al 1996). Antireflux surgery has been shown to improve not only symptoms, but also quality-of life (QoL) (Velanovich et 1999; Trus et al 1999).

Laparoscopic Nissen fundoplication constructs an antireflux barrier in the cardia region and effectively controls the typical symptoms of gastroesophageal reflux disease in approximately 85-90\% (Bammer et al 2001; Beldi et al 2002; Eubancs et 2000; Carlson et al 2001) of cases at 5 to 10 years follow-up and has low morbidity and mortality rates.

Poor surgical results are caused by mechanical problems or persistence of symptoms (Campos et al 1999; Rice et al 2000).

A proportion of patients have persistent reflux symptoms and require use of proton pump inhibitors despite normal functional studies (Eubancs et al 2000; Khajanchee et al 2002; Galvani et al 2003).

In our study only 5.9\% required PPI post-operatively and the satisfaction rate was $63.8 \%$ at 6 months and $83.3 \%$ at 12 months, with a Johnson\&DeMeester score of 8.05 (IQR: 6.95-10.20) at 6 months and to 7.60 (IQR: 7.60-9.50) at the 12 months follow-up.

After antireflux surgery we observed a significant reduction in both severity and frequency scores of heartburn, epigastric pain, regurgitation, and respiratory symptoms, but an increase of dysphagia for solids and/or liquids. This point of view may affect the improvement of quality of life after Nissen-Rossetti fundoplication. The dysphagia was reported especially during the first 3 postoperative months, and in most cases it could be controlled by diet modifications as it gradually subsided (Loustarinen et al 2001; Mungan et al 1999; Balci et al 2007).

In our series we observed $19.4 \%$ of dysphagia for solids and/or liquids at 1 months postoperatively and only 2 readmissions, with need for endoscopic ballon dilatation in one case, but at 3 months all symptoms had disappeared.

Trus et al reported a significant and durable improvement in all 8 scales of the SF-36 at 6 weeks and 1 year after laparoscopic antireflux surgery (Trus et al 1999).

Amato et al reported that dysphagia for solids and/or liquids was the only significant symptom associated with 3 of 8 scales (physical function, role physical and bodily pain) (Amato et al 2008). A border line association was found between bloating and other 3 of 8 scales (social function, role-emotional and mental health).

Peters et al found no improvement in all 8 scales, with the exception of bodily pain, in 46 patients at a median of 21 months after laparoscopic Nissen fundoplication (Peters et al 1998).

In our series we observed good results of surgical procedures with a DeMeester score after 6 months like 8.05 and after 12 months 7,60 ( $p<0.0001)$ and an improvement of quality of life measured in all subdomains of SF-36 at 6 months and 12 months.

In our series, also, we evaluated the impact of Nissen-Rossetti fundoplication with GERDHRQL to measure the relation between symptoms and the quality of life of patients before and after surgery.

Balci et al measured QoL with SF-36 and GERD-HRQL in 60 patients at 1 months and 6 months which showed that QoL increased significantly for all their patients after surgery (Balci et al 2007). 
In all subdomains of the SF-36 the patients score increased, showing an improved quality of life in the related aspects of each item and the GERD-HRQL score showed a corresponding increase.

In another study Velanovich compared SF-36 and GERD-HRQL (Velanovich 1998). In that study, multivariate analysis showed that the only significant predictor of patient satisfaction was GERD-HRQL.

Several studies have suggested an influence of psychopathological disorders on the results of laparoscopic fundoplication (Watson et al 1997; Velanovich 2006). Kamolz et al compared the postoperative results of laparoscopic fundoplication in 21 patients with anxiety disorders diagnosed with the International Classification of Diseases 10 (ICD-1) and 21 controls. Although patients with anxiety disorders showed improvements in both their clinical parameters and the postoperative quality of life using the GIQLI questionnaire, such improvements were lower than those seen in control subjects. However, authors stated that these patients should not be excluded from surgery, and that an improvement in panic attacks was seen in one-third of them (Kamolz et al 2001).

These same authors conducted a similar case-control study on 38 patients diagnosed with major depression according to the ICD-10 classification and found that, despite adequate preoperative selection and normalization of functional parameters, these patients showed less symptomatic relief and poorer results in the postoperative GIQLI quality-of-life questionnaire as compared with control cases (Kamolz et al 2003). The same group documented improvement of results and quality of life in patients undergoing surgery for GERD with stress-related symptoms depending on whether or not they had also received psychological therapy (Kamolz et al 2001).

In our study all patients after surgery presented an improvement in quality of life measured by GERD-HRQL, with 25 (IQR 10-35.5) preoperatively, and 7 (IQR 3.5-10.15) at 6 months, and 5 (IQR 2.5-9.5) at 12 months.

In conclusion laparoscopic Nissen-Rossetti fundoplication is a safe and effective surgical procedure for treatment of GERD, generally offering an improvement of quality of life.

Finally, all patients of this series experienced an improvement in their postoperative quality of life.

\section{References}

Allison PR \& Johnstone AS (1953). The oesophagus lined with gastric mucous membrane. Thorax;8:87-101.

Amato G, Limongelli P, Pascariello A, Rossetti G, Del Genio G, Del Genio A et al (2008). Association between persistent symptoms and long-term quality of life after laparoscopic total fundoplication. Am J Sur; 196: 582-586.

Anderson LA, Murray LJ, Murphy S J et al(2003). Mortality in Barrett's oesophagus: results from a population based study. Gut; 52: 1081-1084.

Anvari M \& Allen C (2003). Five-year comprehensive outcomes evaluation in 181 patients after laparoscopic Nissen fundoplication. J Am Coll Surg.;196:51-57

Anvari M \& Allen CJ (1996). Prospective evaluation of dysphagia before and after laparoscopic Nissen fundoplication without routine division of short gastrics. Surg Laparosc Endosc.;6:424-429. 
Attwood SE, Lundell L, Ell C, Galmiche JP, Hatlebakk J, Fiocca R, Lind T, Eklund S, Junghard O; The LOTUS Trial Group (2008). Standardization of Surgical Technique in Antireflux Surgery: The LOTUS Trial Experience. World J Surg.;32:995-998.

Avidan B, Sonnenberg A, Schnell T G, Chejfec G, Metz A \& Sontag S J (2002). Hiatal hernia size, Barrett's length and severity of acid reflux are all risk factors for oesophageal adenocarcinoma. Am J Gastroenterol; 97: 1930-1936.

Bajbouj M, Becker V, Neuber M, et al (2007). Combined pH-metry/impedance monitoring increases the diagnostic yield in patients with atypical gastroesophageal reflux symptoms. Digestion;76(3 4):223-8.

Balci D \& Turkcapar AG (2007). Assessment of Quality of Life after Laparoscopic Nissen Fundoplication in Patients with Gastroesophageal Reflux Disease, World J Surg; 31: 116-121.

Bammer T, Hinder RA, Klaus A \& Klingler PJ (2001). Five- to eight-year outcome of the first laparoscopic Nissen fundoplications. J Gastrointest Surg;5:42-48.

Barham CP, Gotley DC,Mills A \& Alderson D (1995). Precipitating causes of acid reflux episodes in ambulant patients with gastro-oesophageal reflux disease. Gut;36:50510.

Barrett N (1950). Chronic peptic ulcer of the oesophagus and 'oesophagitis'. Br. J. Surg.;38:175-182.

Bartelsman JFWM, Hameeteman W \& Tytgat GN (1992). Barrett's oesophagus. Eur J Cancer Prev; 1: 323-325.

Beldi G \& Glattli A. (2002) Long-term gastrointestinal symptoms after laparoscopic Nissen fundoplication. Surg Laparosc Endosc Percutan Tech;12:316-319.

Biertho L, Dallemagne B, Dewandre JM, Jehaes C, Markiewicz S, Monami B, Wahlen C \&Weerts J. (2007) Laparoscopic treatment of Barrett's esophagus: long-term results. Surg Endosc; 21: 11-15.

Blomqvist A, Dalenbäck J, Hagedorn C, Lönroth H, Hyltander A \& Lundell L. (2000) Impact of complete gastric fundus mobilization on outcome after laparoscopic total fundoplication. J Gastrointest Surg.;4:493-500.

Bloom S.; McCartney S. \& Langmead L. (2009) The modern investigation and management of gastro-oesophageal reflux disease (GORD). Clinical Medicine, 9, 6: 600-4.

Bonatti H, Achem SR \& Hinder RA. (2008) Impact of changing epidemiology of gastroesophageal reflux disease on its diagnosis and treatment. J Gastrointest Surg.;12:373-381.

Booth MI, Jones L, Stratford J \& Dehn TC. (2002)Results of laparoscopic Nissen fundoplication at 2-8 years after surgery. Br J Surg.;89:476-481.

Booth MI, Stratford J, Jones L \& Dehn TC (2008). Randomized clinical trial of laparoscopic total (Nissen) versus posterior partial (Toupet) fundoplication for gastrooesophageal reflux disease based on preoperative oesophageal manometry. $\mathrm{Br} \mathrm{J}$ Surg.;95:57-63.

Bowers SP, Mattar SG, Smith CD, Waring JP \& Hunter JG. (2002) Clinical and histologic follow-up after antireflux surgery for Barrett's esophagus. J Gastrointest Surg; 6: 532-538. 
Cameron AJ, Lomboy CT, Pera M \& Carpenter HA (1995). Adenocarcinoma of the esophagogastric junction and Barrett's esophagus. Gastroenterology; 109: 15411546.

Cameron AJ \& Lomboy CT (1992). Barrett's esophagus: Age, prevalence, and extent of columnar epithelium. Gastroenterology;103:1241-1245.

Campos GM, Peters JH, DeMeester TR, Oberg S, Crookes PF, Tan S et al (1999). Multivariate analysis of factors predicting outcome after laparoscopic Nissen fundoplication. J Gastrointest Surg; 3:292-300

Carlson MA \& Frantzides CT (2001). Complications and results of primary minimally invasive antireflux procedures: a review of 10735 reported cases. J Am Coll Surg; 193: 428-39.

Catarci M, Gentileschi P, Papi C, Carrara A , Marrese R, Gaspari AL \& Grassi GB (2004). Evidence-based appraisal of antireflux fundoplication. Ann Surg; 239 :325-337.

Chang E Y, Morris C D, Seltman A K, O'Rourke R W, Chan B K, Hunter J G \& Jobe B A (2007) The effect of antireflux surgery on esophageal carcinogenesis in patients with barrett esophagus: a systematic review. AnnSurg 246:11-21

Chrysos E, Tzortzinis A, Tsiaoussis J, Athanasakis H, Vasssilakis J \& Xynos E (2001). Prospective randomized trial comparing Nissen to Nissen-Rossetti technique for laparoscopic fundoplication. Am J Surg.;182:215-221.

Cooper BT, Chapman W, Neumann CS \& Gearty JC (2006). Continuous treatment of Barrett's oesophagus patients with proton pump inhibitors up to 13 years: observations on regression and cancer incidence. Aliment Pharmacol Ther; 23:727733

Csendes A, Burdiles P, Braghetto I \& Korn O (2004). Adenocarcinoma appearing very late after antireflux surgery for Barrett's esophagus: long-term follow-up, review of the literature, and addition of six patients. J Gastrointest Surg; 8: 434-441.

Csendes A, Smok G, Quiroz J et al (2002). Functional studies in 408 patients with Barrett's esophagus, compared to 174 cases with intestinal metaplasia of the cardia. Am J Gastroenterol;97: 554-560.

Dallemagne B, Weerst JM, Jehaes C, Markiewicz S \& Lombard R (1991). Laparoscopic Nissen Fundoplication: preliminary report. Surg Laparosc Endosc; 1: 138-143.

Dallemagne B, Weerts J, Markiewicz S, Dewandre JM, Wahlen C, Monami B \& Jehaes C (2006). Clinical results of laparoscopic fundoplication at ten years after surgery. Surg Endosc.;20:159-165.

DeMeester TR, Bonavina L \& Albertucci M (1986). Nissen fundoplication for gastroesophageal reflux disease. Evaluation of primary repair in 100 consecutive patients. Ann Surg.;204:9-20.

Dent J, El-Serag HB, Wallander MA \& Johansson S (2005). Epidemiology of gastrooesophageal reflux disease: a systematic review. Gut.;54:710-717.

Dodds WJ, Dent J, Hogan WJ et al (1982). Mechanisms of gastroesophageal reflux in patients with reflux esophagitis. $N$ Engl J Med;307:1547-52.

Donahue PE, Samelson S, Nyhus LM \& Bombeck CT (1985). The floppy Nissen fundoplication. Effective long-term control of pathologic reflux. Arch Surg.;120:663668. 
Engström C, Blomqvist A, Dalenbäck J, Lönroth H, Ruth M \& Lundell L (2004). Mechanical consequences of short gastric vessel division at the time of laparoscopic total fundoplication. J Gastrointest Surg.;8:442-447.

Eubanks TR, Omelanczuk P, Richards C, Pohl D \& Pellegrini CA (2000). Outcomes of laparoscopic antireflux procedures. Am J Surg; 179: 391-5.

Farrell TM, Archer SB, Galloway KD, Branum GD, Smith CD \& Hunter JG (2000). Heartburn is more likely to recur after Toupet fundoplication than Nissen fundoplication. Am Surg;;66:229-236.

Fass R, Quan SF, O'Connor GT, Ervin A \& Iber C (2005). Predictors of heartburn during sleep in a large prospective cohort study. Chest;127:1658-66.

Fein M, Bueter M, Thalheimer A, Pachmayr V, Heimbucher J, Freys SM \& Fuchs KH (2008). Ten-year outcome of laparoscopic antireflux surgery. J Gastrointest Surg.;12:18931899.

Fernando HC, Luketich JD, Christie NA, Ikramuddin S \& Schauer PR (2002). Outcomes of laparoscopic Toupet compared to laparoscopic Nissen fundoplication. Surg Endosc.;16:905-8.

Fornari F \& Sifrim D (2007). Gastroesophageal reflux and atypical symptoms: the role of impedance-pH monitoring. Digestion;76(3-4):221-2.

Galvani C, Fisichella PM, Gorodner MV, Perretta S \& Patti MG (2003). symptoms are a poor indicator of reflux status after fundoplication for gastroesophageal reflux disease. Role of esophageal functions test. Arch Surg; 138:514-9

Gastroenterology TBSO (2005). Guidelines for the diagnosis and management of Barrett's columnar-lined oesophagus . cited; Available from: http://www.bsg.org.uk.

Gatenby PA, Caygill CPJ, Ramus JR, Charlett A \& Watson A (2008). Barrett's columnar-lined oesophagus: demographic associations and adenocarcinoma risk. Dig Dis Sci; 53: 1175-1185.

Gatenby PA, Ramus JR, Caygill CPJ, Shepherd NA, Watson A (2008). The relevance of detection of intestinal metaplasia in columnar-lined esophagus. Scand J Gastroenterol;43:524-530.

Gatenby PA, Ramus JR, Caygill CP, Charlett A, Winslet MC \& Watson A (2009). Treatment modality and risk of development of dysplasia and adenocarcinoma in columnarlined esophagus. Dis Esophagus; 22: 133-142.

Guérin E, Bétroune K, Closset J, Mehdi A, Lefèbvre JC, Houben JJ, Gelin M, Vaneukem P \& El Nakadi I (2007). Nissen versus Toupet fundoplication: results of a randomized and multicenter trial. Surg Endosc;21:1985-1990.

Hampel H, Abraham NS \& El-Serag HB (2005). Meta-analysis: obesity and the risk for gastroesophageal reflux disease and its complications. Ann Intern Med;143:199-211.

Harrison R, Perry I, Haddadin W et al (2007). Detection of intestinal metaplasia in Barrett's esophagus: An observational comparator study suggests the need for a minimum of eight biopsies. Am. J. Gastroenterol.;102:1154-1161.

Hillman LC, Chiragakis L, Shadbolt B, Kaye GL \& Clarke AC (2004). Proton-pump inhibitor therapy and the development of dysplasia in patients with Barrett's oesophagus. Med J Aust;180: 387-391

Hirota WK, Loughney TM, Lazas DJ, Maydonovitch CL, Rholl V \& Wong RK (1999). Specialized intestinal metaplasia, dysplasia, and cancer of the esophagus and 
esophagogastric junction: Prevalence and clinical data. Gastroenterology;116:277285.

Hofstetter WL, Peters JH, DeMeester TR, Hagen JA, De-Meester SR, Crookes PF, Tsai P, Banki F \& Bremner CG (2001). Longterm outcome of antireflux surgery in patients with Barrett's esophagus. Ann Surg; 234: 532-538.

Horvath KD, Jobe BA, Herron DM \& Swanstrom LL (1999). Laparoscopic Toupet fundoplication is an inadequate procedure for patients with severe reflux disease. J Gastrointest Surg.;3:583-591.

Hüttl TP, Hohle M, Wichmann MW, Jauch KW \& Meyer G (2005). Techniques and results of laparoscopic antireflux surgery in Germany. Surg Endosc.;19:1579-87.

Iftikar SY, James PD, Steele RJC, Hardcastle JD \& Atkinson M (1992). Length of Barrett's oesophagus: an important factor in the development of dysplasia and adenocarcinoma. Gut; 33:1155-1158.

Japan Esophageal Society (2009). Japanese Classification of Esophageal Cancer, tenth edition: Part I. Esophagus ;6:1-26.

Jobe BA, Wallace J, Hansen PD \& Swanstrom LL (1997). Evaluation of laparoscopic Toupet fundoplication as a primary repair for all patients with medically resistant gastroesophageal reflux. Surg Endosc.;11:1080-1083.

Johnson LF (1980). 24-hour $\mathrm{pH}$ monitoring in the study of gastroesophageal reflux. J Clin Gastroenterol;2:387.

Jones RH, Hungrin ADS \& Phillips J (1995). Gastroesophageal reflux disease in primary care in Europe: clinical presentation and endoscopic findings. Eur J Gen Pract;1:149-54.

Kahrilas PJ, Dodds WJ, Hogan WJ et al (1986). Esophageal peristaltic dysfunction in peptic esophagitis. Gastroenterology;91:897-904.

Kahrilas PJ, Dodds WJ, Hogan WJ et al (1986). Esophageal peristaltic dysfunction in peptic esophagitis. Gastroenterology;91:897-904.

Kalmoz T, Bammer T, Granderath FA \& Pointer R (2001). Laparoscopic antireflux surgery in gastroesophageal reflux disease patients with concomitant anxiety disorders. Dig Liver Dis; 33: 659-664.

Kalmoz T, Granderath FA, Bammer T, Pasiut M (2001) Pointner R. Psycological intervention influences the outcome of laparoscopic antireflux surgery in patients with stressrelated symptoms of gastroesophageal reflux disease. Scand J Gastroenterol ; 36: 800-805.

Kalmoz T, Granderath FA \& Pointner R (2003). Does major depression in patients with gastroesophageal reflux affects the outcome of laparoscopic antireflux surgery? Surg Endosc; 17:55-60.

Kamolz T, Granderath F \& Pointner R (2003). Laparoscopic antireflux surgery: diseaserelated quality of life assessment before and after surgery in GERD patients with and without Barrett's esophagus. Surg Endosc.;17:880-885.

Kamolz T, Granderath FA, Bammer T, Wykypiel H Jr \& Pointner R (2002). "Floppy" Nissen vs. Toupet laparoscopic fundoplication: quality of life assessment in a 5-year follow-up (part 2). Endoscopy.;34:917-922.

Katzka D A \& Castell D O (1994). Successful elimination of reflux symptoms does not insure adequate control of acid reflux in patients with Barrett's esophagus. Am J Gastroenterol; 89:989-991. 
Kelly JJ, Watson DI, Chin KF, Devitt PG \& Game PA (2007) Jamieson GG. Laparoscopic Nissen fundoplication: clinical outcomes at 10 years. J Am Coll Surg.;205:570-575.

Khajanchee YS. O'Rourke RW, Lockhart B, Patterson EJ, Hansen PD \& Swanstrom LL (2002). Postopearive symptoms and failure after antireflux surgery. Arch Surg;137:1008-14.

Kösek V, Wykypiel H, Weiss H, Höller E, Wetscher G, Margreiter R \& Klaus A (2009). Division of the short gastric vessels during laparoscopic Nissen fundoplication: clinical and functional outcome during long-term follow-up in a prospectively randomized trial. Surg Endosc. Oct;23:2208-13.

Laffullarde T, Watson DI, Jamieson GG, Myers JC, Game PA \& Devitt PG (2001). Laparoscopic Nissen Fundoplication: five-year results and beyond. Arch Surg; 136: 180-184.

Lagergren J, Bergstrom R, Lindgren A \& Nyren O (1999) Symptomatic gastroesophageal reflux as a risk factor for esophageal adenocarcinoma. N Engl J Med 340:825-831

Lagergren J \& Viklund P (2007). Is esophageal adenocarcinoma occurring late after antireflux surgery due to persistent postoperative reflux? World J Surg; 31: 465-469

Laws HL, Clements RH \& Swillie CM (1997). A randomized, prospective comparison of the Nissen fundoplication versus the Toupet fundoplication for gastroesophageal reflux disease. Ann Surg.;225:647-653.

Leggett PL, Bissell CD, Churchman-Winn R \& Ahn C (2000). A comparison of laparoscopic Nissen fundoplication and Rossetti's modification in 239 patients. Surg Endosc.;14:473-477.

Lieberman D A, Oehlke M, Helfand M \& the GORGE Consortium (1997). Risk factors for Barrett's esophagus in community-based practice. Am J Gastroenterol; 92:12931297.

Locke G R, Zinsmeister A R \& Talley N J (2003). Can symptoms predict endoscopic findings in GERD? Gastrointest Endosc; 58: 661-670.

Long JD \& Orlando RC (1999). Esophageal submucosal glands: Structure and function. Am. J. Gastroenterol.;94:2818-2824.

Loustarinen M, Vurtanen J, Koskinen M, Matikainen M \& Isolauri J (2001). Dysphagia and oesophageal clearance after laparoscopic versus open Nissen fundoplication. A randomized, prospective trial. Scand J Gastroenterol;36:565-571.

Loustarinen ME \& Isolauri JO (1999). Randomized trial to study the effect of fundic mobilization on long-term results of Nissen fundoplication. Br J Surg.;86:614-618.

Lundell L, Miettinen P, Myrvold HE, Hatlebakk JG, Wallin L, Malm A, Sutherland I, Walan A \& Nordic GORD Study Group (2007). Seven-year follow-up of a randomized clinical trial comparing proton-pump inhibition with surgical therapy for reflux oesophagitis Br J Surg.;94:198-203.

Lundell L, Miettinen P, Myrvold HE, Pederson SA, Liedman B, Hatlebakk JG, Julkonen R, Levander K, Carlsson J, Lamm M \& Wiklund I (2001). Continued (5-year) follow-up of a randomized clinical study comparing antireflux surgery and omeprazole in gastroesophageal reflux disease. J Am Coll Surg ; 192: 172-179; discussion 179:181.

Manifold D K, Marshall R E K, Anggiansah A \& Owen W J (2000). Effect of omeprazole on antral duodenogastric reflux in Barrett oesophagus. Scand J Gastroenterol; 8: 796801. 
Mardani J, Lundell L, Lönroth H, Dalenbäck J \& Engström C (2009). Ten-year results of a randomized clinical trial of laparoscopic total fundoplication with or without division of the short gastric vessels. Br J Surg.;96:61-65.

McKenzie D, Grayson T \& Polk HC Jr (1996). The impact of omeprazole and laparoscopy upon hiatal hernia and reflux esophagitis. J Am Coll Surg; 183: 413-418.

Morgenthal CB, Shane MD, Stival A, Gletsu N, Milam G, Swafford V, Hunter JG \& Smith CD (2007). The durability of laparoscopic Nissen fundoplication: 11-year outcomes. J Gastrointest Surg.;11:693-700.

Mungan Z, Demir K, Onuk MD, Göral V, Boztaş G, Beşışık F al (1999). Characteristics of gastroesophageal reflux disease in our country. Turk J Gastroenterol;10:101-106.

Naef AP, Savary M \& Ozzello L (1975). Columnar-lined lower esophagus: an acquired lesion with malignant predisposition. Report on 140 cases of Barrett's esophagus with 12 adenocarcinomas. J. Thorac. Cardiovasc. Surg.;70:826-835.

Nguyen DM, El-Serag HB, Henderson L, Stein D, Bhattacharyya A \& Sampliner RE (2009). Medication usage and the risk of neoplasia in patients with Barrett's esophagus. Clin Gastroenterol Hepatol; 7: 1299-1304.

O'Boyle CJ, Watson DI, Jamieson GG, Myers JC, Game PA \& Devitt PG (2002). Division of short gastric vessels at laparoscopic nissen fundoplication: a prospective doubleblind randomized trial with 5-year follow-up. Ann Surg;235:165-170.

Oelschlager B K, Eubanks T R, Oleynikov D, Pope C \& Pellegrini C A (2002) Symptomatic and physiologic outcomes after operative treatment for extraesophageal reflux. Surg Endosc 16:1032-1036

Oelschlager BK, Barreca M, Chang L, Oleynikov D \& Pellegrini CA (2003). Clinical and pathologic response of Barrett's esophagus to laparoscopic antireflux surgery. Ann Surg; 238: 458-464.

Oelschlager BK, Quiroga E, Parra JD, Cahill M, Polissar N \& Pellegrini C A (2008). Longterm outcomes after laparoscopic antireflux surgery. Am J Gastroenterol.;103:280287.

O'Riordan JM, Byrne PJ, Ravi N, Keeling PW \& Reynolds JV (2004). Long-term clinical and pathologic response of Barrett's esophagus after antireflux surgery. Am J Surg; 188: 27-33.

Ouatu-Lascar R \& Triadafilopoulos G (1998). Complete elimination of reflux symptoms does not guarantee normalisation of intraesophageal acid reflux in patients with Barrett's esophagus. Am J Gastroenterol; 93: 711-716.

Pandolfino JE, El-Serag HB, Zhang Q et al (2006). Obesity: a challenge to esophagogastric junction integrity. Gastroenterology;130:639-49.

Pandolfino JE, Shi G, Trueworthy B \& Kahrilas PJ (2003). Esophagogastric junction opening during relaxation distinguishes nonhernia reflux patients, hernia patients, and normal subjects. Gastroenterology;125:1018-24

Paraf F, Flejou JF, Pignon JP, Fekete F \& Potet F (1995). Surgical pathology of adenocarcinoma arising in Barrett's esophagus. Analysis of 67 cases. Am. J. Surg. Pathol.;19:183-191.

Parker SL, Tong T, Bolden S \& Wingo PA (1997). Cancer statistics, 1997. CA Cancer J. Clin.;47:5-27. 
Parrilla P, Martínez de Haro LF, Ortiz A, Munitiz V, Molina J, Bermejo J \& Canteras M (2003). Long-term results of a randomized prospective study comparing medical and surgical treatment of Barrett's esophagus. Ann Surg; 237: 291-298.

Pessaux P, Arnaud JP, Delattre JF, Meyer C, Baulieux J \& Mosnier H (2005). Laparoscopic antireflux surgery: five-year results and beyond in 1340 patients. Arch Surg.;140:946-951.

Peters JH, DeMeester TR, Crookes P, Oberg S, de Vos Shoop M, Hagen JA et al (1998). The treatment of gastroesophageal reflux disease with laparoscopic Nissen fundoplication: prospective evaluation of 100 patients with "typical" symptoms. Ann Surg;228:40-50.

Peters MJ, Mukhtar A, Yunus RM, Khan S, Pappalardo J, Memon B \& Memon MA (2009). Meta-analysis of randomized clinical trials comparing open and laparoscopic antireflux surgery. Am J Gastroenterol;104:1548-61.

Playford RJ (2006). New British Society of Gastroenterology (BSG) guidelines for the diagnosis and management of Barrett's oesophagus . Gut; 55:442.

Pohl H \& Welch HG (2005). The role of overdiagnosis and reclassification in the marked increase of esophageal adenocarcinoma incidence. J. Natl. Cancer Inst.; 97:142-146.

Rakita S, Villadolid D, Thomas A, Bloomston M, Albrink M, Goldin S \& Rosemurgy A (2006) Laparoscopic Nissen fundoplication offers high patient satisfaction with relief of extraesophageal symptoms of gastroesophageal reflux disease. Am Surg 72:207212.

Rice T W (2000). Why antireflux surgery fails? Dig Dis;18:43-7.

Rice T. W. \& Blackstone E. H (2008). Surgical Management of Gastroesophageal Reflux Disease Gastroenterol Clin N Am 37: 901-919.

Roberts KE, Duffy AJ \& Bell RL (2006). Controversies in the treatment of gastroesophageal reflux and achalasia. World J Gastroenterol.;12:3155-3161.

Rossetti M \& Hell K (1977). Fundoplication for the treatment of gastroesophageal reflux in hiatal hernia. World J Surg.;1:439-443.

Rossi M, Barreca M, de Bortoli N, Renzi C, Santi S, Gennai A, Bellini M, Costa F, Conio M \& Marchi S (2006). Efficacy of Nissen fundoplication versus medical therapy in the regression of low-grade dysplasia in patients with Barrett esophagus: a prospective study. Ann Surg 243:58-63.

Salminen PT, Hiekkanen HI, Rantala AP \& Ovaska JT (2007). Comparison of long-term outcome of laparoscopic and conventional nissen fundoplication: a prospective randomized study with an 11-year follow-up. Ann Surg.;246:201-206.

Sampliner R E (1994). Effect of up to 3 years of high-dose lansoprazole on Barrett's esophagus. Am J Gastroenterol; 89:1844-1848.

Sampliner RE (2002). The Practice Parameters Committee of the American College of Gastroenterology. Updated guidelines for the diagnosis, surveillance, and therapy of Barrett's esophagus. Am J Gastroenterol.; 97:1888-95.

Sato K, Awad ZT, Filipi CJ, Selima MA, Cummings JE, Fenton SJ \& Hinder RA (2002). Causes of long-term dysphagia after laparoscopic Nissen fundoplication. JSLS.;6:35-40. 
Sgromo B, Irvine LA, Cuschieri A \& Shimi SM (2008). Long-term comparative outcome between laparoscopic total Nissen and Toupet fundoplication: Symptomatic relief, patient satisfaction and quality of life. Surg Endosc.;22:1048-1053.

Sharma P, Sampliner R E \& Camargo E (1997). Normalisation of esophageal pH with highdose proton pump inhibitor therapy does not result in regression of Barrett's esophagus. Am J Gastroenterol.; 92:582-585.

Shepherd NA (2003). Barrett's esophagus: its pathology and neoplastic complications. Esophagus; 1:17-29.

Smith RR, Hamilton SR, Boitnott JK \& Rogers EL (1984). The spectrum of carcinoma arising in Barrett's esophagus.A clinicopathologic study of 26 patients. Am. J. Surg. Pathol.;8:563-573.

Society of American Gastrointestinal and Endoscopic Surgeons (2010). Guidelines for surgical treatment of gastro-oesophageal reflux disease (GERD). Avaible at: http://www.sages.org/publication/id/22/

Solaymani-Dodaran M, Logan RFA, West J, Card T \& Coupland C (2004). Risk of oesophageal cancer in Barrett's oesophagus and gastro-oesophageal reflux. Gut; 53 : 1070-1074.

Spechler S J \& Goyal R K (1996) The columnar-lined esophagus, intestinal metaplasia, and Norman Barrett. Gastroenterology 110:614-621

Spechler SJ, Lee E, Ahnen D, Goyal RK, Hirano I, Ramirez F, Raufman JP, Sampliner R, Shnell T, Sontag S, Vlahcevic ZR, Young R \& Williford W (2001). Long-term outcome of medical and surgical therapies for gastroesophageal reflux disease: follow-up of a randomized controlled trial. JAMA; 285: 2331-2338.

Stanciu C \& Bennett JR (1974). Alginate/antacid in the reduction of gastro-oessophageal reflux. Lancet;I:109-111.

Strate U, Emmermann A, Fibbe C, Layer P \& Zornig C (2008). Laparoscopic fundoplication: Nissen versus Toupet two-year outcome of a prospective randomized study of 200 patients regarding preoperative esophageal motility. Surg Endosc.;22:21-30.

Takubo K, Aida J, Naomoto Y et al (2009). Cardiac rather than intestinal-type background in endoscopic resection specimens of minute Barrett adenocarcinoma. Hum. Pathol.;40: 65-74.

Takubo K, Nixon JM \& Jass JR (1995). Ducts of esophageal glands proper and paneth cells in Barrett's esophagus: Frequency in biopsy specimens. Pathology;27:315-317.

Takubo K, Sasajima K, Yamashita K, Tanaka Y \& Fujita K (1991). Double muscularis mucosae in Barrett's esophagus. Hum. Pathol.;22:1158-1161.

Trus TL, Laycock WS, Waring JP, Branum GD \& Hunter JG (1999). Improvement in qualityof-life measures after laparoscopic antireflux surgery. Ann. Surg.;229:331-336.

Vaezi MF \& Richter JE (1996). Role of acid and duodenogastroesophageal reflux in gastroesophageal reflux disease. Gastroenterology.; 111:1192-1199.

Vakil N, van Zanten SV, Kahrilas P, Dent J \& Jones R (2006). The Montreal definition and classification of gastroesophageal reflux disease: a global evidence-based consensus. Am J Gastroenterol.;101:1900-1920.

Van der Veen AH, Blankenstein JD \& van Blankenstein M (1989). Adenocarcinoma in Barrett's oesophagus: an overrated risk. Gut; 30: 14-18. 
Velanovich V (1998). Comparison of generic (SF-36) versus disease specific (GERD-HRQL) quality of life scales for gastroesophageal reflux disease. J Gastrointest Surg.; 2:141145.

Velanovich V (1999). Comparison of symptomatic and quality-of-life outcomes of laparoscopic and open antireflux surgery. Surgery.; 126:782-789.

Velanovich V (2006). Nonsurgical factors affecting symptomatic outcomes of antireflux surgery. Dis Esophagus. ;19: 1-4.

Wang KK \& Sampliner RE (2008). Updated guidelines 2008 for the diagnosis, surveillance and therapy of Barrett's esophagus. Am. J. Gastroenterol.; 103:788-797.

Ware JE \& Sherbourne CD (1992). The MOS 36-item short-form health survey (SF-36). I. Conceptual framework and item selection. Med Care; 30:473-83

Wassenaar EB \& Oelschlager BK (2010). Effect of medical and surgical treatment of Barrett's metaplasia. World J Gastroenterol.;16:3773-3779.

Watson A, Heading RC \& Shepherd NA (2005). Guidelines for the diagnosis and management of Barrett's columnar-lined oesophagus: a report of the working party of the British Society of gastroenterology. British Society of Gastroenterology; London, UK; 1-42.

Watson D I, Pike G K, Baigrie R J et al (1997). Prospective doubleblind randomized trial of laparoscopic Nissen fundoplication with division and without division of short gastric vessels. Ann Surg.; 226: 642-652.

Watson DI, Chan AS, Myers JC \& Jamieson GG (1997). Illness behaviour influences the outcome of laparoscopic antireflux surgery. J Am Coll Surg.; 184:44-48.

Watson DI \& de Beaux AC (2001). Complications of laparoscopic antireflux surgery. Surg Endosc.;15:344-52.

Watson DI, Jamieson GG, Baigrie RJ, Mathew G, Devitt PG, Game PA, et al (1996). Laparoscopic surgery for gastroesophageal reflux : beyond the learning curve. $\mathrm{Br} \mathrm{J}$ Surg; 83:1284-1287.

Watson DI, Jamieson GG, Devitt PG, Matthew G, Britten-Jones RE, Game PA \& Williams RS (1995). Changing strategies in the performance of laparoscopic Nissen fundoplication as a result of experience with 230 operations. Surg Endosc.;9:961-6.

Watson DI, Pike GK, Baigrie RJ, Mathew G, Devitt PG, Britten-Jones R \& Jamieson GG (1997). Prospective double-blind randomized trial of laparoscopic Nissen fundoplication with division and without division of short gastric vessels. Ann Surg.;226:642-652.

Winters C Jr, Spurling TJ, Chobanian SJ et al (1987). Barrett's esophagus. A prevalent, occult complication of gastroesophageal reflux disease. Gastroenterology.;92:118-124.

Yang H, Watson DI, Lally CJ, Devitt PG, Game PA \& Jamieson GG (2008). Randomized trial of division versus nondivision of the short gastric vessels during laparoscopic Nissen fundoplication: 10-year outcomes. Ann Surg.;247:38-42.

Zagari RM, Fuccio L,Wallander MA et al (2008). Gastro-oesophageal reflux symptoms, oesophagitis and Barrett's oesophagus in the general opoulation: the LoianoMonghidoro study. Gut;57:1354-9.

Zaninotto G, Portale G, Costantini M, Rizzetto C, Guirroli E, Ceolin M, Salvador R, Rampado S, Prandin O, Ruol A \& Ancona E (2007). Long-term results (6-10 years) of laparoscopic fundoplication. J Gastrointest Surg.;11:1138-1145. 
Zaninotto G, Portale G, Parenti A, Lanza C, Costantini M, Molena D, Ruol A, Battaglia G, Costantino M, Epifani M \& Nicoletti L (2002). Role of acid and bile reflux in development of specialised intestinal metaplasia in distal oesophagus. Dig Liver Dis.;3:251-257.

Zügel N, Jung C, Bruer C, Sommer P \& Breitschaft K (2002). A comparison of laparoscopic Toupet versus Nissen fundoplication in gastroesophageal reflux disease. Langenbecks Arch Surg.;386:494-498. 


\title{
Laparoscopic One-Stage vs Endoscopic Plus Laparoscopic Management of Common Bile Duct Stones - A Prospective Randomized Study
}

\author{
Giuseppe P. Ferulano et al.* \\ Department of Systemic Pathology General and \\ Miniinvasive Surgical Unit \\ University of Naples "Federico II"
} Italy

\section{Introduction}

The incidence of gallstones is rather high and is referred as approximately $13 \%-17 \%$ among the western population, [Bateson, 2000; Barbara et al., 1987; Everhart et al., 1999; Pixley et al., 1985]. It is well known that most of the people with gallstones are asymptomatic and often they are absolutely unaware of their presence, it is even referred that no more than $15-20 \%$ of them has the probability of suffering from a biliary colic later on [Attili et al., 1995], which, once occurred, could recur more easily causing sometime serious complications, such as pancreatitis by stone's migration and biliary obstruction, that over a 10-year period can be expected to occur in $2-3 \%$ of patients with initially silent gallbladder stones [Gracie \& Ransohoff, 1982].

The incidence of common bile duct (CBD) stones has been reported as ranging between $5 \%$ to $18 \%$ of patients undergoing cholecystectomy for gallstones, and patients with symptoms suggestive of choledocholithiasis have an even higher incidence, also increasing with age [Martin et al., 2006]. Because of the continuous developing of the diagnostic and therapeutic techniques from the introduction of intra-operative cholangiography by Mirizzi in 1932, the choose of the most effective strategy in the management of the common bile duct (CBD) stones associated with gallstones is object of close discussions far from any conclusive agreement. The new diagnostic techniques as magnetic resonance cholangiography (MRC) and endoscopic ultrasound (EUS), give the opportunity to visualize the biliary tree without any invasive exploration of the ducts and share the same idea as the minimally invasive laparoscopic surgical approach. They are progressively evolving as well as the standard of care for the management of common bile duct (CBD) stones, historically performed via

\footnotetext{
* Saverio Dilillo, Michele D'Ambra, Ruggero Lionetti, Piero Di Silverio, Stefano Capasso, Domenico Pelaggi and Michele Rutigliano

Department of Systemic Pathology General and miniinvasive Surgical Unit (Prof. G.P.Ferulano) University of Naples "Federico II", Italy
} 
laparotomy, which over the past decade-and-a-half has changed from open cholecystectomy with common bile duct exploration through intraoperative cholangiography or choledocoscopy, to the routine availability of endoscopic retrograde cholangioscopy (ERC) with endoscopic sphincterotomy (EST) for common bile duct (CBD) stone extraction performed before or after surgery, open in the past and laparoscopic from almost fifteen years, [Clayton et al., 2006]. However, endoscopic sphincterotomy for bile duct stones complains about a disappointing $8 \%-10 \%$ rate of long-term biliary complications including recurrent or residual ductal stones, cholangitis, stenosis of the papilla, and biliary pancreatitis [Paganini et al., 2007]. Macadam\&Goodall, [2004] referred a high $28 \%$ rate of late, rather frequent symptoms related to low-grade cholangitis following papillosphincterotomy. Consequently the potential sequence of late persistent cholangitis should be regarded as a matter of concern, particularly in fertile female patients.

More recently laparoscopic exploration of the common bile duct (LCBDE) has been introduced for managing patients with suspected CBD stones, which allows the intraoperative definite diagnosis and the treatment at the same time, if necessary. As referred in the New Guidelines Address Management of Common Bile Duct Stones [Williams et al., 2008] the consequences are that "clinicians are now faced with a number of potentially valid options for managing patients with suspected CBDS".

Consequently the primary challenge in the management of common bile duct stones in association with gallstones nowadays is to select the best strategy with regard to success, morbidity and cost-effectiveness, [Clayton et al., 2006].

Endoscopy for common bile duct stones and surgery, mainly laparoscopic, for gallstones have been widely adopted as the preferred approach, because the results in terms of success rate, morbidity and mortality tend to overlap those of the whole surgical open approach for gallstones and common bile duct stones offering the undeniable advantages of being less invasive. The ultimate evolution of the association of laparoscopic cholecystectomy with endoscopic retrograde cholangioscopy (ERC) + endoscopic sphincterotomy (EST) was the rendez vous approach performed in a single stage operative procedure together by the surgical and the endoscopic teams, which has shown an overlapping outcome compared to other kinds of association between surgical and endoscopic procedures. Since it is commonly accepted that only a low rate of patients suffering from gallstones and undergoing laparoscopic cholecystecomy are likely to have bile duct stones identified, this procedure to be cost effective needs a definite preoperative diagnosis of common bile duct stones using the modern techniques of imaging such as MR and EUS, which can improve the likelihood of stones being found to over $90 \%$, [Liu et al., 2001; Williams et al., 2008].

Nevertheless in the literature some limits concerning the use of endoscopy are referred like the number and the size of stones, the incidence of complications of ERC + EST occurring in $5 \%-8 \%$ of cases, with mortality rates of $0.2 \%$ to $0.5 \%$ from more difficult procedures or the necessity of multiple sessions to clear completely the common bile duct requiring the use of expensive equipment and accessories. This strategy statistically increased the likelihood of complications as two or more procedures sometime should be performed in a patient to clear up successfully the duct, [Byrne et al., 2009]. As recently referred by Sjer et al. [2010], the ideal technique of common bile duct stones clearing should be minimally invasive, easy to perform, reliably clear all stones from the CBD, obtaining as well the earliest possible discharge from the hospital and leaving the patient with an undisturbed function of the 
papilla Vateri. The routine adoption of laparoscopic common bile duct exploration (LCBDE) associated with cholecystectomy has been promoted by the constant improvement in techniques and expertise of surgeons who are increasingly confident with laparoscopic hepato-biliary surgery and are deeply interested to bring back the whole procedure within the surgical approach. This approach seems to fulfill almost all the previous issues, nevertheless some negative aspects should be considered as the evidence that laparoscopic common bile duct surgery is time consuming and requires a rather long lasting learning curve of the whole staff of an advanced laparoscopic procedure, as well as fluoroscopic equipment and expensive accessories for the procedure that moreover may not be feasible in cases where the CBD diameter is $<6 \mathrm{~mm}$., [Fitzgibbons \& Gardner, 2001]. Our group, as other centers, adopted the procedure of single-stage laparoscopic cholecystectomy (LC) plus common bile duct exploration, with stone extraction performed by different techniques, and a randomized prospective study has been designed to compare it with the standard doublestage procedure based on preoperative endoscopic clearance followed by a laparoscopic cholecystectomy, with the aim of assessing the more safe and successful therapy for the patient.

\section{Materials and methods}

In our unit from January 1996 until June 2010, 918 consecutive patients underwent elective or acute laparoscopic cholecystectomy, and ductal biliary stones were detected in 121 patients $(13.1 \%)$. These patients were treated with the two-stage procedure until 2002, at that time laparoscopic common bile duct exploration and treatment, if necessary, was introduced following a decision analysis performed with the aim of evaluating cost/benefits, efficacy, recurrence, compliance of the patients as referred in the literature, [Urbach et al., 2001].

Consequently 124 out of 534 consecutive patients with evidence (36 patients) or suspicion (88 patients) of duct stones as result of a diagnostic program including US scanning, MR cholangiography and biochemical investigations, were randomly assigned to one of the two selected procedures: one-stage surgical procedure (group 1) and two-stages endoscopic + surgical procedure (group 2). All patients were informed about each procedure and involved technology and they were also asked for their consent to be randomized in the group 1 or in the group 2 and signed the consent forms. Exclusion criteria against laparoscopic common bile duct exploration were suspicion of malignancy, stone impaction, evidence of severe pancreatitis and/or cholangitis or unfitness for general anesthesia, consequently from this series were excluded three patients.

The two groups had comparable demographic and clinical profiles, (tab. 1). The presence of stones was confirmed in 39 out of 62 pts. of the two-stage group who underwent preoperative ERCP and sphincterotomy for clearing the CBD and after 2-5 days underwent a successful laparoscopic cholecystectomy. In the one-stage group stones were found in $45 / 62$ pts. in whom an intraoperative ductal exploration was attempted via the cystic duct that was successful in 55 patients, $(88.7 \%)$ and required a choledochotomy in 7 patients $(11.3 \%)$, because of the size of the stones or unexpected intraoperative difficulties.

Stones were completely removed through the cystic duct in 29 patients while in 16 patients through the previous or a newly performed choledochotomy, using Dormia and/or Fogarty catheters. The transcystic approach failed because of the following reasons: the cystic duct was too small or frale, the stones were larger than $1 \mathrm{~cm}$ or in a number greater than five or proximal to the confluence into the hepatic duct. The techniques of transcystic catheter 
insertion to extract the stones include cystic duct dilation, washing, exploration with biliary balloon catheters or wire baskets, final check with a cholangiography at the end of the procedure. In case of choledocotomy a biliary endoscopy was also performed in 9/16 patients, with an Olympus flexible choledocoscope CHF-CB 30S, in order to remove stones with a catheters under direct vision or to check the duct after the removal of the stones. All patients underwent a control cholangiogram to ensure that duct's clearance was successfully done and that the papilla was patent to contrast dye passage into the duodenum. External biliary drainage with $\mathrm{T}$ tube and postoperative cholangiography was performed in 9 patients. All patient had an external transparietal subhepatic drainage at least for 24 hours, at most for four-five days in those patients in which T-tube drainage was inserted, to control potential early or late persistent biliary leak. T tube was removed after a negative control with a transKehr cholangiography performed within 3-4 weeks from the previous surgery. The laparoscopic procedure was completed in 121 patients (97.6\%). Patients were followed up for 1-9 years (mean 4.5 yrs.), visiting them in the outpatient clinic or interviewing by telephone calls after the first year.

\subsection{Operative procedure}

The laparoscopic cholecystectomy was performed with a standard four-trocar technique using the transumbilical open approach according to the Hasson technique. In case of preoperative evidence or suspicion of CBD stones, a small quantity of diluted contrast solution was injected, (Ultravist-300, Schering A.G., Berlin, Germany, 50\% diluted with a $0.9 \%$ saline solution), performing the first cholangiography through the incision of the cystic duct made close to the confluence into the common duct to facilitate the passage of the operative cholangiogram catheter $4.5 \mathrm{Fr} \times 45.7 \mathrm{~cm}$ (TAUT inc. Geneva, Il 60134 USA).

\section{Preoperative clinical variables $\quad \operatorname{LCBDE}(n=62) \quad$ ERCP $+\operatorname{LC}(n=62) \quad p$ Value}

$\begin{array}{llll}\text { Age (years) } & 53 \pm 13 & 55 \pm 15 & \text { NS } \\ \text { Gender (females, \%) } & 76 & 79 & \text { NS } \\ \text { ASA } & 2 \pm 1 & 2 \pm 1 & \text { NS } \\ \text { Biliary symptoms (\%) } & 75 & 70 & 0.01 \\ \text { Cholecystitis (\%) } & 14.8 & 18.1 & 0.05 \\ \text { Jaundice (\%) } & 6 & 20 & 0.01 \\ \text { Pancreatitis (\%) } & 6.4 & 4.8 & \text { NS } \\ \text { Cholangitis (\%) } & 0 & 1.6 & 0.05 \\ \text { Previous abdominal surgery (\%) } & 29 & 32 & \text { NS }\end{array}$

Table 1. Comparison of clinical demographics of patients in LCBD exploration and treatment group and in ERCP and LC group.

When CBD stones were detected a non-Radiopaque Karlan Balloon Catheter: 4 Fr. 2-Lumen, $60 \mathrm{~cm}$, (Arrow percutaneous laparoscopic cholangiography set CS-01701; Arrow International Europe) was introduced on the anterior axillary line under the right costal margin to allow an appropriate access to the cystic duct, to remove the stones, using a flexible wire guide, if necessary, through the curved guide catheter. The choledochotomy was done after a good exposure of the liver hilus pulling up and to the right the gallbladder and lifting up the round ligament, exposing the anterior wall of the duct making a longitudinal incision sometime helped by two $4 / 0$ prolene stitches lifting up the 
supraduodenal choledocus. The primary closure of the incision was done mainly with a running 4/0 prolene suture. Details of the surgical procedure (exploration, stones extraction, radiological and endoscopic control etc.), timing of the two-stage procedure, results and complications of surgical and endoscopic treatment were recorded.

\subsection{Statistical analyses}

The procedures performed in the two groups were recorded as success or failure according to the complete clearance of the $\mathrm{CBD}$ as showed by the final intraoperative cholangiography. The outcome of the procedures was evaluated as well, looking at different parameters: common bile duct diameter, number of stones, stone size, presence of intrahepatic stones, mean operating time, length of hospital stay. Some of these where splitted in two categories: the limit of $6 \mathrm{~mm}$. was identified for the bile duct diameter, the size of $5 \mathrm{~mm}$. for the stones, and the number of three for the stones. In significance testing Fischer's exact test was used for dichotomized discrete variables and the nonparametric Wilcoxon method for comparisons between means, [Stromberg et al., 2008].

\subsection{Definition of success}

It has been defined as primary outcome measure the successful removal of gallbladder and common bile duct clearance performing the procedures of treatment, and as secondary outcome the results in terms of specific and generic complications such as bleeding, cholangitis, bile leak or fistula, surgical-site infection, late recurrency and other medical complications.

\section{Results}

Removal of the stones in the two groups was successfully done in 79 patients $(94 \%)$, mortality directly related to the procedures was nil (1 cardiac failure at 6 months) nor occurred major intra-operative complications in either group. In two patients, a conversion into laparotomy was necessary for intraoperative haemorrhage caused in the first by an accessory cystic artery and by a severe haemobilia in the second one. The average diameter of the common bile duct was $10.7 \mathrm{~mm}$ (range 6-22 $\mathrm{mm}$ ). The mean number of stones was 3. 4 (1-10). The mean operating time in the group 1 was $160 \mathrm{~m}^{\prime}$ (range 100-280 $\mathrm{m}^{\prime}$ ), the operation lasted significantly more time in the unsuccessful procedures and in patients undergone choledocal exploration, either as first choice or in case of failure of the transcystic approach.

Obviously patients who underwent laparoscopic common bile duct exploration had a longer operating time compared with the group undergone laparoscopic cholecystectomy alone (mean time $70 \mathrm{~m}^{\prime}$ ). $\mathrm{T}$ tubes were applied to patients with multiple stones $(>5)$ and $\mathrm{CBD}$ diameter greater than $6 \mathrm{~mm}$., at risk for retained sludge, previous attacks of cholangitis or pancreatitis, poor tissue quality secondary to duct's infection. It was removed within 3-4 weeks after a trans-Kehr cholangiography without complications neither difference in comparison with primary suture of the choledocal incision, (tab. 2).

Residual CBD stones were detected in the two groups at different intervals of time, following a routine control by an abdominal ultrasonography or magnetic resonance cholangiography. In two patients of the group 1 the stones were removed successfully by 
ERC and endoscopic sphincterotomy after 6 and 8 months from previous surgery, in one patient a new laparoscopic approach (LCBDE) was performed after 30 months because suffering from symptoms referred to recurrent stones. The residual stones in the two patients of the group 2 were successfully removed by a new endoscopic approach and sphincterotomy (EST), without any local and systemic complication.

\begin{tabular}{|c|c|c|c|c|}
\hline & $\mathrm{N}^{\circ}$ & $\begin{array}{l}\text { Successful } \\
(\%)\end{array}$ & $\begin{array}{c}\text { Failure } \\
(\%)\end{array}$ & Pvalue \\
\hline Total number of patients & 84 & $79(94)$ & $5(6)$ & \\
\hline CBD diameter & & & & n.s. \\
\hline$\leq 6 \mathrm{~mm}$ & 31 & $29(93.5)$ & $2(6.5)$ & \\
\hline$>6 \mathrm{~mm}$ & 53 & $50(94)$ & $3(6)$ & \\
\hline Mean number of stones & 3.4 & 2.9 & 5.2 & $0.0053^{*}$ \\
\hline Number of stones & & & & $<0.001^{\wedge}$ \\
\hline$\leq 3$ & 58 & $57(98)$ & $1(2)$ & \\
\hline$>3$ & 26 & $22(85)$ & $4(15)$ & \\
\hline Mean stone size (mm) & 5.4 & 5.1 & 8.3 & $0.0045^{*}$ \\
\hline Stone size & & & & $<0.001^{\wedge}$ \\
\hline$\leq 5 \mathrm{~mm}$ & 61 & $60(98)$ & $1(2)$ & \\
\hline$>5 \mathrm{~mm}$ & 23 & $19(83)$ & $4(17)$ & \\
\hline Intrahepatic stones & & & & n.s. \\
\hline Yes & 3 & $3(100)$ & 0 & \\
\hline No & 81 & $76(94)$ & $5(6)$ & \\
\hline $\begin{array}{l}\text { Mean operating } \\
\text { time (minutes) }\end{array}$ & $170 \mathrm{~m}^{\prime}$ & $150 \mathrm{~m}^{\prime}$ & $230 \mathrm{~m}^{\prime}$ & $<0.001^{\wedge}$ \\
\hline $\begin{array}{l}\text { Mean length of } \\
\text { hospital stay (2-16days) }\end{array}$ & 7.1 & 4.5 & 9.0 & $<0.001^{\wedge}$ \\
\hline
\end{tabular}

Table 2. Overall results of the procedures of CBD stones removal

There was a significant increasing risk among patients with stones of diameter greater than $5 \mathrm{~mm}$. compared to patients with stones of $5 \mathrm{~mm}$. or less. One-stage management of duct stones was associated with a significant less morbidity than two-stage approach $(8.1 \%$ vs. $14.2 \%)$, which is increasingly significant for multiple stones or stones $>5 \mathrm{~mm}$. Haemorrhage occurred in $4.8 \%$ (2.2\% vs. $7.7 \%)$, pancreatitis in $2.4 \%$ (2.2\% vs. 2.6$)$, port site infection and cholangitis in $1.1 \%$ (in the group 2). The mean postoperative hospital stay was 7.1 days (range 2-16), and depended mainly on the surgical outcome in terms of clearing of the common bile duct i.e. success or failure of the procedure.

In the group 1, one patient, who underwent a transcystic stone extraction had a biliary leak not requiring reoperation. After 13 months one patient of the group 2 underwent a new endoscopic treatment, as she was referred to our Day Surgery Unit for a symptomatic cholangitis with evidence of biliary sludge by ultrasonographic examination at the casualty department, caused by a stenosis of the papilla Vateri as showed by a following magnetic resonance, (tab. 3). 


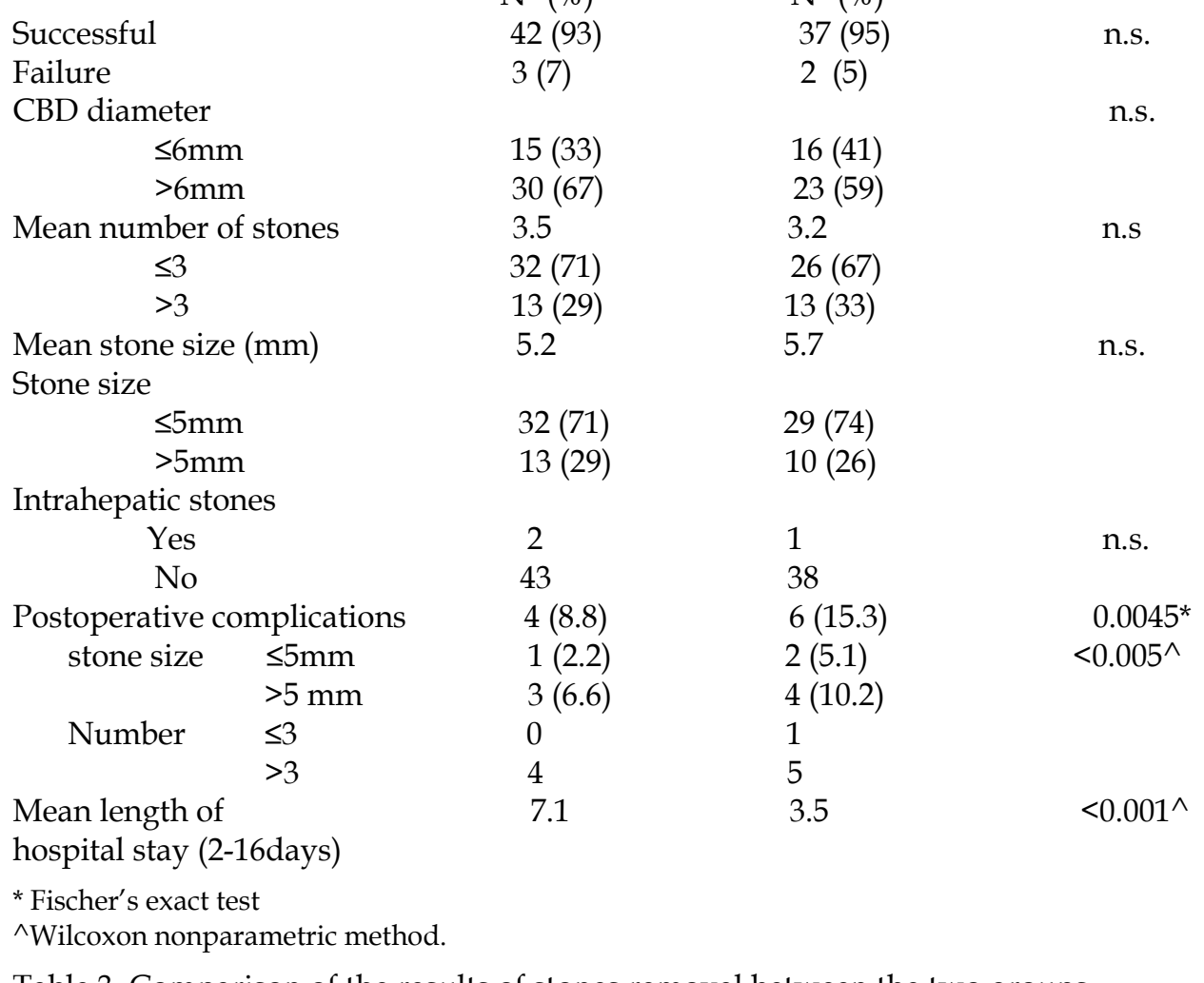

LCBDE $(n=45)$

$\mathrm{N}^{\circ}(\%)$

$42(93)$

$15(33)$

$30(67)$

$32(71)$

$13(29)$

$32(71)$

2

$4(8.8)$

$1(2.2)$

$3(6.6)$

0

7.1

ERC

p Value

$\mathrm{N}^{\circ}(\%)$

$37(95)$

(5)

$16(41)$

$23(59)$

3.2

$13(33)$

$29(74)$

$10(26)$

38

$6(15.3)$

$2(5.1)$

$4(10.2)$

1

5

3.5

$<0.001^{\wedge}$

Table 3. Comparison of the results of stones removal between the two groups

\section{Discussion}

The aim of this study was to evaluate the results of the treatment of common bile duct stones in patients undergoing single-stage laparoscopic management of gallstones and CBD stones performing either transcystic common bile duct exploration (TC-CBDE) or laparoscopic choledochotomy, compared to the two-stage well established and more widely used endoscopic retrograde cholangioscopy + endoscopic sphincterotomy followed by laparoscopic cholecystectomy. The analysis of the results of this prospective study, based on a randomized distribution of 124 consecutive patients in which suspicion or evidence of CBD stones was reported, emphasizes the role of mininvasive treatment of gallbladder and CBD stones, that has become the main focus of biliary surgery. Though a single center study, a comparison has been done between two procedures, with the removal of stones from $\mathrm{CBD}$ as primary end-point, recruiting consecutive patients affected by common bile duct stones or highly suspected of stones presence, without any selection criteria, except the exclusion caused by malignant lesions, high surgical risks or patient's refusal to undergo surgery. They were randomly assigned either to a totally laparoscopic approach including cholecystectomy and duct exploration and treatment, if necessary, or to a double procedure: endoscopic (ERC \pm EST) as first step, followed by laparoscopic cholecystectomy at different interval of time depending mainly on the outcome of the endoscopic treatment. 
These are scheduled among the accepted procedures for an elective treatment, since the conservative or wait and see strategy have been ruled out. Other procedures as the association of the laparoscopic cholecystectomy with ERC + endoscopic sphincterotomy known as the rendezvous approach [Morino et al., 2006; Tricarico et al., 2002;], or the endoscopic treatment after a positive intra-operative cholangiography need more experience and good cooperation between different teams, particularly in the second issue [Hong et al., 2006], and could increase the risk of postoperative complications in both cases, included the need of a second operation if the endoscopic sphincterotomy (EST) fails, [Patel et al., 2003].Laparoscopic common bile duct exploration as single approach, requires a longer learning curve mainly because of the possibility that the procedure could become more demanding if a laparoscopic suture should be performed when the removal of stones is done via a choledocal incision, with or without T-tube placement. The other single stage procedure is the laparoendoscopic rendezvous associating laparoscopic cholecystectomy with intraoperative endoscopic retrograde cholangiography with stone extraction as a onetime therapy for gallstones and CBD stones, that according to Morino et al., (2006) had a higher success rate $(95.6 \%$ v. $80 \%)$, shorter hospital stay (4.3 days v. 8 days) and lesser cost ( $€$ 2829 v. $€ 3834)$, compared with the two stage procedure. It was recently referred that the rendezvous technique can warrant a successful treatment even in cases complicated by cholangitis or pancreatitis, with the help of a guidewire introduced through the cystic duct into the papilla that may reduce the complications secondary to the endoscopic cannulation. With this device is possible to reduce the failure rate of the retrograde cholangiography as well as the incidence of the major complication, i.e. acute pancreatitis, of the laparoendoscopic rendezvous technique compared with the sequential ERCP and LC (5\% vs. 20\%), as referred by El Geidie et al., (2011) who had worse results (no significant difference in failure rate) probably due the non use of the guidewire. Nevertheless with this approach it is impossible to avoid the potential complications linked to the endoscopic sphincterotomy, [Borzellino et al.,2010]. As reported before the major limits lie on the management of endoscopy together with surgery in the operating theatre, and these problems have discouraged the diffusion of this combined approach throughout surgeons interested to this disease, [Meyer et al., 1999]. It should be outlined that the rendezvous procedure should be adopted only in patients with a positive evidence of common bile duct stones, and the ideal would be to predict CBD stones without invasive tests in order to avoid unnecessary and sometime risky procedures, as today magnetic resonance cholangiography actually can obtain. It is likewise necessary to refer that skilled surgeons are able to achieve an overall satisfying outcome, performing the rendez vous procedure, which is quite overlapping with those of the one stage total laparoscopic approach, also from the point of view of the residual stones' rate, [Tranter \& Thompson, 2002].

On the other hand in the literature the A.A. generally agree that the first endoscopic step of the two stage procedure is associated with a high complication rate of about $10 \%$, mainly acute pancreatitis (3\%) and a mortality rate of $4 \%$, which could increase respectively to a maximum of $16 \%$ and $6 \%$, by the addition of the potential complications following the surgical step, [Hong et al., 2006]. This difference could be partially explained by the length of the interval between the two procedures, which is not well defined, as even in the multicenter trial by the European Association for Endoscopic Surgery (EAES) the interval between endoscopic papillosphincterotomy and laparoscopic cholecystectomy was not specified, [Cuschieri et al.,1999]. It is referred that patients awaiting for laparocopic cholecystectomy risk a high rate of readmissions and complications due to acute cholecystitis, pancreatitis, empyema and cholangitis; de Vries et al., [2005 ], showed that in 
case of delayed cholecystectomy, done more than two weeks after endoscopic sphincterotomy, there is a higher conversion rate, increasing from $4 \%$ when LC was done within 2 weeks, to $31 \%$ between 2 and 6 weeks, and 16\% after 6 weeks. A consequence of these considerations was the policy of leaving in situ the gallstones, avoiding the second laparoscopic approach, a sort of wait and see strategy based on the results of retrospective studies which described a relatively low incidence (5-12\%) of biliary complications or recurrent symptoms in patients not undergone routine cholecystectomy following the endoscopic removal of common bile duct stones, [Byrne et al., 2009]. Nevertheless there is a positive consensus on the indication to primary endoscopic sphincterotomy in case of suppurative cholangitis, severe pancreatitis, high-risk patients, and patients who had previous cholecystectomy, [NIH Consensus Statements, 2002]. In our study patients with gallstones did not undergo any invasive diagnostic exploration if not in case of history of jaundice, gallstone pancreatitis with elevated amylase or lipase, elevated bilirubin level, abnormal liver function test results, dilated CBD on preoperative ultrasonography. Magnetic resonance cholangiography was performed when were present one or more criteria above referred, which as well indicated the necessity to perform an intraoperative cholangiography (IOC). The presence of stones was confirmed in 84 out of 124 patients $(68 \%)$ who entered in the prospective trial and subsequently were explored by IOC or ERCP, the rate of stones was similar in the two groups without significant difference between groups, $(72 \%$ vs. $60 \%$ : $p<0.05)$, confirming that they were substantially homogeneous. The overall evaluation of the outcome of the two procedures shows that two factors mainly influence the results of the treatment: the number and the size of stones, neither CBD diameter neither intrahepatic stones influenced the outcome of the procedures. Strömberg et al., [2008] confirmed previous results of Petelin, [2003], who referred that patients with stones larger than $5 \mathrm{~mm}$ had a significant threefold increased risk of failure in stone clearance compared to patients with stones $\leq 5 \mathrm{~mm}$., and suggested a causal relation between large stone size and an increased risk of failure in stone clearance during LTCE. As consequence of the difficulties come across the procedures, postoperative complications were significantly higher in patients unsuccessfully treated. Nevertheless in the overall series postoperative morbidity was reasonably low and there was no postoperative mortality among the patients enrolled in this study. All these data agree with most of the past and recent reports in the literature [Campbell et al., 2004; Kharbutli \& Velanovich, 2008], confirming the indications of the European Association for Endoscopic Surgery for TC-CBDE that are limited to stones that are smaller than the size of the cystic duct [Paganini et al., 2007]. However in our experience the dilation of the cystic duct with a balloon catheter, as usually done to easy the passage into the choledochus, allows to carry out successfully the transcystic procedure for extracting stones even larger than cystic duct, moreover becase of their friability. Nevertheless the choledocothomy, performed by elective choice or compelled by intraoperative complication or difficult removal, did not imply an increase of risks and the rate of successful extraction of stones in the two groups is quite similar, without any difference statistically significant (93\% vs. 95\%). The learning curve of laparoscopic duct exploration (LCBDE) through choledocothomy is not negligible, but once achieved a sufficient expertise it can be safely performed during the one-stage procedure without any evidence for longer hospitalization caused directly by the surgical maneuvers on common duct. No biliary peritonitis or postoperative cholangitis were observed in the one stage group and some minor complications (hyperamylasemia, port site infection, biliary leak etc.) were treated by a conservative therapy and did not require surgical 
measures, as well as they did not lengthen significantly the mean hospital stay, as also showed by the results of different authors, who did not report significant increase of common duct lesions by surgical and/or instrumental maneuvers [Decker et al., 2003; Lezoche \& Paganini, 1995; Paganini et al., 2007] . However the transcystic cannulation of the common bile duct must be regarded as the primary approach to explore $\mathrm{CBD}$, and it can be done as showed by our experience in agreement with several authors, because it is less invasive than laparoscopic choledochotomy.

Nevertheless when stones are larger than $6 \mathrm{~mm}$. or located above the cystic duct choledocothomy could be indicated or sometime compelled by the failure of the transcystic exploration and/or stones removal. The extraction of the stones can be very difficult when they are impacted but in most cases gentle maneuvers with atraumatic Croce forceps through the choledochotomy or irrigation with saline solutions can achieve, after some efforts, a successful duct clearing. However in case of failure biliary Fogarthy catheters or Dormia basket could be used blindly or under vision introducing a choledocoscope, through the cystic duct or more easily through the choledochotomy, depending, of course from the diameter of the endoscope available. It was referred that the mean rate of failure because of residual stones after laparoscopic exploration and treatment of duct stones is about $5 \%-7 \%$, that is quite similar to the rate referred by Moreaux, [1995], following open biliary surgery. The use of choledochoscopy can reduce to $2.8 \%$ the rate of residual stones according to the experience of Berthou et al., [2007], which is remarkably lower than the incidence ranging from $17 \%$ to $35 \%$ of residual stones following endoscopic treatment [Lenriot et al., 1993; Tranter \& Thompson,2002]. Recently it has been confirmed that employing the Dormia laparoscopic basket under control of a choledochoscope the CBD removal is safer and more effective as far as postoperative complication and residual stones are concerned, particularly in comparison with endoscopic procedure burdened by a $10 \%$ rate of residual stones, which is significantly higher and advised to perform non surgical treatment only in case of high risk patients, [Campagnacci et al., 2010], . There is no doubt that the direct approach to CBD can eliminate any problem caused by high number or large diameter of the stones, or their intrahepatic placement. In our experience about $35 \%$ of stones extraction was accomplished performing a choledochotomy, sutured at the end of the procedure mainly with a primary running suture. A closure over a $\mathrm{T}$ tube with an external biliary drainage and postoperative cholangiography was done in 9 patients, without differences in postoperative complication rate, except the necessity of a cholangiographyc control following surgery, to check the duct's patency with normal flow of the contrast into the duodenum, and even the potential presence of residual stones, sludge or fragments of stones, which were flushed down through the papilla with saline injection, provided that there was no leak around the catheter. The procedure was repeated before the removal of the $\mathrm{T}$ tube, which did not cause any major problem, such as peritonitis or biliary fistula, nor minor local or general complication.

In our experience the use of endobiliary $\mathrm{T}$ tube did not affect the outcome in terms of complications, even if we realize that the number of our patients is relatively poor and does not allow any definite conclusion. However in the literature it is referred that the most frequent early complications after LCBDE derive from biliary leaks or infections and are caused mainly by the presence of biliary drainage, that could also cause late biliary stricture, [Decker et al., 2003; Thompson \& Tranter,2002; Alhamdani et al., 2008]. Thompson and Tranter [2002] reported a complication rate of $16 \%$ following the use of the T tube vs. $5 \%$ for primary closure. However our results support those of Paganini and Lezoche , [1998] and 
Berthou et al., [2007], who found a similar incidence of biliary complications following both the procedures. It is generally accepted that a closure over a $\mathrm{T}$ tube is to prefer when the CBD is inflamed because of recurrent cholangitis, [Karaliotas et al., 2008], or dilated and consequently at risk of postoperative atonia and leakage, it could likewise allow for postoperative radiographic control and in case even for extraction of missed or retained stones. There are also some studies comparing primary closure versus $\mathrm{T}$ tube drainage which refer similar rates of complications, but definitely it was showed a shorter operating times and a consistent trend toward shorter hospital stays in favour of the primary closure, [Kanamaru et al., 2007; Jameel et al., 2008].

The incidence of residual or recurrent biliary stones, which has been referred as failure of the procedures in table 1 , is quite similar in the two groups ( $7 \%$ vs. $5 \%$ ), with a rate of residual stones sensibly lower in the LCBDE group (4.1\%). These data are slightly higher than those referred by Chander et al., with a rate of 2.7\%, [2011], and by Berthou et al.,[2007], of 2.8\%, Paganini et al., [2007], of 3.1\%, Hong et al., [2006], of 3.5\%, but are lower than the rate of $6.3 \%$ of Schreurs et al., [36], and all the same are significantly lower than the data referred in the literature of the CBD stones recurrent rate of $9 \%-12 \%$ found at IOC after previous ERCP+EST and LC, [Pierce et al., 2008; Campagnacci et al., 2010].

Nowadays the patients suffering from gallstones with CBD stones scheduled to undergo laparoscopic cholecystectomy may be treated by peri-operative ERCP or managed by LCBDE associated with cholecystectomy in a single surgical step. The "Guidelines on the management of common bile duct stones (CBDS)" [Williams et al., 2008], asserted that "There is no evidence of a difference in efficacy, morbidity or mortality when these approaches are compared, though LCBDE is associated with a shorter hospital stay. It is recommended that the two approaches are considered equally valid treatment options, and that training of surgeons in LCBDE is to be encouraged. (Evidence grade I b. Recommendation grade A.)". It seems that it is widely accepted the evidence from randomised control trials that the outcomes of the one- and two-stage procedures are comparable, some arguments in favour of laparoscopic exploration of the biliary duct could be the evidence of a shorter hospital stay and a better cost-effectiveness as showed by Urbach et al., [2001]. However data from the Cochrane Hepato-Biliary Group, [Martin et al., 2006], don't support any definite evidence of superiority in terms of efficacy, morbidity and mortality of one procedure over another, while the metanalysis of the literature had showed clearly that open biliary surgery was significantly superior to ERC+Endoscopic sphincterotomy in achieving CBD stone clearance.

Recently the Practice/Clinical Guidelines published on 01/2010 by the Society of American Gastrointestinal and Endoscopic Surgeons: "SAGES guidelines for the clinical application of laparoscopic biliary tract surgery" in the chapter dedicated to the management of choledocholithiasis stated that:

- There are several approaches and current data does not suggest clear superiority of any one approach. (Level I, Grade A).

- Laparoscopic transcystic common bile duct exploration is frequently successful, but may be hampered by analomous anatomy, proximal stones, strictures and large or numerous stones. (Level II, Grade B).

- Laparoscopic choledochotomy requires advanced laparoscopic skills, but has good clearance rates; the incision may be closed over a $\mathrm{T}$ tube, an exteriorized transcystic drain, or primary closure with or without endoluminal drainage. (Level II, Grade B). 
- ERCP with stone extraction may be performed selectively before, during or after cholecystectomy with little discernable difference in morbidity and mortality and similar clearance rates when compared to laparoscopic common bile duct exploration, though routinely performed preoperative ERCP will likely result in unnecessary procedures with higher than acceptable mortality and morbidity rates. (Level I, Grade A).

On the basis of this evidence based medicine, our experience from the results of this trial suggests that biliary stones should be treated again by surgeons in first approach, as the endoscopic procedures do not automatically guarantee the complete cleansing of choledocus from stones or the absence of endoscopically related complications. Consequently a surgeon used to perform laparoscopic advanced procedures and dedicated to the management of hepato-biliary diseases, should improve his skill in the intraoperative management of the common bile duct, because the treatment of the individual patient needs an available and expert surgical team to assure good results in terms of success, costs, and length of hospital stay.

The experience resulting from this prospective study supports the aim of demonstrating that laparoscopic surgery of cholecystocholedocal stones is as safe as the procedure associating LC with endoscopic removal of ductstones, but in the great majority of cases it avoids an unnecessary double admission to the hospital services, lowers the risks connected with a double procedure, and as far as the outcome of the follow-up, though not too extended, it involves a low recurrence rate, as already showed in a study with a long-term follow-up (118 mo.) by Paganini et al., [2007]. Looking at the clinical effectiveness, and at the cost/benefits ratio, these two procedures should be considered therefore between the most useful treatment of biliary stones disease, but the single surgical approach has the advantage of taking care of the papilla Vateri avoiding unnecessary and sometimes dangerous sphincterotomy, [Sugiyama \& Atomi, 2002].

Differently from the observation referred by Hong et al., [2006], about the use of the cholangioscope to remove the stones, because it could cause a waste of time, we would stress the opinion that all the techniques and devices used in the open approach to common bile duct, which are currently available in the up-to-date models, should be as well at disposal of the laparoscopic exploration of the bile duct, and used by the surgeon depending on the needs more than on predisposed patterns. The ability of managing even difficult situations consists in choosing the better way to explore the duct and to remove the stones if identified, without rejecting any helpful option. Actually most of the authors agree on the necessity of an adequate training of the surgeon facing with laparoscopic exploration of common duct in order to allow that this procedure could become the first choice approach to biliary stones disease, preventing the occurrence of early and late complications. Our study reaches a collateral not prevented aim, demonstrating that it is possible to obtain a successful surgical treatment adopting the transcystic exploration as first-line approach, that was successful in managing common bile duct stones in almost $70 \%$ of cases and that opening the common duct in case of difficulties or failure actually increases the overall success rate of the surgical approach. This confirms the conclusion of the study of Hanif et al., [2010], who encouraged surgeons to learn and apply both the procedures when they perform the one stage laparoscopic common bile duct exploration.

\section{Conclusions}

The significant and progressive improvements during the last decade of the diagnostic equipments associated with a definite trend to limit, as far as possible, any invasive 
instrumental exploration in favour of the digital work out of the images registered by ultrasonography and magnetic resonance, has shown that it is possible to achieve excellent diagnostic results which allow a correct therapeutic approach. In a similar way the applications of new technologic devices, mainly dedicated to laparoscopic surgery, in association with the increasing diffusion of intraoperative surgical maneuvers borrowed from open surgery such as cholangiography, X-guided explorations, US scanning and others, allow the surgeons to increase their confidence with advanced laparoscopic surgery, while keeping the concepts related to a miniinvasive attitude. This study demonstrates that it is possible to deal with gallstones and CBD stones at the same time, treating them with only one surgical procedure, avoiding unnecessary damage to the papilla Vateri as well as the risk of increasing complications caused by the potential addition of the complications of the endoscopic sphincterotomy to the laparoscopic cholecystectomy, (sphincterotomy, incidentally, was a matter of violent discussions between surgeons in the past decades), and achieving a complete clearing of the common bile ducts with a low rate of residual or recurrent stones. In our study CBD clearing was done in some cases with the help of the choledocoscope, which is a safe but not crucial procedure, provided that several tricks can be used to achieve the same results, from gentle papillary pneumatic dilatation, to flush saline irrigations in association with intraductal lidocaine or intravenous glucagon administration. The peculiar friability of bile stones in the majority of cases helps the happy outcome of the whole procedure, which can be done for the most part by the transcystic route, as clearly showed by our results. Particularly the comparison between the totally surgical and the mixed endoscopic plus surgical treatment in this study did not showed a definite statistical advantage of one over the other, but it demonstrates that the one stage laparoscopic approach is able to solve the problem without mortality, with a low rate of morbidity and long distance sequences, residual stones included and finally with an earlier recovery and return to the normal activity of the patient. It is useful to outline that the one stage surgery does not complain of any of the peculiar biliary complication as cholangitis, papillary stenosis or recurrent pancreatitis referred to the endoscopic sphincterotomy, as the results our follow-up show. However we agree with the opinion that the two procedures are not in conflict each other, because it is possible to distinguish different indications, namely the general conditions of the patients, which could contraindicate a longer surgical approach, such as the laparoscopic exploration and cleaning of the common bile duct, particularly in case of previously recognized necessity of performing a choledocotomy because of size, number, position of the stones, or the local acute complications like cholangitis or stone impaction, with whom endoscopic treatment with sphincterotomy and or naso-biliary drainage more easily can deal successfully. In conclusion nowadays LCBDE is a safe and effective procedure that can be regarded as the first option approach to the treatment of patients affected by gallstones in association with CBD stones, in the hands of well experienced miniinvasive surgeons.

Disclosures: Prof. Giuseppe P. Ferulano, Drs. Saverio Dilillo, Michele D’Ambra, Ruggero Lionetti, Piero Di Silverio, Stefano Capasso, Domenico Pelaggi, Michele Rutigliano, have no conflicts of interest or financial ties to disclose.

\section{References}

Alhamdani A, Mahmud S, Jameel M, Baker A. Primary closure of choledochotomy after emergency laparoscopic common bile duct exploration. Surg Endosc 2008; 22: 2190-5. 
Attili AF, De Santis A, Capri R, Repice AM, Maselli S. The natural history of gallstones: the experience. The GREPCO Group. Hepatology 1995;21:655-60.

Barbara L, Sama C, Morselli-Labate AM, Taroni F, Rusticali AG. A population study on the prevalence of gallstone disease: the Sirmione study. Hepatology 1987;7:913-917.

Bateson MC. Gallstones and cholecystectomy in modern Britain. Postgrad Med J 2000;76:700-3.

Berthou J C, Dron B, Charbonneau P, Moussalier K, Pellissier L. Evaluation of laparoscopic treatment of common bile duct stones in a prospective series of 505 patients: indications and results. Surg Endosc 2007; 21:1970-1974.

Borzellino G, Rodella L, Saladino E, Catalano F, Politi L, Minicozzi A, Cordiano C.

Treatment for retrieved common bile duct stones during laparoscopic cholecystectomy: the rendezvous technique. Arch Surg. 2010; 145(12):1145-9.

Byrne MF, McLoughlin MT, Mitchell RM, Gerke H, Pappas TN, Branch MS, Jowell P S, Baillie J. The fate of patients who undergo "preoperative" ERCP to clear known or suspected bile duct stones. Surg Endosc 2009; 23:74-79.

Campagnacci R, Baldoni A, Baldarelli M, Rimini M, De Sanctis A, Di Emiddio M, Guerrieri M. Is laparoscopic fiberoptic choledochoscopy for common bile duct stones, a fine option or a mandatory step? Surg Endosc 2010; 24:547-553.

Campbell S, Mee A, Thompson MH. Common bile duct calculi-ERCP versus laparoscopic exploration. Ann R Coll Surg Engl 2004; 86:470-473.

Chander J, Vindal A, Lal P, Gupta N, Kumar Ramteke V. Laparoscopic management of CBD stones: an Indian experience. Surg Endosc 2011; 25:172-181.

Clayton ESJ, Connor S, Alexakis N, Leandros E. Meta-analysis of endoscopy and surgery versus surgery alone for common bile duct stones with the gallbladder in situ. $\mathrm{Br} \mathrm{J}$ Surg 2006;93:1185-91.

Cuschieri A, Lezoche E, Morino M, Croce E, Lacy A, Toouli J, Faggioni A, Ribeiro VM, Jakimowicz J, Visa J, Hanna GB. E.A.E.S. multicenter prospective randomized trial comparing two-stage vs single-stage management of patients with gallstone disease and ductal calculi. Surg Endosc 1999; 13:952-957.

Decker G, Borie F, Millat B, Berthou JC, Deleuze A, Drouard F, Guillon F, Rodier JG, Fingerhut A. One hundred laparoscopic choledochotomies with primary closure of the common bile duct. Surg Endosc 2003; 17: 12-18.

de Vries A, Donkervoor SC, van Geloven AAW, Pierik EGJM. Conversion rate of laparoscopic cholecystectomy after endoscopic retrograde cholangiography in the treatment of choledocholithiasis. Does the time interval matter? Surg Endosc 2005; 19:996-1001.

ElGeidie AA, ElEbidy GK, Naeem YM. Preoperative versus intraoperative endoscopic sphincterotomy for management of common bile duct stones. Surg Endosc 2011; 25:1230-1237.

Everhart JE, Khare M, Hill M, Maurer KR., Prevalence and ethnic differences in gallbladder disease in the United States. Gastroenterology 1999;117:632-9.

Fitzgibbons RJ Jr, Gardner GC Laparoscopic surgery and the common bile duct. World J Surg 2001; 25:1317-132.

Jameel M, Darmas B, Baker AL. Trend towards primary closure following laparoscopic exploration of the common bile duct. Ann R Coll Surg Engl 2008; 90:29-35. 
Kanamaru T, Sakata K, Nakamura Y, Yamamoto M, Ueno N, Takeyama Y. Laparoscopic choledochotomy in management of choledocholithiasis. Surg Laparosc Endosc Percutan Tech 2007;17:262-6.

Karaliotas C, Sgourakis G, Goumas C, Papaioannou N, Lilis C, Leandros E. Laparoscopic common bile duct exploration after failed endoscopic stone extraction. Surg Endosc 2008; 22:1826-31.

Kharbutli B, Velanovich V. Management of preoperative suspected choledocholithiasis: a decision analysis. J Gastrointest Surg 2008; 12:1973- 1980 .

Gracie WA, Ransohoff DF. The natural history of silent gallstones: the innocent gallstone is not a myth. N Engl J Med 1982;307:798-800.

Hanif F, Ahmed Z, Samie AM, Nassar AHM. Laparoscopic transcystic bile duct exploration: the treatment of first choice for common bile duct stones. Surg Endosc 2010; 24:1552-1556.

Hong DF, Xin Y, Chen DW (2006) Comparison of laparoscopic cholecystectomy combined with intraoperative endoscopic sphincterotomy and laparoscopic exploration of the common bile duct for cholecystocholedocholithiasis. Surg Endosc 20:424-427.

Lenriot JP, Le Neel JC, Hay JM, Jaeck D, Millat B, Fagniez PL. Cholangio-pancreatographie retrograde et sphincterotomie endoscopique pour lithiase biliaire. Gastroenterol Clin Biol 1993; 17:244-250.

Lezoche E, Paganini AM. Single-stage laparoscopic treatment of gallstones and common bile duct stones in 120 unselected, consecutive patients. Surg Endosc 1995; 9: 10701075.

Liu TH, Consorti ET, Kawashima A. Patient evaluation and management with selective use of magnetic resonance cholangiography and endoscopic retrograde cholangiopancreatography before laparoscopic cholecystectomy. Ann Surg 2001;234:33-40.

Macadam RCA, Goodall RJR Long-term symptoms following endoscopic sphincterotomy for common bile duct stones. Surg Endosc 2004; 18: 363-366

Martin DJ, Vernon DR, Toouli J. Surgical versus endoscopic treatment of bile duct stones. Cochrane Database Syst Rev 2006 (2): CD003327.

Meyer C, Vo Huu Le J, Rohr S. Management of common bile duct stones in a single operation combining laparoscopic cholecystectomy and perioperative endoscopic sphincterotomy. Surg Endosc 1999; 13:874-87.

Moreaux J. Traditional surgical management of common bile duct stones: a prospective study during a 20 year experience. Am J Surg 1995; 169: 220-226.

Morino M, Baracchi F, Miglietta C, Furlan N, Ragona R, Garbarini A. Preoperative endoscopic sphincterotomy versus laparoendoscopic rendezvous in patients with

gallbladder and bile duct stones. Ann Surg 2006;244:889-96.

National Institutes of Health (NIH) state of the science on endoscopic retrograde cholangiopancreatography (ERCP) for diagnosis and therapy. NIH Consens Sci Statements 2002; 19: 1-26.

Paganini AM, Lezoche E. Follow-up of 161 unselected consecutive patients treated laparoscopically for common bile duct stones. Surg Endosc 1998; 12: 23-29.

Paganini A M, Guerrieri M, Sarnari J, De Sanctis A, D’Ambrosio G, Lezoche G, Perretta S, Lezoche E. Thirteen years experience with laparoscopic transcystic common bile 
duct exploration for stones. Effectiveness and long-term results. Surg Endosc (2007) 21: 34-40.

Patel AP, Lokey JS, Harris JB, Sticca RP, McGill ES, Arrillaga A, Miller RS, Kopelman TR. Current management of common bile duct stones in a teaching community hospital. Am Surg 2003;69:555-560.

Petelin JB. Laparoscopic common bile duct exploration. Surg Endosc 2003; 17: 1705-1715.

Pierce RA, Jonnalagadda S, Spitler JA, Tessier DJ, Liaw JM, Lal SC, Melman LM, Frisella MM, Todt LM, Brunt LM, Halpin VJ, Eagon JC, Edmundowicz SA, Matthews BD. Incidence of residual choledocholithiasis detected by intraoperative cholangiography at the time of laparoscopic cholecystectomy in patients having undergone preoperative ERCP. Surg Endosc 2008; 22:2365- 2372.

Pixley F, Wilson D, McPherson K, Mann J., Effect of vegetarianism on development of gall stones in women. BMJ (Clin Res Ed) 1985;291:11-2.

Schreurs WH, Juttmann JR, . Stuifbergen WNHM, Oostvogel HJM and Vroonhoven TJMV. Management of common bile duct stones . Surg Endosc 2002; 16, 10681072.

Sjer A. EB, Boland DM, van Rijn PJJ, Mohamad S. A decade of washing out common bile duct stones with papillar balloon dilatation as a one-stage procedure during laparoscopic cholecystectomy. Surg Endosc 2010; 24:2226-2230.

Stromberg C, Nilsson M, Leijonmarck CE. Stone clearance and risk factors for failure in laparoscopic transcystic exploration of the common bile duct. Surg Endosc 2008; 22:1194-1199.

Sugiyama M, Atomi Y. Risk factors predictive of late complications after endoscopic sphincterotomy for bile duct stones: long-term (more than 10 years) follow-up study. Am J Gastroenterol 2002; 97: 2763-2767.

Thompson MH, Tranter SE. All-comers policy for laparoscopic exploration of the common bile duct. Br J Surg 2002; 89: 1608-1612.

Tranter SE, Thompson MH Comparison of endoscopic sphincterotomy and laparoscopic exploration of the common bile duct. Br J Surg 2002; 89: 1495-1504.

Tricarico A, Cione G, Sozio M, Di Palo P, Bottino V, Tricarico T,Tartaglia A, Iazzetta I, Sessa E, Mosca S, De Nucci C, Falco P. Endolaparoscopic rendezvous treatment: a satisfying therapeutic choice for cholecystocholedocholithiasis. Surg Endosc 2002; 16: 585-588.

Urbach DR, Khajanchee YS, Jobe BA, Standage BA, Hanson PD, Swanstrom LL. Costeffective management of common bile duct stones. A decision analysis of the use of endoscopic retrograde cholangiopancreatography (ERCP), intraoperative cholangiography and laparoscopic bile duct exploration. Surg Endosc 2001; 15:4-13.

Williams EJ, Green J, Beckingham I, Parks R, Martin D, Lombard M. Guidelines on the management of common bile duct stones (CBDS). Gut 2008;57:1004-21. 


\title{
Endoscopic Ultrasound for Solid and Cystic Neoplasms of the Pancreas
}

\author{
Karim M. Eltawil ${ }^{1}$ and Michele Molinari ${ }^{2}$ \\ ${ }^{1}$ Dalhousie University, Department of Surgery, Queen Elizabeth II \\ Health Sciences Centre, Rm 6-302 Victoria Building,Halifax, Nova Scotia \\ ${ }^{2}$ Associate Professor of Surgery, Rm 6-302 Victoria Building, Halifax, Nova Scotia
}

Canada

\section{Introduction}

Endoscopic ultrasound (EUS) was introduced in clinical practice in $1980^{1}$ and during the last few decades the quality of instrumentation has improved significantly. The first commercially available radial echoendoscope was introduced in Japan ${ }^{2}$ and then in Europe 3 in the mid to late 1980s. At that time, radial probes were used for fine needle aspiration (FNA). Only in the early 1990s EUS-FNA become technologically practical with the introduction of linear echoendoscopes that generated ultrasonic images parallel to the shaft of the instrument. With this modification, needles could be guided into areas of interest. Soon after this improvement, the first report of EUS-FNA of the pancreas was published 4 and numerous other publications have followed ${ }^{5-8}$. Later on, mechanical probes have been replaced by electrical probes that allowed expansion of the diagnostic capacity of EUS. More recently, the addition of color Doppler ultrasonography, injection of contrasts for ultrasound and the application of elastography has further extended the clinical use of EUS for hepatobiliary and pancreatic diseases. The fact that EUS is able to provide direct visualization of the walls of the gastrointestinal tract and direct the placement of needles for cytology or histology specimens 5,9, makes this technique very useful for the diagnosis of benign and malignant diseases of these organs that are difficult to reach percutaneouly. In recent years diagnostic modalities such as multidetector-multiphasic CT with pancreatic protocols, magenetic resonance imaging (MRI) and positron emission tomography (PET) scans have improved significantly the pre-operative tumor staging of pancreatic malignancies. Nevertheless, a significant proportion of patients will benefit from EUS-FNA for confirmation of pancreatic neoplasm and assessment of the planes along the vascular superior mesenteric trunk ${ }^{10}$.

\section{Epidemiology of pancreatic neoplasms}

Pancreatic adenocarcinoma is the fourth leading cause of cancer related mortality in the United States with estimated 42,500 new cases and 35,000 deaths from the disease each year 11. In industrialized countries, the incidence of pancreatic adenocarcinoma (11 per 100,000 individuals) ranks second after colorectal cancer among all gastrointestinal malignancies ${ }^{12}$. More than $80 \%$ of PCs are diagnosed in patients older than 60 and almost $50 \%$ have distant metastases at the time of presentation ${ }^{13,14}$. Men are more frequently affected than women 
(Relative Risk $(\mathrm{RR})=1.3$ ) and individuals of African American descent in comparison to Caucasians $(R R=1.5){ }^{15}$. Analysis of overall survival shows that the prognosis of PC is still quite poor despite the fact that 1-year survival has increased from 15.2\% (period between 1977-1981) to $21.6 \%$ (period between 1997-2001) and 5-year survival has increased from $3 \%$ (period between 1977-1986) to 5\% (period between 1996-2004)16.

\section{Classification of pancreatic neoplasms}

The vast majority (90\%) of pancreatic cancers (PC) are malignant tumors originating from pancreatic ductal cells ${ }^{17}$. Anatomically, $78 \%$ of PCs are located in the head, and the remaining $22 \%$ are equally distributed in the body and in the tail 18 . The most common

\begin{tabular}{|c|c|}
\hline EPITHELIAL TUMORS & NON-EPITHELIAL TUMORS \\
\hline Benign Pancreatic Tumors & Endocrine Tumors \\
\hline Serous Cystoadenoma & Insulinoma (Incidence: 70-80\%) \\
\hline Mucinous Cystoadenoma & Gastrinoma (Incidence: 20-25\%) \\
\hline Intraductal Papillary Mucinous & \\
\hline Neoplasm (IPMN) & VIPoma (Incidence: 4\%) \\
\hline Mature Teratoma & Glucagonoma (Incidence: 4\%) \\
\hline Borderline Pancreatic Tumors & Somastatinoma (Incidence: $<5 \%$ ) \\
\hline Mucinous Cystic Neoplasm with & Carcinoid (Incidence: $<1 \%$ ) \\
\hline Moderate Dysplasia & \multirow[b]{2}{*}{ ACTHoma (Incidence: $<1 \%$ ) } \\
\hline $\begin{array}{l}\text { Intraductal Papillary Mucinous } \\
\text { Neoplasm with Moderate Dysplasia }\end{array}$ & \\
\hline $\begin{array}{l}\text { Solid-pseudopapillary Neoplasm } \\
\text { (SPPN) }\end{array}$ & GRFoma (Incidence: $<1 \%$ ) \\
\hline Malignant & PTH-like-oma (Incidence: <1\%) \\
\hline Ductal Adenocarcinoma & Neurotensinoma (Incidence: $<1 \%$ ) \\
\hline Mucinous non-cystic carcinoma & Non-functional tumors (Incidence $30-50 \%$ ) \\
\hline Signet ring cell carcinoma & Mesenchymal Neoplasms \\
\hline Undifferentiated (anaplastic) carcinoma & Leiomyoma \\
\hline $\begin{array}{l}\text { Undifferentiated carcinoma with } \\
\text { osteoclast-like giant cells }\end{array}$ & Lipoma \\
\hline Mixed ductal-endocrine carcinoma & Neurofibroma - Ganglioneuromas \\
\hline Serous Cystoadenocarcinoma & Hemangyoma - Lymphangioma \\
\hline Mucinous Cystoadenocarcinoma & Granular Cell Tumors \\
\hline Intraductal Papillary-Mucinous & Schwann Cell Tumors \\
\hline Carcinoma & schwann Cell I umors \\
\hline Acinar Cell Carcinoma & Gastrointestinal Stroma Tumors (GIST) \\
\hline Medullary Carcinoma & B-cell Lymphomas \\
\hline Acinar Cell Cystoadenocarcinoma & Metastatic Tumors \\
\hline Pancreatoblastoma & Renal Cell Carcinoma \\
\hline Solid-pseudopapillary Carcinoma & Melanoma \\
\hline Others & Breast Cancer \\
\hline & Squamous Cell Carcinoma \\
\hline Secondary Tumors & Endometrioid Adenocarcinoma \\
\hline & Osteosarcoma \\
\hline
\end{tabular}

Table 1. Tumor Classification 
clinical presentations are progressive weight loss and anorexia, mid abdominal pain and jaundice. Pancreatic neoplasms are classified in benign or malignant according to the cytological characteristics. These can be further divided into endocrine or exocrine tumors according to the function of their cells and into cystic or solid according to the macroscopic features of the lesion. Recent advances in surgical pathology techniques integrated with molecular biology have allowed advances in the modern classification of pancreatic neoplasms. A summary of the clinico-pathological features of the different categories of pancreatic lesions is shown in Table 1.

\section{Conventional diagnostic modalities for pancreatic cancer}

\subsection{Abdominal ultrasound}

Trans-abdominal ultrasound (US) is often used as a screening test ${ }^{19}$. Its sensitivity ranges between $48 \%{ }^{8}$ and $89 \%{ }^{20}$ with accuracy between $46 \%{ }^{8}$ and $64 \% 21$. Small tumors measuring less than $1 \mathrm{~cm}$ are detected by US in only $50 \%$ of cases while the sensitivity increases to $95.8 \%$ for tumors larger than $3 \mathrm{~cm}^{20}$. US has a relatively low performance profile for staging of pancreatic tumors as its sensitivity for lymph nodes involvement ranges only between 8 17 to $57 \%$ 20. Color Doppler US has been used to assess possible involvement of the portal vein and superior mesenteric vessels with a sensitivity ranging between $50 \% 22$ and $94 \% 23$ specificity between $80 \%$ and $100 \% 22$ and accuracy between $81 \%$ and $95 \% 19$.

\subsection{Computed Tomography}

On contrast CT, pancreatic adenocarcinoma appears as an ill-defined, hypo attenuating focal mass with dilatation of the upstream pancreatic and or biliary duct 24 . The optimum visualization of the pancreas requires imaging acquisition obtained during both arterial and portal phases 25 with sensitivity and specificity of $77 \%$ and $100 \%$ respectively for lesions less than $2 \mathrm{~cm}^{26}$. In a multicentric trial, the diagnostic accuracy of contrast CT for resectability was $73 \%$ with $90 \%$ PPV for non resectability 27 . With the advent of multi detector CT scanners (MDCT) the pancreas can be imaged at a very high spacial and temporal resolution ${ }^{28,29}$. Recent studies have shown that NPV for respectability was $87 \%$ for MDCT compared to $79 \%$ for conventional helical CT 30 and with accuracy between $85 \%$ and $95 \% 31,32$.

\subsection{Magnetic Resonance Imaging (MRI-MRCP)}

In most institutions, MRI is performed when other imaging modalities provide insufficient data for the clinical staging of the tumor or when treatment planning can not be based on the images obtained by other techniques. Several studies have shown that MRI is superior to CT scan for the detection and staging of pancreatic adenocarcinoma ( $100 \%$ vs. $94 \%$ respectively) $33-36$. The use of MRI-MRCP for pancreatic malignancies is supported by a prospective analysis showing that MRI-MRCP was superior to CT in differentiating malignant from benign lesions and MRI-MRCP had better sensitivity (92\% vs. 76\%), specificity $(85 \%$ vs. $69 \%)$, accuracy $(90 \%$ vs. $75 \%)$, PPV (95\% vs. $88 \%)$ and NPV $(79 \%$ vs. $50 \%$ ) compared to CT 37 .

\subsection{Positron Emission Tomography}

${ }^{18}$ F-2fluoro-2-deoxy-D-glucose (FDG) accumulated by tumor cells provides PET the advantage of combining metabolic activity and imaging characteristics. Newly developed PET scanners can detect small PC up to $7 \mathrm{~mm}$ in diameter and diagnose metastatic disease in 
about $40 \%$ of cases $^{38,39}$. A Japanese study found that the overall sensitivity of PET-CT was superior to contrast CT $(92 \%$ vs. $88 \%)$ and that PET was better at detecting bone metastases $(100 \%$ vs. $12 \%)$. However, CT scan was superior for the evaluation of vascular invasion $(100 \%$ vs. $22 \%)$, involvement of para-aortic regional lymph nodes (78\% vs. 57\%), identification of peritoneal dissemination $(57 \%$ vs. $42 \%)$ and hepatic metastases $(73 \%$ vs. $52 \%) 40$.

\subsection{Treatment of pancreatic neoplasms}

Solid tumors of the pancreas are typically associated with malignancy, whereas cystic tumors more often tend to be benign ${ }^{41}$. Due to the difficulties in differentiating benign from malignant lesions, resection is often indicated when patient's conditions and tumor stage allows it ${ }^{42}$. Surgical resection with negative margins is the only potential curative treatment for pancreatic malignancies but unfortunately, even when surgery is performed successfully, recurrent disease is frequent and long term survival is expected only for $5-15 \%$ of patients ${ }^{17}$. According to the United States Surveillance and Epidemiology End Results registries, the 5-year relative survival for the period between 1999 and 2006 was $22.5 \%$ for localized and $1.9 \%$ for metastatic tumors ${ }^{43}$. The majority of tumors are diagnosed when locally advanced or with early metastases, and only $20 \%$ are suitable for resection at the time of diagnosis. Despite the improvements in surgical techniques and advances in perioperative supportive care that have reduced the mortality rates to less than $5 \%$ in high volume centers, pancreatic surgery remains challenging $44-46$. Therefore, pre-operative accurate staging is fundamental in identifying patients who would benefit from surgery. EUS has been shown to play an important role in preoperative diagnosis and tumor staging as it provides high resolution images of the pancreas without interference of bowel gas 47 .

\subsection{EUS equipment and techniques}

EUS is usually performed with patients positioned in the left dcubitus and under conscious sedation. The transducer located in the tip of the oblique-viewing fiberscope is inserted as far as the second portion of the duodenum, and scanning is done with a de-areated water filled ballon applied to the tip of the echoendoscope. After examination of the pancreatic head, the ecoendoscope is drawn backward to the stomach, and EUS of the body and tail of the pancreas is performed. The frequency usually used to assess the pancreas and surrounding organs during EUS ranges between 5 and $12 \mathrm{MHz}{ }^{48}$. During the last decade, intraductal US (IDUS) has been possible by the introduction of miniprobes measuring 1.7$2.4 \mathrm{~mm}$ in outer diameter that can be advanced in the common bile and pancreatic ducts utilizing scanning frequencies ranging between $10-30 \mathrm{MHz}$ and obtaining a maximum tissue view penetration of approximately $2 \mathrm{~cm} \mathrm{1,48}$. The miniprobe is initially introduced into the papilla of Vater and advanced into the pancreatic or bile duct beyond the area of interest and then it is slowly pulled back. The location of the miniprobe can be confirmed by using fluoroscopy as it is usually done during regular endoscopic retrograde cholangiopancreatography (ERCP). IDUS is able to visualize only limited parts of the pancreas and surrounding structure such as the splenic vessels, portal vein, superior mesenteric artery and vein and extrahepatic duct.

\subsection{Indications for Endoscopic Ultrasound and Fine Needle Aspiration (EUS-FNA)}

The most common indication for EUS-FNA of the pancreas is for evaluation of pancreatic masses with atypical characterisitics on cross sectional images or for optimal pre-operative 
staging (Table 2). Differential diagnosis of pancreatic masses includes malignant and benign neoplasms, chronic pancreatitis, lymphoma and metastases. Approximately $90 \%$ of pancreatic neoplasms are adenocarcinomas, 5\% are cystic lesions, and $2-5 \%$ are neuroendocrine tumors. Metastatic lesions to the pancreas, primarily from renal cancer, lung cancer, and lymphomas represent a small percentage. Because cystadenocarcinomas ${ }^{49}$ and neuroendocrine tumors ${ }^{50}$ have a significantly better prognoses than pancreatic adenocarcinoma, accurate cytologic preoperative identification can significantly alter the subsequent management of these patients ${ }^{51}$. In general, EUS has been shown to be superior to CT, MRI, and ERCP 52 in the diagnosis of pancreatic diseases as an imaging modality $2,5,53,54$. The current sensitivity of EUS is in the range of $95-100 \%$. During the last decades, the diagnostic advantages of EUS for pancreatic pathology have been challenged by the advances of other cross sectional modalities such as CT, MRI and PET scans 55 56. When combined with FNA capabilities, EUS has the advantage of being able to sample suspicious lesions. The one area where malignancies can be still easily missed by EUS, even with EUSFNA, is in the setting of underlying chronic pancreatitis $4,53,57-59$. No single or combination of imaging modalities has yet proven accurate in definitively determining when a patient with chronic pancreatitis has developed pancreatic cancer. The technique of EUS-guided FNA involves passing an 18 to 22 gauge metal needle through the biopsy port of a linear echoendoscope under real-time guidance into an endosonographically visualized pancreatic mass. The needle is then moved back and forth several times (5-10 passes) with varying degrees of negative pressure to collect cells or small tissue samples that are then deposited on cytology slides for immediate fixation and staining ${ }^{60}$. EUS-FNA of primary pancreatic malignancies is able to provide a definitive diagnosis in $80-93 \%$ of cases $8,54,57,61-63$. The ability to have a cytopathologist on site who can provide immediate feedback on the quality and adequacy of the specimens obtained by FNA is extremely important for the accurate diagnosis ${ }^{62,63}$. Choosing what part of a pancreatic mass to aspirate is something of an art and comes with experience. The most difficult pancreatic masses to aspirate are the ones located near the uncinate process as it can be very hard to direct the needle to enter the lesion around the second and third portion of the duodenum. The best yield of diagnostic cells usually seems to come from $1 \mathrm{~cm}$ to $2 \mathrm{~cm}$ deep to the margin of the tumor. Color flow Doppler can be used prior to EUS-FNA to help avoid vessels overlying the proposed path of the aspiration needle such as are seen when there is underlying portal vein or splenic vein obstruction. Similarly to CT and ultrasound-guided FNA or biopsy, the overall complication rate secondary to EUS-FNA of the pancreas is about 1-2\% 6,8,58,61,64. The major complications reported with EUS-FNA are bleeding, pancreatitis, and infection but mortality is very rare

\section{Indications for EUS}

Acute onset of diabetes in elderly patients

Involuntary weight loss

Presence of epigastric or back pain

Acute or chronic pancreatitis

Suspected pancreatic cancer on other cross sectional imaging modalities

Family history of pancreatic cancer or presence of genetic predisposition to pancreatic cancer

Table 2. Common Indications for Endoscopic Ultrasound 
and usually caused by uncontrollable hemorrhage 65 that is more likely when the patient has portal hypertension. Pancreatitis after EUS-FNA is most likely to occur in patients already being evaluated for recurrent pancreatitis and when the FNA needle is passed through more than $2 \mathrm{~cm}$ to $3 \mathrm{~cm}$ of normal pancreas to obtain a specimen. Bacteremia following EUS-FNA for solid tumors is quite uncommon while EUS-FNA of cystic pancreatic lesions has a higher risk of infectious complications and broad-spectrum intravenous antibiotics are routinely recommended. The risk of cancer seeding by EUS-FNA appears to be significantly lower when compared to percutaneous FNA ${ }^{6}$.

\subsection{EUS-FNA for benign pancreatic lesions}

Indications and impact of EUS-FNA for benign disease other than pancreatic cystic lesions is still in evolution as it appears to be safe but does not add significantly to the diagnostic accuracy of EUS or other cross sectional imaging tests 67 .

\subsection{Ultrasonographic characteristics}

Pancreatic adenocarcinoma often appears as a mass with irregular echogenicity due to the irregulary arranged carcinomatous canaliculi or coagulative necrosis of the neoplastic cells superimposed on a hypoechoic background ${ }^{6}$. Small pancreatic cancers instead, can often have homogeneous and hypoechoic echogeneicity that can mimic benign diseases such as focal pancreatitis, pseudotumors or islet cell neoplasms that have clear margins, smooth contour and regular central echogenicity ${ }^{48}$. When compared to CT scan and ERCP, EUS performs better for the detection of small pancreatic cancers (less than $2 \mathrm{~cm}$ in diameter) and it is currently indicated when patients are suspected to have early stage tumors or when undergoing screening for familial pancreatic cancer as it is the most sensitive diagnostic test for lesions measuring less than $1 \mathrm{~cm}$ in diameter 69,70 .

\section{Differential diagnosis of pancreatic lesions}

Differentiation between pancreatic malignancies from inflammatory masses has been very challenging with the use of cross sectional imaging modalities such as US, CT scans, MRI and ERCP. EUS-FNA appears to be the best diagnostic strategy as it combines the ability of ultrasound imaging of the pancreatic lesions and the ability of obtaining samples for cytological or histological evaluation. Recent studies have reported that adequate specimen acquisition is possible in $97 \%$ of cases with accurate differential diagnosis in $87 \%$ of patients 48. Sensitivity, specificity, positive predictive value and negative predictive value for EUSFNA were: $85 \%, 100 \%, 100 \%$ and $53 \%$ respectively 48 (Table 3). One of the major pitfalls is over interpretation of a lesion as positive for malignancy as a result of contamination of dysplastic cells when the needle traverses an area of high-grade dysplasia of the gastrointestinal tract mucosa. It is equally important that benign mucosal glandular cells in the aspirate of the lymph node not be over interpreted as metastasis. Although EUS-FNA is a very useful diagnostic technique for the differential diagnosis of patients with pancreatic lesions, it has to be kept in mind that even if the results of the test are negative for malignancy, pancreatic cancer can not be completely excluded. Currently, EUS-FNA should be still used in conjuction with other imaging modalities and repeated when clinical suspicion is suggestive for the possibility of malignancy (Table 4). 


\begin{tabular}{lccc}
\hline Diagnostic Performance & CT & EUS & EUS-FNA \\
\hline \hline Sensitivity & $63-84 \%$ & $95-100 \%$ & $79-95 \%$ \\
Specificity & $35-93 \%$ & $19-81 \%$ & $69-100 \%$ \\
Negative Predictive Value & $12-49 \%$ & $48-100 \%$ & $31-78 \%$ \\
Positive Predictive Value & $89-99 \%$ & $85-98 \%$ & $94-100 \%$ \\
Accuracy & $70-88 \%$ & $97-98 \%$ & $81-96 \%$ \\
\hline \hline
\end{tabular}

Table 3. Diagnsostic Performance of Endoscopic Ultrasound and Computerized Tomography for Solid Pancreatic Cancers

\begin{tabular}{|c|c|c|c|c|}
\hline & & CT & EUS & EUS-FNA \\
\hline \multirow{4}{*}{ 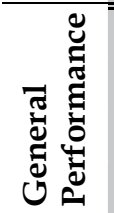 } & Sensitivity & 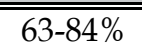 & $995-100 \%$ & $\overline{779-95 \%}$ \\
\hline & Negative Predictive Value & $12-49 \%$ & $48-100 \%$ & $31-78 \%$ \\
\hline & Positive Predictive Value & $89-99 \%$ & $85-98 \%$ & $94-100 \%$ \\
\hline & Accuracy & $70-88 \%$ & $97-98 \%$ & $81-96 \%$ \\
\hline \multirow{5}{*}{ 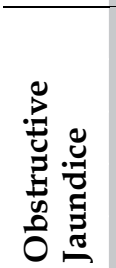 } & Sensitivity & $51-80 \%$ & $92-100 \%$ & $71-94 \%$ \\
\hline & Specificity & $16-100 \%$ & $1-99 \%$ & $16-100 \%$ \\
\hline & Negative Predictive Value & $1-36 \%$ & $25-100 \%$ & $3-60 \%$ \\
\hline & Positive Predictive Value & $88-100 \%$ & $88-100 \%$ & $91-100 \%$ \\
\hline & Accuracy & $53-81 \%$ & $89-100 \%$ & $72-94 \%$ \\
\hline \multirow{5}{*}{ 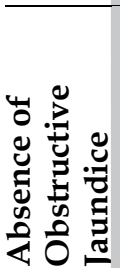 } & Sensitivity & $70-98 \%$ & $87-100 \%$ & $80-100 \%$ \\
\hline & Specificity & $24-91 \%$ & $16-84 \%$ & $63-100 \%$ \\
\hline & Negative Predictive Value & $24-91 \%$ & $40-100 \%$ & $52-100 \%$ \\
\hline & Positive Predictive Value & $70-98 \%$ & $69-96 \%$ & $86-100 \%$ \\
\hline & Accuracy & $65-93 \%$ & $73-97 \%$ & $85-100 \%$ \\
\hline
\end{tabular}

Table 4. Diagnostic Performance of Endoscopic Ultrasound with and without Fine Needle Aspiration in Comparison to Computerized Tomography for Pancreatic Cancers in the Presence and Absence of Obstructive Jaundice

\subsection{Cancer staging}

The accuracy of EUS for the stage of patients with pancreatic cancer is superior to US and CT scans with values ranging between 85 and $100 \%$ in comparison to $64-66 \%$ for CT and 61$64 \%$ for US 55,71. The EUS accuracy in staging pancreatic cancer does not depend on the use of radial or linear scanners 65 . Radial scanners offer a better overview of surrounding structures, whereas linear scanners allow the safe execution of tissue sampling. Initial studies showed excellent accuracy up to $94 \%$, but later publication reported lower values ranging between $63 \%$ and $78 \% 55,72-76$. Overall, EUS-FNA is highly sensitive (84\%), specific $(97 \%)$, accurate $(84 \%)$ and has a high positive predictive value $(99 \%)$, but relatively low negative predictive value $(64 \%)^{77}$ (Table 5). A major problem in staging pancreatic cancer is the prediction of resectability as the best chance for long-term survival occurs in patients with localized disease undergoing resection. The primary goal of surgical therapy is to achieve a margin-negative $\mathrm{R} 0$ resection with minimal postoperative complications and a secondary important goal is to avoid unnecessary laparotomies for unresectable tumors ${ }^{78}$. 
Combining the pre-operative utilization of CT and EUS proved to be the method with the highest accuracy compared to each single technique to predict tumor resectability ${ }^{79}$.

In this context, a preoperative assessment of $\mathrm{R} 0$ resectability becomes critically important. Radiologic staging with EUS and CT or MRI is currently used to identify patients who may be resectable. The criteria of unresectability of pancreatic cancer include evidence of distant metastasis, tumor enchroachment (defined as tumor surrounding the vessel more than 180 degrees) of arteries such as the celiac artery, hepatic artery, superior mesenteric artery (SMA) or massive venous invasion with thrombosis. Portal or superior mesenteric venous invasion without thrombosis or obliteration of vessels can still be classified as resectable tumors. A recent study comparing the roles of EUS, CT, MRI and angiography in the assessment of pancreatic cancer staging and respectability, has shown that CT scan was the most accurate in assessing the stage of the tumor (73\%), locoregional invasion (74\%), vascular involvement $(83 \%)$, distant metastases (88\%), final TNM stage (46\%) and overall tumor resectablity $(83 \%)^{76}$. Although EUS appeared to be superior to detect smaller tumors not visualized by CT scan it is important to recognize that most surgeons would probably not rely on EUS alone before making important therapeutic decisions about surgical resections ${ }^{80}$.

\begin{tabular}{l|cc|cc|}
\cline { 2 - 5 } \multicolumn{2}{c|}{} & \multicolumn{2}{c|}{ No Discrete Mass on CT } & \multicolumn{2}{c|}{ Discrete Mass on CT Scan } \\
\hline \hline Sensitivity & EUS & EUS-FNA & EUS & EUS-FNA \\
Specificity & $82-100 \%$ & $65-99 \%$ & $93-100 \%$ & $77-96 \%$ \\
Negative Predictive Value & $29-96 \%$ & $59-100 \%$ & $0-71 \%$ & $29-100 \%$ \\
Positive Predictive Value & $48-100 \%$ & $40-97 \%$ & na & $7-70 \%$ \\
Accuracy & $68-99 \%$ & $79-100 \%$ & $85-99 \%$ & $92-100 \%$ \\
\hline \hline
\end{tabular}

Table 5. Diagnostic Value of Performing EUS-FNA Along with Spiral CT in Patients with Suspected Pancreatic Cancer

\subsection{Conclusions}

EUS-FNA has greatly impacted the diagnostic management of patients affected by pancreatic masses in conjunction with other cross sectional imaging tests. EUS is the best

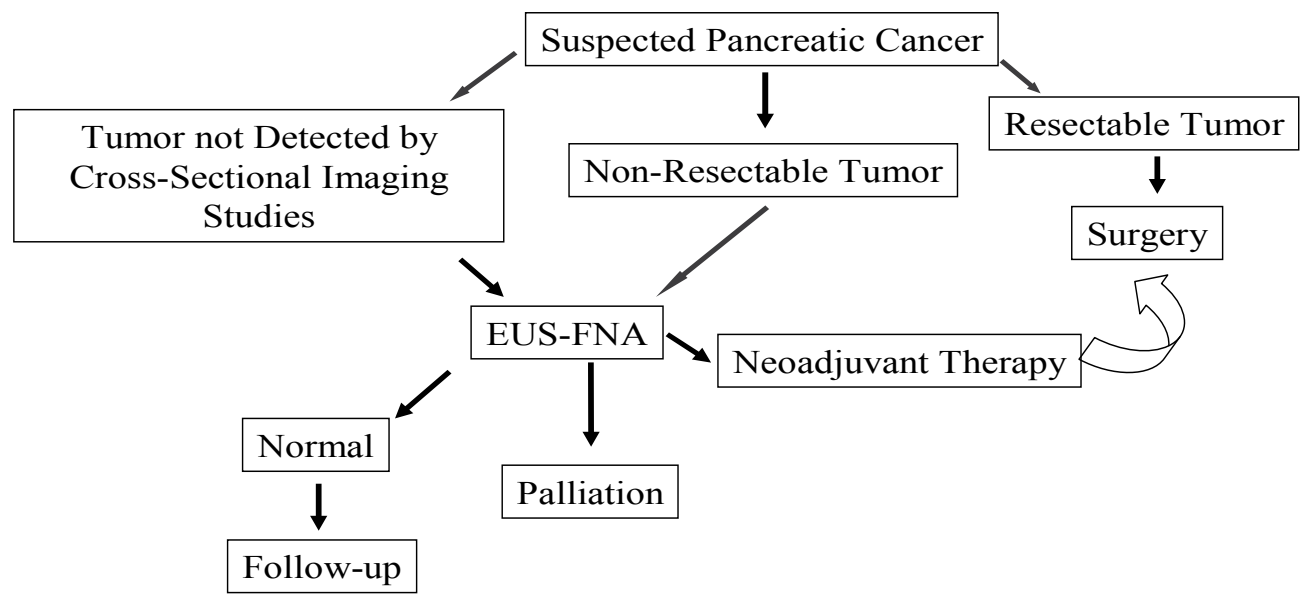

Fig. 1. Flow Chart for the Evaluation and Management of Patients with Suspected Pancreatic Malignancies 
method for tissue diagnosis and it is extremely useful for the management of patients who are not surgical candidates and who require neo-adjuvant or palliative chemoradiation therapy. Conversely, pre-operative tissue diagnosis is not indispensable for resectable tumors and therefore EUS plays a lesser role in this group of patients. In recent years, EUS has assumed an important interventional role in the management of patients who are affected by debilitating pancreatic cancer pain as it is extremely useful in obtaining celiac plexus block for long term analgesic effects. Current role of EUS and EUS-FNA is summarized in Figure 1.

\section{Pancreatic cystic lesions}

Pancreatic cystic lesions (PCLs) are commonly identified due to increased use of crosssectional imaging in patients with non-specific abdominal complaints. Although their exact prevalence is unknown, it is estimated to range from $1 \%$ to $2.6 \%$ of the general population based on large scale observational imaging studies using MDCT81,82. Pancreatic cystic lesions are strongly associated with increasing age and Asian race ${ }^{82}$. Their relative frequency varies substantially geographically and by institution (primary vs. secondary) due to different practices and patient populations ${ }^{83}$. In addition, the prevalence of PCLs including intraductal papillary mucinous neoplasms (IPMNs) was found to be higher among patients on hemodialysis compared to the normal population ${ }^{84}$.

Inflammatory pseudocysts represent about $75 \%$ of pancreatic cysts; however they are not classified as true PCLs since they are non-epithelial inflammatory fluid collections associated with acute or chronic pancreatitis ${ }^{85}$. Based on surgical pathology, PCLs are classified according to the characteristics of the cells lining the cyst in benign, premalignant and malignant 86 (Table 6).

Serous cystadenomas (SCAs), mucinous cystadenocarcinomas (MCAs) and IPMNs represent $90 \%$ of PCLs and only the mucin producing neoplasms have been described to have risk of malignancy ${ }^{87}$. In a small percentage of patients, solid malignant tumors such as adenocarcinoma, neuroendocrine tumors and other less common causes can present with cystic degeneration 88 .

\subsection{Limitations of radiological imaging modalities for the diagnosis of PCLs}

Trans-abdominal ultrasound (US) is a safe and cheap diagnostic modality that can be used to diagnose PCLs, however, its sensitivity and specificity are often affected by the operator's experience and the technical characteristics of the machine ${ }^{89}$. In addition, the deep position of the pancreas and the interposition of other organs such as the stomach and the transverse colon limit the capacity of US to identify small PCLs. The overall sensitivity of US in detecting pancreatic lesions ranges from $48 \% 8$ to $89 \% 20$.

The majority of studies describing the role of CT and MRI in the diagnosis of PCLs have been small and retrospective. Relying on radiological characteristics alone can be misleading, with up to $40 \%$ of serous and mucinous lesions being misdiagnosed as pseudocysts 85,90 . Reported overall diagnostic accuracy for these lesions has been highly variable ranging between $20 \%$ and $83 \%$ 91-93. (Table 7 )

In a large group of patients, accurate preoperative diagnosis of PCLs was reported in $20 \%$ for serous cystadenoma, $30 \%$ for mucinous cystadenoma and $29 \%$ for mucinous cystadenocarcinoma, as the majority of these lesions were misdiagnosed as pseudocysts 92 . MRI is equal or slightly superior to CT in diagnosing PCLs except in its limited ability to demonstrate calcifications in the tumor wall or septa ${ }^{94}$. 
Pseudocysts (no epithelial lining)

Conventional pseudocysts

Paraduodenal wall cyst (cystic dystrophy)

Infection-related pseudocysts

Cysts with mucinous epithelium

Intraductal papillary mucinous neoplasms

Intraductal oncocytic papillary neoplasms

Mucinous cystic adenoma

Mucinous cystic adenocarcinoma

Mucocele

Retention cysts

Serous (clear-cell) cystic tumors

Serous cystadenoma

VHL-associated pancreatic cysts

Serous cystadenocarcinomas (Extremely rare, case reports)

Squamous-lined cysts

Lymphoepithelial cysts

Epidermoid cysts within intrapancreatic accessory spleen

Dermoid cysts

Squamoid cyst of pancreatic ducts

Cysts lined by acinar cells

Acinar cell cystadenocarcinomas

Acinar cell cystadenomas (cystic acinar transformation)

Endothelial-lined cysts

Lymphangiomas

Degenerative or necrotic changes in solid tumors

Solid-pseudopapillary tumor

Cystic change in ordinary ductal adenocarcinoma

Cystic pancreatic endocrine neoplasia (islet cell tumors)

Cystic mesenchymal neoplasms

Other rare cystic lesions

Cystic hamartomas

Endometriotic cyst

Metastatic cystic neoplasms

Congenital or developmental cysts

Others

VHL $=$ Von Hippel-Lindau

Table 6. Pancreatic cystic lesions classified by cells lining the cavity 
For IPMN, magnetic resonance cholangiopancreatography (MRCP) seems to be superior to endoscopic retrograde cholangiopancreatography (ERCP) in detecting cysts communicating with the main pancreatic duct ${ }^{95}$.

\subsection{Endoscopic ultrasonography}

Endoscopic ultrasound (EUS) allows high resolution imaging of the pancreas with the ability to provide fine morphological details. The combination of fine-needle aspiration (FNA) cytology with the other recently available diagnostic markers has further increased its diagnostic accuracy ${ }^{96}$. Indications for EUS-FNA should be considered based on diagnostic accuracy of alternative modalities, costs, patient comfort and safety 97 and should be performed only when the information obtained has the potential to alter patient's management 98 . EUS-FNA should not be performed when there is inability to clearly visualize the target lesion, presence of large vessels interposed in the path between the needle and the lesion, bleeding diathesis and for patients at high risk of tumor seeding 98 such as those with cholangiocarcinoma who are considered for liver transplantation ${ }^{99}$. Once a cystic lesion is identified, the main clinical issue is the characterization and eventual therapeutic approach. Diagnostic accuracy of EUS imaging alone for differentiating malignant versus premalignant or benign lesions is ranging between $82 \%$ and $96 \% 100-103$.

The endosonographic features suggestive of malignancy are: wall thickness of $3 \mathrm{~mm}$ or greater, macroseptations with cystic compartments greater than $10 \mathrm{~mm}$, presence of a mass or intramural growth or cystic dilation of the main pancreatic duct. These features have a sensitivity of $91 \%$, a specificity of $60 \%$ and an accuracy of $72 \%$ in predicting malignant or potentially malignant PCLs 104 . The sensitivity, specificity, positive predictive value (PPV), negative predictive value (NPV) and accuracy of EUS in comparison to other imaging modalities is summarized in Table 7

Despite EUS alone is a very sensitive test, EUS-guided fine needle aspiration (FNA) provides additional information for the characterization of PCLs. In a study by Frossard et al., the results of EUS and EUS guided FNA were compared with the final surgical pathology report. The sensitivity, specificity, PPV and NPV of EUS-guided FNA in this study were $97 \%, 100 \%, 100 \%$ and $95 \%$ respectively 102 . On the other hand, corresponding values for EUS imaging alone were $71 \%, 30 \%, 49 \%$ and $40 \%$ respectively 102 . Yet, the overall sensitivity and accuracy of EUS-FNA cytology remains widely variable with average sensitivity and accuracy around 50\% 62,103,112,113.

\subsection{Cytology}

The aspirated cyst fluid is generally analyzed for tumor markers, chemical and molecular analysis. Solid component associated with PCLs or regional lymph nodes can be aspirated for cytology or histology. EUS guided FNA is safe and rare complications include pancreatitis $(2-3 \%)$, intracystic hemorrhage $(<1 \%)$ and infection $(<1 \%)$. The administration of antibiotic during the procedure is a common practice even if there are no data to support it 114. Tumor cell seeding has been a significant concern; there is limited evidence on the actual rise of tumor spread by EUS-FNA 115. Recent study has shown that EUS-FNA has a decreased risk of peritoneal tumor spread as compared with computed tomography-guided FNA $(2.2 \%$ vs. $16.3 \%){ }^{66}$. During EUS-FNA, the operator should always avoid to insert the needle through malignant tissue to reach a suspicious lesion. An example of this may be a patient with gastric or esophageal cancer and a suspicious lymph node adjacent to the primary tumor as the needle would have tumor entrapped into the channel that could contaminate the final results. 


\begin{tabular}{|c|c|c|c|c|c|c|c|}
\hline $\begin{array}{l}\text { Diagnostic } \\
\text { modality }\end{array}$ & Author & Year & $\begin{array}{c}\text { Sensitivity } \\
(\%)\end{array}$ & $\begin{array}{c}\text { Specificity } \\
(\%)\end{array}$ & $\begin{array}{l}\text { PPV } \\
(\%)\end{array}$ & $\begin{array}{l}\text { NPV } \\
(\%)\end{array}$ & $\begin{array}{c}\text { Accuracy } \\
(\%)\end{array}$ \\
\hline \multirow{6}{*}{ US } & Giovanni et als & 1994 & \multirow{6}{*}{$48-95$} & \multirow{6}{*}{$40-91$} & \multirow{6}{*}{92} & \multirow{6}{*}{100} & \multirow{6}{*}{$46-64$} \\
\hline & Bottger et al 20 & 1998 & & & & & \\
\hline & Rosch et al ${ }^{3}$ & 1991 & & & & & \\
\hline & Niederau et al105 & 1992 & & & & & \\
\hline & Palazzo et al 21 & 1993 & & & & & \\
\hline & Tanaka et al106 & 1996 & & & & & \\
\hline \multirow{3}{*}{ Doppler US } & Candiani et al107 & 1998 & \multirow{3}{*}{$50-94$} & \multirow{3}{*}{$80-100$} & \multirow{3}{*}{79} & \multirow{3}{*}{88} & \multirow{3}{*}{$81-95$} \\
\hline & Casadei et al 23 & 1998 & & & & & \\
\hline & calculli et al 108 & 2002 & & & & & \\
\hline \multirow{3}{*}{ EUS } & Akahoshi et al 72 & 1998 & \multirow{3}{*}{98} & \multirow{3}{*}{97} & \multirow{3}{*}{94} & \multirow{3}{*}{100} & \multirow{3}{*}{90} \\
\hline & Sedlack et al104 & 2002 & & & & & \\
\hline & Legmann et al55 & 1998 & & & & & \\
\hline $\begin{array}{l}\text { Contrast } \\
\text { enhanced US }\end{array}$ & Dietrich et al 109 & 2008 & 90 & 100 & 100 & 86 & 93 \\
\hline \multirow{2}{*}{ CT } & Bronstein et al 26 & 2004 & \multirow{2}{*}{77} & \multirow{2}{*}{100} & \multirow{2}{*}{ na } & \multirow{2}{*}{ na } & \multirow{2}{*}{73} \\
\hline & Megibow et al 27 & 1995 & & & & & \\
\hline \multirow{4}{*}{ MDCT } & Park et al110 & 2009 & \multirow{4}{*}{$83-91$} & \multirow{4}{*}{$63-75$} & \multirow{4}{*}{80} & \multirow{4}{*}{87} & \multirow{4}{*}{$85-95$} \\
\hline & Vargas et al30 & 2004 & & & & & \\
\hline & Diehl et al ${ }^{31}$ & 1998 & & & & & \\
\hline & Schima et al 33 & 2002 & & & & & \\
\hline MRI-MRCP & Anderssonet al 37 & 2005 & $83-92$ & $63-85$ & 95 & 79 & 89 \\
\hline \multirow{2}{*}{ PET } & Maemura et al38 & 2006 & \multirow{2}{*}{$87-100$} & \multirow{2}{*}{$67-77$} & \multirow{2}{*}{94} & 100 & 5-95 \\
\hline & Delbeke et al111 & 1999 & & & & 100 & $03-93$ \\
\hline
\end{tabular}

Abbreviations: Ultrasound (US), Endoscopic ultrasound (EUS), Computed tomography (CT), Multi detector computed tomography (MDCT), Positron emission tomography (PET)

Table 7. Summary of the performance characteristics of imaging tests for the diagnosis of pancreatic cancer

\subsection{Cystic fluid markers}

Several markers can be measured in the fluid aspirated from the pancreatic lesions during EUS to differentiate mucinous from non mucinous cysts. The most commonly used are; CEA, carbohydrate antigen (CA) 19-9, CA 72-4, and CA 15-3 116. CEA appears be the most useful as levels higher than $192 \mathrm{ng} / \mathrm{ml}$ had an accuracy of $79 \%$ for mucinous lesion characterization and was superior to cytology and EUS morphology 103.

Other markers such as amylase and lipase are important in the evaluation of cystic pancreatic lesions. Amylase is usually elevated in inflammatory cysts like pseudocysts but also in IPMN due to communication between the cystic lesion and the pancreatic duct. Amylase level less than $250 \mathrm{U} / \mathrm{L}$ favors the diagnosis of benign or malignant cystic neoplasms versus pancreatic pseudocysts (sensitivity $44 \%$, specificity $98 \%$ ) 117 . 
Molecular markers are recently considered a more reliable alternative. A multicenter study on pancreatic cyst fluid DNA analysis demonstrated a strong association of mucinous cystic neoplasms with K-ras mutations occurring with other loss of heterozygosity ( $\mathrm{LOH}$ ) mutations ${ }^{118}$. Shen et al ${ }^{119}$ assessed the correlation between this molecular diagnosis with a clinical consensus diagnosis for PCLs defined by histology, malignant cytology, or two concordant tests (such as EUS, cytology, or CEA>_192 ng/ml for mucinous cysts). The study showed that the two diagnostic methods correlated well and molecular analysis of pancreatic cyst fluid added diagnostic value to the preoperative diagnosis.

\section{Conclusion}

Pancreatic cystic lesions are detected more frequently than in the past due to more sensitive imaging modalities. The differentiation between benign and malignant cystic lesions is often challenging. EUS and EUS-FNA have become a leading modality for the differential diagnosis of these lesions as it provides imaging characteristics and the possibility of obtaining cytology or fluid samples with high sensitivity and specificity. Characterization of cystic morphology by other imaging studies should be supplemented by EUS-FNA as cytology, tumor markers and DNA analysis can further characterize these lesions and increase the diagnostic accuracy of premalignant and malignant cysts.

\section{Summary}

Despite the advancement of other cross sectional imaging tests, EUS appears to have a higher sensitivity in detecting small pancreatic neoplasms in comparison to CT. On the other hand EUS does not appear to be accurate enough in assessing the invasion of SMA and SMV and respectability of locally advanced tumors. Recent studies have shown improved diagnostic performance of EUS with the use of parenteral contrast agents and EUS-FNA plays a key role when. tissue diagnosis is needed.

\section{References}

[1] DiMagno EP, Buxton JL, Regan PT, et al. Ultrasonic endoscope. Lancet 1980;1:629-31.

[2] Yasuda K, Mukai H, Cho E, Nakajima M, Kawai K. The use of endoscopic ultrasonography in the diagnosis and staging of carcinoma of the papilla of Vater. Endoscopy 1988;20 Suppl 1:218-22.

[3] Rosch T, Lorenz R, Braig C, et al. Endoscopic ultrasound in pancreatic tumor diagnosis. Gastrointest Endosc 1991;37:347-52.

[4] Vilmann P, Hancke S. [Endoscopic ultrasound scanning of the upper gastrointestinal tract. Preliminary results]. Ugeskr Laeger 1991;153:422-5.

[5] Bhutani MS, Hawes RH, Baron PL, et al. Endoscopic ultrasound guided fine needle aspiration of malignant pancreatic lesions. Endoscopy 1997;29:854-8.

[6] Chang KJ, Katz KD, Durbin TE, et al. Endoscopic ultrasound-guided fine-needle aspiration. Gastrointest Endosc 1994;40:694-9.

[7] Gress FG, Savides TJ, Sandler A, et al. Endoscopic ultrasonography, fine-needle aspiration biopsy guided by endoscopic ultrasonography, and computed tomography in the preoperative staging of non-small-cell lung cancer: a comparison study. Ann Intern Med 1997;127:604-12. 
[8] Giovannini M, Seitz JF. Endoscopic ultrasonography with a linear-type echoendoscope in the evaluation of 94 patients with pancreatobiliary disease. Endoscopy 1994;26:579-85.

[9] Chang KJ, Nguyen P, Erickson RA, Durbin TE, Katz KD. The clinical utility of endoscopic ultrasound-guided fine-needle aspiration in the diagnosis and staging of pancreatic carcinoma. Gastrointest Endosc 1997;45:387-93.

[10] Santo E. Pancreatic cancer imaging: which method? JOP 2004;5:253-7.

[11] Jemal A, Siegel R, Ward E, Hao Y, Xu J, Thun MJ. Cancer statistics, 2009. CA Cancer J Clin 2009;59:225-49.

[12] Lynch SM, Vrieling A, Lubin JH, et al. Cigarette smoking and pancreatic cancer: a pooled analysis from the pancreatic cancer cohort consortium. Am J Epidemiol 2009;170:403-13.

[13] Hassan MM, Bondy ML, Wolff RA, et al. Risk factors for pancreatic cancer: case-control study. Am J Gastroenterol 2007;102:2696-707.

[14] Iodice S, Gandini S, Maisonneuve P, Lowenfels AB. Tobacco and the risk of pancreatic cancer: a review and meta-analysis. Langenbecks Arch Surg 2008;393:535-45.

[15] Permert J, Ihse I, Jorfeldt L, von Schenck H, Arnqvist HJ, Larsson J. Pancreatic cancer is associated with impaired glucose metabolism. Eur J Surg 1993;159:101-7.

[16] Shaib YH, Davila JA, El-Serag HB. The epidemiology of pancreatic cancer in the United States: changes below the surface. Aliment Pharmacol Ther 2006;24:87-94.

[17] Sharma C, Eltawil KM, Renfrew PD, Walsh MJ, Molinari M. Advances in diagnosis, treatment and palliation of pancreatic carcinoma: 1990-2010. World J Gastroenterol 2011;17:867-97.

[18] Sener SF, Fremgen A, Menck HR, Winchester DP. Pancreatic cancer: a report of treatment and survival trends for 100,313 patients diagnosed from 1985-1995, using the National Cancer Database. J Am Coll Surg 1999;189:1-7.

[19] Gandolfi L, Torresan F, Solmi L, Puccetti A. The role of ultrasound in biliary and pancreatic diseases. Eur J Ultrasound 2003;16:141-59.

[20] Bottger TC, Boddin J, Duber C, Heintz A, Kuchle R, Junginger T. Diagnosing and staging of pancreatic carcinoma-what is necessary? Oncology 1998;55:122-9.

[21] Palazzo L, Roseau G, Gayet B, et al. Endoscopic ultrasonography in the diagnosis and staging of pancreatic adenocarcinoma. Results of a prospective study with comparison to ultrasonography and CT scan. Endoscopy 1993;25:143-50.

[22] Baarir N, Amouyal G, Faintuch JM, Houry S, Huguier M. [Comparison of color Doppler ultrasonography and endoscopic ultrasonography for preoperative evaluation of the mesenteric-portal axis in pancreatic lesions]. Chirurgie 1998;123:445-9.

[23] Casadei R, Ghigi G, Gullo L, et al. Role of color Doppler ultrasonography in the preoperative staging of pancreatic cancer. Pancreas 1998;16:26-30.

[24] Tamm EP, Silverman PM, Charnsangavej C, Evans DB. Diagnosis, staging, and surveillance of pancreatic cancer. AJR Am J Roentgenol 2003;180:1311-23.

[25] Choi BI, Chung MJ, Han JK, Han MC, Yoon YB. Detection of pancreatic adenocarcinoma: relative value of arterial and late phases of spiral CT. Abdom Imaging 1997;22:199-203.

[26] Bronstein YL, Loyer EM, Kaur H, et al. Detection of small pancreatic tumors with multiphasic helical CT. AJR Am J Roentgenol 2004;182:619-23. 
[27] Megibow AJ, Zhou XH, Rotterdam H, et al. Pancreatic adenocarcinoma: CT versus MR imaging in the evaluation of resectability--report of the Radiology Diagnostic Oncology Group. Radiology 1995;195:327-32.

[28] Gangi S, Fletcher JG, Nathan MA, et al. Time interval between abnormalities seen on CT and the clinical diagnosis of pancreatic cancer: retrospective review of CT scans obtained before diagnosis. AJR Am J Roentgenol 2004;182:897-903.

[29] Ohwada S, Ogawa T, Tanahashi Y, et al. Fibrin glue sandwich prevents pancreatic fistula following distal pancreatectomy. World J Surg 1998;22:494-8.

[30] Vargas R, Nino-Murcia M, Trueblood W, Jeffrey RB, Jr. MDCT in Pancreatic adenocarcinoma: prediction of vascular invasion and resectability using a multiphasic technique with curved planar reformations. AJR Am J Roentgenol 2004;182:419-25.

[31] Diehl SJ, Lehmann KJ, Sadick M, Lachmann R, Georgi M. Pancreatic cancer: value of dual-phase helical CT in assessing resectability. Radiology 1998;206:373-8.

[32] Lu DS, Reber HA, Krasny RM, Kadell BM, Sayre J. Local staging of pancreatic cancer: criteria for unresectability of major vessels as revealed by pancreatic-phase, thinsection helical CT. AJR Am J Roentgenol 1997;168:1439-43.

[33] Schima W, Fugger R, Schober E, et al. Diagnosis and staging of pancreatic cancer: comparison of mangafodipir trisodium-enhanced MR imaging and contrastenhanced helical hydro-CT. AJR Am J Roentgenol 2002;179:717-24.

[34] Ichikawa T, Haradome $H$, Hachiya J, et al. Pancreatic ductal adenocarcinoma: preoperative assessment with helical CT versus dynamic MR imaging. Radiology 1997;202:655-62.

[35] Irie H, Honda H, Kaneko K, Kuroiwa T, Yoshimitsu K, Masuda K. Comparison of helical $\mathrm{CT}$ and $\mathrm{MR}$ imaging in detecting and staging small pancreatic adenocarcinoma. Abdom Imaging 1997;22:429-33.

[36] Romijn MG, Stoker J, van Eijck CH, van Muiswinkel JM, Torres CG, Lameris JS. MRI with mangafodipir trisodium in the detection and staging of pancreatic cancer. J Magn Reson Imaging 2000;12:261-8.

[37] Andersson M, Kostic S, Johansson M, Lundell L, Asztely M, Hellstrom M. MRI combined with MR cholangiopancreatography versus helical CT in the evaluation of patients with suspected periampullary tumors: a prospective comparative study. Acta Radiol 2005;46:16-27.

[38] Maemura K, Takao S, Shinchi H, et al. Role of positron emission tomography in decisions on treatment strategies for pancreatic cancer. J Hepatobiliary Pancreat Surg 2006;13:435-41.

[39] Higashi T, Saga T, Nakamoto $Y$, et al. Diagnosis of pancreatic cancer using fluorine-18 fluorodeoxyglucose positron emission tomography (FDG PET) --usefulness and limitations in "clinical reality". Ann Nucl Med 2003;17:261-79.

[40] Wakabayashi H, Nishiyama Y, Otani T, et al. Role of 18F-fluorodeoxyglucose positron emission tomography imaging in surgery for pancreatic cancer. World J Gastroenterol 2008;14:64-9.

[41] Reese SA, Traverso LW, Jacobs TW, Longnecker DS. Solid serous adenoma of the pancreas: a rare variant within the family of pancreatic serous cystic neoplasms. Pancreas 2006;33:96-9. 
[42] Stern JR, Frankel WL, Ellison EC, Bloomston M. Solid serous microcystic adenoma of the pancreas. World J Surg Oncol 2007;5:26.

[43] Institute TUNC. Surveiilance Epidemiology and End Results (SEER) database. 2007. Available from: URL:http://seer.cancer.gov/. 2007.

[44] Buchler MW, Wagner M, Schmied BM, Uhl W, Friess H, Z'Graggen K. Changes in morbidity after pancreatic resection: toward the end of completion pancreatectomy. Arch Surg 2003;138:1310-4; discussion 5.

[45] Birkmeyer JD, Siewers AE, Finlayson EV, et al. Hospital volume and surgical mortality in the United States. N Engl J Med 2002;346:1128-37.

[46] Cameron JL, Riall TS, Coleman J, Belcher KA. One thousand consecutive pancreaticoduodenectomies. Ann Surg 2006;244:10-5.

[47] Sahani DV, Shah ZK, Catalano OA, Boland GW, Brugge WR. Radiology of pancreatic adenocarcinoma: current status of imaging. J Gastroenterol Hepatol 2008;23:23-33.

[48] Yamao K, Okubo K, Sawaka A, et al. Endolumenal ultrasonography in the diagnosis of pancreatic diseases. Abdom Imaging 2003;28:545-55.

[49] Moesinger RC, Talamini MA, Hruban RH, Cameron JL, Pitt HA. Large cystic pancreatic neoplasms: pathology, resectability, and outcome. Ann Surg Oncol 1999;6:682-90.

[50] Oberg K. Neuroendocrine gastrointestinal tumours. Ann Oncol 1996;7:453-63.

[51] Fritscher-Ravens A, Izbicki JR, Sriram PV, et al. Endosonography-guided, fine-needle aspiration cytology extending the indication for organ-preserving pancreatic surgery. Am J Gastroenterol 2000;95:2255-60.

[52] Baron PL, Kay C, Hoffman B. Pancreatic imaging. Surg Oncol Clin N Am 1999;8:35-58.

[53] Muller MF, Meyenberger C, Bertschinger P, Schaer R, Marincek B. Pancreatic tumors: evaluation with endoscopic US, CT, and MR imaging. Radiology 1994;190:745-51.

[54] Chang KJ. Endoscopic ultrasound-guided fine needle aspiration in the diagnosis and staging of pancreatic tumors. Gastrointest Endosc Clin N Am 1995;5:723-34.

[55] Legmann P, Vignaux O, Dousset B, et al. Pancreatic tumors: comparison of dual-phase helical CT and endoscopic sonography. AJR Am J Roentgenol 1998;170:1315-22.

[56] Mertz HR, Sechopoulos P, Delbeke D, Leach SD. EUS, PET, and CT scanning for evaluation of pancreatic adenocarcinoma. Gastrointest Endosc 2000;52:367-71.

[57] Bhutani MS, Gress FG, Giovannini M, et al. The No Endosonographic Detection of Tumor (NEST) Study: a case series of pancreatic cancers missed on endoscopic ultrasonography. Endoscopy 2004;36:385-9.

[58] Erickson RA, Sayage-Rabie L, Avots-Avotins A. Clinical utility of endoscopic ultrasound-guided fine needle aspiration. Acta Cytol 1997;41:1647-53.

[59] Barthet M, Portal I, Boujaoude J, Bernard JP, Sahel J. Endoscopic ultrasonographic diagnosis of pancreatic cancer complicating chronic pancreatitis. Endoscopy 1996;28:487-91.

[60] Binmoeller KF, Thul R, Rathod V, et al. Endoscopic ultrasound-guided, 18-gauge, fine needle aspiration biopsy of the pancreas using a $2.8 \mathrm{~mm}$ channel convex array echoendoscope. Gastrointest Endosc 1998;47:121-7.

[61] Gress F, Gottlieb K, Sherman S, Lehman G. Endoscopic ultrasonography-guided fineneedle aspiration biopsy of suspected pancreatic cancer. Ann Intern Med 2001;134:459-64. 
[62] Wiersema MJ, Vilmann P, Giovannini M, Chang KJ, Wiersema LM. Endosonographyguided fine-needle aspiration biopsy: diagnostic accuracy and complication assessment. Gastroenterology 1997;112:1087-95.

[63] Erickson RA, Garza AA. Impact of endoscopic ultrasound on the management and outcome of pancreatic carcinoma. Am J Gastroenterol 2000;95:2248-54.

[64] Bhutani MS. Endoscopic ultrasonography in pancreatic disease. Semin Gastrointest Dis 1998;9:51-60.

[65] Gress F, Savides T, Cummings O, et al. Radial scanning and linear array endosonography for staging pancreatic cancer: a prospective randomized comparison. Gastrointest Endosc 1997;45:138-42.

[66] Micames C, Jowell PS, White R, et al. Lower frequency of peritoneal carcinomatosis in patients with pancreatic cancer diagnosed by EUS-guided FNA vs. percutaneous FNA. Gastrointest Endosc 2003;58:690-5.

[67] Hollerbach S, Klamann A, Topalidis T, Schmiegel WH. Endoscopic ultrasonography (EUS) and fine-needle aspiration (FNA) cytology for diagnosis of chronic pancreatitis. Endoscopy 2001;33:824-31.

[68] Hayashi Y, Nakazawa S, Kimoto E, Naito Y, Morita K. Clinicopathologic analysis of endoscopic ultrasonograms in pancreatic mass lesions. Endoscopy 1989;21:121-5.

[69] Canto MI, Goggins M, Hruban RH, et al. Screening for early pancreatic neoplasia in high-risk individuals: a prospective controlled study. Clin Gastroenterol Hepatol 2006;4:766-81; quiz 665.

[70] Canto MI, Goggins M, Yeo CJ, et al. Screening for pancreatic neoplasia in high-risk individuals: an EUS-based approach. Clin Gastroenterol Hepatol 2004;2:606-21.

[71] Rosch T, Braig C, Gain T, et al. Staging of pancreatic and ampullary carcinoma by endoscopic ultrasonography. Comparison with conventional sonography, computed tomography, and angiography. Gastroenterology 1992;102:188-99.

[72] Akahoshi K, Chijiiwa Y, Nakano I, et al. Diagnosis and staging of pancreatic cancer by endoscopic ultrasound. Br J Radiol 1998;71:492-6.

[73] Cannon ME, Carpenter SL, Elta GH, et al. EUS compared with CT, magnetic resonance imaging, and angiography and the influence of biliary stenting on staging accuracy of ampullary neoplasms. Gastrointest Endosc 1999;50:27-33.

[74] Ahmad NA, Lewis JD, Ginsberg GG, Rosato EF, Morris JB, Kochman ML. EUS in preoperative staging of pancreatic cancer. Gastrointest Endosc 2000;52:463-8.

[75] Meining A, Dittler HJ, Wolf A, et al. You get what you expect? A critical appraisal of imaging methodology in endosonographic cancer staging. Gut 2002;50:599-603.

[76] Soriano A, Castells A, Ayuso C, et al. Preoperative staging and tumor resectability assessment of pancreatic cancer: prospective study comparing endoscopic ultrasonography, helical computed tomography, magnetic resonance imaging, and angiography. Am J Gastroenterol 2004;99:492-501.

[77] Eloubeidi MA, Chen VK, Eltoum IA, et al. Endoscopic ultrasound-guided fine needle aspiration biopsy of patients with suspected pancreatic cancer: diagnostic accuracy and acute and 30-day complications. Am J Gastroenterol 2003;98:2663-8.

[78] Bao PQ, Johnson JC, Lindsey EH, et al. Endoscopic ultrasound and computed tomography predictors of pancreatic cancer resectability. J Gastrointest Surg 2008;12:10-6; discussion 6. 
[79] Helmstaedter L, Riemann JF. Pancreatic cancer--EUS and early diagnosis. Langenbecks Arch Surg 2008;393:923-7.

[80] Hartwig W, Schneider L, Diener MK, Bergmann F, Buchler MW, Werner J. Preoperative tissue diagnosis for tumours of the pancreas. Br J Surg 2009;96:5-20.

[81] Spinelli KS, Fromwiller TE, Daniel RA, et al. Cystic pancreatic neoplasms: observe or operate. Ann Surg 2004;239:651-7; discussion 7-9.

[82] Laffan TA, Horton KM, Klein AP, et al. Prevalence of unsuspected pancreatic cysts on MDCT. AJR Am J Roentgenol 2008;191:802-7.

[83] Volkan Adsay N. Cystic lesions of the pancreas. Mod Pathol 2007;20 Suppl 1:S71-93.

[84] Ishikawa T, Takeda K, Itoh M, et al. Prevalence of pancreatic cystic lesions including intraductal papillary mucinous neoplasms in patients with end-stage renal disease on hemodialysis. Pancreas 2009;38:175-9.

[85] Warshaw AL, Rutledge PL. Cystic tumors mistaken for pancreatic pseudocysts. Ann Surg 1987;205:393-8.

[86] Kloppel G, Luttges J. WHO-classification 2000: exocrine pancreatic tumors. Verh Dtsch Ges Pathol 2001;85:219-28.

[87] Friedel DM, Abraham B, Georgiou N, Stavropoulos SN, Grendell JH, Katz DS. Pancreatic cystic neoplasms. South Med J;103:51-7.

[88] Bose D, Tamm E, Liu J, et al. Multidisciplinary management strategy for incidental cystic lesions of the pancreas. J Am Coll Surg;211:205-15.

[89] Karlson BM, Ekbom A, Lindgren PG, Kallskog V, Rastad J. Abdominal US for diagnosis of pancreatic tumor: prospective cohort analysis. Radiology 1999;213:107-11.

[90] Mathieu D, Guigui B, Valette PJ, et al. Pancreatic cystic neoplasms. Radiol Clin North Am 1989;27:163-76.

[91] Le Borgne J, de Calan L, Partensky C. Cystadenomas and cystadenocarcinomas of the pancreas: a multiinstitutional retrospective study of 398 cases. French Surgical Association. Ann Surg 1999;230:152-61.

[92] Procacci C, Biasiutti C, Carbognin G, et al. Characterization of cystic tumors of the pancreas: CT accuracy. J Comput Assist Tomogr 1999;23:906-12.

[93] Bassi C, Salvia R, Molinari E, Biasutti C, Falconi M, Pederzoli P. Management of 100 consecutive cases of pancreatic serous cystadenoma: wait for symptoms and see at imaging or vice versa? World J Surg 2003;27:319-23.

[94] Minami M, Itai Y, Ohtomo K, Yoshida H, Yoshikawa K, Iio M. Cystic neoplasms of the pancreas: comparison of MR imaging with CT. Radiology 1989;171:53-6.

[95] Koito K, Namieno T, Ichimura T, et al. Mucin-producing pancreatic tumors: comparison of MR cholangiopancreatography with endoscopic retrograde cholangiopancreatography. Radiology 1998;208:231-7.

[96] Adler DG, Jacobson BC, Davila RE, et al. ASGE guideline: complications of EUS. Gastrointest Endosc 2005;61:8-12.

[97] Mizuno N, Bhatia V, Hosoda W, et al. Histological diagnosis of autoimmune pancreatitis using EUS-guided trucut biopsy: a comparison study with EUS-FNA. J Gastroenterol 2009;44:742-50.

[98] Hawes RH. Indications for EUS-directed FNA. Endoscopy 1998;30 Suppl 1:A155-7.

[99] Rosen CB, Heimbach JK, Gores GJ. Liver transplantation for cholangiocarcinoma. Transpl Int;23:692-7. 
[100] Ahmad NA, Kochman ML, Lewis JD, Ginsberg GG. Can EUS alone differentiate between malignant and benign cystic lesions of the pancreas? Am J Gastroenterol 2001;96:3295-300.

[101] Ahmad NA, Kochman ML, Brensinger C, et al. Interobserver agreement among endosonographers for the diagnosis of neoplastic versus non-neoplastic pancreatic cystic lesions. Gastrointest Endosc 2003;58:59-64.

[102] Frossard JL, Amouyal P, Amouyal G, et al. Performance of endosonography-guided fine needle aspiration and biopsy in the diagnosis of pancreatic cystic lesions. Am J Gastroenterol 2003;98:1516-24.

[103] Brugge WR, Lewandrowski K, Lee-Lewandrowski E, et al. Diagnosis of pancreatic cystic neoplasms: a report of the cooperative pancreatic cyst study. Gastroenterology 2004;126:1330-6.

[104] Sedlack R, Affi A, Vazquez-Sequeiros E, Norton ID, Clain JE, Wiersema MJ. Utility of EUS in the evaluation of cystic pancreatic lesions. Gastrointest Endosc 2002;56:543-7.

[105] Niederau C, Grendell JH. Diagnosis of pancreatic carcinoma. Imaging techniques and tumor markers. Pancreas 1992;7:66-86.

[106] Tanaka S, Kitamra T, Yamamoto K, et al. Evaluation of routine sonography for early detection of pancreatic cancer. Jpn J Clin Oncol 1996;26:422-7.

[107] Candiani F, Meduri F, Norberto L, Calderone M. [Contrast media in ultrasonography. Venous involvement in tumors of the head of the pancreas]. Radiol Med 1998;95:29-33.

[108] Calculli L, Casadei R, Amore B, et al. The usefulness of spiral Computed Tomography and colour-Doppler ultrasonography to predict portal-mesenteric trunk involvement in pancreatic cancer. Radiol Med 2002;104:307-15.

[109] Dietrich CF, Braden B, Hocke M, Ott M, Ignee A. Improved characterisation of solitary solid pancreatic tumours using contrast enhanced transabdominal ultrasound. J Cancer Res Clin Oncol 2008;134:635-43.

[110] Park HS, Lee JM, Choi HK, Hong SH, Han JK, Choi BI. Preoperative evaluation of pancreatic cancer: comparison of gadolinium-enhanced dynamic MRI with MR cholangiopancreatography versus MDCT. J Magn Reson Imaging 2009;30:586-95.

[111] Delbeke D, Rose DM, Chapman WC, et al. Optimal interpretation of FDG PET in the diagnosis, staging and management of pancreatic carcinoma. J Nucl Med 1999;40:1784-91.

[112] Bruno M, Bosco M, Carucci P, et al. Preliminary experience with a new cytology brush in EUS-guided FNA. Gastrointest Endosc 2009;70:1220-4.

[113] Al-Haddad M, Gill KR, Raimondo M, et al. Safety and efficacy of cytology brushings versus standard fine-needle aspiration in evaluating cystic pancreatic lesions: a controlled study. Endoscopy;42:127-32.

[114] Jacobson BC, Baron TH, Adler DG, et al. ASGE guideline: The role of endoscopy in the diagnosis and the management of cystic lesions and inflammatory fluid collections of the pancreas. Gastrointest Endosc 2005;61:363-70.

[115] Shah JN, Fraker D, Guerry D, Feldman M, Kochman ML. Melanoma seeding of an EUS-guided fine needle track. Gastrointest Endosc 2004;59:923-4. 
[116] Repak R, Rejchrt S, Bartova J, Malirova E, Tycova V, Bures J. Endoscopic ultrasonography (EUS) and EUS-guided fine-needle aspiration with cyst fluid analysis in pancreatic cystic neoplasms. Hepatogastroenterology 2009;56:629-35.

[117] van der Waaij LA, van Dullemen HM, Porte RJ. Cyst fluid analysis in the differential diagnosis of pancreatic cystic lesions: a pooled analysis. Gastrointest Endosc 2005;62:383-9.

[118] Khalid A, Zahid M, Finkelstein SD, et al. Pancreatic cyst fluid DNA analysis in evaluating pancreatic cysts: a report of the PANDA study. Gastrointest Endosc 2009;69:1095-102.

[119] Shen J, Brugge WR, Dimaio CJ, Pitman MB. Molecular analysis of pancreatic cyst fluid: a comparative analysis with current practice of diagnosis. Cancer 2009;117:217-27. 


\section{Part 5}

\section{Urogynecological Endoscopy}





\title{
Urology: The Home of Endoscopy
}

\author{
Rastislav Hejj, Marie McNulty and John G. Calleary \\ Department of Urology, \\ North Manchester General Hospital, \\ Pennine Acute Hospitals NHS Trust \\ Crumpsall, Manchester M8 5RB \\ United Kingdom
}

\section{Introduction}

\subsection{Place of endoscopy in urology}

Urology is truly the home of endoscopy. Starting with the introduction of first cystoscope, Urology has been at the forefront of endoscopic use in clinical practice. Endoscopy is used in both diagnosis and therapeutic settings (Table 1). Currently, endoscopy is used for diagnosis of bladder pathology and this is primarily with the use of flexible instruments under local anaesthesia. It is the gold standard for the identification of urethral stricture disease and diagnostic standard for the identification of intravesical Transitional Cell Carcinoma (TCC) and the primary follow-up tool for non-muscle invasive TCC. Transurethral Resection of Bladder Tumour (TURBT) is the mainstay for pathological diagnosis of Transitional Cell Carcinoma (TCC). Its efficacy has been improved by modern technical developments such as blue-light cystoscopy. This staging provides information on whether more radical therapies are necessary or whether continued endoscopic surveillance can be continued.

Relief of ureteric obstruction often involves stenting of the relevant ureter. Above the bladder, rigid endoscopy is used diagnose ureteric stone disease. Treatment of identified stones is usually endoscopic, using either Holmium laser or lithoclast technology. In recent times this practice seemed to have shifted toward a greater role for Shock wave lithotripsy.

In the sphere of organ ablative surgery, since the introduction of laparoscopy to Urology it has been possible to apply endourological techniques to ablative procedures. Thus laparoscopic nephrectomy is now the standard for radiological T1-T2 lesions where partial ablation is inappropriate. This chapter proposes to track the use of endoscopy in Urology and to show why Endoscopy and Endoscopic techniques have become central to Urological diagnosis and treatment.

\subsection{History of endoscopy in urology}

This section is not meant to be the didactic history of Urological Endoscopy. For that we would suggest Mr J Shah`s review (Shah 2002) or Dr Herr`s review in the Journal of Endourology (Herr 2006). Rather it is an attempt to provide an insight into developments and timelines in the evolution of Urological endoscopy. 


\begin{tabular}{|c|c|c|c|}
\hline Organ & $\begin{array}{l}\text { Endoscopy used } \\
\text { for Diagnosis D/ } \\
\text { Therapy T / } \\
\text { Both B }\end{array}$ & $\begin{array}{l}\text { Open or } \\
\text { Endoscopic } \\
\text { Techniques as the } \\
\text { prime Surgical } \\
\text { modality }\end{array}$ & $\begin{array}{l}\text { Conditions } \\
\text { (examples) }\end{array}$ \\
\hline Urethra & B & $E>O$ & $\begin{array}{l}\text { Stricture, HUA, Pain, } \\
\text { Foreign bodies, } \\
\text { Malignancy }\end{array}$ \\
\hline $\begin{array}{l}\text { Prostate / } \\
\text { Bladder neck } \\
\text { Benign } \\
\text { Malignancy }\end{array}$ & $\begin{array}{l}\mathrm{B} \\
\mathrm{T}\end{array}$ & $\begin{array}{l}E \gg>O \\
O=E\end{array}$ & $\begin{array}{l}\text { Obstruction, } \\
\text { Malignancy, }\end{array}$ \\
\hline $\begin{array}{l}\text { Bladder } \\
\text { Benign } \\
\text { Malignancy }\end{array}$ & $\begin{array}{l}\text { B but } \mathrm{D}>>\mathrm{T} \\
\mathrm{B}\end{array}$ & $\begin{array}{l}E>>>O \\
E>O\end{array}$ & $\begin{array}{l}\text { Strorage symptoms, } \\
\text { HUA, Malignancy, } \\
\text { Foreign bodies }\end{array}$ \\
\hline $\begin{array}{l}\text { Ureter including } \\
\text { renal collecting } \\
\text { system } \\
\text { Benign } \\
\text { Malignant }\end{array}$ & $\begin{array}{l}\text { B } \\
\text { B but } \mathrm{T}>>\mathrm{D}\end{array}$ & $\begin{array}{l}E>>>O \\
O=E\end{array}$ & $\begin{array}{l}\text { Urolithiasis, HUA, } \\
\text { Malignancy, Stricture, } \\
\text { Functional obstruction } \\
\text { eg Pelvi-ureteric- } \\
\text { junction }\end{array}$ \\
\hline $\begin{array}{l}\text { Kidney } \\
\text { Benign } \\
\text { Malignant }\end{array}$ & $\begin{array}{l}\text { Little place } \\
\mathrm{T}\end{array}$ & $E=O$ & $\begin{array}{l}\text { Glomerulonephritis } \\
\text { Renal Cell malignancy }\end{array}$ \\
\hline
\end{tabular}

Table 1. The place of Endoscopic Urological procedures in the treatment of Urological disease

The first to attempt to visualise the urogenital tract was by Bozzini in 1806. His "Lichtleiter" consisted of a funnel, candle and a reflector. Its problem was of poor illumination so much so that from the practical viewpoint it was unusable. Further improvements made by Sėgales and Fischer led to the development by Desormeaux of a cystoscope where illumination was provided by an alcohol and turpentine lamp. This was the first instrument to be used for a therapeutic manoeuvre. Desormeaux, has been called "the Father of Cystoscopy", in part because he was one of the first to introduce a Lichleiter into a patient. Cruise, Newman and especially Nitze were responsible for modifications which improved illumination including the use of intra-corporeal bulbs. In 1887 Nitze introduced a cystoscope which did not require a cooling apparatus and through which biopsies could be taken.

In 1889 Boisseau du Rocher introduced a twin sheath modification which allowed simultaneous visualisation and instrumentation/ irrigation. Albarran in 1896 introduced a catheter deflector. Further developments in both the USA and Europe in illumination allowed the use of prisms as lenses. These developments led to the resectoscope which was first introduced in 1926 by Stern and modified by McCarthy in 1931 to produce the SternMcCarthy resectoscope. This was the first instrument recognisable as a modern resectoscope. Trans-Urethral Resection of the Prostate / Bladder lesions was made possible 
through the work of Hertz and DeForest who described the use of high frequency current for and vacuum tube that made tissue resection possible. Their work was further progressed by Wappler and Wyeth (cutting current) and W.T. Bovie and G.H. Leibel (coagulating current). The true modern resection instrument was then developed by Frederick Wappler in 1931 and resection of the prostate (TURP) was first described in a published account by Nesbitt in 1943.

Flexible instruments were initially developed by Mikulicz in 1881 who attached a 30 degree deflection mechanism to a cystoscope and performed an oesohagoscopy. The first semiflexible cystoscopy was performed using a Wolf and Schindler instrument in 1936. Ureteroscopy was first performed in the early 1900`s using a Brandford Lewis instrument. As alluded to above, the earliest instruments used external combustible light sources. Their main disadvantage was of poor illumination and a certain danger of burns to their operator and the patient. Candles gave way to alcohol burners which produced more light but the heat they produced required the addition of a cooling device. The field of view was still limited to the diameter of the instrument although the light intensity was increased.

The major improvement came from Edison`s invention of the bulb in 1880. The development of the "Mignon" lamp allowed better illumination of the bladder from its position at the distal end of the cystoscope. Distal illumination remained the main stay until fibre-optics was applied to endoscope design in the 1950`s. Fibre-optics use the phenonomen of Multiple Total Internal Reflection. Professor Harold Hopkins and Narinder Kampany published in Nature in January 1954 on image transmission through unclad fibres. In the same edition Van Heel reports on image transmission through clad light fibres. Hence light could be transmitted through fibre-optic bundles and the target image viewed through the same telescope. To see a true image the fibres had to be coherenty arranged i.e. the fibres had to occupy the same relative position at either end. Hopkins also worked on rigid systems and the application of glass fibre innovations led to the rod-lens system currently used in most rigid endoscopes. The advantage of this system is the increased light intensity delivered to the target and the better image due to a reduction in light loss within the lens casing.

The development which will further revolutionise endoscopy is the development of distal sensors. These are based on either CCD (charge coupled device) or CMOS (complementary metal-oxide semi-conductor) technology. Both technologies register an image as an electrical charge proportional to the light intensity of the image. These charges / impulses are then processed and reformatted as a colour image. CCD cameras and telescopes are further along in development. Three chip (one for each of the prime colours) systems have resulted in increased visualisation and resolution. Its introduction has led to NBI (Narrow Band Imaging) technology which is discussed later. In a head to head comparison between fibreoptic and distal sensor endoscopes, Okhunov et al showed that distal sensor technology was superior in the 1000 procedures tested (Okhunov 2009).

\section{Endoscopy of lower urinary tract}

\subsection{Flexible cystoscopy (figure 1)}

Flexible cystoscopy permits direct visual inspection of the urethra and bladder following instillation of lubricant local anaesthetic gel. The instruments used currently predominantly use fibre-optic technology for light and image transmission. Hence they need a separate light source and usually a camera system. The indications are principally diagnostic (investigation of haematuria, the storage lower tract symptoms of frequency and urgency) 
where intravesical pathology is suspected and in the surveillance of patients with previously diagnosed and treated bladder cancer. The gradual introduction of newer technology such as distal sensor all digital endoscopes and narrow band imaging will improve resolution and thus detection of recurrent bladder TCC (Transitional Cell Carcinoma).

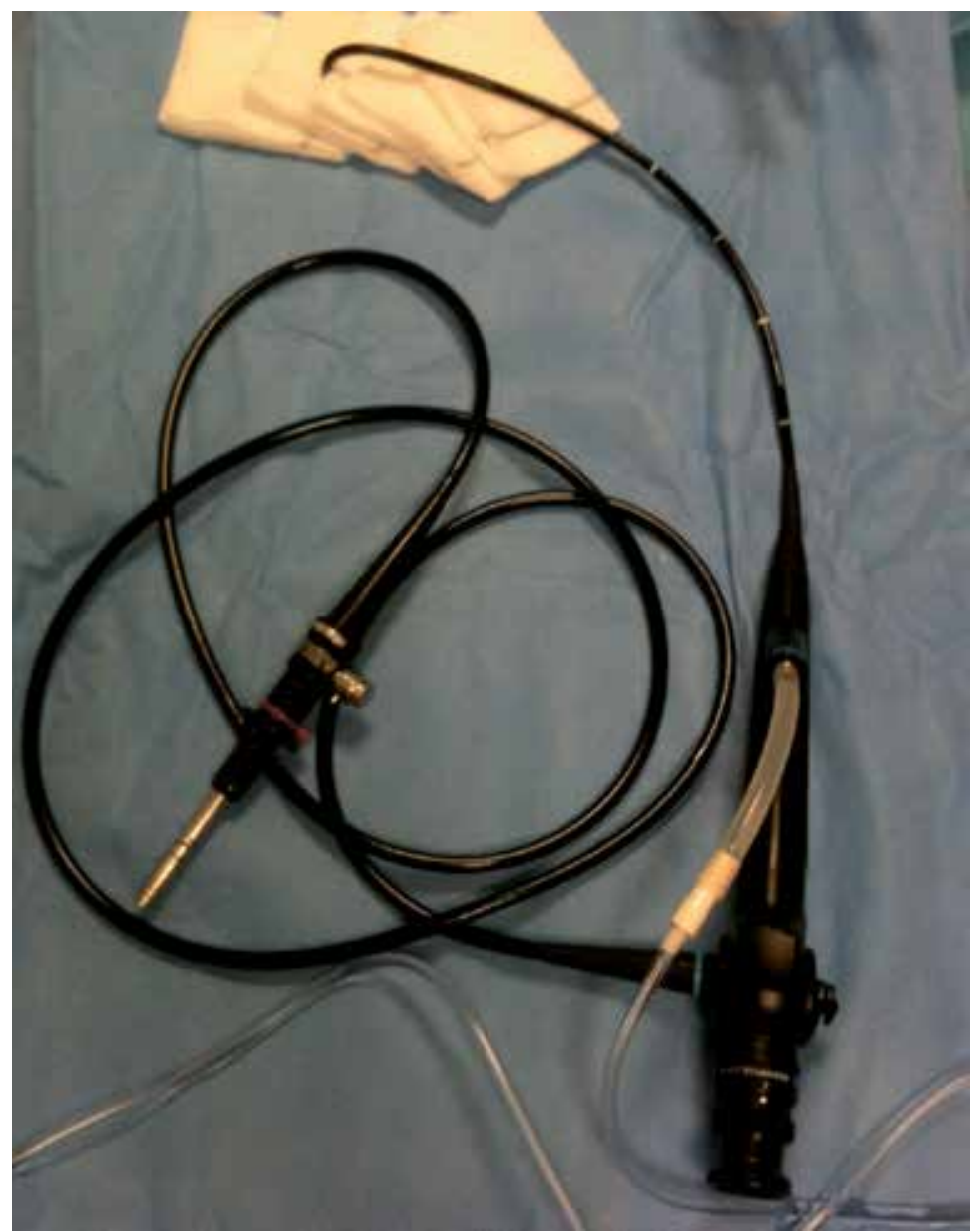

Fig. 1. Modern fibre-optic flexible cystoscope

In terms of therapeutic indications flexible cystoscopy can be used for biopsy and destruction of small tumours using diathermy fulguration or holmium laser vaporisation. Polymer based stents (eg JJ stents) inserted to relieve ureteric obstruction are usually removed at flexible cystoscopy and in some cases can even be inserted (Reynard et al., 2009). However the majority of such procedures are performed at rigid cystoscopy. Intravesical Botulinium-A toxin sub-mucosal injection performed through the flexible cystoscope is now a standard treatment for intractable storage symptoms resistant to oral anti-muscarinic agents . Complications of flexible cystoscopy include mild burning or bleeding on passing urine for a short period (common) and urinary tract infection requiring antibiotics $(<5-10 \%-$ this rate can be reduced by prophylactic antibiotics). 


\subsection{Rigid Cystoscopy and Trans Urethral Resection of Bladder Tumour; TURBT (Figure 2 shows instruments in common use)}

Rigid cystoscopy as a diagnostic tool has been virtually replaced by flexible cystoscopy. However it is the main vehicle through which reasonable bladder biopsies are taken and a pre-request to many endoscopic procedures. Instruments are available for paediatric and adults and the adult instruments usually are 18-22 Ch in diameter.

TURBT is the most important diagnostic procedure for bladder tumours as the histological evaluation of the resected tissue allows a clinician to distinguish between non-muscle invasive ("superficial") and muscle invasive bladder cancer. The most common histological sub-type of bladder cancer is Transitional Cell Carcinoma (TCC). 75-85\% of newly diagnosed bladder TCC are non-muscle invasive ("superficial") and for these TURBT is also the definitive treatment. The remaining $15-25 \%$ are muscle invasive, requiring radical treatment (either surgical- radical cystectomy, or radical radiotherapy).

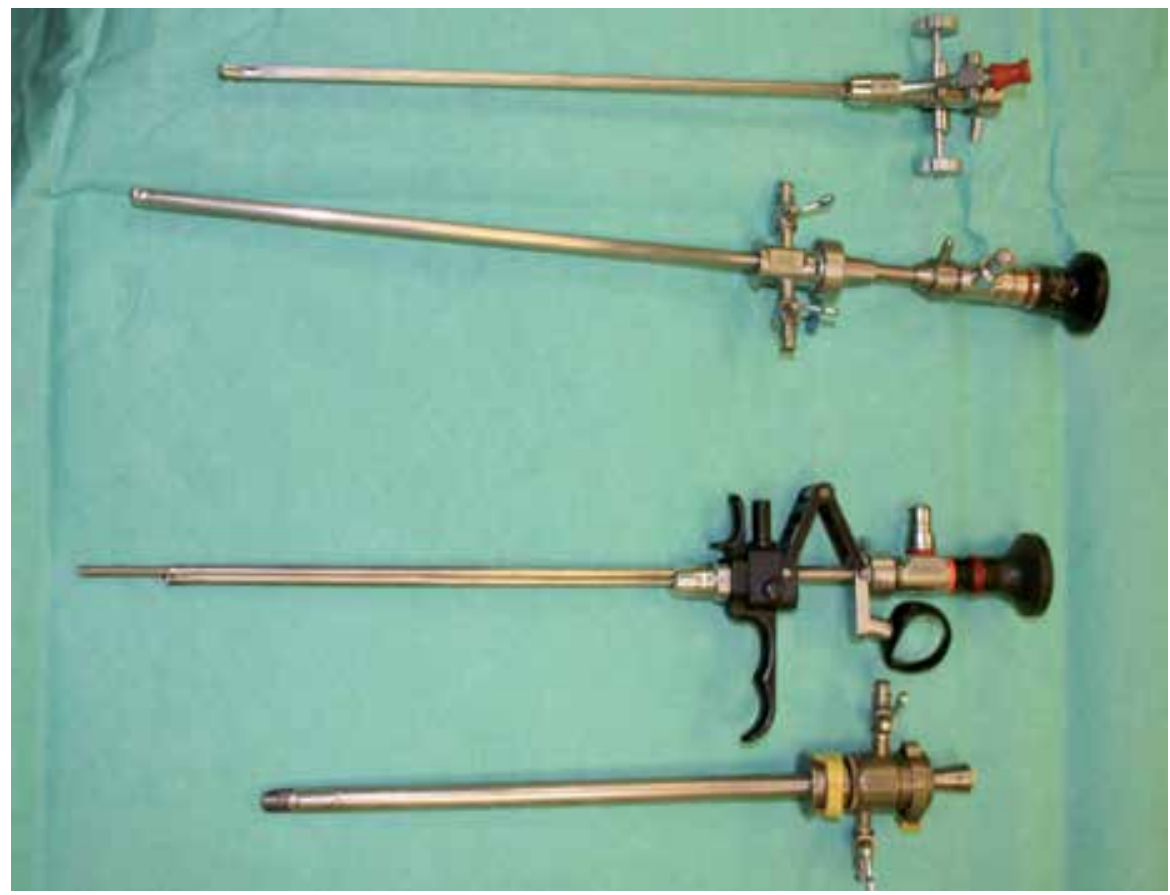

Fig. 2. Instruments used in Lower tract endoscopy

From top to bottom; Modern Albarran catheterizing bridge, Rigid cystoscope in its $22 \mathrm{f}$ sheath, Resectoscope working element with its lens and resectoscope sheath and obturator.

TURBT starts with bimanual palpation to assess whether a tumour mass is palpable and to ascertain whether its mobile. A fixed mass implies a more locally advanced disease process. Following this, a rigid cystoscopy is performed to visualise the bladder tumour and assess the size (in $\mathrm{cm}$ ), appearance (?papillary, ?solid, ?flat/red area), position (base, lateral wall, posterior wall, dome) and their relationship to the ureteric orifici.

A resectoscope is then placed per-urethrally with an external diameter $18 \mathrm{Ch}$ and $28 \mathrm{Ch}$. Identified lesions are then resected using a loop electrode and sent for histology. Bladder muscle (muscularis propria) should be present in resected tissue as the presence of TCC in 
muscle is the hallmark of muscle invasive disease. The resected tissue is then removed using an Ellik evacuator and diathermy to bleeding points performed with a rollerball electrode. At the end of the procedure, repeat bimanual palpation is performed as a means of ensuring complete resection of the lesion. A 3-way irrigation catheter is inserted and removed within 24-48 hours unless an extra-peritoneal perforation has occurred. For tumours which appear superficial, Intravesical Mitomycin C within 24 hours of resection has been shown to reduce number of recurrences by 39\% (Sylvester et al., 2004). Currently the only place for open procedures in TCC diagnosis and management is in radical cystectomy and a urinary diversion procedure. However, the technique of laparoscopic cystectomy has been developed (Parra 1992) and is gaining acceptance.

\subsection{Trans-Urethral Resection of Prostate (TURP)}

While the majority of men are managed medically, TURP is the gold standard surgical treatment for lower urinary tract symptoms (LUTS) due to benign prostatic hyperplasia $(\mathrm{BPH})$ causing bladder outlet obstruction (BOO). TURP involves resection of prostatic adenomatous tissue to the level of the prostatic capsule. Prostatic chippings are flushed into the bladder during resection by irrigation fluid (usually 1,5\% glycine) and at the end of the procedure washed out from the bladder (using Ellik bladder evacuator) and sent for histological examination. The most important landmarks are the ureteric orificii proximally and the Veru montanum distally. The Veru is the marker of the proximal limit of the external sphincter, damage to which results in stress urinary incontinence.

TURP is performed for men with voiding symptoms which fail to respond to medical treatment (alpha-blockers, 5-alpha reductase inhibitors), for men with urinary retention (failed trial of catheter removal on medical treatment) and for those who develop complications of bladder outlet obstruction especially renal impairment. Complications are as follows severe bleeding requiring blood transfusion (1-2\%), sepsis (3\%), TUR syndrome $(<1 \%)$, urinary incontinence $(<1 \%)$, bladder neck stenosis/ urethral stricture $(3-5 \%)$, retrograde ejaculation (80-100\%) and erectile dysfunction (approximately 10\%). Alternative surgical treatments include "Greenlight" laser ablation or Holmium laser enucleation, both of which are performed under cystoscopic guidance. Smaller prostates are also managed endoscopicaly using a Bladder Neck Incision (BNI). Open surgery for benign prostatic disease is now extremely rare and performed only when the prostate is $>100$ gr or if there is a concurrent bladder stone (McAllister 2010).

\subsection{Technological advances in the endoscopic diagnosis of bladder cancer}

The development of distal sensor technologies has led to development of techniques which aim to increase the diagnostic capability of white-light cystoscopy (WLC) in the detection of TCC. Probably the two landmark developments are Photodynamic diagnosis (PDD), also referred to as "fluorescence cystoscopy" and Narrow-Band Imaging (NBI).

\subsubsection{Photodynamic diagnosis (PDD)}

PDD requires the intravesical instillation of a fluorescent agent (e.g. 5-aminolaevulinic acid (5-ALA), or its ester hexaminolevulinate) before performing blue-light cystoscopy. The principle of PDD is based on the difference in uptake of fluorescence molecules in normal and pathologic tissue. Absorption of light of an appropriate wavelength causes excitation of the fluorophore molecule which on returning to its ground state emits a photon equivalent 
to the energy difference between these states. Endoscopes with specially developed light sources and filters are used, and with the aid of a foot pedal or push-button on the camera one can easily switch from WLC to PDD. By illuminating the bladder wall with blue light, the malignant tissue appears intensely pink or red on a blue background (Cauberg et al., 2009). Clinical trials have shown that the detection of TCC with PDD is superior to WLC with reported sensitivities of $82-97 \%$ for PDD versus $62-84 \%$ for WLC. With regard to the detection of CIS lesions, PDD performs significantly better than WLC with detection rates of $92-97 \%$ versus $56-68 \%$, respectively. PDD use may also have an impact on recurrence rate. This has been studied by cystoscopic re-evaluation six weeks following initial resection using WLC or Blue light cystoscopy. Results of published studies seem to show a statistically significant reduction in residual tumour if resection was performed with PDD (25-53\% for white-light resection vs 4-32\% for PDD-assisted resection). However, PDD has a relatively low specificity, ranging from 41 to $98 \%$ and false-positive fluorescence can be induced by inflammation, scarring after TUR, prior intravesical therapy and tangential illumination of mucosa (Cauberg et al., 2009). It is hoped that the initial cost of the technology will be recouped by an increased recurrence-free survival or progression-free survival. This remains to be proven in larger randomised clinical trials.

\subsubsection{Narrow-band imaging (NBI)}

NBI is an optical image enhancement technique designed to enhance the contrast between mucosal surfaces and microvascular structures without the use of dyes. It is based on the phenomenon that the depth of light penetration into the mucosa increases with increasing wavelength. The tissue surface is illuminated with light of a narrow bandwidth, with centre wavelengths in the blue $(415 \mathrm{~nm})$ and green $(540 \mathrm{~nm})$ spectrum of light. Since these specific wavelengths are strongly absorbed by haemoglobin, the vascular structures appear dark brown or green against a pink or white mucosal background. Systems that have integrated NBI and WLC are commercially available. With the push of a button, the NBI mode is activated by mechanical insertion of the narrow-band filter in front of the white-light source. NBI has not been as extensively investigated as PDD and therefore it is hard to know the effect of inflammation, previous intra-vesical instillations or scarring on its sensitivity (Cauberg et al., 2009). The inherent advantage of NBI over PDD is that it avoids the physical discomfort and extra cost associated with requirement for instillation of the intra-vesical agent pre-operatively. The small number of studies performed comparing NBI to WLC suggested a non-significant improvement in the detection of bladder cancer, but more studies are needed to assess the benefit of NBI further.

\subsection{Optical urethrotomy (figure 3)}

Modern Urethral stricture diagnosis is primarily by flexible endoscopy. The initial endoscopy can identify the number of, diameter of and the rigidity of any identified strictures. Previously diagnosis was primarily by radiological means prompted by clinical suspicion. It is possible to definitively treat a stricture by endoscopic means by performing a Direct Visual Internal Urethrotomy, using an optical urethrotome.

An Optical urethrotome has a straight (0 degree) lens, cold knife and a channel for the introduction of a guide-wire into the bladder. This guidewire allows a urethral catheter to be passed if bleeding obscures visualisation of the lumen. A cold-knife incision is made at the 12 o'clock position until the lumen of the strictured segment is approximately the same 
as of the remaining urethra. A 16-18 Fr catheter is inserted post-operatively and removed after 2-5 days. Optical urethrotomy is most suitable for strictures less than $1 \mathrm{~cm}$ in length, of the Bulbar urethra (71\% success rate) and for strictures with a calibre of greater than $15 \mathrm{~F}$ (69\% vs 36\%). Recurrences, if they occur, will do so within $12(56 \%)$ or 24 months (26\%) months (Pansadoro 1996). For all other strictures the definitive treatment should still be open reconstructive surgery.

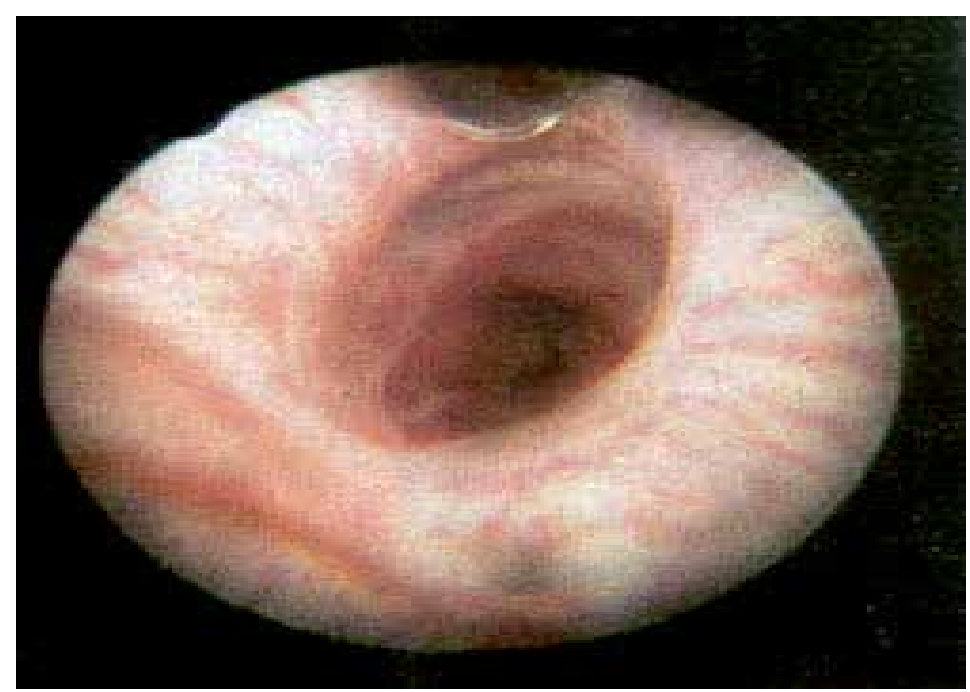

Fig. 3. Anterior Urethral stricture at Direct Visual Optical Urethrotomy (optical urethrotomy with a cold knife seen at 12 o'clock)

\section{Endoscopy of upper urinary tract}

\subsection{Ureteroscopy (figure 4)}

Ureteroscopy can be performed using semi-rigid or flexible instruments to allow visual inspection of ureter and renal pelvis (semi-rigid ureteroscope) and major and minor renal calyces (flexible ureteroscope). Semi-rigid ureteroscopes have high-density fibre-optic bundles for light (non-coherently arranged) and image transmission (coherently arranged to maintain image quality). The instrument can be bent by several degrees without the image being distorted, hence the description semi-rigid. The working tip of most current models is 7-8 $\mathrm{Ch}$ with the proximal end being 11-12 $\mathrm{Ch}$. There is usually at least one working channel of at least 3 or $4 \mathrm{Ch}$ (Reynard et al., 2009). In a flexible instrument, the operator can control the degree of deflection of the distal end (active deflection). Behind the actively deflecting tip is a segment of greater flexibility than the rest of the shaft. This section is able to undergo passive deflection (when the tip is fully actively deflected, by advancing the scope further, this flexible segment allows even more deflection). The fibre-optic bundles in flexible instruments are identical to those in semi-rigid scopes, only of smaller diameter. The price of the extra mobility is reduced image quality and light transmission (Reynard et al., 2009). The working tip of most current models is 7-8 Ch, with the proximal end of the scope 9-10 Ch. There is usually at least one working channel of at least 3.6 Ch. Flexible instruments are more expensive and less durable than semi-rigid scopes. 


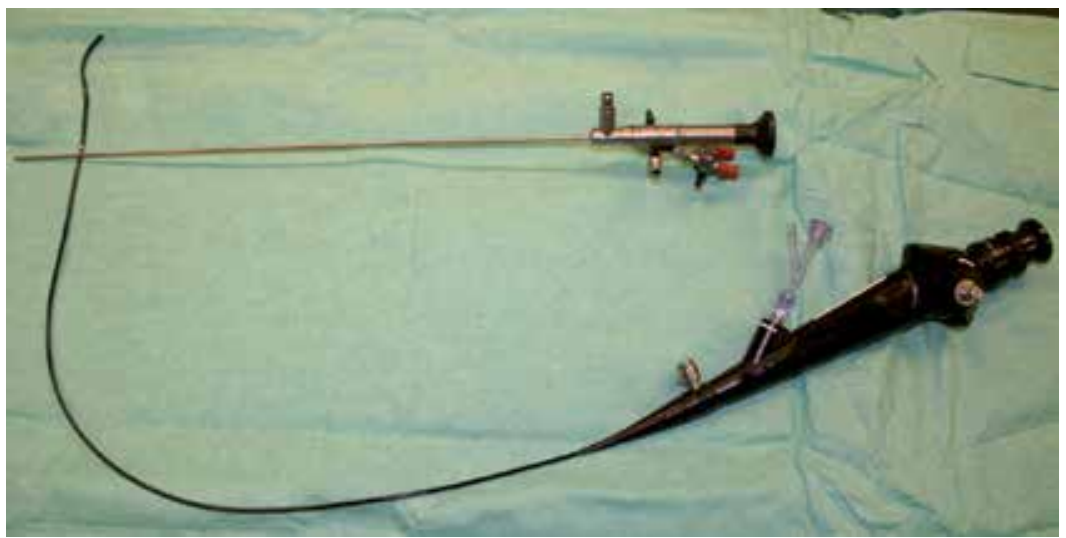

Fig. 4. Examples of Modern Upper Tract instruments in routine Urological use. Top: SemiRigid Ureteroscope. Bottom: Flexible Ureteroscope (fibre-optic technology)

The indications for ureteroscopy are either diagnostic (e.g. patient with haematuria and filling defect in upper urinary tract on contrast study) or therapeutic. The primary therapeutic use of Ureteroscopy is in upper tract stone disease (figure 5) where stone destruction can be achieved with laser, ultrasonic or EHL (electrohydrolic) technology. Fragments can be removed using a variety of baskets introduced through the working channel. Potential complications include stone migration $(4 \%)$, ureteric injury $(3.5 \%)$, sepsis (1\%) and failure to reach stone (3.7\%) (Geavlete 2006).

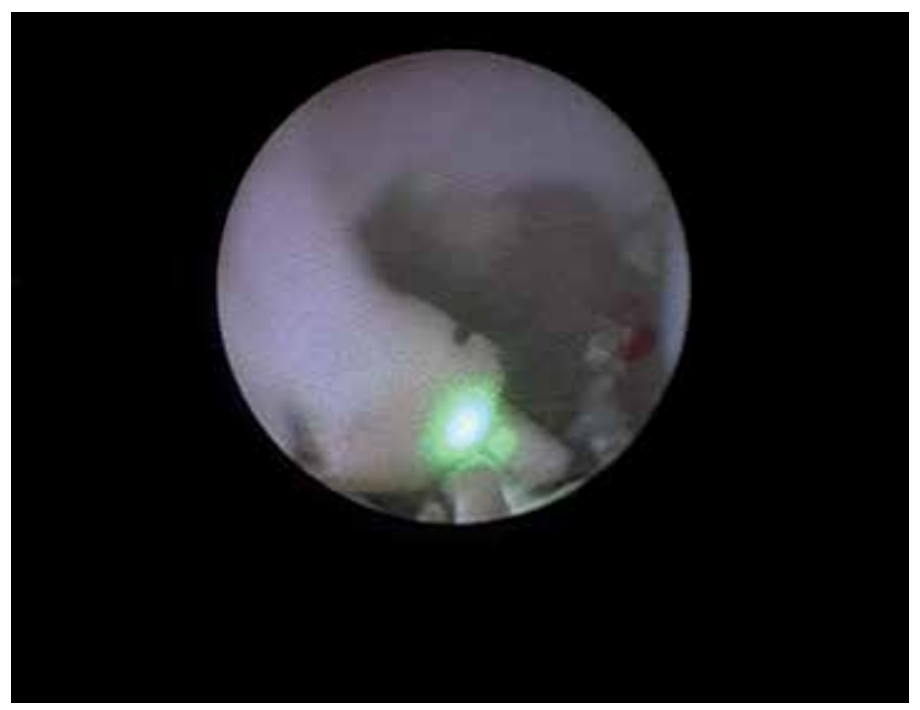

Fig. 5. Ureteroscopic holmium laser lithotripsy. Laser fibre at 6 o`clock with green aiming beam shining on the stone.

to the extent that, for stones requiring surgical treatment, open stone surgery is now the exception rather than the normal. The other main therapeutic indication is in selected cases of upper urinary tract transitional cell carcinoma (TCC). 


\subsection{Per-Cutaneous NephroLithotomy (PCNL)}

The first PCNL was performed in 1976. Since then open nephrolithotomy has reduced in frequency to the current rate of $<1 \%$ of all stone procedures. The current treatment options are PCNL, Shock-wave lithotripsy and flexible ureteroscopy. PCNL is the $1^{\text {st }}$ line treatment option for staghorn and other large renal stones ( $>2 \mathrm{~cm}$ in maximum diameter). A Staghorn stone is defined as a stone filling the entire renal pelvis and at least one (partial staghorn) or all of the calyces (complete staghorn). PCNL is also indicated for smaller stones in specific circumstances such as abnormal anatomy (e.g. horshoe kidney, calyceal diverticulum), failure of other treatment options (ureteroscopy, ESWL) or patient preference. PCNL is more invasive (higher morbidity) than ureteroscopy or ESWL but has a higher efficacy in single treatment stone clearance (especially for larger stones). Treatment of lower pole calyceal stones measuring 1-2 cm in maximum diameter remains a controversial issue. As stated above, the available options are flexible ureteroscopy, ESWL or PCNL. Access to the lower pole calyx by flexible ureteroscopy can be difficult and the stone-free rates after ESWL are lower than for stones in other parts of the kidney. This has led some to favour PCNL as the first line option for 1-2 cm stones in lower pole calyx. Two prospective, randomised studies tried to resolve this problem; Lower Pole Study I (Albala et al., 2001) and Lower Pole Study II (Pearle et al., 2005). Lower Pole Study I compared ESWL to PCNL for 1-3 cm stones localised in lower pole calyx. PCNL was more successful (95\% vs 37\%) but had higher morbidity. Lower Pole Study II compared ESWL to Ureteroscopy for $<1 \mathrm{~cm}$ stones in lower pole calyx. Ureteroscopy was more successful in stone clearance (50\% vs $35 \%)$ but due to relatively small number of patients $(n=67)$ this result failed to achieve statistical significance. Despite these two studies the question of the best treatment for $<2 \mathrm{~cm}$ lower pole calyceal stones remains difficult to answer and proper patient counselling about pros and cons of each therapeutic option is crucial.

How is PCNL performed? The patient has been traditionally placed prone, but over the recent years it has been demonstrated that PCNL is also feasible in supine position. Ureteric catheterisation (with or without balloon) is performed primarily to fill the collecting system which facilitates puncture (contrast and methylene blue injected through ureteric catheter dilates and opacifies collecting system) but also to minimise migration of stone fragments into the ureter. The puncture can be made under combined ultrasound and X-ray control or under fluoroscopy. Ultrasound makes it easier to identify, and therefore avoid damage to, neighbouring organs. In rare cases with complex anatomy, CT-guided renal access may be an option (Turk et al., 2010). The most common access is through the posterior lower pole calyx. It is the safest access point due to absence of major blood vessels (so called Brodel's avascular zone) and low risk of pleural injury. In selected cases (e.g.complex staghorn stones) a supra-costal upper pole access or even multiple punctures have been used. After successful puncture of the renal collecting system, dilatation of renal tract is performedusing Amplatz system, balloon or metallic dilators (choice depends on availability, experience and cost). After insertion of the nephroscope, the stone is disintegrated using ultrasound, laser, or pneumatic energy. Fragments are removed using suction (via ultrasound probe) or specially designed forceps. In complicated cases (or when second intervention is necessary), a self-retaining balloon nephrostomy tube tamponades the tract and maintains access to the collecting system. Although standard nephroscopes have shaft calibres of 24-30 F, "mini-perc" instruments are now available with shaft calibres of 12-20 F. 
Mini-perc is the method of choice for PCNL in children (Turk et al., 2010). The value of miniperc in adults hasn't been established (as treatment time increases with stone size and also with decreased instrument size- mini-perc in adults would be recommended only for $<2 \mathrm{~cm}$ stones where many experts would argue that alternative options- ESWL or URS would be preferred). In uncomplicated cases, tubeless PCNL (with or without application of sealant or JJ stent) has been mentioned over the last few years as an alternative (Turk et al., 2010). The percutaneous route has also been used to treat PUJ (Pelvi-ureteric Junction) obstruction as a concurrent procedure to stone treatment.

The major complications of PCNL are infection, bleeding and internal organ injury. Many of the larger stones are either infection stones or cause obstruction. In view of this, perioperative antibiotic prophylaxis is a standard of practice. The choice of antibiotic is guided by preoperative urine cultures and local antibiotic policies.

Bleeding following PCNL can be severe enough to require blood transfusion but rarely needs intervention such as embolisation or Nephrectomy. Internal organ injury can affect pleura (risk higher if attempting upper pole puncture), bowel (risk especially if bowel lies behind kidney- CT is therefore important to show the bowel position and plan the access) and rarely liver or spleen.

\subsection{Relief of ureteric obstruction}

Endoscopic placement of ureteric stents (ie Retrograde stent insertion) is the commonest method of relieving ureteric obstruction. Stents are either barium impregnated polymer compounds or metallic. Drainage occurs either through or around the tubes. The JJ shape contains coils at both ends to help retain the stent in its position. Retrograde stenting is used for both intrinsic and extrinsic malignant obstruction and less successfully for retroperitoneal obstruction due to fibrotic benign pathology. Prophylactic stenting is used prior to ESWL for large stones where the fragments could obstruct the ureter (ie Steinstrasse). Post-operatively stent placement is used to ensure urinary drainage following prolonged/ complicated ureteroscopy or PUJ procedures.

Retrograde stenting is performed usually under anaesthetic. The ureteric orifice is visualised at cystoscopy, it is then cannulated with an open-ended polymer catheter and retrograde studies are performed to outline the ureter and renal collecting system. A guide-wire is passed through the ureteric orifice into the collecting system (ideally upper pole calyx). The appropriate stent (dependent on patient height) is inserted over the guide-wire, under X-ray guidance, so that the upper end of the stent is in the correct position in renal collecting system. The guide-wire is then removed and both ends of stent subsequently coil (they have "memory").

The most common adult stents are 4.8-6 Fr and 22-28 cm long. Some stents have a hydrophilic coating (makes them more slippery and easier to insert), some are multi-length (no need to adjust according to patient's height). Endopyelotomy stents are wider towards the upper end (10-14 Fr)- to keep PUJ wide open while it heals.

The complications commonly associated with stent usage are storage LUTS (frequency, urgency) and haematuria. Stent migration is less common and can occur in both directions. Probably the biggest concern is stent calcification due to "forgotten stent". If only the lower end of the stent is calcified treatment can usually be completed endoscopically. Calcification of a stent above the bladder will require ureteroscopic lithotripsy, PCNL or even open surgery to facilitate removal. 


\section{Organ ablative surgery}

While the majority of lower tract and stone surgical treatments are now performed endoscopically the field of organ ablation remains the domain of the "open" surgeon. However that is changing and started when the first laparoscopic trans-abdominal nephrectomy was performed in 1990 by Clayman and colleagues at Washington University. The progress of endoscopy techniques in organ ablative urological surgery is mirrored in the changed attitudes to laparoscopic nehrectomy and hence this procedure will be the focus of this section.

\subsection{Laparoscopic nephrectomy}

Following the initial nephrectomy, techniques have evolved so that laparoscopic radical nephrectomy is almost the standard procedure for T1- T2 tumours (those limited to the kidney and less than $7 \mathrm{~cm}$ i.e. $\mathrm{T} 1$ or less than $10 \mathrm{~cm}$ i.e. T2) where renal sparing procedures are inappropriate. In experienced hands $\mathrm{T} 3$ tumours (i.e. involving renal vein or vena cava) can also be removed using this endoscopic technique. The Oncological outcomes are almost identical with disease free survival rates of $94 \%$ and $95 \%$ respectively for the open and laparoscopic approaches (Hemal 2007). When morbidity and return to normal function are analysed it appears that the laparoscopic approach to nephrectomy is better in all measured outcomes apart from a prolongation of operative time (Nandis 2008).

Indeed the debate around nephrectomy now centres on whether the NOTES approach to nephrectomy should be the method of choice. Preservation of renal tissue may be associated with an independent reduction in cardio-vascular and all causes mortality rates (Go 2004). This has led to the increased utilisation of partial nephrectomy for T1a (ie $<4 \mathrm{~cm}$ ) lesions. While the open approach to partial nephrectomy is the more popular approach there are an increasing number of centres performing laparoscopic partial nephrectomy. For a more detailed description of laparoscopic techniques I would suggest Bishoff and Kavoussi`s Atlas of Laparoscopic Urologic Surgery (2007) as an introductory text.

Currently, in the field of organ ablative urology, laparoscopic techniques (including robotics) are commonly being used for nephrectomy, nephro-ureterectomy and radical prostatectomy. Their use in procedures such as retro-peritoneal lymph node dissection or radical cystectomy remains to be proven.

\section{Conclusion}

To the reader of this chapter, it may appear that we have only provided a summary of modern urology. Of itself this impression should illustrate clearly the central place of endoscopy and endoscopic techniques in modern urological practise. Obviously we could not describe all endoscopic techniques used in urology but we have tried to describe the more commonly performed procedures. In places we have shown how developments are further improving efficacy of endoscopic techniques and increasing the range of conditions treated by endoscopic means.

\section{References}

Albala, D.M.; Assimoss, D.G.; Clayman, R.V.; Densted, J.D.; Grasso, M. et al. (2001). Lower pole I: a prospective randomised trial of extracorporeal shock wave Lithotripsy and 
percutaneous nephrolithotomy for lower pole nephrolithiasis- Initial results. J Urol, 166, pp. 2072-2080

A. Alcaraz, L. Peri, A. Molina, I. Goicoechea, E. García, L. Izquierdo, M. Ribal. (2010) Feasibility of Transvaginal NOTES-Assisted Laparoscopic Nephrectomy. European Urology, Volume 57, Issue 2, Feb 2010. Pages 233-237 ISSN 03022838

Anger JT, Weinberg A, Suttorp MJ, Litwin MS and Shekelle PG. Outcomes of Intravesical Botulinum Toxin for Idiopathic Overactive Bladder Symptoms: A Systematic Review of the Literature. J Urol 2010; 183(6): pp 2258-2264.

Bishoff JT and Kavoussi LR (eds). (2007) Atlas of Laparoscopic Urological Surgery. 2007 Saunders, ISBN-13: 978-1-4160-2580-1, Philidelphia.

Cauberg, E.C.C.; De Bruin, D.M.; Faber, D.J.; Van Leeuwen, T.G.; De la Rosette,J.M.C.H; De Reijke, T.M.; (2009). A New Generation of Optical Diagnostics for Bladder Cancer: Technology, Diagnostic Accuracy, and Future Applications. European Urology, Vol. 56, Issue 2, August 2009, pp. 287-297, ISSN 03022838

Denstedt, J.; Khoury S. (2008). Stone disease (2nd international consultation on stone disease), Editions 21, ISBN 0-9546956-7-4, Paris, France

Geavlete P, Georgescu D, Niță G, Mirciulescu V, Cauni V. (2006) Complications of 2735 retrograde semi-rigid ureteroscopic procedures: a single centre experience. J Endourol 20, Mar 2006 , pp 179-185.

Go AS, Chertow GM, Fan D, McCulloch CE \& Hsu CY. (2004) Chronic kidney disease and the risks of death, cardiovascular events and hospitalisation. N Engl J Med 351; (13): September 232004 pp 1296-1305.

Hemal AK, Kumar A, Kumar R, Wadhwa P, Seth A \& Gupta NP. (2007) Laparoscopic versus open radical nephrectomy for large renal tumours: a longterm prospective comparison. J Urol; 177(3) March 2007: pp 862-866

Herr H. Early history of treatment of bladder tumours from Grunfeld`s polypenkneipe to the Stern-McCarthy resectoscope. (2006) J Endourl 2006; 20 (2); pp 85-91

McAllister WJ. Benign Prostatic Hyperplasia in Arya, M.; Shergill, I.S.; Kalsi J.S.; Muneer A.; Mundy A.R. (2010). Viva Practice for the FRCS (Urol) Examination, Radcliffe Publishing Ltd, ISBN-13: 978184619317 0, Oxon, United Kingdom

Nanidis TG, Antcliffe D, Kokkinos C, Borysiewicz CA, Darzi AW, Tekkis PP \& Papalois VE. (2008) Laparoscopic versus open donor nephrectomy in renal transplantation: a meta-analysis. Ann Surg 247(1) January 2008; pp 58-70

Okhunov Z, Hruby GW, Mirabile G, Marruffo F, Lehman DS, Benson MC, Gupta M \& Landman J. (2009). Prospective comparison of flexible fibreoptic and digital cystoscopies. Urology 2009; 74: 427-430

Pansadoro V and Emiliozzi P. (1996) Internal Urethrotomy in the management of anterior urethral strictures: long-term followup. J Urol 1996; 156(1):73-75

Parra RO, Andrus CH, Jones JP \& Boullier JA. Laparoscopic cystectomy: Initial report on a new treatment for the retained bladder (1992). J Urol 148: pp 1140-1144

Pearle MS, Lingeman JE, Leveillee R, Kuo R, Preminger GM, Nadler RB, Macaluso J, Monga M, Kumar U, Dushinski J, Albala DM, Wolf JS Jr, Assimos D, Fabrizio M, Munch LC, Nakada SY, Auge B, Honey J, Ogan K, Pattaras J, McDougall EM, Averch TD, Turk T, Pietrow P, \& Watkins S. (2005) Prospective, randomised trial comparing Shock wave lithotripsy and ureteroscopy for lower pole caliceal calculi $1 \mathrm{~cm}$ or less. J Urol ; 173(6) june 2005, pp 2005-2009 
Reynard, J.; Brewster, S.; Biers, S. (2009). Oxford Handbook of Urology (2nd edition), Oxford University Press Inc., ISBN 978-0-19-953494-4, New York, United States

Shah J: (2002) Endoscopy through the ages, BJU 2002: 89:645-652

Sylvester RJ, Oosterlinck W \& van der Meijden AP. (2004) A single immediate postoperative instillation of chemotherapy decreases the risk of recurrence in patients with stage Ta T1 bladder cancer: a meta-analysis of published results of randomized clinical trials J Urol 171(6 pt 1) June 2004: pp 2186-2190.

Turk, C.; Knoll, T.; Petrik, A.; Sarica, K.; Seitz, C.; Straub, M.; Traxer, O. (2010). Guidelines on Urolithiasis, EAU (European Association of Urology 2010) 


\section{Part 6}

Pediatric Endoscopy 



\title{
Diagnostic and Therapeutic Sinonasal Endoscopy in Pediatric Patients
}

\author{
Marco Berlucchi ${ }^{1}$, Barbara Pedruzzi ${ }^{1}$, \\ Michele Sessa ${ }^{2}$ and Piero Nicolai ${ }^{2}$ \\ ${ }^{1}$ Department of Pediatric Otorhinolaryngology, \\ Spedali Civili, Brescia \\ 2Department of Otolaryngology, \\ University of Brescia, Brescia \\ Italy
}

\section{Introduction}

Fifty years ago, the extracorporeal cold light and its transmission by glass fibers, along with the Hopkins rod lens system, were introduced. The development and application of these new technologies to upper airways allowed studying, understanding, and improving knowledge of the anatomy, physiology, and diseases of the nasal cavity and sinuses. In particular, some fundamental concepts of modern rhinology are based on endoscopic nasal findings and Messerklinger's investigations of the pathophysiology of sinus mucosa. These studies radically changed traditional understanding of sinus inflammation and revolutionized its treatment using endoscopic conservative surgical management (Messerklinger, 1966, 1967, 1978). In the 1980s, Kennedy (Kennedy, 1985) first utilized this surgical technique in the United States and termed it functional endoscopic sinus surgery (FESS). At the beginning, the technique was performed only for treatment of rhinosinsusitis in adult patients. In following years, the surgical indications were extended to selected malignant neoplasms (Kennedy \& Senior, 1997; Lund, 1997; Nicolai et al., 2009, 2011). Due to the good results observed by FESS, in 1990s the development of smaller endoscopes and instrumentation adapted for pediatric patients was encouraged. For the treatment of recurrent or chronic rhinosinusitis in children, favorable results were obtained with endoscopic surgery (Lusk \& Muntz, 1990; Wolf et al., 1995). During subsequent years, other diseases of sinuses were treated successfully with a nasal endoscopic surgical approach (Triglia \& Nicollas, 1997: Berlucchi et al., 2003, 2010; Woodworth et al., 2004; Nicollas et al., 2006; Durmaz et al., 2008; Al-Mazrou et al., 2009; Presutti et al., 2009; Nicolai et al., 2010). In this chapter, a description of endonasal diagnostic techniques and a brief report of sinonasal disorders that may be effectively treated by FESS in pediatric patients are presented. Finally, fundamental surgical steps and their relation between pediatric endoscopic sinus surgery (PESS) and facial growth is briefly discussed.

\section{Diagnostic nasal endoscopic procedures}

The availability of adequate equipment such as flexible and rigid nasal endoscopes of various degrees and sizes (Fig. 1,2,3) is fundamental to achieve accurate endonasal diagnoses. 


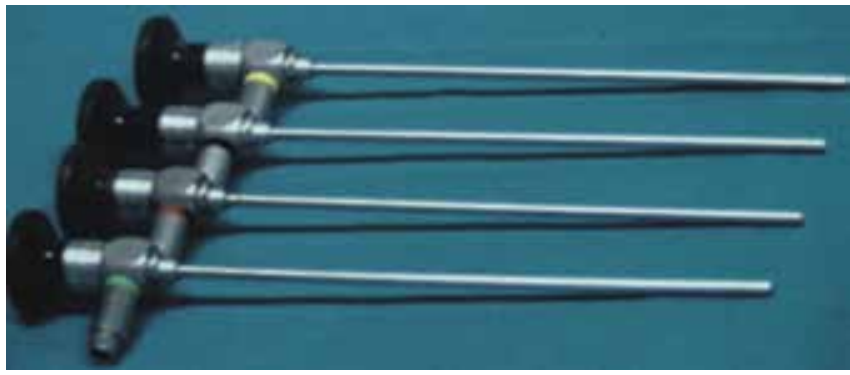

Fig. 1. Nasal rigid endoscopes.

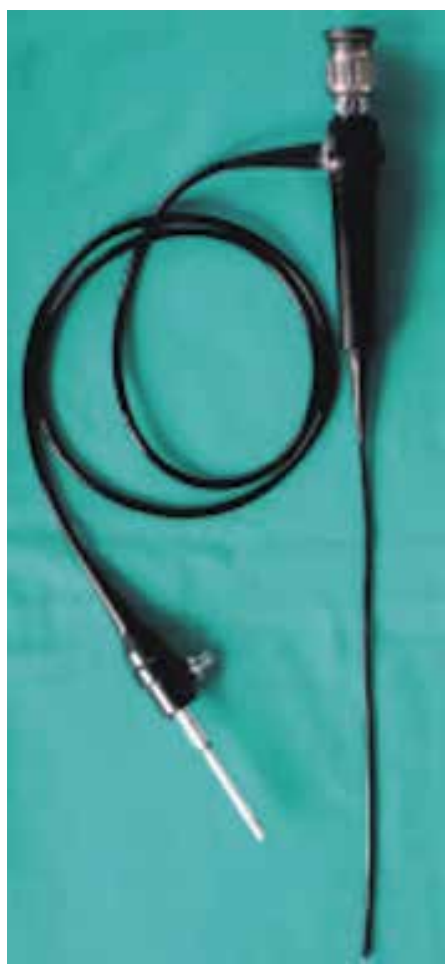

Fig. 2. Flexible endoscope.

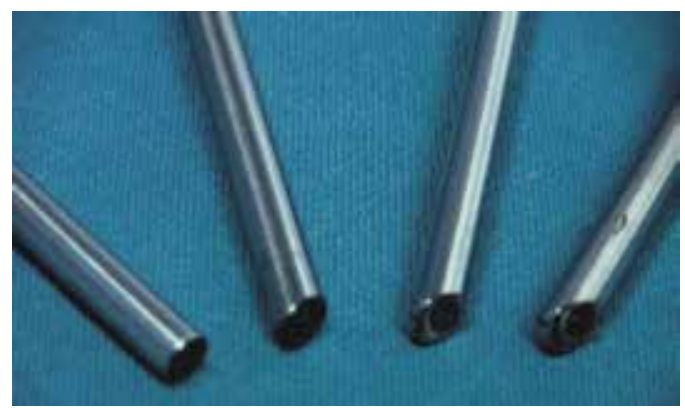

Fig. 3. Tips of the rigid nasal endoscopes of various degrees. 
The choice of nasal endoscope is related to the age and compliance of the pediatric patient. In compliant children and in those older than 8 years, 4-mm and/or 2.7-mm rigid nasal endoscopes are usually well tolerated and provide good endoscopic nasal views. Because of possible traumatic complications, in non-compliant children and in those younger than 8 years, 3.5- $\mathrm{mm}$ and/or $2.5 \mathrm{~mm}$ flexible endoscopes must be utilized even if they provide an endonasal vision that is qualitatively inferior compared to rigid endoscopes. Before performing nasal endoscopy, cottons soaked with decongestant and local anesthetic are placed in the nasal cavities for about 10 minutes. This allows simultaneously augmenting the space of nasal fossae and obtaining a topical anesthetic effect. This may be easily performed in adolescents, whereas in toddlers and non-compliant children a local anesthetic is preferable sprayed in the nasal cavities. In infants and neonates, topical drugs are not generally utilized. During rhinoscopy, the child is placed in either a sitting position or kept in the arms of a nurse in relation to age and compliance. Nasal endoscopy must be performed correctly, meticulously, and accurately to avoid traumatic lesions of endonasal structures. Before starting endoscopic evaluation, whenever possible it is important to explain the diagnostic procedure to the child in the attempt to obtain full collaboration. After removal of cottonoids and treatment of the endoscopic lens with a thin film of anti-fog solution, the endoscope is inserted slowly and delicately in the nasal fossa. First, the floor of the nose and nasal septum, inferior nasal turbinate and its meatus are examined (Fig. 4).

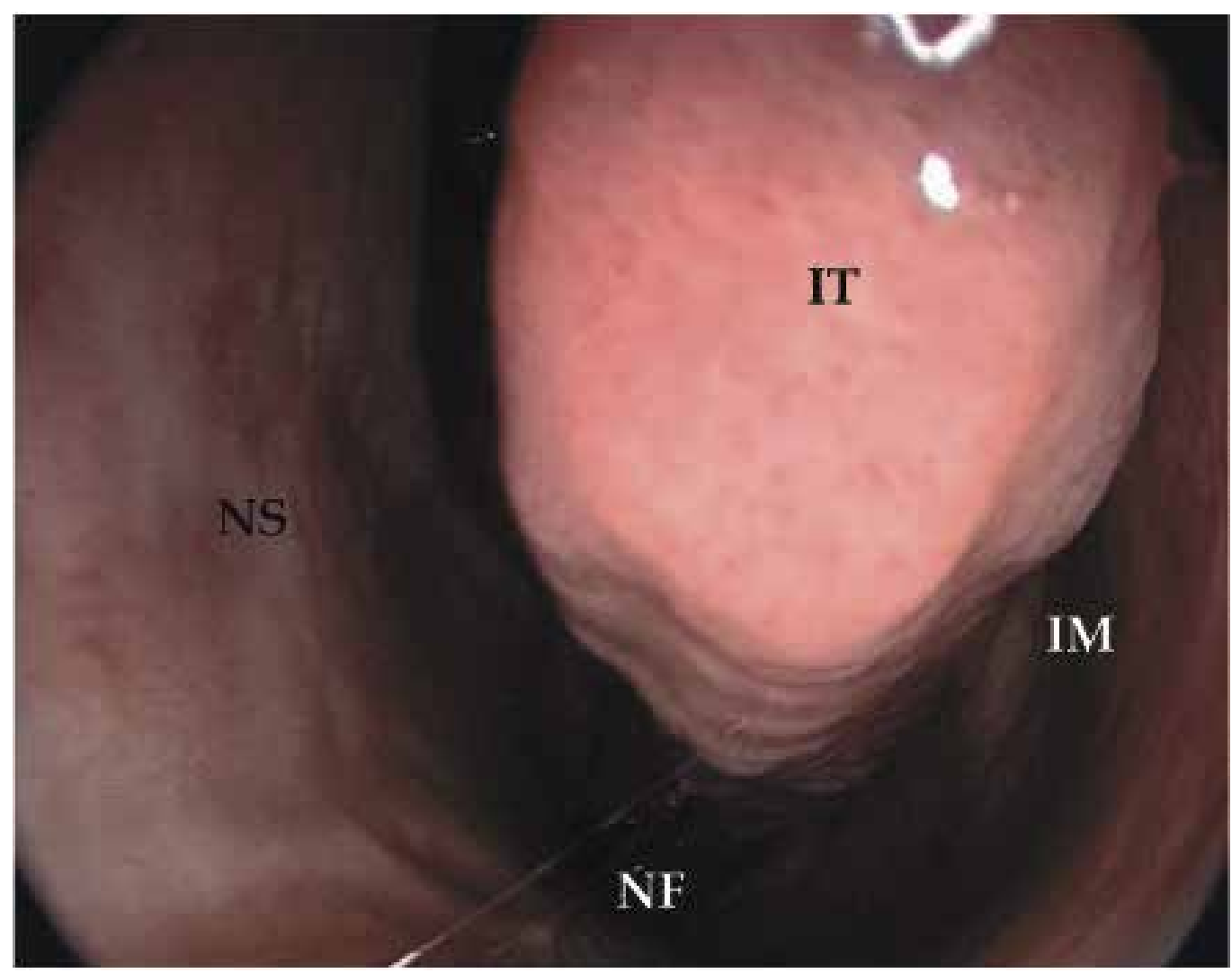

Fig. 4. Endoscopic view of the left nasal cavity: inferior turbinate (IT), inferior meatus (IM), nasal septum (NS), and nasal floor (NF). 
Advancing posteriorly, the entire nasopharynx, Eustachian tube orifices, and torus tubarius can be assessed (Fig. 5).

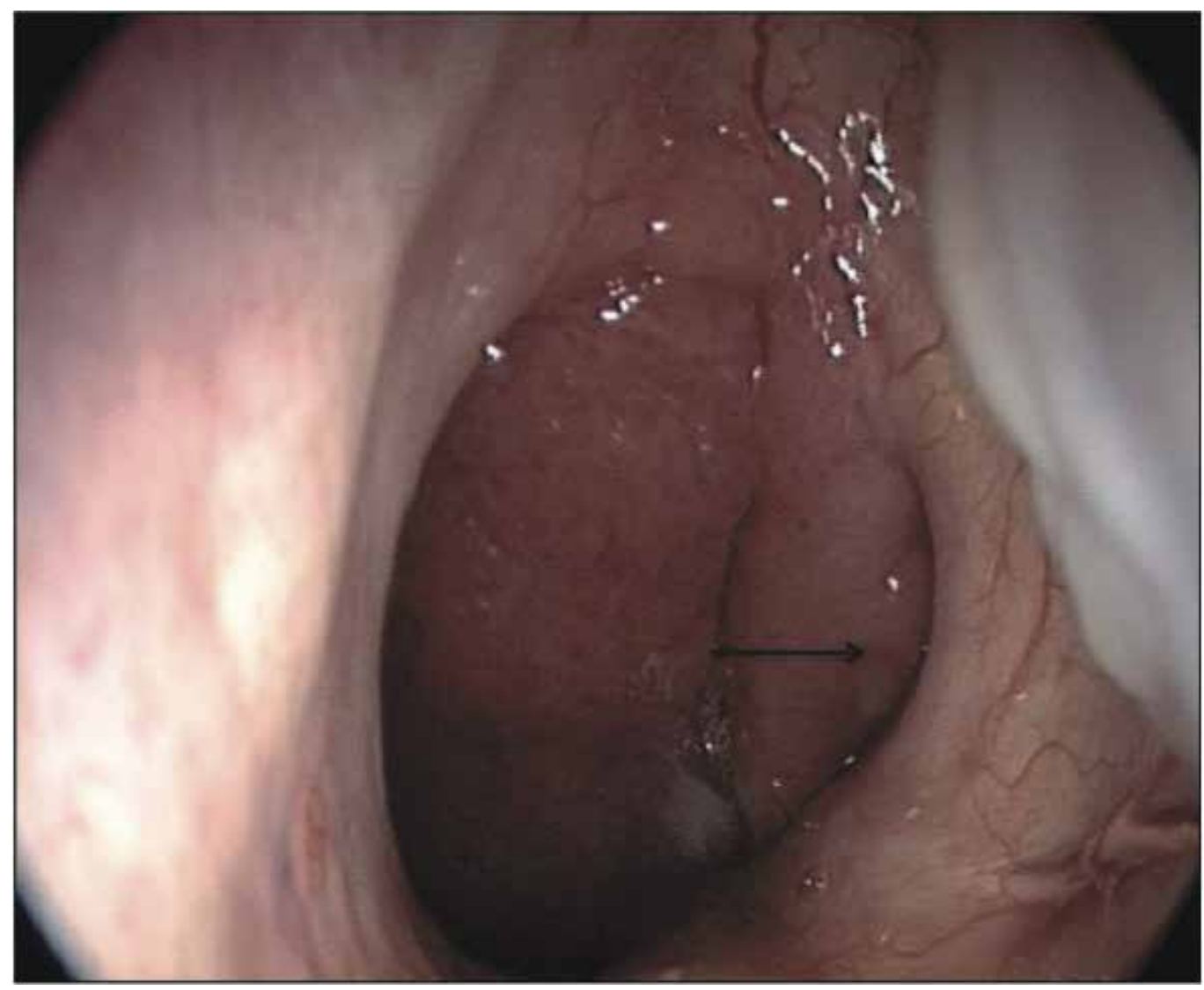

Fig. 5. Nasopharynx and left Eustachian tube orifice (arrow) at endoscopic evaluation.

Afterwards, coming back and turning the endoscope superiorly, the middle nasal turbinate and its meatus are explored (Fig. 6).

When the endoscope moves toward the uncinate process area, fontanellae, accessory maxillary sinus ostia, and sphenoethmoid recess can be assessed. By rotating the endoscope superiorly when it is located anteriorly to the head of middle turbinate, it is possible to observe the anterior olfactory region. In addition to evaluation of nasal anatomy, rhinoscopy allows assessment of mucosa status, the presence and type of endonasal secretions (i.e., serous, mucous, or purulent discharge) and their suspicious origin, associated disorders, and their relationships with surrounding structures. Furthermore, rhinoscopy allows monitoring sinonasal diseases such as rhinosinusitis and adenoid hypertrophy, as well as postsurgical follow-up of nasal sinuses. It can also evaluate response to medical treatment, ease cavity debridement in the post-operative period to favor healing of the sinuses, and identify persistent or early recurrences of sinonasal lesions. 


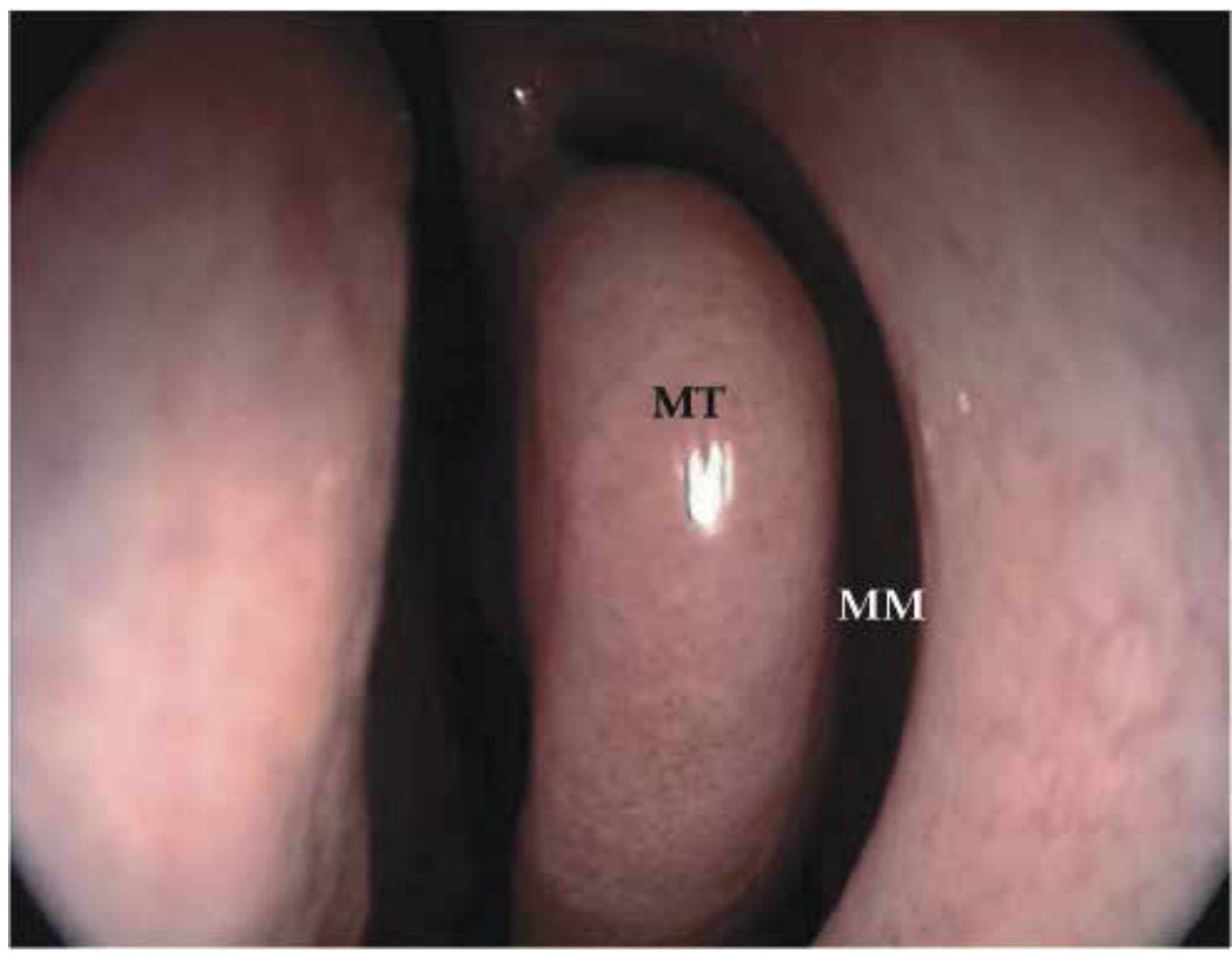

Fig. 6. Endoscopic examination of the head of the middle turbinate (MT) and middle meatus (MM).

\section{Sinonasal disorders treated by endoscopic sinonasal surgery}

Numerous sinonasal diseases can be successfully treated by endoscopic sinus surgery. Extensive surgical experience is mandatory to treat some sinonasal lesions and to obtain good results. Several sinonasal pathologies will be briefly discussed.

\subsection{Inferior turbinate hypertrophy}

Inferior turbinate hypertrophy can be either congenital or acquired. The former is rare, whereas the latter is usually due to septal deviation, allergic rhinitis, or gastroesophageal reflux disease (Kwok et al., 2007; Cingi et al., 2010). The primary presenting symptom is nasal obstruction occasionally associated with seromucosal rhinorrhea, itching, and sneezing. Moreover, chronic nasal obstruction may modify the normal function of the Eustachian tube causing effusion in the middle ear (Pelikan, 2009). Diagnosis is made by nasal endoscopy. Rhinomanometry in basal conditions and after decongestion can be added in selected cases.

\subsection{Adenoid hypertrophy}

Adenoid hypertrophy is probably the most frequent pathology in the pediatric population. This disorder manifests usually between 3 and 6 years of age in both sexes. Children 
complain of bilateral nasal obstruction associated with snoring, rhinorrhea, mouth breathing, hyponasal speech, and cough (Berlucchi et al., 2007). In some cases, obstructive sleep apnea syndrome can also be observed. The pathology may lead to cardiorespiratory syndromes such as cor pulmonale in extreme cases. Furthermore, adenoid hypertrophy may favor other illnesses such as recurrent and effusive otitis media and recurrent/chronic rhinosinusitis. Nasal endoscopy is the gold standard diagnostic technique to evaluate adenoid size, inflammatory and infectious status, and its anatomical relationship with the nasopharyngeal orifice of Eustachian tubes. Moreover, it allows checking changes in adenoid size after medical therapy (Cassano et al., 2003; Berlucchi et al., 2007). At endoscopic assessment, adenoids appear as a single pyramid-shaped aggregation of lymphoid tissue with the apex pointed toward the nasal septum and the base at the level of the superior and posterior wall of the nasopharynx. The adenoid pad appears as a lobulated and pinkish mass, partially or totally occupying the nasopharynx (Fig. 7).

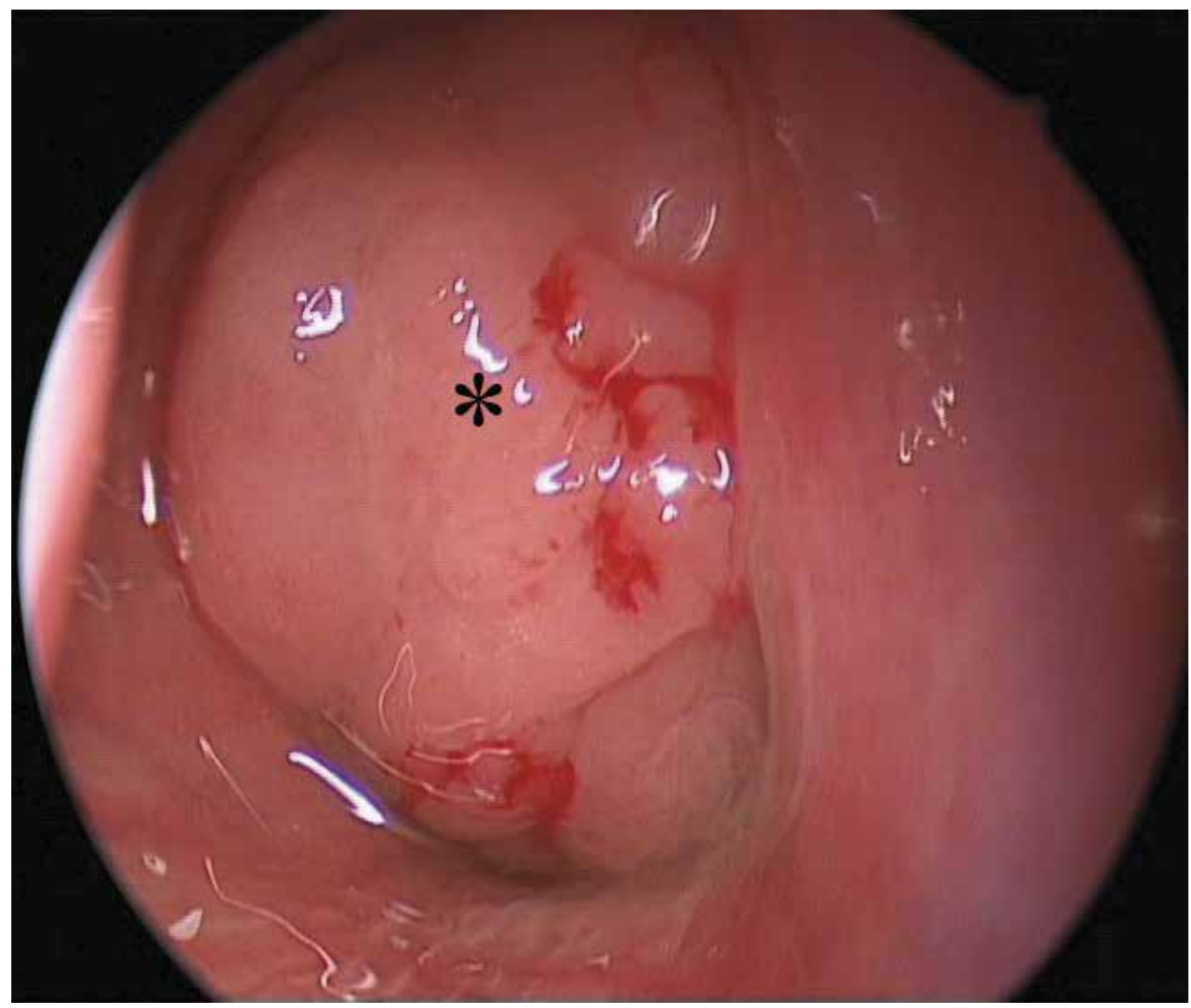

Fig. 7. Adenoid hypertrophy (asterisk) totally obstructing the right nasal fossa.

\subsection{Sinonasal polyposis}

Sinonasal polyposis is an uncommon pathology in pediatric subjects (Triglia \& Nicollas, 1997). In the 1990s, the disorder was classified in 5 subtypes: antrochoanal polyps (this 
lesion will be described separately due to its peculiar characteristics), choanal polyps, polyps associated with chronic rinosinusitis (non-eosinophil dominated), polyps associated with chronic rinosinusitis (eosinophil dominated), and polyps associated with specific illnesses such as cystic fibrosis, Kartagener's Syndrome, and asthma (Stammberger, 1999). Even though the etiology of sinonasal polyposis is unknown, some predisposing factors have been identified. The lesions affect both sexes and can be either monolateral or bilateral. Clinically, children complain of nasal obstruction, rhinorrhea, reduction of the sense of smell or anosmia, headache, and facial pain (Triglia \& Nicollas, 1997). At nasal endoscopy, polyps show a characteristic edematous and translucid appearance (Fig. 8).

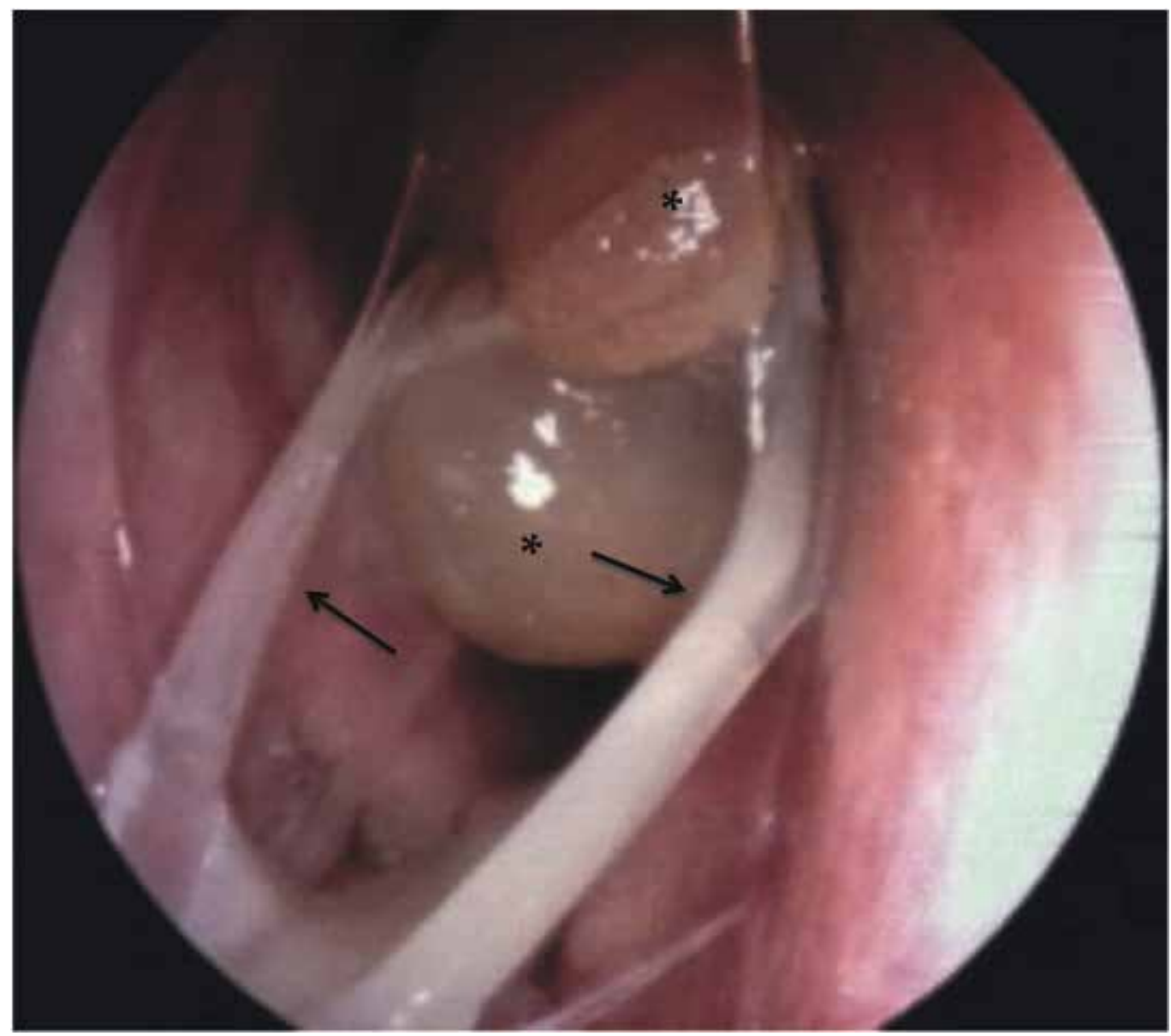

Fig. 8. Nasal polyps (asterisks) associated with mucous secretion (arrows) in a patient with cystic fibrosis.

They can fill partially or totally the nasal cavity and may be associated with a broad or narrow pedicle. Imaging is the diagnostic technique of choice, and CT of the sinuses is the gold standard procedure as it shows exact extension of disease and presence of anatomic anomalies, which may favor sinonasal polyps and/or influence surgical strategy (Triglia \& Nicollas, 1997). 


\subsection{Antrochoanal polyp}

First described by Paefyn in 1753 (Paefyn, 1753), antrochoanal polyp (ACP) or Killian's polyp is a benign, solitary, nasal polypoid lesion. It represents $4-6 \%$ of all nasal polyps in the general population (Yaman et al., 2010). It is also is prevalent in the pediatric age, and ACP is found in about one-third of pediatric cases with polyps (Schramm \& Effron, 1980; Basak et al., 1998; Ozdek et al., 2002; Yaman et al., 2010). The mass originates inside the maxillary sinus and as it grows it extends from the accessory or natural ostium of maxillary sinus to the middle meatus (Fig. 9), finally protruding toward the choana in the nasopharynx.

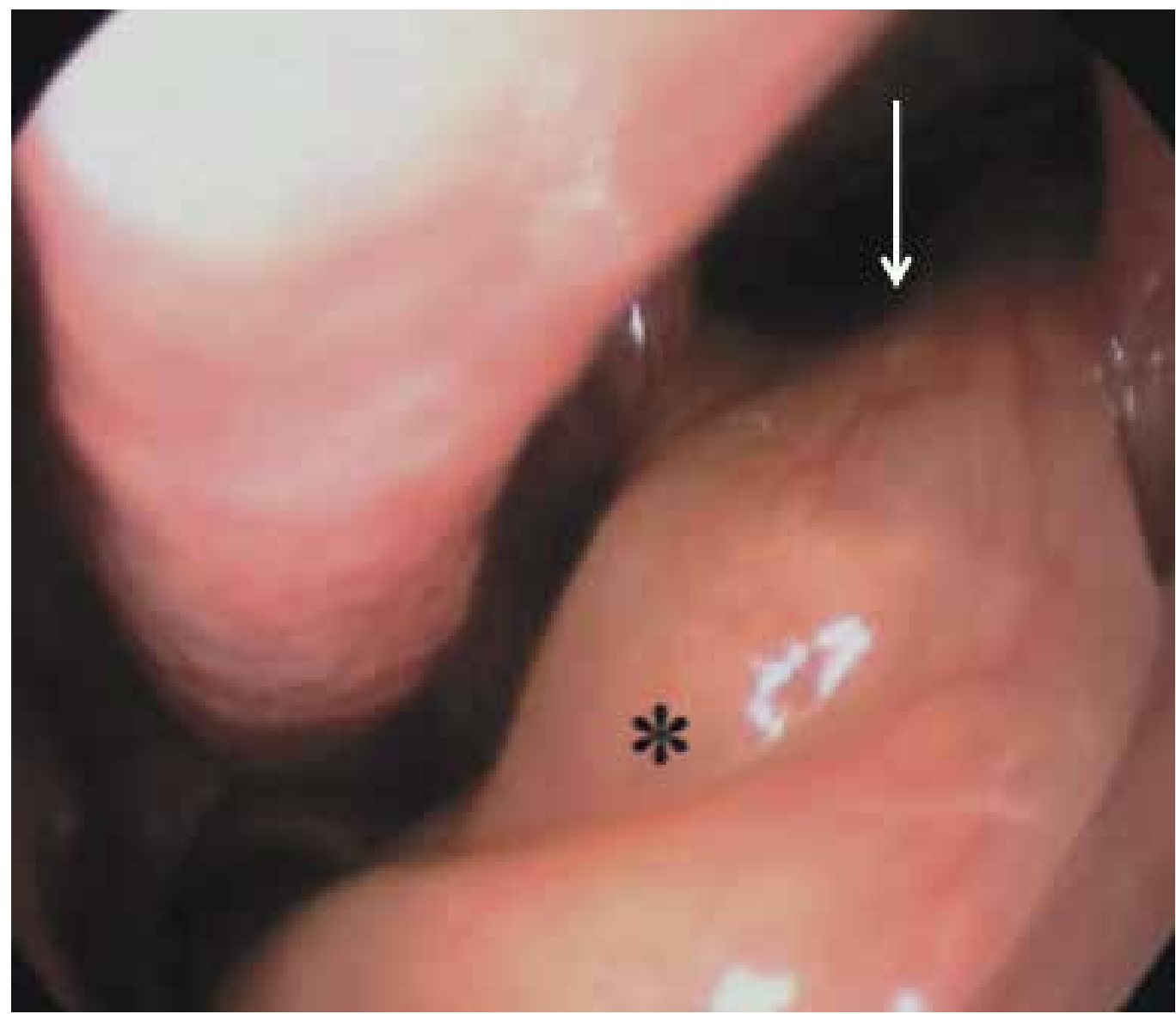

Fig. 9. Left antrochoanal polyp (asterisk) that come out from natural ostium (white arrow) of maxillary sinus.

From an etiological point of view, ACP develops from intramural Tornwaldt's cyst in the wall of the maxillary sinus. This particular origin reflects the presence of cysts in the antral portion of the polyp (Berg et al., 1988; Skladzień, 2001). Chronic sinus inflammation and allergy are other factors favoring formation of ACP (Skladzień et al., 2001). The disorder, whose etiology is still unknown, is usually unilateral and more frequent in males (M:F=2.1:1). ACP is composed of cystic and solid portions. The former occupies the maxillary sinus, while the latter, which generally emerges through an enlarged maxillary 
accessory ostium, is found in the nasal fossa. The most common symptoms are unilateral nasal obstruction, rhinorrhea, bleeding, headache, snoring, and foreign body sensation (Orvidas et al., 2001; Aydil et al., 2008). Moreover, in 20-25\% of cases nasal obstruction may be bilateral, in relation to complete blockage of the nasopharynx. Moreover, some reports have described dysphagia and dyspnea correlated with mouth extension. Nasal endoscopy and $\mathrm{CT}$ are the gold standard diagnostic procedures. At endoscopic examination, the lesion appears as a white and bright mass located in the middle meatus. This mass juts out the maxillary sinus and occupies the nasal fossa (Frosini et al., 2009). At imaging, ACP fills the maxillary sinus growing through the accessory or natural ostium into the middle meatus to the choana (Pruna et al., 2000). By MR, the lesion reveals hypointense T1 and enhanced T2 signals, and the cystic part is enhanced in the peripheral area after intravenous gadolinium administration (De Vuysere et al., 2001).

\subsection{CSF leak}

Cerebrospinal fluid (CSF) leak occurs when there is abnormal communication between the space containing CSF around the brain (subarachnoid space) and the sinonasal tract and/or the ear (middle ear/mastoid system) (Pianta et al., 2005). It implies a breach of the underlying dura mater and adherent pia-arachnoid mater resulting in a pathological communication between the intracranial cavity and either the nasal or middle ear cavity (Lloyd et al., 2008; Presutti et al., 2009). According to Ommaya's classification (Ommaya, 1976), CSF leaks can be divided into non-traumatic (with high or normal CSF pressure) and traumatic (accidental or iatrogenic lesion). About $80 \%$ and $16 \%$ of CSF leaks are due to head trauma and sinuses or skull base surgery, respectively (Beckhardt et al., 1991). Spontaneous fistulae, which are more frequent in obese females in the fourth decade of life (Pianta et al., 2005), represent 3-4\% of cases (Beckhardt et al., 1991; Yerkes et al., 1992; Nachtigal et al., 1999; Schlosser \& Bolger, 2002). Moreover, skull base tumors or other congenital lesions (such as untreated aqueductal stenosis) may cause CSF leaks directly through erosion of the skull base or indirectly through the development of hydrocephalus. Other congenital causes of CSF leak are the developmental of skull base defects with associated meningoceles, meningoencephaloceles, large arachnoid granulations or cysts, or congenital inner ear anomalies (Lloyd et al., 2008). If the pathogenesis of traumatic fistula is intuitive, spontaneous leaks may have a multifactorial origin. Among these, intracranial pressure, brain pulsation, cranial base pneumatization, and arachnoid pits are thought to play a major role (Pianta et al., 2005). Spontaneous CSF fistula occurs commonly at the ethmoid roof, cribriform plate, perisella of sphenoid sinus, or inferolateral or pterygoid recess (Lloyd et al., 2008). Patients with CFS leak suffer from unilateral or bilateral watery persistent or intermittent rhinorrhea with positive history for a previous head trauma or surgery of the sinonasal tract, middle ear/mastoid, or skull base. Increase of postnasal drip in the supine position may be reported. Moreover, patients can complain of a salty or sweet taste in the mouth. Recurrent meningitis should alert the physician to a diagnosis of CSF leak (Pianta et al., 2005). Intermittent clear nasal discharge may be exacerbated by the Valsalva maneuver and/or compression of both internal jugular veins (Pianta et al., 2005). When the lesion is located in the temporal bone, CSF reaches the nasopharynxc via the Eustachian tube and becomes evident in most cases as bilateral clear rhinorrhea (Pianta et al., 2005). Patients with intermittent CSF leak complain frequently of headache, which appears whenever rhinorrhea stops and the CSF pressure increases (Beckhardt et al., 1991). Finally, signs and symptoms 
such as headache, vomit, or edema of the papilla are suggestive for intracranial hypertension (Pianta et al., 2005). "Reservoir sign" is a feature suggestive for the presence of a CSF fistula at the sphenoid, and is due to accumulation of CSF in the sphenoid sinus when the patient is recumbent. It remains in the sinus until the patient resumes an erect position and the head is leaned forward. At that moment, fluid exits from sphenoid ostium and sudden profuse rhinorrhea becomes evident (Nuss \& Costantino, 1995). Diagnosis of CSF leak includes laboratory testing, imaging, and fluorescein test. The former includes dosage of several proteins (i.e., beta-2 transferrin or beta-trace protein) on the watery fluid collected from the nose. These are polypeptides produced in the brain, leptomeninges, or choroid plexus that may be identified in nasal mucus when CFS leak is present (Bachmann et al., 2000; Lloyd et al., 2008). Radiological procedures such as CT and MR are used to localize and characterize the involved site, to evaluate for an underlying cause, and to exclude an associated meningocele or meningoencephalocele (Lloyd et al., 2008). Finally, fluorescein test is performed by intra- or peri-operative intrathecal injection of dye solution diluted with $10 \mathrm{ml}$ of CFS (Pianta et al., 2005). This can allow localization of the site of leak and ensure successful closure during surgical intervention.

\subsection{Rhinosinusitis}

As rhinitis and sinusitis are usually simultaneous, the use of the term rhinosinusitis is medically correct. This disorder is a common upper airway infection in the pediatric age. It is an inflammation of nasal cavity and sinuses and is characterized by two o more symptoms one of which should be either nasal obstruction or nasal discharge associated or no with facial pain/pressure and reduction or loss of smell. Based on duration of symptomatology, rhinosinusitis can be divided into: 1) acute rhinosinusitis, when total resolution of aforementioned symptoms may take up to 12 weeks; 2) chronic rhinosinusitis, when clinical picture persists for more than 12 weeks; and 3) recurrent acute rhinosinusitis, when multiple acute rhinosinusitis occurs with total resolution of each acute episode (Fokkens et al., 2007). Several predisposing factors such as allergy, adenoid mass, gastroesophageal reflux disease, sinonasal anomalies (i.e., septal deviation, concha bullosa, Haller cell, choanal atresia, and paradoxical middle turbinate), immunological disorders, primary ciliary dyskinesia, cystic fibrosis, exposure to tobacco, and daycare attendance have been noted to favor rhinosinusitis (Lusk, 1992, 1997; Clement 2008). The classical triad, which is generally responsible for upper respiratory infections (i.e., Streptococcus pneumoniae, Haemophilus influenzae, and Moraxella catarrhalis), has been shown to be involved in most acute rhinosinusitis as well. Staphylococcus aureus and anaerobes can be occasionally found (Lieser \& Derkay 2005). Clinically, rhinosinusitis is characterized by rhinorrhea, nasal obstruction, cough, headache, and facial pain. Purulent rhinorrhea, periorbital edema, and high fever may be observed in severe form. Signs and symptoms of chronic rhinosinusitis are those of the non-severe acute form, but they persist for more than 12 weeks. At rhinoscopy, diffuse mucosal inflammation associated with turbinate congestion is the typical endonasal endoscopic appearance of acute rhinosinusitis (Fig. 10).

Mucopurulent secretions can be also present and, in relation to their site, it is possible to suspect which sinuses may be affected. Purulent secretions located at middle meatus or sphenoethmoid recess are a sign of involvement of maxillary, ethmoid, and/or frontal sinus and sphenoid sinus, respectively. Under endoscopic control, cultures can be taken directly 


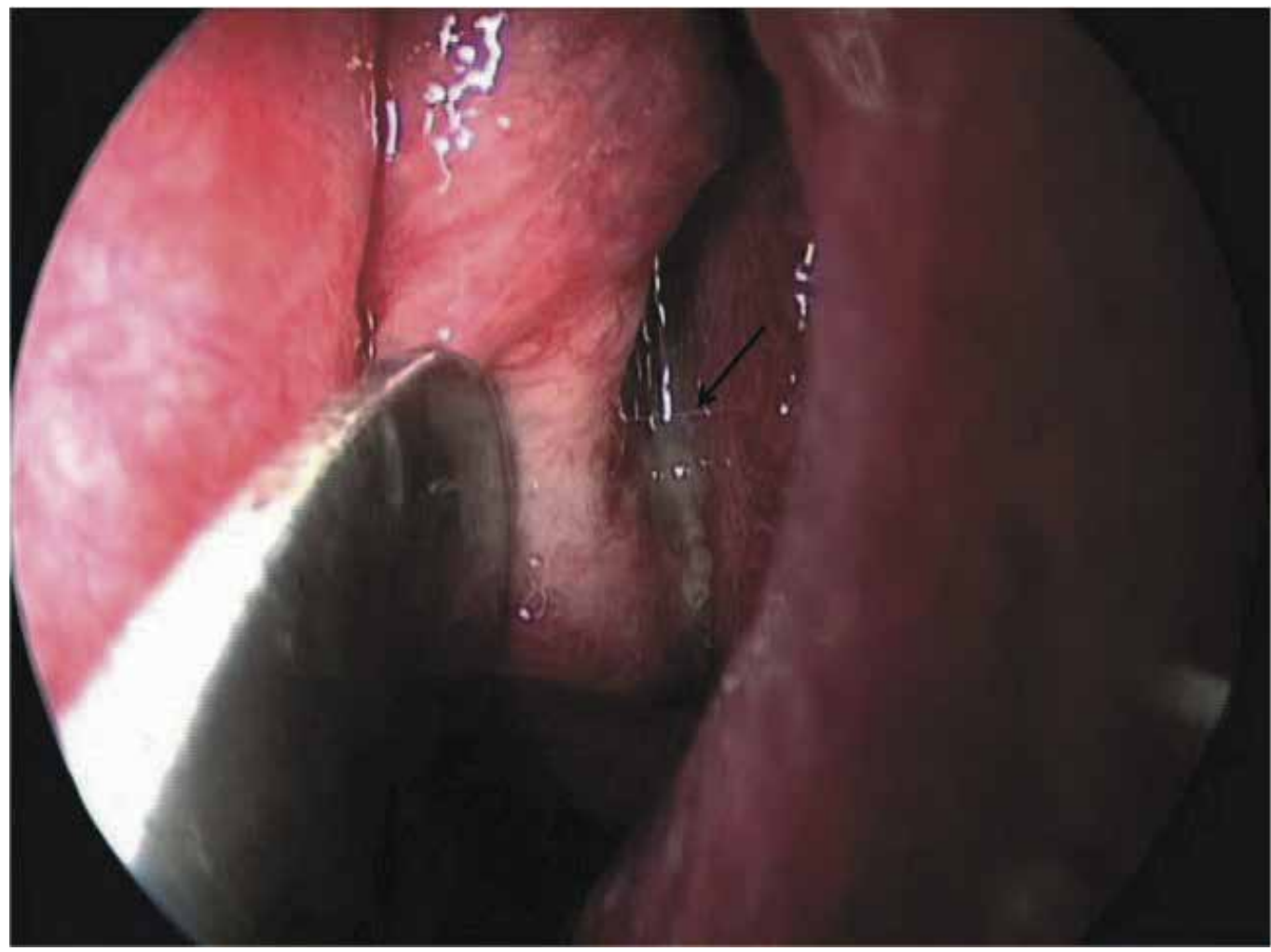

Fig. 10. Diffuse nasal mucosal inflammation and congestion of middle turbinate. After medial dislocation of middle turbinate, purulent secretion is found in the middle meatus (arrow).

from the involved meatus. Polypoid changes around the middle turbinate insertion is indicative of inflammation of the frontal sinus, whereas the presence of polyps suggests chronic rhinosinusitis (Joe et al., 2001). Moreover, nasal endoscopy allows monitoring inflammation and objectively evaluating the response to treatment. For this reason, serial endoscopic nasal examinations are mandatory to individualize therapy and, eventually, to modify antibiotic administration when no improvement is observed. Diagnosis is based on careful assessment of the patient's history and clinical picture. In dubious cases, endoscopy of nasal cavity can confirm clinical suspicion. Microbiological cultures are not routinely necessary, but when sinus infection does not improve using antibiotic therapy within 48-72 hours, occurs in an immunocompromised patient, the child is toxic or extremely ill, suppurative complications are evident, or when infectious sinonasal illness recurs 1-2 weeks after the end of medical therapy, microbiological evaluation is mandatory (Lusk \& Stankiewicz, 1997; Clement, 2008). Imaging is not indicated to confirm a diagnosis of rhinosinusitis. CT is performed after failure of medical therapy and, therefore, in the planning of surgery or when surgical treatment may be considered as in the aforementioned pathological situations (Lusk \& Stankiewicz, 1997). Furthermore, examinations for allergy, cystic fibrosis, immunological disease, gastroesophageal reflux, and primary ciliary dyskinesia can be performed as necessary. 


\subsection{Choanal atresia}

Choanal atresia (CA) is a rare, congenital disease characterized by complete obstruction of the posterior nasal passages. Its incidence is 1:5000-8000 live births (Teissier et al., 2008).

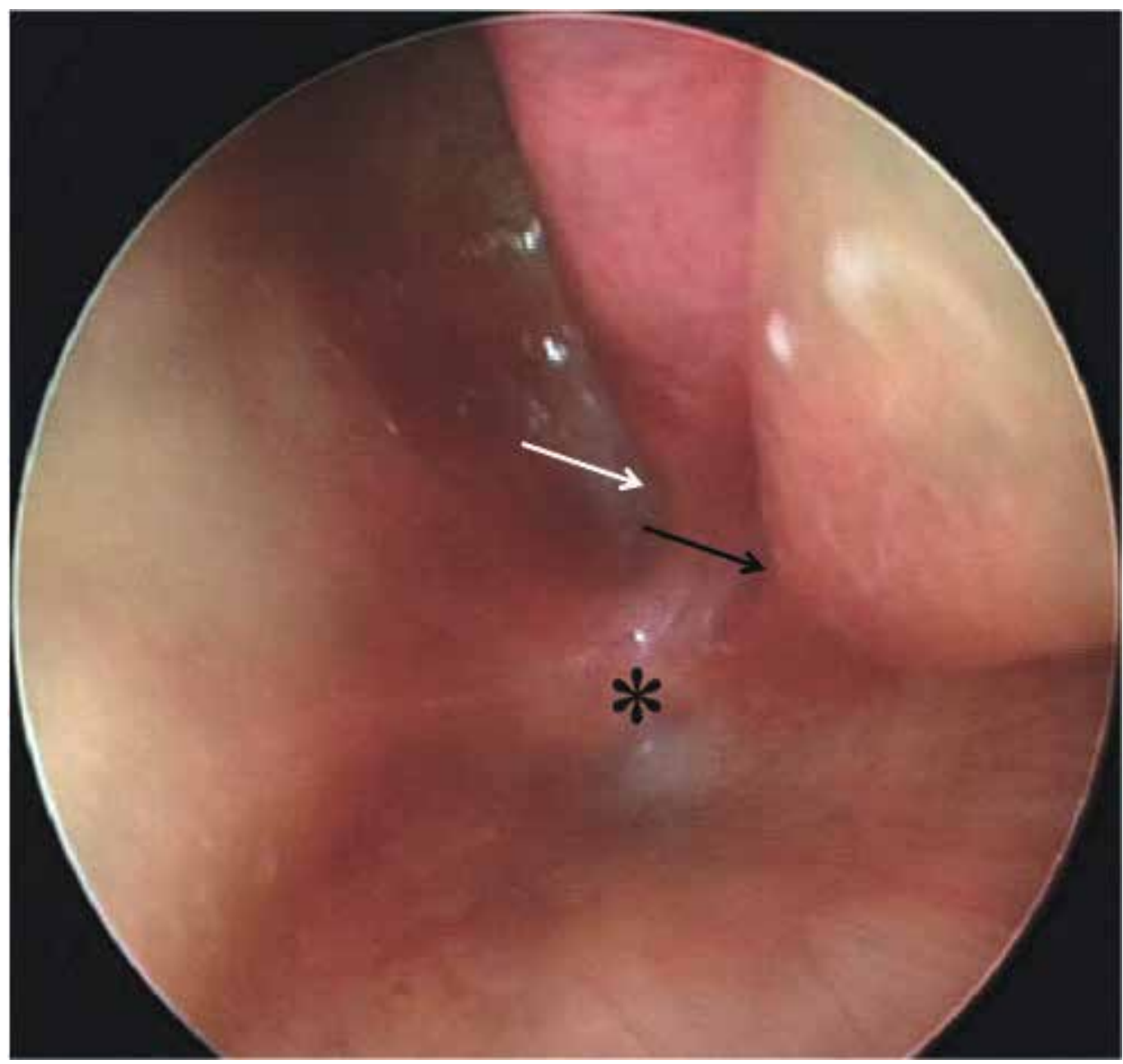

Fig. 11. Endoscopic view of the left choanal atresia (asterisk). The inferior turbinate (black arrow) and middle turbinate (white arrow) are also evident.

There is a female predominance with a F/M ratio of 5/1 among Caucasians. The lesion may be unilateral $(60 \%)$ or bilateral $(40 \%)$ and can subdivided into bony $(90 \%)$ or membranous $(10 \%)$ types (Vatansever et al., 2005). The genetic aspect of CA remains unclear and is likely multifactorial. In $50 \%$ of patients, CA is associated with other anomalies as in the CHARGE syndrome (coloboma, heart abnormalities, CA, retarded growth and development of central nervous system, genitourinary anomalies, ear defects) (Jyonouchi et al., 2009). Several theories such as persistence of the buccopharyngeal membranes, failure of the oronasal membrane to rupture either the nasobuccal membrane of Hochstetter or buccopharyngeal membrane of the foregut, incomplete resorption of nasopharyngeal mesoderm, or locally misdirected mesodermal flow have been proposed to explain the occurrence of CA, but 
none have been universally accepted. This process occurs between the 4th and 11th fetal week (Dunham \& Miller, 1992; Keller \& Kacker, 2000; Samadi et al., 2003). Since neonates are obligate nasal breathing, at birth bilateral choanal atresia can manifest with dyspnea, cyanosis, severe hypoxia, and suckling difficulties, whereas the unilateral form presents monolateral rhinorrhea. Its diagnosis can be late and, often, occasional. Endoscopic examination with flexible nasal endoscope is mandatory when CA is suspected. Endonasal evaluation shows complete closure of involved choana that may be associated with inflammation of nasal mucosa and mucous stagnation (Fig. 11).

Imaging is the next, fundamental diagnostic procedure. CT performed in axial and coronal projections provides a thorough assessment of CA, reveals the bony or membranous nature of the disease, and shows the narrowing of posterior nasal cavity and the thickening of the vomer (Schweinfurth, 2002).

\subsection{Mucocele}

Mucocele is a benign, cyst-like, locally expansile paranasal sinus mass. The pathology consists of accumulation of secretion products, aseptic slimy mucus, desquamation, and inflammation lined by the respiratory mucosa (Marks et al., 1997; Busaba \& Salman, 1999), developing within a paranasal sinus associated with expansion of its bony walls as a consequence of ostium blockage. A mucocele grows slowly and expands by eroding the surrounding bony walls. The obstruction can result from congenital anomalies, chronic rhinosinusitis, previous radiotherapy and/or surgical treatment, trauma, and sinonasal neoplasms (Johnson \& Ferguson, 1998; Maroldi et al., 2005). Moreover, congenital illnesses such as cystic fibrosis and primary ciliary dyskinesia are considered predisposing factors for occurrence of mucoceles (Guttenplan \& Wetmore, 1989; Thomé et al., 2000; Nicollas et al., 2006; Olze et al., 2006; Berlucchi et al., 2010). Mucoceles occur more frequently in the fourth and fifth decade of life, with a similar distribution in both sexes. Paranasal sinuses mucoceles are extremely rare in a pediatric age and most cases described have been associated with cystic fibrosis (Olze et al., 2006). The frontal sinus is involved in $60 \%$ of cases, followed by the ethmoid labyrinth and maxillary sinus with $30 \%$ and less than $10 \%$ of cases, respectively. Few cases are localized in the sphenoid sinus (Som \& Brandwein, 1996; Arruè et al., 1998; Lloyd et al., 2000; Caylakli et al., 2006). The higher incidence of mucoceles in the frontal sinus seems to be related to anatomical variations of the frontal recess (Arruè et al., 1998; Martin et al., 2000). Mucoceles are usually monolateral, whereas bilateral mucoceles are infrequently observed (Varghese et al., 2004). The clinical picture, which varies in relation to the sinus involved, includes nasal obstruction, rhinorrhea, headache, cheek pressure or pain associated with or without check swelling, maxillary nerve hyperesthesia, infra-orbital anesthesia, dental pain, loosening of teeth, periorbital pain, proptosis, blurred vision, alteration of visual acuity, diplopia, and sudden loss of vision (Avery et al., 1983; Hayasaka et al., 1991; Moriyama et al., 1992; Curtin \& Rabinov, 1998; Busaba \& Salman 1999; Maroldi et al., 2005; Tseng et al., 2005). Whenever erosion of the anterior or posterior wall of the frontal sinus is present, a Pott's puffy tumor or neurological symptoms may be evident (Maroldi et al., 2005). At nasal endoscopy, the appearance varies according to the site of the mucocele and the phase of growth. During the intrasinusal phase, no alterations are generally visible. The subsequent expansion of the mucocele may alter the paranasal sinus bony walls. In a maxillary localization, medialization of the middle turbinate, anterior dislocation of the uncinate process (Fig. 12), 


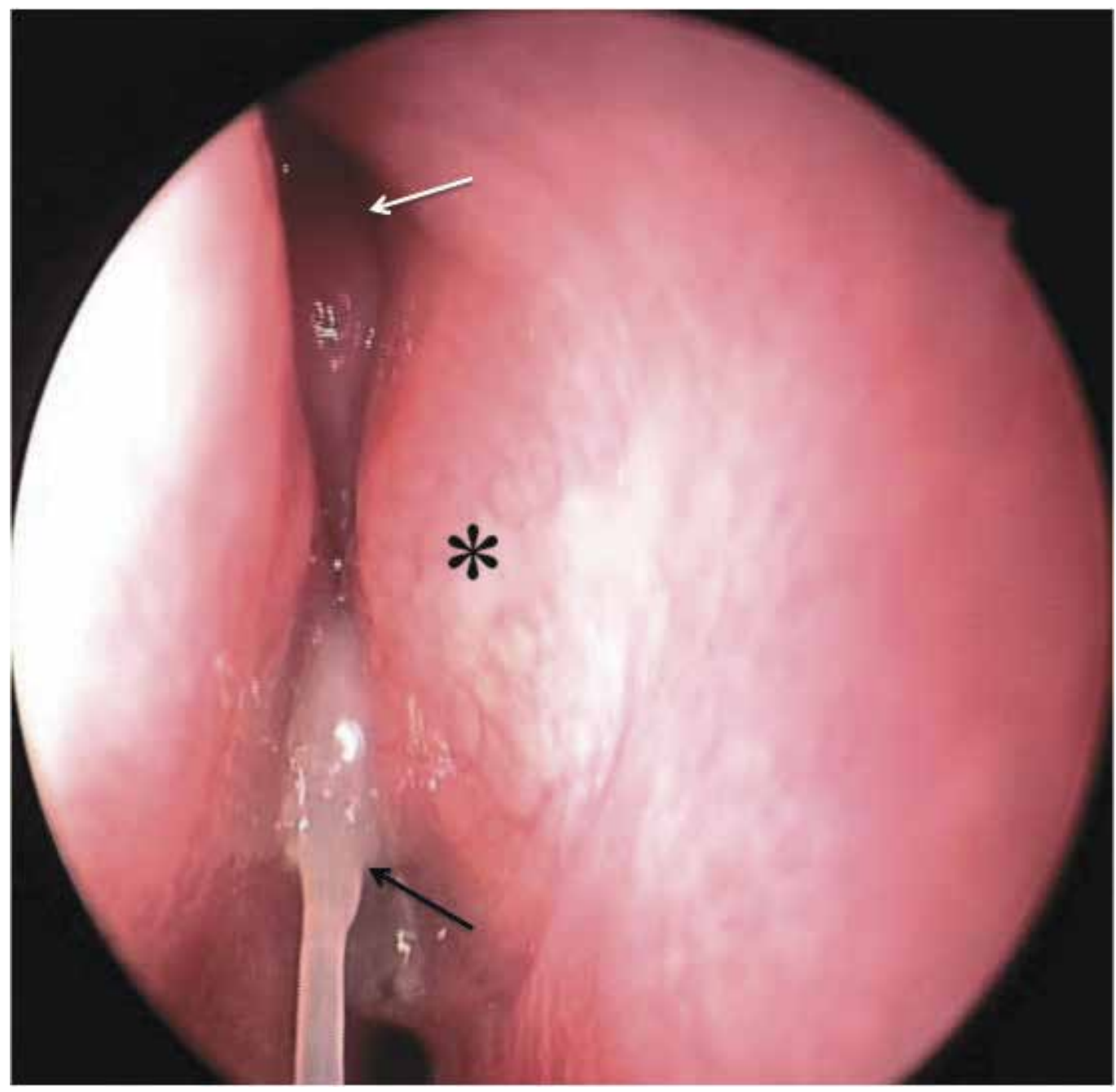

Fig. 12. Anterior displacement of the left uncinate process (asterisk) due to mucocele of the maxillary sinus at endoscopic examination. Mucous secretion (black arrow) and middle turbinate (white arrow) are also observed.

and bulging of the agger nasi cells or the infundibular area can be observed, whereas submucosal remodeling or bulging of the sphenoethmoid recess or posterior ethmoid can be evident in sphenoidal mucoceles. In frontal mucoceles, endoscopic examination is usually negative (Maroldi et al., 2005) since the lesion has expanded inferiorly to involve the agger nasi. Diagnosis is based on signs and symptoms, nasal endoscopic evaluation, and imaging. By CT, the disease appears as a homogenous lesion that completely occupies the involved sinus with smooth clear-cut margins of bone erosion of its walls (Han et al., 1995; Busaba \& Salman, 1999). Moreover, CT shows the site and extension of the disease, remolded cortex, bony erosion entity, anatomical variants, and hyperostotic changes, (Maroldi et al., 2005). MR is usually performed when mucocele formation is secondary to sinonasal soft tissue tumors in which the lining membrane of the mucocele will enhance after intravenous contrast (Jayaraj et al., 1999). 


\subsection{Meningoencephalocele}

Cephalocele or encephalocele (EC) is an extracranial extension of any intracranial structure through a congenital or acquired defect of the skull base (Pianta et al., 2005). Such herniation may be represented by the leptomeninges associated with cerebrospinal fluid or it can also include the brain. The former is defined meningocele (MC), whereas the latter is termed meningoencephalocele (MEC) (Naidich et al., 1992). The incidence of EC ranges from 1 case/5,000 live births in Thailand to about 1 case/40,000 live births in western countries (Mahapatra \& Suri, 2002). The disorder may be divided into occipital, parietal, basal, and syncipital types (Mc Carty et al., 1990). The latter group is subdivided into fronto-ethmoidal and interfrontal subtypes, and those associated with craniofacial clefts (C. Suwanwela \& N. Suwanwela, 1972). The fronto-ethmoidal form, which accounts for about $10 \%$ of all meningoceles, includes: 1) naso-ethmoidal form that is the herniation of meninges with or without brain tissue through the anterior cranial base at the level of the foramen caecum between nasal bone and nasal cartilage; 2) naso-frontal form that occurs between nasal and frontal bones; and 3) naso-orbital form that develops between the maxilla and lacrimal bones. MEC is located at the occipital region in $75 \%$ of cases, followed in order of frequency by the frontoethmoidal and parietal area in about $15 \%$ and $10 \%$ of patients, respectively. (Hoving, 2000; Mahapatra \& Aqrawal, 2006). The neural tissue in MEC was initially considered dysplastic and non-functioning, but since functioning brain has been found in some occipital and trans-sphenoidal MEC, this concept has been recently revisited (Pianta et al., 2005). MEC may cause nasal obstruction and CSF rhinorrhea. This latter symptom can be unilateral or bilateral, persistent or intermittent, and it increases or may be elicited by maneuvers elevating CSF pressure such as compression of the internal jugular veins or the Valsalva maneuver (Pianta et al., 2005). Moreover, MEC can promote alterations and distortions of surrounding facial structures such as displacement of the medial orbital wall, orbit, telecanthus, broad nasal bridge, nasal and/or glabellar swelling, and hypertelorism. Ocular and lacrimal signs and symptoms (i.e., decrease of visual acuity, strabismus, epiphora and/or dacryocystitis) can be observed (Lello et al., 1989; Morris et al., 1989). At nasal endoscopic evaluation, the lesion may appear as a smooth, isolated, pulsatile polypoid mass arising from the olfactory fossa or sphenoid sinus (Samii \& Draf 1989; Pianta et al., 2005). The site of the lesion may increase upon jugular vein compression (Furstenberg sign). In addition to evaluation of the clinical picture and nasal endoscopy, diagnostic work-up of MEC must include imaging. CT can show bony defects of the craniofacial junction and the sclerotic margins of the bone defect (Pianta et al., 2005), whereas MR may reveal the relationship with brain.

\subsection{Lacrimal duct stenosis}

With an incidence ranging from 6 to $84 \%$, congenital lacrimal duct obstruction is a common disorder at birth. Fortunately, most cases resolve spontaneously within the first months of life. The remaining patients will require conservative procedures (lacrimal probing and intubation) and, if symptomatology persists, non-conservative management (dacryocystorhinostomy) will be performed (Berlucchi et al., 2003). The pathology is due to lack of canalization of the lacrimal system that generally intervenes at the distal end (Hasner's valve). Epiphora and recurrent dacryocystitis represent the typical clinical picture observed. Rarely, some patients present bulging of the medial canthus that corresponds to dacryocystocele. This cystic lesion of the lacrimal sac is due to both proximal (Rosenmuller's valve) and distal (Hasner's valve) obstruction. When the lesion expands in the nasal fossa at the level of inferior meatus (Fig. 13), the patient may also complain of different degrees of nasal obstruction in relation to its size (Wong \& VanderVeen, 2008); respiratory distress can also be observed in bilateral localization. 


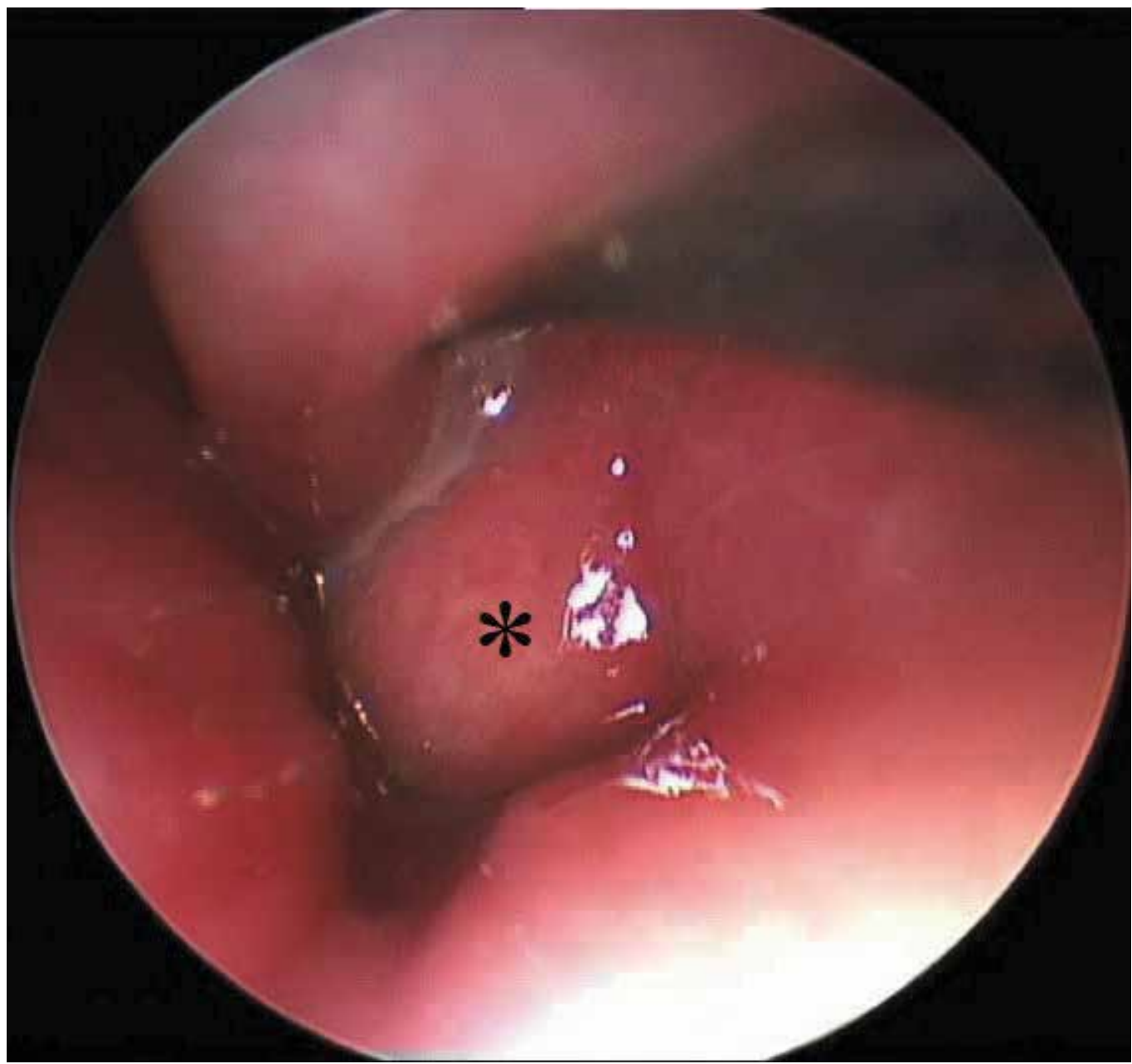

Fig. 13. Endoscopic view of a nasolacrimal duct cyst (asterisk).

At nasal endoscopy, the nasal cavity can be completely normal or, in some cases, a nasolacrimal duct cyst can be identified in the inferior meatus. Ophthalmologic and otorhinolaryngologic evaluation, dacryocystography, and CT of sinuses are the diagnostic procedures indicated or required (Berlucchi et al., 2003).

\subsection{Lobular capillary hemangioma}

Also known as pyogenic granuloma, telengiectasic granuloma, granuloma pedunculatum, and infected granuloma, lobular capillary hemangioma (LCH) is a benign, rapidly growing, painless, easily-bleeding, solitary lesion, which occurs in the skin and mucous membranes (Maroldi et al., 2005). Although several factors (i.e., nasal trauma, hormonal influences, viral oncogenes, underlying microscopic arteriovenous malformations, and the production of angiogenic growth factors) have been advocated to favor this disorder, its etiopathogenesis remains unknown (Puxeddu et al., 2006). In the head and neck area, the lesion commonly occurs in the oral cavity (gingiva, lips, tongue, and buccal mucosa), whereas involvement of the nasal cavity is rare (Simo et al., 1998; Ozcan et al., 2004). Sinonasal localization ranges 
from $7 \%$ to $29 \%$, and the lesion more frequently involves the anterior portion of the nasal septum and the tip of the turbinates (Maroldi et al., 2005). The disease most often occurs in the third decade of life, with a female predominance (El-Sayed \& al-Serhani, 1997; Maroldi et al., 2005), whereas its occurrence in pediatric populations has been only rarely reported (Berlucchi et al., 2010). The most common symptoms of LCH of the nasal cavity are recurrent unilateral epistaxis, nasal obstruction, and nasal discharge; facial pain, hyposmia and alteration of smell, and headache are rarely present (Ozcan et al., 2004; Puxeddu et al. 2006). At nasal endoscopy, the lesion usually appears as a single reddish hypervascularized polypoid mass that bleeds easily (Fig. 14).

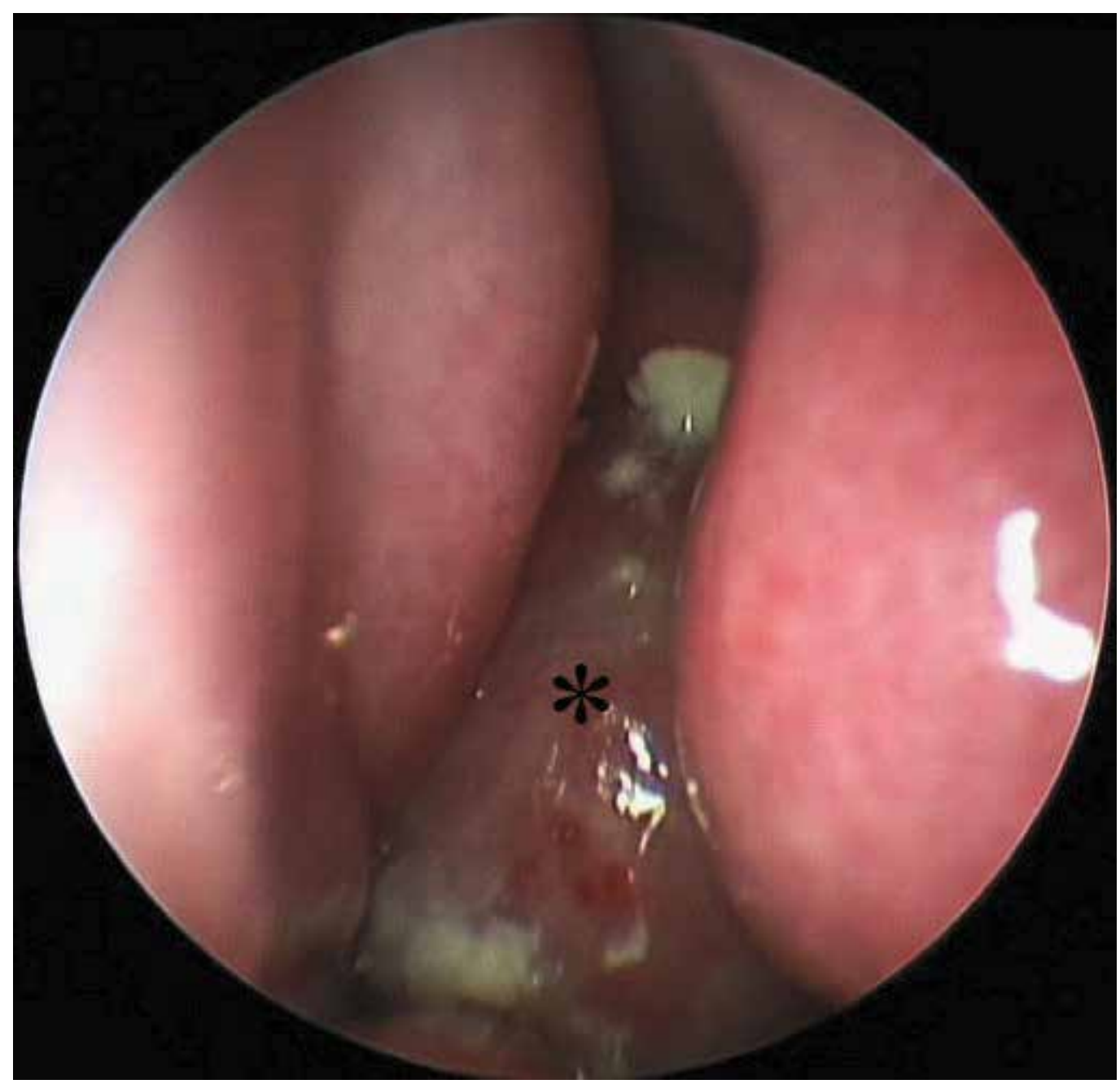

Fig. 14. Lobular capillary hemangioma (asterisk) completely occluding left nasal cavity.

When a nasal LCH is small, diagnosis is not difficult, while problems occur when the mass is relatively large and its macroscopic appearance is unclear. In these situations, imaging is mandatory (Berlucchi et al., 2010) as it reveals important features of the lesion such as size, probable site of origin, and vascularization pattern. CT shows a soft-tissue density nasal 
lesion with lobulated contours. MR reveals masses with an intermediate to hyperintense signal on T2-weighted images and a hypointense signal on T1-weighted images. Enhancement after contrast administration can be helpful (Berlucchi et al., 2010).

\subsection{Nasal glioma}

Nasal glioma (NG), also known as nasal glial heterotopias, brain-like heterotopia, glial hamartoma, heterotopic neuroglial tissue, nasal cerebral heterotopias, cephalic brain-like heterotopias, and nasal heterotopic brain tissue (Rahbar et al., 2003; Pakkasjärvi et al., 2008), is a rare benign developmental abnormality of neurogenic origin. The peak of occurrence is between 5 and 10 years of age, with a male-to-female ratio of 3:2 (Puppala et al., 1990; Vuckovic et al., 2006). The disorder represents $0.25 \%$ of all nasal tumors and accounts for approximately 5\% of all congenital nasal swellings (Dabholkar et al., 2004, Vuckovic et al., 2006). The most widely accepted etiopathogenetic theory is that NG represents an encephalocele that becomes sequestered from the brain early in gestation. This is probably due to an abnormal closure of the nasal and frontal bone (fonticulus frontalis) that can lead to an ectopic remnant of glial tissue that remains extracranially (Ma \& Keung, 2006). Since it is not a true neoplasm, the term NG is actually not correct. The lesion consists of ectopic/heterotopic neural tissue with neuroglial elements and glial cells in a matrix of connective tissue with or without a fibrous connection to the subarachnoid space or dura. It can grow within the nasal region and is covered by skin or respiratory mucosa (Lowe et al., 2000, Vuckovic 2006). Moreover, $90 \%$ of NG do not contain neurons and its benign nature is demonstrated by a low proliferative activity (Dimov et al., 2001). NG can be extranasal (60\% of cases), lying external to the nasal bones and cavities; intranasal (30\%), lying within the nasal cavity (Fig. 15), mouth, or pterygopalatine fossa; or mixed $(10 \%)$, communicating through a defect of nasal bones. Extranasal gliomas that are usually paramedian are generally located at the glabella, but can be also present laterally or at the nasal tip (Uzunlar et al., 2001; Vuckovic et al., 2006). Intranasal lesions are usually located within the nasal passage medially to the middle turbinate bone. The intranasal type is more often associated with dural attachment $(35 \%)$ than the extranasal type (9\%) (Kennard \& Rasmussen, 1990). Finally, combined intra/extranasal gliomas have a typical dumbbell shape with a connecting band (Vuckovic et al., 2006). Patients with NG may complain of nasal obstruction, epistaxis, and cerebrospinal fluid rhinorrhea. Moreover, the lesion can be associated with deformities of the adjacent bones and nasal cartilage such as widened nose and obstruction of the nasolacrimal duct. Hypertelorism, broadening of the nasal bridge, airway obstruction, and epiphora are secondary to growth of the mass (Bradley \& Singh, 1982; Fitzpatrick \& Miller, 1996). At endoscopic view, NGs appear as nonpulsatile, uncompressible, gray or reddish-blue to purple, soft or firm at touch, and polypoid-like lesion. The mass, which is present on the nasal dorsum and/or arises from the lateral nasal wall, may be associated with telangiectasias of the overlying skin (Hengerer \& Newburg, 1990). Neuroimaging is mandatory to identify nasal lesions, to exclude its possible intracranial connection, and to plan the optimal surgical approach (Harley 1991; Hoeger et al., 2001). Because of its potential intracranial connection, excisional biopsy or fine needle aspiration cytology should not be performed due to the risk of meningitis or cerebrospinal fluid (CSF) leak (Claros 1998). 


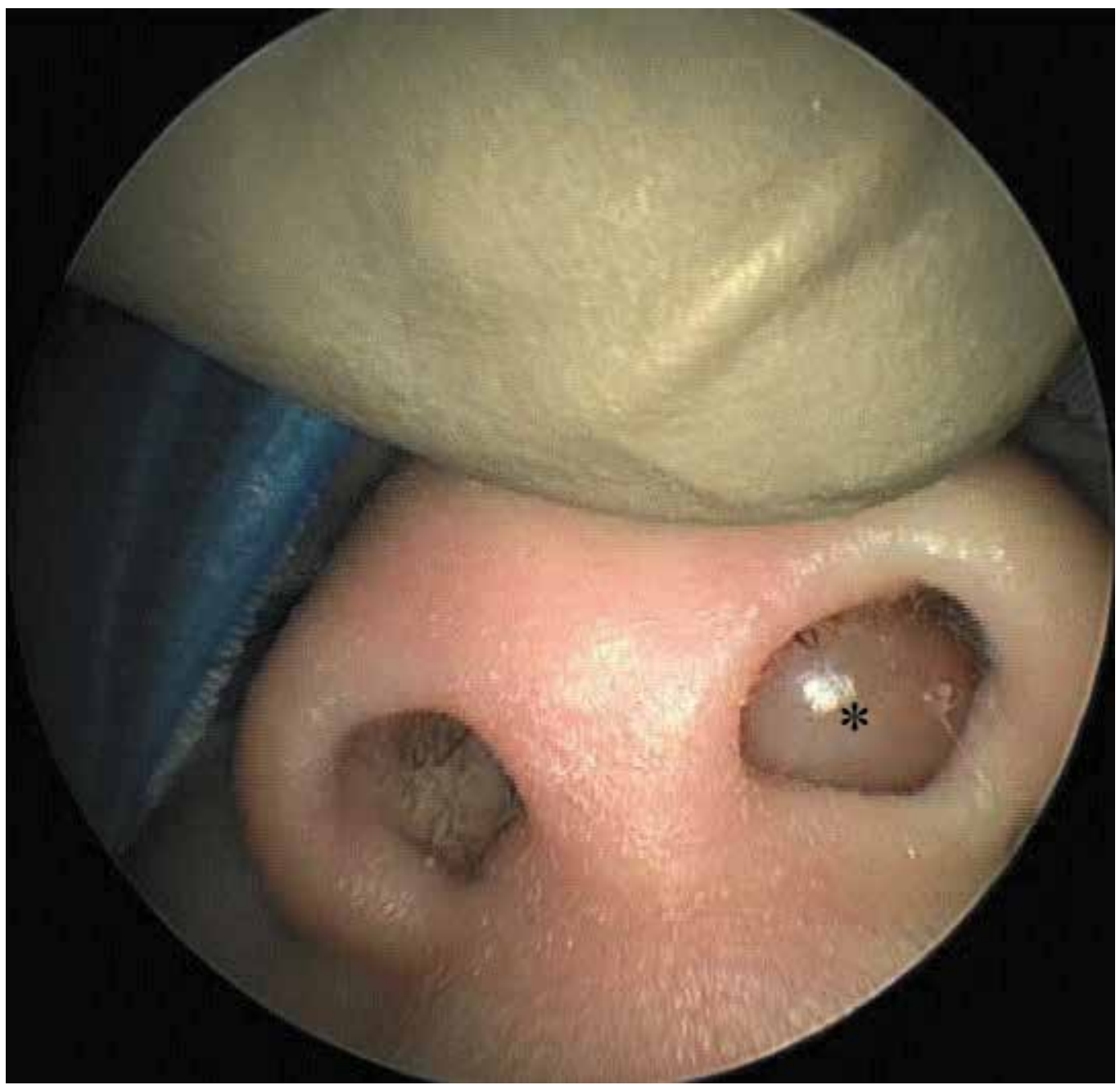

Fig. 15. Intranasal glioma (asterisk) occupying the left nasal pyriform aperture.

\subsection{Juvenile angiofibroma}

Juvenile angiofibroma (JA) is a highly vascular benign and locally invasive lesion that accounts around $0.05 \%$ of all head and neck neoplasms. The disorder typically occurs in adolescent males. Recently, some studies have reported that the lesion has an immunohistological and electron microscopic profile more consistent with a vascular malformation rather with a tumor (Beham et al., 1997, 2000). The site of origin of JA appears to be the sphenopalatine foramen or the bone of the vidian canal. From there, the lesion can expand to the nasopharynx, nasal fossa, paranasal sinuses, and pterygopalatine and infratemporal fossa. In some cases, involvement of the orbit and middle and anterior cranial fossa by bone erosion may be observed (Nicolai et al., 2003). Most patients present nasal obstruction associated with discharge and recurrent, spontaneous epistaxis. Due to enlargement of the tumor, facial swelling, proptosis, headache, cranial nerve palsies, and conductive hearing loss secondary to otitis media with effusion may also be observed. At 
nasal endoscopic evaluation, JA appears as sessile, lobulated, rubbery and red-pink to gray mass covered by several vascular structures (Fig. 16).

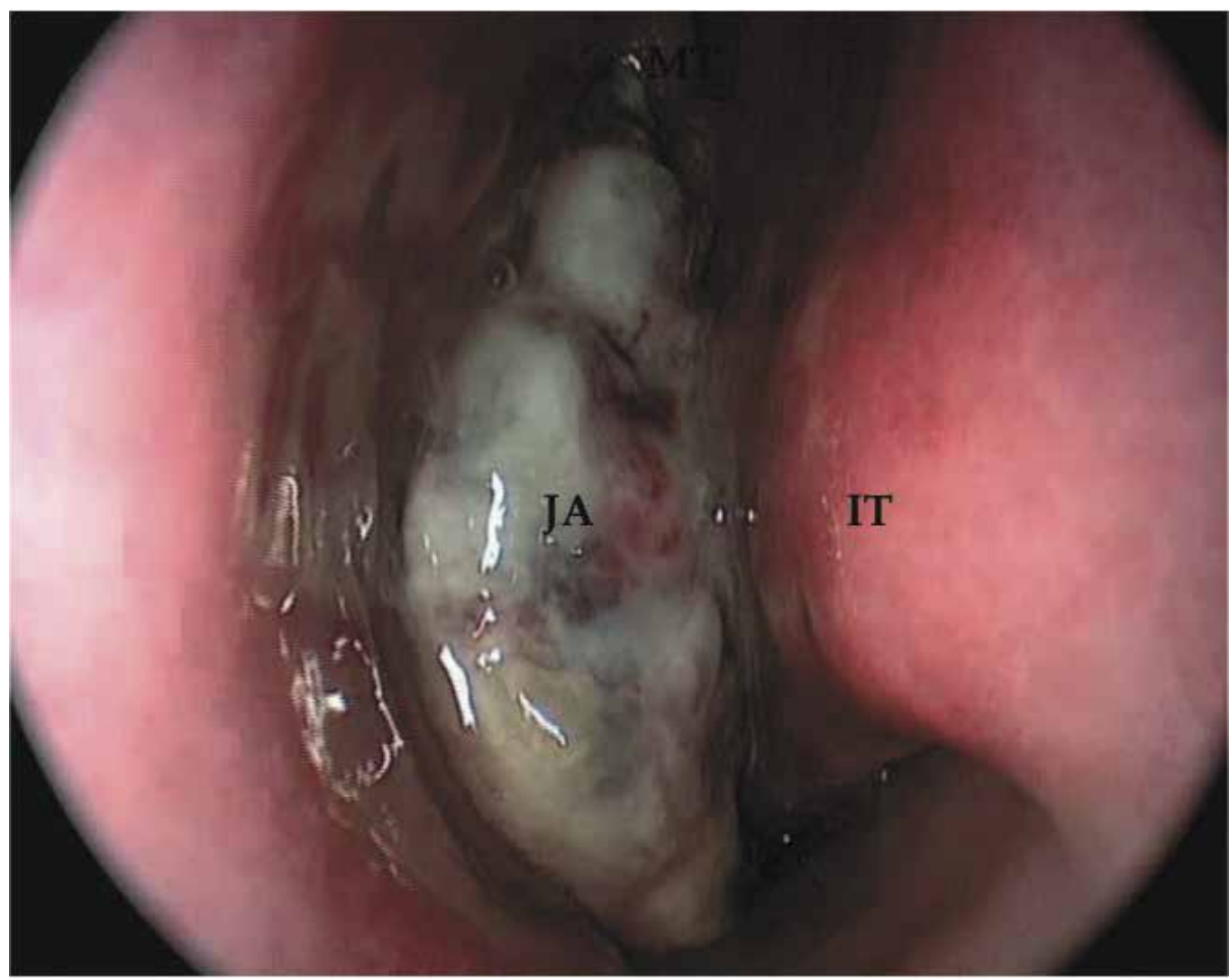

Fig. 16. Juvenile angiofibroma (JA) covered by several fibrin due a recent bleeding in the left nasal fossa. Inferior turbinate (IT).

It occupies usually the nasopharynx and nasal cavity, and it bleeds easily when touched. It may sometimes have a polypoid or pedunculated aspect. Because multiplanar evaluation of the disease and detailed information on the relationship between the lesion and important adjacent structures are needed, MR is considered the gold standard diagnostic procedure. Moreover, before surgical treatment, preoperative diagnostic assessment of the vascular pattern of the lesion by angiography is required, which should be associated with angiographic embolisation to decrease intraoperative bleeding and, consequently, the risk of perioperative transfusion (Nicolai et al., 2003). A biopsy of the lesion is not indicated due to profuse bleeding (Antonelli et al., 1987).

\section{Surgical technique and its influence on facial growth}

Before describing the main surgical procedures, it is fundamental to highlight some general aspects of PESS. 1) The patient must undergo preoperative CT of sinuses to evaluate anatomy, likely type of disease, and extension to plan surgical management. 2) Preoperative 
antibiotic and steroid therapy is also added to reduce inflammation and infection in the sinuses. 3) PESS is always performed under general anesthesia. 4) Endoscopes of different degrees $\left(0^{\circ}, 30^{\circ}, 45^{\circ}\right.$, and $\left.70^{\circ}\right)$ and size $(4$ and $2.7 \mathrm{~mm})$, adult and pediatric instrumentation sets for PESS, and microdebrider must all be available in the operating room. 5) Application of cotton decongestant pledgets in nasal fossae for 10 minutes before surgical management is helpful to increase the nasal space. 6) Surgical management must be conservative and involves only the pathological sinuses. Herein, basal procedures about PESS are reported. Since extensive and advanced endoscopic sinus procedures are beyond the scope of the present chapter, these surgical treatments will not be presented.

\subsection{Middle antrostomy}

Submucosal injection of 1\% mepivacaine chlorohydrate and 1:200,000 epinephrine is given at the level of the root of the middle turbinate and uncinate process. The posterior edge of uncinate process and, when evident, the main ostium of maxillary sinus are probed with a small seeker. Next, partial uncinectomy with conservation of its upper third is performed usually with back-biting forceps. When necessary, the natural ostium of the maxillary sinus can be wided both posteriorly and inferiorly. The risk-areas are nasolacrimal duct, sphenopalatine foramen, and lamina papyracea sited anteriorly, posteriorly, and superiorly, respectively.

\subsection{Anterior and posterior ethmoidectomy}

After removal of the uncinate process, the anterior wall of the ethmoid bulla is evident and may be opened. This surgical step may be performed by a microdebrider or Weil forceps, and must be achieved medially and inferiorly avoiding damage to the orbit and roof of sinus. At this point, basal lamella is exposed. When needed, the basal lamella is perforated by Weil forceps or microdebrider at the infero-medial portion to prevent damage to the lamina papyracea and fovea ethmoidalis which are situated laterally and superiorly, respectively. Next, each bony lamella is opened and removed. During this surgical step, the optic nerve, located posteriorly and superiorly, can be identified.

\subsection{Sphenoidotomy}

This is performed through transnasal, transethmoidal, or trans-septal approach, and the opening of sphenoid sinus is achieved only if the pathology involves this sinus. In this surgical procedure, instruments are utilized at an infero-medial angle to avoid injury of the optic nerve and internal carotid artery, which lie at the lateral wall of the sinus.

\subsection{Frontal sinusotomy}

Frontal sinusotomy is only rarely performed in pediatric patients as sinusotomy of the frontal sinus is highly challenging due to its small recess and anatomical position. A standard anterior ethmoidectomy associated with opening of agger nasi is usually sufficient to identify the frontal recess. If necessary, it can be enlarged using angle circular-biting forceps. It is mandatory do not to strip mucosa to avoid a secondary frontal stenosis.

\subsection{Potential effects of PESS on midfacial and sinus development}

Even though the use of PESS is diffuse worldwide, its potential effects on sinus development and midfacial growth are still object of discussion. In 1995, Wolf et al. 
reviewed 124 children undergoing PESS for chronic recurrent rhinosinusitis. The mean age of patients was 12 years, with 3 children under 5 years. Based on a questionnaire about patient satisfaction and symptomatic relief, it was found that endoscopic surgical sinus surgery had no clinically relevant effects on facial bone development. In our opinion, these results might be influenced by the fact that only $25 \%$ of patients were under the age of 5 years, an age during which there is rapid growth of the sinuses. In 1996, Kosko et al. described 5 children who underwent PESS for recurrent rhinosinusitis at a median age of 30 months. After a mean follow-up of 42 months, these patients still complained of signs and symptoms of recurrent rhinosinusitis. For this reason, CT was performed in all children. Imaging revealed maxillary sinus hypoplasia in all patients without clinically apparent facial asymmetry. The authors concluded that this radiological finding might be related to endoscopic sinus surgery. In 2000, Senior et al. assessed the quantitative long-term impact of PESS on sinus development. In this study, 8 children who underwent PESS for periorbital or orbital sinusitis were reviewed after a mean follow-up of 6.9 years. Control groups included 9 adults without signs of rhinosinusitis on imaging and 10 adult patients with a clinical history of childhood sinus symptoms and CT-positive for rhinosinusitis. No significant differences in sinus volumes were observed among groups. In 2002, Bothwell et al. analyzed the long-term outcome of facial growth after PESS in a retrospective age-matched study. The study and control groups included 46 children who underwent PESS for chronic rhinosinusitis and 21 children who did not undergo intervention, respectively. Quantitative anthropomorphic and qualitative analyses were performed in all cases. No statistical differences in facial growth were identified between the two patient groups.

In 2006, Van Peteghem \& Clement evaluated the influence of PESS on facial growth in a prospective study. The patient cohort consisted of 23 children with cystic fibrosis of whom 13 underwent endonasal surgical treatment for massive nasal polyposis. After a follow-up of at least 10 years, cephalometric measurements were performed in the surgical patients and compared with those obtained in non-surgical group. No significant differences were found. Thus, even if the available evidence seems to indicate that PESS does not significantly affect growth and development of sinuses, analysis of potential surgical effects during rapid growth on facial skeleton has not been well assessed and warrants further investigation.

\section{Conclusion}

The introduction of rigid and flexible endoscopes has radically changed both diagnosis and therapeutic approaches to sinonasal diseases in pediatric patients. Endoscopy of the nasal cavities and nasopharynx permits observation of important anatomical areas that were previously not visible, evaluating macroscopic characteristics of the sinonasal lesions and their relationship with the endonasal structures. When associated with imaging of the sinuses, it may influence therapeutic planning. Consecutive endoscopic nasal procedures can also monitor sinonasal illnesses and their response to medical therapy. Subsequent development of PESS permitted the possibility to perform targeted and conservative treatments. In the post-operative period, rhinoscopy facilitates accurate debridement of nasal fossae and sinuses, promoting their healing. Finally, the performance of regular endoscopic nasal follow-up may identify early recurrences of sinonasal pathologies. 


\section{References}

Al-Mazrou, K.A.; Al-Qahtani, A. \& AL-Fayez, A.L. (2009). Effectiveness of transnasal endoscopic powered adenoidectomy in patients with choanal adenoids. Int J Pediatr Otorhinolaryngol, Vol.73, No.12, (December 2009), pp. 1650-2

Alvarez, R.J. \& Liu, N.J. (1997). Pediatric ethmoid mucoceles in cystic fibrosis: long-term follow-up of reported cases. Ear Nose throat J, Vol.76, No.8, (August 1997), pp. 538-9, pp. 543-6

Arrué, P.; Kany, M.T.; Serrano, E.; Lacroix, F.; Percodani, J.; Yardeni, E.; Pessey, J.J. \& Manelfe, C. (1998). Mucoceles of the paranasal sinuses: uncommon location. J Laryngol Otol, Vol.112, No.9, (September 1998), pp. 840-4

Avery, G.; Tang, R.A. \& Close, L.G. (1983). Ophthalmic manifestations of mucoceles. Ann Ophthalmol, Vol.15, No. 8, (August 1983), pp. 734-7

Aydil, U.; Karadeniz H. \& Sahin C. (2008). Choanal polyp originated from the inferior nasal concha. Eur Arch Otorhinolaryngol, Vol.265, No.4, (April 2008), pp. 477-479

Bachmann, G.; Nekic, M. \& Michel, O. (2000). Clinical experience with beta-trace protein as a marker for cerebrospinal fluid. Ann Otol Rhinol Laryngol, Vol.109, No.12 Pt 1, (December 2000), pp. 1099-102

Basak, S.; Karaman, C.Z.; Akdilli A. \& Metin K.K. (1998). Surgical approaches to antrochoanal polyps in children. Int J Pediatr Otorhinolaryngol, Vol.46, No.3, (December 1998), pp. 197-205

Beckhardt, R.N.; Setzen M. \& Carras R. (1991). Primary spontaneous cerebrospinal fluid rhinorrhea. Otolaryngol Head Neck Surg, Vol.104, No.4, (April 1991), pp. 425-432

Beham, A.; Kainz, J.; Stammberger, H.; Auböck, L. \&. Beham-Schmid, C. (1997). Immunohistochemical and electron microscopical characterization of stromal cells in nasopharyngeal angiofibromas. Eur Arch Otorhinolaryngol, Vol.254, No.4, (April 1997), pp. 196-9

Beham, A.; Beham-Schmid, C.; Regauer, S.; Auböck, L. \& Stammberger, H. (2000). Nasopharyngeal angiofibroma: true neoplasm or vascular malformation? Adv Anat Pathol, Vol.7, No.1, (January 2000), pp. 36-46

Berg, O.; Carnfelt, C.; Silfversward C. \& Sobin A. (1988). Origin of the choanal polyp. Arch Otolaryngol Head Neck Surg, Vol.114, No. 11, (November 1988), pp. 1270-1

Berlucchi, M.; Staurenghi, G.; Rossi Brunori, P.; Tomenzoli, D. \& Nicolai, P. (2003). Transnasal endoscopic dacryocystorhinostomy for the treatment of lacrimal pathway stenoses in pediatric patients. Int J Pediatr Otorhinolaryngol, Vol.67, No.10, (October 2003), pp. 1069-74

Berlucchi, M.; Salsi, D.; Valetti, L.; Parrinello, G. \& Nicolai P. (2007). The role of mometasone furoate aqueous nasal spray in the treatment of adenoid hypertrophy in the pediatric age: preliminary results of a prospective randomized study. Pediatrics, Vol.119, No. 6, (June 2007), pp. e1392-7

Berlucchi, M.; Maroldi, R.; Aga, A.; Grazzani, L. \& Padoan, R. (2010). Ethmoid mucocele: a new feature of primary ciliary dyskineesia. Pediatr Pulmonol, Vol.45, No. 2, (February 2010), pp. 197-201 
Berlucchi, M.; Pedruzzi, B. \& Farina, D. (2010). Radiology quiz case 2. Lobular capillary hemangioma (LCH). Arch Otolaryngol Head Neck Surg, Vol.136, No. 11, (November 2010), pp. 1141, 1143

Bothwell, M.R.; Piccirillo, J.F.; Lusk, R.P. \& Ridenour B.D. (2002). Long-term outcome of facial growth after functional endoscopic sinus surgery. Otolaryngol Head Neck Surg, Vol.126, No.6, (June 2002), pp. 628-34

Bradley, P.J. \& Singh S.D. (1982). Congenital nasal masses: diagnosis and management. Clin Otolaryngol Allied Sci, Vol.7, No.2, (April 1982), pp. 87-97

Busaba, N.Y. \& Salman, S.D. (1999). Maxillary sinus mucoceles: clinical presentation and long-term results of endoscopic surgical treatment. Laryngoscope, Vol.109, No.9, (September 1999), pp. 1446-9

Cassano, P.; Gelardi, M.; Cassano, M.; Fiorella, M.C. \& Fiorella R. (2003) Adenoid tissue rhinopharyngeal obstruction grading based on fibroendoscopic findings: a novel approach to therapeutic management. Int J Ped Otorhinolaryngol, Vol.61, No.12, (December 2003), pp. 1303-9

Caylakli, F.; Yavuz, H.; Cagici, A.C. \& Ozluoglu L.N. (2006). Endoscopic sinus surgery for maxillary sinus mucoceles. Head Face Med, Vol.6, No.2, (September 2006), pp. 29

Cingi, C.; Ure, B.; Cakli, H. \& Ozudogru E. (2010). Microdebrider-assisted versus radiofrequency-assisted inferior turbinoplasty: a prospective study with objective and subjective outcome measures. Acta Otorhinolaryngol Ital, Vol.30, No.3, (June 2010), pp. 138-43

Clarós, P.; Bandos, R.; Clarós, A. Jr; Gilea, I.; Clarós, A. \& Real, M. (1998). Nasal gliomas: main features, management and report of five cases. Int J Pediatr Otorhinolaryngol, Vol.46, No.1-2, (November 1998), pp. 15-20

Clement, P.A.R. (2008). Rhinosinusitis in children. In: Pediatric ENT, J.M. Graham; G.K. Scadding \& P.D. Bull, (Eds.), 307-25, Springer, Berlin, Germany

Curtin, H.D. \& Rabinov J.D. (1998). Extension to the orbit from paraorbital disease. The sinuses. Radiol Clin North Am, Vol.36, No.6, (November 1998), pp. 1201-13

Dabholkar, J.P.; Sathe N.U. \& Patole A.D. (2004). Nasal Glioma - A diagnostic challenge. Ind J Otolaryngol Head Neck Surg, Vol.56, No.1, (January 2004), pp. 27-28

De Vuysere S.; Hermans R. \& Marchal G. Sinochoanal polyp and its variant, the angiomatous polyp: MRI findings. Eur Radiol, Vol.11, No.1, pp. 55-8

Dimov, P.; Rouev, P.; Tenev, K.; Krosneva, R. \& Valkanov, P. (2001). Endoscopic surgery for the removal of a nasal glioma: case report. Otolaryngol Head Neck Surg Vol.124, No.6, (June 2001), pp. 690

Dunham, M.E. \& Miller R.P. (1992). Bilateral choanal atresia associated with malformation of the anterior skull base: embryogenesis and clinical implications. Ann Otol Rhinol Laryngol, Vol.101, No.11, (November 1992), pp. 916-9

Durmaz, A.; Tosun, F.; Yldrm, N.; Sahan, M.; Kvrakdal, C. \& Gerek M. Transnasal andoscopic repair of choanal atresia: results of 13 cases and meta-analysis. J Craniofac Surg, Vol.19, No.5, (September 2008), pp. 1270-4

el-Sayed, Y. \& al-Serhani A. (1997). Lobular capillary haemangioma (pyogenic granuloma) of the nose. J Laryngol Otol, Vol.111, No.10, (October 1997), pp. 941-5 
Fitzpatrick, E. \& Miller R.H. (1996). Congenital midline nasal masses: dermoids, gliomas, and encephaloceles. J La State Med Soc, Vol.148, No.3, (March 1996), pp. 93-6

Fokkens, W.; Lund, V.; Mullol, J. \& European Position Paper on Rhinosinusistis and Nasal Polyps group. (2007). European position paper on rhinosinusistis and nasal polyps 2007. Rhinol Suppl 20, pp.1-136

Frosini, P.; Picarella, G. \& De Campora E. (2009). Antrochoanal polyp: analysis of 200 cases. Acta Otorhinolaryngol Ital, Vol.29, No.1, (February 2009), pp. 21-6

Guttenplan, M.D. \& Wetmore, R.F. (1989). Paranasal sinus mucocele in cystic fibrosis. Clin Pediatr (Phila), Vol.28, No.9, (September 1989), pp. 429-30

Han, M.H.; Chang, K.H.; Lee, C.H.; Na, D.G.; Yeon K.M. \& Han M.C. (1995). Cystic expansile masses of the maxilla: differential diagnosis with CT and MR. AJNR Am J Neuroradiol, Vol.16, No.2, (February 1995), pp. 333-8

Harley, E.H. (1991). Pediatric congenital nasal masses. Ear Nose Throat J, Vol.70,No.1, (January 1991), pp. 28-32

Hayasaka, S.; Shibasaki, H.; Sekimoto, M.; Setogawa, T. \& Wakutani, T. (1991). Ophthalmic complications in patients with paranasal sinus mucopyoceles. Ophthalmologica, Vol.203, No.2, pp. 57-63

Hengerer, A.S. \& Newburg J.A. (1990). Congenital malformations of the nose and paranasal sinuses. In: Pediatric Otolaryngology, 2nd edition, C.D. Bluestone; S.E. Stool \& M.D. Scheetz, (Eds.),718-28, W.B. Saunders Company, Philadelphia, U.S.A.

Hoeger PH, Schaefer H, Ussmueller J, Helmke K. Nasal glioma presenting as capillary haemangioma. Eur J Pediatr 2001;160: 84-7

Hoving, E.W. (2000). Nasal Encephaloceles. Child's Nerv Syst, Vol.16, pp. 702-6

Jayaraj, S.M.; Patel, S.K.; Ghufoor, K. \& Frosh, A.C. (1999). Mucoceles of the maxillary sinus. Int J Clin Pract, Vol.53, No.5, (July-August 1999), pp. 391-3

Jyonouchi, S.; McDonald -Mc-Ginn, D.M.; Bale, S.; Zackai, E.H. \& Sullivan, K.E. (2009). CHARGE (coloboma, heart defect, atresia choanae, retarded growth and development, genital hypoplasia, ear anaomalies/deafness) syndrome and chromosome 22q11.2 deletion syndrome: a comparison of immunologic and nonimmunologic phenotypic features. Pediatrics, Vol.123, No.5, (May 2009), pp. e871-7

Joe, S.A.; Bolger, W.E.; \& Kennedy, D.W. (2001). Nasal endoscopy: diagnosis and staging of inflammatory sinus disease, In: Diseases of the sinuses. Diagnosis and management, D.W. Kennedy; W.E. Bolger \& S.J. Zinreich, (Eds.), 119-128, B.C. Decker Inc, London, England

Johnson, J.T. \& Ferguson, B.J. (1998). Infection. In: Otolaryngology, Head and Neck Surgery, 3rd edition, C.W. Cummings; J.M. Fredrickson, L.A. Harker; C.J. Krause; D.E. Schuller; M.A. Richardson, (Eds.), 1115-6, Mosby, St. Louis, U.S.A.

Keller, J.L. \& Kacker, A. (2000). Choanal atresia, CHARGE association, and congenital nasal stenosis. Otolaryngol Clin North Am, Vol.33, No.6, (December 2000), pp. 1343-51

Kennard, C.D. \& Rasmussen J.E. (1990). Congenital midline nasal masses: diagnosis and management. J Dermatol Surg Oncol, Vol.16, No.11, (November 1990), pp. 1025-36 
Kennedy, D.W. (1985). Functional endoscopic sinus surgery. Technique. Arch Otolaryngol, Vol.111, No.10, (October 1985), pp. 643-9

Kennedy, D.W. \& Senior B.A. (1997). Endoscopic sinus surgery. A review. Otolaryngol Clin North Am, Vol.30, No.3, (June 1997), pp. 313-30

Kwok, J.; Leung, MK. \& Koltai, P. (2007). Congenital inferior turbinate hypertrophy: an unsual cause of neonatal nasal obstruction. Int J Pediatr Otorhinolaryngol Extra, Vol.2, No.1, (March 2007), pp. 26-30

Kosko, J.R.; Hall, B.E. \& Tunkel, D.E. (1996). Acquired maxillary sinus hypoplasia: a consequence of endoscopic sinus surgery? Laryngoscope, Vol.106 No.10, (October 1996), pp. 1210-3

Lello, G.E.; Sparrow, O.C. \& Gopal, R. (1989). The surgical correction of fronto-ethmoidal meningo-encephaloceles. J Craniomaxillofac Surg Vol.17, No.7, (October 1989), pp. 293-8

Lieser, J.D. \& Derkay, C.S. (2005). Pediatric sinusitis: when do we operate? Curr Opin Otolaryngol Head Neck Surg, Vol.13,No.1, (February 2005), pp. 60-6.

Lloyd, G.; Lund, V.J.; Savy L. \& Howard D. (2000). Optimum imaging for mucoceles. J Laryngol Otol, Vol.114, No.3, (March 2000), pp. 233-6

Lloyd, K.M.; Del Gaudio, J.M. \& Hudgins, P.A. (2008). Imaging of skull base cerebrospinal fluid leaks in adults. Radiology, Vol.248, No.3, (September 2008), pp. 725-36

Lowe, L.H.; Booth, T.N.; Joglar, J.M. \& Rollins, N.K. (2000). Midface anomalies in children. Radiographics, Vol.20, No.5, (September-October 2000), pp. 907-22

Lund, V.J. Extended applications of endoscopic sinus surgery - the territorial imperative. J Laryngol Otol Vol.111, No.4, (April 1997), pp. 313-5

Lusk, R.P. \& Muntz, H. (1990). Endocopic sinus surgery in children with chronic sinusitis: a pilot study. Laryngoscope, Vol.100, No.6, (June 1990), pp. ,654-8

Lusk, R.P. (1992). Pediatric sinusitis. Raven, New York, U.S.A.

Lusk, R.P. \& Stankiewicz, J.A. (1997). Pediatric rhinosinusitis. Otolaryngol Head Neck Surg, Vol.117,No.2, (September 1997), pp. S53-S57

Ma, K.H. \& Keung, K.L. (2006). Nasal glioma. Hong Kong Med J, Vol.12, No.6, (December 2006), pp. 477-9

Mahapatra, A.K. \& Suri, A. (2002). Anterior Encephaloceles: a study of 92 Cases. Pediatr Neurosurg, Vol.36, No.3, (March 2002), pp. 113-8

Mahapatra, A.K. \& Agrawal D. (2006). Anterior encephaloceles: a series of 103 cases over 32 years. J Clin Neurosci, Vol.13, No.5, (June 2006), pp. 536-9

Marks, S.C.; Latoni J.D. \& Mathog R.H. (1997). Mucoceles of the maxillary sinus. Otolaryngol Head Neck Surg, Vol.117, No.1, (july 1997), pp. 18-21

Maroldi, R.; Berlucchi, M.; Farina, D.; Tomenzoli, D.; Borghesi, A. \& Pianta, L. Benign neoplasms and tumor-like lesions. In: Imaging in treatment planning for sinonasal diseases, R. Maroldi \& P. Nicolai, (Eds.), 107-158, Springer, Berlin, Germany

Martin, R.J.; Jackman, D.S.; Philbert, R.F. \& McCoy J.M. (2000). Massive proptosis of the globe. J Oral Maxillofac Surg, Vol.58, No.7, (July 2000), pp. 794-9

Mc Carty, J.G.; Thorne, C.H.M.; Wood-Smith D. (1990). Principles of craniofacial surgery: Orbital hypertelorism. In: Plastic Surgery, J.G. Mc Carty, (Ed.), 2974-3012, WB Saunders Company, Philadelphia, U.S.A. 
Messerklinger, W. (1966). On the drainage of the human paranasal sinuses under normal and pathological conditions. 1. Monatsschr Ohrenheilkd Laryngorhinol, Vol.100, pp. 56-68

Messerklinger, W. (1967). On the drainage of the human paranasal sinuses under normal and pathological conditions. 2. The frontal sinus and its evacuation system. Monatsschr Ohrenheilkd Laryngorhinol, Vol.101, pp. 313-26

Messerklinger, W. (1978). Endoscopy of the nose. Urban and Schwarzenberg, Munchen, Germany

Moriyama, H.; Hesaka, H.; Tachibana, T. \& Honda, Y. (1992). Mucoceles of ethmoid and sphenoid sinus with visual disturbance. Arch Otolaryngol Head Neck Surg, Vol.118, No.2, (February 1992), pp. 142-6

Morris, W.M.; Losken, H.W. \& le Roux P.A. (1989). Spheno-maxillary meningoencephalocele. A case report. J Craniomaxillofac Surg Vol.17, No.8, (November 1989), pp. 359-62

Nachtigal, D.; Frenkiel, S.; Yoskovitch, A. \& Mohr, G. (1999). Endoscopic repair of cerebrospinal fluid rhinorrhea: is it the treatment of choice? J Otolaryngol, Vol.28, No.3, (January 1999), pp. 129-33

Naidich, T.P.; Altman N.R.; Braffman, B.H.; McLone, D.G. \& Zimmerman, R.A. (1992). Cephaloceles and related malformations. AJNR Am J Neuroradiol, Vol.13, No2, (March-April 1992), pp. 655-90

Nicolai, P.; Berlucchi, M.; Tomenzoli, D.; Cappiello, J.; Trimarchi, M.; Maroldi, R.; Battaglia, G. \& Antonelli, A.R. (2003) Endoscopic surgery for juvenile angiofibroma: when and how. Laryngoscope, Vol.113,No.5, (May 2003), pp. 775-82

Nicolai, P.; Lombardi, D.; Tomenzoli, D.; Villaret, A.B.; Piccioni, M.; Mensi, M. \& Maroldi, R. (2009). Fungus ball of the paranasal sinuses: experience in 160 patients treated with endoscopy surgery. Laryngoscope, Vol.119, No.11, (November 2009), pp. 2275-9

Nicolai, P.; Villaret, A.B.; Farina, D., Nadeau, S.; Yakirevitch, A.; Berlucchi, M. \& Galtelli, C. (2010). Endoscopic surgery for juvenile angiofibroma: a critical review of indications after 46 cases. Am J Rhinol Allergy Vol.24, No.2, (March 2010), pp. e67-72

Nicolai, P.; Castelnuovo, P. \& Bolzoni Villaret A. (2011). Endoscopic resection of sinonasal malignancies. Curr Oncol Rep Vol.13, No.2, (April 2011), pp. 138-144

Nicollas, R.; Facon, F.; Sudre-Levillain, I.; Forman, C.; Roman, S. \& Triglia J.M. (2006). Pediatric paranasal sinus mucoceles: etiologic factors, management, and outcome. Int J Pediatr Otorhinolaryngol, Vol.70, No.5, (May 2006), pp. 905-8

Nuss, D.W. \& Costantino P.D. (1995). Diagnosis and Management of Cerebrospinal Fluid Leaks. In: Highlights of the Instructional Courses of the American Academy of Otolaryngology-Head and Neck Surgery. F.E. Lucente, (Ed.), Volume 8, MosbyYearbook Publishers, St. Louis, U.S.A.

Ohta, N.; Ito, T.; Sasaki, A. \& Aoyagi, M. (2010). Endoscopic treatment of intranasal glioma in an infant presenting with dyspnea. Auris Nasus Larynx, Vol.37, No.3, (June 2010), pp. 373-6

Olze, H.; Matthias, C. \& Degenhardt, P. (2006), Paediatric paranasal sinus mucoceles. Eur J Pediatr Surg, Vol.16, No.3, (June 2006), pp. 192-6. 
Ommaya, A.K. (1976). Spinal fluid fistulae. Clin Neurosurg, Vol. 23, (1976), pp. 363-92

Orvidas, L.J.; Beatty, C.W. \& Weaver, A.L. (2001). Antrochoanal polyps in children. Am J Rhinol, Vol.15, No.5, (September-October 2001), pp. 321-5

Ozcan, C.; Apa, D.D. \& Görür, K. (2004). Pediatric lobular capillary hemangioma of the nasal cavity. Eur Arch Otorhinolaryngol, Vol.261, No.8, (September 2004), pp. 449-51

Ozdek, A.; Samim, E.; Bayiz, U.; Meral, I.; Safak, M.A. \& Oguz, H. (2002). Antrochoanal polyps in children. Int J Pediatr Otorhinolaryngol, Vol.65, No.3, (September 2002), pp. 213-8

Palfyn J. Anatomie chirurgicale. Paris, 1753.

Pakkasjärvi, N.; Salminen, P.; Kalajoki-Helmiö, T.; Rintala, R. \& Pitkäranta, A. (2008). Respiratory distress secondary to nasopharyngeal glial heterotopia. Eur J Pediatr Surg, Vol.18, No.2, (August 2008), pp. 117-8

Pelikan, Z. (2009). Role of nasal allergy in chronic secretory otitis media. Curr Allergy Asthma Rep, Vol.9, No.2, (March 2009), pp. 107-13

Pianta, L.; Pinelli, L.; Nicolai, P. \& Maroldi, R. Cerebrospinal fluid leak, meningocele and meningoencephalocele. In: Imaging in treatment planning for sinonasal diseases, R. Maroldi \& P. Nicolai, (Eds.), 93-106, Springer, Berlin, Germany

Presutti, L.; Mattioli, F.; Villari, D.; Marchioni, D. \& Alicandri-Ciufelli, M. (2009). Transnasal endoscopic treatment of cerebrospinal fluid leak: 17 years' experience. Acta Otorhinolaryngol Ital, Vol.29, No.4, (August 2009), pp. 191-6

Pruna, X.; Ibanez, J.M.; Serres, X.; Garriga, V; Barber, I. \& Vera, J. (2000) Antrochoanal polyps in children: CT findings and differential diagnosis. Eur Radiol, Vol.10, No.5, (2000), pp. 849-51

Puppala, B.; Mangurten, H.H.; McFadden, J.; Lygizos, N.; Taxy, J. \& Pellettiere, E. (1990). Nasal glioma presenting as neonatal respiratory distress. Definition of the tumor mass by MRI. Clin Pediatr (Phil), Vol.29, No.1, (January 1990), pp. 49-52

Puxeddu, R.; Berlucchi, M.; Ledda, G.P.; Parodo, G.; Farina, D. \& Nicolai, P. (2006). Lobular capillary hemangioma of the nasal cavity: a retrospective study on 40 patients. Am J Rhinol, Vol.20, No.4, (July-August 2006), pp. 480-4

Rahbar, R.; Resto, V.A.; Robson, C.D.; Perez-Atayde, A.R.; Goumnerova, L.C.; McGill, T.J. \& Healy, G.B. (2003). Nasal glioma and encephalocele: diagnosis and management. Laryngoscope, Vol.113, No.12, (December 2003), pp. 2069-77

Samadi, D.S.; Shah, U.K.; Handler, S.D. (2003). Choanal atresia: a twenty-year review of medical comorbidities and surgical outcomes. Laryngoscope, Vol.113, No.2, (February 2003), pp. 254-8.

Samii, M. \& Draf, W. (1989). Surgery of malformations of the anterior skull base. In: Surgery of the Skull Base, M. Samii \& W. Draf (Eds), 114-26, Springer-Verlag, Berlin, Germany

Schlosser, R.J. \& Bolger, W.E. (2002). Nasal cerebrospinal fluid leaks. J Otolaryngol, Vol.31, Suppl.1, (August 2002), pp. S28-S37

Schramm, V.L. Jr. \& Effron, M.Z. (1980). Nasal polyps in children. Larygoscope, Vol.90, No.9, (September 1980), pp. 1488-95

Schweinfurth, J.M. (2002). Image guidance-assisted repair of bilateral choanal atresia. Laryngoscope, Vol.112, No.11, (November 2002), pp. 2096-8 
Senior, B.; Wirtschafter, A.; Mai, C.; Becker, C. \& Belenky,W. (2000). Quantitative impact of pediatric sinus surgery on facial growth. Laryngoscope, Vol.110, No.11, (November 2000), pp. 1866-70

Składzień, J.; Litwin, J.A.; Nowogrodzka-Zagórska, M. \& Wierzchowski, W. (2001). Morphological and clinical characteristics of antrochoanal polyps: comparison with chronic inflammation-associated polyps of the maxillary sinus. Auris Nasus Larynx, Vol.28, No.2, (April 2001), pp. 137-41

Som, P.M. \& Brandwein, M. (1996). Sinonasal cavities. Inflammatory disease, tumors, fractures, and postoperative findings. In: Head and Neck Imaging, P.M. Som \& H.D. Curtin, (Eds.), 3rd edition, 126-85, Mosby, St. Louis, U.S.A.

Stammberger, H. (1999). 2. Surgical treatment of nasal polyps: past, present and future. Allergy, Vol.54, Suppl.53, (1999), pp. 7-11

Suwanwela, C. \& Suwanwela, N. (1972). A morphological classification of sincipital encephalomeningocele. J Neusurg, Vol.36, No.2, (February 1972), pp. 201-11

Teissier, N.; Kaguelidou, F.; Couloigner, V.; Francois, M. \& Van Den Abbeele, T. (2008). Predictive factors for success after transnasal endoscopic treatment of coanal atresia. Arch Otolaryngol Head Neck Surg, Vol.134, No.1, (January 2008), pp. 57-61

Thomé, D.C.; Voegels, R.L.; Cataldo de la Cortina, R.A. \& Butugan, O. (2000). Bilateral ethmoidal mucocele in cystic fibrosis: report of a case. Int J Pediatr Otorhinolaryngol, Vol.55, No.2, (September 2000), pp. 143-8

Triglia, J.M. \& Nicollas, R. (1997). Nasal and sinus polyposis in children. Laryngoscope, Vol.107, No.7, (July 1997), pp. 963-6

Tseng, C.C.; Ho, C.Y. \& Kao, S.C. (2005). Ophthalmic manifestations of paranasal sinus mucoceles. J Chin Med Assoc, Vol.68, No.6, (June 2005), pp. 260-4.

Uzunlar, A.K.; Osma, U.; Yilmaz, F. \& Topcu, U. (2001). Nasal glioma: report of two cases. Turk J Med Sci, Vol.31, pp. 87-90

Van Peteghem, A. \& Clement, P.A. (2006). Influence of extensive functional endoscopic sinus surgery (FESS) on facial growth in children with cystic fibrosis. Comparison of 10 cephalometric parameters of the midface for three study groups. Int J Pediatr Otorhinolaryngol, Vol.70, No.8, (August 2006), pp. 1407-13

Varghese, L.; John, M. \& Kurien, M. (2004). Bilateral asymmetric mucoceles of the paranasal sinuses: a first case report. Ear Nose Throat J, Vol.83, No.12, (December 2004), pp. 834-5

Vatansever, U.; Duran, R.; Acunas, B.; Koten, M. \& Adali, M.K. (2005). Bilateral choanal atresia in premature monozygotic twins. J Perinatol, Vol.25, No.12, (December 2005), pp. 800-2

Vuckovic, N.; Vuckovic, D.; Dankuc, D. \& Jovancevic, L. (2006). Nasal glioma. Arch Oncol, Vol.14, No.1-2, (June 2006), pp. 57-9

Wolf, C.; Greistorfer, K. \& Jebeles, J.A. (1995). The endoscopic endonasal surgical technique in the treatment of chronic recurring sinusitis in children. Rhinology, Vol.33, No.2, (June 1995), pp. 97-103

Wong, R.K. \& VanderVeen, D.K. (2008). Presentation and management of congenital dacryocystocele. Pediatrics, Vol.122, No.5, (November 2008), pp. e1108-12. 
Woodworth, B.A.; Schlosser, R.J.; Faust, R.A. \& Bolger, W.E. (2004). Evolutions in the management of congenital intranasal skull base defects. Arch Otolaryngol Head Neck Surg, Vol.130, No.11, (November 2004), pp. 1283-88

Yaman, H.; Yilmaz, S.; Karali, E.; Guclu, E. \& Ozturk, O. (2010). Evaluation and management of antrochoanal polyps. Clin Exp Otorhinolaryngol, Vol.3, No.2, (June 2010), pp. 110-4

Yerkes, S.A.; Thompson D.H. \& Fisher, W.S. (1992). Spontaneous cerebrospinal fluid rhinorrhea. Ear Nose Throat J, Vol. 71, No.7, (July 1992), pp 318-20 


\title{
Endoscopy in the Evaluation and Management of the Pediatric Airway
}

\author{
Kris R. Jatana ${ }^{1}$ and Jeffrey C. Rastatter ${ }^{2}$ \\ ${ }^{1}$ Nationwide Children's Hospital and The Ohio State University Columbus, Ohio \\ ${ }^{2}$ Children's Memorial Hospital and Northwestern University Chicago, Illinois
}

USA

\section{Introduction}

The airway starts at the anterior nasal vestibule and ends at the lung parenchyma. This includes the nasal cavity, nasopharynx, oral cavity, oropharynx, larynx, hypopharynx, trachea, and bronchi. When evaluating a child with noisy breathing, it is important to assess the airway in a systematic manner.

A thorough history is important. This includes assessment of the severity or progression of symptoms, and whether the symptoms are worse with feeding or increased exertion. The severity determines the urgency of complete evaluation. Apparent life-threatening events (ALTE) warrant prompt multidisciplinary evaluation (McMurray, 1997).

Inspiratory stridor generally originates from the larynx, while expiratory stridor from the trachea or bronchi. Stridor that is biphasic (both inspiratory and expiratory) typically indicates a fixed obstructive lesion is present. Depending on symptomatology, the addition of endoscopic evaluation of the airway to the basic physical examination can be extremely beneficial. A definitive diagnosis is critical to proper treatment intervention.

\section{Types of endoscopy}

Endoscopic evaluation of the pediatric airway can be accomplished with both rigid and flexible endoscopes. It can be done awake in the office or under general anesthesia. In the office, awake flexible endoscopic examination of the nasal cavity, nasopharynx, oropharynx, hypopharynx, and larynx can be performed. Rigid endoscopy, using various sizes and degrees of scopes, is used to evaluate the nasal cavity and nasopharynx. Transoral rigid endoscopy with an angled scope to evaluate the oropharynx, hypopharyx, or larynx is not well-tolerated in children. Awake flexible fiberoptic endoscopy allows for full assessment of the upper airway to the level of the true vocal cords. An advantage to awake exanimation is that direct visualization of the airway can be correlated with the noisy breathing of the child, helping to identify the cause. Vocal cord function is best assessed using awake flexible fiberoptic laryngoscopy. In the past, awake evaluation of the upper airway in children was limited to the level of the true vocal cords, however there is some evidence that flexible fiberoptic laryngoscopy with tracheoscopy can be performed in the office setting without complications (Hartzell, 2010). Due to potential risk of laryngospasm and airway 
compromise, such evaluation is generally done in the operating room. Under general anesthesia, the assessment of the airway is best for static lesions, however, if the patient can be kept breathing spontaneously, a more dynamic assessment of the lower airways (trachea and bronchi) can be performed. A rigid bronchoscope allows for ventilation (providing adequate time for complete inspection) as well as visualization of the segmental bronchi. While some advocate using a telescope alone to evaluate the trachea and proximal main bronchus, it is difficult to examine the distal portions of each main bronchus and the segmental bronchi.

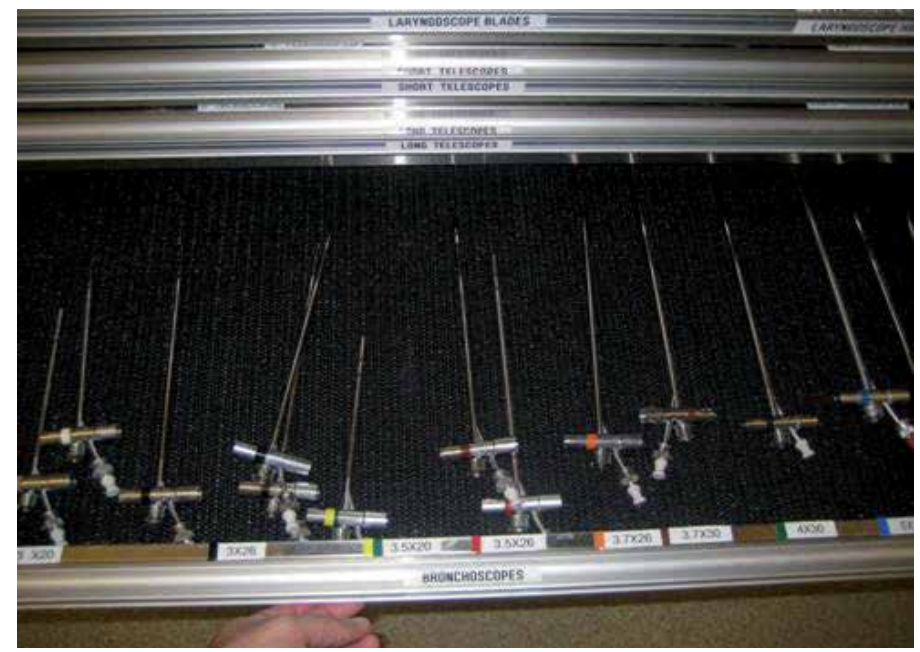

Fig. 1. Equipment

An operating room with systematic organization of various sizes of rigid bronchoscopes, telescopes, laryngoscopes, and flexible fiberoptic scopes.

\section{Abnormalities of the nasal cavity}

\subsection{Nasal vestibular stenosis and pyriform aperature stenosis}

Nasal vestibular stenosis results from disruption of the vestibular lining with proliferation of granulation and fibrous tissue. It has occurred in association with nasal CPAP use (Jatana 2008 ,2010), prior nasal surgical procedures, nasal packing (Karen 2000), excessive cautery for epistaxis (Bajaj, 1969), birth trauma (Jablon, 1997), and flash burn injury (Salvado, 2008). Anterior nasal endoscopy may be required to look for vestibular stenosis, and it can be significant in a neonate who is an obligate nasal breather.

Congenital nasal pyriform aperature stenosis, is a different entity causing a narrowing at the level of the pyriform aperature (Ramadan, 1995).

\subsection{Nasal septal deviation}

This is a common finding on endoscopy of the nasal cavity. The nasal septum is composed of cartilage and bone, and deformities can contribute to nasal obstruction. This can be developmental or acquired due to nasal trauma. A statistically significant higher incidence of nasal septal deformities has been shown with a history of nasal injury (Zielnik-Jurkiewicz, 2006) 


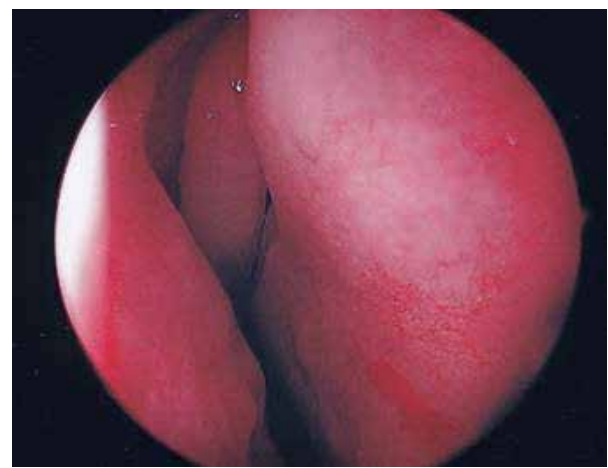

Fig. 2. Nasal septal deviation

Nasal endoscopy using a 0 degree endoscope in the right nasal cavity, showing a nasal septal deformity to the right compromising the nasal airway.

\subsection{Nasal polyposis}

Nasal endoscopy can be useful in the diagnosis of nasal polyps which generally result from chronic inflammation. The development may be related to chronic rhinosinusitis, asthma, allergic rhinitis, or cystic fibrosis (Triglia, 1997). Patients with cystic fibrosis, an autosomal recessive condition, frequently develop chronic rhinosinusitis with or without nasal polyps (Franco, 2009; Weber, 2008). Imaging is critical prior to any surgical intervention as tumors or masses with intracranial extension can be present in the sinonasal cavity of children. Endoscopic sinus surgery can be used to remove polyps as indicated.

\subsection{Anterior cranial fossa masses}

Dermoid sinus (with/without intracranial extension), encephaloceles, and gliomas are the most common midline nasal masses (Hughes, 1980), and imaging must be performed prior to any surgical intervention. If intracranial extension is not known, there is risk of CSF leak, meningitis, or intracranial injury (Hedlund, 2006). If a skull base defect is present, it may need to be repaired simultaneously which can be done either endoscopically or by craniotomy depending on the size and intracranial extent.

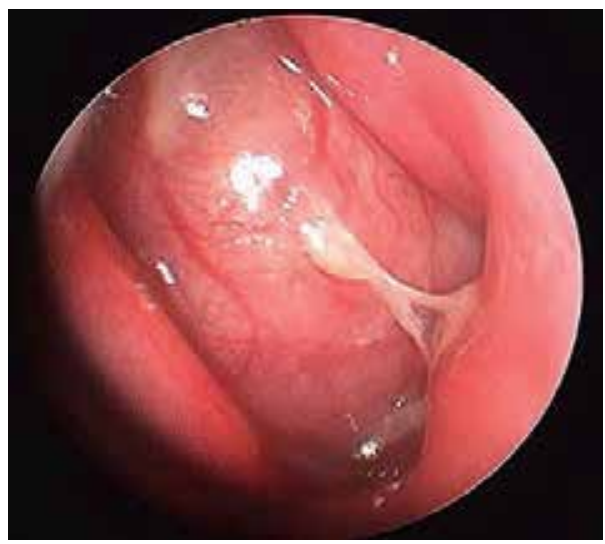

Fig. 3. Cystic mass, nasal cavity 
This rare nasal mass, a chondromesencymal hamartoma, is a benign congenital lesion that has known propensity to have intracranial extension. This can mimic the appearance of a meningoencephalocele (Kim 2004).

\subsection{Choanal atresia}

A failure of the posterior nasal aperature to canalize results in choanal atresia, and this can be unilateral or bilateral. It was first described in 1755 by Roederer and occurs in 1 of 50007000 live births. A newborn is an obligate nasal breather. Neonates with bilateral choanal atresia require endotracheal intubation at the time of birth (Deutsch, 1997). It has been shown that $71 \%$ of these are mixed (bony and membranous), and $29 \%$ are bony atresia (Brown, 1996). Nasal endoscopy can be used to show the atretic plate in the back of the nasal cavity along the nasal floor. In most cases, repair can be successfully performed endoscopically (Hengerer, 2008; Ramsden, 2009).

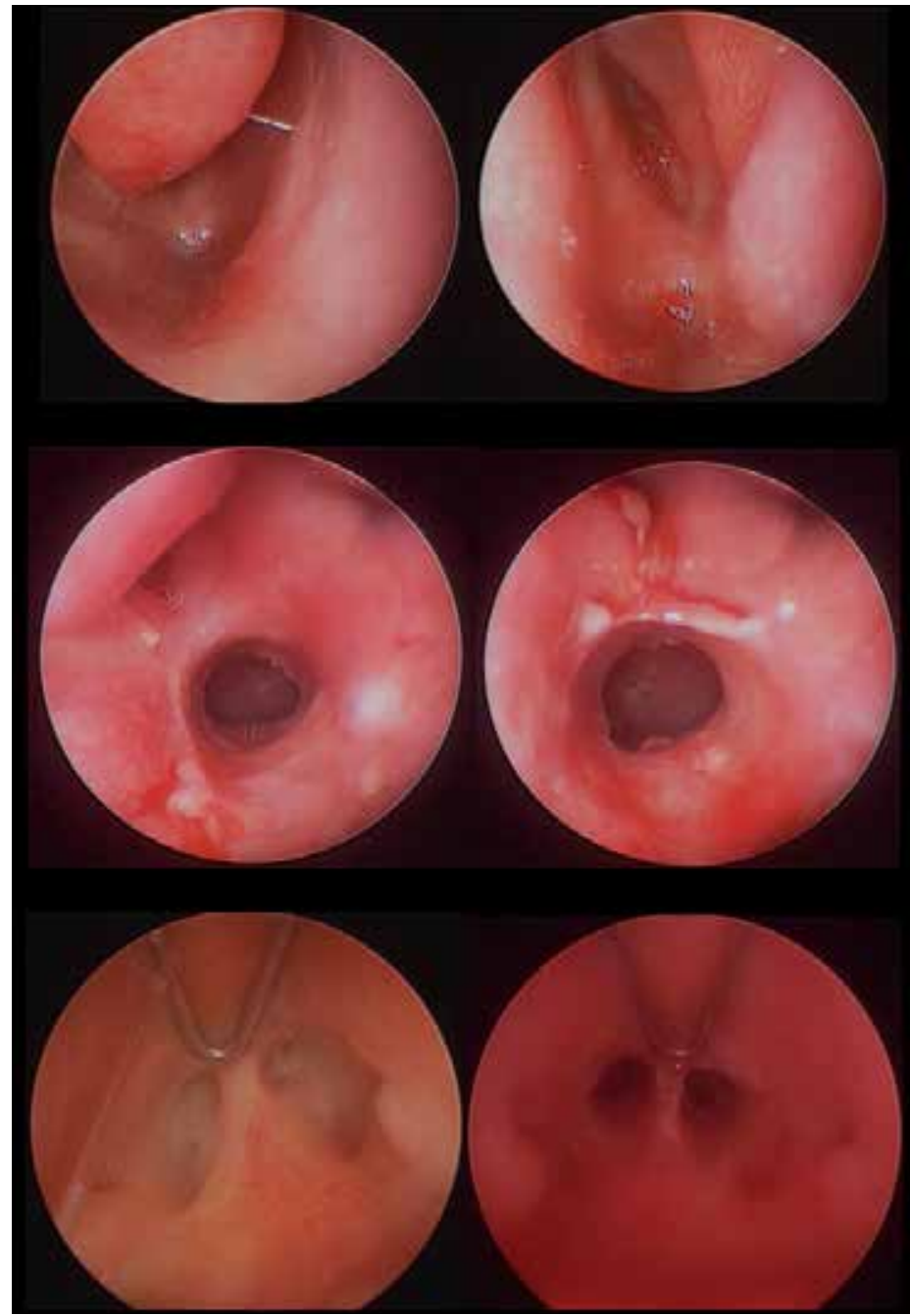

Fig. 4. Bilateral choanal atresia 
At the top, a 0 degree endoscope in the nasal cavity of a newborn child who had respiratory distress immediately at birth. Note the blocked choana bilaterally (right \& left). The middle image shows the right and left posterior nasal cavity, 2 weeks after endoscopic repair. At the bottom is a view through a 120 degree endoscope with the soft palate retracted anteriorly. Note the posterior choana before (left) and after (right) endoscopic repair.

\subsection{Nasal septal perforation}

The nasal septum, consisting of both cartilage and bone, divides the left and right nasal cavities. A perforation, or hole, in the septum can cause non-specific symptoms, including epistaxis, obstruction, crusting, whistling, and pain. Causes of perforation include: chronic trauma, piercings, intranasal placement of button batteries, drug use (including cocaine), industrial metal plating solutions, intranasal steroid use, surgical trauma, nasal cautery, Wegener granulomatosis, sarcoidosis, and syphilis (Diamantopoulos, 2001; Lanier, 2009). Some large nasal septal perforations have been repaired using an endoscope-assisted approach (Giacomini, 2011).

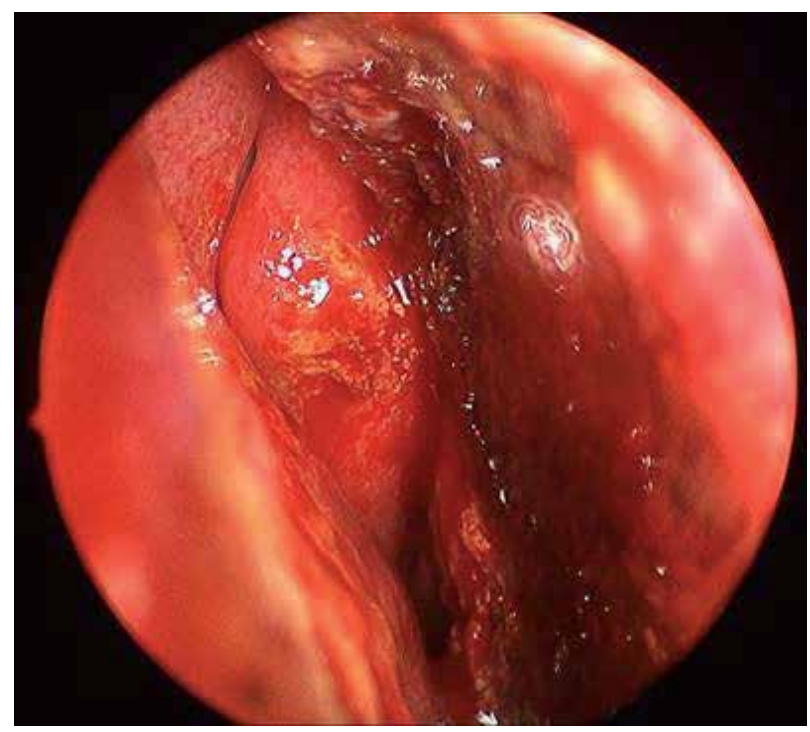

Fig. 5. Right nasal cavity. When placed inside of the body, button batteries need to be removed as soon as possible (emergently), to avoid serious complications.

Note early tissue necrosis (black) after removal of button battery, often resulting in a nasal septal perforation.

\section{Abnormalities of the nasopharynx}

\subsection{Adenoid hypertrophy}

The adenoid tissue in the nasopharynx, consists of lymphoid tissue, which is typically small at birth and enlarges to various degrees during early childhood. It often involutes during late childhood. Obstructive adenoid tissue can be related to nasal obstruction symptoms (snoring), eustachian tube dysfunction, and chronic sinusitis. Adenoidectomy is one of the most common surgical procedures done in children. 


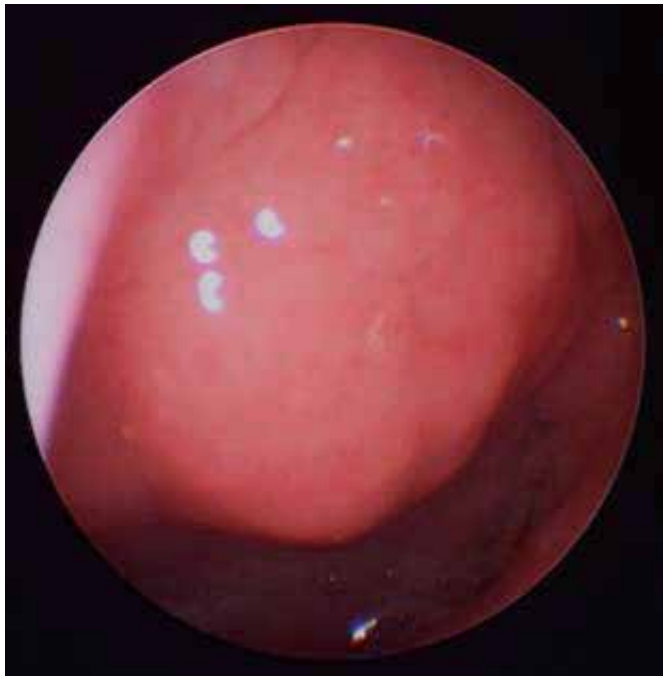

Fig. 6. Adenoid tissue, nasopharynx

The adenoid tissue can be easily visualized transnasally with a 0 degree endoscope, note the moderate obstruction in this patient.

\subsection{Meningocele}

A meningocele in the nasopharynx must be kept in the differential diagnosis of pediatric nasopharyngeal masses. It has a cystic appearance, originates intracranially from herniation of meninges, and is filled with cerebral spinal fluid (CSF). A congenital defect in the skull base is usually present, and both CT and MRI are critical for surgical planning.

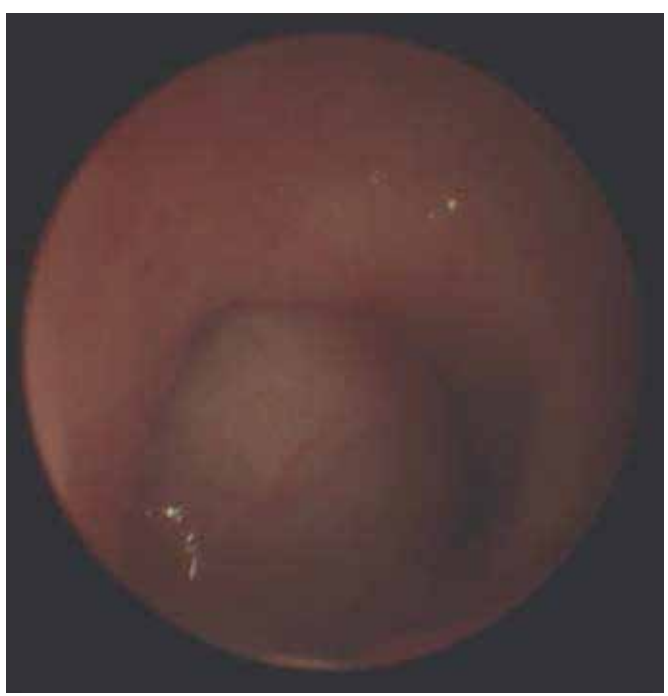

Fig. 7. Cystic mass, nasopharynx

This was a skull base meningocele in a 6 week old child. It was filled with clear fluid (CSF), repair of the skull base defect is necessary to avoid CSF leak and reduce risk of meningitis. 
Note the limitation of picture definition and brightness when the surgeon has to use a smaller diameter telescope lens.

\subsection{Juvenile Nasopharyngeal Angiofibroma (JNA)}

JNA is a benign vascular tumor that arises in the nasopharynx of adolescent males. It often presents with unilateral recurrent epistaxis or nasal obstruction. JNAs originate from the sphenopalatine foramen, commonly extend to the nasal cavity and nasopharynx, and can also extend to the pterygopalatine fossa, infratemporal fossa, orbit, or intracranially. Transnasal rigid or flexible endoscopy typically gives good visualization of the lesion. JNAs can often be removed with minimally invasive endoscopic surgery (Douglas, 2006), while some require traditional open surgical approaches (Bales, 2002).

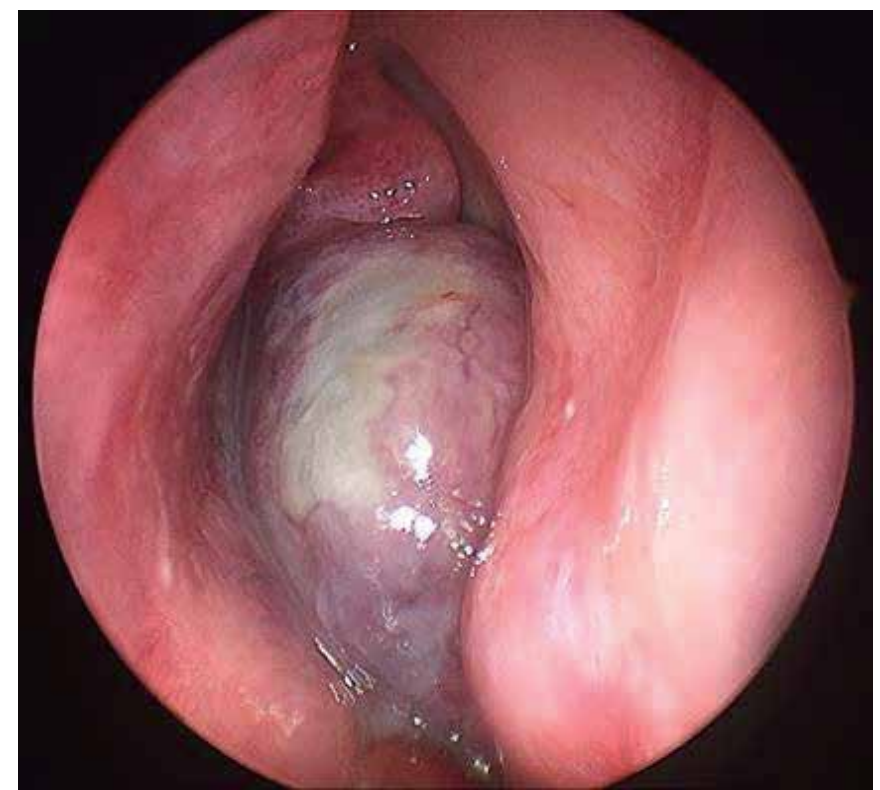

Fig. 8. Right nasal cavity

A JNA originating from the right sphenopalatine foramen, some tumor necrosis is present (white) after pre-resection arterial embolization.

\subsection{Nasopharyngeal hamartoma}

A hamartoma is a benign, abnormal proliferation of normal tissue. It can be composed of tissue from all 3 germ layers. In the head and neck region they can be found in the oral cavity, nasal cavity, and nasopharynx (Hulsmann, 2009). The overall incidence of nasal hamatomas is 1 in 20,000 to 40,000 live births (Harley, 1991).

\section{Abnormalities of the oropharynx}

\subsection{Lingual Thyroglossal Duct Cyst}

Thyroglossal duct cysts (TGDCs) are congenital and can arise at any point along the typical path of the thyroid gland during embryogenesis. Initially formed in the midline base of 
tongue, the thyroid gland descends to the final location low in the anterior midline of the neck. TGDC is the most common congenital pediatric neck mass (Koeller, 1999). When found in the oropharynx at the base of tongue, these lesions are termed lingual TGDCs. Transnasal flexible fiberoptic laryngoscopy can often visualize these at the tongue base. Endoscopic surgery for removal has also been described (Burkhart, 2009). Proper diagnosis and treatment is important as swelling or growth can lead to airway obstruction (Kuint, 1997; Fu, 2008).

\subsection{Vallecular cyst}

A vallecular cyst is simple cyst, typically lined with respiratory epithelium and mucous glands, which forms on the lingual surface of the epiglottis (Gutierrez, 1999). They typically present with stridor, feeding problems, and upper airway obstruction (Gluckman, 1992, LaBagnara, 1989). They can be visualized and diagnosed with flexible fiberoptic laryngoscopy. Marsupialization of the cyst is typically curative.

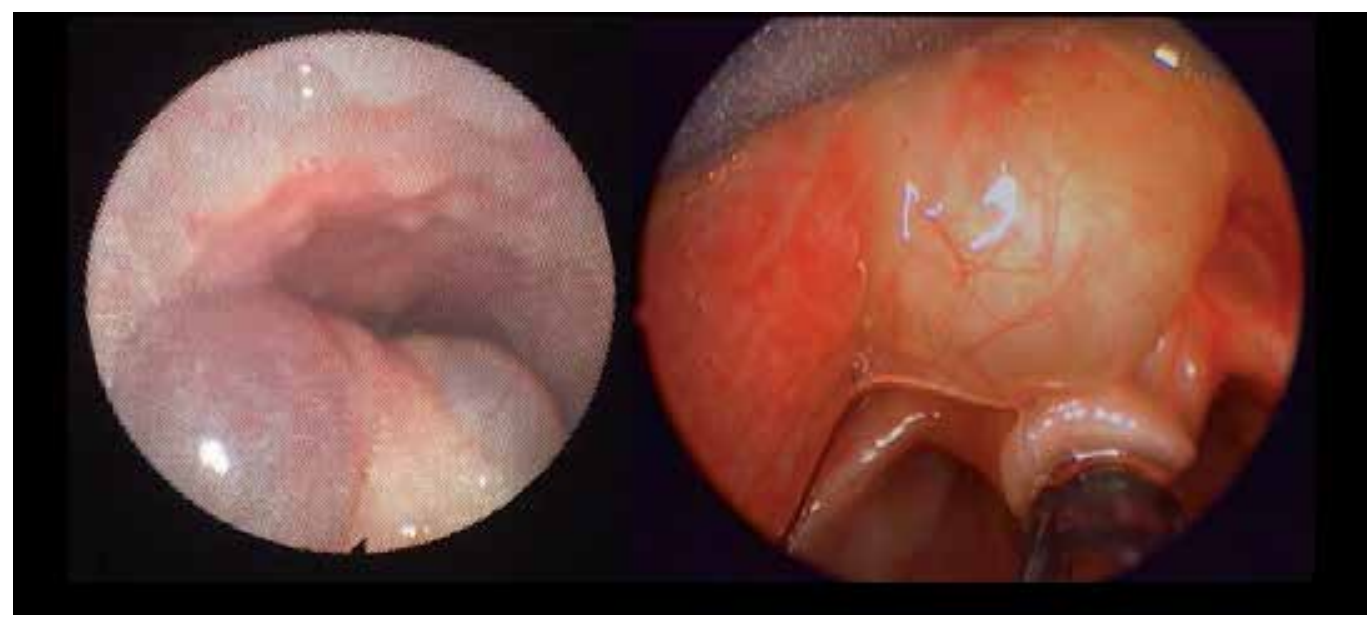

Fig. 9. Vallecular Cyst

On left, using a flexible fiberoptic laryngoscope placed into the nasopharynx (looking down at the tongue base), a large cystic mass originating from vallecula. Posteriorly there is evidence of cobblestoning of the pharyngeal wall. On right a vallecular cyst in a neonate which caused an apparent life-threatening event (ALTE).

\section{Abnormalities of the hypopharynx}

\subsection{Pyriform sinus tracts}

Tracts originating from the pyriform sinus within the hypopharynx have also been referred to as third or fourth branchial sinuses, fistulas, or remnants. The vast majority are left-sided, but rarely can occur on the right or be bilateral. These can be the etiology of recurrent neck abscesses or thyroiditis. Treatment options include cautery of the sinus orifice and definitive surgical excision of the tract including thyroid lobectomy. Recently, endoscopic cautery has been advocated as the initial treatment option as it carries less potential morbidity than open excision. (Chen, 2009; Verret, 2004) 


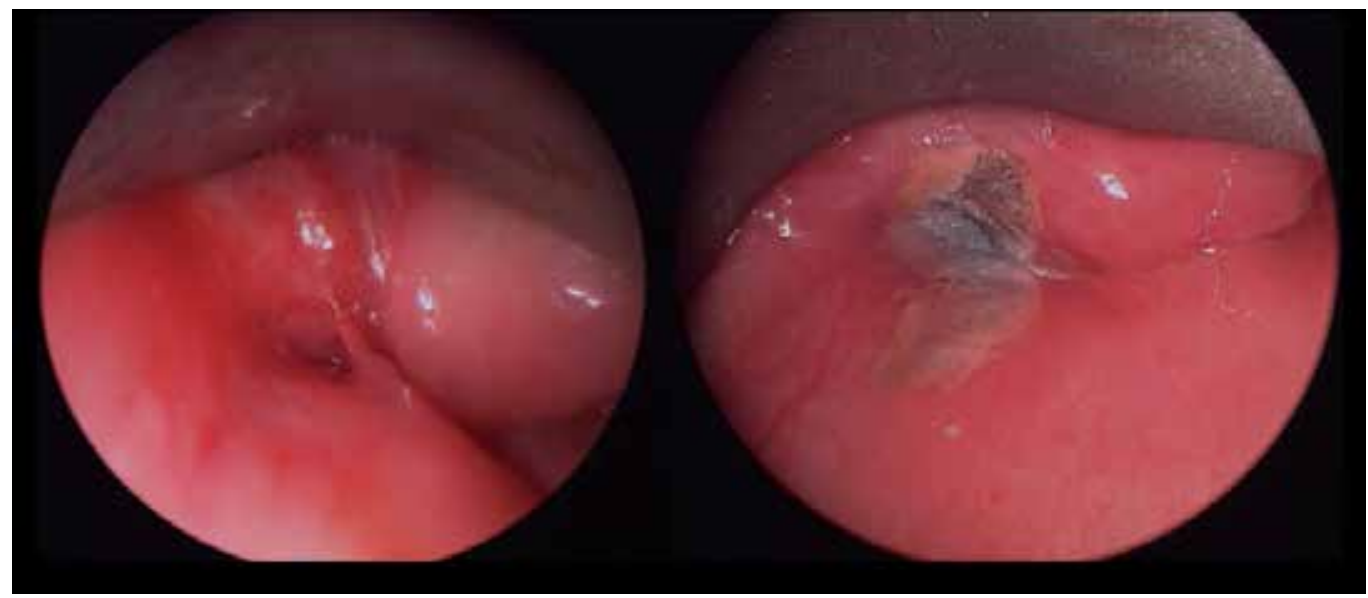

Fig. 10. Pyriform sinus fistula

On the left, note the fistula opening in the left pyriform sinus. On the right, endoscopic cautery was performed using silver nitrate to obliterate the opening which is the source of bacterial contamination of the tract.

\section{Abnormalities of the larynx}

\subsection{Supraglottis and glottis}

Flexible fiberoptic laryngoscopy can be used for excellent visualization of the supraglottis and glottis. This is often done initially in the outpatient clinic setting as this procedure is very well tolerated in awake infants and children. Laryngomalacia is the most common congenital laryngeal anomaly causing stridor in infants (Thompson, 2007). Inspiration leads to dynamic collapse of supraglottic structures, the cause of the upper airway obstruction and stridor. Failure of the primitive larynx to recanalize during the tenth week of embryogenesis can lead to laryngeal web formation (McGill, 2000). As they are most

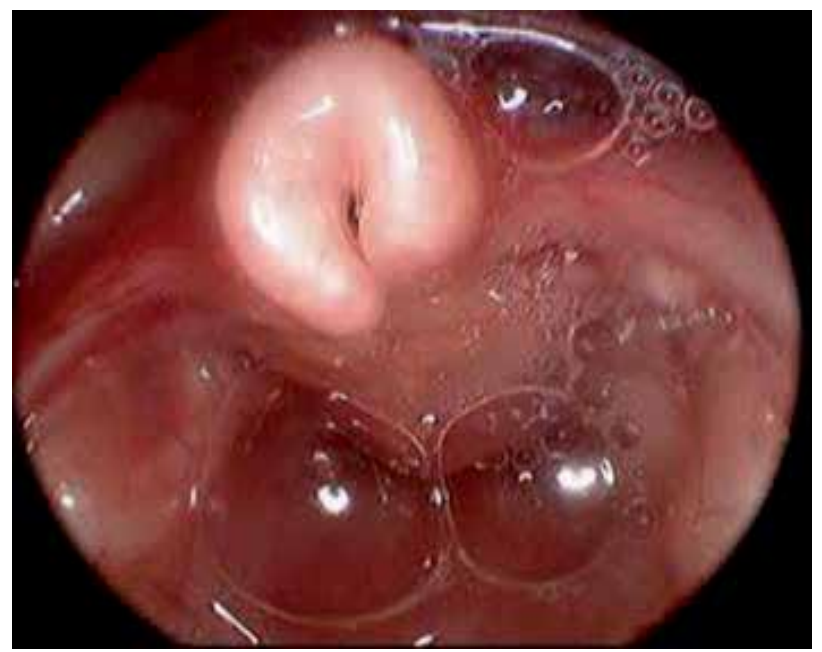

Fig. 11. Laryngomalacia 
common at the level of the glottis with variable extension to the subglottis, symptoms include a weak voice and/or upper airway obstruction. Saccular cysts and laryngoceles also are abnormal dilations of the laryngeal saccule that contain either mucoid fluid or air respectively. They can present as swellings in the area of the false vocal fold as seen on flexible fiberoptic laryngoscopy. These relatively rare lesions have been characterized in detail in the literature (Holinger, 1978; DeSanto, 1970). Vocal cord paralysis is best assessed on awake flexible fiberoptic laryngoscopy. Static evaluation of the posterior larynx with a probe is necessary to look for a laryngeal cleft (type I-IV). Children can present with symptoms of stridor, aspiration, and/or respiratory distress (Rahbar 2006). While all type IV clefts require open surgical repair, correction can be done endoscopically in type I-II clefts, and even some type III clefts (Garabedian 2010).

Note the "omega" or "tubular" shaped epiglottis. The aryepiglottic folds are shortened, pulling arytenoids anteriorly. Collapse on inspiration causes stridor and can be directly correlated in the office setting. In severe cases, on awake flexible fiberoptic laryngoscopy the vocal cords cannot be visualized due to this supraglottic obstruction. Supraglottoplasty can be performed in severe cases with failure to thrive or respiratory compromise.

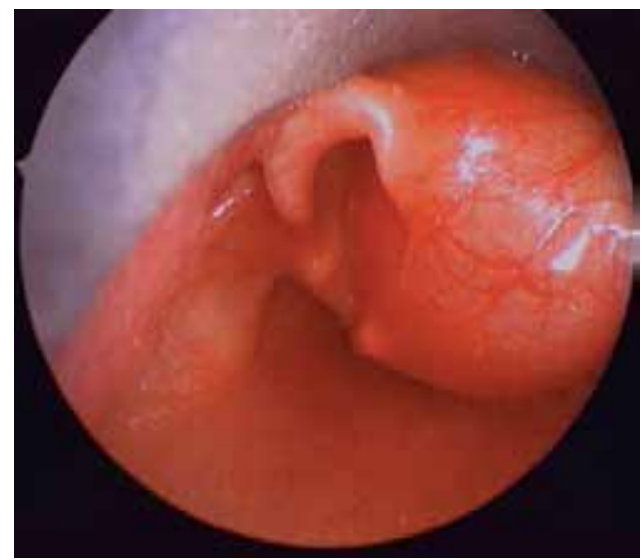

Fig. 12. Right saccular cyst

Note the significant upper airway obstruction that can be present.

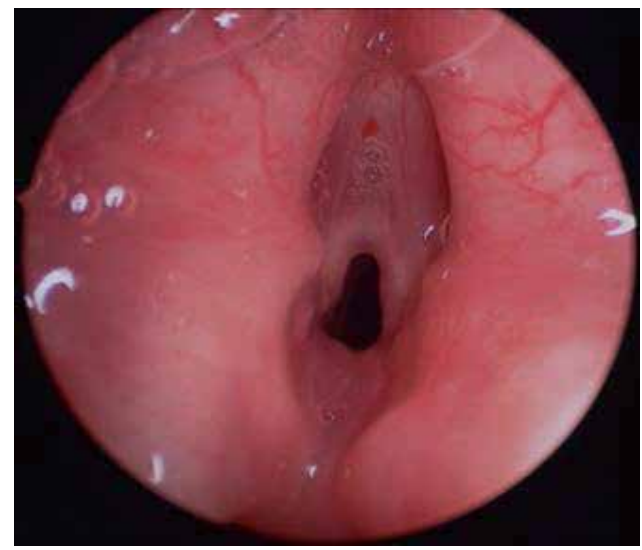

Fig. 13. Glottic web 
The vocal cords have limited mobility due to an anterior glottic web. This narrows the glottic airway causing biphasic stridor. Endoscopic surgical repair can be performed.

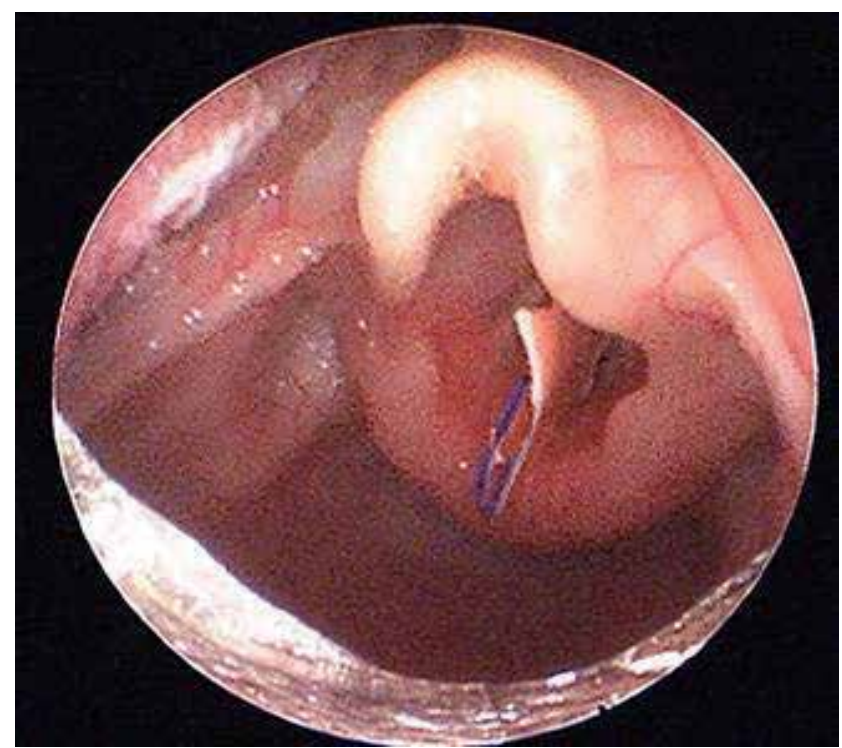

Fig. 14. Glottic foreign body

A pencil shaving lodged in the glottis of a child with severe stridor. This was emergently removed in the operating room.

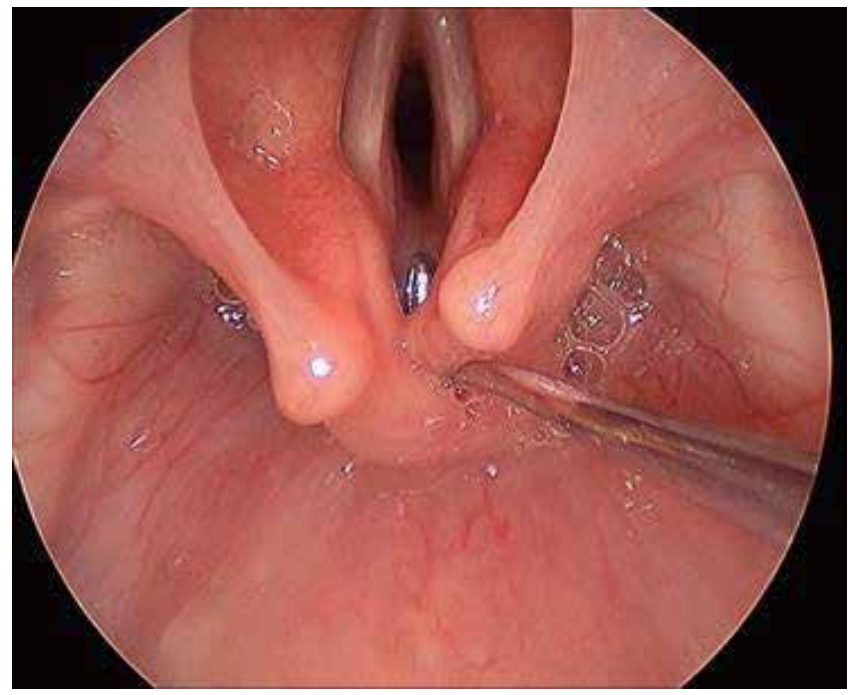

Fig. 15. Normal larynx

A right angle probe can be used to palpate the interarytenoid notch to check for a posterior laryngeal cleft. It is also helpful to palpate the posterior cricoid lamina with a right angle probe to ensure there is no divot or absence of cartilage. 


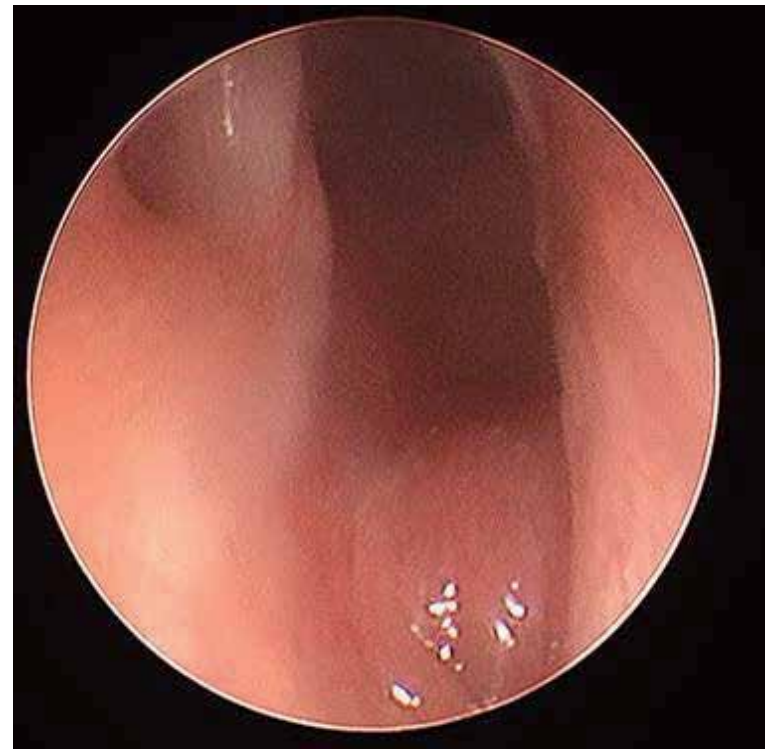

Fig. 16. Type I posterior laryngeal cleft

The interarytenoid notch is below the level of the true vocal cord. With palpation, the cricoids cartilage is intact. Aspiration of thin liquids often can occur, but many infants do well with a thickened diet. If aspiration fails to resolve with time, endoscopic repair can be performed.

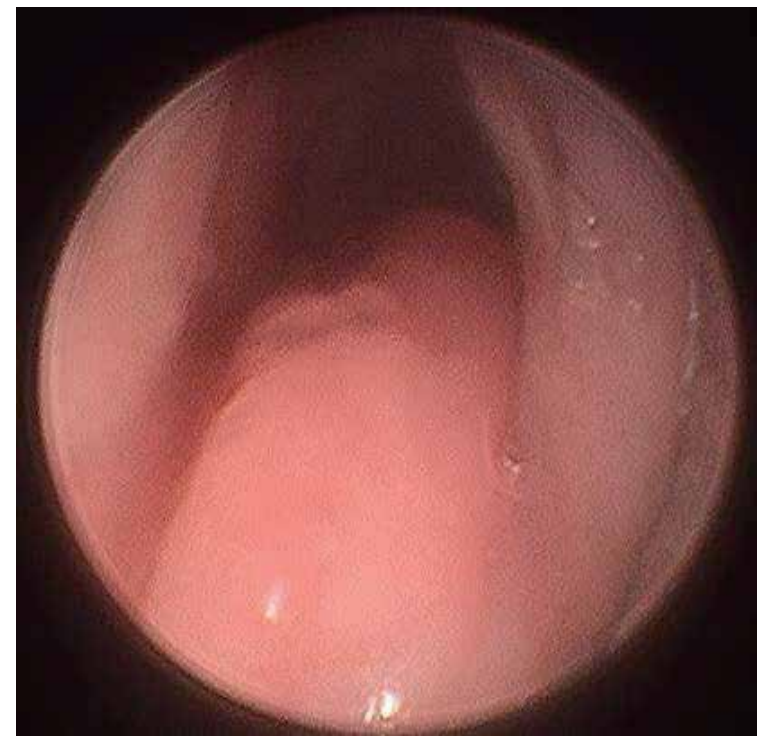

Fig. 17. Type III posterior laryngeal cleft

The cleft extends down through the cricoid cartilage below the vocal cords. With palpation, there is only a cleft of soft tissue posteriorly, no posterior cricoid lamina. It does not enter the thoracic cavity, so is therefore a type III. This cleft is a direct connection between the esophagus and trachea leading to aspiration with feeding. 


\subsection{The subglottis}

The subglottic region is generally difficult to visualize on awake flexible fiberoptic laryngoscopy in the office setting. Subglottic stenosis can be congenital or acquired, and open laryngotracheal reconstruction has been successful (Cotton 2000). Endoscopic management including dilation, laser, mitomycin c, and steroids have also been used (Quensel 2011).

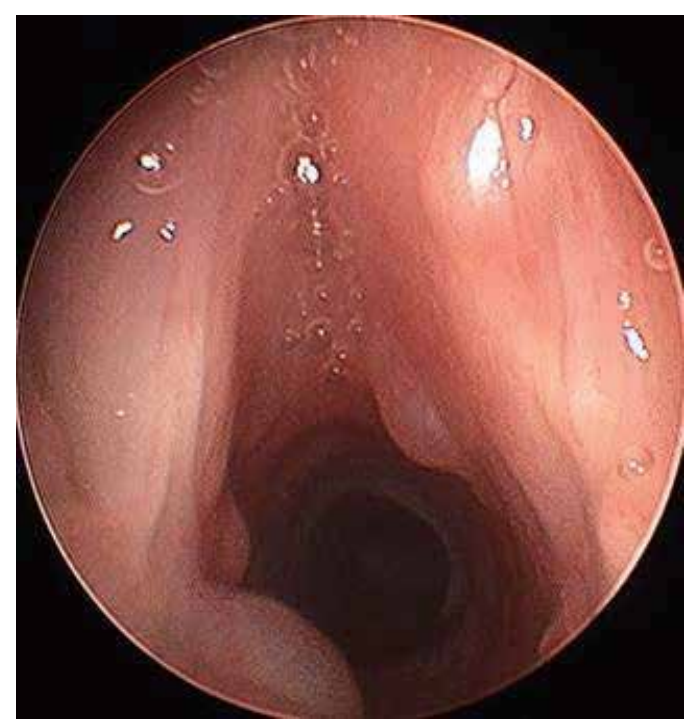

Fig. 18. Glottic and subglottic cysts

Commonly as a result of intubation, sometimes ductal cysts form from occluded submucosal glands. These can be asymptomatic if small.

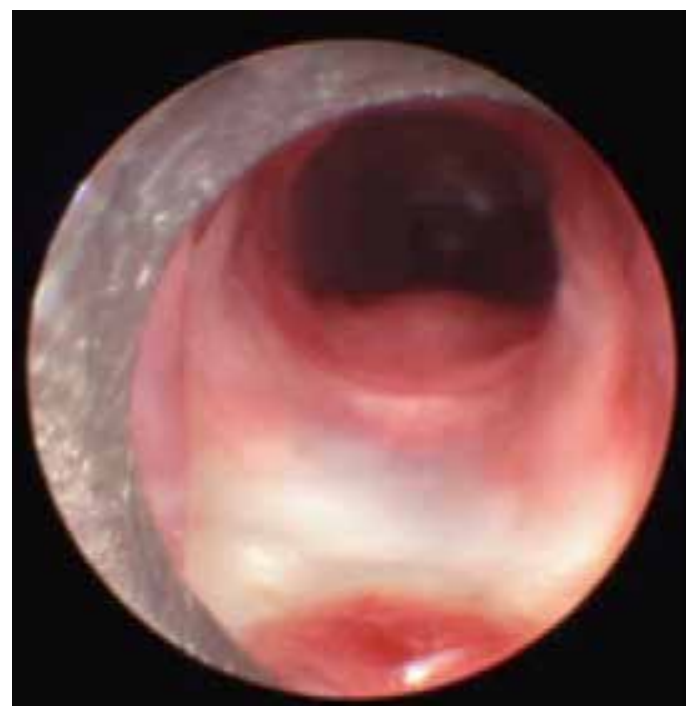

Fig. 19. Intubation injury 
Erosion of mucosa overlying posterior cricoid lamina, due to direct pressure from endotracheal tube. Exposed cartilage is seen posteriorly (white). This can lead to subglottic stenosis after intubation.

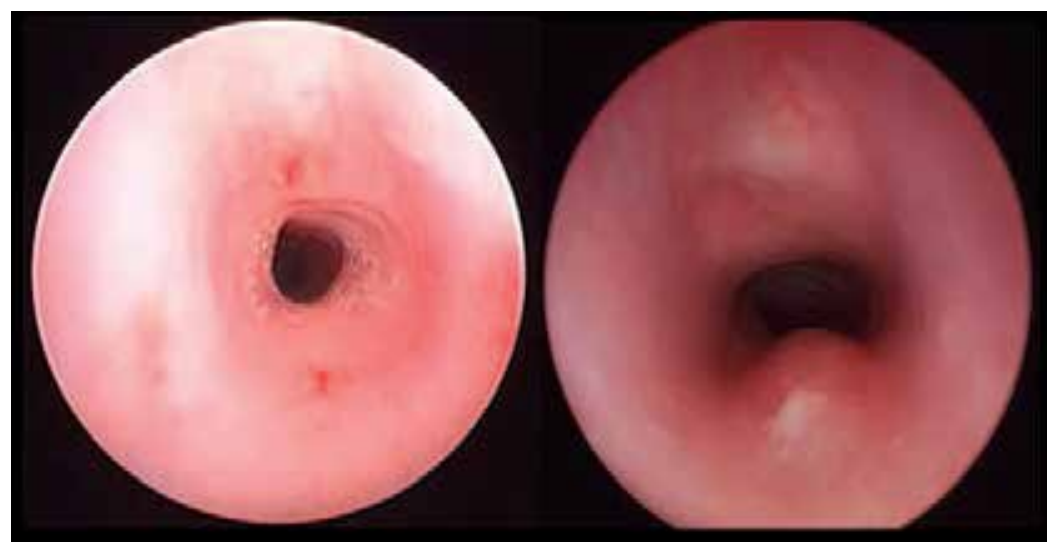

Fig. 20. Congenital subglottic stenosis

Note the narrowing below the vocal cords at the level of the cricoid cartilage and first tracheal ring, Grade III in this patient. This same patient, after a laryngotracheal reconstruction with anterior thyroid ala cartilage graft, the post-operative bronchoscopy shows a well-mucosalized graft.

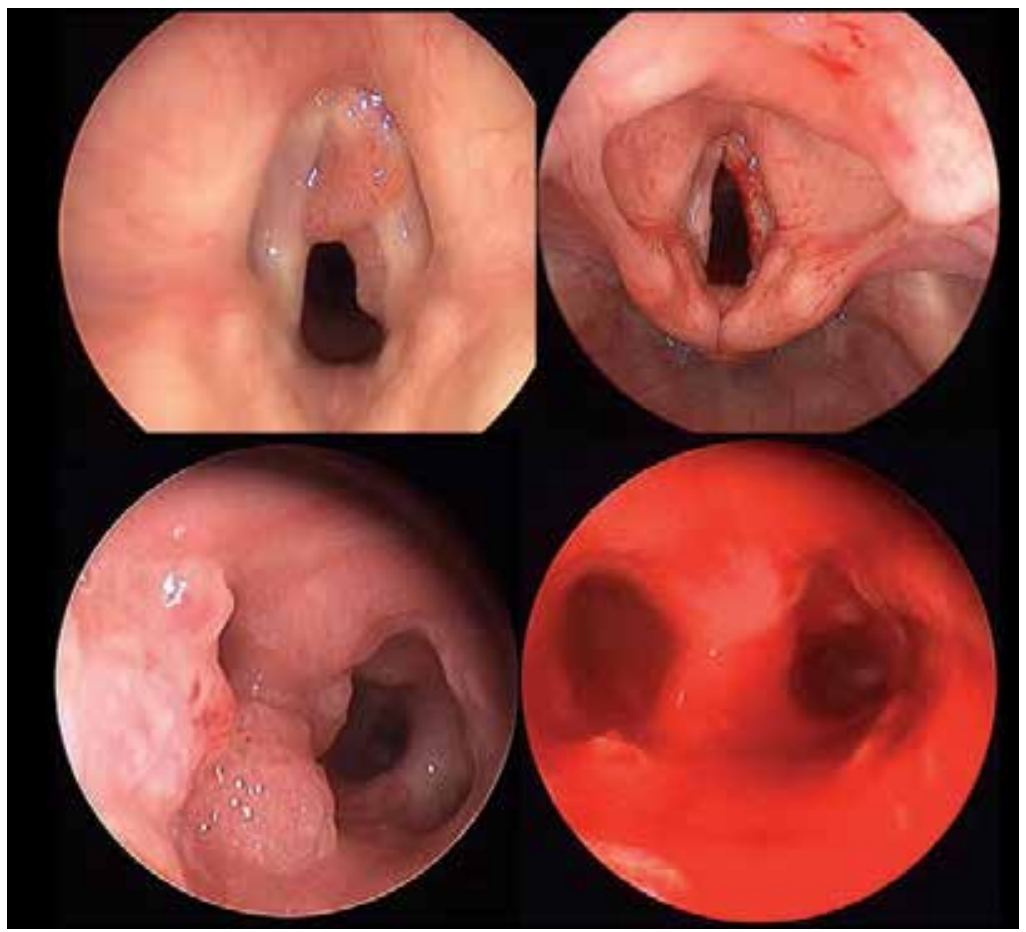

Fig. 21. Laryngeal and tracheal papilloma 
Top left: Endoscopic view of the true vocal cords, showing laryngeal papilloma of the right true vocal cord; Top right: After endoscopic removal using microdebrider. Bottom left, tracheal papilloma at the carina; bottom right, after endoscopic surgical removal.

\subsection{Recurrent Respiratory Papillomatosis (RRP)}

RRP is the most common benign neoplasm of the larynx and the second most common cause of hoarseness in children. It is caused by human papilloma virus and can have either childhood or adult onset. There is no cure for RRP and malignant transformation can rarely occur. Due to location and airway obstruction, surgical debulking is necessary in symptomatic patients. Adjuvant therapies include cidofovir, acyclovir, ribavirin, interferon, photodynamic therapy, indole-3-carbinol, cox-2 inhibitors, and retenoids. A quadravalent HPV vaccine is available and may help reduce the incidence of RRP in the future. (Derkay, 2008). Extra-esophageal reflux has been linked to the disease course in some patients with RRP (McKenna, 2005; Pignatari, 2007).

\section{Abnormalities of the tracheobronchial tree}

\subsection{Tracheobronchomalacia}

A condition where there is collapse of the lumen due either intrinsic (primary) or extrinsic factors (secondary). The extraluminal pressure exceeds the intraluminal pressure and can be most significant on expiration. This can cause significant airway obstruction (Austin, 2003; Boogaard 2005). Tracheomalacia is commonly seen in patients with tracheoesophageal fistula.

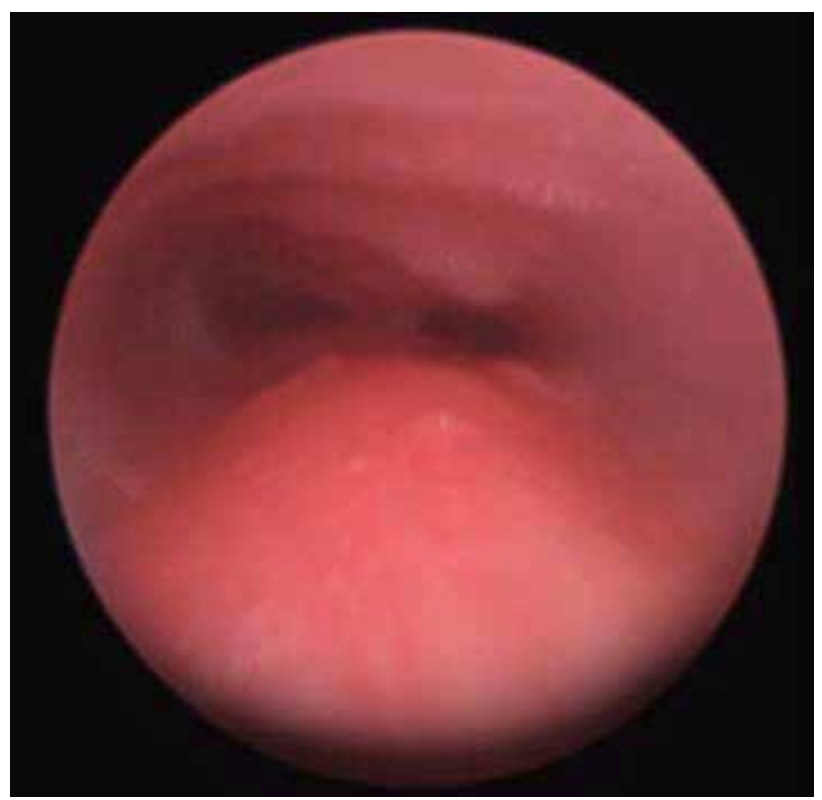

Fig. 22. Severe tracheomalacia

Narrowing of tracheal lumen is seen in a child with tracheoesophageal fistula. 


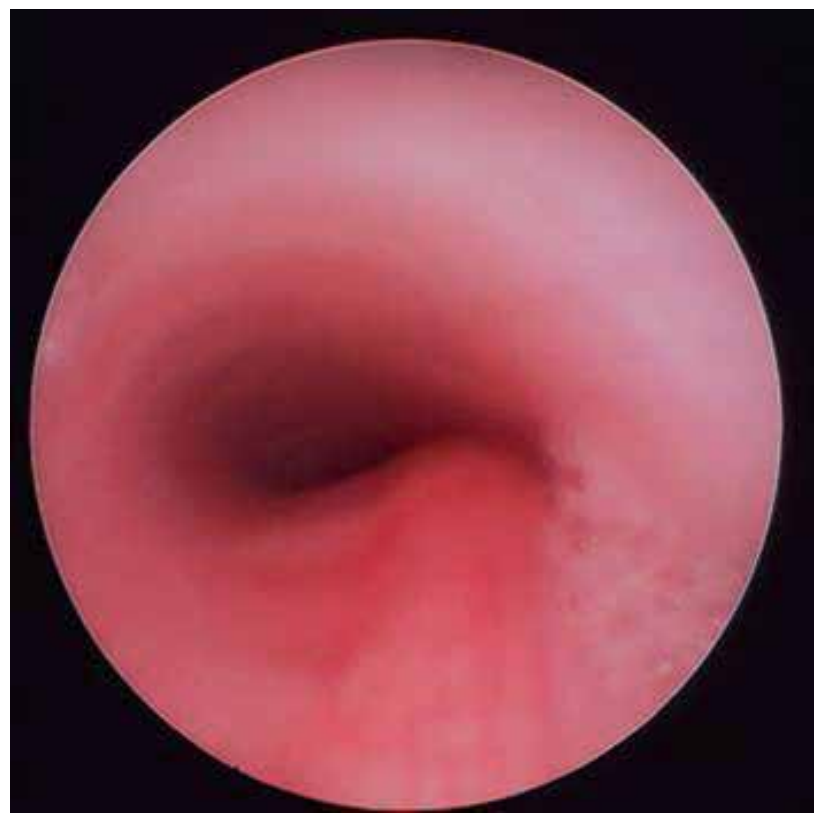

Fig. 23. Severe tracheomalacia

Narrowing of the tracheal lumen is seen with a coin in the esophagus causing external compression of the tracheal airway.

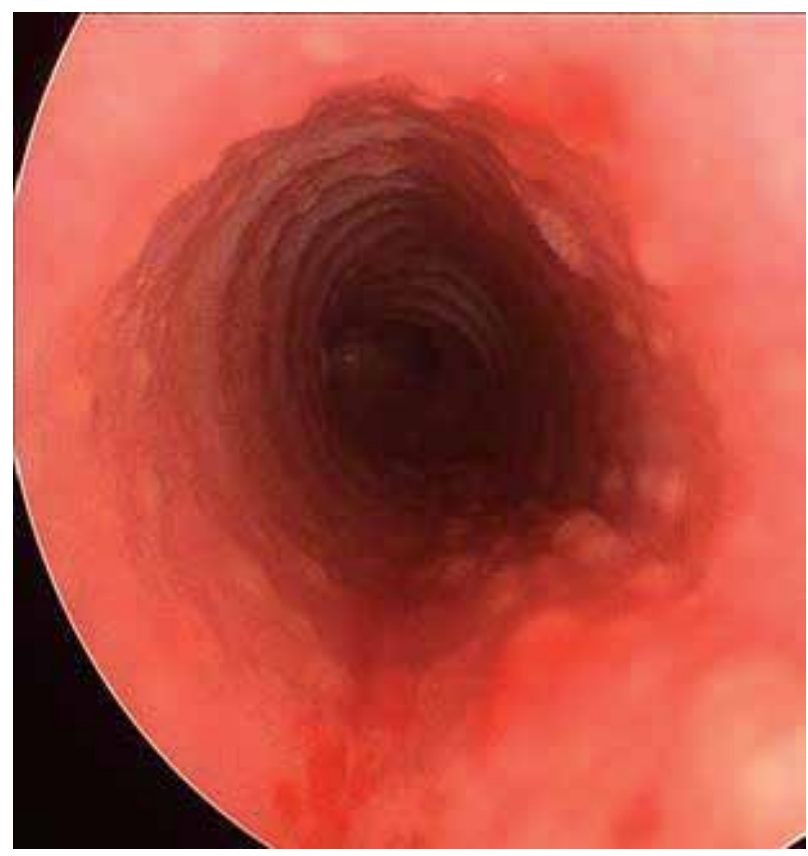

Fig. 24. Chronic follicular tracheitis

Raised mucosal follicles, often seen with acid reflux into the airway. 


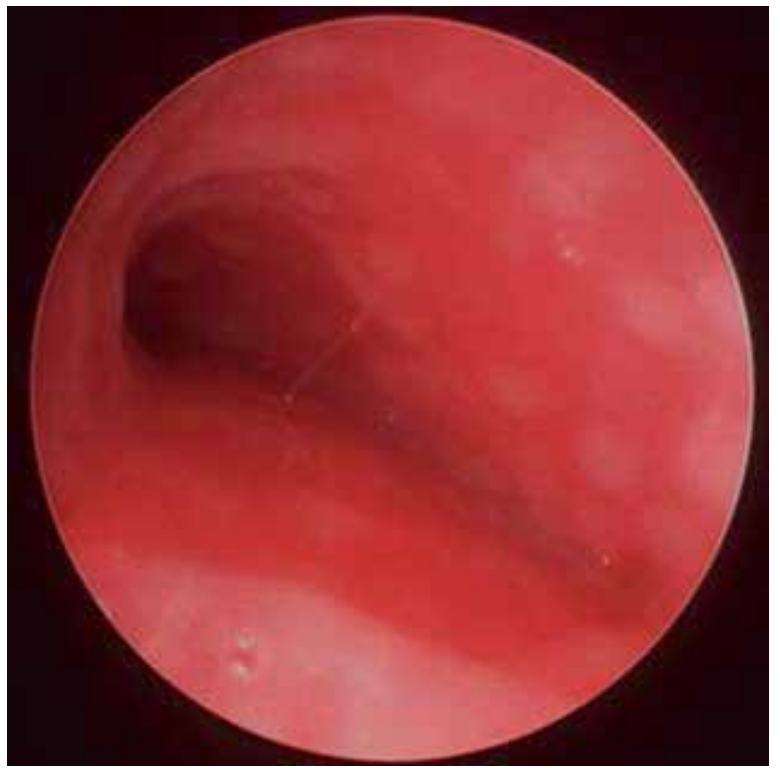

Fig. 25. Bronchomalacia

Narrowing of the left bronchial lumen is seen through a rigid bronchoscope as well as follicular changes of the mucosa.

\subsection{Complete tracheal rings}

A rare condition characterized by complete cartilaginous rings of the trachea, which can be of short segments or the entire length of the trachea. The surgical management of this condition has evolved over the past two decades (Backer, 2001). Currently, reconstruction methods can include excision of short segments with end-to-end anastomosis and slide tracheoplasty for longer segments (Russell, 2010). A slide tracheoplasty, unless done in a cervical tracheal location, is often done on cardiopulmonary bypass. Often a vascular anomaly, such as pulmonary artery sling is present, and it can be repaired simultaneously (Rutter 2003). Tracheal reconstruction has been successful in even patients with unilateral lung agenesis or severe hypoplasia (Backer, 2009).

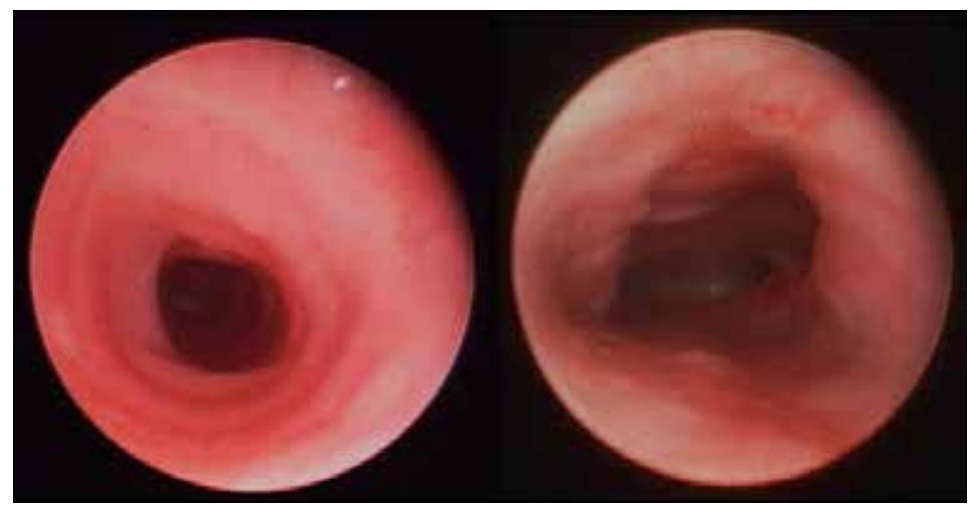

Fig. 26. Complete tracheal rings 
Note the concentric complete rings, absent membranous trachea posteriorly, resulting in a distal tracheal stenosis. On the right, the same patient 6 weeks after undergoing a slide tracheoplasty

\subsection{Airway hemangioma}

A hemangioma is a benign, vascular tumor. Rarely, these arise in the airway and can cause airway obstruction in the first year of life.Treatment options have included endoscopic resection, open resection, systemic or injectable steroids, tracheostomy (to bypass obstruction), interferon, observation (Rahbar, 2004) and more recently, propanolol therapy (Maturo, 2010).

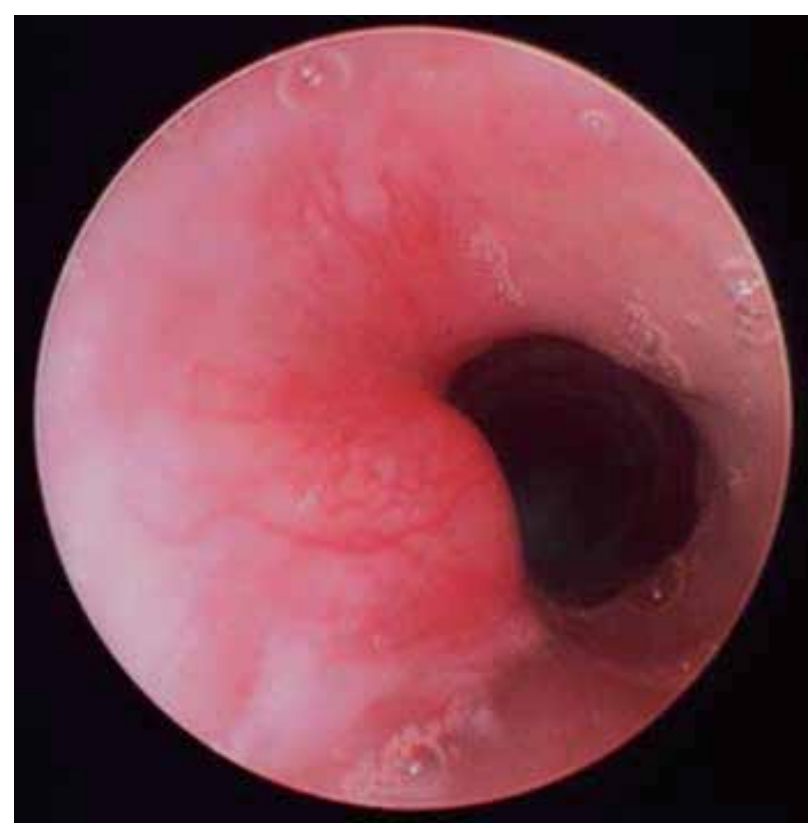

Fig. 27. Tracheal hemangioma

A left-posterior tracheal hemangioma at the level of the $3^{\text {rd }}$ tracheal ring. These are more commonly seen in the subglottis.

\subsection{Tracheobronchial foreign body and plastic bronchitis}

Bronchial foreign bodies can be life-threatening due to acute airway obstruction. Anatomically, an inhaled foreign body is most likely to enter the right bronchial tree in children of all ages as the proximal right main bronchus is generally steeper and wider than the left (Tahir, 2009). Rigid bronchoscopy is not only diagnostic but also therapeutic using optical forceps to remove foreign bodies under direct visualization. While ventilating the patient through the scope. Communication between the surgeon and anesthesiologist is critical for optimal results (Zur, 2009).

Plastic bronchitis is rare condition where bronchial casts form resulting in lifethreatening obstruction. This has been associated with congenital heart disease, particularly after patients undergo a Fontan procedure (Tzifa,2005; Ishman, 2003; Preciado, 
2010). Other causes of bronchial casts include cystic fibrosis, asthma, and influenza H1N1 (Terano 2010). The exact etiology for these is unknown, and treatment includes immediate endoscopic removal of the airway obstruction. Various adjuvant therapies including chest physiotherapy, aerosolized urokinase and tissue plasminogen activator, corticosteroids, DNase, and macrolides have been used with limited success (Brogan, 2002; Preciado, 2010)

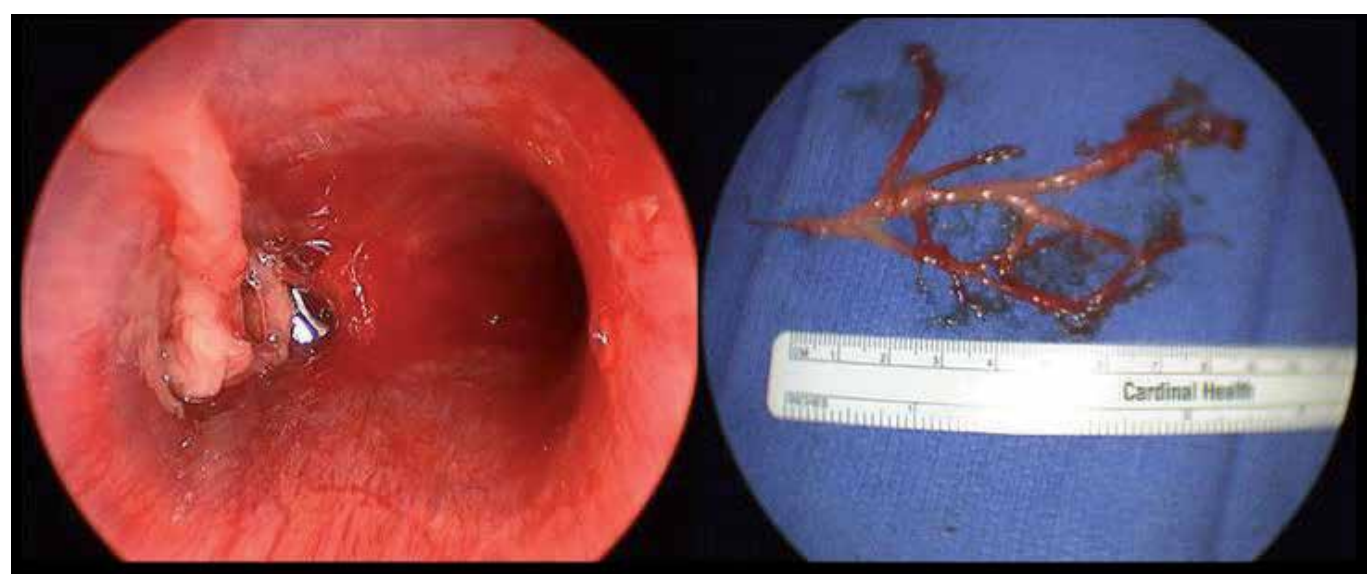

Fig. 28. Plastic bronchitis

Cast formation within the left bronchus before (left) and after (right) endoscopic removal.

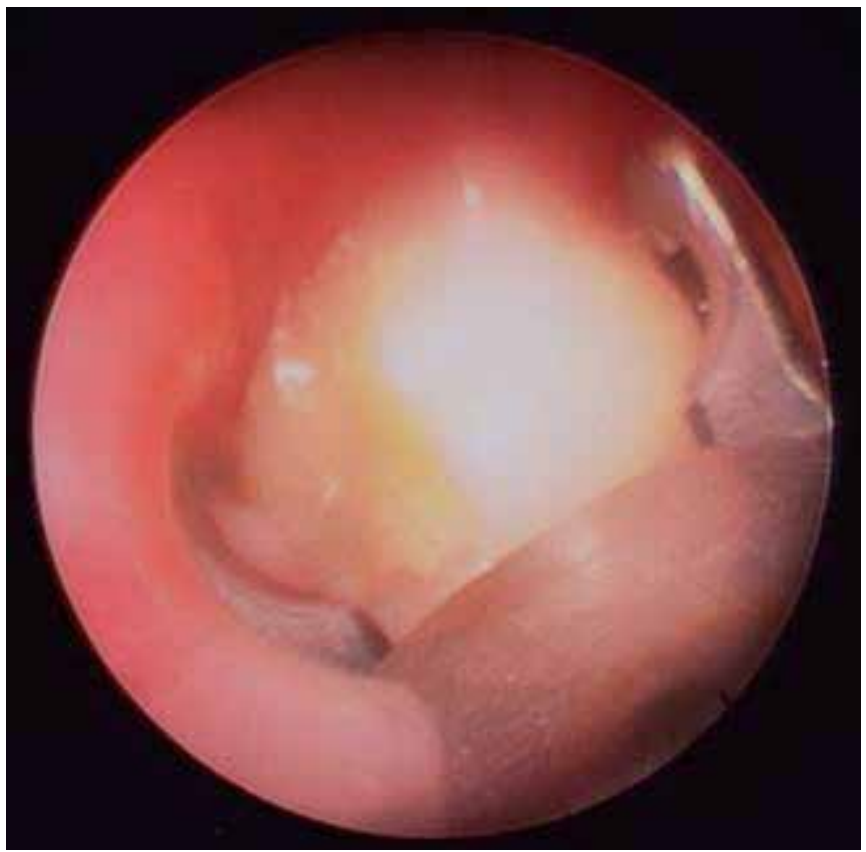

Fig. 29. Bronchial foreign body removal 
The optical graspers (telescope with attached camera) through a rigid bronchoscope are used to remove a molar tooth unusually lodged in the the left upper bronchus.

\subsection{Vascular rings}

A "vascular ring" refers to any anomaly of the aortic arch that leads to compression of the trachea, and/or esophagus. Most children will present early in life with breathing and feeding difficulties. Only the double aortic arch and right aortic arch with left ligament are true complete vascular rings; the innominate artery compression and pulmonary artery sling are incomplete from an anatomical standpoint. Endoscopic evaluation with laryngoscopy and bronchoscopy is helpful to assess the degree of airway compression (Shah, 2007; Russell, 2010).

The double aortic arch occurs when two arches arise at the ascending aorta, pass around both sides of the trachea and esophagus, to join the descending aorta. The right aortic arch occurs when the apex of the arch is to the right side of the trachea; various configurations are possible. Innominate artery compression of the anterior trachea is often due to a more left and posterior origination from the aorta (Russell 2010). If severe, suspension of the innominate artery to the posterior aspect of the sternum has been performed (Moes, 1975). Bronchoscopic evaluation after suspension confirms adequate relief. Pulmonary artery sling occurs when the left pulmonary artery originates from the right pulmonary artery, coursing around the right main bronchus and distal trachea, between the trachea and esophagus, to return to the left side. Pulmonary artery sling is often associated with complete tracheal rings and surgical correction of both can be performed simultaneously (Russell 2010).

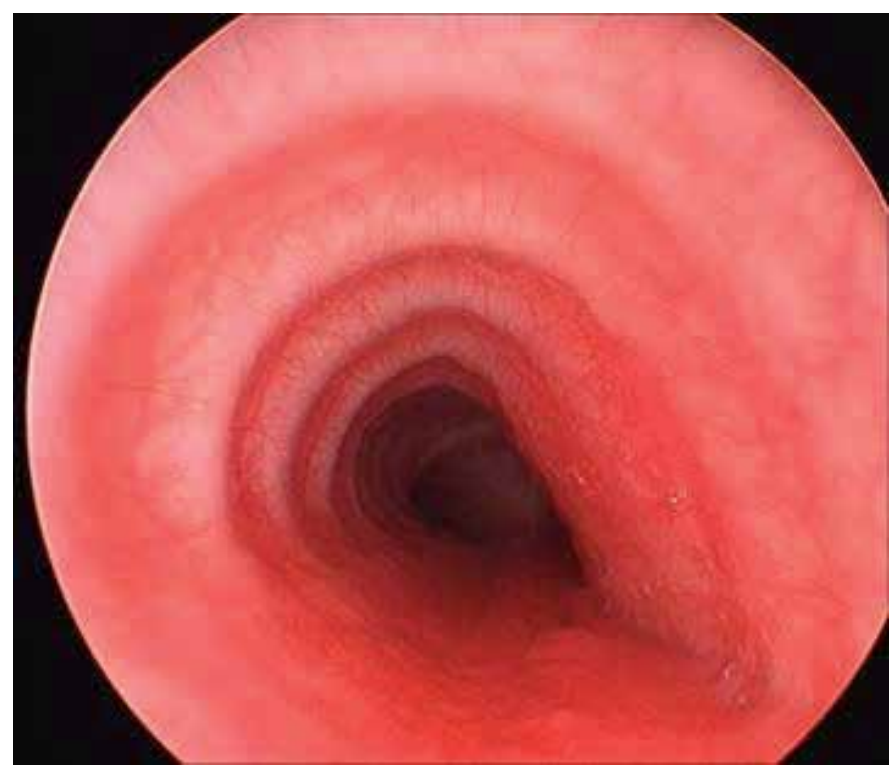

Fig. 30. Tracheomalacia, secondary to vascular ring

A child with a double aortic arch, prior to surgical repair, note the external tracheal compression causing airway obstruction. 


\subsection{Blunt laryngotracheal trauma}

Blunt trauma to the anterior neck can be a life-threatening injury. Significant injury in children is not common as the larynx is higher in location within the neck, and the laryngeal cartilage structures are more flexible than adults. Symptoms can include hoarseness, stridor, respiratory distress, subcutaneous emphysema, dysphagia, neck pain, and hemoptysis. In a stable child with suspected injury, flexible endoscopic evaluation of the larynx can be performed to determine if further surgical intervention would be needed (Gold, 1997). Endotracheal intubation of such cases can be catastrophic resulting in laryngotracheal separation and death (Bernat, 2005). In severe injury, tracheotomy is required for obtaining a stable airway. Children often cannot undergo an awake tracheotomy procedure under local anesthesia, so rigid bronchoscopy using a ventilating scope is critical in securing the airway prior to tracheotomy and open surgical repair.

\section{Conclusion}

The advancement in endoscopic technology combined with contemporary surgical applications has helped progress the field of diagnostic airway evaluation in children, as well as therapeutic interventions. Certainly, with enhanced visualization, the ability to more safely manage the airway allows for optimal care of children with congenital and acquired anomalies. There is a definite role for endoscopic surgery in pediatric otolaryngology.

\section{References}

Austin J, Ali T. Tracheomalacia and bronchomalacia in children: pathophysiology, assessment, treatment and anaesthesia management. Paediatr Anaesth 2003;13:3-11.

Backer CL, Kelle AM, Mavroudis C, Rigsby CK, Kaushal S, Holinger LD. Tracheal reconstruction in children with unilateral lung agenesis or severe hypoplasia. Ann Thorac Surg 2009;88:624-30; discussion 30-1.

Backer CL, Mavroudis C, Gerber ME, Holinger LD. Tracheal surgery in children: an 18-year review of four techniques. Eur J Cardiothorac Surg 2001;19:777-84.

Bajaj PS, Bailey BN. Stenosis of the nostrils: a report of three cases. Br J Plast Surg 1969;22:269-73.

Bales C, Kotapka M, Loevner LA, et al. Craniofacial resection of advanced juvenile nasopharyngeal angiofibroma. Arch Otolaryngol Head Neck Surg 2002;128:1071-8.

Bernat RA, Zimmerman JM, Keane WM, Pribitkin EA. Combined laryngotracheal separation and esophageal injury following blunt neck trauma. Facial Plast Surg 2005;21:187-90.

Boogaard R, Huijsmans SH, Pijnenburg MW, Tiddens HA, de Jongste JC, Merkus PJ. Tracheomalacia and bronchomalacia in children: incidence and patient characteristics. Chest 2005;128:3391-7.

Brogan TV, Finn LS, Pyskaty DJ, Jr., et al. Plastic bronchitis in children: a case series and review of the medical literature. Pediatr Pulmonol 2002;34:482-7. 
Brown OE, Pownell P, Manning SC. Choanal atresia: a new anatomic classification and clinical management applications. Laryngoscope 1996;106:97-101.

Burkart CM, Richter GT, Rutter MJ, Myer CM, 3rd. Update on endoscopic management of lingual thyroglossal duct cysts. Laryngoscope 2009;119:2055-60.

Chen EY, Inglis AF, Ou H, et al. Endoscopic electrocauterization of pyriform fossa sinus tracts as definitive treatment. Int J Pediatr Otorhinolaryngol 2009;73:1151-6.

Cotton RT. Management of subglottic stenosis. Otolaryngol Clin North Am 2000;33:111-30.

Derkay CS, Wiatrak B. Recurrent respiratory papillomatosis: a review. Laryngoscope 2008;118:1236-47.

DeSanto LW, Devine KD, Weiland LH. Cysts of the larynx--classification. Laryngoscope 1970;80:145-76.

Deutsch E, Kaufman M, Eilon A. Transnasal endoscopic management of choanal atresia. Int J Pediatr Otorhinolaryngol 1997;40:19-26.

Diamantopoulos, II, Jones NS. The investigation of nasal septal perforations and ulcers. J Laryngol Otol 2001;115:541-4.

Douglas R, Wormald PJ. Endoscopic surgery for juvenile nasopharyngeal angiofibroma: where are the limits? Curr Opin Otolaryngol Head Neck Surg 2006;14:1-5.

Franco LP, Camargos PA, Becker HM, Guimaraes RE. Nasal endoscopic evaluation of children and adolescents with cystic fibrosis. Braz J Otorhinolaryngol 2009;75:806-13.

Fu J, Xue X, Chen L, Fan G, Pan L, Mao J. Lingual thyroglossal duct cyst in newborns: previously misdiagnosed as laryngomalacia. Int J Pediatr Otorhinolaryngol 2008;72:327-32.

Garabedian EN, Pezzettigotta S, Leboulanger N, et al. Endoscopic surgical treatment of laryngotracheal clefts: indications and limitations. Arch Otolaryngol Head Neck Surg 2010;136:70-4.

Giacomini PG, Ferraro S, Di Girolamo S, Ottaviani F. Large Nasal Septal Perforation Repair by Closed Endoscopically Assisted Approach. Ann Plast Surg 2011;66(6):633-6.

Gluckman PG, Chu TW, van Hasselt CA. Neonatal vallecular cysts and failure to thrive. J Laryngol Otol 1992;106:448-9.

Gold SM, Gerber ME, Shott SR, Myer CM, 3rd. Blunt laryngotracheal trauma in children. Arch Otolaryngol Head Neck Surg 1997;123:83-7.

Gutierrez JP, Berkowitz RG, Robertson CF. Vallecular cysts in newborns and young infants. Pediatr Pulmonol 1999;27:282-5.

Harley EH. Pediatric congenital nasal masses. Ear Nose Throat J 1991;70:28-32.

Hartzell LD, Richter GT, Glade RS, Bower CM. Accuracy and safety of tracheoscopy for infants in a tertiary care clinic. Arch Otolaryngol Head Neck Surg 2010;136:66-9.

Hedlund G. Congenital frontonasal masses: developmental anatomy, malformations, and MR imaging. Pediatr Radiol 2006;36:647-62; quiz 726-7.

Hengerer AS, Brickman TM, Jeyakumar A. Choanal atresia: embryologic analysis and evolution of treatment, a 30-year experience. Laryngoscope 2008;118:862-6.

Holinger LD, Barnes DR, Smid LJ, Holinger PH. Laryngocele and saccular cysts. Ann Otol Rhinol Laryngol 1978;87:675-85. 
Hughes GB, Sharpino G, Hunt W, Tucker HM. Management of the congenital midline nasal mass: a review. Head Neck Surg 1980;2:222-33.

Hulsmann AR, de Bont N, den Hollander JC, Borgstein JA. Hamartomas of the oro- and nasopharyngeal cavity in infancy: two cases and a short review. Eur J Pediatr 2009;168:999-1001.

Ishman S, Book DT, Conley SF, Kerschner JE. Plastic bronchitis: an unusual bronchoscopic challenge associated with congenital heart disease repair. Int J Pediatr Otorhinolaryngol 2003;67:543-8.

Jablon JH, Hoffman JF. Birth trauma causing nasal vestibular stenosis. Arch Otolaryngol Head Neck Surg 1997;123:1004-6.

Jatana KR, Oplatek A, Elmaraghy CA. Bilateral vestibular stenosis from nasal continuous positive airway pressure/cannula oxygen administration. Otolaryngol Head Neck Surg 2008;138:690-1.

Jatana KR, Oplatek A, Stein M, Phillips G, Kang DR, Elmaraghy CA. Effects of nasal continuous positive airway pressure and cannula use in the neonatal intensive care unit setting. Arch Otolaryngol Head Neck Surg 2010;136:287-91.

Karen M, Chang E, Keen MS. Auricular composite grafting to repair nasal vestibular stenosis. Otolaryngol Head Neck Surg 2000;122:529-32.

Kim B, Park SH, Min HS, Rhee JS, Wang KC. Nasal chondromesenchymal hamartoma of infancy clinically mimicking meningoencephalocele. Pediatr Neurosurg 2004;40:136-40.

Koeller KK, Alamo L, Adair CF, Smirniotopoulos JG. Congenital cystic masses of the neck: radiologic-pathologic correlation. Radiographics 1999;19:121-46; quiz 52-3.

Kuint J, Horowitz Z, Kugel C, Toper L, Birenbaum E, Linder N. Laryngeal obstruction caused by lingual thyroglossal duct cyst presenting at birth. Am J Perinatol 1997;14:353-6.

LaBagnara J, Jr. Cysts of the base of the tongue in infants: an unusual cause of neonatal airway obstruction. Otolaryngol Head Neck Surg 1989;101:108-11.

Lanier B, Kai G, Marple B, Wall GM. Pathophysiology and progression of nasal septal perforation. Ann Allergy Asthma Immunol 2007;99:473-9; quiz 80-1, 521.

Maturo S, Hartnick C. Initial experience using propranolol as the sole treatment for infantile airway hemangiomas. Int J Pediatr Otorhinolaryngol;74:323-5.

McGill TJ. Congenital anomalies of the larynx. London: Arnold Publisher; 2000.

McKenna M, Brodsky L. Extraesophageal acid reflux and recurrent respiratory papilloma in children. Int J Pediatr Otorhinolaryngol 2005;69:597-605.

McMurray JS, Holinger LD. Otolaryngic manifestations in children presenting with apparent life-threatening events. Otolaryngol Head Neck Surg 1997;116:575-9.

Moes CA, Izukawa T, Trusler GA. Innominate artery compression of the Trachea. Arch Otolaryngol 1975;101:733-8.

Pignatari SS, Liriano RY, Avelino MA, Testa JR, Fujita R, De Marco EK. Gastroesophageal reflux in patients with recurrent laryngeal papillomatosis. Braz J Otorhinolaryngol 2007;73:210-4.

Preciado D, Verghese S, Choi S. Aggressive bronchoscopic management of plastic bronchitis. Int J Pediatr Otorhinolaryngol;74:820-2. 
Quesnel AM, Lee GS, Nuss RC, Volk MS, Jones DT, Rahbar R. Minimally invasive endoscopic management of subglottic stenosis in children: Success and failure. Int J Pediatr Otorhinolaryngol 2011;75:652-656.

Rahbar R, Nicollas R, Roger G, et al. The biology and management of subglottic hemangioma: past, present, future. Laryngoscope 2004;114:1880-91.

Rahbar R, Rouillon I, Roger G, et al. The presentation and management of laryngeal cleft: a 10-year experience. Arch Otolaryngol Head Neck Surg 2006;132:1335-41.

Ramadan H, Ortiz O. Congenital nasal pyriform aperture (bony inlet) stenosis. Otolaryngol Head Neck Surg 1995;113:286-9.

Ramsden JD, Campisi P, Forte V. Choanal atresia and choanal stenosis. Otolaryngol Clin North Am 2009;42:339-52, x.

Russell HM, Backer CL. Pediatric thoracic problems: patent ductus arteriosus, vascular rings, congenital tracheal stenosis, and pectus deformities. Surg Clin North Am;90:1091-113.

Rutter MJ, Cotton RT, Azizkhan RG, Manning PB. Slide tracheoplasty for the management of complete tracheal rings. J Pediatr Surg 2003;38:928-34.

Salvado AR, Wang MB. Treatment of complete nasal vestibule stenosis with vestibular stents and mitomycin C. Otolaryngol Head Neck Surg 2008;138:795-6.

Shah RK, Mora BN, Bacha E, et al. The presentation and management of vascular rings: an otolaryngology perspective. Int J Pediatr Otorhinolaryngol 2007;71:57-62.

Tahir N, Ramsden WH, Stringer MD. Tracheobronchial anatomy and the distribution of inhaled foreign bodies in children. Eur J Pediatr 2009;168:289-95.

Terano C, Miura M, Fukuzawa R, et al. Three Children with Plastic Bronchitis Associated with 2009 H1n1 Influenza Virus Infection. Pediatr Infect Dis J. 2011;30(1):80-2.

Thompson DM. Abnormal sensorimotor integrative function of the larynx in congenital laryngomalacia: a new theory of etiology. Laryngoscope 2007;117:1-33.

Triglia JM, Nicollas R. Nasal and sinus polyposis in children. Laryngoscope 1997;107:963-6.

Tzifa A, Robards M, Simpson JM. Plastic bronchitis; a serious complication of the Fontan operation. Int J Cardiol 2005;101:513-4.

Verret DJ, McClay J, Murray A, Biavati M, Brown O. Endoscopic cauterization of fourth branchial cleft sinus tracts. Arch Otolaryngol Head Neck Surg 2004;130:465-8.

Weber SA, Ferrari GF. Incidence and evolution of nasal polyps in children and adolescents with cystic fibrosis. Braz J Otorhinolaryngol 2008;74:16-20.

Zielnik-Jurkiewicz B, Olszewska-Sosinska O. The nasal septum deformities in children and adolescents from Warsaw, Poland. Int J Pediatr Otorhinolaryngol 2006;70:731-6.

Zur KB, Litman RS. Pediatric airway foreign body retrieval: surgical and anesthetic perspectives. Paediatr Anaesth 2009;19 Suppl 1:109-17. 


\section{Part 7}

Concepts in Endoscopic Surgery 



\title{
Laparoscopic Surgery: An Almost Scarless Approach
}

\author{
Peng Soon Koh and Kin Fah Chin \\ Department of Surgery, Faculty of Medicine, \\ University of Malaya, Kuala Lumpur \\ Malaysia
}

\section{Introduction}

Minimally invasive surgery is gaining popularity worldwide as it contributes to less postoperative pain, shorter hospital stay and reduced morbidity. Since laparoscopic cholecystectomy was first performed in the 80's, a wide array of different types of laparoscopic surgery was performed and this has been extended to colorectal resection, where the first reported case of laparoscopic colorectal resection was made in 1991. Conventionally, a mini laparotomy is required to extract the specimen following laparoscopic colorectal resection, which may increase post-operative pain, wound infection, a bigger scar and other pain related morbidities. Thus, the use of natural orifice specimen extraction (NOSE) for the extraction of colorectal specimen is slowly gaining worldwide acceptance and evolving.

Single Incision laparoscopic Surgery or Single Port Access Surgery is also gaining popularity as an alternative approach to NOSE and Natural Orifice Transluminal Endoscopic Surgery (NOTES) in order to provide an almost scarless surgery. Often at times, such procedures are complementary to one another.

However, there is a significant cost implication due to the expensive disposable port and hand instruments but the main aim of having such procedures is the retrieval of resected surgical specimens through exploitation of natural body orifices such as the mouth, anus, vagina or urethra.

In this chapter, we described a technique for retrieval of the colonic specimen via a natural orifice or transvaginal for a 78 year old lady who had sigmoid carcinoma and underwent a total laparoscopic high anterior resection, hence avoiding a mini-laparotomy for retrieval of the specimen. We also described various instrumentation and developments in Single Incision Laparoscopic Surgery, NOSE or NOTES, including the latest technique to make this surgery safe. This is concluded with a Health Technology Assessment (HTA) of this emerging technology in health care.

\section{Case illustration}

\subsection{Clinical history}

Madam A is a 78 year old lady who complained of per rectal bleeding for 2 months. She had no history of altered bowel habit, loss of appetite or weight and symptoms of bowel 
obstruction. Examination was unremarkable except for haemorrhoids on proctoscopy. She had a colonoscopy which showed a fungating polypoidal mass at sigmoid colon and biopsy confirmed the lesion to be malignant. Computed Tomography (CT) scan for staging was done and showed no evidence of extra-colonic metastases.

She underwent a total laparoscopic high anterior resection with natural orifice specimen extraction (NOSE) after obtaining an informed consent.

\subsection{Operative technique}

In the operating theatre, the necessary laparoscopic equipments were readily available. The use of various intracorporeal and extracorporeal stapling devices was also necessary for the operation to be carried out. We also find that the use of Harmonic scalpel ${ }^{\circledR}$ or ultrasonic dissector to be very useful for tissue dissection during the procedure (Figure 1).

Once patient was placed under general anaesthesia, she was positioned into Lloyd-Davies position. This position allows access to the vagina for specimen retrieval as well as via the anal canal for bowel end to end anastomosis (Figure 2).

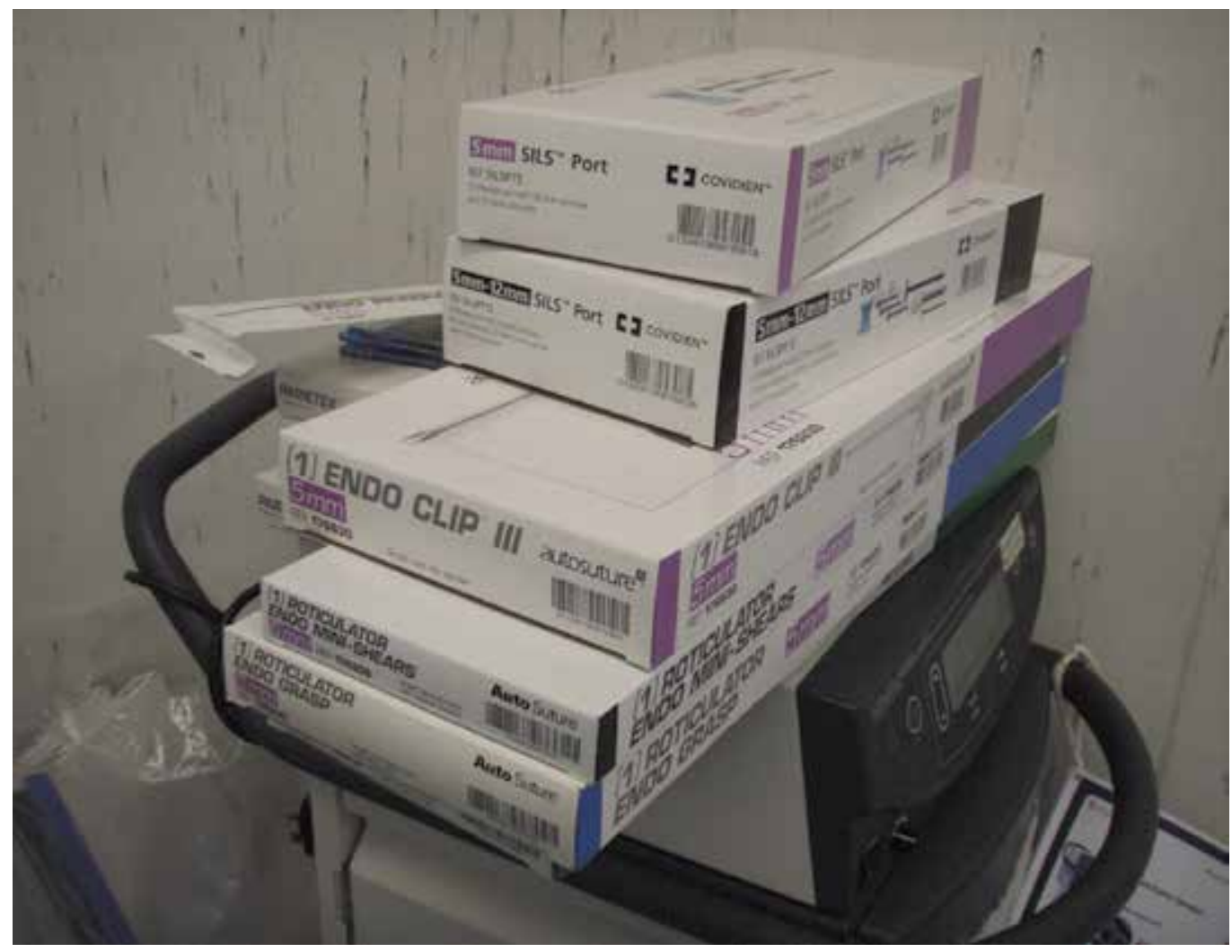

Fig. 1. The various stapling devices, equipments and ultrasonic dissector required for the surgery 


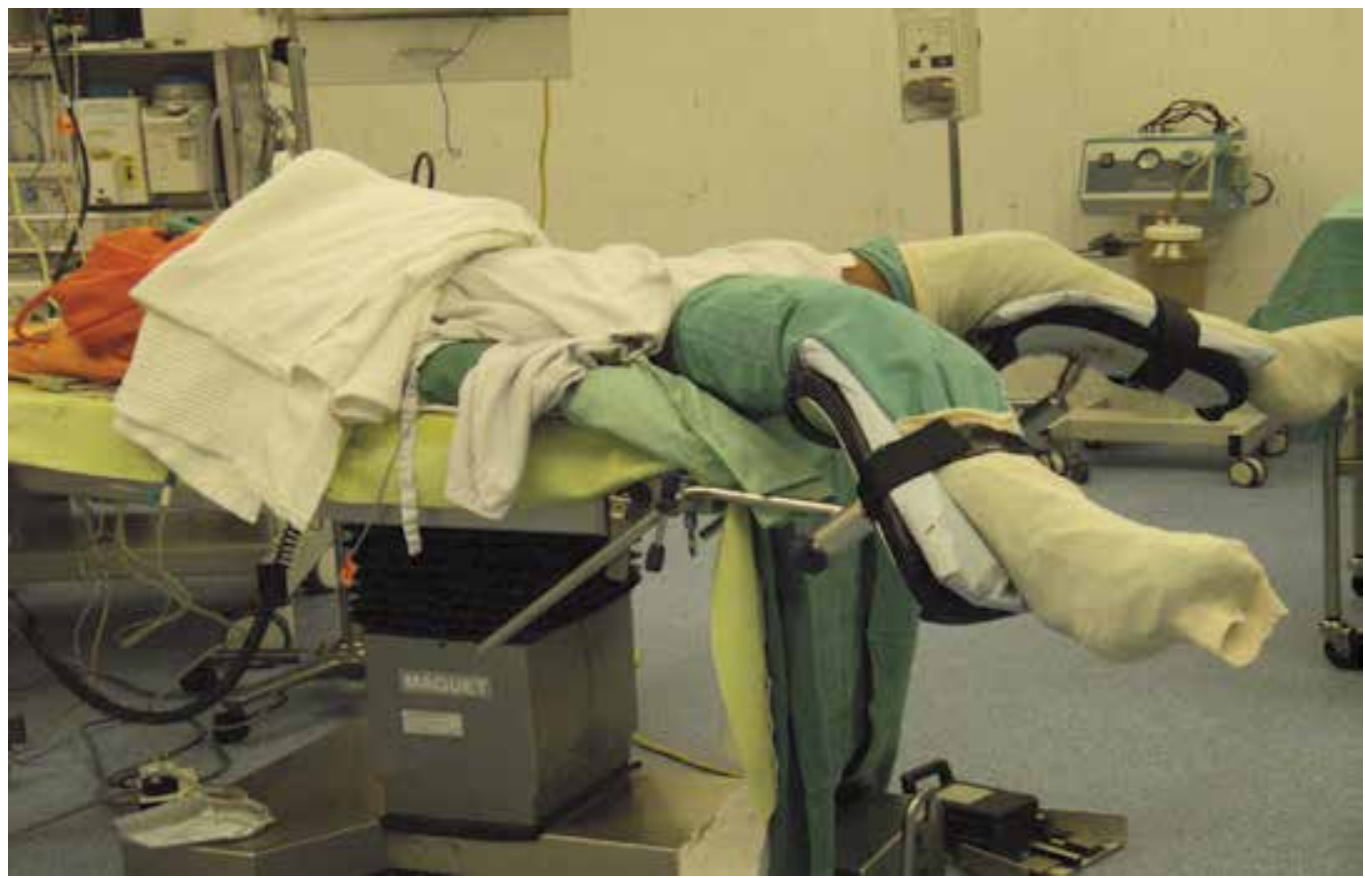

Fig. 2. Positioning of patient for the procedure to be carried out on the operating table.

Intra-operatively, laparoscopic ports were inserted at the appropriate place following pneumoperitoneum. The sigmoid colon was found to be redundant and the tumour was noted to be at the lower sigmoid colon. We began by hitching up the uterus by using a straight needle inserted percutaneously and into the broad ligament of the uterus and retrieving back the needle from the skin where the suture was tied hence lifting the uterus up onto the anterior abdominal wall (Figure 3).

Once there was adequate exposure, we began mobilizing the sigmoid colon from medially to laterally and the left ureter was identified and preserved. The inferior mesenteric vessels were then identified and ligated using vascular staplers. Mesorectal excision was then performed with the tumour further mobilized. The bowel distal to the tumour is then transected using a stapler.

Next, with the assistance from the gynaecologist, the posterior fornix was lifted up using a swab on a sponge forcep that was inserted via the vagina. With the posterior fornix lifted up, a transverse incision is made across the posterior fornix (Figure 4).

The sponge forcep is removed and a laparoscopic hand-port is inserted transvaginally to maintain pneumoperitoneum (Figure 5). The tumour is then delivered transvaginally where it is resected proximally (Figure 6). 


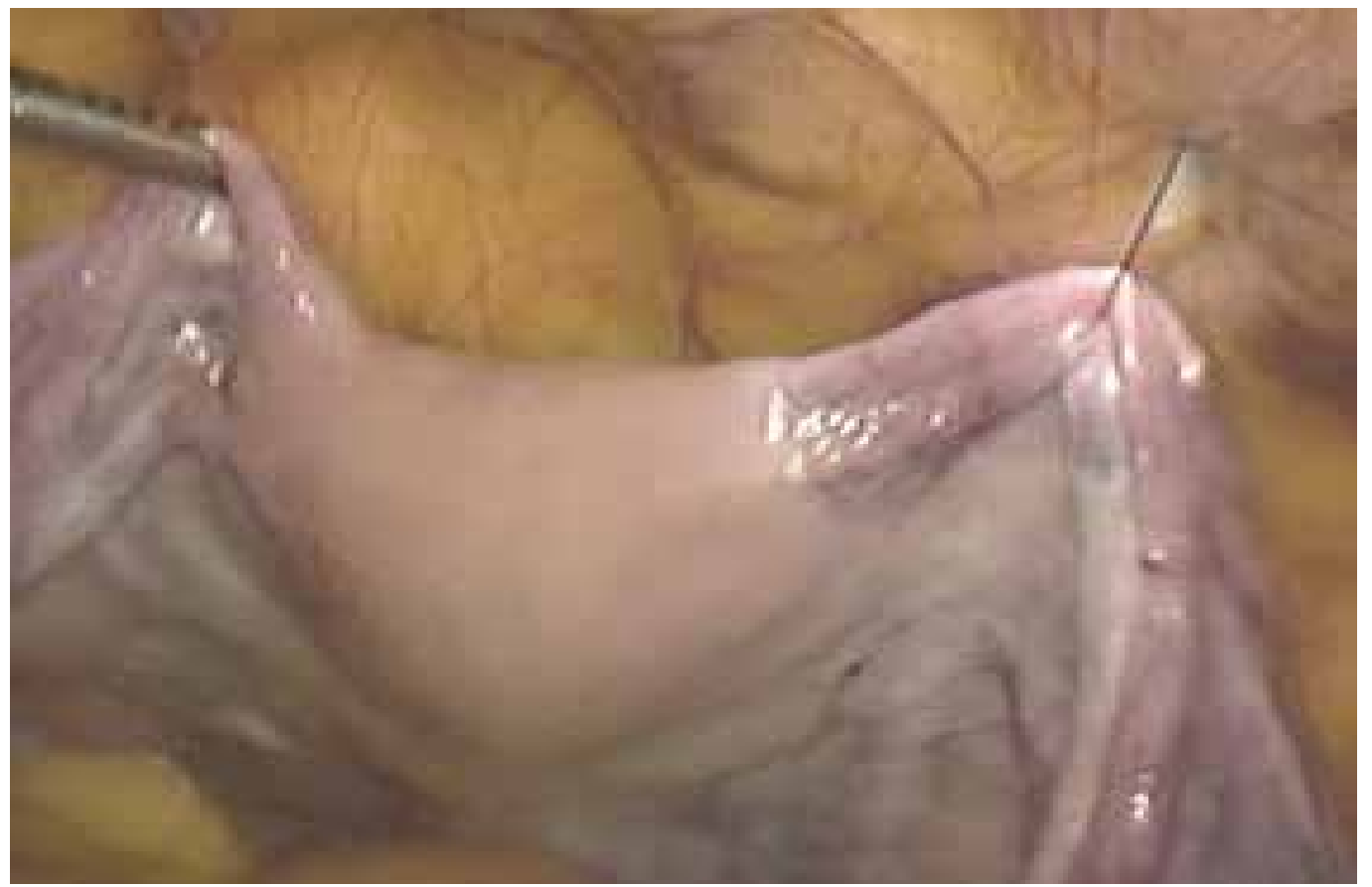

Fig. 3. The uterus hitched to the anterior abdominal wall using sutures passed through the broad ligament

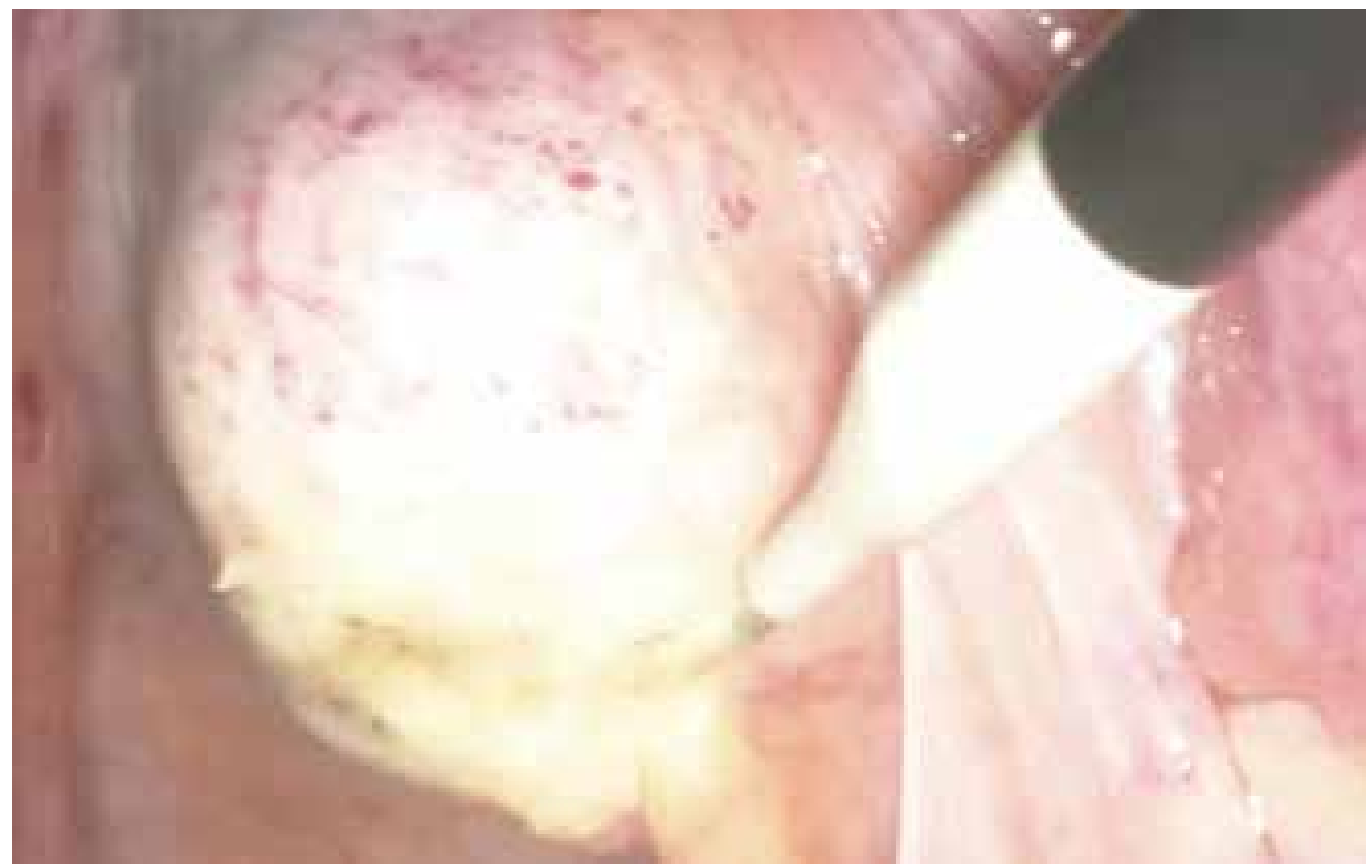

Fig. 4. Incision made across posterior fornix using diathermy following lifting up by the assistant transvaginally 


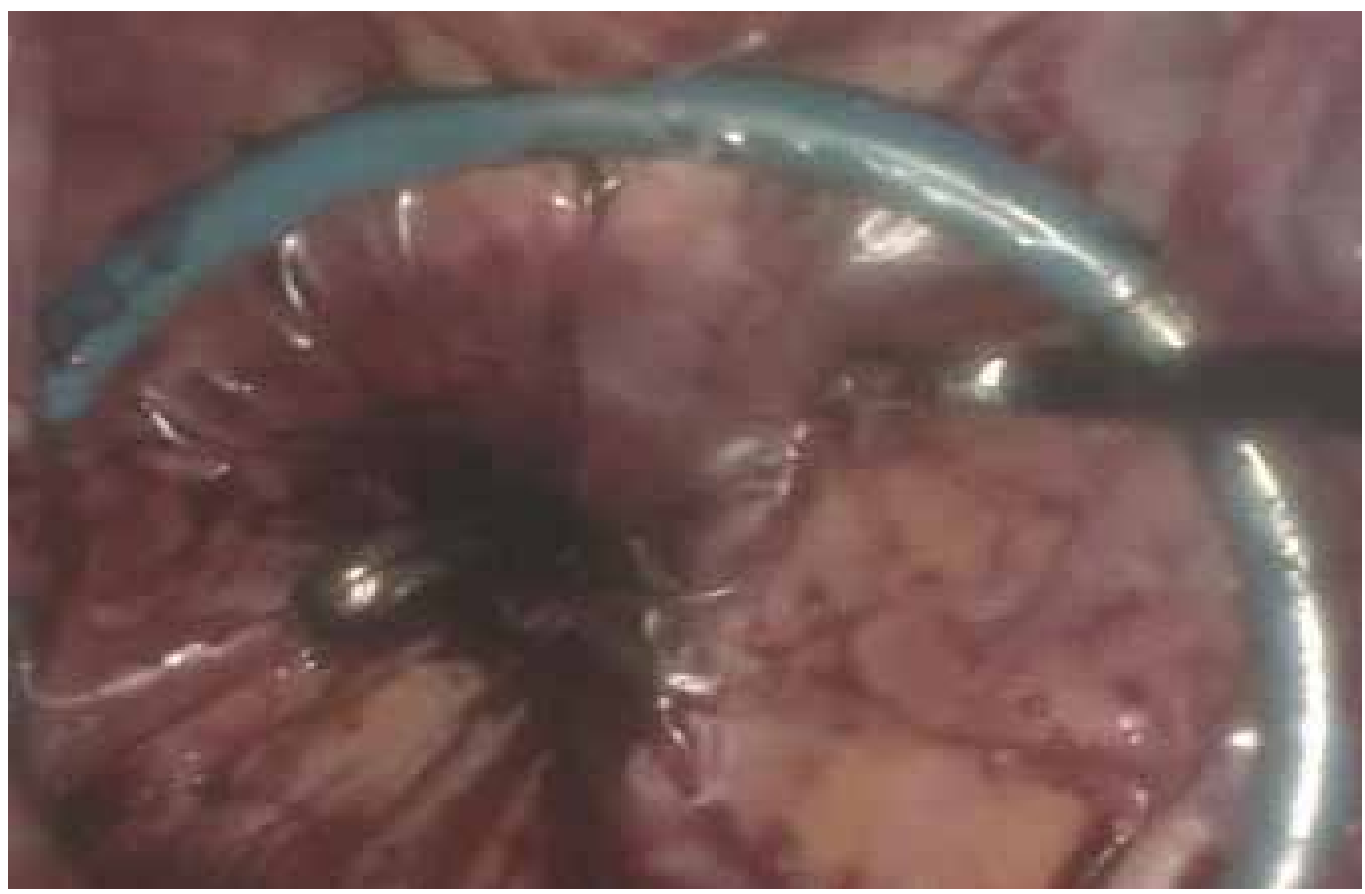

Fig. 5. Hand port inserted via vagina and through the incision made across the posterior fornix. Seen here within the abdomen.

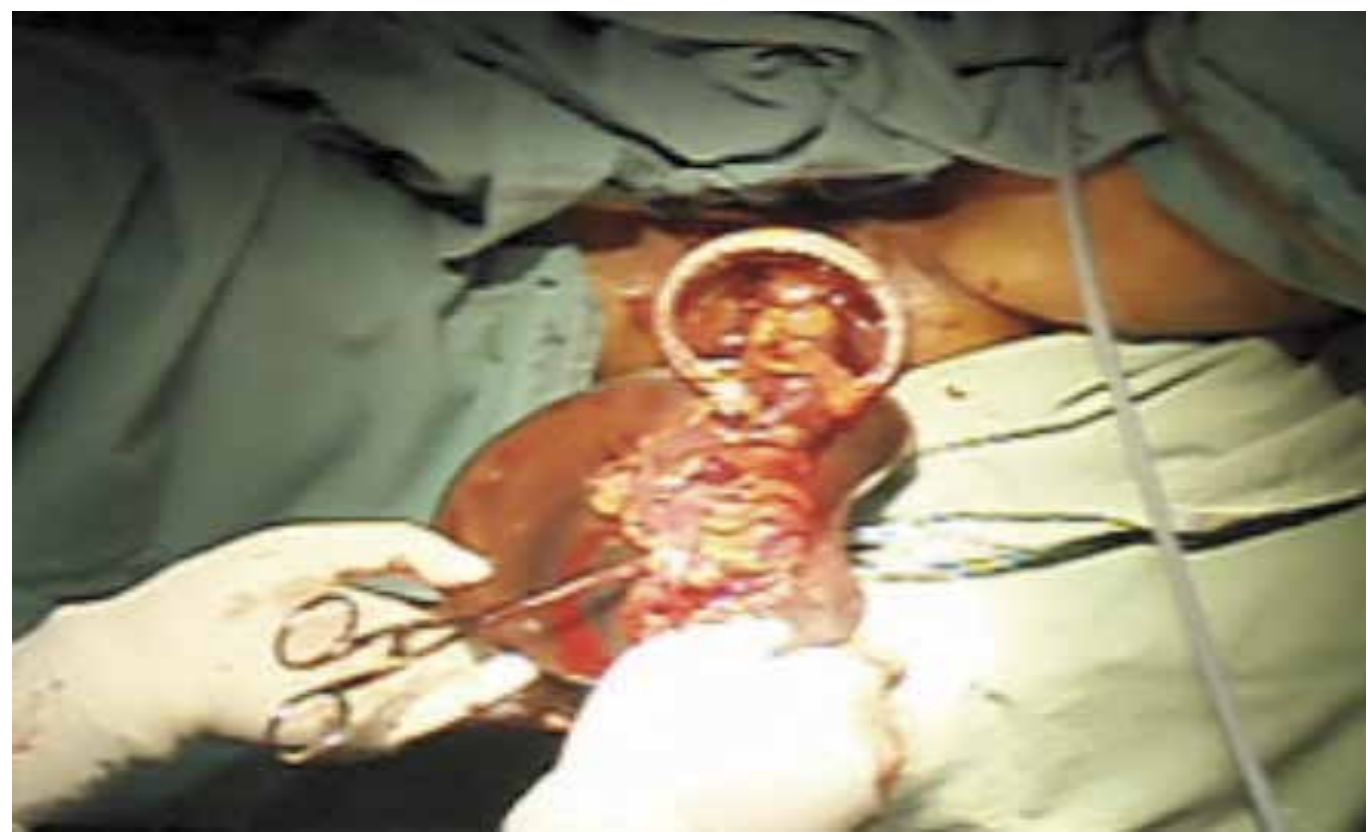

Fig. 6. Tumour extracted out via the vagina and transected proximally to tumour. 
The anvil of the circular stapler is then inserted into the proximal colon and closed using purse string suture. The proximal colon is then returned into the abdomen with the anvil. The hand port is removed and the posterior fornix is closed via intracorporeal suturing (Figure 7). Bowel end to end anastomosis was completed by inserting the $\mathrm{CDH} 29 \mathrm{~mm}$ circular stapler via anal canal (Figure 8). No leak was encountered intraoperatively when checked post anastomosis.

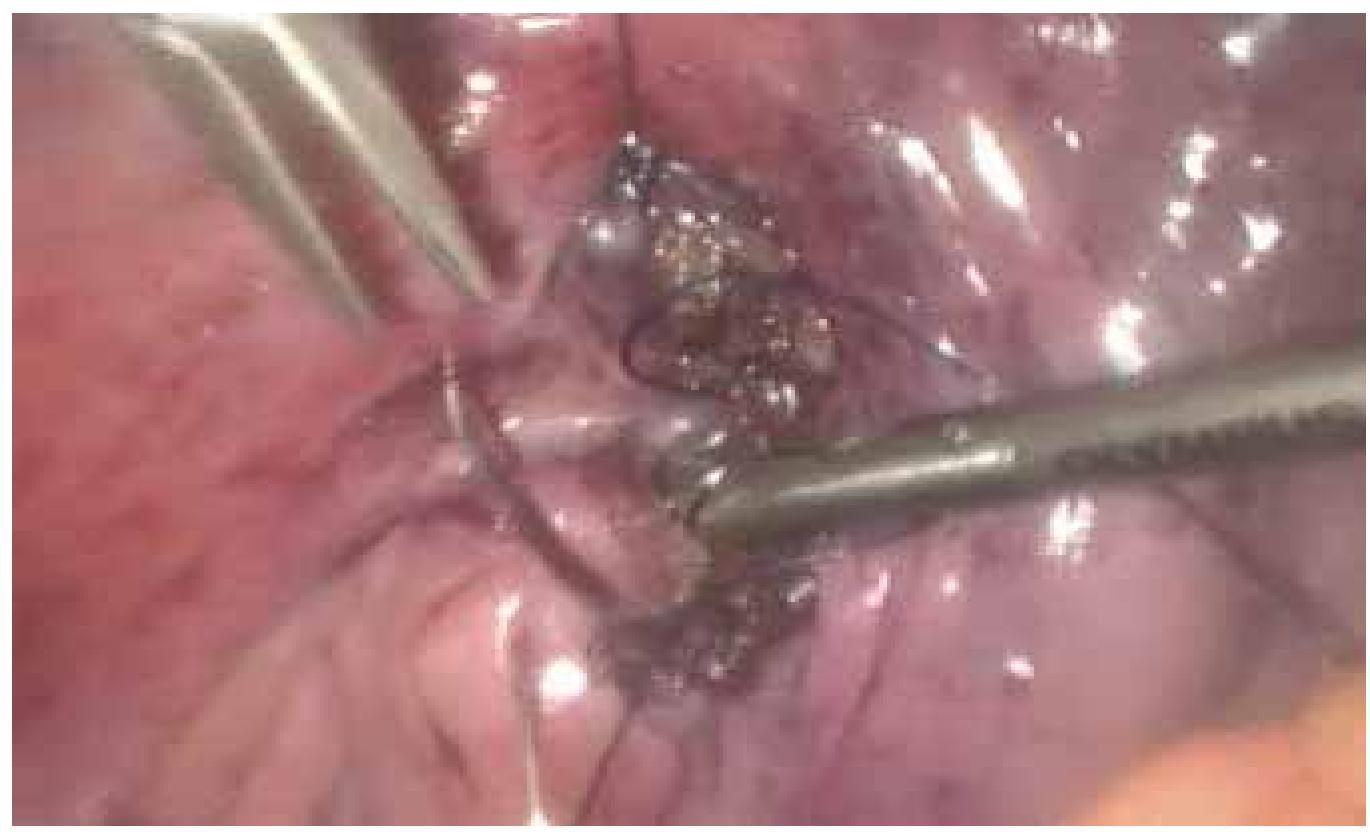

Fig. 7. Posterior fornix is stitched via intracorporeal technique after removal of specimen 


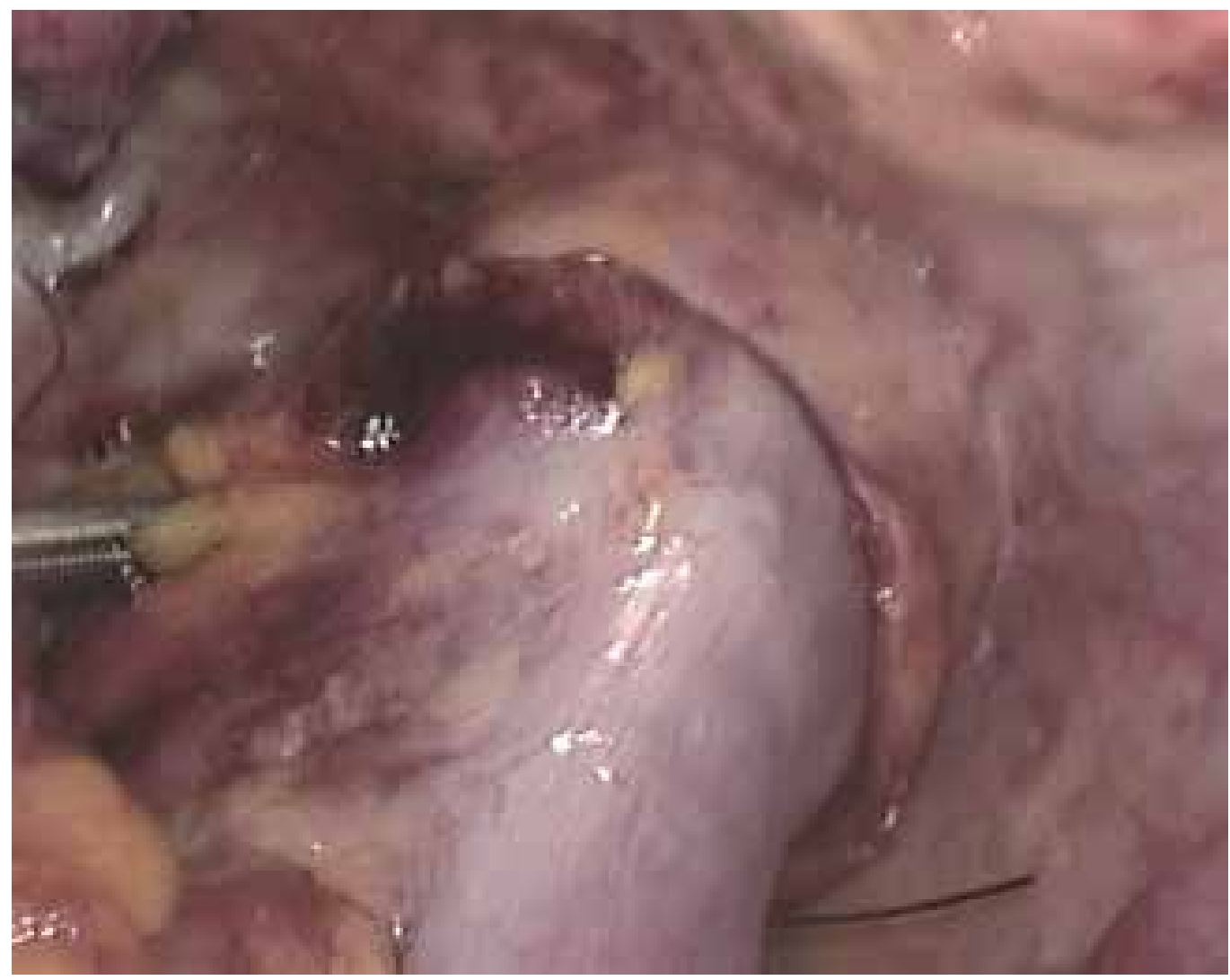

Fig. 8. Bowel end to end anastomosis performed and shown here.

\subsection{Clinical progress}

Postoperative recovery was uneventful. Pain score was reported to be VAS 0 to 1 throughout her stay. Her post-operative bloods taken did not show any significant changes and blood loss was very minimal. She was put on thrombo-embolic deterrent (TED) stockings, low molecular weight heparin for thromboprophylaxis and was encouraged for incentive spirometry post operatively. We allowed her liquids on the 1st postoperative day and resume normal diet on the $2^{\text {nd }}$ day. There was hardly any post-operative ileus experienced. Her drains were removed at $3^{\text {rd }}$ post-operative day (Figure 9). She began ambulating at day 3 and was discharged on the $5^{\text {th }}$ post-operative day.

On follow up, patient was very happy with the outcome of surgery and was impressed that there was hardly any major scar associated with the operative approach.

\subsection{Histopathological result}

Histopathological examination showed an $18 \mathrm{~cm}$ length of colon was removed on gross examination (Figure 10). On microscopic examination, there was no lymphovascular permeation and the tumour only involved the mucosa layer. The margins were clear of tumour. All lymph nodes harvested were negative for malignancy. Proximal and distal donuts were clear of tumour. Pathological staging was noted to be T1, N0, M0 (Stage 1). The final result showed a moderately differentiated adenocarcinoma. 


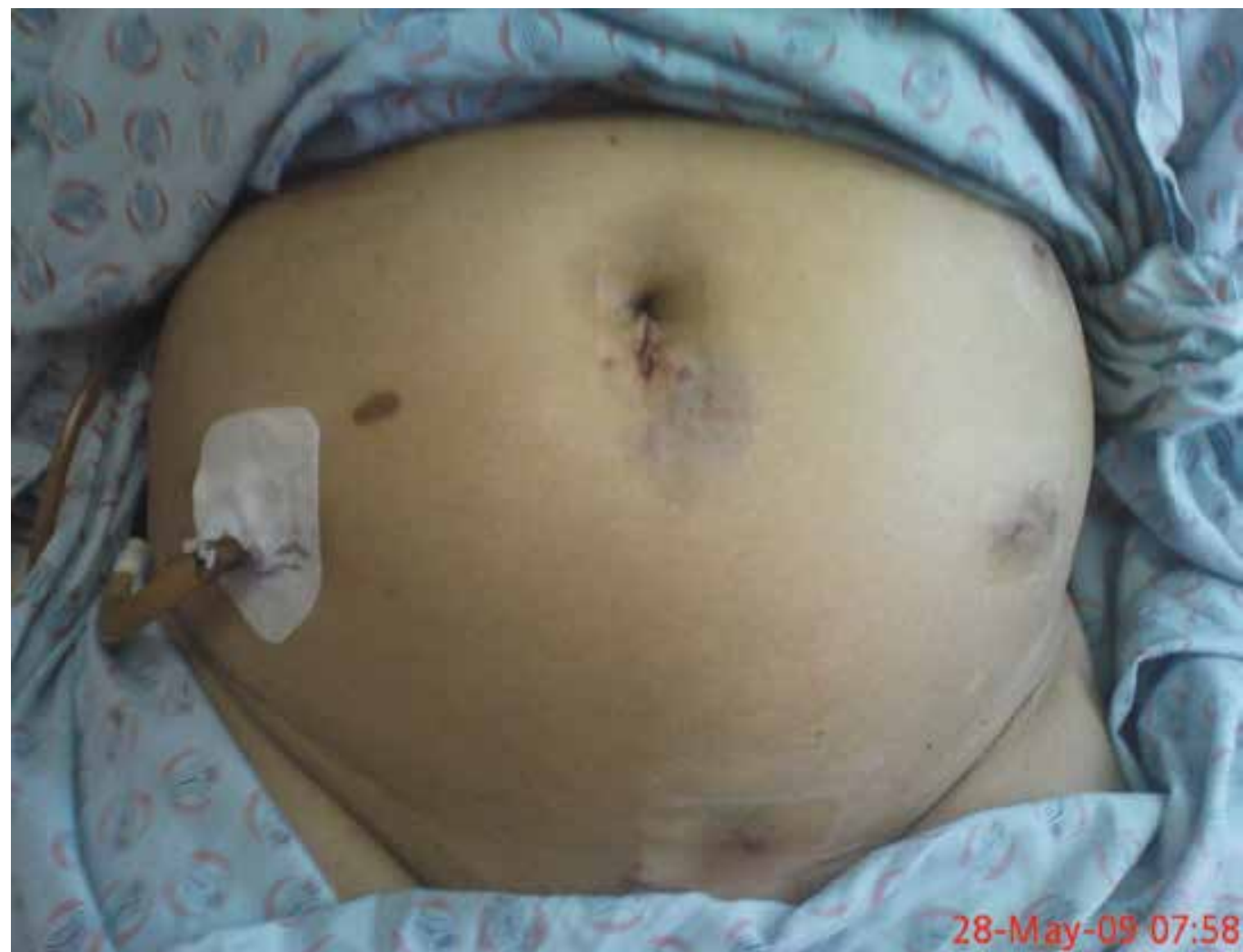

Fig. 9. Wound inspection at post-operative Day 2 showing an almost scarless abdomen

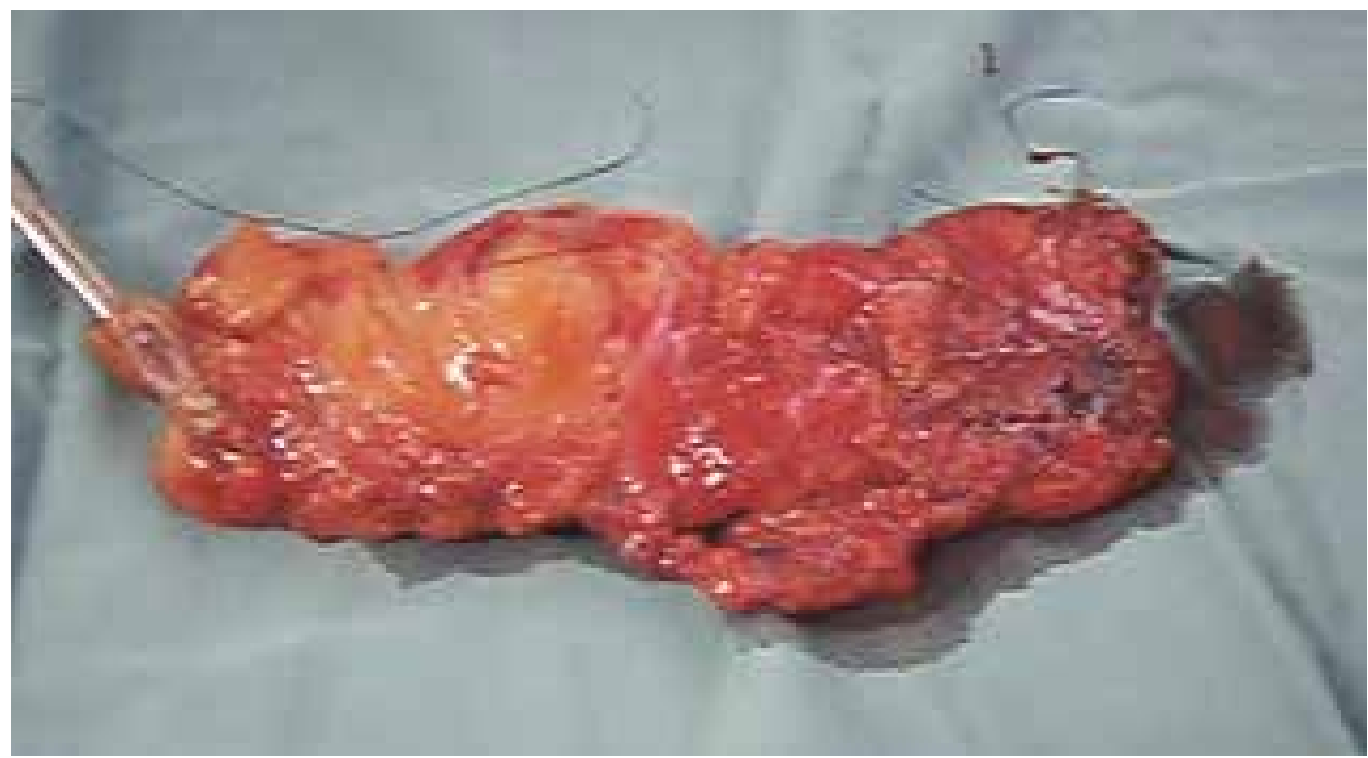

Fig. 10. The entire length of bowel removed along with the tumour. 


\section{Laparoscopic surgery with Natural Orifice Specimen Extraction (NOSE) in colorectal surgery: The way forward in the scarless surgical approach?}

In laparoscopic colorectal resection, it was conventional to retrieve the resected colonic specimen via a mini-laparotomy. This is rather a frustrating situation for most laparoscopic surgeons who took pain to ensure minimal scarring for patients following a long and arduous laparoscopic dissection. Such situation can now be overlooked with the advent of natural orifice surgery, which is gaining popularity and evolving.

It is without doubt that a mini-laparotomy which is required for the retrieval of colonic specimen following resection is associated with a bigger scar hence poor cosmetic appearance, increase in pain outcome, increase risk of wound infection and other pain related complications [1]. This can now be avoided with the technique of retrieving specimens via natural orifices via the transvaginal, transanal or transgastric routes.

In our patient, we have described the retrieval of specimen via the transvaginal route. This is feasible in female patients with colonic cancers. This technique had also been described in other literature. Palanivelu et al. had described the transvaginal route for a series of patients with familial adenosis polyposis (FAP) with early rectal adenocarcinoma who had laparoscopic proctocolectomy with ileoanal pouch anastomosis who had the specimen retrieved transvaginally [2].

Extraction of specimen via the transvaginal route has also been described in other operations besides colorectal surgery such as nephrectomy, cholecystectomy, appendecetomy, spleen and so forth which will be elaborated much later in this chapter [3,4].

Retrieval of colonic specimen via other natural orifice such as the transgastric or transanal routes had also been described in literature but not without limitations. In this case, the transgastric approach would be limited by the restriction point in the oesophagus, the long travel distance and lack of adequate instruments for extraction as described by Franklin et al [5]. Similarly, the transanal approach also had its limitations such as the risk of fecal contamination, wound infection, injury to the anal sphincters and limitation to the size of the specimen extracted [2,5]. At this moment, the transanal approach can only be recommended for small benign tumours or early colorectal cancers [6]

One of the reasons for choosing the transvaginal route is the advantage of the elasticity of the vagina, hence allowing specimen of various sizes to be able to retrieve. Literature has shown that transvaginal extraction is most ideal and patients undergoing this technique had good post-operative outcome with clinically no significant impact on orgasm, pregnancy, dyspareunia or sexual function [7].

One of the main concerns regarding retrieval of specimen is port site or retrieval incision metastasis which was reported to be 0.9 to $3.4 \%$ by Schaeff et al [8]. However, this fear is allayed as was described in our patient where the use of a handport, which was inserted transvaginally, was used to retrieve the specimen. We also found that the advantage of using the handport also allowed us to minimize infection or contamination, and to maintain pneumoperitoneum throughout the operation.

As can be seen in our patient, the post-recovery was excellent. Pain outcome was good with almost no pain reported. Normal diet was able to resume from the $2^{\text {nd }}$ post-operative day onwards. By the $3^{\text {rd }}$ day, patient was ambulating. On hindsight, patient was actually able to go home on the $3^{\text {rd }}$ post-operative day with the drains removed. We have kept her longer as this was the first case performed in our centre, hence the longer observation period.

The end result of this technique is very satisfactory and patient was highly satisfied with the post-operative outcome. 


\section{Application of NOSE or NOTES in other surgical disciplines}

The sudden rise in interest towards NOSE or NOTES have also seen its application flourished and boomed in various surgical discipline as we moved into the $21^{\text {st }}$ century. Although still in its infancy, it is slowly gaining popularity and clinical acceptance as can be seen in its application in non-colorectal surgery.

\subsection{Other general surgical procedures}

Although much has been reported regarding the retrieval of colonic specimens be it a right sided colectomy, left sided colectomy or an anterior resection via the more popular natural orifices such as transvaginal or transanal routes, the application of NOSE and NOTES can also be seen in other non-colorectal procedures.

Similar to retrieval of colonic specimen where feasibility is not an option via the transgastric or the transurethral route, the use of NOSE in retrieval of stomach specimen via the transvaginal route had been described by Jeong et al in 2010. This was the first ever description of retrieval of stomach specimen using NOSE method published for early gastric cancer where patients in its series undergone a subtotal gastrectomy [9].

When compared to NOSE in colorectal surgeries, it has been almost 15 years from the first reported extraction of colonic specimen via natural body orifices to its first stomach specimen extraction via NOSE today. Even then, the first NOSE was first described in 1993 by Delvaux et al who removed the gallbladder via the transvaginal route [10]. What have happened along those years?

One can only wonder that such a procedure must have slowly gained acceptance and changed in perception among the clinical community and public acceptance towards NOSE where the procedure can now extend to extraction of other bodily specimens as the people are slowly more inclined towards a scarless surgery.

With regards to NOTES, the use of natural orifices to perform surgery without any bodily incision thus providing patients with a scarless surgery have also been described although it has its limitations. Nevertheless, the future looks promising with the advent of such newer techniques.

At the moment NOTES have been described using an endoscope which is channeled through various bodily orifices and perforating the viscus to perform the intended procedure. Here, examples of peritoneoscopy, appendecectomy, cholecystectomy and so forth had been performed via the transgastric route with retrieval of the specimen. Other routes such as the transcolonic or transvesical or transvaginal route have also been described where once the instruments is in the peritoneal cavity, pneumoperitoneum is created and surgery is performed [11].

In the present, there are still limitations for NOTES when it comes to performing more complex surgery and as for now, most procedures are still performed on animal models. With better technological advancement and improvement in the development of equipments used in NOTES the future indeed looks promising.

\subsection{Urology}

The use of natural body orifices in Urology began first with the extraction of resected specimen. This was first reported in 1993 by Breda et al following a laparoscopic 
nephrectomy where the kidney was removed via the transvaginal route [12]. Following this, numerous application of NOSE and NOTES began the flourish in the urological world with popularity began to soar.

The use of transvaginal route for specimen extraction in females was also widely reported by Gill et al who extended the procedure to include radical nephrectomy [13].

Further evidence in the use of other natural orifices slowly took place where the transanal route was used for the extraction of bladder following cystectomy for bladder tumours where a rectosigmoid pouch was created for urinary diversion and the tumour removed via the transanal route [14].

The above described techniques often combined the use of laparoscopic techniques coupled with natural orifice specimen extraction often known as the Hybrid technique. However, pure NOTES where performing surgery via natural orifices without the possibility of any skin incision in Urological procedure began to emerge thus making Urological procedures a complete scarless surgery.

This was first described in animal models but Lima et al in 2006 described the first pure NOTES where peritoneoscopy was done via the transvesical route which allow peritoneal assessment and liver biopsy [15]. The very same author had gone on to describe other surgical procedures using the same transvesical route.

Another urological option towards an almost scarless surgery seems possible with the introduction of Single Incision Laparoscopic Surgery (SILS) making most urological procedure a possibility in using this approach. Of course, we will be touching on about SILS much later in the chapter.

Although it seem like such urological procedures are still a long way from gaining routine acceptance and at times seem experimental, nevertheless the road ahead looks promising. Nevertheless, limitations abound and there is still more work to be done.

\subsection{Gynaecological procedures}

The emergence of NOSE and NOTES can also be seen in the field of gynaecology. The use of endoscopic procedure via the transvaginal route has established both diagnostic and therapeutic needs in the field of gynaecology. This is better known as culdoscopy among the gynaecology fraternity.

This further transcends towards the use of fertility medicine such as investigation for infertility in both diagnostic and therapeutic means known as Transvaginal Hydrolaparoscopy (THL) [16]. THL is a modification of culdoscopy and was first introduced by Gordts in 1998. It enables the surgeon to assess or evaluate the posterior uterus, the pelvic side walls and adnexae structures. Such procedure acts as a diagnostic tool and can often times be performed under local anaesthesia without subjecting patients to the risk of general anaesthesia.

THL when coupled with chromotubation also allows assessment of infertility and can replace the conventional hysterosalpingography (HSG) which is often used as the first line of diagnostic modality in the assessment of infertility. It is able to show tubal patency as accurately as an HSG.

Besides diagnostic yield, it also allows various operative procedures to be performed such as ovarian drilling, adhesiolysis, treatment of ovarian cyst as well as endometriosis and salpingostomy. 


\section{Single Incision Laparoscopic Surgery (SILS) the almost scarless surgical approach and the transumbilical route as another natural body orifice?}

\subsection{SILS experience}

Laparoscopic surgery when first introduced in the early 80 's brought a revolutionary change to how surgery is performed. Since then, it has evolved from a simple cholecystectomy or appendecectomy to a more complex surgical procedures such as colorectal resection, bariatric surgery, liver and pancreatic resection and so forth.

It is the intention of every laparoscopic surgeon to offer patients with a more feasible form of surgery in the hope of reducing pain, hospital stay and morbidity. Of utmost importance is the reduction or prevention of the conventional surgical scars associated with open surgery. Hence, laparoscopic surgery has evolved from the conventional laparoscopic ports used in certain laparoscopic surgical procedure to usage of much lesser ports for similar procedure.

A fine example is the conventional laparoscopic cholecystectomy with the use of four laparoscopic ports. From thence, literature has been published regarding use of three or even two ports for laparoscopic cholecystectomy with the intention of further reducing the amount of scar as well as tissue damage associated with port insertion [17, 18].

From here, laparoscopic surgery has further evolved when used in combination with NOSE or NOTES to further reduce surgical scars to an almost scarless surgery as was described in this chapter earlier. This is sometimes known as a hybrid procedure. Patients can now be offered this form of surgery as large specimens that cannot be retrieved via the small laparoscopic ports can be retrieved via the natural orifice. This enables us to avoid giving patient a big scar as it made no sense to make a big scar to retrieve specimen that is resected after going through many hours of performing such a complex surgery.

It is only a matter of time when laparoscopic surgery is performed via a single port, hence the introduction of single incision laparoscopic surgery (SILS). This may allow one to do away with some of the combined procedures with NOSE and NOTES as surgery can now be done via one incision with retrieval of specimen from the same incision.

When the single incision or port is inserted via the umbilicus or transumbilical, postsurgical scars is almost invisible when made over the umbilicus. Our experience in our centre has seen SILS being performed for operation such as cholecystectomy (Figure 11), excision of gastric Gastrointestinal stromal tumour (Figure 12), right hemi-colectomy (Figure 13), appendecectomy, SILS anterior resection, SILS spleno-distal pancreatectomy and inguinal hernia repair where the port is inserted via theumbilicus. Post operatively, these scars were almost invisible or in other words scarless! Patients who have undergone these procedures were very satisfied with the outcome of this surgery and were happy that almost no scars were noticeable. Recovery is often uneventful and patients have experienced lower amount of pain.

In some literature, the umbilical route has also been described as a natural orifice as it is considered once as a natural orifice during embryological development. Hence, the term natural orifice transumbilical surgery (NOTUS) has been coined [19].

Not to rest on its laurels, SILS has also seen itself being evolved into more complex surgery such as colorectal resections or even bariartric surgery. The uses of SILS have also been described in various urological and gynaecological procedures as well with favourable outcomes. 
Chambers et al have described the use of SILS in various colorectal resections ranging from appendecectomy to hemicolectomies and anterior resection and proved that it was a safe and feasible surgery to perform in the hands of experience surgeons [20]. With regards to bariartric surgery, Reavis et al have described how sleeve gastrectomy was performed in obesity surgery via a single incision for laparoscopic ports placement and retrieval of the stomach specimen via the same incision [21].

The various literatures published to date regarding SILS augurs well for this surgical technology to further develop and as an offering to patients seeking a scarless surgery in the near future.

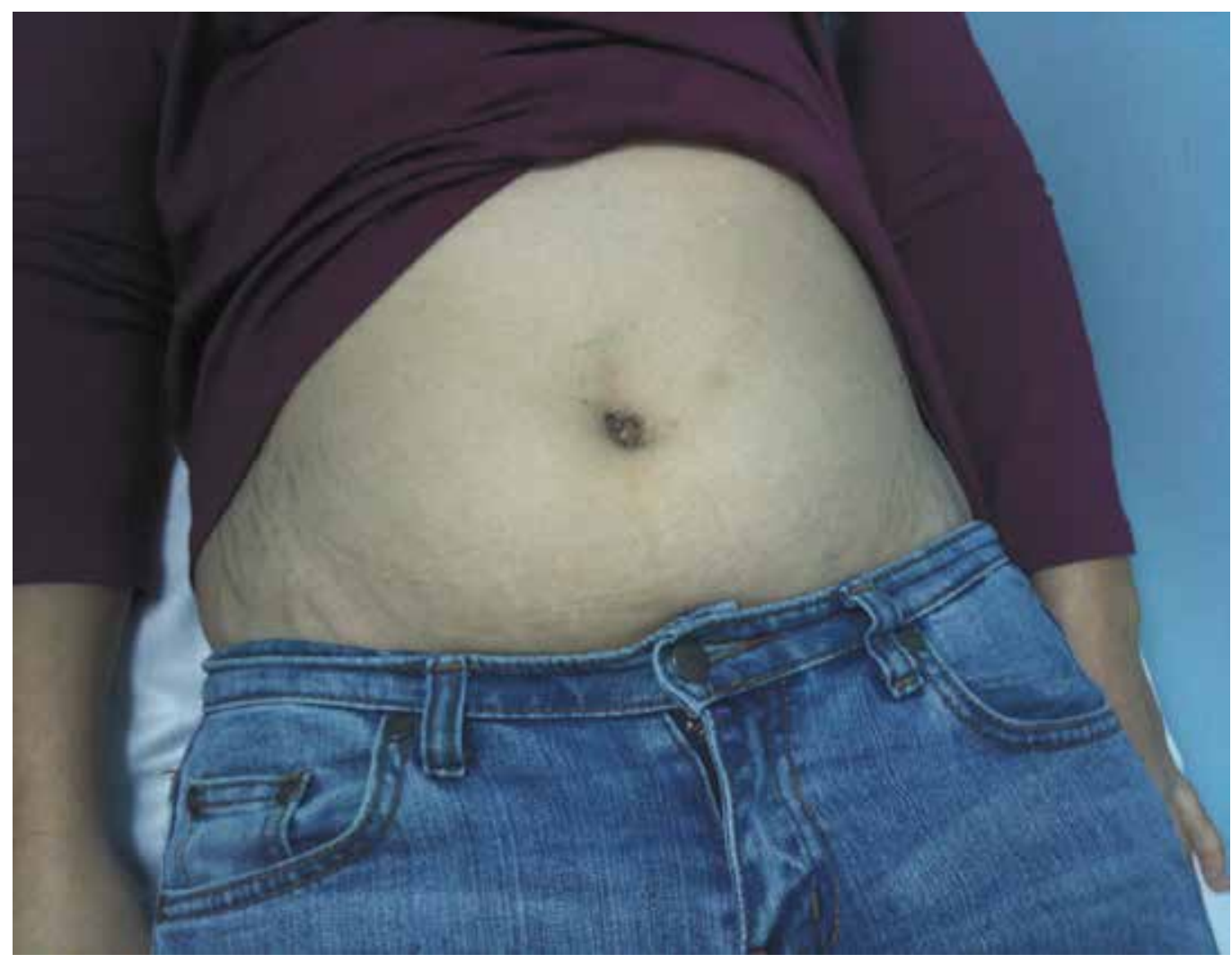

Fig. 11. Almost scarless wound at the umbilicus after SILS cholecystectomy 


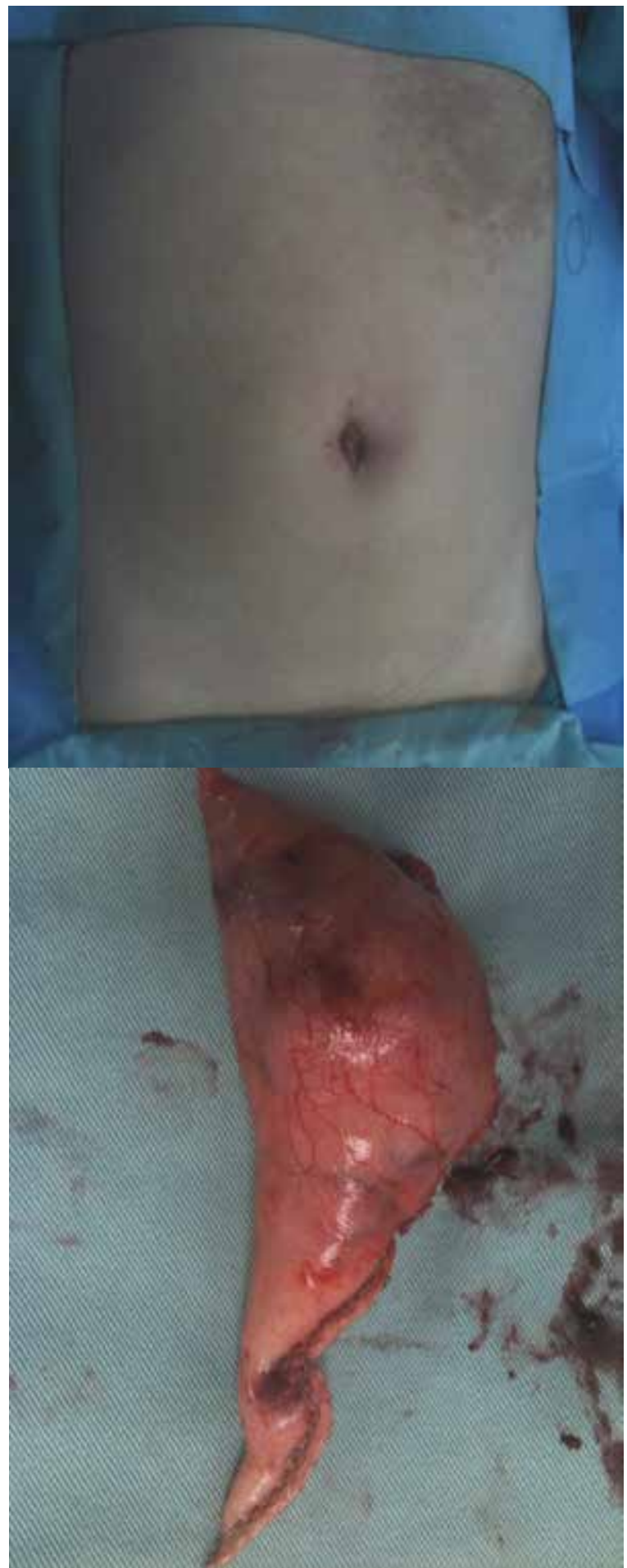

Fig. 12. Almost scarless wound at the umbilicus after SILS wedge excision of gastric GIST 


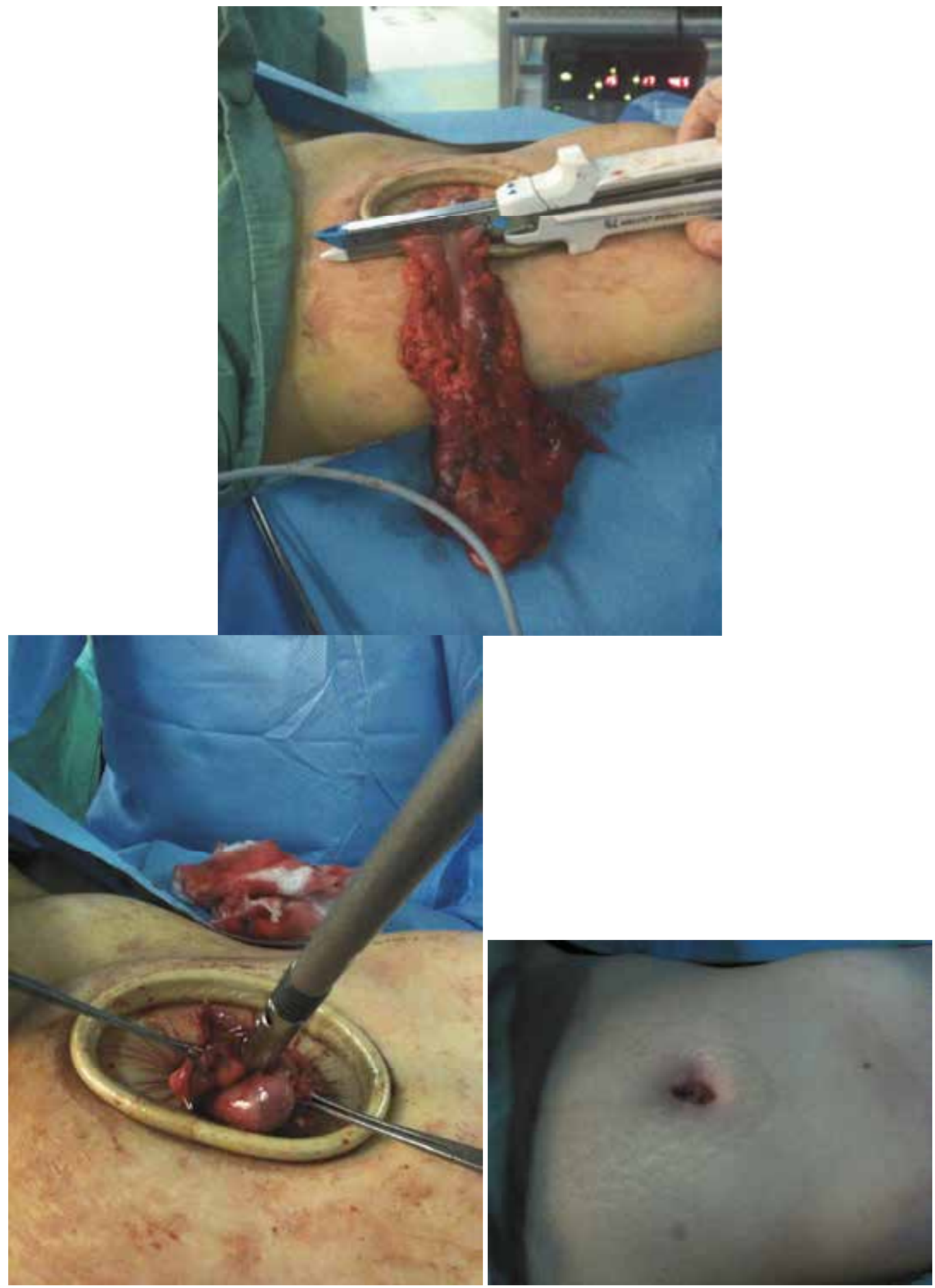

Fig. 13. An almost scarless wound at the umbilicus after a SILS right hemi-colectomy with extracorporeal anastomosis as demonstrated above. 


\subsection{The challenge ahead for SILS}

Like any new surgical technology, SILS is not without its limitations. Among the many challenges faced by surgeons taking up SILS is the associated long learning curve. It is believed that one may overcome this limitation as more experience is gained from performing SILS on a regular basis.

More young surgeons should also be encouraged to take up this procedure as the longer exposure to SILS will in turn be gained as an experience over a period of time.

Next would be the instrumentation associated with SILS. The smaller degree of instrument triangulation often hampers or discourages one from taking up SILS. In our experience, this is one major drawback to SILS as oftentimes, surgeons finds it difficult to perform SILS with the conventional and existing available instruments. It is not without doubt that smaller degrees of instrument triangulation often occurs compared to the conventional laparoscopic procedures when a single port is used. However, this may be overcome with better laparoscopic ports, platforms and devices which may allow flexibility and angulation to facilitate the use of SILS. This has actually encouraged industry players to inject funds into research and development for better instruments and equipment related to SILS as can be seen in today's modern day of surgical practice.

Is the future looking bright for SILS? We believe so, albeit a learning curve is associated with it and future development of newer laparoscopic instruments may further enhance and ease the use of this new surgical technology as more and more patients demand for a scarless approach to surgery in the near future.

\section{Conclusion}

The transvaginal route is most ideal for the retrieval of colonic specimens in laparoscopic colorectal surgery due to the advantage of its anatomical and physiological properties. Our experience with this technique showed that it is feasible for selected patients. It is also a safe procedure with low morbidity. Pain outcome is good and patient satisfaction is excellent.

The use of NOSE can also been seen in its application in other surgical disciplines allowing specimen extraction using various routes described.

Other techniques such as NOTES and SILS have also gained popularity and acceptance in this fast moving day and age of surgical technologies. These techniques described above often times complement each other or may be accomplished on its own in the case of SILS.

In conclusion, the advancement and evolution in surgical techniques this present day and for the future gives patients the satisfaction of an almost a scarless surgery.

\section{References}

[1] Cheung, H.Y., et al., Endo-laparoscopic colectomy without mini-laparotomy for left sided colonic tumors. World J Surg, 2009. 33(6): p. 1287-1291. 
[2] Palanivelu, C., et al., An innovative technique for colorectal specimen retrieval: A new era for Natural Orifice Specimen Extraction (NOSE). Diseases of the Colon and Rectum, 2008. 51: p. 1120-1124.

[3] Gill, I.S., et al., Vaginal extraction of the intact specimen following laparoscopic radical nephrectomy. J Urol, 2002. 167: p. 238-241.

[4] Vereczkei, A., et al., Transvaginal extraction of the laparoscopically removed spleen. Surg Endosc, 2003. 17: p. 157.

[5] Franklin, M.E., et al., Transvaginal extraction of the specimen after total laparoscopic right hemicolectomy with intracorporeal anastomosis. Surg Laparosc Endosc Percutan Tech, 2008. 18(3): p. 294-298.

[6] Ooi, B.S., et al., Laparoscopic high anterior resection with natural orifice specimen extraction (NOSE) for early rectal cancer. Tech Coloproctol 2009. 13: p. 61-64.

[7] Roovers, J.P., et al., A randomized comparison of post operative pain, quality of life, and physical performance during the first six weeks after abdominal or vaginal surgery correction of the uteri. Neurourol Urodyn, 2005. 24: p. 334-340.

[8] Schaeff, B., V. Paolucci, and J. Thomopoulos, Port site recurrences after laparoscopic surgery. A review. Dig Surg 1998. 15: p. 124-134.

[9] Jeong, S.H., et al., Trans-vaginal specimen extraction following totally laparoscopic subtotal gastrectomy in early gastric cancer. Gastric Cancer, 2011. 14(1): p. 91-6.

[10] Delvaux, G., et al., Transvaginal removal of gallbladders with large stones after laparoscopic cholecystectomy. Surg Laparosc Endosc, 1993. 3(4): p. 307-9.

[11] Pearl, J.P. and J.L. Ponsky, Natural orifice translumenal endoscopic surgery: a critical review. J Gastrointest Surg, 2008. 12(7): p. 1293-300.

[12] Breda, G., et al., Laparoscopic nephrectomy with vaginal delivery of the intact kidney. Eur Urol, 1993. 24(1): p. 116-7.

[13] Gill, I.S., et al., Vaginal extraction of the intact specimen following laparoscopic radical nephrectomy. J Urol, 2002. 167(1): p. 238-41.

[14] DeGer, S., et al., Laparoscopic radical cystectomy with continent urinary diversion (rectosigmoid pouch) performed completely intracorporeally: an intermediate functional and oncologic analysis. Urology, 2004. 64(5): p. 935-9.

[15] Lima, E., et al., Transvesical endoscopic peritoneoscopy: a novel $5 \mathrm{~mm}$ port for intra-abdominal scarless surgery. J Urol, 2006. 176(2): p. 802-5.

[16] Escobar, P.F., et al., Laparoendoscopic single-site and natural orifice surgery in gynecology. Fertil Steril, 2010. 94(7): p. 2497-502.

[17] Kagaya, T., Laparoscopic cholecystectomy via two ports, using the "Twin-Port" system. J Hepatobiliary Pancreat Surg, 2001. 8(1): p. 76-80.

[18] Leggett, P.L., et al., Three-port microlaparoscopic cholecystectomy in 159 patients. Surg Endosc, 2001. 15(3): p. 293-6.

[19] Nguyen, N.T., et al., Laparoscopic transumbilical cholecystectomy without visible abdominal scars. J Gastrointest Surg, 2009. 13(6): p. 1125-8.

[20] Chambers, W.M., et al., Single-incision laparoscopic surgery (SILS) in complex colorectal surgery: a technique offering potential and not just cosmesis. Colorectal Dis, 2011. 13(4): p. 393-8. 
[21] Reavis, K.M., et al., Single-laparoscopic incision transabdominal surgery sleeve gastrectomy. Obes Surg, 2008. 18(11): p. 1492-4. 


\title{
Endoscopic Monitoring of Postoperative Sinonasal Mucosa Wounds Healing
}

\author{
Ivana Pajić-Penavić \\ Department of ENT, Head and Neck Surgery, General \\ Hospital "Dr Josip Benčević", Slavonski Brod
}

Croatia

\section{Introduction}

Nasal epithelium lies on the basement membrane, situated on the lamina propria. Pseudostratified columnar (respiratory) epithelium is composed of four major types of cells: ciliated cells, nonciliate cells, goblet cells and basal cells, ensuring mucus production and transport, resorption of surface materials, and formation of new epithelial cells. Lamina propria consists of two layers of seromucous glands, i.e. superficial and deep layers. Just beneath the basement membrane, lymphocytes and plasma cells form a lymphoid layer.

Maintenance of normal ventilation/aeration of sinus spaces is necessary for normal functioning of paranasal sinuses. The sinus labyrinth spaces and ostia of various sinus areas can be visualized by use of endoscopic techniques, e.g., in functional endoscopic sinus surgery (FESS). Ventilation and normal sinus function can be maintained by this minimally invasive method. Endoscopic sinus surgery (ESS) is the superior surgical method of treatment for recurrent acute sinusitis, chronic sinusitis, obstructive nasal polyposis, extramucous fungal sinusitis, periorbital abscess, rhinoliquorrhea, antrochoanal polyp, foreign body extraction, mucocele, dacryocystorhinostomy, excision of various tumors of the sinuses, nose, anterior, middle and posterior cranial fossa, epistaxis control, optic nerve decompression, choanal atresia, and orbit decompression. . Functional endoscopic sinus surgery (FESS), a minimally invasive technique, remains the most widely accepted therapy for chronic rhinosinusitis (CRS) and nasal polyposis (NP) after failure of medical treatment. FESS aims to remove inflammatory mucosa and to restore both ventilation and drainage of the sinus cavities. However, healing quality significantly influences the functional outcome. The exact mechanism of mucosal healing after sinus operation remains unclear. Postoperative wound healing is a highly coordinated process that includes coagulation, i.e. clot formation, inflammatory stage, and tissue formation and remodeling. During the process of healing, the extracellular matrix of nasal mucosa may be directly influenced by the growth factor (GF), while the expression of GF receptors may influence the cell phenotype and its adhesion. Endoscopic observation of the nasal and sinus mucosa healing after FESS revealed four clinical stages: stage 1 characterized by the formation of abundant crusts, lasting for 1-10 days; stage 2 characterized by obstructive lymphedema, with pronounced swelling of residual mucosa, lasting for up to 30 days; stage 3 characterized by mesenchymal growth, when pale, edematous mucosa is transformed to red mucosa, lasting for up to 3 months; and stage 4 characterized by cicatrix formation, lasting for 3-6 months. 
The duration of particular stages can be reduced or prolonged by postoperative treatment. Any derangement in the process of healing may result in the formation of hypertrophic scar or impaired tissue differentiation, thus reducing functioning capacity of the organ involved. Healing defects of the respiratory mucosa regularly lead to development of infection or obstruction scar formation, making revision surgery necessary. Iatrogenic complication after FESS appears in $5 \%$ to $30 \%$ of patients, and recurrence is reported in about $18 \%$ of patients (Tan BK. 2010). Proper treatment of postoperative cavity is a significant segment in the process of mucosal healing, and thus part of the FESS. Due knowledge of the healing stages can help recognize a mucosal healing impairment and introduce appropriate therapy depending on the stage of the healing process. The healing stages and planning of postoperative therapy after endoscopic sinus surgery are presented.

\subsection{Structure of sinonasl cavity}

The nasal cavity and sinusies are covered with respiratory epithelium which is composed of ciliated pseudostratified columnar epithelium (respiratory epithelium) composed of four major types of cells: ciliated cells, nonciliated cells, goblet cells and basal cells assuring mucus production and transports, absorbtion of surface materials and formation of new epithelial cells. The lamina propria consists of two layers of seromucous glands: the superficial layer is situated just underneath the epithelium and the deep layer is under the vascular layer. Just beneath the basement membrane, lymphocytes and plasma cells form a lymphoid layer. The basal cells are intermediate stem cells capable of differentiation into ciliated columnar or goblet cells. Occasional cuboidal and squamous cells are also found the epithelium. The columnar cells are $25 \mu \mathrm{m}$ long and $7 \mu \mathrm{m}$ wide, tapering to $2-4 \mu \mathrm{m}$ at the base. They are separated from each other by tight junctions. Each cell is always covered by $300-$ 400 microvilli, and may or may not have cilia. Microvillies increase the surface area, thereby preventing drying. The number of cilia on each cell and size of the cilia varies between different species. The goblet cells produce mucus. The size and staining characteristics of the cell will depend on the phase of the secretory cycle of each individual cell. The number of cells varies throughout the nose and sinuses. On the septum, there is gradual increase in numbers passing from anterior to posterior and increase from superior to inferior. The seromucinous glands are found in the submucosa of the respiratory epithelium. They are relatively few in number and are more numerous in the mucosa near the choanae. The mucosa of the nasal cavity is much thicker than that of the sinuses. Mucosa tends to be thin and dry over bony excrescences and outcroppings that are characteristically notable over the nasal septum and in nasal valve area. Sinus mucosa is much thinner than that lining the rest of the nasal cavity. Epithelium tends to be lower; there are generally few goblet cells; and seromucinous glands are extremely scarce. The basement membrane is attenuated or not readily discernible and the lamina propria is often absent. The basic cells are columnar ciliated epithelial cells. They average $5 \mu \mathrm{m}$ long and $0.2 \mu \mathrm{m}$ thick and carry between 100 and 150 cilia on each luminal cell surface. Microvilli are much smaller, averaging around $1.5 \mu \mathrm{m}$ long and $0.08 \mu \mathrm{m}$ in diameter. Goblet cells are shorter except during the active phase of secretion. The maxillary sinus is lined with ciliated columnar respiratory epithelium containing goblet cells and glands. The mucous membrane is relatively thin, less vascular and more loosely adherent to the bony walls than in the nasal cavity. Density of goblet cells in the maxillary sinus is the highest of any in the paranasal sinuses, and similar to that on the inferior turbinate. There is no obvious increase in goblet cell density around the ostium. The seromucinous glands, though few in number compared with the goblet cells, are again 
more numerous in the maxillary sinus compared with the other sinuses, and are more concentrated around the ostium. Ethmoid sinuses are also lined with ciliated columnar respiratory epithelium. The density of goblet cells is lower then in the maxillary sinus. Tubuloalveolar seromucinous glands are found trough out the mucosa and are actually more numerous in the ethmoid than in the other sinuses. In sphenoid sinuses the respiratory epithelium contains goblet cells and glands, as in the other sinuses. Goblet cells are of similar density as in the ethmoids, though the glands are last numerous in these sinuses and are therefore not found on all walls. The frontal sinus respiratory epithelium has the fewest number of goblet cells and very few glands. Mucus has a gel and sol layer with the narrow sol layer covering the cilia, facilitating their movement, and the gel layer on top to which foreign material will stick. Mucus blanket sweeps from the nares to the choanae and in the sinus cavities toward their ostia. The only exception to this is the frontal sinus in which the mucus blanket sweeps from the ostium, arcs over the roof of the sinus, and progresses along the floor to empty into the lateral aspect of the frontal sinus ostium. Vasculature of the nose is characterized by capacitance vessels. With these vascular specificities, nasal mucosa can regulate the airflow, adapt the nasal resistance, filter and condition the inspired air, and organize the first line of immune defense. Ethmoid bloc is the most complex of the sinuses. It often appears to be pivotal sinus in pathophysiology of sinus inflammatory disease (Donald PJ.1995).

\subsection{Cytokines and GFs in nasal repair}

The transforming growth factor-beta (TGF- $\beta$ ) is the most relevant growth factor in wound healing, affecting nearly all the phases of the process. Besides its immunosuppressive effects, TGF- $\beta 1$ influences cell proliferation and myofibroblast differentiation. GFs are mediators produced by cells, tissue or blood products that activate target cells to proliferate by binding to their high-affinity surface membrane receptors. Transforming GF (TGF) $\beta$ is released by major cell types participating in the repair process (epithelial cells, inflammatory cells, fibroblast, etc.) and nearly all cells express TGF- $\beta$ receptors. More than $85 \%$ of TGF- $\beta$ in adult wound fluid is of the type 1 isoform. TGF- $\beta$ had important activities such as an adverse effect in vitro on reepithelization, immunosuppression, or stimulation of ECM deposition. PDGF isoforms have potent mitogenic and chemotactic activities on dermal fibroblasts, endothelial cells, smooth muscle cells, neutrophils, and macrophages and stimulates collagen synthesis and collagenase acitivity. PDGF isoforms have potent mitogenic and cemotactic activities on fibroblasts, endothelial cells, smooth muscle cells.

Epidermal GF (EGF) family, including aphiregulin and TGF-a has been shown to induce epithelial development and differentiation, to promote angiogenesis, and, in vivo, to accelerate wound healing. Cells associated with wound healing such as inflammatory cells (macrophages and T lymphocytes), vascular endothelial cells, and fibroblasts can produce fibroblast GF (FGF), which are mitogens for a wide variety cell types. Insulin-like GF (IGF) I and II have an amino acid sequence homologous to proinsulin and are secreted by a wide range of adult tissues. IGF-I (known as Somatomedin-C) stimulates, in vitro mitosis of fibroblasts, osteocytes and chondrocytes. Because its combination with other growth hormones is more effective than either peptide alone, it frequently acts in synergy with PDGF to enhance epidermal regeneration. GFs enhance the deposition of extracellular matrix (ECM). Extracellular matrix provides nutrients support, and adhesion for the inflammatory on structural cells participating in repair (Watelet JB. 2002). 


\subsection{Neutrophils in wound healing}

Neutrophils play an important role in tissue remodeling occurring after tissue damage. The particular inverse relationship between eosinophils and fibrosis found at baseline persists during the healing process. On the other hand, macrophages and eosinophil cells are highly associated not only with the tissue remodeling characteristic of chronic sinus disease but also with the neutrophilic inflammation occurring during wound repair. Macrophages and eosinophil cells were highly associated not only with the tissue remodeling characteristic of chronic sinus diseases but also with the neutrophilic inflammation occurring during wound repair. The selective recruitment of eosinophils into sinonasal inflamed tissue involves priming, activation, and recruitment mediated by chemoattractants, cell adhesion molecules, and cytokines. Activated eosinphils contribute to the production of cytokines and inflammatory molecules, which damage nasal mucosa, leading to edema and inflammation.

The differentiation of mast cells occurs by the effects of released cytokines from inflammatory cells. Histamine, prostaglandine E2, and leukotriens are released from degranulate mast cells. Sensory nerve stimulation by these mediators attracts eosinophils to the inflammatory areas. Substance $\mathrm{P}$ released by mast cells causes increased vasodilatation, increased vascular permeability, mucous secretion, and eosinophil chemotaxis; increase mast cells degranulation; and enhances the response to allergens in atopics patients (Watelet JB. 2006).

\subsubsection{Neutrophil-derived Metalloproteinase-9}

Metalloproteinase-9 (MM-9) expression in ECM was also significantly correlated with healing quality. MM-9 is actively expressed by eosinophils, monocytes-macrophages, epithelial-derived cells and is stored by neutrophils. It degrades collagen fibres, basement membrane, fibronectin and elastin. MM-9 activity is controlled at different levels: transcription of the gene under control of cytokines or cellular interaction, activation of the proenzyme by serin proteases or other MMPs, and finally, activity regulation by natural tissue inhibitors (TIMPs). The amounts of MMP-9 in nasal fluid are linked to ECM expression of MMP-9. The MMP-9 deposition inside ECM correlates with inflammatory cells. The number of neutrophils and lesser extent, macrophages could predict MMP-9 release in nasal fluid. There is close relationship between MMP-9 and neutrophils and they establish a direct link between the severity of the inflammatory reaction and the consequent tissue damage. MMP-9 is considered as effector but also as a regulator of leukocyte function. It is stored in granules of mature neutrophils and has been shown to be a specific marker of neutrophils and has been shown to be a specific marker of neutrophils maturation. Transcription of the gelatinase- $B$ gene is stimulated in leukocytes by cytokines, viral or bacterial products, or cellular interactions. In response to lipopolysaccharide, the neutrophil is responsible for the rapid secretion of MMP-9 as a result of release of preformed enzymes stored in granules. The level of MMPs parallels the severity of clinical condition. Release of gelatinase-B by degranulation of neutrophils occurs within the first hour when these cells are stimulated by chemotactic factors. During wound healing, the association beween neutrophils and macrophages observed in tissue suggests that the amounts of MMP-9 requested are high and that a conjunction of rapid release and continuous production is needed. MMP-9 has been shown to clip many cytokines or chemokines such as interleukin (IL) $-1 \beta$ or IL- 8 . On the other hand, the binding of MMP-9 to the plasma membrane of neutrophils enables it to be inhibited by TIMPs and thereby may alter the pericellular 
proteolytic balance in favor of ECM degradation. The MMP-9 production by neutrophils participates actively in airway remodeling (Watelet JB. 2005)

\section{General principles of wound healing}

Wound healing of the mucosa lining consists a few phases such as: inflammation, cell proliferation, matrix deposition and remodeling (Watelet JB.2002).

\subsection{Coagulation}

Injury to the nasal epithelium causes hemorrhage with exposure of platelets to the connective tissue, which activates the platelets and results in an almost immediate release of numerous vasoactive substances (serotonin, bradykinin and histamine). The subsequent transient (5-10 minutes) vasoconstriction helps to control bleading and is followed by the formation of a primary hemostatic plug with aggregation of platelets in the mucosal effect. Platelets are critical elements during this early response, not only because of their concurrent release of numerous cytokines. Damaged nasal cells release PDGF, TGF- $\alpha$ and TGF- $\beta$ and mast cells represent another source of biologically active substance or GFs that regulate the early repair sequences. Fibrin, in conjunction with fibronectin, acts as a provisional matrix for the influx of monocytes and fibroblasts. Fibrin also stimulates the agranules within the aggregated platelets to release PDGF, EGF, IGF-I, TGF- $\beta$ and FGF.

\subsection{Inflamation}

In the nasal lamina propria, an intense inflammatory reaction starts simultaneously with the coagulation phase. This inflammation is marked by an infiltration of leukocytes, which migrate through vessel walls by a process known as diapedesis. Polymorphonuclear neutrophils predominate during the first 24-48 hours and stimulate release of elastase and collagenase molecules, which facilitate cell penetration into the ECM. Three to five days after injury, the neutrophilic population in the wound is replaced by monocyte predominance. Unlike neutrophils, the influx of macrophages is essential for the continuation of nasal wound repair. Macrophages contribute to cellular debridement and secret a number of GFs: TGF- $\beta$, basic FGF (b FGF), EGF, TGF- $\alpha$ and PDGF. They amplify and sustain the wound healing process. Lymphocytes and their products, TGF- $\beta$, interleukin, tumor necrosis factor and interferons also interact with the macrophages during the inflammatory process, linking the immune response to wound healing. In a typically clean surgical wound, this inflammatory reaction subsides over a period of several days.

\subsection{Tissue formation}

New stroma or granulation tissue consisting of fibroblasts, macrophages and neovasculature can be observed 4 days after injury within a loose connective tissue matrix of collagen, hyaluronic acid and fibronectin. Macrophages of the nasal lamina propria provide a continuing source of cytokines necessary to stimulate proliferation of fibroblast and angiogenesis.

\subsubsection{Fibroplasia}

This term reflects fibroblast cell migration or proliferation and ECM deposition. Through a variety of cytokines from platelets and macrophages or through an autocrine regulation, 
fibroblasts are attracted to the nasal wound. Structural molecules of the early ECM also contribute to tissue formation by providing a network for cell mobility and guidance (fibronectin, collagen and hyaluronic acid) and by acting as a cytokine reservoir. Once the nasal fibroblasts have migrated into the wound, they gradually switch their major function to protein synthesis and GF release. The composition and structure of granulation tissue depends both on the time course since tissue injury and on the distance from the wound margin.

\subsubsection{Angiogenesis}

Nasal endothelial cells start to proliferate through fragmented basement membranes. They migrate into the perivascular space, and other endothelial cells follow. Angiogenic GFs: FGF, TGF- $\beta$, EGF, TGF $-\alpha$ and PDGF, released from injured nasal cells, platelets and ECM, induced vascularization, resulting in delivering oxygen to the wound bed. Endothelial cell migration depends on continuous collagen secretion and is accompanied by proteoglycan synthesis.

\subsubsection{Reepithelization}

Migration of new respiratory cells from the undamaged areas starts within a few hours, with an estimated velocity of $4 \mu \mathrm{m} /$ hour in the sinuses. The nasal epithelial cells at the wound edge lose their apical-basal polarity and develop cytoplasmic extensions into the wound. Four different processes are operative during regeneration: migration from adjacent epithelium, multiplication of undifferentiated cells, reorientation and differentiation. Undifferentiated respiratory basal cells from adjacent non-traumatized areas seem to serve as the main source of new cells. Different hypotheses are proposed to explain the initiation of reepithelialization: absence of neighbor cells at the wound margin, local release of GFs (TGF- $a$ and EGF) or increase of GF receptors.

\subsection{Tissue remodeling}

Nasal ECM remodeling, cell maturation and cell apoptosis overlapping with tissue formation and wound remodeling may continue up to 6 month after surgery. Most cells produce proteinases able to degrade the ECM. These enzymes can be subdivided into three groups: the serine proteinase, the matrix metalloproteinases ad the cysteine proteinase (cathepsins). The matrix metalloproteinases need an active $\mathrm{Zn}+$ site for their catalytic mechanism and they can be categorized in function of their degradation abilities in the ECM: interstitial collagenases 1-3, stromelysins 1-3, gelatinases A and B, matrilysin, macrophage metalloelastase and transmembrane metalloproteinase. Their proteolytic activity is controlled by tissue-erived metalloproteinase inhibitors 1-3.

In the remodeling or maturation phase, the inflammators response and angiogenesis diminish, whereas the intense fibroblast proliferation starts to attenuate. The composition of ECM changes as the wound matures. Initially, The ECM is composed mainly of hyaluronic acid, fibronectin and collagen types I, III and V. During remodeling, the ratio of collagen type I to III changes until type I is the dominant form; elastin fibers or proteglycans are actively produced within the matrix. This dynamic balance between collagen synthesis and lysis is responsible for the maturation of the wound. This phase increases wound tensile strength and resilience to deformation. 


\section{Endoscopic observations of wound healing}

Endoscopic observation of normal wound healing revealed four different phases (Hosemann W. 1991, Xu G.2008):

\subsection{Phase of blood crusting (day 1-10)}

During the first stage / peak the operative cavity is clean or dry, which is called "stage of clean cavity". In the 3-5 days after the filled nasal material was taken away, oozed blood clotted and formed a dry and hard black crust. During the first 10 days the endoscopic picture was dominated by blood crusts. After $12^{\text {th }}$ days the whole wound was covered by blood crusts. There was no change of the residual mucosa underneath these crusts within the first 2-3 days. Due to shortage of mucosa clearance, viscous secretion was gathered in the bottom of the sinus, and mucosa gap and fibrous pseudomembrane was observed on the surface of mucosa with responsive edema. The edematous swelling became more marked after detachment of the crusts in the second phase. On days 7-10, the edema was relieved and secretion was reduced, and clots and crusts decreased or disappeared after cleaning. After 10 days, the operative cavity became clean.

\subsection{Phase of obstructive lymphedema (up $\mathbf{t} 30$ days)}

During this period, the residual mucosa showed edematous swelling. This is secondary peak which occurs in the third to tenth weeks. Edema reoccurred in the operative cavity mainly due to lymphatic obstruction. Vesicles, mini-polyps and granulation tissue began to grow in the mucosa gap, which is called "reaction to mucosa removal". Hyperplasia and adhesion of connective tissue were also observed in this stage. In the meantime, regeneration and epithelialization were also happening, competing against mucosa diseases. After the vesicles, granulation tissue, mini-polyps and fibrous adhesion had been cleaned and mucosa regeneration and epithelialization expanded little by little, the scope of disease got smaller and smaller, and complete epithelialization was attained in the end. This secondary edema regresses spontaneously. If this phase is not handled carefully, the diseases would expande gradually and hamper epithelialization, resulting in deferred inflammation, leading to adhesion, constriction and blockage of the operative cavity and sinus ostium. In view of stage characterized by coexistence and rivalry of mucosa regeneration and disease, it is called "stage of mucosal transitional competition". The generation of vesicles or polyps during this stage was simply regarded as "recurrence of disease" instead of an inevitable mucosal transitional process. When the local reaction to mucosal removal was under control, mucosa restored well.

\subsection{Phase of mesenchymal growth (up to 3 months)}

After the $30^{\text {th }}$ day, mucosal reorganization took place preferably below the regenerated epithelial covering. This is the third stage/ peak. After 10 weeks, implying finished epithelialization of the operative cavity, which was called "stage of complete epithelialization". Though epithelialization started in the first 2 weeks and extended to the second stage, only a small number of cases finished epithelialization within 5 weeks, the majority was after 10 weeks and had a benign outcome. The color of the mucosa changed from a yellowish-pale edema to a more reddish color.

Infection with additional destruction of the mucosa or excessive granulation allergic factors, hyperplasia of connective tissue and lack of control of regenerated polyps could slow down this epithelialization. 


\subsection{Phase of scarification (after 3 months)}

At this time reorganization of tissue in the operated area had nearly finished. Subepithelial changes are noted after 6 months.

During these four phases in operative field can be seen:

1. Clean cavity: no oozing, fibrous pseudomembrane nearly disappeared, secretion decreased, clots and brown-yellow crusts diminished or disappeared

2. Mucosal edema: the whole inflammatory cavity and mucosa swelled, with smooth surface and indistinct boundary

3. Edematous vesicle: single or multiple or patches of polyp-like edematous vesicle appeared, with a grey, smooth and thin wall.

4. Polyp: it exists singly and locally

5. Granulation tissue: local or scattered hyperplasia occurred, with unsmooth papillary-like surface, fragile and easy to bleed

6. Polyp-like mucosal edema: extensive polyp-like changes and severe mucosal edema with plenty of purulent secretion were found

7. Cicatricial tissue hyperplasia: extensive connective tissue was yielded, mostly connected to patches, which was thick, tough and easy bleeding

8. Adhesion: fibrous membranous or cicatricial bridges resulted, mostly lying between the anterior verge of the middle turbinate and the lateral wall of the nasal cavity

9. Empyema: mostly was a viscous purulent secretion difficult to exudate

10. Narrow or blocked sinus ostium: fibrous cicatricial bridges occurred around the sinus ostium or connective tissue hyperplasia appeared

11. Constriction and blockage of sinus cavity: caused by adhesion of the laterally moved middle turbinate or connective tissue hyperplasia of the sinus wall

12. Epithellization: thin and smooth mucosa was found, which was closely linked with the preserved sinus bone wall and anatomical processes, and well-opened sinus ostia were clearly observed.

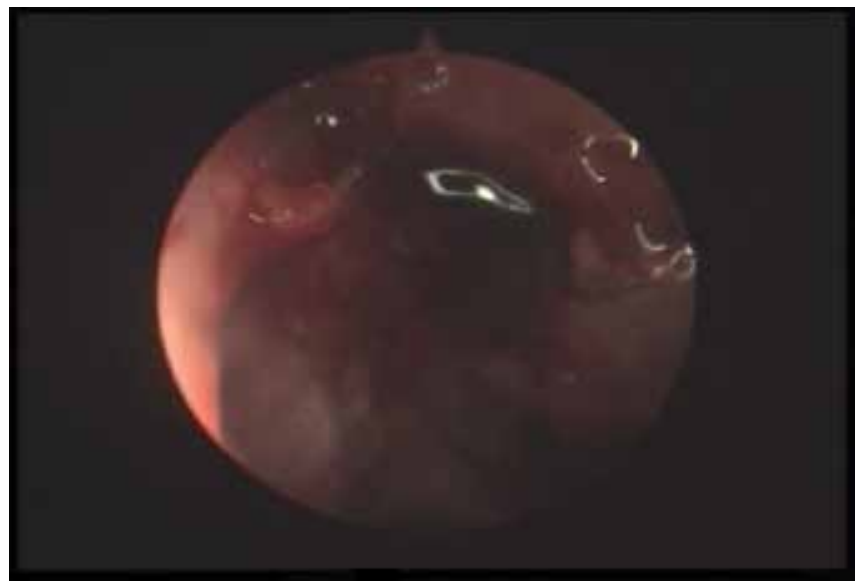

Fig. 1. Clean cavity (blood clot) 


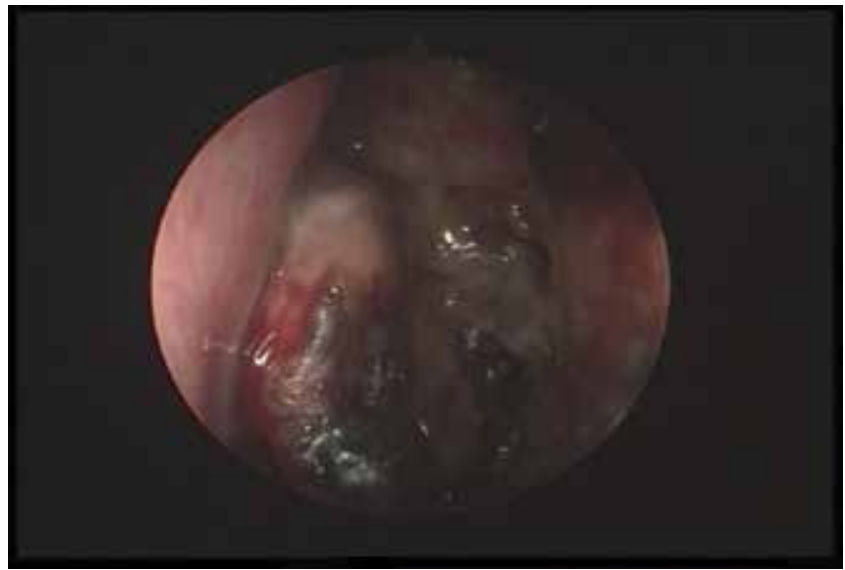

Fig. 2. Clean cavity (hard black crusts)

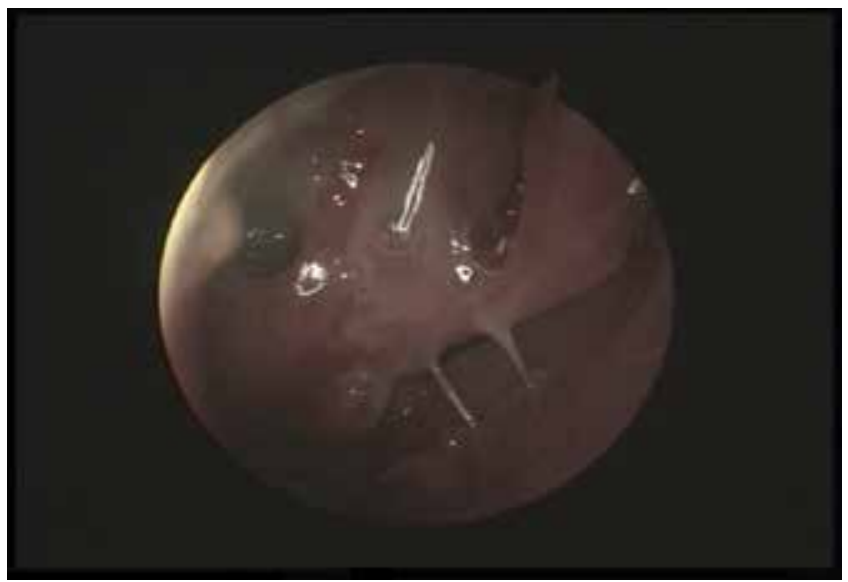

Fig. 3. Mucosal edema

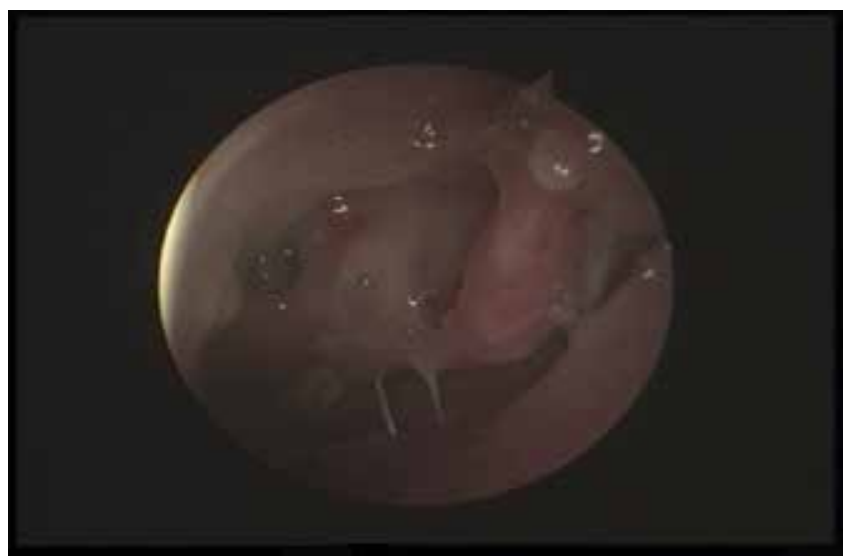

Fig. 4. Edematous vesicle 


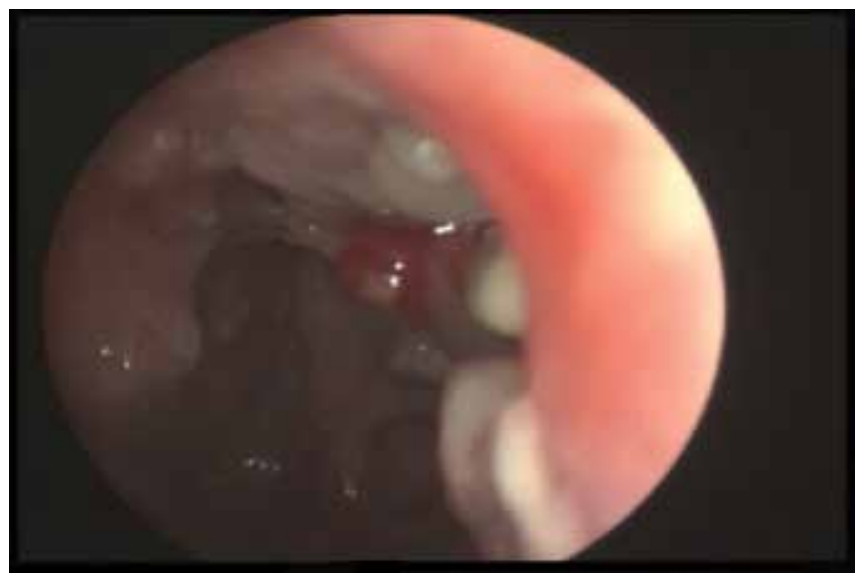

Fig. 5. Polyp formation

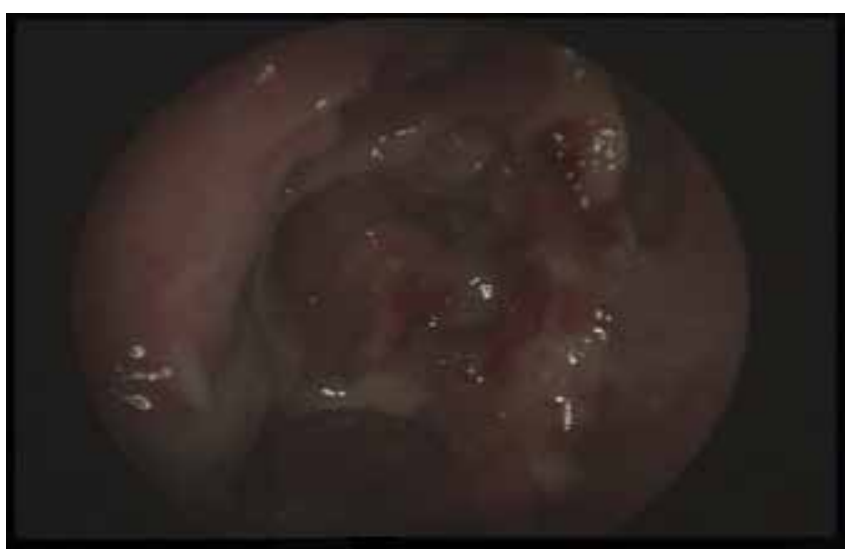

Fig. 6. Granulation tissue

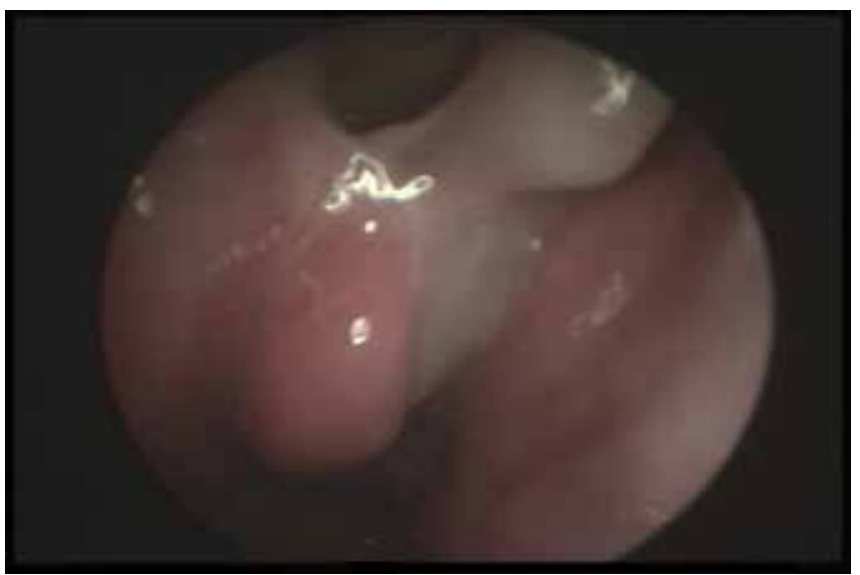

Fig. 7. Polyp like mucosal edema 


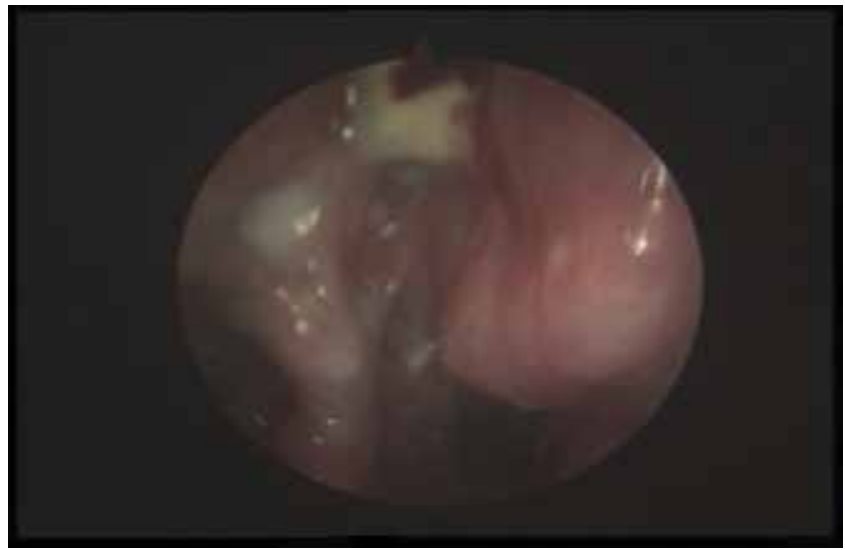

Fig. 8. Cicatrical tissue

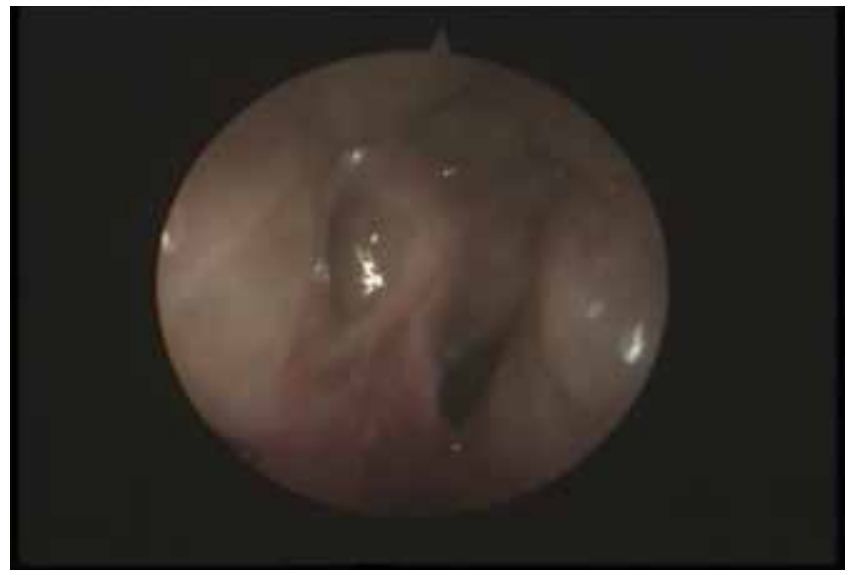

Fig. 9. Adhesion

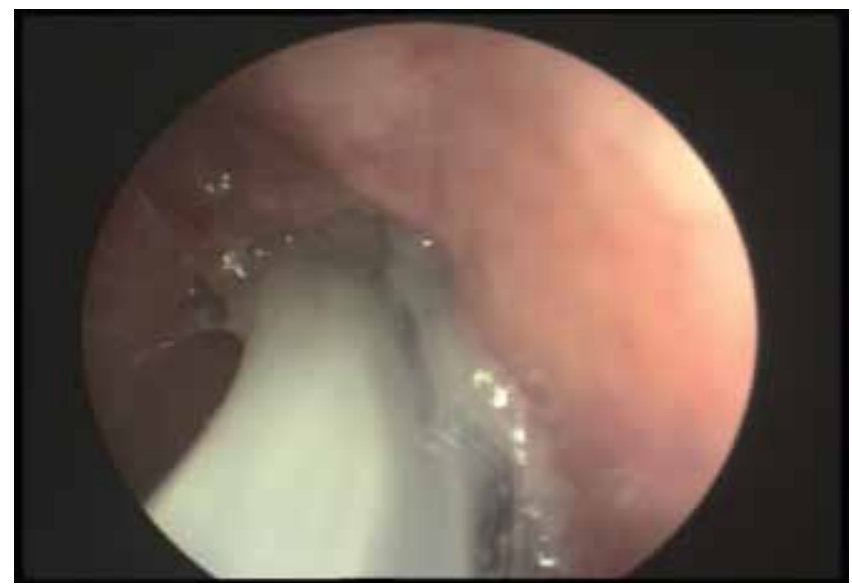

Fig. 10. Empyema 


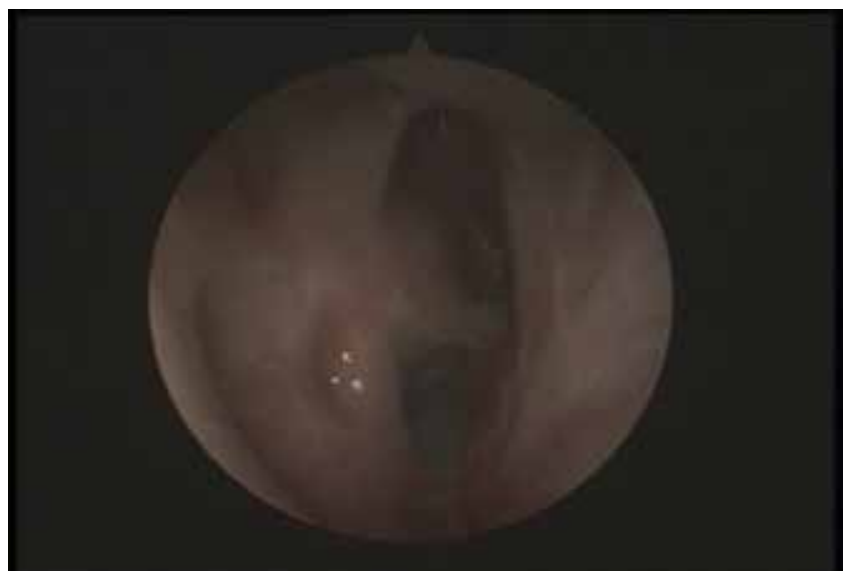

Fig. 11. Narrow or blocked sinus cavity

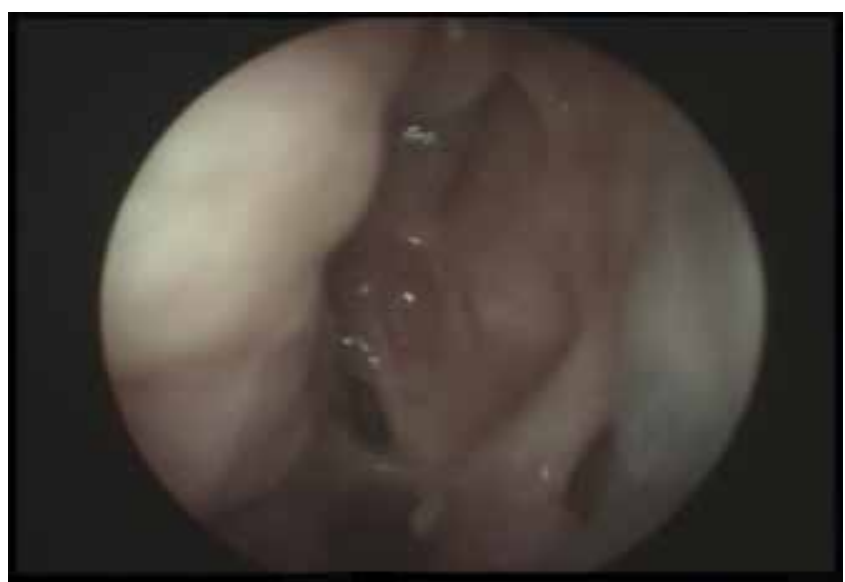

Fig. 12. Constriction and blockage of sinus cavity

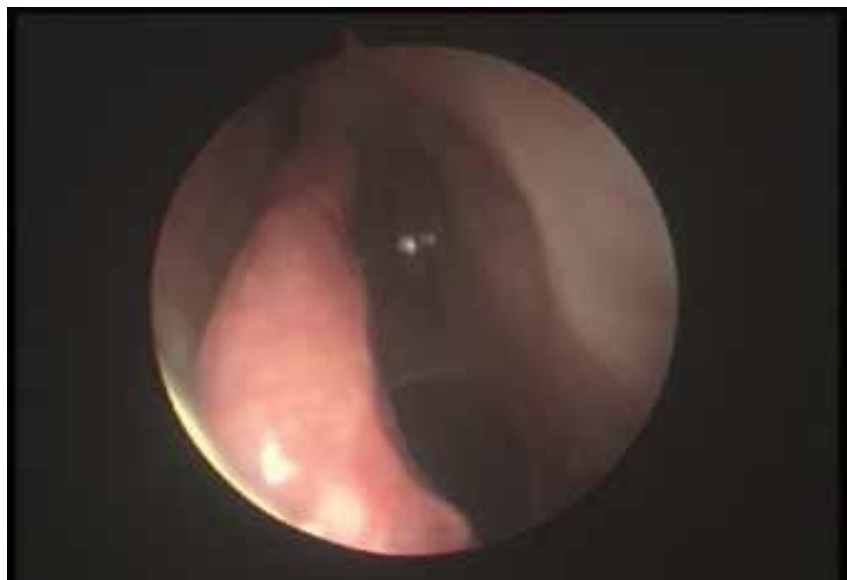

Fig. 13. Epithelization 
Clinical observations of wound healing in human beings have shown predisposed areas of reduced healing, suggesting that local microanatomical factors may play a decisive role in the reparation process. The closure of a defined wound of the respiratory mucous membrane is independent of the direction of blood, lymph or mucociliary flow. The management of the operative cavity especially in the first and second stages was essential to the whole curative effect, making up an important part of ESS.

Functional postoperative results are directly dependent on the healing quality of the nasal or sinusal mucosa and it can end differently. If the healing is dominant it can end with completed epithelial metaplasia, but if there is dominance of regenerative phase, epithelial metaplasia will be incomplete with adhesion, partial or total obstruction of the ostium and sinus cavity. Despite advances in instrumentation and surgical technique, postoperative synechia formation continues to occur in between $1-27 \%$ of patients. When sinechia occur in the middle meatus, the maxillary, ethmoid and frontal sinuses may become obstructed resulting in recurrent problems. The reported complications following ESS can be classified broadly into immediate postoperative complications such as bleeding and crusting; short-term complications such as infection, synechiae formation, and turbinate lateralization; and long-term complications such as ostial stenosis, refractory disease, and disease recurrence.

Mucosal sparing techniques, middle turbinate resection or medialization and frequent postoperative debridement have been used to varying degrees and with varying success to prevent postoperative synechia.

\section{Principles of management in different stages after operation}

Different author suggests different time of postoperative observation and care after extensive paranasal surgery. It is from 1 to 12 month. The crucial care of postoperative sinonasal mucosa is topical care and it should be:

Nasal packing, whether absorbable or nonabsorbable, has little effect on postoperative bleeding but more importantly, plays a more important role in postoperative healing. Considering the average duration of postoperative bleeding and the infection risk because of the "foreign body" there is no reason to leave a packing for more than 3 days. Blood surrounding the packing may (re)organize and the fibrin deposits around the packing could lead to scar tissue and adhesions. Packing moreover may obstruct evacuation of blood and secretions from the paranasal sinuses (Jorissen M. 2004.). Gelatin antibiotic and glucocorticosteroid can accelerate mucosa regeneration.

Rinse with enzymes contributes to the dissolution of fibrous pseudo membrane, clots and crusts, thus promoting the cleaning and healing of the sinus mucosa. Irrigation with saline solution is conductive to improve local nasal blood circulation and ciliary clearance. Using small volume (up to $1 \mathrm{ml}$ ) only dampens the nasal mucosa, and the paranasal sinuses can not be reached. Only with large volume $(300 \mathrm{ml}$ for a nose can) can the paranasal sinuses be reached, rinsed and washed. In addition to the pure mechanical rinsing, the saline will mix with secretions and decrease viscosity, propagating evacuation by mucociliary transport. High volume-low pressure rinsing of the nose and paranasal sinuses is the preferred technique for cleaning the surgical cavity and improving wound healing (Jorissen 2004). Welch recently published a study of the irrigation bottles used by patients after ESS and found that bacteria could be cultured from the irrigation bottles in $29 \%$ of studied patients including Pseudomonas aerugionosa, Acinetobacter baumanni, and Klebsiella pneumoniae 
although fortunately no clinically significant postoperative infections were noted. Frequent changing and sterilization of nasal irrigation bottles is advocated (Welch2009). Nasal irrigation with sulfurous-arsenical-ferruginous solution locally reduce the eosinophil number and may limit eosinophil-mediated production of cytokines and inflammatory molecules, which damage nasal mucosa, leading to edema and sinonasl inflammation. As eosinophils play an important role in allergic response through the release of mediators as eosinophilic cationic protein, major basic protein, and leukotrien C4, which causes extracellular matrix deposition, epithelial denudation, and basement membrane disruption. Sulfurous-arsenical ferruginous solution nasal irrigation significantly reducing the local eosinophil count should be suggested for allergic patients (Staffieri A. 2008). Local steroid spray has potent regional anti-allergy anti-inflammatory and anti-edema effects, which can control the generation of vesicles and mini polyps. The incidence of morphological changes in the nasal mucosa of patients with perennial rhinitis following treatment for 1 year with MFNS (mometasone furoate nasal spray) $100-200 \mu \mathrm{g}$ b.i.d. has demonstrated that this agent did not lead to any significant adverse tissue changes including increase in epithelial thickness or tissue atrophy, although the numbers of inflammatory cells in the epithelium and lamina propria were significantly decreased, compared to baseline (Minshall E. 1998). Subjects with an initial diagnosis of nasal polyps (CRSwNP) were more likely to experience MFNS-mediated improvements in wound healing than subjects with an initial diagnosis of chronic rhinosinusitis without polyposis (CRSsNP). At a subcellular level, corticosteroids have an antiinflammatory effect by activating glucocorticoid receptors, which interact with inflammatory transcription factors resulting in suppression of proinflammatorymolecules. At a cellular level, corticosteroids reduce the quantity of inflammatory cells (eosinophils, $\mathrm{T}$ lymphocytes,mast cells, and dendritic cells), the degree of inflammatory suppression correlates with the tissue concentration of steroid (Jorissen M. 2009). Oral steroids used preoperatively in patients undergoing endoscopic sinus surgery for nasal polyposis have been shown to reduce vascularity and improve surgical nasal field conditions resulting in shorter operating time. Postoperative administration of intranasal corticosteroids has also been demonstrated to reduce nasal polyp recurrence after endoscopic sinus surgery. The effect of oral steroids was investigated in the UZ Leuven in prospective, randomized comparative study: 1 tablet of betamethasone $0,25 \mathrm{mg}$ during 20 days versus a reducing regimen $(4 \mathrm{co} / \mathrm{d} \mathrm{d} 1-\mathrm{d} 5,3 \mathrm{co} / \mathrm{d} \mathrm{d} 5-\mathrm{d} 10,2 \mathrm{co} / \mathrm{d}$ d11-d15 and finally $1 \mathrm{co} / \mathrm{d} \mathrm{d} 16-\mathrm{d} 20)$. For all groups of patients beneficial effects of a higher dose of steroids during the first 3 weeks after ESS were found and the systemic and local side effects of the higher dose oral regimen were minor (Jorissen 2004). Gelomyrtol forte capsules are conductive to loosen viscous mucus secretion, dissolve fibrous adhesion and increase mucociliary clearance. Inhibitor of proton pump once / at night or twice a day is recommended to reduce gastric fluid acidity. Gastroesophageal reflux may play a significant role in wound healing. This is illness that includes the most of population. In GERB the lower esophageal sphincter doesn't work well, but in LPR (laryngopharyngeal reflux) the problem is in upper esophageal sphincter. Laryngopharyngeal reflux is defined as the retrograde movement of gastric contents into the larynx, pharynx, and upper aerodigestive tract. Mucosa of the respiratory tract is more sensitive on acid and pepsin instead of esophageal mucosa. Acids can locally harm the mucosa and increase mucosal reaction that leads to prolonged inflammation with granulation. Night reflux phases are much longer than day phases and they can lead to mucosal damages. The nasal symptoms aggravate during nights and in the mornings which can be connected with LPR. So, LPR can aggravate healing of sinonasal mucosa (Kleemann 2005). 
Crusting or cloting may trap mucus, resulting in reinfection of the sinuses, and the old blood itself may serve as a culture medium for bacteria. The crusts may act as bridges in which scar formation may occur, leading to an obstructed postoperative cavity and synechia formation in the middle meatus. Retained bone fragments that are denuded of mucosa may act as base for reinfection. Removing the crust, suctioning the retained secretion, and preventing lateral synechia formation and the obstruction of ostia and air cells are essential for healing and successful surgical outcome. Blood crusts cover the mucosal wound in the first 2 to 3 weeks and mucosal edema continues about 4 to 6 weeks after the operation. Although frequent postoperative debridement may remove the loose crusts and small devitalized bone fragments. Hard or fixed crusts cannot be cleaned because of bleeding from the underlying mucosal surface, discomfort and pain, and reformation of blood crusts. Loose, removable crusts were observed 2 weeks after the operation, indicating that mucosal re-epithelization requires at least 2 weeks. Mucosal edema was increased and sustained for 4 to 6 weeks. Mechanical wound care and the removal of blood crusts was avoided for at least 10 days. Gentle suction cleaning for several mounts. Violent tearing and too much cutting, which may cause injury to the epithelium, should be avoided. Sharp curette, electric cutter (debrider) and laser are recommended to lean the regenerated lesions. During the process of epithelization, the sinus was managed once at week, and any invalid surgery should be avoided unless the above-mentioned diseases occur because newly formed epithelium is loosely connected to bone, which is easy to tear away (Lee JY. 2008). The postoperative cleaning should continue until the operative site is mucosally covered. Presence of cultured bacteria from post-ESS patient cavities remains unclear. Several investigators have described the presence of biofilms in post-ESS cavities, and a retrospective pathologic study by Psaltis found that bacterial biofilms were found in $20(50 \%)$ of the 40 CRS patients. Patients with biofilms also had significantly worse preoperative radiological scores and, postoperatively, had statistically worse postoperative symptoms and mucosal outcomes (Psaltis AJ. 2008). The use of an antibiotic (amoxicillin/clavulanate) in the postoperative period is able to improve the outcome in the early blood crust healing phase: nasal obstruction and drainage are reduced and the endoscopic score objectively showed a faster recovery (Albu S. 2010). Patients recover in 9 to 10 days after ESS when provided with appropriate pain management. Postoperative pain after ESS can be controlled effectively with acetaminophen 665mg modified-release tablets three times a day during the first five postoperative days (Kempainen TP. 2007). Postoperative treatment after endoscopic surgery of frontal sinus or frontal recess is a little bite different of others sinuses. No nasal packing needs to be used. The frontal sinus cannulae are left in place for up to 5 days if there has been inadvertent trauma to the frontal ostium mucosa, if the natural frontal sinus ostium is very narrow $(<3 \mathrm{~mm})$, if there was evidence of osteitis with new bone formation in the frontal recess or ostium, and if there was extensive polyposis resulting in significant traumatized mucosa after the polyp removal. Frontal sinus saline douches are started through the frontal cannulae within 2 hours of completion of the operation. This will wash any blood clot out of the frontal ostium. The frontal cannulae are flushed with $5 \mathrm{~mL}$ normal saline every 2 hours starting immediately after surgery. If prednisolone drops are to be used 0,5 to $1 \mathrm{~mL}$ is instilled with a syringe into the frontal sinus after every second douche. The aim of the prednisolone is to dampen the inflammatory response of the mucosa. Immediately prior to removal of the frontal sinus cannulae, $5 \mathrm{~mL}$ of steroid and antibiotic cream (not ointment) is injected through each cannulae. This coats the newly created frontal sinus ostium and tends to decrease the amount of adherent crusts (Wormald PJ2008). Every operation, no matter how minor, is accompanied by swelling of the surrounding tissues. The 
amount of swelling varies from person to person, but it always seems more dramatic when involving the face. We suggest that you keep your head elevated as much as possible. The swelling itself is normal and is not an indication that something is wrong with the healing phase of your operation. Swelling after sinus surgery is not usually seen on the face itself; rather, it manifests itself as a stuffy or blocked nasal passage. Any swelling of the face will be limited to the area around the eyes and will last for only a few days. Symptoms of pain and pressure will be relieved in the very early postoperative period while thick postnasal drainage will continue until the mucosa within the sinuses has returned to normal. This may take weeks to months depending on the severity of the disease and the rapidity of healing. The patients should be warned of this preoperatively.

Proposed therapy

1. The packing, tamponades, should be removed from first till fifth day (as soon as possible)

2. Irrigation with saline solution 3 times a day for 3 weeks, high volume $(>100 \mathrm{ml})$, low pressure rinsing

3. Irrigation with sulfurous-arsenical-ferruginous solution for 3 weeks (for patients who has allergy)

4. The frontal cannulae are flushed with $5 \mathrm{~mL}$ normal saline every 2 hours starting immediately after surgery and Prednisolon drops 0,5 to $1 \mathrm{~mL}$ instilled with syringe into the frontal sinus after every second douche (for frontal Sinus surgery)

1. Rinse with enzymes can promote cleaning and healing of sinus mucosa

2. Bloody sediment and the crusts in the nasal cavity should drained with sucker after 10 th day

3. Endoscopic treatment once a week in weeks 2-6

4. Topical drugs: glucocorticosteroids nasal spray apply once or twice- daily for 6 months (for patients who has allergy)

5. Topical drugs: glucocorticosteroids can stop once healing has occur (for patients who has no other problems than chronic infection)

6. Oral glucocorticosteroids: Prednison $1 \mathrm{mg} / \mathrm{kg} 5$ days before surgery and higher dose of steroids during the first 3 weeks ( for patients with nasal polyposis)

7. Mucus eliminator such as Gelomyrtol forte capsules are recommended for 10 weeks

8. Proton pump inhibitors once / at night or twice a day is recommended to reduce gastric fluid acidity for 3 months (for patients who has LPR)

9. Pain killer, Acetaminophen $665 \mathrm{mg}$ modified-release tablets three times a day for first 5 days

10. Antibiotics (Amoxicillin/Clavulanate) for 2 weeks

Some tips to shorten the duration of the swelling and improve the ability to breathe through your nose include:

1. Stay vertical. Sit, stand and walk around as much as is comfortable beginning on your second postoperative day. Of course, you should rest when you become tired but keep your upper body as upright as possible.

2. Gentle blowing of the nose is allowed without closing the nasal vestibule.

3. Avoid bending over or lifting heavy things for one week. In addition to aggravating swelling, bending and lifting may elevate blood pressure and start bleeding.

4. Sleep with the head of the bed elevated 45 degrees for 7 - 10 days following your surgery. To accomplish this, place two or three pillows under the head of the mattress and one or two on top of the mattress. It is helpful if you sleep on your back for 30 nights. 
5. Avoid straining during elimination. If you need a laxative. Proper diet, plenty of water and walking are strongly recommended to avoid constipation.

6. Avoid sunning of your face for one month. Always use a sunscreen with SPF15 or above.

7. Avoid exercise for one week following surgery.

\section{References}

Albu S, Lucaciu R. Prophylactic antibiotics in endoscopic sinus surgery: a short follow up study. Am J. Rhinol Allergy.2010; 24:306-9.

Donald PJ, Gluckman JL, Rice DH (1995) The Sinuses. Raven Press New York, ISBN 0-78170041-8.

Hosemann W, Wigand ME, Göde U, Länger F, Dunker I (June1990) Normal wound healing of the paranasal sinuses: clinical and experimental investigations. Eur Arch Otorhinolaryngol, 1991; 248:390-394.

Jorissen M (2004) Postoperative care following endoscopic sinus surgery.Rhinology, 2004;42:114-120.

Jorissen M, Bschert C (December 2008) Efects of corticosteroids on wound healing after endoscopic sinus sugery.Rhinology, 2009;47: 280-286. DOI:10.4193/Rhin08.227.

Kemppainen TP, Toumilehto H, Kokki H, Seppä J, Nuutinen J. Pain treatment and recovery after endoscopic sinus surgery. Laryngoscope, 2007; 117:1434-8.

Kleeman D, Nofz S, Plank I, Schlottmann A (November 2004) Prolongierte Heilungsverläufe nach endonasalen Nasen-nebenhöhlenoperationen. HNO, 2005; 53:333-36.DOI 10.1007/s00106-004-1108y.

Lee JY, Byun JY. Relationship between the frequency of postoperative debridement and patient discomfort, healing period, surgical outcomes, and compliance after endoscopic sinus surgery.Larynoscope, 2008;118:1868-72. DOI: 10.1097/MLGOb013e31817f93d3.

Minshall E, Ghaffar O, Cameron L (1998) Assesment by nasal biopsy of long -term use of mometasone furoate aqueous nasal spray in the treatment of perennial rhinitis. Otolaryngol Head Neck Surg, 118: 648-654.

Psaltis AJ, Weitzel EK, Ha KR (2008) The effect of bacterial biofilms on postsinus surgical outcomes. Am J Rhinol 2008; 22:1-6.

Xu G, Jiang H, Li H, Shi J, Chen H ( April 2008) Stages of nasal mucosal transitional course after functional endoscopic sinus surgery and their clinical indications ORL,2008;70:118-123. DOI: 10.1159/0014535.

Staffieri A, Marino F Staffieri C, Giacomelli L, D’Alessandro D, Ferraro SM, Fedrazzoni U, Marioni G (2008) The effects of sulphurous-arsenical ferruginous thermal water nasal irrigation in wound healing after functional endoscopic sinus surgery for chronic rhinosinusitis: a prospective randomized study. American Journal of Otolaryngology-Head and Neck Medicine Surgery, 2008; 29:223-229.

Tan BK, Chandra RK (2010) Postoperative prevention and treatment of complications after sinus suregry. Otolaryngol Clin N Am.2010; 43: 769-779. DOI: 10.1016/j.otc.2010.04.004.

Watelet JB, Gevaert P, Bachart C (Avgust 2001) Secretion of TGF-ßI, TGF-ß32, EGF and PDGF into nasal fluid after sinus surgery. Eur Arch Otorhinolaryngol, 2002; 259:234-238. DOI: 10.1007/s00405-002-0448-z. 
Watelet JB, Bachert C, Gevaert P, Van Cauwenberge P (2002) Wound Healing of the nasal and paranasal mucosa: A review. American Journal of Rhinology, 2002; 16: 77-84.

Watelet JB, Demeter P, Claeys C, Van Cauwenberge P, Cuvelier C, Bachert C (2005) Neutrophil-derived Metalloprotenase-9 predicts healing quality after sinus surgery. The laryngoscope, 2005; 115:56-61. DOI: 10.1097/01.mlg.0000150674.30237.3f.

Watelet JB, Demetter P, Claeys C, Van Cauwenberge P, Cuvelier C, Bachert C ( April 2005) Wound healing after paranasal sinus surgery: neutrophilic inflammation influences the outcome. Histopatology, 2006; 48: 174-181.D=I: 10.1111(j.1365-2559.2005.02310.x

Welch KC, Cohen MB, Doghramji LL (2009). Clinical correlation between irrigation bottle contamination and clinical outcomes in postfunctional endoscopic sinus surgery patients. Am J Rhinol Allergy.2009; 23:401-4.

Wormald PJ (2008). Endoscopic sinus surgery anatomy,three-dimensional reconstruction and surgical technique. Second edition. Thieme New York. Stuttgart, The Americas ISBN:978-1-58890-603-8, Rest of World ISBN: 978-3-13-139422-4. 


\title{
Microbial Contamination of Suction Tubes Attached to Suction Instrument and Its Preventive Methods
}

\author{
Katsuhiro Yorioka ${ }^{1}$ and Shigeharu Oie ${ }^{2}$ \\ ${ }^{1}$ Department of Pharmacy, Shunan Municipal Shinnanyo \\ Citizen Hospital, 2-3-15 Miyanomae, Shunan \\ 2Department of Pharmacy, Yamaguchi University \\ Hospital; 1-1-1 Minamikogushi, Ube \\ Japan
}

\section{Introduction}

We investigated the microbial contamination of suction tubes attached to wall-type suction instrument. Microbial contamination of suction tubes used for endoscopy or sputum suction in wards was examined before and after their disinfection. In addition, disinfection and washing methods for suction tubes were evaluated. Suction tubes $(\mathrm{N}=33)$ before disinfection were contaminated with 102-108 colony-forming units $(\mathrm{cfu}) /$ tube. The main contaminants were Pseudomonas aeruginosa, Acinetobacter baumannii, and Stenotrophomonas maltophilia. The suction tubes were disinfected with sodium hypochlorite $(\mathrm{N}=11)$ or hot water $(\mathrm{N}=11)$, or using an automatic tube cleaner $(\mathrm{N}=11)$. After 2-hour immersion in $0.1 \%(1,000 \mathrm{ppm})$ sodium hypochlorite, $10^{3}-10^{7} \mathrm{cfu} /$ tube of bacteria were detected in all 11 tubes examined. After washing in hot running water $\left(65^{\circ} \mathrm{C}\right), 10^{3}-10^{7} \mathrm{cfu} /$ tube were detected in 3 of 11 examined tubes. The bacteria detected in the suction tubes after disinfection with sodium hypochlorite or hot water were P. aeruginosa, A.baumannii, and S.maltophilia. On the other hand, after washing with warm water $\left(40^{\circ} \mathrm{C}\right)$ using the automatic tube cleaner, the contamination were < $20 \mathrm{cfu} /$ tube (lower detection limit: $20 \mathrm{cfu} / \mathrm{tube}$ ) in all 11 tubes examined. These results suggest the usefulness of washing using the automatic tube cleaners.

\section{Background}

In hospitals in Japan, the suction of body fluid such as sputum or blood is performed daily using wall-type suction instrument in wards and outpatient clinics such as endoscopy rooms (Fig.1-a,2-b). Wall-mounted suction instrument are used being connected to a suction tube. Suction instruments are used for procedures such as sputum suction, endoscopy using a suction tube connected to a gastrofiberscope, and bronchoalveolar lavage (BAL) using a suction tube connected to a bronchofiberscope. In sputum suction and suction in gastrofiberscopy, sucked body fluid (such as sputum and saliva) flows from the patient's 


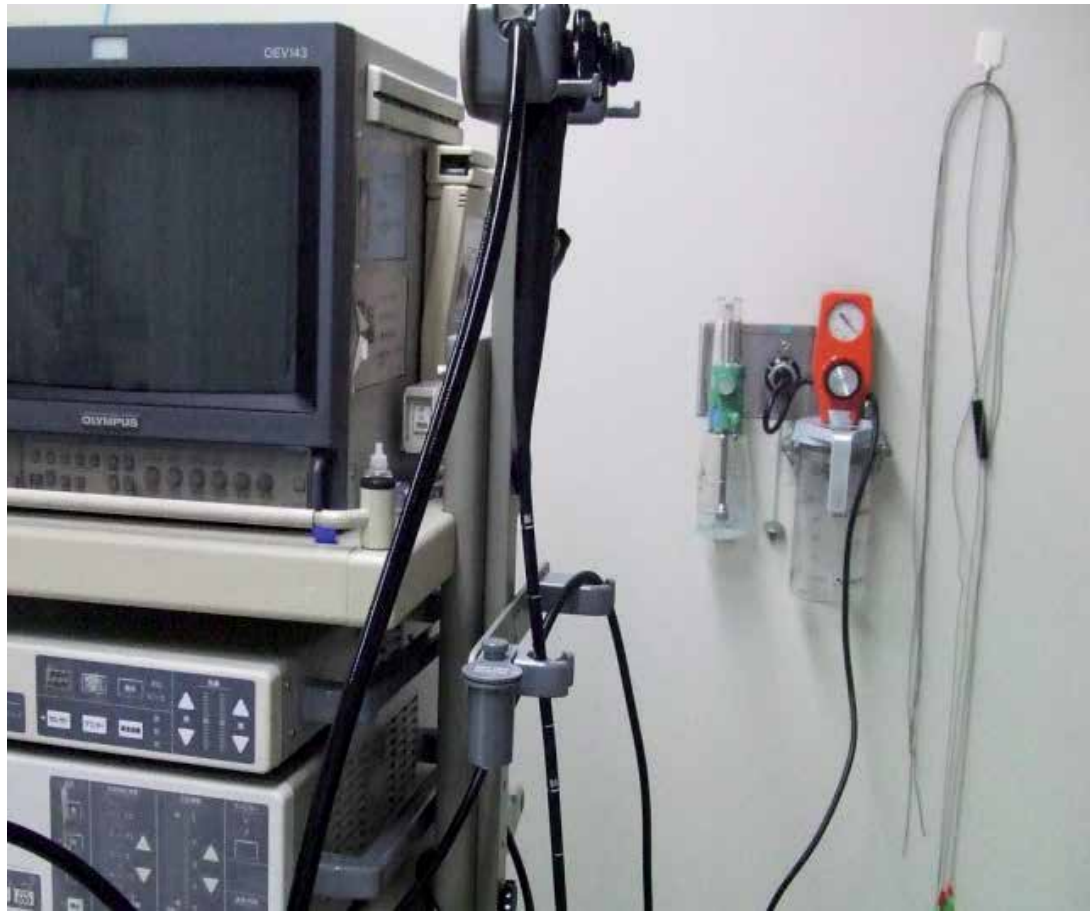

a)

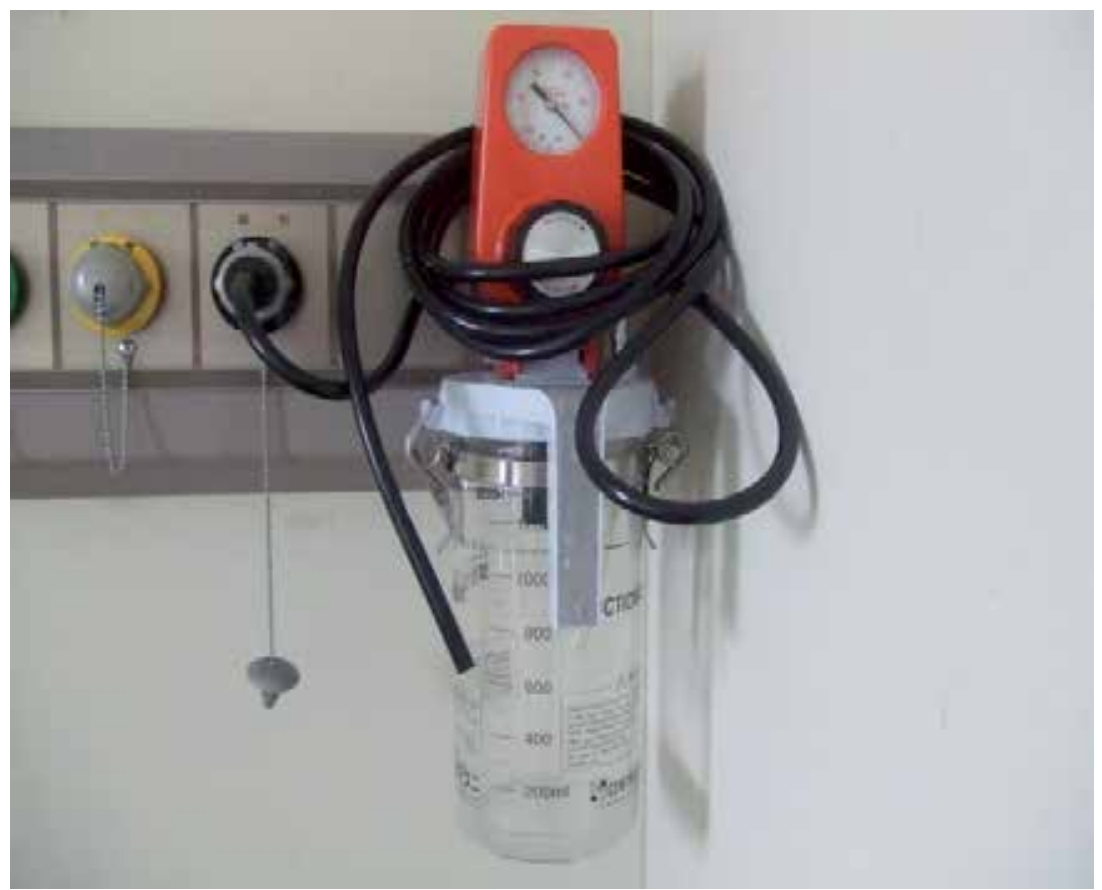

b)

Fig. 1. 
side toward the suction tube (suction instruments). However, in BAL, regurgitation from the suction tube side toward the bronchofiberscope or bronchoalveolar lavage fluid (BALF) sometimes occurs (1). Indeed, we experienced regurgitation from the suction tube side toward the BALF side several times during BAL. BAL using suction tubes that are contaminated or have not been disinfected runs the risk of the contamination of patients and BALF, which may induce nosocomial infection $(2,3)$. When suction tubes are washed or disinfected in sink such as the ward or outpatient clinic, water drops containing patients' body fluid and microorganism's splash on health care workers, which runs the risk of exposure and infection (4-6). The use of disposable (single-use) suction tubes or washing/disinfection of suction tubes in each patient is necessary. However, at present, there are no guidelines (or recommendation) regarding the washing/disinfection methods for suction tubes as non-critical instruments. In addition, there are no clinical data on the relationship between the microbial contamination of suction tubes and their disinfection methods. Therefore, we evaluated microbial contamination of suction tubes and methods for their disinfection.

\section{Methods}

We investigated the microbial contamination of suction tubes that are used, being connected to wall-type suction instruments (Central Uni Co., Tokyo, Japan), and evaluated their disinfection/washing methods. Microbial contamination in a total of 33 suction tubes used for endoscopy or sputum suction in wards was compared before and after disinfection/washing. Tubes were disinfected with sodium hypochlorite $(\mathrm{N}=11)$ or hot water $(\mathrm{N}=11)$, or washed using an automatic tube cleaner $(\mathrm{N}=11)$. Per one patient, we used one suction tube. The suction tube is $3 \mathrm{~m}$ in length, $4 \mathrm{~mm}$ in internal diameter and made of high-purity latex (Deluxe type latex tubing: Central Uni Co., Tokyo, Japan). The washing methods using sodium hypochlorite, hot water, or an automatic tube cleaner are as follows.

Disinfection with sodium hypochlorite solution: Suction tubes after use were washed under running water, immersed in $0.1 \%(1,000 \mathrm{ppm})$ sodium hypochlorite for 2 hours (Fig.2-a), and dried naturally in the ward or endoscopy room.

Disinfection with hot water: Suction tubes were washed under running water and immersed in an enzyme detergent (Biotect ${ }^{\circledR} 55$, Sakura Seiki Co.,Tokyo, Japan) at $40^{\circ} \mathrm{C}$ for 30 minutes. Subsequently, hot water $\left(65^{\circ} \mathrm{C}\right)$ was run into the suction tubes for 5 minutes (Fig.2-b). In addition, the tubes were flushed with $20 \mathrm{~mL}$ of $80 \%$ (v/v) ethanol for disinfection (Yoshida Pharmaceutical Co., Tokyo, Japan) using a syringe, and dried naturally in the ward.

Washing using an automatic tube cleaner: Suction tubes were washed using an automatic tube cleaner in the central supply room, flushed with $20 \mathrm{~mL}$ of $80 \%$ (v/v) ethanol for disinfection, and dried using an automatic drier at $70^{\circ} \mathrm{C}$ for 2 hours. This automatic tube cleaner automatically performs the cleaning process consisting of washing with an enzyme detergent, washing without a detergent, rinsing, and drying (Fig.2-c: Automatic tube cleaner MU-72 K: Sharp System Product Co.,Tokyo, Japan). Warm water at $40^{\circ} \mathrm{C}$, with which the optimal effects of the enzyme detergent can be expected, was used for the automatic tube cleaner. 


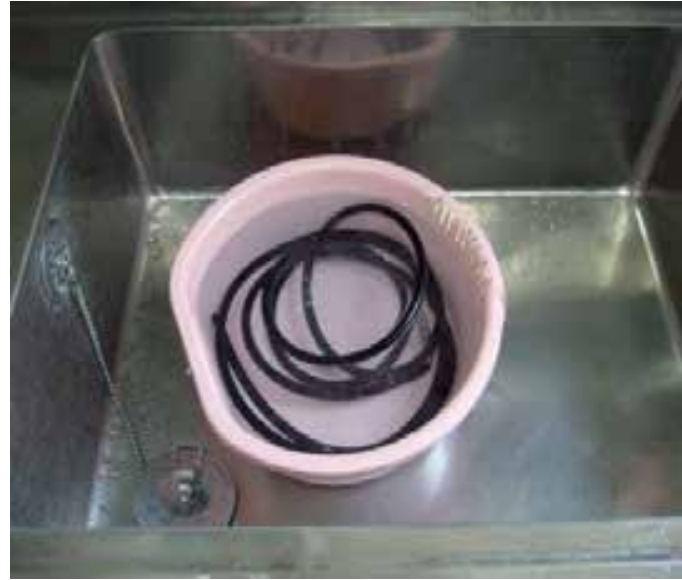

A)

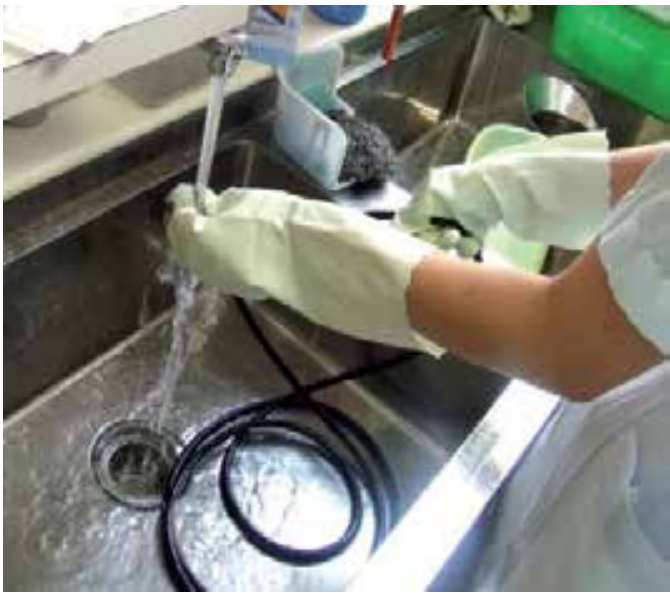

B)

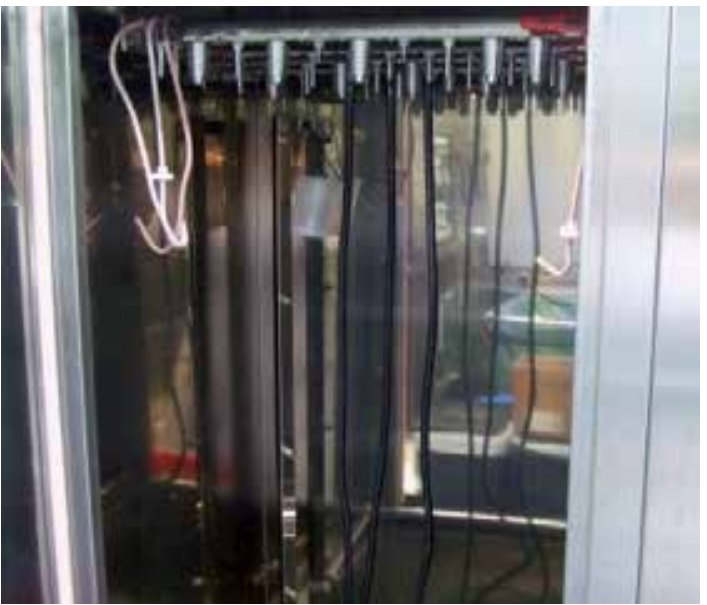

C)

A: Disinfection by sodium hypochlorite solution

Suction tubes after use were washed under running tap water, immersed in $0.1 \%(1,000 \mathrm{ppm})$ sodium hypochlorite for 2 hours.

B: Disinfection with hot water

Suction tubes were washed under running tap water and immersed in an enzyme detergent at $40^{\circ} \mathrm{C}$ for 30 minutes. Subsequently, hot water $\left(65^{\circ} \mathrm{C}\right)$ was run into the suction tubes for 5 minutes.

C: Washing with an automatic tube cleaner

This automatic tube cleaner automatically performs the cleaning process consisting of washing with an enzyme detergent, washing without a detergent, rinsing, and drying.

Fig. 2. Immersion in sodium hypochlorite (a), washing under running hot water (b) and washing with an automatic tube cleaner (c)

\section{Results}

Table 1 shows the results of microbial contamination in suction tubes before disinfection with immersion in sodium hypochlorite solution, washing with hot water, and washing 
with an automatic tube cleaner. Suction tubes before disinfection with sodium hypochlorite solution or hot water were contaminated with $10^{3}-10^{8} \mathrm{cfu} /$ tube, and the main contaminants were Pseudomonas aeruginosa, Acinetobacter baumannii, and Stenotrophomonas maltophilia. Table 2 shows the results of microbial contamination in suction tubes after disinfection by immersion in sodium hypochlorite solution, those after washing by hot running water, and those after washing with warm water using an automatic tube cleaner. Bacteria were detected in all 11 examined tubes after 2-hour immersion in $0.1 \%(1,000 \mathrm{ppm})$ sodium hypochlorite solution and 3 of 11 after washing in hot running water. The contaminant after disinfection was $10^{3}-10^{8} \mathrm{cfu} /$ tube, and the contaminants detected in the suction tubes were glucose non-fermentative gram-negative rods such as $P$. aeruginosa, A. baumannii, Sphingomonas paucimobilis, and Stenotrophomonas maltophilia. The contaminant was $<20$ $\mathrm{cfu} /$ tube (lower detection limit, $20 \mathrm{cfu} /$ tube) in all 11 examined tubes after washing using the automatic tube cleaner.

After disinfection by immersion in sodium hypochlorite solution or washing in hot running water, $14(63.6 \%)$ of the 22 tubes examined were contaminated with $10^{3}-10^{7} \mathrm{cfu} /$ tube. The main contaminants were glucose non-fermentative gram-negative rods such as Pseudomonas aeruginosa, Acinetobacter baumannii, and Stenotrophomonas maltophilia.

\section{Discussion}

This inadequate disinfection may be because the inside of the tubes was not immersed in sodium hypochlorite solution due to the thin long tube structure $(\geq 3 \mathrm{~m})$, and organic matter and microorganisms in the tubes could not be removed or diluted, and remained. Indeed, in a suction tube after disinfection by immersion in sodium hypochlorite solution, a mass of body fluid was discovered (Fig.3). On the other hand, all 11 automatic tube cleaners examined were contaminated with $<20 \mathrm{cfu} /$ tube, showing accurate disinfection effects. Automatic cleaners can reduce microorganisms and organic matter inside suction tubes by a mean of $4 \log (99.9 \%)$ (7). The use of automatic cleaners is a useful disinfection method that has marked disinfection effects without causing side effects due to residual toxicity, as are observed with disinfectants (8).

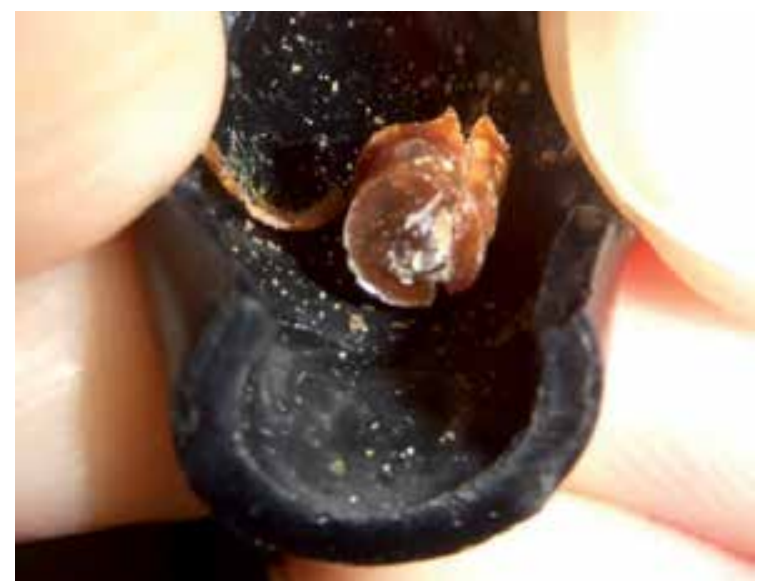

Fig. 3. A mass of body fluid discovered in the suction tube after disinfection with sodium hypochlorite solution. 


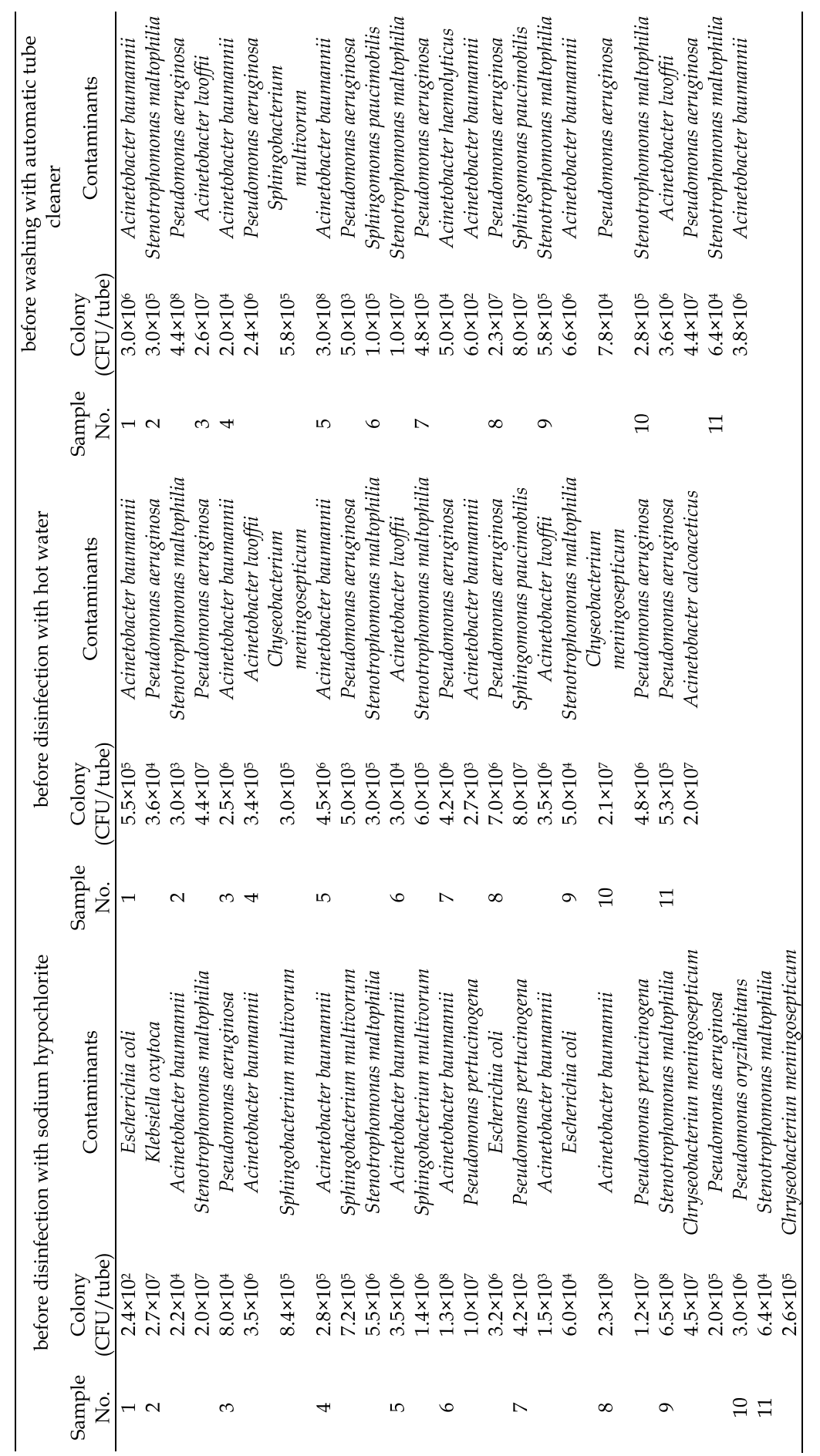

Table 1. Microbial contamination inside suction tubes before disinfection with sodium hypochlorite solution, disinfection with hot water, or washing using automatic tube cleaner 


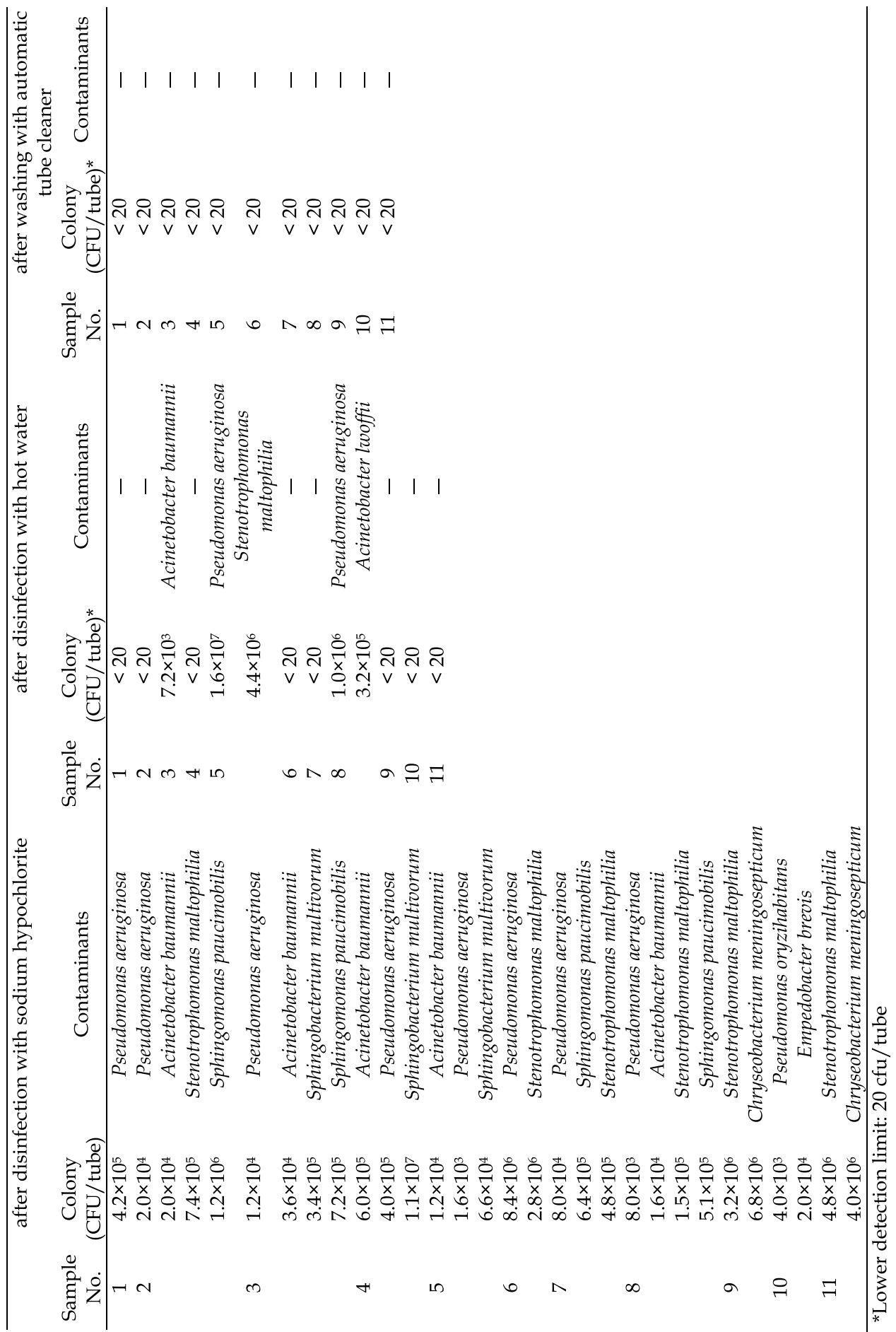

Table 2. Microbial contamination inside suction tubes after disinfection with sodium hypochlorite solution, disinfection with hot water, or washing using automatic tube cleaner 
The present status survey in the 18 institutions revealed 3 institutions (16\%) using disposable tubes and 2 (11\%) (including our hospital) where the disinfection of tubes is performed (by immersion in sodium hypochlorite at the ward/outpatient clinic in both institutions). When moist/respiratory tract medical instruments such as suction tube are disinfected at the ward or outpatient clinic, medial workers or sinks are contaminated with water droplets from suction tubes, which may cause occupational infection (9-11). On the other hand, washing with automatic tube cleaners is certain decontamination/washing effects than the disinfection method performed at the ward or outpatient clinic, and is also desirable in terms of the prevention of occupational contamination of medical workers performing washing/disinfection (12-13). Therefore, it is necessary to recommend the use of disposable suction tubes or washing disinfection using automatic tube cleaners by medical staff members of the central supply room.

\section{References}

[1] The European society of pneumology task group on BAL. (1990): Eur. Respir. J., 3, 937974.

[2] Wishart, M.M., Riley, T.V. (1967): Infection with Pseudomonas maltophilia hospital outbreak due to contaminated disinfectant. Med. J. Aust., 2, 710-712.

[3] Pokrywka, M., Viazanko, K., Medvick, J. et al. (1993): A Flavobacterium meningosepticum outbreak among intensive care paitients. Am. J. Infect. Control., 21, 139-145.

[4] Ferroni, A., Nguyen, L., Pron, B., Quesne, G., Brussent, M.C., Berche, P. (1998): Oubreak of nosocomial urinary tract infection due to Pseudomonas aeruginosa in a pediatric sur-gical unit associated with tap-water contamination. J. Hosp. Infect., 39, 301-307.

[5] Widmer, A.F., Wenzel, R.P., Trilla, A., Bale, M.J., Jones, R.N., Doebbeling, B.N. (1993): Outbreaks of Pseudomonas aeruginosa infections in a surgical intensive care unit: probable transmission via hands of a health care worker. Clin. Infect. Dis., 16, 372376.

[6] Miller, D.M., Youkhana, I., Karunaratne, W.U., Pearce, A. (2001): Presence of protein deposits on 'cleaned' re-usable anaesthetic equipment. Anaesthesai., 56, $1069-$ 1072.

[7] Rutala, W.A. (1996): APIC guideline for selection and use of disinfectants. Am. J. Infect. Control, 24, 313-342.

[8] Block, C., Baron, O., Bogokowski, B., et al. (1990): An in-use evaluation of decontamination of polypropylene versus steel bedpans. J. Hosp. Infect., 16, 331-338.

[9] Tordoff, S.G., Scott, S. (2002): Blood contamination of the laryngeal mask airways and laryngoscopes-what do we tell our patients? Anaesthesai., 57, 505-506.

[10] (Coetzee, G.J. (2003): Eliminating protein from reusable laryngeal mask airways. A study comparing routinely cleaned masks with three alternative cleaning methods. Anaesthesai., 58, 346-353.

[11] Bodey, G.P., Bolivar, R., Fainstein, V., Jadeja, L.(1983): Infection caused by Pseudomonas aeruginosa. Rev. Infect. Dis., 5, 279-313.

[12] Quinn, J.P. (1998): Clinical problems posed by multiresistant nonfermenting gram negative pathogens. Clin. Infect. Dis., 27, 117-124.

[13] Bergogne-Berezin, E., Towner, K.J. (1996): Acinetobacter spp. as nosocomial pathogens: microbiological, clinical, and epidermiological features.Clin. Microbiol. Rev., 148-165. 



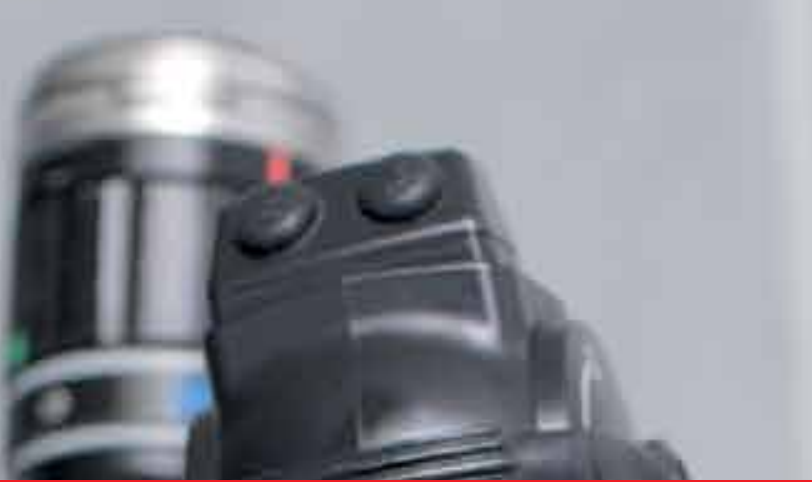

\section{Edited by Cornel Iancu}

Surgeons from various domains have become fascinated by endoscopy with its very low complications rates, high diagnostic yields and the possibility to perform a large variety of therapeutic procedures. Therefore during the last 30 years, the number and diversity of surgical endoscopic procedures has advanced with many new methods for both diagnoses and treatment, and these achievements are presented in this book. Contributing to the development of endoscopic surgery from all over the world, this is a modern, educational, and engrossing publication precisely presenting the most recent development in the field. New technologies are described in detail and all aspects of both standard and advanced endoscopic maneuvers applied in gastroenterology, urogynecology, otorhinolaryngology, pediatrics and neurology are presented. The intended audience for this book includes surgeons from various specialities, radiologists, internists, and subspecialists.

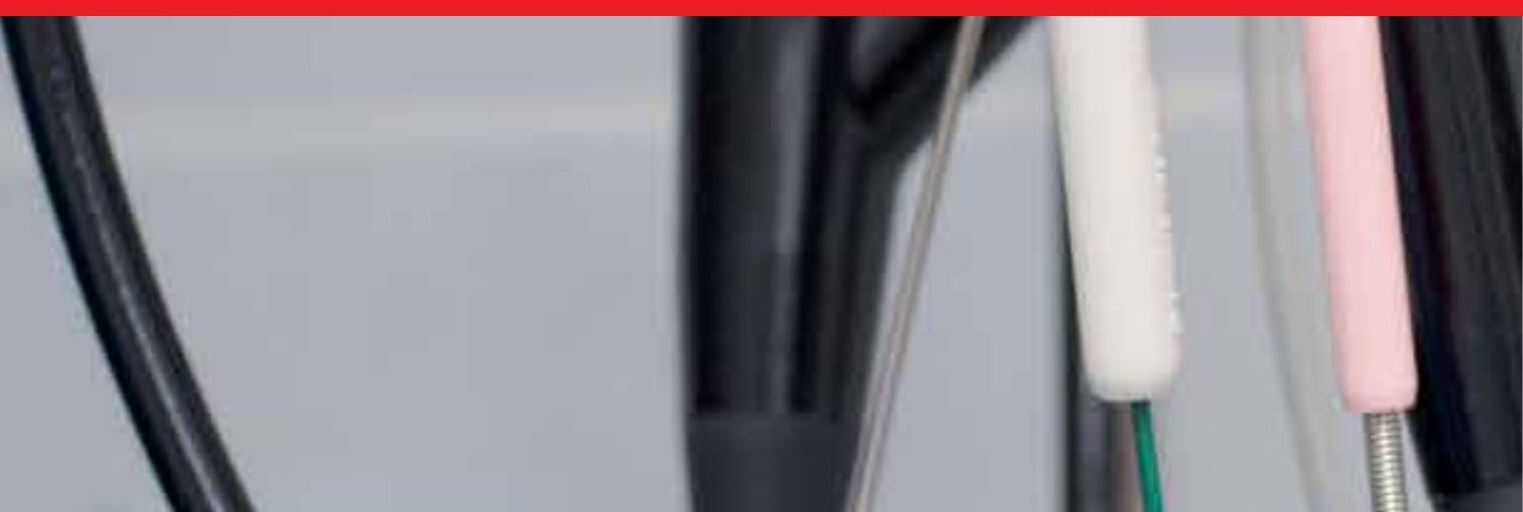

